\title{
QUANTITATIVE ASPECTS OF
}

\section{COMPUTED TOMOGRAPHY}

\section{$6 y$}

Alexander William Mitchell

\begin{abstract}
$\mathcal{A}$ thesis submitted for the degree of DOCTOR OF PHILOSOPHY IN PHYSICS at the Victoria University of Wellington
\end{abstract}




\section{ABSTRACT}

Computed tomographic (C.T.) images are stored digitally as arrays of C.T. numbers, the elements of which are strongly related to the linear X-ray attenuation coefficients of the corresponding tissue volumes in the patient. This thesis is concerned with this quantitative information. Relating measured C.T. numbers to the properties of materials requires the ability to compute attenuation coefficients from compositions and densities. Numerical techniques have been used in the present work to produce formulae which are much simpler and more accurate than those currently used. Dual energy computed tomography permits the computation of parameters such as tissue densities and effective atomic numbers which may have more clinical relevance than the attenuation coefficient. A dual energy capability has been developed for the Wellington Hospital G.E. 8800 scanner. A simple algorithm has been developed for computing electron densities from dual energy C.T. scans. A spine mineral measurement procedure has been developed for the 8800 scanner which reports both single and dual energy bone measurements. A computationally efficient algorithm has been derived for the calculation of true mineral concentration and cancellous tissue density from dual energy C.T. measurements. Measurements on 31 volunteers compare well with published normal ranges for single energy C.T. Both single energy C.T. spine mineral densitometry and dual photon absorptiometry are prone to errors due to variable fat content, leading to ambiguity in interpreting the results of measurements on patients with anorexia nervosa. Measurements on 68 anorexic patients gave an average single energy value $18 \%$ lower than that for the volunteers. The average mineral concentration determined from dual energy measurements was $19 \%$ lower, confirming that there is a genuine loss of

mineral. Neither the single or dual energy mineral measurements showed any protective effect from the use of estrogen contraceptive pills, but both measurements correlated positively with the level of exercise at the time of examination, and with the duration of high calcium intake during adolescence. The dual energy mineral measurement showed no significant improvement in patients who had recovered from anorexia nervosa. Differences in image texture seen in C.T. images of the vertebral body reflect differences in the gross structure of the cancellous bone tissue. An in vivo study on vertebrae from 9 sheep was not useful in correlating the C.T. appearances of the cancellous tissue with scanning electron microscope (SEM) images of the same tissue because the characteristic appearances noted in humans were not present in the sheep specimens. However correlations between C.T. measurements of sheep vertebrae and trabecular bone volumes determined from SEM images of the same sections give linear regression correlation coefficients of 0.87 for the single energy measurement and 0.81 for the dual energy measurement. A study using human tissue is under way, but insufficient specimens had become available at the time of writing to be reported in detail here. 


\section{PREFACE}

A computed tomography (C.T.) scanner is an instrument which produces images of the human body for the purposes of medical diagnosis. This technology has its roots in conventional radiography, in which the image is effectively a projection of the distribution of attenuation within the patient onto a single plane, but produces much more sophisticated images. In a C.T. scanner the X-ray tube and detectors are moved about the patient in various ways so that several thousand different projections of the pattern of attenuation within the patient are obtained. Following computer reconstruction of the projection data, an image is produced of a transverse section of the patient. The image is stored in the computer as an array of numbers (called C.T. numbers) whose values are related to the $\mathrm{X}$-ray attenuation coefficients of the tissue volumes that they represent. Thus the appearance of an image may be quantified in terms of the average C.T. number of the tissue within a lesion, for example. This thesis is concerned with the use of such quantitative information.

The processes by which the C.T. image is produced are discussed in chapter one, where particular reference is made to those factors which influence the accuracy of the C.T. numbers. The relationship between measured C.T. numbers and the X-ray attenuation coefficients of the materials scanned are discussed in chapter two. The linear attenuation coefficient of a material depends on both its chemical composition and density. Change in the linear attenuation coefficient due to a change in one of these factors may be masked by a compensating change in the other. For this reason the linear attenuation coefficient (and hence the C.T. number) does not always correlate well with disease processes. Dual energy C.T. techniques have been developed in an attempt to minimise such problems. These techniques, which are discussed in chapter three, make use of C.T. measurements at two different energies to obtain estimates of two tissue properties such as effective atomic number and density. Selection of the most appropriate technique for use with the Wellington Hospital C.T. scanner is made.

Perhaps the most successful application of dual energy C.T. to date has been the measurement of bone mineral content in the lumbar spine. This measurement has great clinical significance since this is one site where the effects of bone demineralisation are felt severely. Application of this technique at Wellington Hospital is the subject of chapter four. In this chapter it is shown that the derivations of some existing algorithms for the calculation of mineral concentration from dual energy C.T. scans are not strictly valid, and they do not yield the true mineral concentration. A derivation is presented of a new formula which does give the true mineral concentration, and which also yields the density of the tissues examined. 
Clinical applications of quantitative techniques are presented in chapter five. The first of these is a novel method for the computation of electron densities from C.T. images, to be used in radiotherapy treatment planning. To this end the formalism of chapter two is used to develop a simple, computationally efficient algorithm which is sufficiently accurate for its intended purpose. The rest of chapter five is concerned with bone mineral measurements on patients with a history of anorexia nervosa, a project which has been run jointly between the Departments of Radiology and Endocrinology of Wellington Hospital and the Department of Psychological Medicine, Wellington School of Medicine. The normal range of bone measurements is first established by scanning volunteer subjects, following which the results of measurements on the patient group are presented, and the chapter concludes with a discussion of the correlations observed between the bone measurements and several clinical factors relevant to anorexia nervosa.

While bone measurements can identify groups of patients which are at increased risk of crush fractures, the measurements cannot predict which individuals within the 'at risk' groups will sustain fractures. Better predictive capability may be obtained if information regarding the structural arrangement of the mineral, as well as its quantity, can be extracted from C.T. images. In chapter six various attempts to quantify the appearance of cancellous bone in C.T. images are described, and proposed methods for relating the parameters so obtained to the underlying bone structure are discussed.

It is a pleasure to acknowledge the help of the following people:

Dr Peter Johnson of the Physics Department, Victoria University of Wellington, and Dr John Delahunt of the Department of Medicine, Wellington School of Medicine, who were primarily reponsible for supervision. Professor Noel Chapman, and Dr Denis Sullivan of the Physics Department, V.U.W., were also supervisors in the earlier stages, and additional clinical supervision was provided by Dr Rodney Allen of the Radiology Department, Wellington Hospital. Drs Guy Harper and Allen Moore of the Radiology Department, Wellington Hospital, also helped with their advice and encouragement, as did Mrs Louise Laming, Miss Heather Samson, Miss Lyn Williams and the C.T. radiographers of Wellington Hospital, who also assisted with the scanning of the controls and anorexic patients.

Dr Phillipa Hay and Professor Anne Hall of the Department of Psychological Medicine, Wellington School of Medicine, who were co-workers in the anorexia nervosa study, and Mr Frank Golder of the Department of Physiology \& Anatomy, Massey University, who was the principal co-worker on the sheep project.

Advice on electron microscopy and specimen preparation techniques has been given by $\mathrm{Dr}$ Martin Sage of the Malaghan Research Institute, Dr Euan Pearce of the Dental Research 
Unit, Mr Rob Thomson of the Electron Microscope Facility, V.U.W., and Don St John and Peter Bastable of the Inorganic Chemistry Section, Chemistry Division, D.S.I.R. Gracefield.

Trevor Knopp provided a program which computes pixel histograms from C.T. images, Adam Zyskowski assisted with the development of the image processing software, Arthur Chapman made many of the imaging phantoms, and Frank Manning formerly of General Electric) provided technical information and assistance.

Assistance with the production of the thesis has been provided by several groups of people. The staff of the Audiovisual Unit, Wellington School of Medicine, assisted with the figures: especially Wendy Forsyth, Louise Goosens, Cecylia Ciechanowski and John Whalan. The staff of the Wellington Medical Library, particularly Jenny Buxton, Caroline Cole, Margot Herzer and Mary-Jane Ansell, who assisted with the references. The staff of the Community Health Department, Wellington School of Medicine, especially Shanthi Chelliah, Sue Payne and Trevor Williams for the use of their computer facilities. Clare Salmond of the same department provided statistical advice. Bruce McIntosh assisted with the photocopying.

The interest and support of the medical physics community is gratefully acknowledged, particularly Ian Ross of Wellington Hospital, and Brian Williamson, John Le Heron, John Poletti and Peter Cartwright of the National Radiation Laboratory, Christchurch.

My grateful thanks to all of the above people. On a personal level I would like to thank my wife, Magi, for her encouragement and support, and I would also like to acknowledge the assistance of Mary Greenhalgh, Teresa Callahan and Athol and Anne Mitchell. 


\section{CONTENTS}

$\begin{array}{lr}\text { Abstract } & \text { ii } \\ \text { Preface } & \text { iii } \\ \text { Contents } & \text { vi } \\ \text { List of Figures } & \text { ix }\end{array}$

CHAPTER ONE: The Computed Tomography Scanner.

1.1 Basic principles. 1

$\begin{array}{lll}1.2 & \text { Image acquisition. } & 7\end{array}$

1.3 Image reconstruction. 14

1.4 Image display. $\quad 24$

$\begin{array}{ll}1.5 & \text { Image quality. } \\ 1.6 & 27\end{array}$

1.6 Reprise. 32

CHAPTER TWO: X-ray Attenuation Coefficients.

2.1 Definition of the linear attenuation coefficient. 33

2.2 Relationship to the Hounsfield number. 33

2.3 Attenuation processes in the diagnostic energy range. 34

2.4 Comparision of published attenuation coefficient data. 36

2.5 Correlation between attenuation coefficients and C.T. measurements. 40

2.6 Computation of attenuation coefficients in the diagnostic energy range. 41

2.7 Computation of the Klein-Nishina cross section. 45

2.8 Simple empirical formulae for calculating attenuation coefficients. $\quad 49$

2.9 A semi-empirical expression for the attenuation coefficient. 53

2.10 Accurate interpolation of attenuation coefficient data. 56

2.11 Calculation of X-ray spectra. 59

2.12 Estimation of the spectrum of the Wellington Hospital C.T. scanner. 67

2.13 Reprise. 68

\section{CHAPTER THREE: Dual Energy Computed Tomography.}

3.1 Limitations of single energy computed tomography. 71

$\begin{array}{ll}3.2 & \text { Theoretical basis of dual energy C.T. } \\ & 72\end{array}$

3.3 Equipment modifications for dual energy C.T. 73

$\begin{array}{lll}3.4 & \text { Algorithms for dual energy C.T. } & 74\end{array}$ 
3.5 A method for obtaining dual energy C.T. scans using the G.E. 8800 C.T. scanner.

3.6 Imaging performance tests 88

$\begin{array}{lll}3.7 & \text { Reprise } & 95\end{array}$

\section{CHAPTER FOUR: C.T. Spine Mineral Densitometry.}

4.1 The clinical need for bone mineral measurement. 98

4.2 Conventional bone mineral measurement. 101

4.3 Single energy C.T. bone mineral measurement. 104

4.4 Dual energy C.T. bone mineral densitometry. 107

4.5 The effect of varying marrow composition. 109

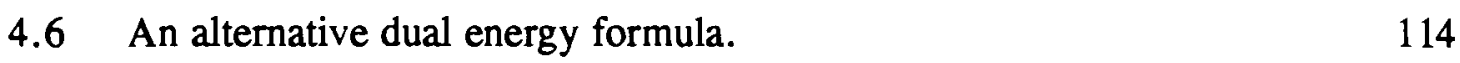

4.7 A spine mineral measurement protocol for Wellington Hospital. 116

4.8 Comparison between C.T. spine mineral densitometry and $\begin{array}{ll}\text { dual photon absorptiometry. } & 121\end{array}$

4.9 Patient dose. 124

$\begin{array}{lll}4.10 & \text { Reprise. } & 125\end{array}$

CHAPTER FIVE: Clinical Applications of Quantitative C.T.

5.1 Computation of electron densities. $\quad 128$

5.2 Normal range for bone mineral measurements. 138

5.3 Measurements on patients with a history of anorexia nervosa. 151

5.4 Correlations between bone measurements and clinical parameters. 162

$\begin{array}{lll}5.5 & \text { Reprise. } & 175\end{array}$

CHAPTER SIX: Correlation of C.T. Images with Bone Structure.

6.1 Introduction.

$\begin{array}{lll}6.2 & \text { The structure of bone. } & 180\end{array}$

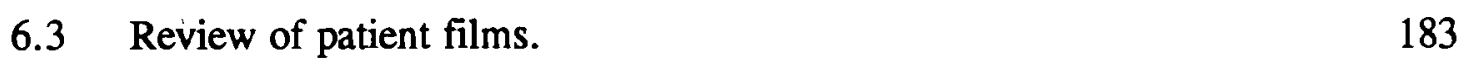

$\begin{array}{llr}6.4 & \text { Pixel distributions. } & 189\end{array}$

$\begin{array}{ll}\text { 6.5 Pilot study on sheep bones. } & 194\end{array}$

6.6 Measurements on human vertebrae. 196

$\begin{array}{lll}6.7 & \text { Reprise. } & 201\end{array}$

CHAPTER SEVEN: Conclusion. 205 


\section{Appendices}

Appendix A: $\quad$ Proof of the central section theorem. 212

$\begin{array}{lll}\text { Appendix B: } & \text { Curve fitting techniques. } & 214\end{array}$

Listing: NEWTPOLY. 216

Listing: TCHEB. 219

Listing: INTCF. $\quad 220$

Appendix C: $\quad$ Computation of X-ray attenuation coefficients. 225

C. 1 Program XATTEN. 225

C.2 Program MCOEF. 232

C.3 Coefficients for Simple Empirical formula. 240

C.4 Interpolation Coefficients (5 parameter). 241

Appendix D: $\quad$ Computation of X-ray spectra. 242

D. 1 Program XSPECT. 242

D.2 Spectrum of the Wellington C.T. scanner. 253

Appendix E: $\quad$ Computation of the modulation transfer function. 254

Appendix F: $\quad$ Wellington protocol for C.T. spine mineral densitometry. 258

Appendix G: $\quad$ Spine mineral software. $\quad 261$

$\begin{array}{lll}\text { Appendix H: } & \text { Patient dose calculations. } & 263\end{array}$

$\begin{array}{lll}\text { Appendix I: } & \text { Statistical techniques. } & 265\end{array}$

$\begin{array}{lll}\text { Appendix J: } & \text { Clinical correlations for anorexics. } & 297\end{array}$

$\begin{array}{lll}\text { Appendix K: } & \text { Program PIXPLOT: Plotting pixel distributions. } & 303\end{array}$ 


\section{LIST OF FIGURES}

Figure

page

1.1 Principles of conventional radiography and axial tomography. 1

1.2 Comparison of a projection radiograph with a computed tomograph. 2

1.3 Relationship between the pixel and the voxel. 4

1.4 Block diagram of the Wellington Hospital C.T. scanner. 5

1.5 The Wellington Hospital C.T. scanner. 6

1.6 The gantry of the E.M.I. scanner. $\quad 8$

1.7 Scanning movements of the E.M.I. scanner. $\quad 8$

1.8 Scanning movements of the third and fourth generation C.T. scanners. 9

1.9 The ray sum. $\quad 13$

1.10 Linearisation of transmission readings. 16

1.11 Principle of the iterative reconstruction technique. 18

1.12 Co-ordinate system used for analytical reconstructions. 19

1.13 The central section theorem.

1.14 Simple back projection. $\quad 21$

1.15 Special windowing methods.

1.16 Contrast-detail-dose relationship. 29

2.1 Relative importance of attenuation processes for oxygen. 35

2.2 Comparison of tabulated attenuation coefficients for oxygen. 37

2.3 Typical constant potential X-ray spectrum generated at $120 \mathrm{kV}$. 37

2.4 Sources of attenuation coefficient data. 38

2.5 Comparison of tabulated attenuation coefficients for selected elements. 39

2.6 Comparison of "mass Hounsfield units" calculated from different tables of attenuation coefficients. $\quad 42$

2.7 Cross sections for carbon and calcium. 44

2.8 Variation of the Klein-Nishina cross section over the

2.9 The minimax fitting technique. $\quad 48$

2.10 Accuracy of the equation of Weber \& Van Den Berge. 51

2.11 Values for the two parameter fit to the attenuation coefficient (50 - $100 \mathrm{keV})$. 53

2.12 Geometry of the X-ray target. 61

2.13 Accuracy of computed spectra. 65

2.14 Comparison of measured and computed spectra: Birch \& Marshall. 66

2.15 Computed spectrum of the Wellington Hospital C.T. scanner at $120 \mathrm{kVp}$. 67

3.1 Exit spectrum of the G.E. 8800 at $120 \mathrm{kVp}$. 75

3.2 Effective energy of the G.E. 8800 as a function of atomic number. 76 
$\begin{array}{lll}3.3 & \text { Energy dependence of the Brooks parameters. } & 78\end{array}$

3.4 Energy dependence of the Alvarez parameters. 80

3.5 Energy dependence of the Alvarez parameters using different basis materials. 81

3.6 Energy dependence of the Hawkes parameters. 85

3.7 Modulation transfer function curves at $120 \mathrm{kVp}$ and $80 \mathrm{kVp}$. 92

3.8 Sensitivity to artefact formation at $120 \mathrm{kVp}$ and $80 \mathrm{kVp}$. 94

4.1 Variation of the composition of vertebral bone with age. 99

4.2 Reference frame for the human body. 99

4.3 C.T. spine mineral densitometry. 105

4.4 Mineral content of cancellous bone. 107

4.5 Attenuation coefficients of bone mineral and marrow. 108

4.6 Effect on single energy mineral estimate of varying marrow composition \& density.

4.7 Effect on the dual energy mineral estimate of varying marrow composition \& density.

4.8 Simulated effect on the estimated fat concentration of varying density. 113

$\begin{array}{lll}4.9 & \text { Effective dose equivalents for some diagnostic examinations. } & 125\end{array}$

5.1 Calibration curve of atomic number versus ratio of attenuation coefficients at two energies.

5.2 Calibration curve of electronic cross section versus atomic number.

5.3 Calibration curve of electronic cross section versus ratio of attenuation coefficients.

5.4 Distributions of the dual energy measurements of the control group. $\quad 140$

5.5 Distributions of the single energy measurements of the control group. 141

5.6 Published single energy C.T. measurements of the normal population. $\quad 142$

5.7 Distributions of the coefficients of variation of the dual energy measurements of the control group.

5.8 Distributions of the coefficients of variation of the single energy measurements of the control group.

5.9 Typical abdominal cross sections at the levels of L2, L3 \& L4. 150

5.10 Locations of measurements taken from films. 150

5.11 Distributions of the dual energy measurements of the anorexics. 153

5.12 Distributions of the single energy measurements of the anorexics. 154

5.13 Distributions of the coefficients of variation of the dual energy measurements of the anorexic group.

5.14 Distributions of the coefficients of variation of the single energy measurements of the anorexic group. 
5.15 Distribution of the total duration of anorexia nervosa.

5.16 Distribution of the total duration of either anorexia nervosa or bulimia nervosa.

5.17 Correlations of bone measurements with ANBULT.

6.1 Micrograph of cancellous bone tissue.

6.2 Micrograph showing cortical and cancellous remodelling systems.

6.3 Bone remodelling sequence.

6.4 Standard image for granularity grade IV.

6.5 Standard image for ' $Y$ ' pattern, grade IV.

6.6 Standard image for stellate pattern, grade IV.

6.7 Distribution of granularity classifications.

6.8 Distribution of ' $Y$ ' structure classifications.

6.9 Distribution of stellate pattern classifications.

6.10 Histogram of C.T. numbers in the cancellous region. 190

6.11 Histograms for a complete examination.

6.12 Mid-sagittal section through sheep vertebrae.

6.13 Variation of single energy measurements along the long axis of the vertebra.

6.14 Variation of dual energy measurements along the long axis of the vertebra.

6.15 Simple method for obtaining Fourier spectrum from C.T. images.

A.1 The central section theorem. 


\section{CHAPTER ONE: THE COMPUTED TOMOGRAPHY SCANNER}

\subsection{Basic Principles}

"Computed tomography" (C.T.) may be viewed as an extension of conventional radiography, in which an X-ray beam is directed through the body onto a film cassette. The image is formed by denser objects such as bone casting shadows on the film. The nature of the improvements offered by C.T. is contained in the name of the technique. Taking the second word first, "tomography" means to obtain an X-ray image of a section of the body. In principle such sections can be taken along any plane, although in computed tomography the images are normally taken along planes (called axial planes) at right angles to the long axis of the body. Tomographic images are sometimes necessary since the conventional $\mathbf{X}$-ray image is simply a two dimensional projection of a body part. A comparison of the two imaging methods is given in figure 1.1, and images of both types are reproduced in figure 1.2.

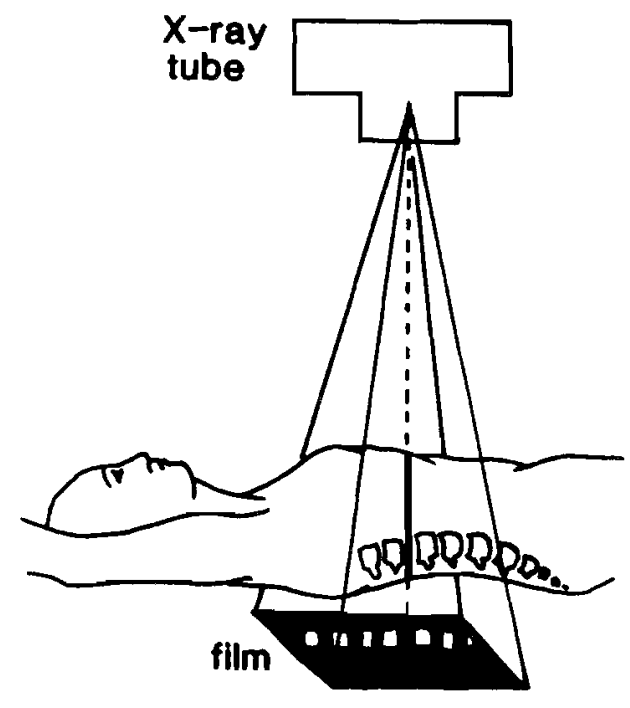

Conventional radiography

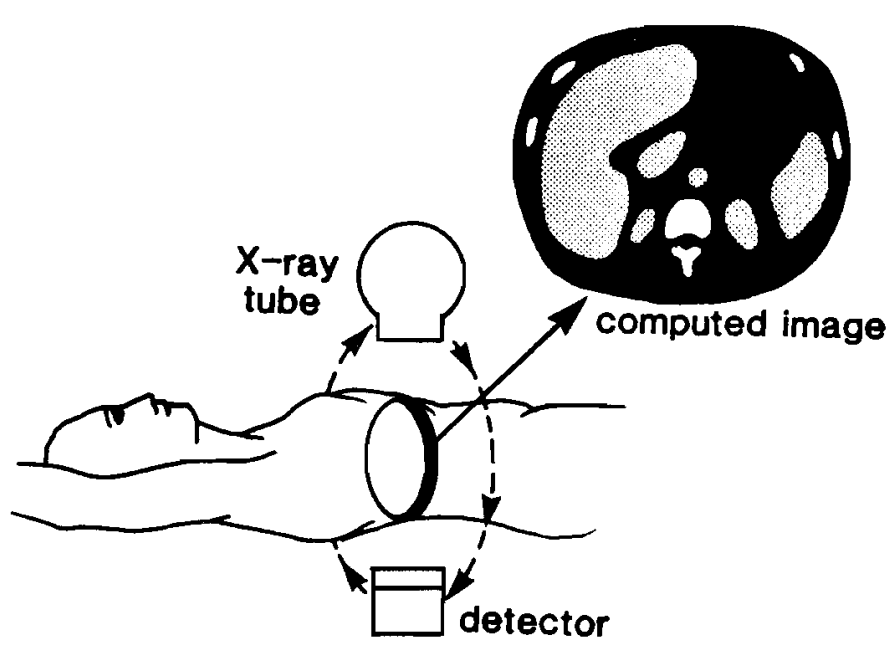

Axial tomography

Figure 1.1: Principles of conventional radiography and axial tomography.

Mathematically, the density of each point on the conventional projection image represents the line integral of the attenuation coefficients of the overlying tissues:

$$
D=\text { const } x \int \mu(1) \cdot d l
$$

where $\mathrm{D}$ is the optical density, $\mu$ is the linear $\mathrm{X}$-ray attenuation coefficient of each tissue element overlying the point on the image and 1 represents distance along the 


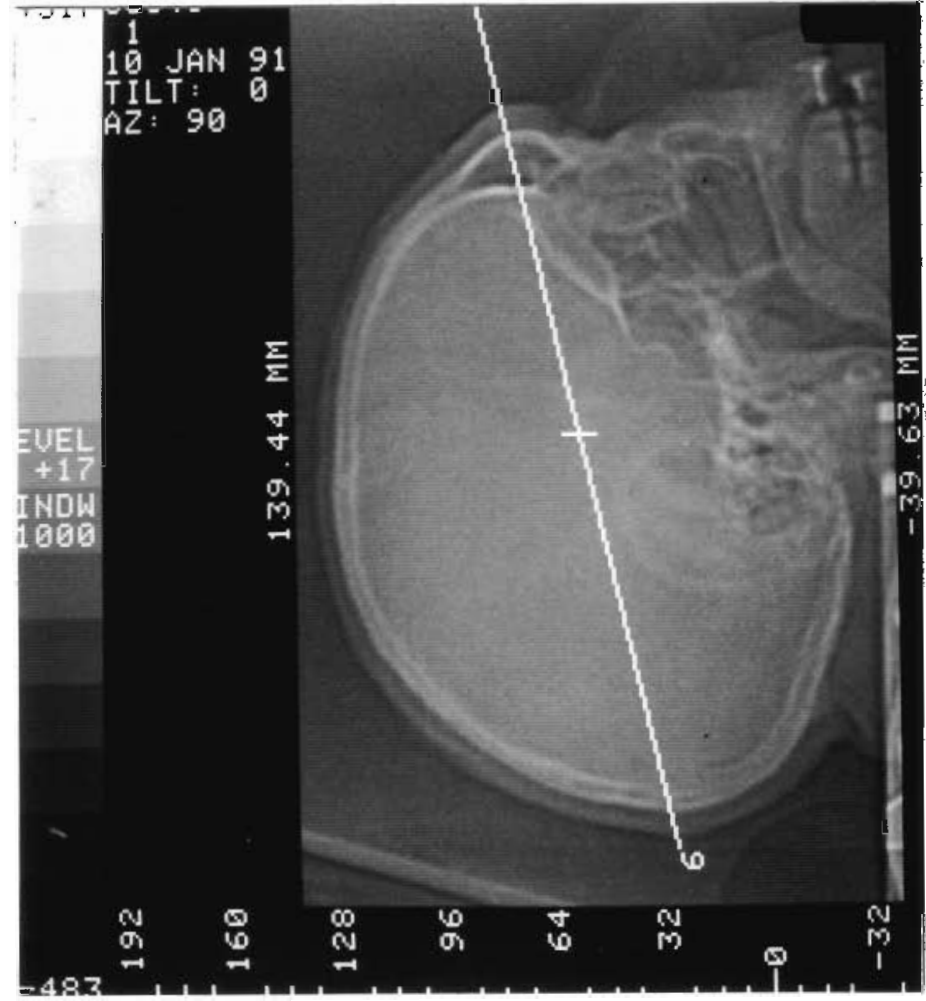

Projection Radiograph

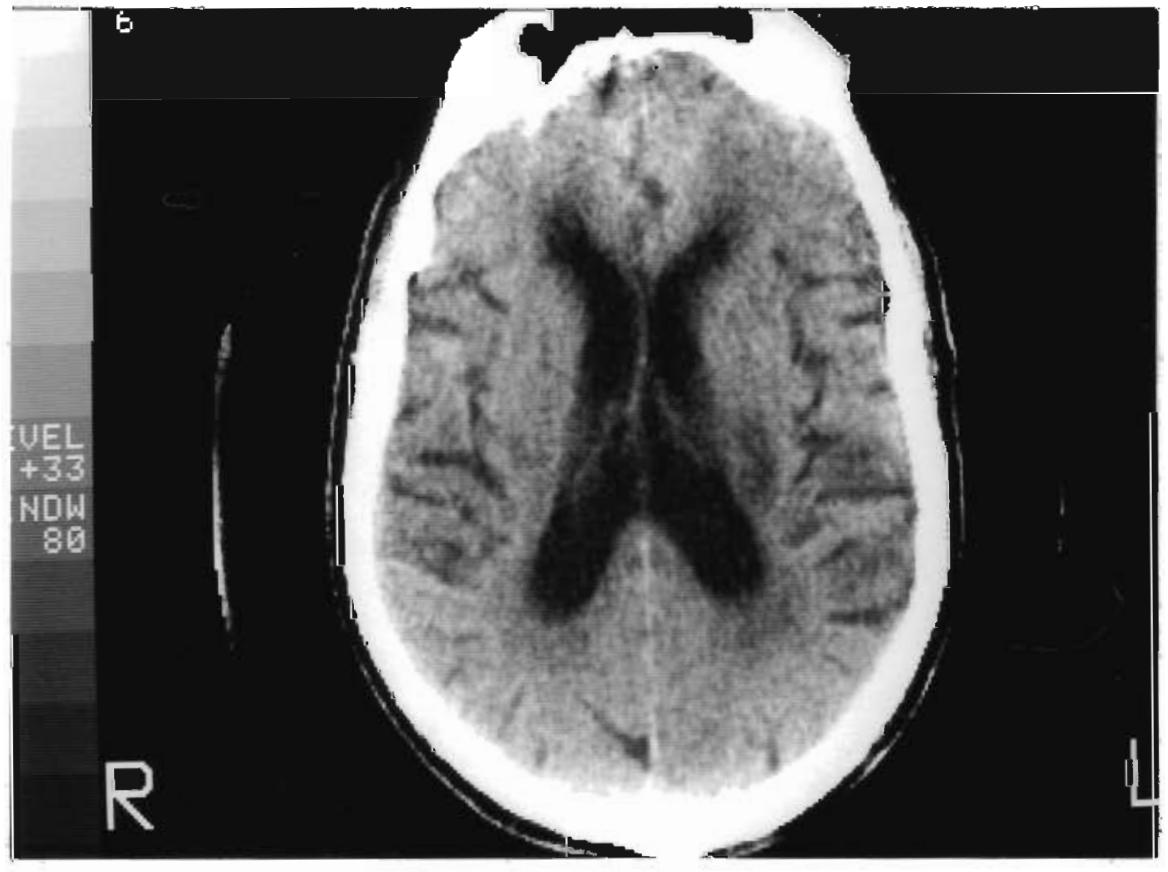

Axial C.T. Image

Figure 1.2: Comparison of a projection radiograph with a computed tomograph.

The projection radiograph is a 'planning scan' or 'scout view' taken by the C.T. scanner itself and is comparable to a conventional radiograph. Brain tissue is not visible on the projection radiograph because it is obscured by the cranium. The location of the axial image is marked on the projection image. 
direction of the beam. The degree of X-ray transmission depends on both the linear attenuation coefficient of the tissue, and the thickness of tissue overlying each point on the film. Objects of low attenuation coefficient which lie either above or below highly attenuating objects will not be visible on the film. To avoid losing diagnostic information in this way it is necessary to obtain tomographic images. Conventional tomography, which was first described Ziedses des Plantes in 1931 [1], is achieved by movement of both the X-ray source and the film during the exposure. The images of tissues lying above or below the plane containing the fulcrum are blurred, leaving just the focal plane in focus. This technique delivers a high dose, and can only image in certain planes. There is never complete blurring of the unwanted areas, contributing to a background density or fog, and structures in the focal plane suffer from slight blurring. More importantly, the differentiation between "soft tissues" (ie those other than bone) is poor.

In computed tomography a very thin X-ray beam is used, usually $10 \mathrm{~mm}$ thick or less, so that only a thin slice of tissue is irradiated and therefore able to contribute to the image. The $\mathrm{X}$-ray beam is directed through the slice of tissue from several different angles. The number of X-rays transmitted at each angle is detected not by film but by either a solid state radiation detector or a high pressure gas detector. The outputs of the detector at the different angles are digitised and passed to the computer which produces (reconstructs) an image from these readings.

A digital image is composed of an array of boxes called "pixels", an acronym for picture element. It should be noted that in computed tomography each two dimensional pixel represents a three dimensional volume of tissue in the patient, as illustrated in figure 1.3. To emphasise this point the tissue volume represented is called a voxel.

The other advantages of computed tomography stem from the use of a computer. Since several X-ray transmission measurements, taken at different angles, are used to produce the image it is possible for the computer to calculate a parameter (the C.T. number: see section 1.4.1) which is related to the average linear attenuation coefficient of the tissue in each voxel. Voxels of highly attenuating tissue have large C.T. numbers and are represented by lighter shaded pixels on the video display, whereas dark pixels represent tissues of low attenuation. C.T. numbers are estimated with good precision so that C.T. scanners can display differences between tissues whose linear attenuation coefficients differ by as little as $0.3 \%$ or less [2]. This is a vast improvement on conventional radiography where the differences in $X$-ray transmission must be greater than $10 \%$ to produce visible differentiation on the film [3]. It is for this reason that soft tissues are imaged much better by C.T. than they are by conventional radiography. 


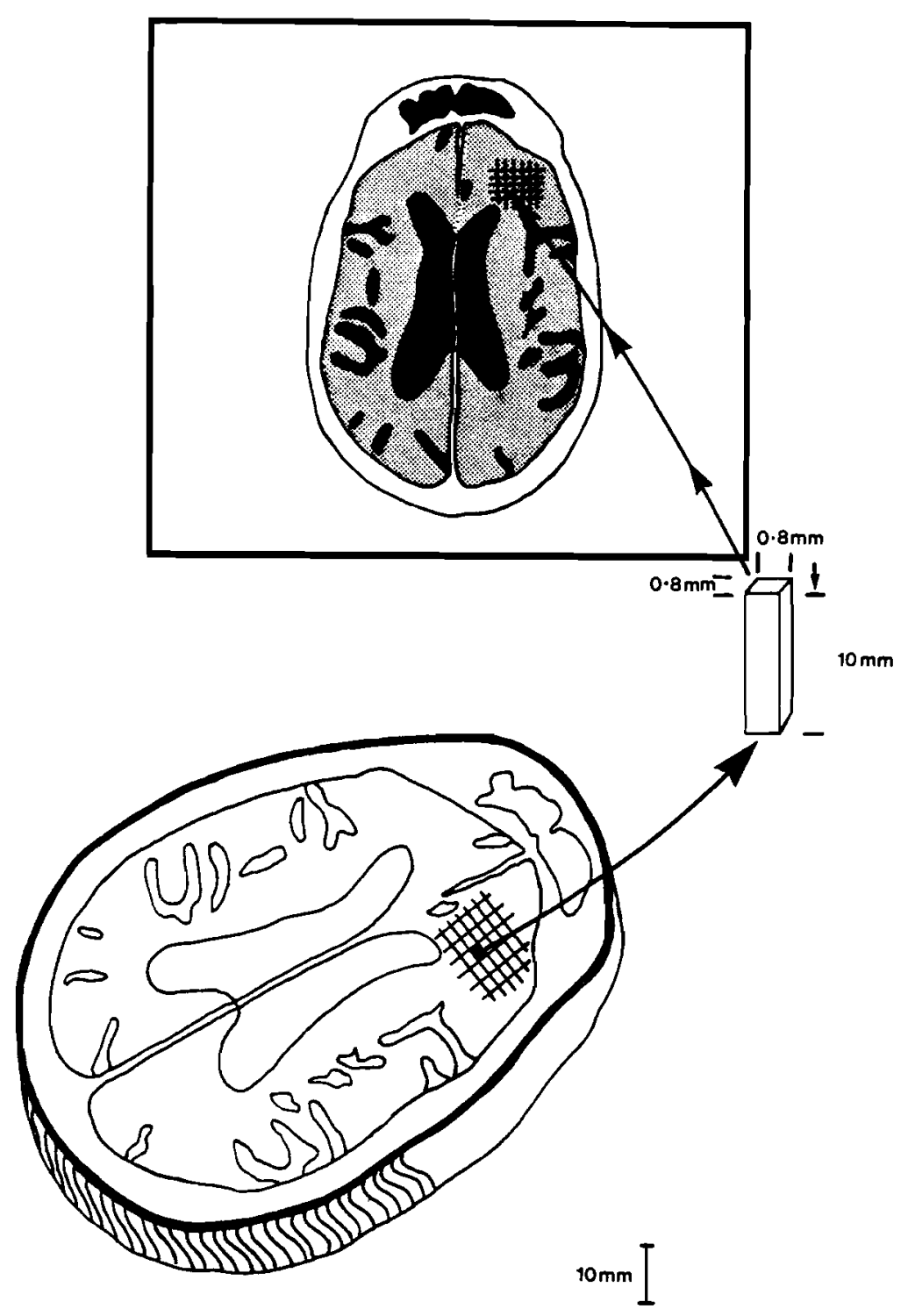

Figure 1.3: Relationship between the pixel and the voxel.

A further advantage of the C.T. scanner is that, since the image is stored electronically and viewed on a cathode ray tube (C.R.T.), the brightness and contrast of the image may be altered to provide optimal viewing conditions for any portion of the image. This is a marked improvement over conventional radiography where the exposure settings must be determined by the radiographer before the image is produced. The contrast and brightness cannot be altered and so must be selected so that all structures of interest are visible on the film, so that the settings would not be optimum for most organs.

Many of the principles involved in C.T. scanning have been known for several decades. In 1917 Radon, an Austrian mathematician, proved that a three dimensional object could be reconstructed from an infinite set of its projections [4]. In 


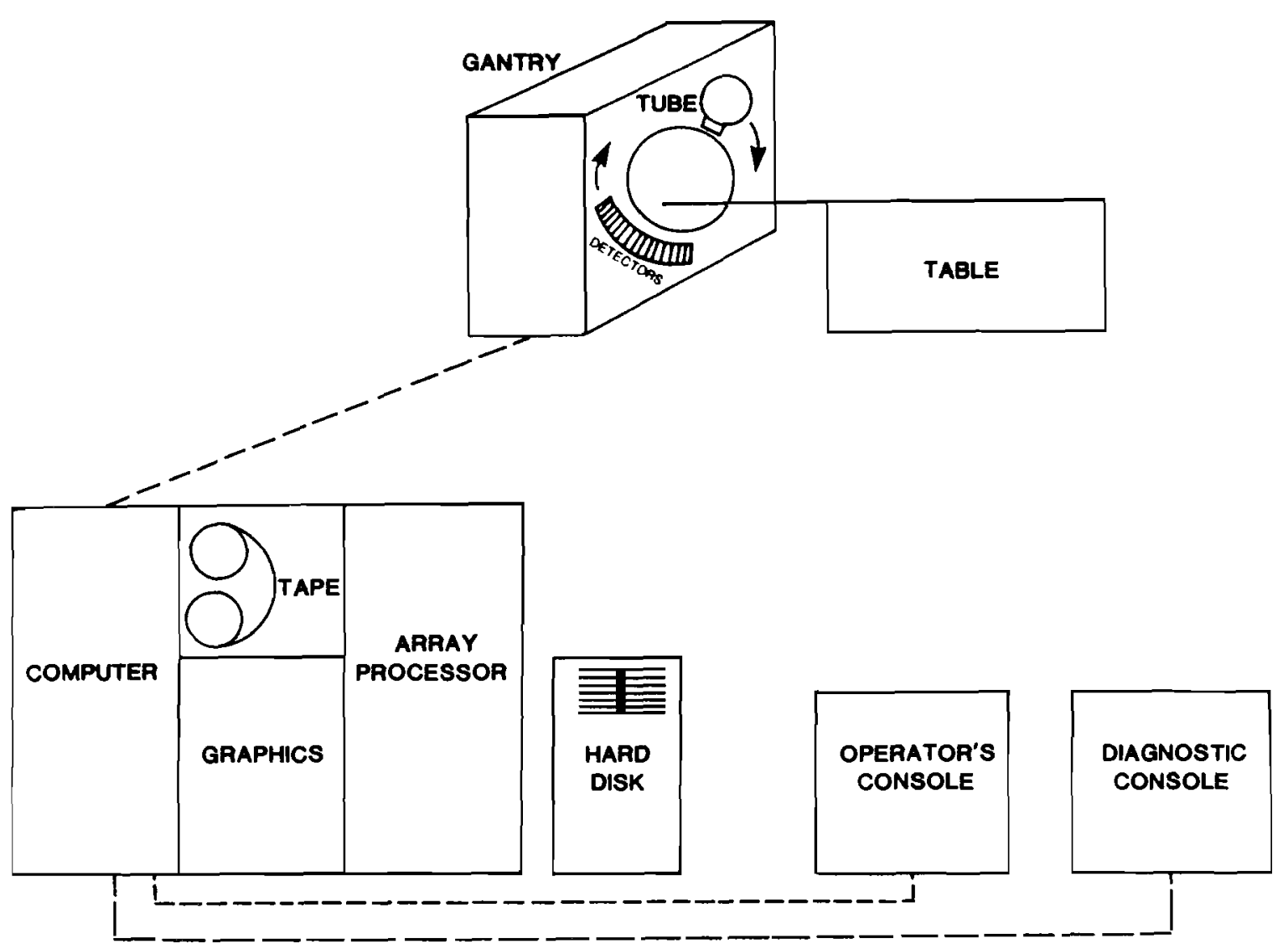

Figure 1.4: Block Diagram of the Wellington Hospital C.T. Scanner.

The Wellington C.T. scanner is a General Electric CT/T 8800. It is a third generation scanner with a 523 element high pressure xenon detector. Brief specifications are as follows:

Gantry aperture:

Scan circle diameter:

Maximum tilt angle:

Scan times:

Slice thickness:

Peak kilovoltage:

Beam filter:

Tube current:

Resolution:

High contrast:

Low contrast:
$60 \mathrm{~cm}$.

$20 \mathrm{~cm}$ (infant), $35 \mathrm{~cm}$ (medium body), $42 \mathrm{~cm}$ (large body). Head field is a $20 \mathrm{~cm} \times 25 \mathrm{~cm}$ ellipse.

$\pm 15^{\circ}$.

$5.8,9.5 \& 11.5$ seconds.

$1.5,5$ or $10 \mathrm{~mm}$.

$120 \mathrm{kVp}$ pulsed. 288,342 or 576 pulses per image.

Pulse duration 1.3, 2.7 or $4 \mathrm{~ms}$.

$1.5 \mathrm{~mm}$ Al plus shaped perspex filter.

Selectable from $20 \mathrm{~mA}$ to $500 \mathrm{~mA}$ (peak).

$1.3 \mathrm{~mm}$ standard. $0.8 \mathrm{~mm}$ with bone algorithm.

$3.4 \mathrm{~mm}$ at $0.5 \%$ contrast \& $45 \mathrm{mGy}$ skin dose. 

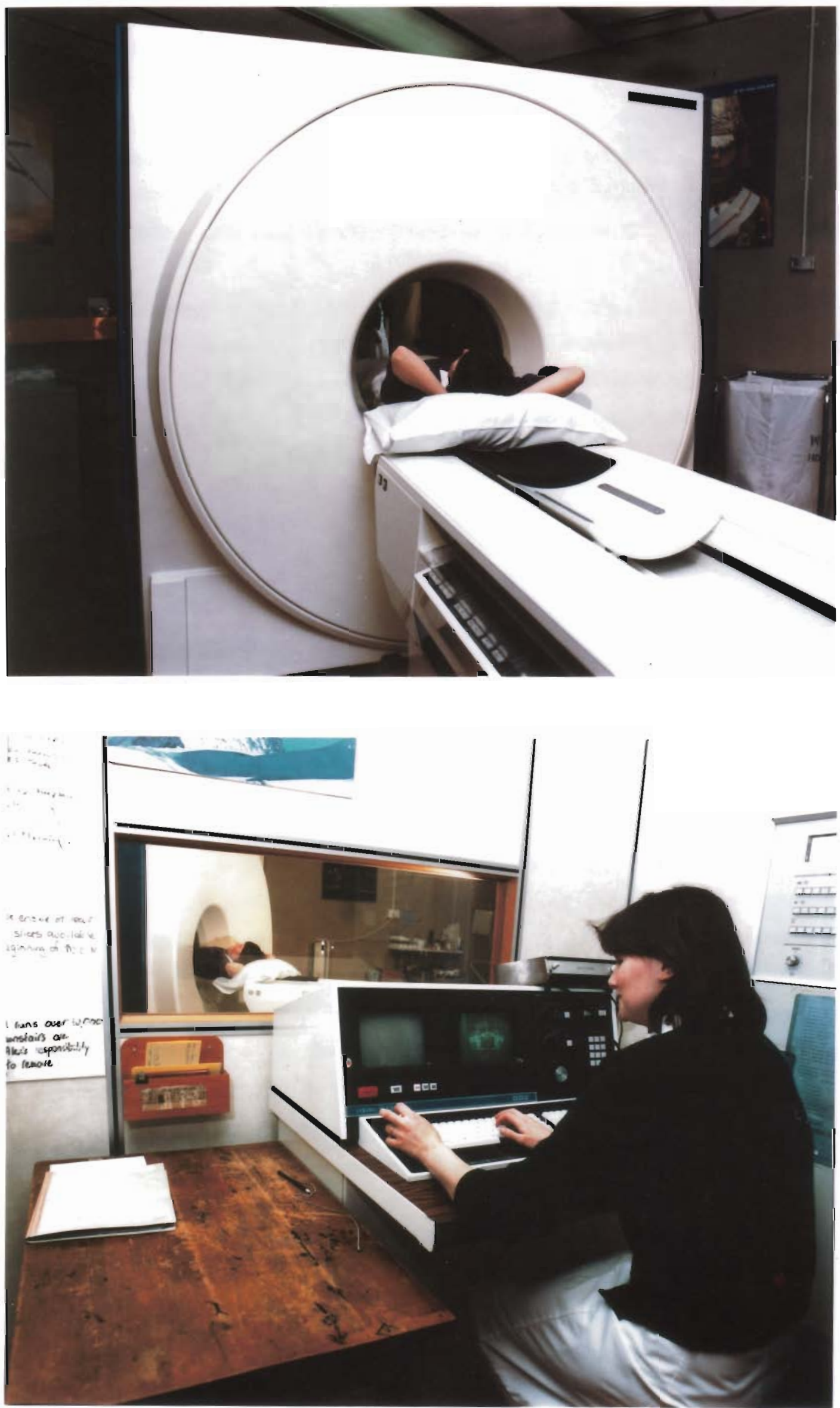

Figure 1.5: The Wellington Hospital C.T. Scanner. 
1956 Bracewell produced a solar map reconstructed from ray projections [5]. Cormack [6] published mathematical work on reconstruction in 1963, and Kuhl [7] described a digital nuclear medicine tomograph in 1968. The C.T. scanner as we now know it results directly from the work of Godfrey Hounsfield [8], a research engineer employed by the English firm E.M.I. The first prototype was installed at AtkinsonMorely's Hospital in 1971. Following successful clinical trials the invention was made public in 1972, and the first production C.T. scanners were delivered in 1973. Hounsfield and Cormack were jointly awarded the Nobel prize in medicine in 1979.

A C.T. scanner consists of a scanning gantry and patient support table (comprising the image acquisition system) and the computer and consoles which perform image reconstruction and display. A block diagram of the Wellington Hospital G.E. 8800 scanner is given in figure 1.4, and in figure 1.5 there are photographs of both the control console and the patient table and gantry.

\subsection{Image Acquisition}

\subsubsection{Scanning Movements}

C.T.scanning involves the computer reconstruction of the pattern of attenuation within the patient from many thousand X-ray transmission measurements through the patient. A diagram of the gantry of the original E.M.I. head scanner is given in figure 1.6. An X-ray tube and detectors, rigidly coupled, were moved transversely across the patient. The gantry mechanism was then rotated one degree about the long axis of the patient and the tube and detectors performed another transverse movement. In the course of each traverse 160 transmission measurements are taken, and a total of 180 traverses each one degree apart were required, as shown in figure 1.7. The reference detector permitted correction for fluctuations in tube output. The complete set of rotate-translate movements took approximately 6 minutes, during which time it was not unusual for a slight amount of patient movement to occur, often with a pronounced degradation of the image. The incorporation of a second imaging detector so that two transverse slices (one above the other) were taken simultaneously reduced the time taken for the number of images making up the examination, lessening the risk of "fidgeting" towards the end of the examination. In what is called the second generation of C.T. scanner the X-ray beam was collimated to a fan shape which was viewed by several detectors, each offset by an angle of approximately one degree. In this way it was possible to obtain in one translational movement the same information as was obtained previously in several movements. The number of rotate-translate movements was reduced in proportion to the number of detectors used, so that scan times of approximately 20 seconds were possible. 


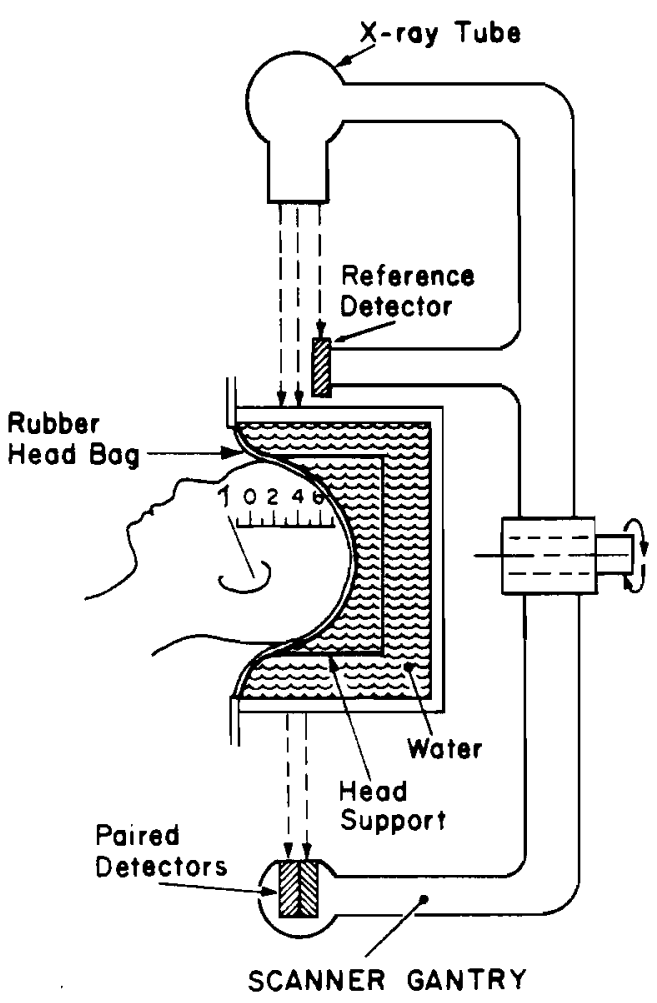

Figure 1.6: The Gantry of the E.M.I. Scanner. (from reference [3]).

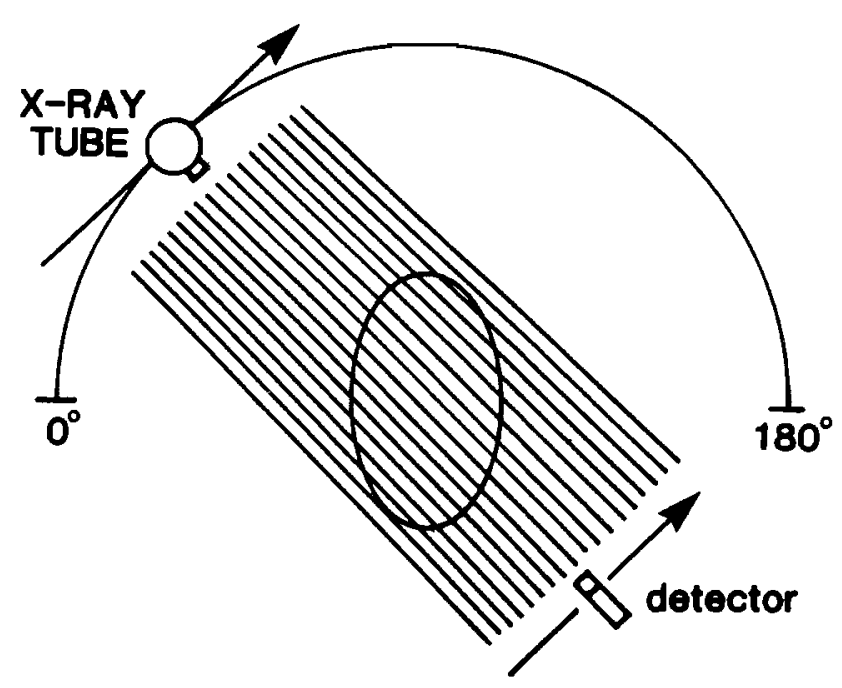

Figure 1.7: Scanning Movements of the E.M.I. Scanner.

Viewed at right angles to the projection in figure 1.6. The scanning motion consists of translational movement of tube and detectors at right angles to the long axis of the patient, followed by a one degree rotation of the tube and detectors about the long axis of the patient. Each image required 180 translational \& rotational movements. 


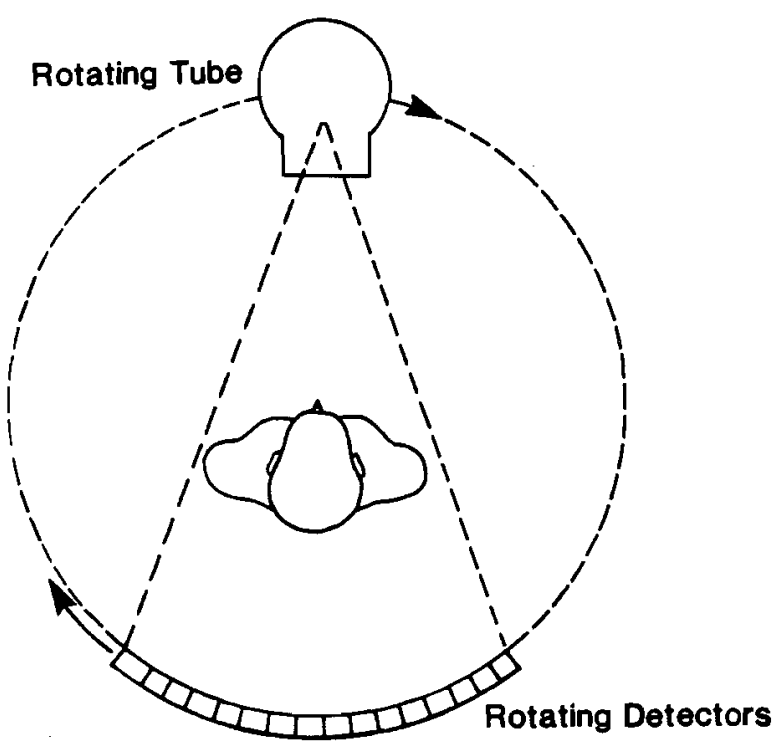

Third Generation

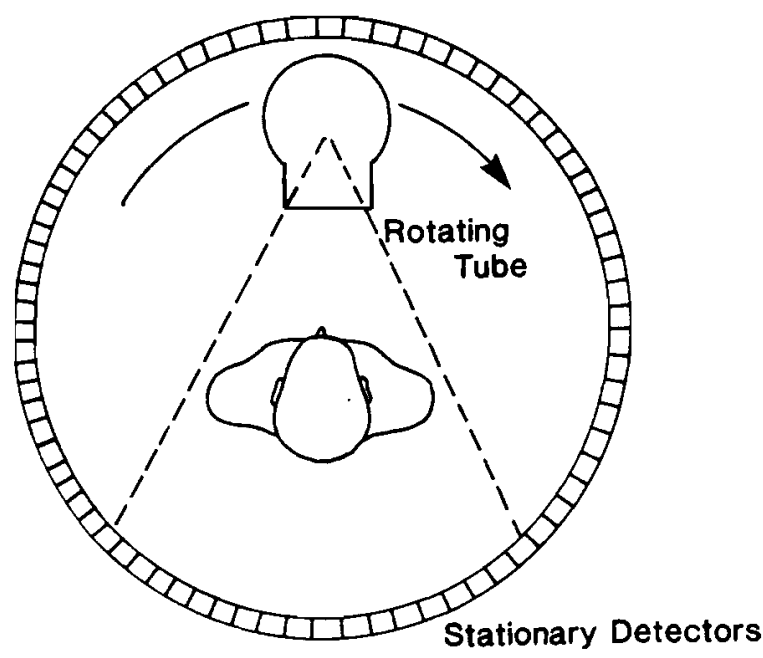

Fourth Generation

Figure 1.8: Scanning movements of the Third and Fourth Generation C.T. Scanners.

The third generation scanner, the scanning method of which is illustrated in figure 1.8, has sufficient detectors to permit the fan angle to be wide enough for the beam to encompass the entire body section to be imaged. This eliminates the need for translational movement, the beam being simply rotated around the patient, permitting a further reduction in scanning time to as little as 1 second in some cases. The number of detectors employed is typically between 500 and 2000. In the early third generation scanners the patient is placed equidistant between the tube and detectors. The geometry is such that patient sizes up to about $60 \mathrm{~cm}$ diameter can be accommodated, but this has the disadvantage that when smaller structures such as the head are imaged only a small fraction of the total number of detectors are used to produce the image. In such cases the number of detectors receiving a useful signal may be increased by positioning the X-ray tube closer to the centre of rotation and moving the detectors further away from it. This principle is referred to as geometrical magnification [9], and results in a significant improvement in the resolution of the image.

In the third generation scanner the beam is kept wider than the patient section so that readings taken at the edge of the beam may be used to estimate the strength of the incident beam. Detectors other than at the edges do not "see" the unattenuated beam at any point of the scan which means that great reliance is placed on detector stability. Detector drift can result in circular artefacts on the image. The fourth generation concept was evolved to overcome this problem. Here a complete ring of stationary detectors encircles the patient, and the tube rotates around the patient on a path 
inside the circle of detectors. Since each detector sees the unattenuated beam during each scan it is possible for computer calibration of each detector to take place frequently enough to compensate for a relatively large degree of detector instability.

A disadvantage of the fourth generation scanners is that a vast number of detectors is required to completely fill the space around the patient. Otherwise radiation is "wasted" passing through the gaps between the detectors [10]. A fifth generation has been proposed in which a smaller ring of detectors, close packed, is placed within the path of the tube. Detectors are displaced when they would lie between the tube and patient, a principle called "nutation".

High performance C.T. scanners are currently available using either the third or fourth generation scanning movements, the success of a particular scanner depending more on how the manufacturer exploits the strengths and minimizes the weaknesses of the design generation selected [10]. The fourth generation scanners are less susceptible to detector drift, but are less dose-efficient, cannot compensate for fluctuations in tube output to the same extent and can be more costly due to the larger number of detectors employed. Some manufacturers have reverted to the third generation design as algorithms to minimize the ring artefact have become available $[2]$.

\subsubsection{Gantry and Table}

The X-ray tube and detectors are contained within the scanner gantry, in the centre of which is a circular aperture for the patient. This aperture is typically $60 \mathrm{~cm}$ in diameter and is sufficient for all but very obese patients. The gantry may be tilted either towards or away from the patient's feet providing some flexibility in the choice of image plane. The amount of gantry angulation possible is frequently $\pm 25^{\circ}$ on modern C.T. scanners. As well as supporting the patient, it is the function of the table to permit accurate movement of the patient into the gantry. It must not attenuate the $\mathrm{X}$ ray beam unduly, and yet it must be strong enough to support heavy patients. Carbon fibre tables come closest to meeting this ideal.

\subsubsection{X-ray Source}

Although a few specialised C.T. scanners use radioisotopes as the source of radiation [11], the overwhelming majority use an $X$-ray tube. The tube is powered by a high quality $\mathrm{X}$-ray generator, often a 3 phase type with full wave rectification (12 pulses per $50 \mathrm{~Hz}$ mains cycle). Tube kilovoltage is usually expressed in terms of the peak value of the high tension waveform, and is denoted $\mathrm{kVp}$. Early scanners used fixed anode tubes with oil cooling, however as scanning times became shorter it was 
necessary to use tubes capable of higher photon fluxes. Good scanners now employ rotating anode tubes for high output while scanning, although such tubes often require a cooling period between scans. Higher heat capacity tubes using graphite anodes are now available which have approximately twice the life of conventional tubes.

Sophisticated collimation of the $\mathrm{X}$-ray beam is needed to maintain a constant slice thickness throughout the patient cross section. Adequate collimation is difficult to achieve since the beam is divergent and originates from a relatively large focal spot, leading to penumbra effects. Usually a multi-plate collimator is used, sometimes with septa (lead plates) aligned parallel to the beam to reduce divergence.

As will be seen in the next section, image reconstruction is based on the assumption of a monochromatic X-ray beam. Currently this can only be achieved using a radioactive source, but there are no isotopes of high activity and long half life which emit gamma rays in the appropriate energy range. The spectrum of $\mathrm{X}$-rays emitted by an $X$-ray tube exhibits a wide range of energies which is reduced by heavily filtering with approximately $4 \mathrm{~mm}$ of aluminium and sometimes $0.1 \mathrm{~mm}$ of copper in addition. The peak kilovoltage is usually $120 \mathrm{kVp}$, representing a compromise between relatively high transmission through the patient thus minimizing dose to the patient and quantum mottle in the image, and the higher degree of contrast which is attainable at lower kilovoltages due to the greater incidence of the photoelectric effect [12]. The monochromatic energy equivalent to such a beam is about $73 \mathrm{keV}$ [13]. In addition to the fixed filtration, shaped filtration is applied to compensate for variations in beam path length across the patient cross section. For example rays passing almost tangentially through the edge of the patient undergo much lower attenuation than rays passing through the centre of the patient, with the result that the central detectors receive a much more heavily filtered beam than those at the edges of the beam. The shaped filters are designed to provide perfect compensation for a circular patient; further correction by computer is needed to compensate for deviations from the "ideal" shape in individual sections. A further reason for using shaped filtration is to limit the dynamic range of the required detector outputs. Considering that the patient can attenuate the beam by factors as high as 6000 or more, it is advantageous to reduce the intensity of the edges of the beam to a level comparable with that at the centre.

\subsubsection{Detectors}

Detectors suitable for C.T. scanning must have high efficiency to reduce patient dose, they must have a fast response, a wide dynamic range with linearity of response, and be very stable. Three types of detector are currently in use in C.T. systems: 
scintillation crystal/photomultiplier combinations, scintillation crystal/photodiode combinations and high pressure gas ionisation detectors.

Scintillation crystal/photomultiplier detectors were used in the original C.T. scanners and are still in common use. The crystal must absorb a high proportion of the incoming photons, and must convert a high proportion of the absorbed energy into light. It is also important that a high proportion of the photons passing through the patient reaches the detectors. This implies that the crystals must not be too bulky and must be able to be packed closely together. The original systems used thallium activated sodium iodide crystals which have high efficiency: approaching $100 \%$ if the crystals are sufficiently thick [14]. However these crystals are not ideally suited to C.T. scanning as they suffer from afterglow (signal persistence after the removal of the radiation impulse) and have limited dynamic range. To overcome the afterglow problems $\mathrm{CaF}_{2}$ doped with europium was introduced, but this material had an efficiency of the order of $60 \%$. A commonly used crystal for this type of detector is bismuth germanate $\left(\mathrm{Bi}_{4} \mathrm{Ge}_{3} \mathrm{O}_{12}\right)$ which combines high efficiency with low afterglow, good dynamic range and long term stability. The disadvantages of this type of detector are its size and cost, and that the gain of the photomultiplier tubes is affected by fluctuations in both temperature and power supply voltage.

The problem of bulk can be overcome by replacing the photomultiplier with a photodiode. These have been successfully coupled with scintillation crystals of either $\mathrm{CdWO}_{4}$ or thallium activated caesium iodide [2]. They have wide dynamic range with close detector spacing and high efficiency. Photodiodes which interact with X-rays directly without the need for a scintillation crystal are under development.

High pressure gas detectors are in use in some third generation scanners. High atomic number gases such as xenon or a mixture of krypton and xenon at pressures of 10 to 30 atmospheres are used to increase the absorption efficiency. A single gas chamber is used, partitioned by thin tungsten electrodes. This type of construction permits a small detector aperture with consequent high spatial resolution. At a polarising voltage of approximately 500 volts, the chambers operate in the ionisation region of their operating characteristic and are therefore relatively insensitive to power supply fluctuations. This type of detector is inherently not as efficient as those employing a scintillation detector, however it does not need a collimator to reduce scatter since its geometry makes it effectively self collimating. Its efficiency of $50 \%$ is comparable with that of a scintillation crystal detector with a collimator. 


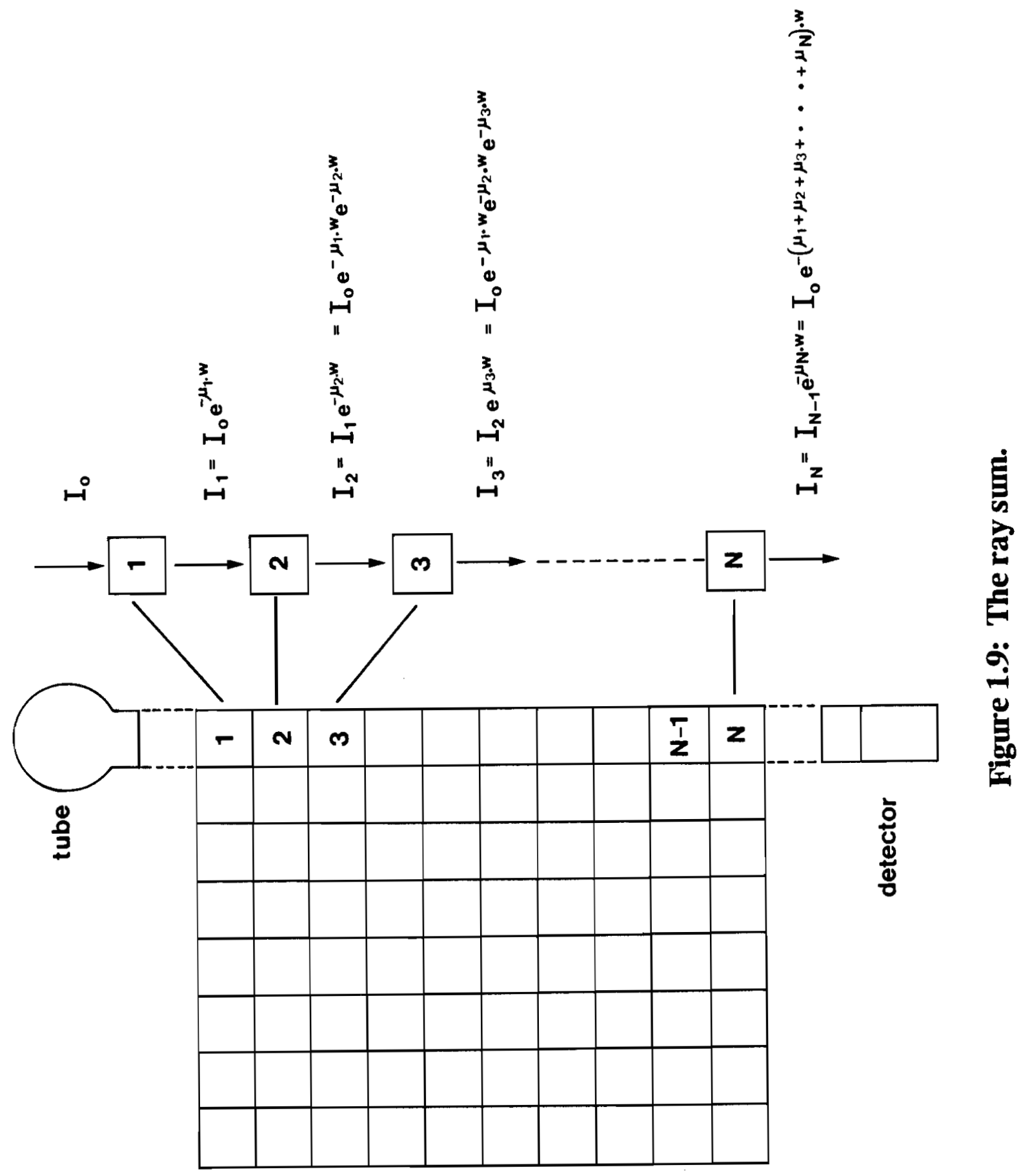




\subsection{Image Reconstruction}

Image reconstruction is the process in which a two dimensional array of attenuation values is obtained from a set of X-ray transmission measurements, or projections. Image reconstruction is a specialised process which cannot normally be modified by people other than the manufacturer of the scanner. Thus the process is usually regarded by the users of C.T. scanners as a "black box", with the proviso that it is important to at least understand the principles of reconstruction to understand the characteristics of the final image. In this work the emphasis will be on principles only, illustrated for simplicity using first generation geometry. Extension to fan beam geometry may be achieved either by interpolation of each fan projection to produce a notional rectangular projection, or by a different mathematical formulation. From figure 1.9 it can be seen that in the idealised case where a monochromatic source of radiation is used, the transmitted $X$-ray intensity, $I_{N}$, is given by:

$$
I_{N}=I_{0} \cdot \exp \left[-w \cdot\left(\mu_{1}+\mu_{2}+\mu_{3}+\ldots+\mu_{N}\right)\right]
$$

where $I_{0}$ is the incident intensity, $w$ is the width of the pixels and $\mu_{1}$ through to $\mu_{\mathrm{N}}$ are the average attenuation coefficients of the volume elements. Equation 1.2 may be rearranged to give:

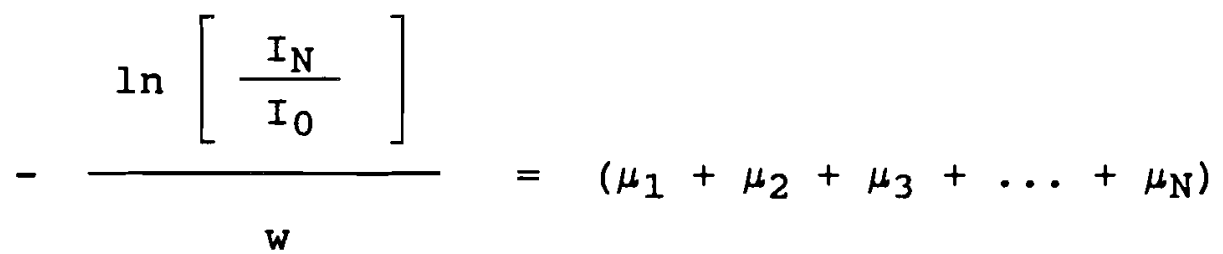

Thus the logarithm of the transmission ratio is proportional to the sum of the pixel attenuation coefficients along the beam direction, the ray sum. In each traverse of the tube and detector across the patient $\mathrm{N}$ ray sums are obtained. Each such set of ray sums is called a profile or view, several profiles being taken at different angles to obtain the information necessary for image reconstruction.

In general the X-ray beam will lie obliquely with respect to the array of pixels, in which case the beam will not be aligned with any one column or row in the pixel array. Each pixel will not contribute fully to that ray sum, so that the $j^{\text {th }}$ ray sum, $p_{j}$, is defined as:

$$
p_{j}=\sum_{i=1}^{N} w_{i j} \cdot \mu_{i}
$$


where the summation is over all pixels in the path of the beam, and $\mathrm{w}_{\mathrm{ij}}$ is the weighting factor for each pixel. The weighting factor for a given pixel along a particular ray is determined exactly by the fraction of its area lying within the beam. This can be time consuming to calculate so approximations are frequently used, such as setting factor $w_{i j}$ to unity if the centre of the pixel lies within the beam and to zero otherwise. Another approximation is to make the weighting factor some function of the distance from the centre of the pixel to the centre of the ray [15]. Solutions for the individual pixel values could in principle be obtained by inverting the weighting matrix:

$$
\mu_{i}=\sum_{j}\left(w^{-1}\right)_{i j} \cdot p_{j}
$$

However with image arrays up to 512 × 512 pixels in size inversion can take an unacceptably long time, and in any case the presence of noise (quantum mottle) on the projection data may render solution in this way impossible due to inconsistencies.

Prior to reconstruction it is necessary to correct the projection data for the beam hardening effect, a consequence of using a polychromatic beam to measure a parameter which is energy dependent. Following a discussion of the various methods of beam hardening correction in the next section, there is a section on each of the three main types of reconstruction technique: the iterative technique, Fourier reconstruction and filtered back projection.

\subsubsection{Beam Hardening Correction}

The problem of polychromaticity may be addressed by defining an effective energy of an inhomogeneous X-ray beam. The effective energy is defined as the energy of a monochromatic beam which would suffer the same degree of attenuation in a given absorber as does the polychromatic beam. However this does not completely solve the problem since the effective energy will still depend on the thickness of the absorber [16]. The thicker the absorber the more the lower energy photons are preferentially absorbed from the beam, and the beam becomes more penetrating or "harder". In the original E.M.I. scanner the effects of beam hardening were reduced firstly by employing substantial tube filtration so that fewer low energy photons are present in the beam as it traverses the patient; and secondly by placing the cranium within a water box so that the path length was always the same [13]. This arrangement gave virtually perfect compensation for soft tissues, but there was still a residual effect due to varying amounts of bone present giving rise to various image artefacts. These residual effects can be reduced by reconstructing twice, using the distribution of bone in the first image to permit a second, corrected, image to be reconstructed [17]. 


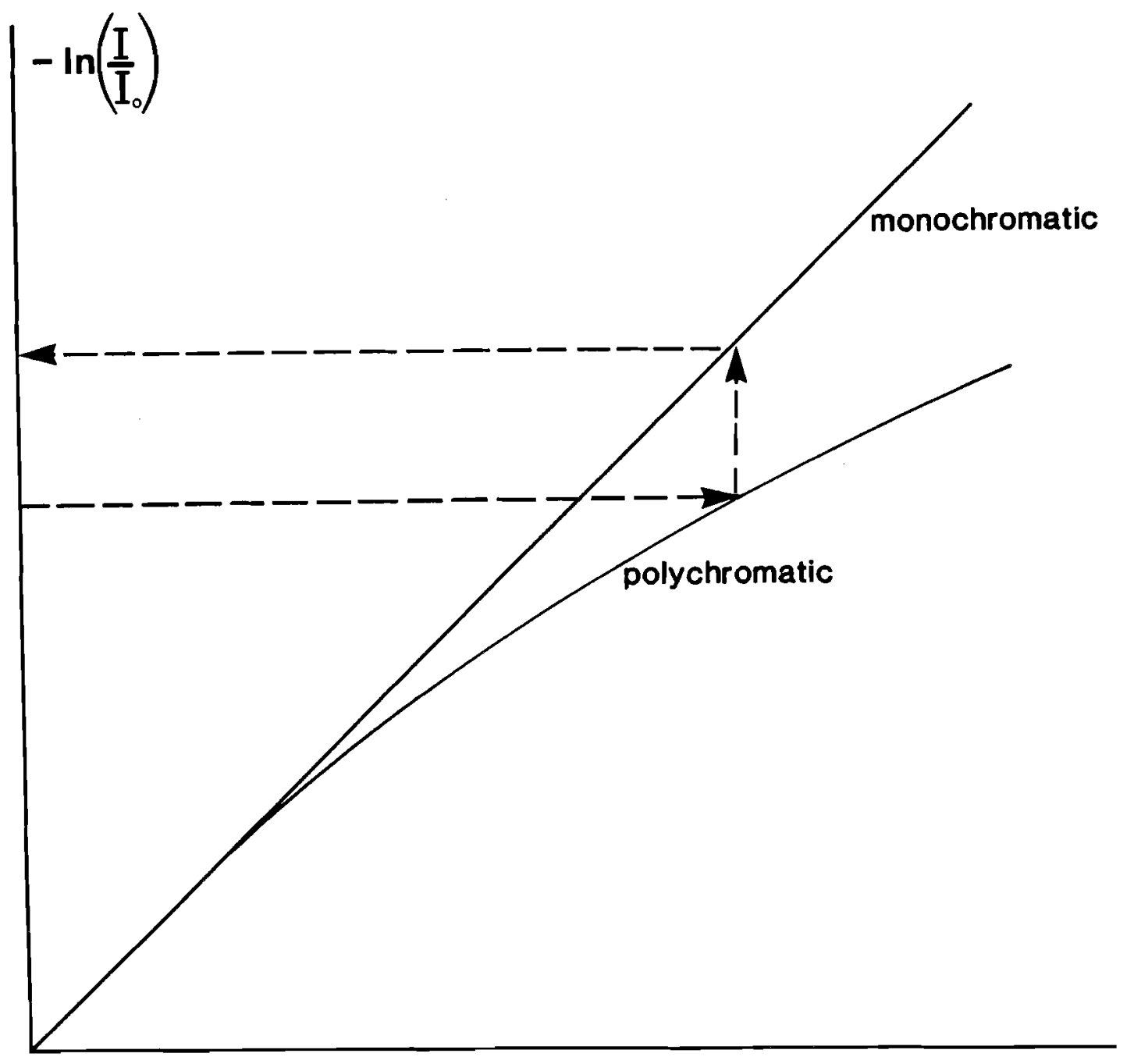

Figure 1.10: Linearisation of Transmission Readings.

For a monochromatic beam the logarithm of the transmission ratio decreases linearly with increased thickness of absorber, $w$. The slope of the curve corresponds to the attenuation coefficient. In the case of a polychromatic beam the effective energy increases with increasing absorber thickness, resulting in a decrease of the attenuation coefficient. Provided that a calibration curve such as the above has been obtained, it is possible to estimate the theoretical monochromatic transmission ratio corresponding to each measured polychromatic transmission ratio.

The use of a water box limited the use of the E.M.I. scanner to examinations of the brain. Extension to whole body scanning required the removal of the water box and its replacement by shaped filters which compensated for differences in path length for the average patient. Further compensation may be achieved by linearising the detector outputs $[18,19]$. In figure 1.10 the logarithm of transmission ratio is plotted against thickness of absorber. For a monochromatic beam a straight line graph is obtained, whereas beam hardening leads to roll off of the attenuation of a polychromatic beam as the thickness increases. Effective monochromatic transmission ratios can therefore be obtained from measurements obtained using a polychromatic 
beam provided that the calibration curve is stored in the computer. This calibration curve would usually be measured for a water absorber, although a separate curve may be used for head scanning which assumes that a small amount of bone is present in each ray. Exact compensation for varying proportions of bone and soft tissue can be obtained using dual energy techniques [20,21], although in the case of the brain this would be of limited value as errors due to increased soft tissue path length tend to cancel with those due to increased bone thickness [18].

In practice the projection values are corrected by comparing them with values obtained in calibration scans on phantoms whose size is similar to that of the patient being scanned. Such phantoms are usually water filled. The parameter reconstructed is now the difference between the attenuation of the object and the attenuation of water under the same conditions $[13,21]$. Beam hardening errors are similar for both the object and the calibration phantom and therefore tend to cancel so that first order correction is normally sufficient.

\subsubsection{Iterative Reconstruction Algorithm}

The iterative reconstruction technique starts by assuming arbitrary values for all pixels in the image. Ray sums are calculated from these values which are then compared with the measured ray sums, and corrections are applied to the individual pixel values to obtain agreement between the calculated and measured ray sums. The process is illustrated for the simple case of a $2 \times 2$ array in figure 1.11. Various modifications of the technique exist. In the Iterative Least Squares Technique (I.L.S.T.) all ray sums are calculated at the beginning of each iteration and corrections are applied to all pixels simultaneously. The technique does not converge unless damping factors are applied to the corrections, the damping factors being chosen to give a best least squares fit to the projection data after each iteration [22]. The Simultaneous Iterative Reconstruction Technique (S.I.R.T.) considers each pixel in turn, correcting the pixel simultaneously for all rays passing through that pixel [23]. The third modification is the so-called Algebraic Reconstruction Technique (A.R.T.) which employs ray by ray correction similar to I.L.S.T. but which corrects the pixels in each ray immediately after calculating the ray sum [24], rather than correcting all rays simultaneously at the end of each iteration.

Each modification has its own advantages and disadvantages. A.R.T. gives faster convergence initially but then tends to wander if noise is present. S.I.R.T. and I.L.S.T. both handle noisy data well but I.L.S.T. is computationally more efficient, so that the optimum strategy would appear to be to start using A.R.T. and then switch to I.L.S.T. [15]. Iterative techniques were used in the E.M.I. scanner. They handle noisy data 


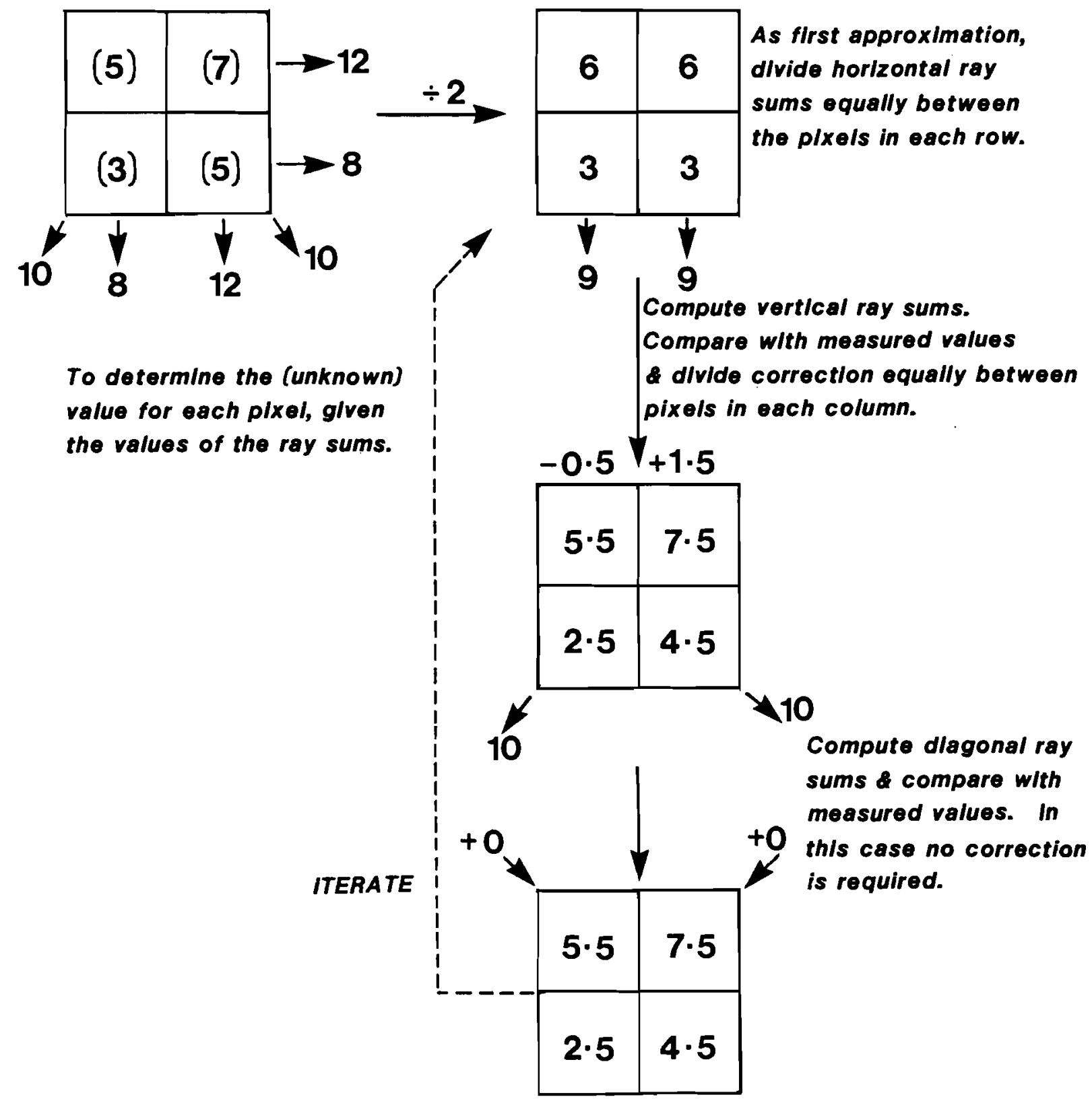

Figure 1.11: Principle of the Iterative Reconstruction Technique

After a single iteration the estimated pixel values are close to the true ones. The process is repeated, successively correcting row, column and diagonal values until all ray sums computed from the estimated pixel values agree with all measured ray sums. 
well but take a long time to produce the image, partly because image reconstruction cannot start until all projection data has been acquired and partly because analytical techniques (sections 1.3.3 and 1.3.4) are much more efficient.

\section{Fourier Transform Technique}

This technique is rendered viable by the central section theorem, which is illustrated in figure 1.13. If a projection $\mathrm{g}(\mathrm{r}, \phi)$ is taken at an angle $\phi$ with respect to the $y$ axis, then the one dimensional Fourier transform of that projection is equal to the values of the (two dimensional) Fourier transform function $F(R, \phi)$ of the object along a line at the same angle in frequency space. This may be stated mathematically as follows:

$$
F(R, \phi)=\int g(r, \phi) \cdot e^{-i 2 \pi R r} \cdot d r
$$

where $\mathrm{R}$ and $\phi$ are the polar co-ordinates in frequency space. The Fourier transform

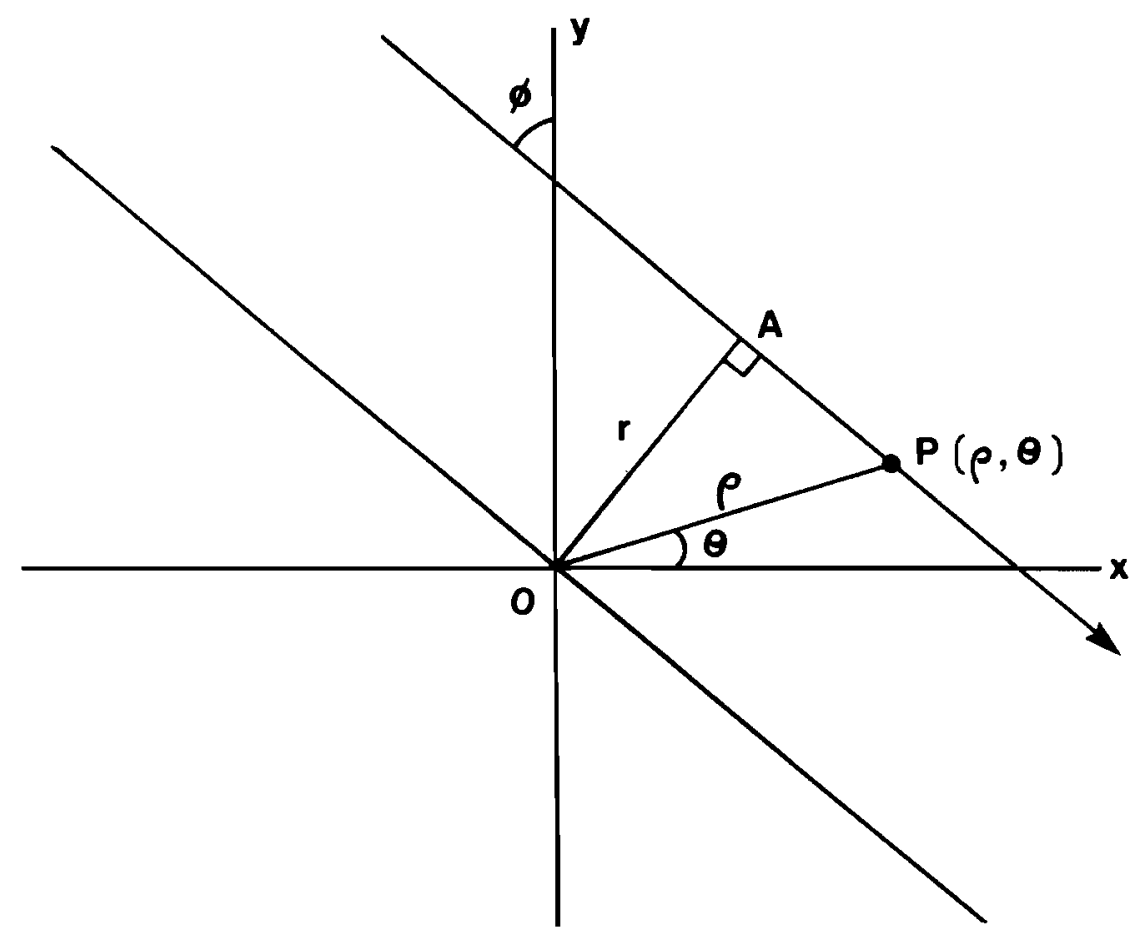

Figure 1.12: Co-ordinate System used for Analytical Reconstructions

Each ray is specified by its angle, $\phi$, with the $y$ axis; and by its perpendicular distance, $r$, from a parallel line through the origin. To find the equation of this line in terms of $\phi$ and $r$, define a general point on the line $P(\rho, \theta)$, specified in polar co-ordinates. Considering triangle OAP:

$$
\begin{aligned}
& \rho \cos (\phi-\theta)=r \\
& \Rightarrow \quad \rho(\cos \theta \cos \phi+\sin \theta \sin \phi)=r \\
& \Rightarrow \quad x \cos \phi+y \sin \phi=r
\end{aligned}
$$




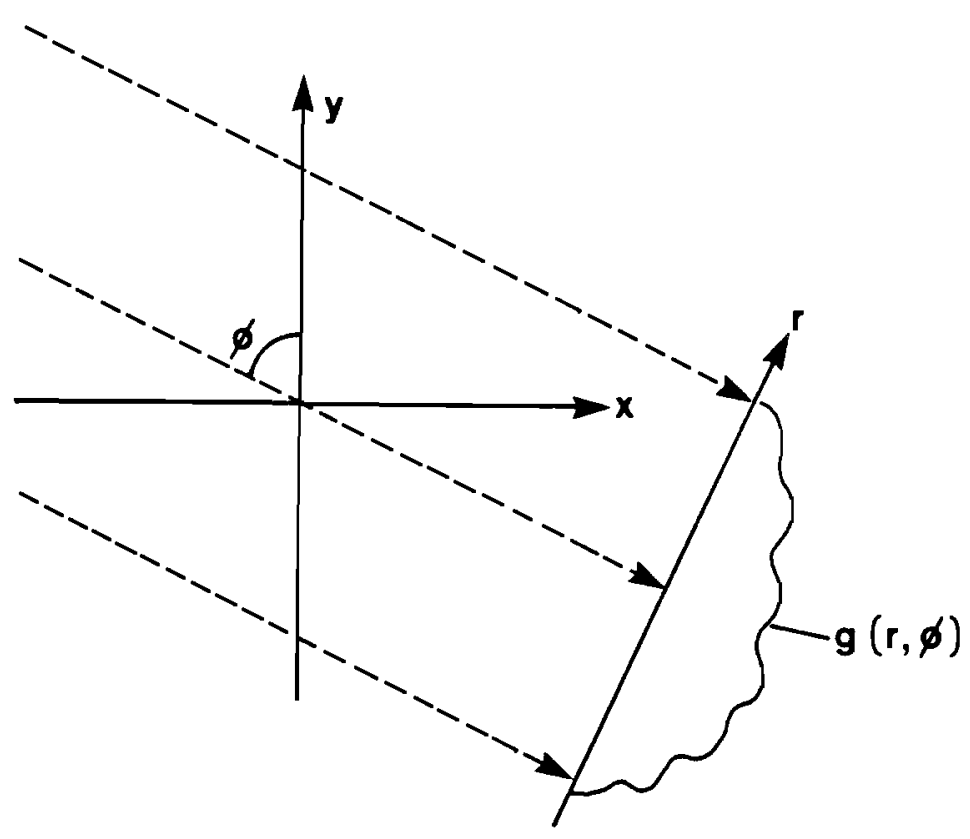

Real space

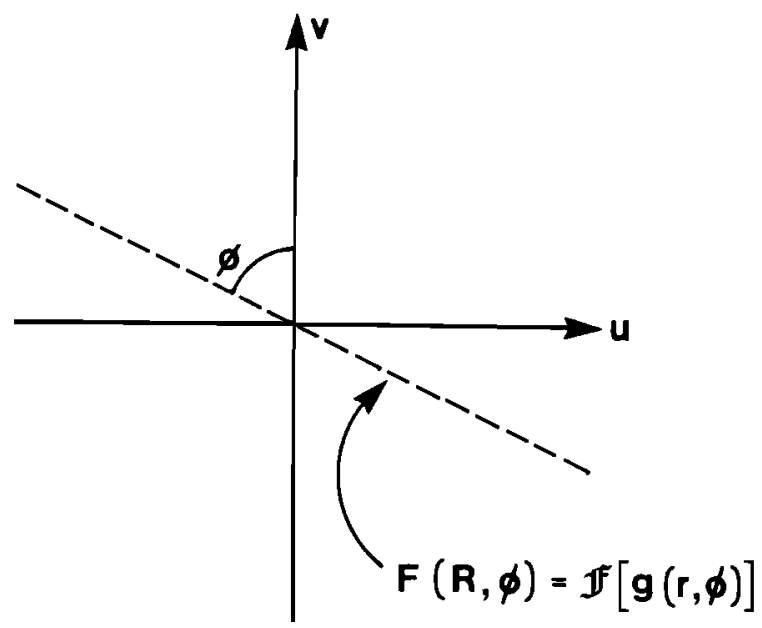

Frequency space

\section{Figure 1.13: The Central Section Theorem}

Function $g(r, \phi)$ is formed from the complete set of ray sums taken at angle $\phi$. This function is a projection of the two dimensional function representing the pattern of attenuation in the object being imaged. The Central Section Theorem states that the one dimensional Fourier transform of this function is equal to the component of the two dimensional Fourier transform of the attenuation function, along the same angle $\phi$.

function of the object can be built up from a set of projections at different angles, and the image obtained by inverse Fourier transformation. Exact solution by this means 


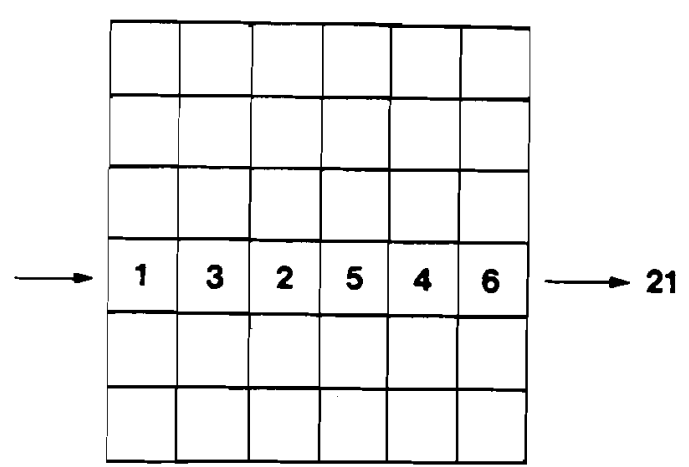

Forming the Ray Sum

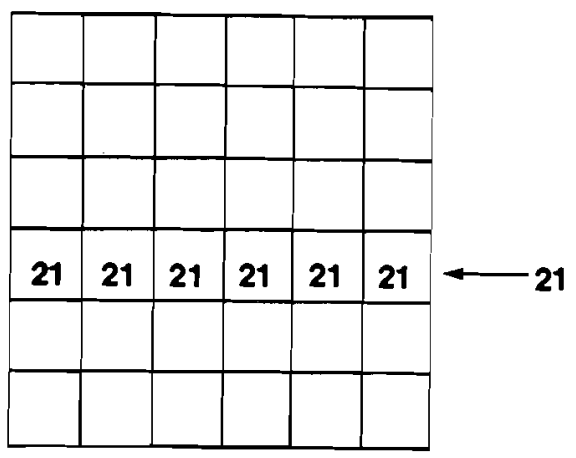

Back projection

Figure 1.14: Simple Back Projection

Iimple back projection involves assigning the full value of the ray sum to each pixel along ine ray.

Fould require an infinite number of projections, but if the image is bandlimited to a :ertain maximum spatial frequency, $f_{m}$, then it is only necessary to take sufficient Frojections to permit accurate interpolation at intervals $\frac{1}{2} \mathrm{f}_{\mathrm{m}}$ in accordance with the Vyquist sampling criterion.

Fourier reconstruction is potentially the fastest technique, but the two $\because$ imensional inverse Fourier transform requires greater computer capacity than is zormally available in a C.T. scanner.

\section{:3.4 Filtered Back Projection}

Simple back projection is a very crude reconstruction technique. It was applied in analogue form to the tomographic reconstruction of radioisotope distribution by Kuhl in 1963 [7], and in digital form for the same technique in 1968 [25]. In simple jack projection the value of each ray sum is back projected along the path of that ray as depicted in figure 1.14. Each pixel along the ray is assigned the value of the ray sum. To give this concept mathematical expression, the co-ordinate system illustrated in figure 1.12 is adopted. The back projected density $\mathrm{b}_{\phi}(\mathrm{x}, \mathrm{y})$ due to a projection $\mathrm{g}_{\phi}(\mathrm{r})$ at a particular angle $\phi$ is given by:

$$
b_{\phi}(x, y)=\int g_{\phi}(r) \cdot \delta(x \cos \phi+y \sin \phi-r) \cdot d r
$$


where the integration is performed over all rays at that angle. The delta function in equation (1.7) selects only those points along the ray which is at distance $r$ from the origin: see figure 1.12. Equation (1.7) back projects the ray sums for all rays at angle $\phi$. Complete reconstruction is obtained by performing this operation for all angles. The estimated attenuation coefficients, $f^{*}(x, y)$, are therefore obtained by integrating over $\phi$ :

$$
f^{*}(x, y)=\int_{0}^{\pi} d \phi \int_{-\infty}^{\infty} \underset{g}{(r, \phi)} \delta(x \cos \phi+y \sin \phi-r) \cdot d r
$$

Filtered back projection involves modification (filtering) of the projections $g(r, \phi)$ prior to back projection. In the rest of this section it is shown how to do this so that the reconstructions obtained are mathematically exact [26]. Using the central section theorem, $g(r, \phi)$ in equation (1.8) can be replaced by the inverse Fourier transform of the component of its frequency space representation, $F(R, \phi)$, at the same angle $\phi$ :

$$
f^{\star}(x, y)=\int_{0}^{\pi} d \phi \int_{-\infty}^{\infty}\left[\int_{-\infty}^{\infty} F(R, \phi) e^{i 2 \pi R r} \cdot d R\right] \delta(x \cos \phi+y \sin \phi-r) \cdot d r
$$

which following integration over $r$ becomes:

$$
f^{*}(x, y)=\int_{0}^{\pi} d \phi \int_{-\infty}^{\infty} F(R, \phi) \exp \{i 2 \pi R(x \cos \phi+y \sin \phi)\} \cdot d R
$$

This may be compared with the two dimensional inverse Fourier transform in polar coordinates [26], in which the true attenuation coefficient function $f(x, y)$ is expressed as the inverse Fourier transform of its frequency space representation $F(R, \phi)$ :

$f(x, y)=\int_{0}^{2 \pi} d \phi \int_{0}^{\infty} F(R, \phi) \exp \{i 2 \pi R(x \cos \phi+y \sin \phi)\} R \cdot d R$

It is clear that the two equations are of the same form. Changing the limits of the integrals in equation 1.11 to conform with those in equation 1.10 gives:

$f(x, y)=\int_{0}^{\pi} d \phi \int_{-\infty}^{\infty} F(R, \phi) \exp \{i 2 \pi R(x \cos \phi+y \sin \phi)\}|R| \cdot d R$

The above may be compared with equation (1.10) which is reproduced below, rewritten in terms of the ray sums $g(r, \phi)$ (central section theorem):

$f^{*}(x, y)=\int_{0}^{\pi} d \phi \int_{-\infty}^{\infty} \frac{f[g(r, \phi)]}{|R|} \exp \{i 2 \pi R(x \cos \phi+y \sin \phi)\}|R| \cdot d R$ 
where $f$ denotes the Fourier transform operation. Comparison of the expression for the back projected estimate of the attenuation coefficients, $f^{*}(x, y)$, in (1.13) with the true attenuation coefficients, $f(x, y)$, in equation (1.12) makes clear the errors involved in simple back projection. Simple back projection gives an image in which the components at each spatial frequency are reduced by the modulus of that frequency. A mathematically exact reconstruction can therefore be obtained if the one dimensional Fourier transforms of the raw projections are obtained, the frequency components increased by the modulus of their spatial frequencies, the inverse Fourier transform is taken and the result back projected according to equation (1.8). This method does not require two dimensional Fourier transformation, and permits spatial smoothing or enhancement to be carried out by variation of the filtering function [26].

The necessity for Fourier transformation can be avoided altogether by making use of convolution techniques [27, 28]. The convolution property of Fourier transforms states that multiplication in Fourier space is equivalent to convolution in real space [29]:

$f^{-1}\left[F_{1}(u, v) \times F_{2}(u, v)\right]=\int_{-\infty}^{\infty} \int_{-\infty}^{\infty} f_{1}(x, y) f_{2}(x-\alpha, y-\beta) \cdot d \alpha \cdot d \beta$

where $\mathrm{u}$ and $\mathrm{v}$ are spatial frequencies in the $\mathrm{x}$ and $\mathrm{y}$ directions respectively, and $F_{1}(u, v)$ and $F_{2}(u, v)$ are the Fourier transforms of functions $f_{1}(x, y)$ and $f_{2}(x, y)$. Convolution is defined by the expression on the right hand side of the equation. In this case the function to be back projected can be obtained as:

$$
f^{-1}[f[g(r, \phi)] \cdot|R|]=g(r, \phi) * f^{-1}[|R|]
$$

where the symbol * is used to denote the convolution operation. The quantity $f^{-1}[|R|]$ is called the convolution kernel. The inverse Fourier transform of $|R|$ is not defined since the function is not integrable [26]. However by invoking the assumption of a bandlimited signal it is possible to find integrable functions which approximate $|R|$ up to the frequency limit of the system. In practice different convolution kernels are used, each involving the product of $|R|$ and some high frequency cutoff filter. Sharp cutoff results in ringing via the Gibbs phenomenon [29], which results in edge enhancement in the image. More gradual cutoff will result in some degree of smoothing. It is common for manufacturers to give the users of C.T. scanners some control over which kernel is used, depending on whether high contrast resolution or low contrast resolution is of greatest importance in a particular clinical situation. 


\subsection{Image Display}

The reconstructed image is stored in the computer as a two dimensional array of attenuation values. This image must be presented to the doctor performing the diagnosis (the medical specialty concerned with the production of diagnoses from images is radiology, its practitioners being called radiologists) as clearly and simply as possible so that he may concentrate on interpretation. The normal presentation of a C.T. image is as a black and white image on a cathode ray tube, having the advantage compared with an X-ray film of permitting the contrast and brightness of the image to be changed at will. Other methods exist which permit more quantitative use of the attenuation values.

\subsubsection{The C.T. Number Scale}

Most C.T. scanners operate at $120 \mathrm{kVp}$ with heavy filtration so that the effective energy of the beam is about $73 \mathrm{keV}$ for water. The attenuation coefficient for water at this energy is $0.910 \mathrm{~cm}^{-1}$, a number which has little meaning for a radiologist. Instead the attenuation values presented to the radiologist are expressed on a scale relative to water:

$$
\mathrm{H}=\mathrm{K} \cdot\left[\frac{\mu-\mu_{\mathrm{W}}}{\mu_{\mathrm{W}}}\right]
$$

$$
\text { where } \begin{aligned}
& \mathrm{H}=\mathrm{C} \cdot \mathrm{T} \text {. number } \\
& \mu_{\mathrm{H}}=\text { measured attenuation coefficient. } \\
& \mu_{\mathrm{W}}=\text { attenuation coefficient for water, } \\
& \quad \text { measured under the same conditions. }
\end{aligned}
$$

The scale factor $\mathrm{K}$ is normally 1000 so that each Hounsfield unit represents an attenuation difference of $0.1 \%$ relative to the attenuation of water.

\subsubsection{Analogue Display Methods}

In principle, display of a digital image consists simply of displaying pixels with large attenuation values in lighter shades of grey and representing low attenuation pixels by darker shades. A normal display system will be capable of displaying between 16 and 64 different shades of grey. However attenuation values in a patient may vary from - 1000 Hounsfield units for air to in excess of 3000 Hounsfield units for very dense bone, with the vital soft tissue information occurring between 0 and 100 . It is therefore impossible to display all the relevant information simultaneously and so it 


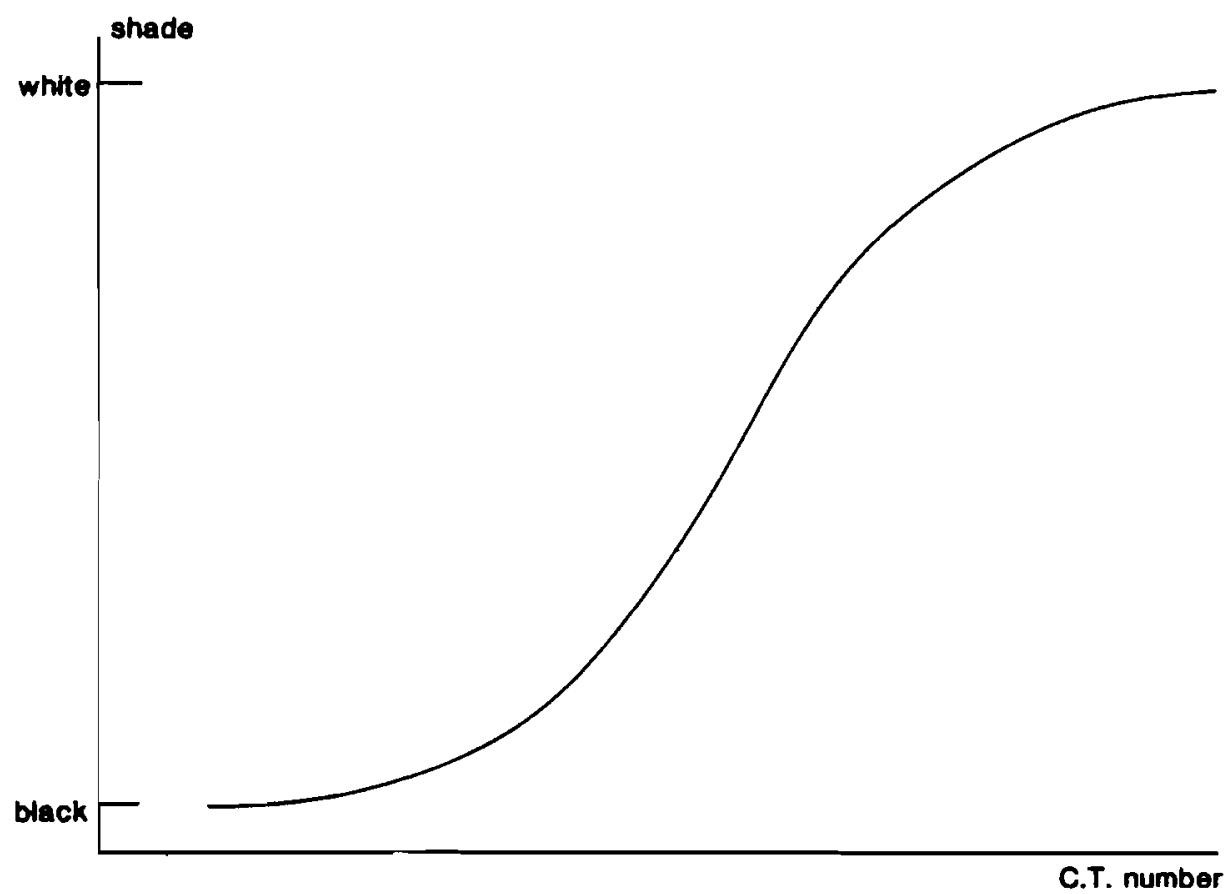

Nonlinear Windowing

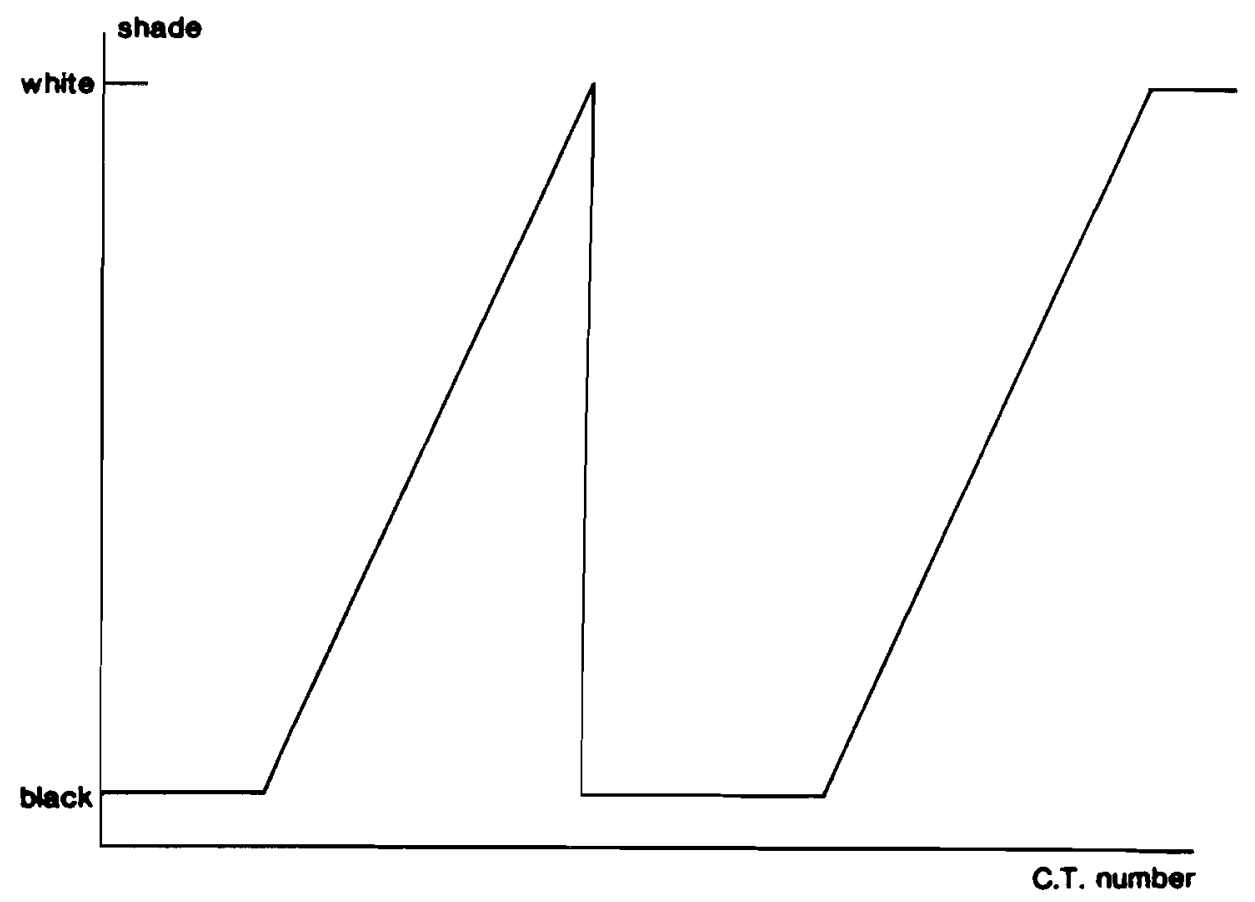

\section{Dual Windowing}

Figure 1.15: Special Windowing Methods.

In nonlinear windowing the contrast is reduced at the two extremes of the brightness scale, permitting a larger range of C.T. numbers to be displayed while retaining full contrast in the central portion of the curve. Ordinary C.T. images of the chest cannot normally show both lung detail and the surrounding tissues because lung tissue is very much less dense. However the fact that there is no overlap in the C.T. number ranges of the two types of tissue permits the full grey scale to be used twice without ambiguity. 
is necessary to use a windowing technique where the entire greyscale is used to display only a limited range of C.T. numbers. The C.T. number interval is referred to as the window width and the C.T. number associated with mid grey is called the window level. Subjectively, adjusting the window width has the effect of altering image contrast while the window level control has the effect of varying brightness. Various attempts have been made to permit simultaneous display of as much information as possible. One such is the use of nonlinear windowing (see figure 1.15) in which the central portion of the grey scale is related to the C.T. numbers in the usual way, but the C.T. scale is compressed at the two extremes of the grey scale. The central section is set to display normal soft tissues, the dark end giving a compressed record of structures containing air and the light end displaying bone, again at reduced contrast. For imaging lungs a technique called dual windowing is used where the entire grey scale is used twice, once to display the range of densities in the lungs themselves and once to display the surrounding soft tissues. Thus each shade is used to represent two very different densities, but the ambiguity does not normally cause problems because the areas represented are quite distinct. There is normally a slight transition effect at the interfaces.

Some C.T. scanners are equipped with colour monitors which give a much more impressive display, but do not add to the amount of information present. Where different shades of grey were previously used to display different ranges of C.T. numbers, colour displays use different colours to achieve the same effect. Such displays have the advantage of better displaying small differences in C.T. number, but have the disadvantage that it is not immediately obvious whether a given change in colour represents an increase or decrease in attenuation value.

A C.T. examination usually consists of one or more sets of contiguous axial slices (ie taken normal to the long axis of the body). Sometimes the required information is better displayed in other planes. Images in other planes can be synthetically obtained from sets of axial slices by reformatting the data. For example a synthetic coronal slice may be obtained by extracting from the set of axial slices all the pixels in the required coronal plane. Moderately good images may be obtained in this way if the original axial slices are of small width, which in turn will require a large number to be taken with a long examination time.

Problems of interpretation occur when examining areas in the image which are surrounded by large areas of bone. As the bone is imaged as white (or clear on a film) it is possible for the radiologist's eyes to become dazzled (or at least tired) by the surrounding light areas. Consequently some scanners make provision for temporarily changing the bone to some more neutral tone of grey while the critical area is being assessed. Software has been written to perform this operation on images from the 
Wellington Hospital C.T. scanner [30].

Conversion of the digital image to a video waveform for C.R.T. display is frequently performed by a specialised graphics computer. A permanent image may be obtained by photographing the display with a polaroid camera, or more commonly by using a multiformat camera. Images may be stored electronically either on magnetic :ape or on floppy discs.

\subsubsection{Quantitative Measurements}

C.T. images permit very accurate measurements of distances and organ sizes, unaffected by magnification as is the case in conventional radiography. Such accuracy permits accurate biopsies to be performed using stereotactic techniques [31, 32]. A stereotaxis technique has been successfully developed at Wellington Hospital [33]. Other quantitative techniques involve C.T. numbers. Individual pixel values can be printed on a line printer, and the calculation of mean C.T. number and standard deviations in selected areas are standard functions available on most systems. Additionally some systems will produce histograms of the pixel values in a selected area [34], and some will produce graphs of C.T. numbers along a line in the image [35]. A graph plotting program has been devised for the G.E. 8800 at Wellington Hospital [36].

Further quantitative techniques may involve the use of dual energy techniques to measure such quantities as effective atomic number and electron density (see chapter 3 ), and the measurement of bone mineral content (see chapter 4).

\subsection{C.T. Image Quality}

As with any imaging procedure using ionising radiation, a C.T. examination carries with it a certain risk (carcinogenesis, genetic effects plus side effects from any contrast agent used $[37,38]$ ) which must be balanced against the possible benefits, mainly a more accurate diagnosis leading to more effective treatment. The accuracy of the diagnosis will be affected by the quality of the images obtained, but it is important to distinguish between the quality of the image per se, and the value of the information that the image conveys [10]. For example the high contrast resolution of a C.T. image is inferior to that of a conventional radiograph, but the C.T. image is likely to have superior information content since the image is not a projection and does not suffer from problems of superimposition. The C.T. scanner can be viewed as made up of three subsystems: data acquisition, image reconstruction and display. Image 
quality depends on all three, but it should be noted that no one system can make up for deficiencies in either of the other two. Thus the display system must be adequate to display the detail present in the image, but beyond that point further improvement in the display system will not improve the image.

Perhaps the most common parameter used to express image quality is image resolution, but this parameter varies according to the conditions under which it is measured. High contrast resolution (sometimes called spatial resolution) is defined as the resolution in the absence of noise, or in the limit where the noise is insignificant in comparison with object contrast. Low contrast resolution refers to the situation where resolution is noise limited due to the small degree of contrast present in the image.

\subsubsection{Image noise}

Noise may be defined as variation in reconstructed attenuation values which does not reflect true variations in the object being imaged [10]. Such variations may be due to random fluctuations in the numbers of detected photons ("quantum mottle") or to such system variables as detector drift or voltage ripple in power supplies ("system noise"). Quantum mottle is inversely related to the square root of the number of photons detected. In theory this mottle may be reduced by increasing the photon flux, but in practice the extent to which this may be achieved is limited by the heat capacity of the X-ray tube used and by the necessity to keep the dose to the patient as low as possible. In a well designed C.T. scanner other sources of noise should be small compared with the quantum mottle.

The amount of noise present in an image is expressed in terms of the standard deviation of C.T. numbers on an image of a uniform phantom (ie no subject variation). The noise level is influenced by the X-ray photon flux, and by the degree of attenuation the beam experiences before reaching the detectors. Factors affecting the $X$-ray photon flux are peak kilovoltage, tube current, exposure time and slice width. In a pulsed system the exposure time is calculated as the number of X-ray pulses per image times the length of each pulse. The slice width affects the photon flux since collimation is effected by removing from the beam those photons lying outside the desired beam profile. Factors affecting $\mathrm{X}$-ray transmission are phantom or patient composition and size, and losses in the table and detector collimators.

The presence of noise has the effect of interfering with the perception of low contrast objects in the image, however the eye is capable of smoothing out noise to a certain extent so that larger low contrast objects may still be perceived. For a given level of contrast there is a limit to how small an object can be and still be detected. The smaller the object is, the greater is the level of contrast required for the object to be 
visible on the image. Provided that all other factors remain constant, decreasing pixel size (increasing the total number of pixels making up the image) results in less information being used to calculate the value of each pixel (ie fewer photons) and increased noise present in the image as a whole. The increase in noise is inversely proportional to the pixel size raised to the power of $3 / 2$ [39].

System noise (as distinct from noise due to quantum mottle) may be estimated by averaging several images of a uniform phantom. The resulting systematic noise "image" may be stored in the system ("calibration") and is subtracted from each image before display [10].

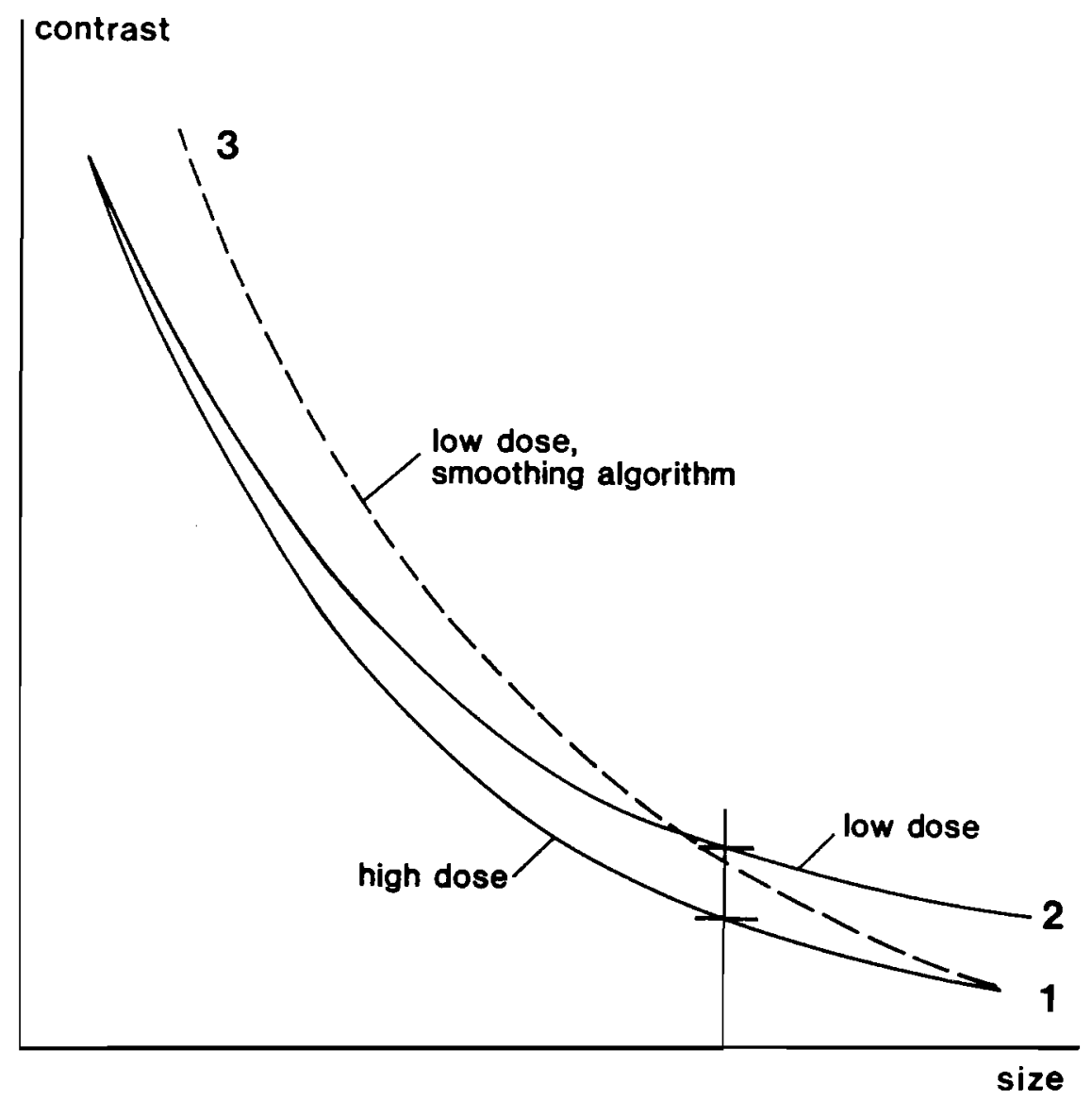

Figure 1.16: Contrast-Detail-Dose Relationship.

Object contrast is plotted against the smallest resolvable object size. Curve 2 represents the response of the same scanner as curve 1, but at a lower dose. At a given dose, the visibility of an object depends on both its size and the degree of contrast between the object and its background. Increasing the dose will permit visualisation of an object of a given size at a lower contrast level.

Curve 3 represents the response at the dose level of curve 2, but using a smoothing algorithm. The visibility of low contrast objects is improved, but the resolution for high contrast objects is reduced. 


\subsubsection{Low Contrast Resolution}

Sometimes simply called contrast resolution. Under low contrast conditions the smallest sized object which may be resolved depends on the X-ray dose and the degree of contrast between the object in question and its background. This information is usually presented as a contrast-detail-dose curve: see figure 1.16. Image contrast (expressed in Hounsfield units) is plotted on the ordinate and the minimum sized object which may be resolved at a particular level of contrast is plotted as the abscissa. Different curves will be obtained depending on the reconstruction algorithm used and on the dose. In the figure curves 1 and 2 represent results obtained using the same algorithm but differing doses, the dose for curve 2 being lower. As would be expected, there is little difference at high contrast levels, but when the contrast is low it can be seen that at low dose (curve 2) an increase in contrast is required in order to be able to resolve objects of the same size as may be resolved using the higher dose (curve 1). Curve 3 represents an algorithm which has a greater degree of smoothing than that of curves 1 and 2, at the same dose level as for curve 2. Consequently it has lower high contrast (spatial) resolution but superior low contrast resolution for a given dose. Image smoothing may also be performed after reconstruction [47]. Suitable software has been written for the Wellington Hospital scanner [30].

Low contrast resolution may also be expressed as a contrast-detail-dose constant. This may be defined in slightly different ways [39, 40], but the simplest approach is to multiply the degree of contrast (expressed as a percentage of the attenuation of water) by the minimum size of object which may be resolved at that contrast level and by the square root of the dose [41].

\subsubsection{High Contrast Resolution}

When the image noise is small compared with the contrast present in the image, factors other than noise determine the resolution of the image. Aspects of the data acquisition step influencing spatial resolution are the sampling frequency (ie the number of projections obtained per revolution of the tube and detectors) and the sampling aperture. The effective sampling aperture in turn depends on tube focal spot size, detector size, detector collimation, averaging due to scan motion and averaging due to detector lag [2]. The type of reconstruction algorithm used influences the spatial resolution, as discussed in section 1.3.4. Algorithms which give best spatial resolution do so at the expense of increased image noise and consequent loss of low contrast resolution. Where resolution tends to be limited by the size of the display matrix, it is possible to improve matters using "zoom" reconstruction techniques where only part of the scan field is reconstructed and 
displayed. This permits the display of a smaller area of the patient over the full size of the display system resulting in a smaller effective pixel size. Enhancement of image detail can also be performed after reconstruction [48], and a suitable routine has been written in Wellington [30].

Several different methods exist for measuring the high contrast resolution of a C.T. scanner. The simplest method is to scan a phantom containing rows of holes of differing diameters, or a phantom containing bars of differing widths and spacings. The resolution may then be expressed in terms of the size of the smallest hole which may be resolved, or in the case of a bar phantom the number of line pairs per centimeter. A line pair is defined as a line or bar and its accompanying space. The width of the space is always the same as that of the bar. These measures of system resolution are necessarily subjective, depending on the visual acuity and judgement of the observer. The perceived resolution will also depend on the viewing conditions set (window width and level) and on the level of ambient lighting [42, 43]. Resolution may alternatively be measured as the degree of blur present, as determined by such measures as the point spread function or the line spread function. However a more general measure of system resolution is the modulation transfer function (M.T.F.) [44 - 46]. The M.T.F. gives an objective indication of how subject contrast (ie in the object being imaged) is manifested as image contrast, as a function of spatial frequency.

\subsubsection{Slice Width}

An important feature of the C.T. scanner is the ability to image a "slice" of the patient. The slice width may in a sense be viewed as the resolution along the ' $\mathrm{Z}$ ' (or long) axis of the patient. Ideally a C.T. scanner would be equally sensitive to objects at any position along the $Z$ axis up to the nominal slice width, and insensitive to objects lying beyond these limits. In fact some rounding of the response curve (the "sensitivity profile") occurs so that the sensitivity to objects lying close to the slice boundaries is less than in the middle of the slice. The sensitivity profile is determined by the dose profile within the patient and by any detector collimation present. The slice thickness will vary with the distance from the isocentre of the gantry $[47,48]$.

\subsubsection{Image. Artefacts}

Image artefacts (alternatively spelt artifacts in some texts) may be defined as structures in the image which do not faithfully reflect the object imaged. In C.T., artefacts frequently appear as streaks on the image. Such artefacts may be caused by patient motion during the scan, by a flash over in the X-ray tube, by the presence of 
metallic objects such as surgical clips, or by the beam hardening effect. Streaking via the beam hardening effect often represents failure of the beam hardening correction due to the presence of an abnormally large amount of bone. A classic example of this is the "Hounsfield dark space" between the petrous bones [10]. A single circular artefact indicates failure of a single detector or its associated electronics, whilst concentric rings can result from a "microphonic" effect where vibration from the tube is transmitted to the Xenon detector causing changes in the effective size of each detector element.

\subsection{Reprise}

The production of a C.T. image involves three key steps: the acquisition of projection data by making several measurements (at several angles) of the transmission of $\mathrm{X}$-rays through the object to be imaged, the use of mathematical algorithms to operate on this data to reconstruct the distribution of $X$-ray attenuation within this object, and suitable hardware and software to produce images from this reconstructed information. Sensible use of quantitative information from C.T. scanning requires intimate knowledge of each of these three processes. In depth knowledge of the image acquisition process is necessary so that the departures from idealised behaviour are known, principally the problems associated with beam hardening. Awareness of the reconstruction process provides insight into methods by which the scanner reduces the impact of these inherent limitations on the quality of the final image produced. Knowledge of the display process facilitates the extraction of quantitative information from images, and permits the display of quantities derived from one or more raw C.T. images.

In the next chapter the relationship between the Hounsfield number and the X-ray attenuation coefficients of tissue is discussed, and in chapter three the use of dual energy techniques to produce estimates of more fundamental tissue properties is described. The production of dual energy images in Wellington has involved non-standard use of the G.E. 8800 scanner which is intended for used at $120 \mathrm{kV}$ only. The discussion on image quality in this chapter provides a platform on which to base the image quality tests contained in chapter three which establish that performance at the lower kilovoltage ( $80 \mathrm{kV}$ in this case) is at least comparable with that at $120 \mathrm{kV}$. 


\section{CHAPTER TWO: X-RAY ATTENUATION COEFFICIENTS}

\subsection{Definition of the Linear Attenuation Coefficient.}

The attenuation of an X-ray beam is found to follow an exponential relationship:

$$
I=I_{0} \cdot e^{-\mu \cdot t}
$$

where $I$ is the intensity of the transmitted beam, $I_{0}$ is the intensity of the incident beam, $t$ is the thickness of the absorbing medium and $\mu$ is a parameter defined by this equation called the linear X-ray attenuation coefficient. The above equation is an idealisation based on the assumption of a monochromatic beam, that is, one in which all the photons have the same energy. Further, equation (2.1) is strictly true only for a pencil beam and for a detector collimated so that no scattered radiation is detected.

The linear attenuation coefficient is found to be dependent on three factors: the density of the attenuating medium, its atomic number, and the energy of the photons in the beam. There is no simple relationship between the linear attenuation coefficient and either atomic number or photon energy, and the relationships vary according to the attenuation processes involved. The linear attenuation coefficient is found to be directly proportional to density, independent of the attenuation process, so a mass attenuation coefficient may be defined which is the linear attenuation coefficient divided by the density. The mass attenuation coefficient is then independent of temperature, since the temperature dependence of the linear attenuation coefficient results solely from the temperature dependence of the density. The mass attenuation coefficient is related to the atomic cross section, $\sigma_{\mathrm{a}}$, in barns per atom as follows [49]:

$$
\frac{\mu}{\rho}=\sigma_{\mathrm{a}}(\mathrm{b} / \mathrm{a}) \times \frac{\mathrm{N}_{\mathrm{A}}}{\mathrm{A}} \times 10^{-24}
$$

where $\rho$ is density, $\mathrm{N}_{\mathrm{A}}$ is Avogadro's number $\left(=6.022045 \times 10^{23}\right)$ and $\mathrm{A}$ is the atomic weight.

\subsection{The Relationship to the Hounsfield Number.}

The tissue property measured by the C.T. scanner is the C.T. number, defined by:

$$
H=K \cdot\left[\frac{\mu-\mu_{W}}{\mu_{W}}\right]
$$


where $H=C . T$. number, $\mu=$ measured attenuation coefficient, and $\mu_{W}=$ attenuation coefficient for water, measured under the same conditions. The factor $K$ is a scaling factor which in the early C.T. scanners was set to 500, but in most modern scanners is set to 1000 . The term E.M.I. scale is used to denote systems where the factor $\mathrm{K}$ is 500 (named after the original scanners which were manufactured by E.M.I. Ltd.) whereas the term Hounsfield unit is applied exclusively to systems where a factor of 1000 is used.

It is important to note that since different C.T. scanners will not produce the same $\mathrm{X}$-ray spectrum, measurements on the same material using different scanners will not necessarily agree. Provided that the relationship between Hounsfield number and linear attenuation coefficient is known for a given scanner, then if the linear coefficients of both normal and diseased tissues are known it ought to be possible to diagnose abnormalities on the basis of the Hounsfield number of the tissues. This in turn implies a knowledge of the compositions of normal and abnormal tissues, and the ability to calculate the linear attenuation coefficient from the composition.

\subsection{Attenuation Processes in the Diagnostic Energy Range.}

The upper limit of photon energy in the spectra produced by diagnostic X-ray equipment is determined by the maximum peak kilovoltage used by such equipment and is usually taken to be $150 \mathrm{keV}$ [50]. The lower limit is determined by both kilovoltage and tube filtration and is sometimes taken to be $30 \mathrm{keV}$ although there may be a small output at energies as low as $10 \mathrm{keV}$ for lightly filtered beams [51]. In the present work the lower limit will be taken to be $20 \mathrm{keV}$. The range so defined is sufficient to encompass general radiography as well as computed tomography, but does not include the full range of energies encountered in mammography.

Three attenuation processes are of significance in the diagnostic energy range: coherent scatter, the photoelectric effect and Compton scatter [16]. The relative importance of the three processes for human soft tissues (that is, tissues other than bone) may be assessed by examining the proportion that each contributes to the total attenuation coefficient of oxygen since soft tissues have a similar effective atomic number [52]. Coherent scatter is sometimes neglected in calculations on the grounds that it contributes only a small proportion of the total attenuation coefficient in the diagnostic energy range, however for oxygen it contributes more than $5 \%$ of the total up to $80 \mathrm{keV}$, and contributes a greater proportion than the photoelectric effect above about $60 \mathrm{keV}$. As these figures are much greater than the precision of a C.T. scanner the contribution of coherent scatter cannot be neglected in the present work. 


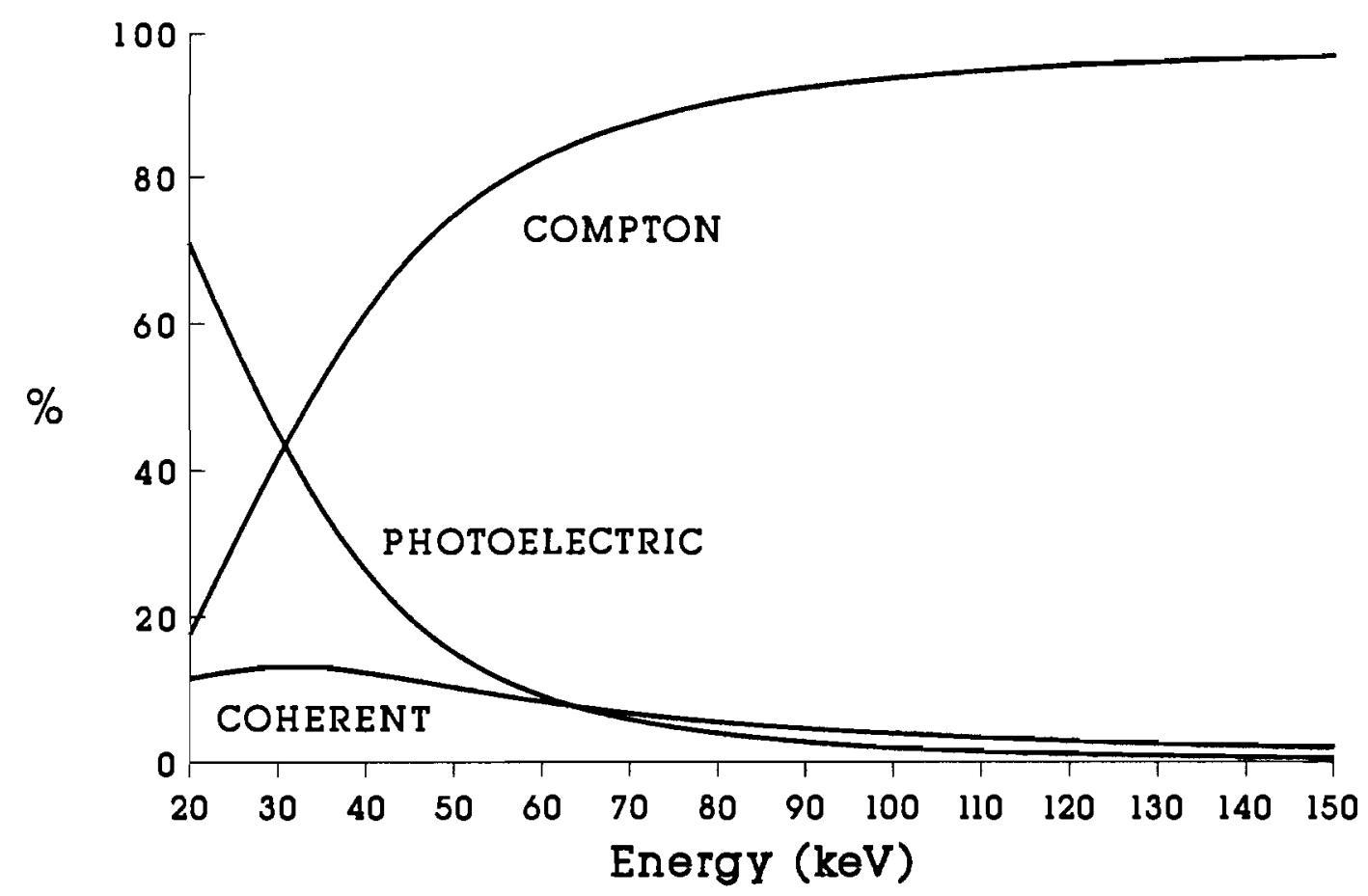

Figure 2.1: Relative Importance of Attenuation Processes for Oxygen.

The term coherent scatter is used to describe those attenuation processes in which there is phase coherence between the incoming and outgoing photon. Such processes are elastic: there is no transfer of energy to the medium. Coherent scatter occurs when the energy of the incoming photon is less than the binding energies of the atomic electrons. The interaction may be regarded as taking place between the photon and a single electron (Thomson scattering) or multiple electrons (Rayleigh scattering) [3].

The photoelectric effect is an interaction between the incoming photon and a single atomic electron, and occurs when the energy of the photon exceeds the binding energy of the electron. The photon gives up all of its energy to the electron, which then has a kinetic energy equal to the difference between that of the incident photon and the binding energy of the electron. When the ejected electron is from an inner orbit the vacancy will be filled by the transition of an outer electron with the emission of a characteristic X-ray photon whose energy is equal to the difference in the binding energies of the two orbitals involved. Alternatively the excess energy may result in the ejection of another outer shell electron (Auger electron). The photoelectric process is most probable when the photon energy does not greatly exceed the binding energy of the electron involved. In human soft tissues, which are principally composed of the elements $\mathrm{H}, \mathrm{C}, \mathrm{N}$ and $\mathrm{O}$, photoelectric interactions in the diagnostic energy range usually involve inner shell electrons. 
Compton scatter is most probable when the photon energy greatly exceeds the binding energy of the electron with which it interacts, so that the electron may be regarded as being essentially unbound. There is a transfer of energy to the ejected electron, and the emission of a scattered photon with lower energy than that of the incident photon. In human tissue such collisions usually involve outer shell electrons when the $\mathrm{X}$-rays are in the diagnostic energy range.

\subsection{Comparison of Published Attenuation Coefficient Data.}

Several published tabulations of X-ray attenuation coefficients are available. Some are based on theoretical calculations, some are from a review of published experimental data, and some are based on theoretical formulae in which some constants are adjusted to give better agreement with experimental values. In figure 2.2 comparison is made between attenuation coefficients for oxygen, taken from several sources, over the diagnostic energy range. Since differences in attenuation of a few tenths of $1 \%$ are of significance in C.T. scanning, this being approximately the difference in attenuation between cerebral white and grey matter, a simple graph of attenuation coefficients would be not suitable for displaying the differences. Instead a set of attenuation values has been selected as a reference, and the other tabulations are plotted as percentage differences with respect to the reference values. In the present work "best" theoretical values are used as the basis for comparison. These values were obtained by adding together photoelectric cross sections taken from Scofield [53], Compton scatter cross sections from Hubbell et al [54] and coherent scatter cross sections from Hubbell and Overbo [55]. The selection of these sources follows that of Jackson and Hawkes [50] who selected these authors as providing the best current theoretical values, with the exception that the coherent cross sections are taken from a more recent work by the authors that they recommend [54].

It can be seen from figure 2.2 that the greatest differences between tabulations occur in the lower portion of the diagnostic energy range where the photoelectric cross section forms a large proportion of the total. The coefficients of Veigele [57] are based on polynomial fits to published experimental data, but the other coefficients are theoretically based with some constants adjusted for better agreement with experiment. Values for oxygen published by Hubbell in 1977 [59] are substantially the same as those published by the same author in 1982 [58] and are not plotted separately. The experimental values plotted exhibit a somewhat different trend from the others in the region in which the transition occurs from predominantly Compton attenuation to predominantly photoelectric attenuation. 


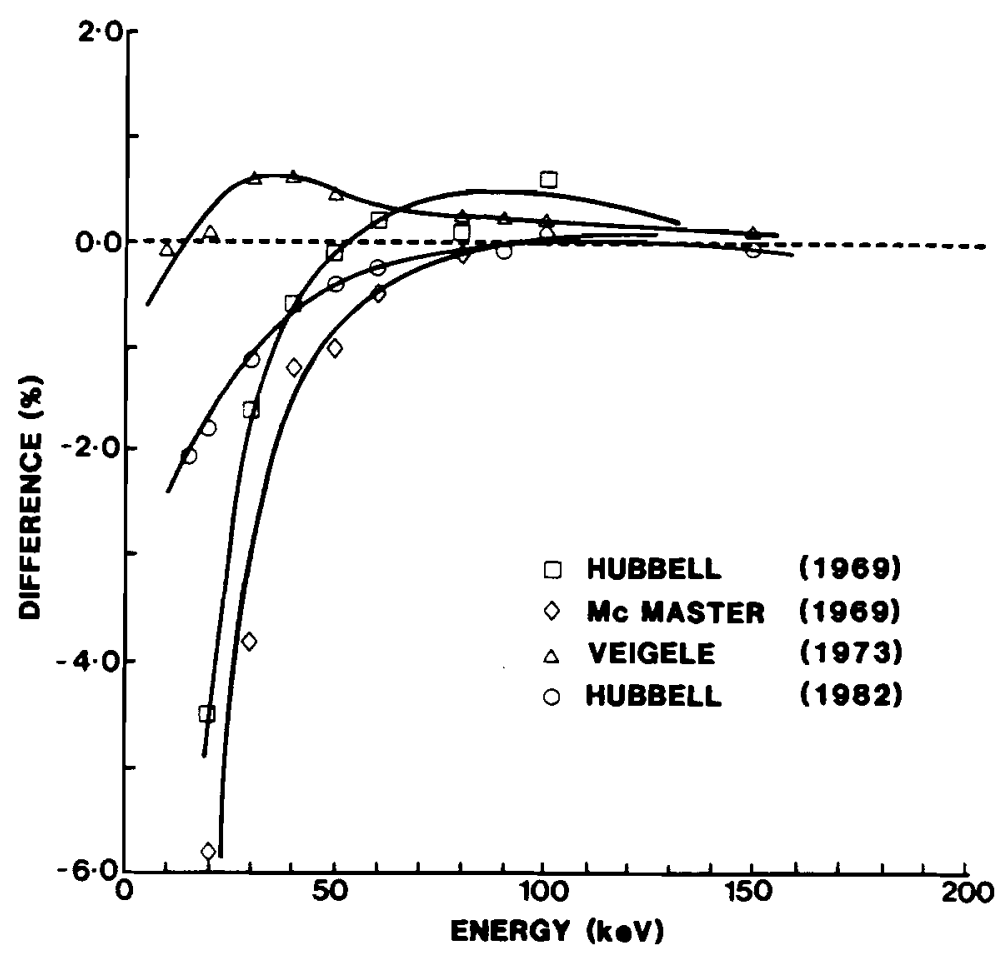

Figure 2.2: Comparison of Tabulated Attenuation Coefficients for Oxygen.

The data of Hubbell [49], McMaster [56], Veigele [57] and Hubbell [58] are compared by plotting percentage differences from "best" theoretical values (see text).

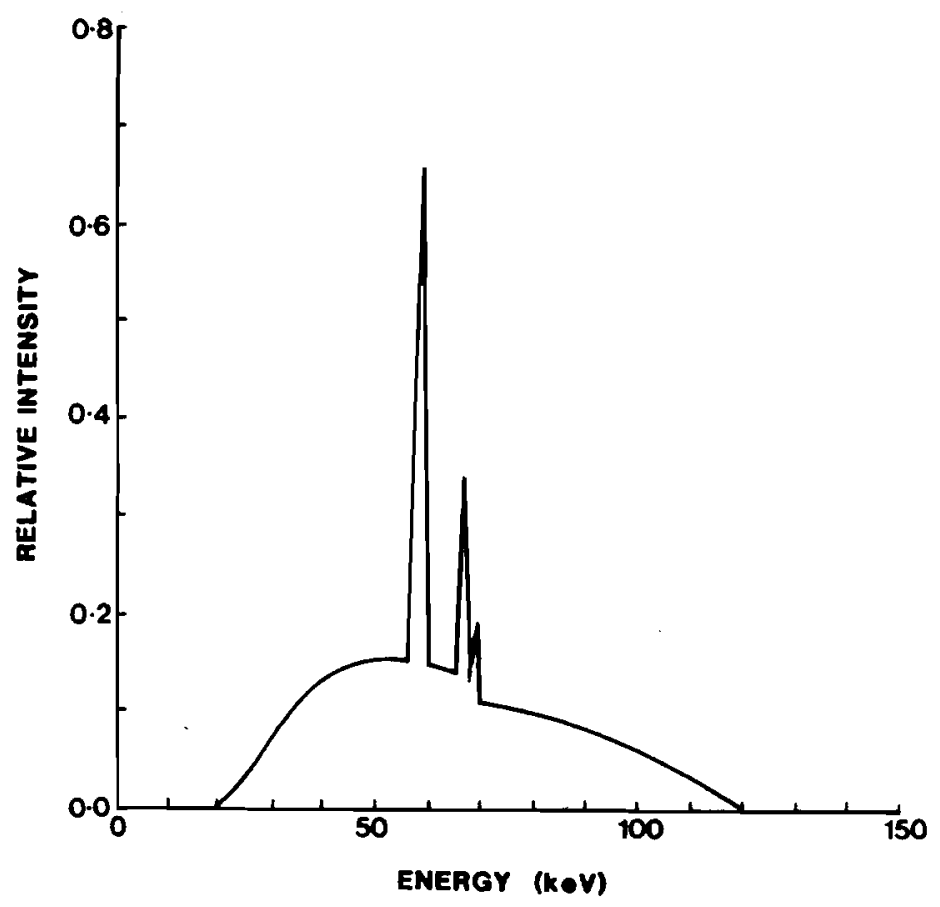

Figure 2.3: Typical Constant Potential X-ray Spectrum Generated at $120 \mathrm{kV}$. 
Since a range of photon energies is present in the X-ray beam used in the C.T. scanner, the significance of the differences in attenuation coefficient can only be assessed with reference to the $\mathrm{X}$-ray spectrum of the scanner. Figure 2.3 shows the intensity spectrum for a constant potential generator operated at $120 \mathrm{kV}$ with a total filtration of $4 \mathrm{~mm}$ aluminium. This spectrum was obtained from a catalogue of spectra published by Birch and Marshall [51], and is similar to the spectra of many C.T. scanners. The peak intensity of the continuum is at $52 \mathrm{keV}$, an energy at which there already significant differences between published attenuation coefficients.

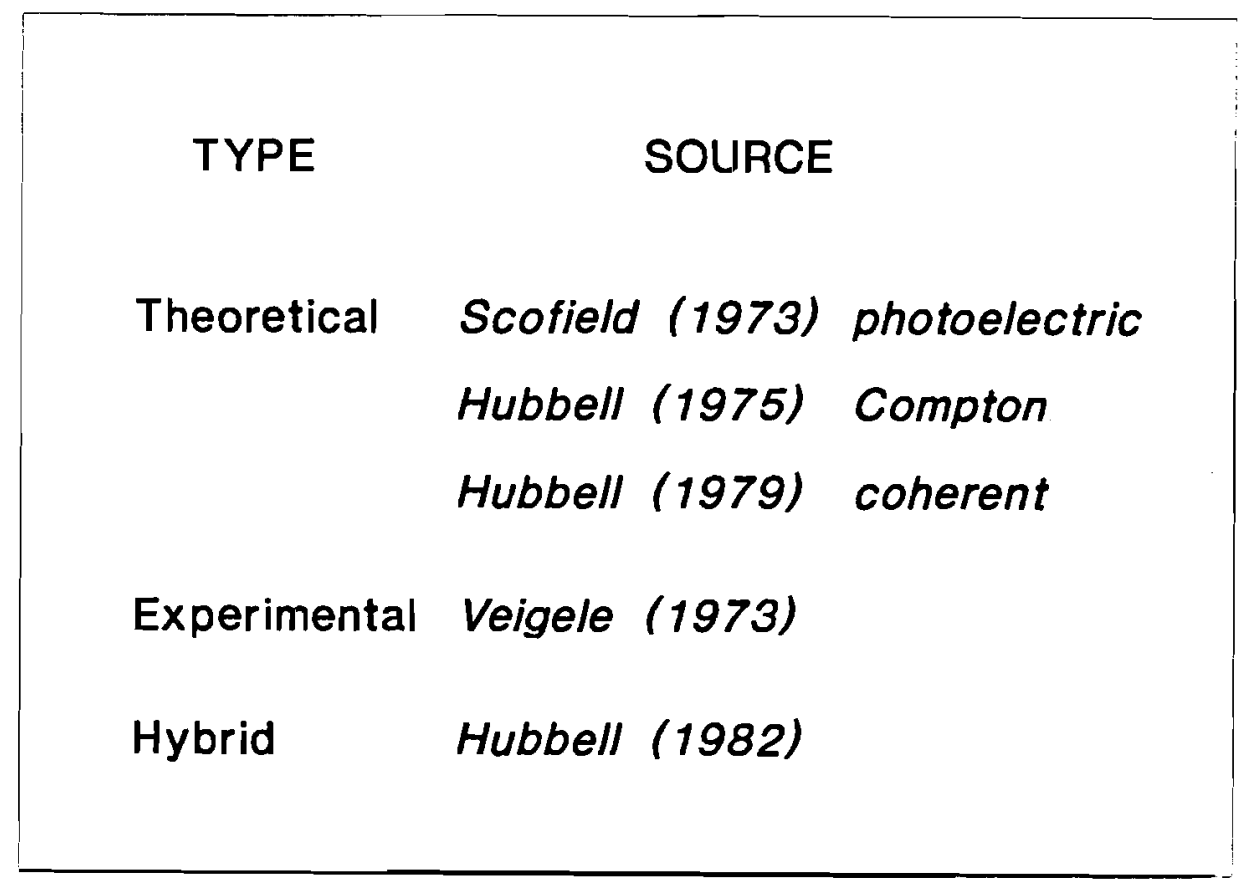

Figure 2.4: Sources of Attenuation Coefficient Data.

Confining attention to the more recent tabulations, in figure 2.5 comparisons are made between the data of Hubbell (1982) [58] and Veigele (1973) [57] with respect to the theoretical base for a number of elements up to $Z=20$. Hydrogen has not been included in these graphs since the differences for this element are too small to plot. It can be seen that for both sets of data there is a systematic trend in the differences from the theoretical baseline as the atomic number increases, the differences being more pronounced for the higher atomic number elements. In view of the magnitude of the differences between the three tabulations it is important to know which is to be preferred. Choice may be made on the basis of which best predicts C.T. measurements. 


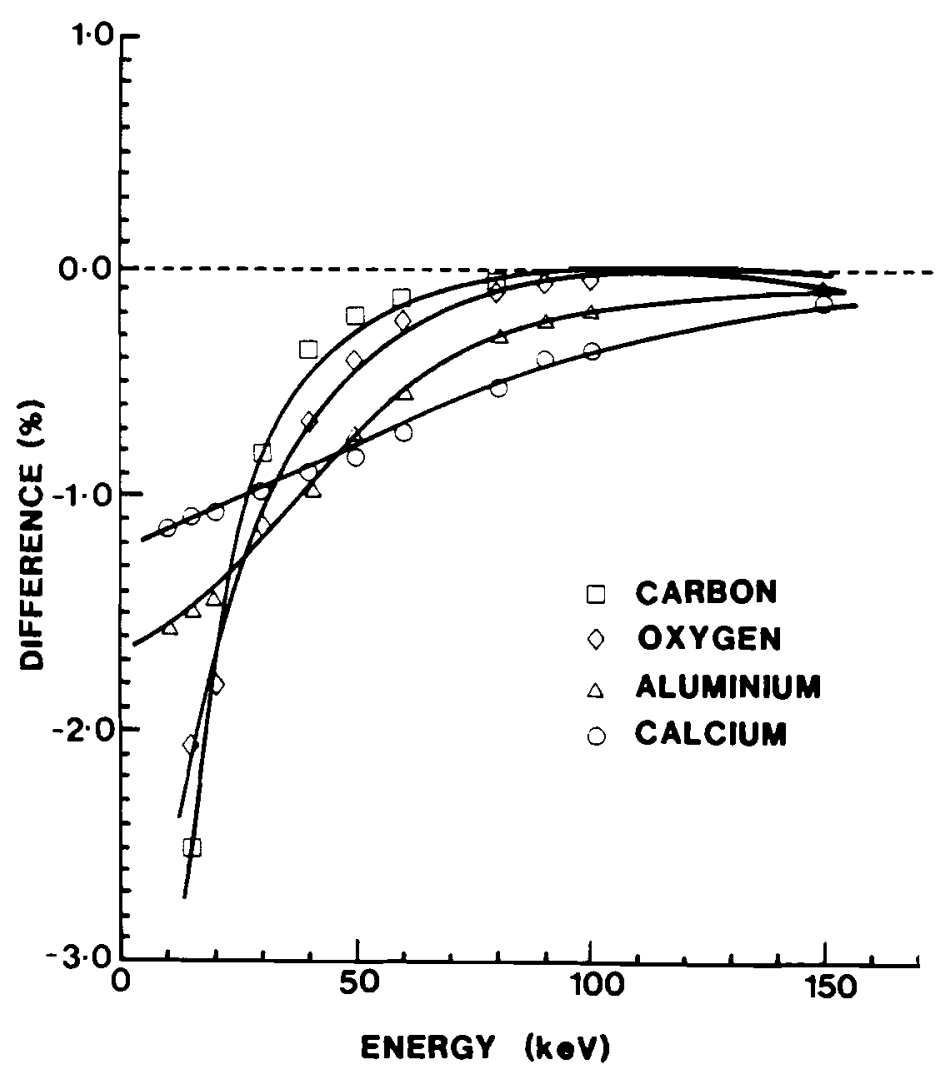

Data of Hubbell [58]

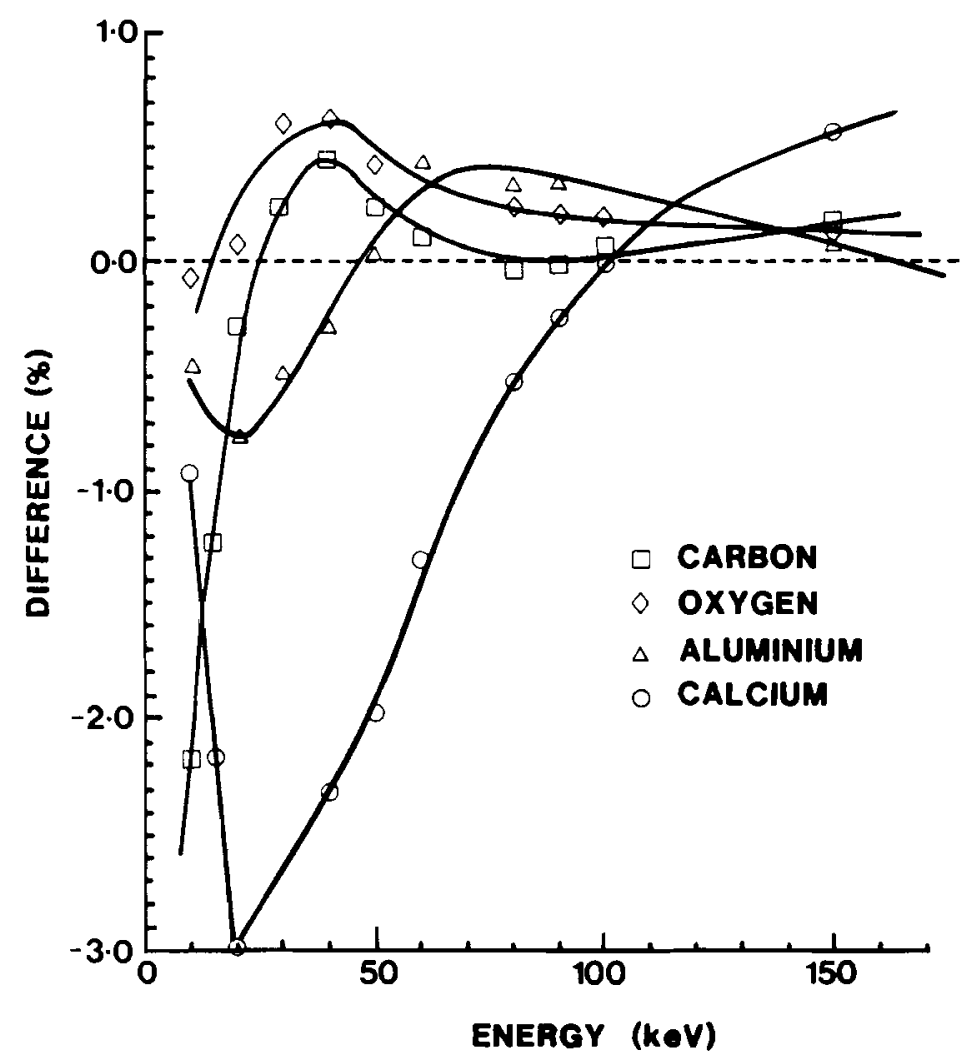

Data of Veigele [57]

Figure 2.5: Comparison of Tabulated Attenuation Coefficients for Selected Elements. 


\subsection{Correlation Between Attenuation Coefficients and C.T. Measurements.}

Since the differences between tables of attenuation coefficients appear to be large enough to significantly affect C.T. scanner measurements, it seems reasonable to expect that the accuracy of a given set of tables could be determined by the extent to which they can be used to predict C.T. measurements taken on samples whose composition and density are known. The approach taken here is a simple extension of a published method for the determination of the "effective" energy of a C.T. scanner [60]. The technique involves scanning a water phantom containing several inserts composed of different types of plastic (A.A.P.M. phantom), and finding the energy at which there is maximum correlation between measured Hounsfield numbers and attenuation coefficients interpolated from tables. Linear regression between the Hounsfield number and the calculated linear attenuation coefficient for the six plastic samples yields a correlation coefficient for each energy value tested. The energy value at which there is maximum correlation is then the "effective" energy. If this exercise is repeated using different tables to calculate the attenuation coefficients of the plastic samples, then the best correlation coefficient obtained using a given set of tables is an index of the accuracy of those tables. In this work interpolation between tabulated values is achieved using 10 point continued fraction interpolation [61], a technique which is discussed in section 2.10 .

Implicit in the above approach is the concept of an "effective" energy of a polychromatic X-ray beam, defined as that energy at which a given material would attenuate a (notional) monochromatic beam by the same amount as it does the polychromatic beam. As has been pointed out by Jackson \& Hawkes [50], this approach is not strictly valid but may be a reasonable approximation when only a limited range of atomic numbers is considered.

This analysis has been applied to measurements taken on the A.A.P.M. phantom using the Wellington Hospital G.E. 8800 scanner, the results of which are given in table 2.1. In the table $r$ is the linear regression correlation coefficient [62]. There is no significant difference in correlation, which may be partly attributed to the fact that the densities of the A.A.P.M. inserts are only quoted to three significant figures $( \pm 0.5 \%)$, a limitation which could be overcome by scanning liquids whose compositions are accurately known, following White \& Speller [63]. Of greater importance is the fact that the measured Hounsfield units are related not simply to the measured attenuation coefficient, but to the ratio of the measured linear attenuation coefficient to that of water. It may be useful therefore to define a "mass Hounsfield unit", $\mathrm{H}_{m}$, by way of analogy with the mass attenuation coefficient: 
Table 2.1: Correlation Between Measured C.T. Numbers and Attenuation Coefficients.

\begin{tabular}{|c|c|c|c|c||}
\hline \hline Source & $\begin{array}{c}E_{\text {eff }} \\
\text { (keV) }\end{array}$ & \multicolumn{2}{|c||}{$\begin{array}{c}\mathrm{K} \\
\text { (see equation 2.3) }\end{array}$} & $\mathrm{r}$ \\
\hline Hubbell (1982) & 72.75 & 1001.2 & 0.1903 & 0.99890 \\
Veigele (1973) & 74.50 & 1001.3 & 0.1892 & 0.99894 \\
"Theoretical " & 73.50 & 1001.1 & 0.1899 & 0.99894 \\
\hline
\end{tabular}

$$
\mathrm{H}_{\mathrm{m}}=\frac{\left[\frac{\mu}{\rho}\right]}{\left[\frac{\mu_{\mathrm{W}}}{e_{\mathrm{W}}}\right]}=\frac{\mu}{\mu_{\mathrm{W}}} \cdot \frac{e_{\mathrm{W}}}{\rho}
$$

The (linear) Hounsfield number may then be calculated simply as follows:

$$
H=K \cdot\left[H_{m} \cdot \frac{\rho}{\rho_{w}}-1\right]
$$

In figure 2.6 comparisons are made between "mass Hounsfield units" calculated from tabulated attenuation coefficients, and calculated "mass Hounsfield units" calculated from "best" theoretical values as discussed previously. Thus figure 2.6 makes the same comparisons as figure 2.5, but using mass Hounsfield units instead of mass attenuation coefficients. It can be seen that the normalisation to water greatly reduces the differences between tabulations for elements close to oxygen, but there is little improvement at $Z=$ 20.

\subsection{Computation of Attenuation Coefficients in the Diagnostic Energy Range.}

As well as being of central importance for quantitative applications of computed tomography, attenuation coefficient data are required for routine quality assurance of C.T. scanners $[60,63]$ and for development work in X-ray imaging generally [51]. However apart from the difficulties created by the differences between the various tabulations, there are also problems in computing the attenuation coefficient of an element at a particular energy from any one set of tables since all of these list attenuation coefficients only at the following points in the diagnostic energy range: $20,30,40,50$, $60,80,100$ and $150 \mathrm{keV}$. There can be no question of linear interpolation between 


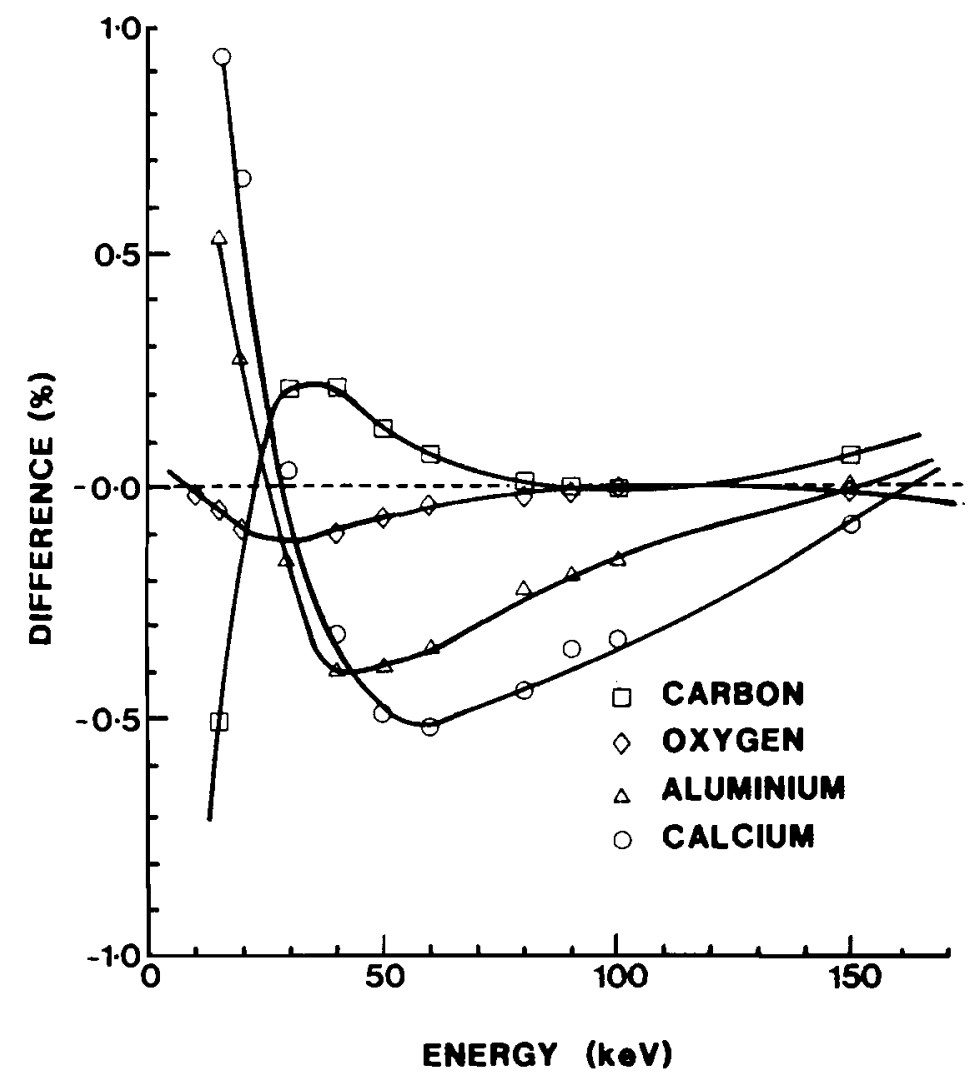

Data of Hubbell [58]

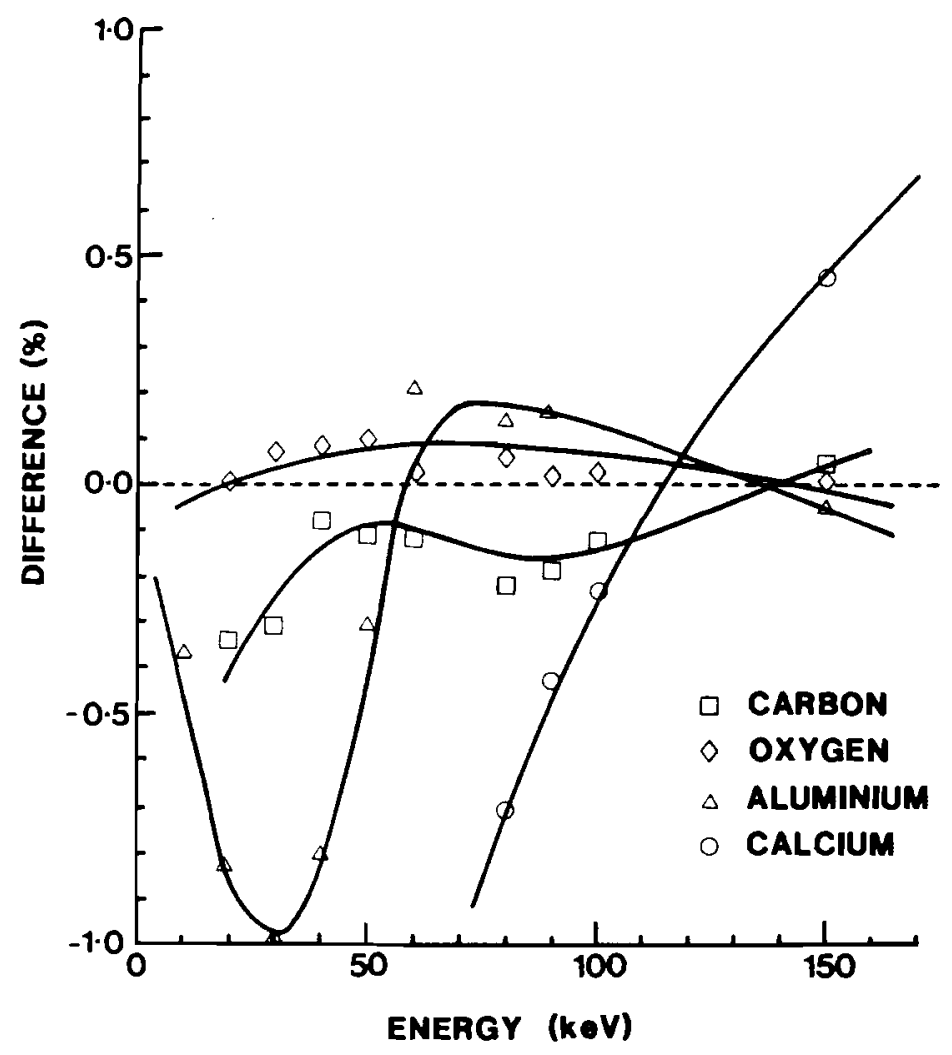

Data of Veigele [57]

Figure 2.6: Comparison of "mass Hounsfield units" calculated from different tables of attenuation coefficients. 
adjacent tabulated values: calculations for oxygen show that, over the range $30-150 \mathrm{keV}$, quadratic interpolation over the three nearest tabulation points to any energy of interest will yield a value within $0.5 \%$ of the true value. With four points (cubic interpolation) the accuracy is $0.2 \%$. To achieve an accuracy of $0.5 \%$ for oxygen right down to $20 \mathrm{keV}$ it is necessary to use a seventh degree polynomial fitted at all eight tabulated points! It should be stressed that the previous figures only apply to local interpolation. Where a single polynomial is required to cover the entire range $30-150$ $\mathrm{keV}$, calculations for oxygen suggest following accuracies: fifth degree $(2 \%)$, sixth degree $(1 \%)$, seventh degree $(0.5 \%)$, eighth degree $(0.2 \%)$. Thus accuracy of $0.2 \%$ requires an expression involving nine constants, since the number of coefficients in a polynomial is one greater than its degree.

In view of the above it is reasonable to consider direct computation of $\mathrm{X}$-ray attenuation coefficients. The problem here is that the theoretical expressions are of great complexity - compare with the expressions of Jackson \& Hawkes in section 2.9 which are simplifications of these - and do not necessarily agree well with reported experimental values. There is a need for readily-accessible means of computing attenuation coefficients for those scientists whose need for them is a means to some end, rather than as an end in itself. Accordingly there have been a number of papers $[16,50,52,64,66]$ which have proposed various simplified expressions for computing attenuation coefficients. This objective is made difficult by the fact that, for the light elements of which the body is principally composed, the diagnostic energy range encompasses the difficult area where there is a transition from the strong (roughly cubic) energy dependence of the photoelectric effect which dominates at low energies to the very weak energy dependence of Compton scatter which is the dominant attenuation process at the top end of the range, as illustrated in figure 2.7.

With simplified expressions there is a trade-off between accuracy, simplicity and the number of constants which must be known. Simplicity is important when large numbers of computations are involved, such as when manipulating images which can frequently be composed of 250,000 pixels and sometimes more, or when performing Monte Carlo simulations. Formulae should be sufficiently accurate to ensure that computational errors are small compared with the uncertainties in the tables upon which they are based so that no new sources of error are introduced. In practice this means that formulae should ideally match the data with accuracy of the order of $0.1 \%$. To approach this level with empirical formulae such as are discussed in section 2.8 requires one or more constants to be separately stored for each element. The best formulae will reduce the number of such constants to a minimum. 


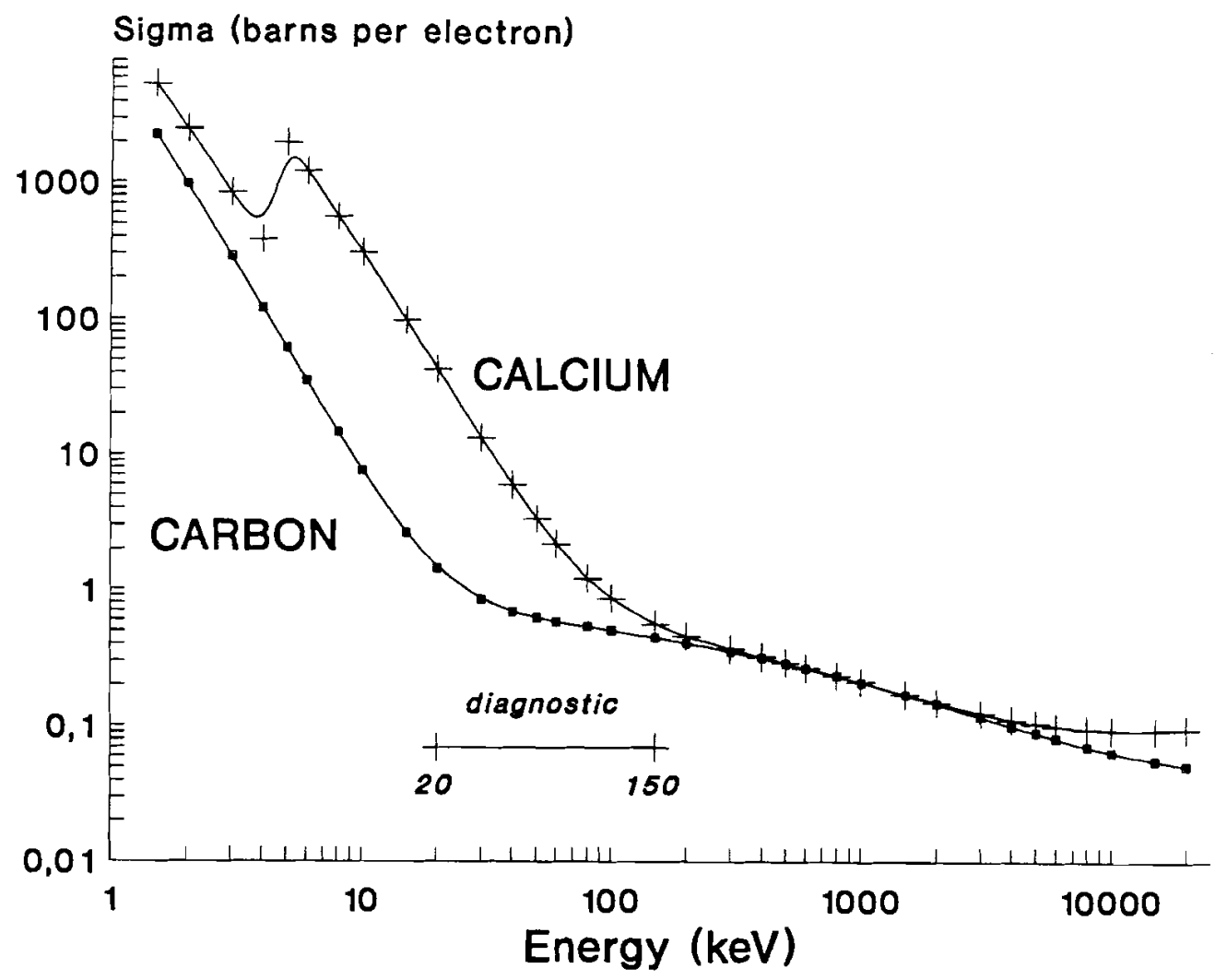

Figure 2.7: Cross Sections for Carbon and Calcium.

Plotted from the data of Hubbell (1982) [58]. For most of the elements of which the human body is composed, the transition between photoelectric dominated attenuation at lower energies and predominantly Compton attenuation at higher energies occurs in the diagnostic energy range. This is one reason why it is difficult find simple formulae which compute attenuation coefficients over this range. Another problem is that since diagnostic energy range is only a small portion of the energy range covered by tables, which only list eight values between 20 and $150 \mathrm{keV}$, it is difficult to interpolate between the values given.

Tables may list atomic cross sections rather than attenuation coefficients, in which case conversion may be performed via equation (2.2). In the diagnostic energy range only collisions between photons and electrons are important so it is useful to be able to compute electron densities. The mass electron density (number of electrons per gram of absorbing medium, denoted $n_{g}$ ) is given by [64]:

$$
\mathrm{n}_{\mathrm{g}}=\frac{\mathrm{z} \cdot \mathrm{N}_{\mathrm{A}}}{\mathrm{A}}
$$

where $\mathrm{z}=$ atomic number, $\mathrm{N}_{\mathrm{A}}=$ Avogadro's number and $\mathrm{A}=$ atomic weight. The volume electron density (number of electrons per $\mathrm{cc}$ ) is obtained by multiplying the mass electron density by the physical density (grams per $\mathrm{cc}$ ) of the medium. The attenuation 
coefficient of a compound or mixture of elements can be computed from the attenuation coefficients of the individual elements provided that the proportion of each by weight is known [49]. For a mixture of $\mathrm{N}$ elements:

$\left[\frac{\mu}{\rho}\right]_{\text {total }}=w_{1} \cdot\left[\frac{\mu}{\rho}\right]_{1}+w_{2} \cdot\left[\frac{\mu}{\rho}\right]_{2}+w_{3} \cdot\left[\frac{\mu}{\rho}\right]_{3}+\ldots+w_{N} \cdot\left[\frac{\mu}{\rho}\right]_{N}$

where $w_{i}$ is proportion by weight of the $i^{\text {th }}$ element.

Since for most of the diagnostic energy range Compton scatter is by far the most important attenuation process (see figure 2.1), the computation of attenuation due to this process is of prime importance when calculating the total attenuation coefficient. For those energies at which Compton scatter is the dominant process, the cross section due to Compton is given to within a few per cent by the Klein-Nishina cross section for a free electron, which is described in the next section. The simplest expressions for the total attenuation coefficient start from the Klein-Nishina formula and apply empirical correction factors which make allowance for the neglect of the other two attenuation processes, and for the neglect of the effect of atomic binding effects on the Compton effect. Such empirical formulae are described in section 2.8. Jackson and Hawkes have tried to both improve the accuracy and reduce the number of constants by deriving improved expressions which are simplifications of theoretical formulae. These are discussed in section 2.9. More accurate formulae involving sophisticated interpolation techniques are presented in section 2.10 , the best of which have been used to compute a set of "true" values against which the simpler expressions have been tested.

\subsection{Computation of the Klein-Nishina Cross Section.}

The Klein-Nishina formula for Compton scattering from a free electron (in $\mathrm{cm}^{2} /$ electron) is given by [67]:

$$
\begin{aligned}
& \sigma_{\mathrm{KN}}=2 \pi \mathrm{r}^{2} . \\
& \cdot\left[\frac{1+\mathrm{k}}{\mathrm{k}^{2}}\left\{\frac{2(1+\mathrm{k})}{1+2 \mathrm{k}}-\frac{\ln (1+2 \mathrm{k})}{\mathrm{k}}\right\}+\frac{\ln (1+2 \mathrm{k})}{2 \mathrm{k}}-\frac{1+3 \mathrm{k}}{(1+2 \mathrm{k})^{2}}\right]
\end{aligned}
$$

where $\mathrm{k}=\mathrm{E} / \mathrm{m}_{\mathrm{e}^{2}} \mathrm{c}^{2}(=\mathrm{E}(\mathrm{keV}) / 511.003) . \quad \mathrm{m}_{\mathrm{e}}=$ rest mass of the electron and $\mathrm{r}_{\mathrm{e}}=$ classical electron radius $\left(=\mathrm{e}^{2} / \mathrm{m}_{\mathrm{e}^{2}}=2.8179380 \times 10^{-15} \mathrm{~m}\right)$. The theoretical 
expression for the Klein-Nishina cross section is not in a form suitable for computation because in the diagnostic energy range the two terms inside the curly bracket are of comparable value but opposite sign, so that the proportional error of the result becomes large. The other two terms are also close to each other in magnitude and of opposite sign but are at least thirty times smaller than the first two terms in the diagnostic energy range. To overcome the computational problem Hubbell proposed the use of a power series [54]:

$$
\begin{aligned}
& \sigma_{\mathrm{KN}}=\frac{8}{3} \cdot \mathrm{r}^{3} \cdot \frac{1}{(1+2 \mathrm{k})^{2}} \cdot \\
& \cdot\left[1+2 \mathrm{k}+\frac{6}{5} \mathrm{k}^{2}-\frac{1}{2} \mathrm{k}^{3}+\frac{2}{7} \mathrm{k}^{4}-\frac{6}{35} \mathrm{k}^{5}+\frac{8}{105} \mathrm{k}^{6}+\frac{4}{105} \mathrm{k}^{7}+\ldots\right]
\end{aligned}
$$

To assess the accuracy of these formulae reference was made to values of the KleinNishina cross section listed by Hubbell [49]. These values were adjusted very slightly $(+0.012 \%)$ to facilitate the use of a more recent value for the classical electron radius [54] than was used in the original work. On a Data General Eclipse S/140 computer, which uses 16 bit floating point arithmetic, the analytical formula (equation (2.8)) was in error by $0.9 \%$ at $20 \mathrm{keV}$. At $10 \mathrm{keV}$ the error was $8 \%$ ! Values obtained using the power series formula were accurate to within \pm 0.000001 , as were values computed in double precision using the analytical formula. Both of the above formulae are cumbersome to use, and are not computationally efficient. For example, sensible evaluation of equation (2.8) involves 17 multiplications or divisions, 8 additions or subtractions and the natural logarithm function.

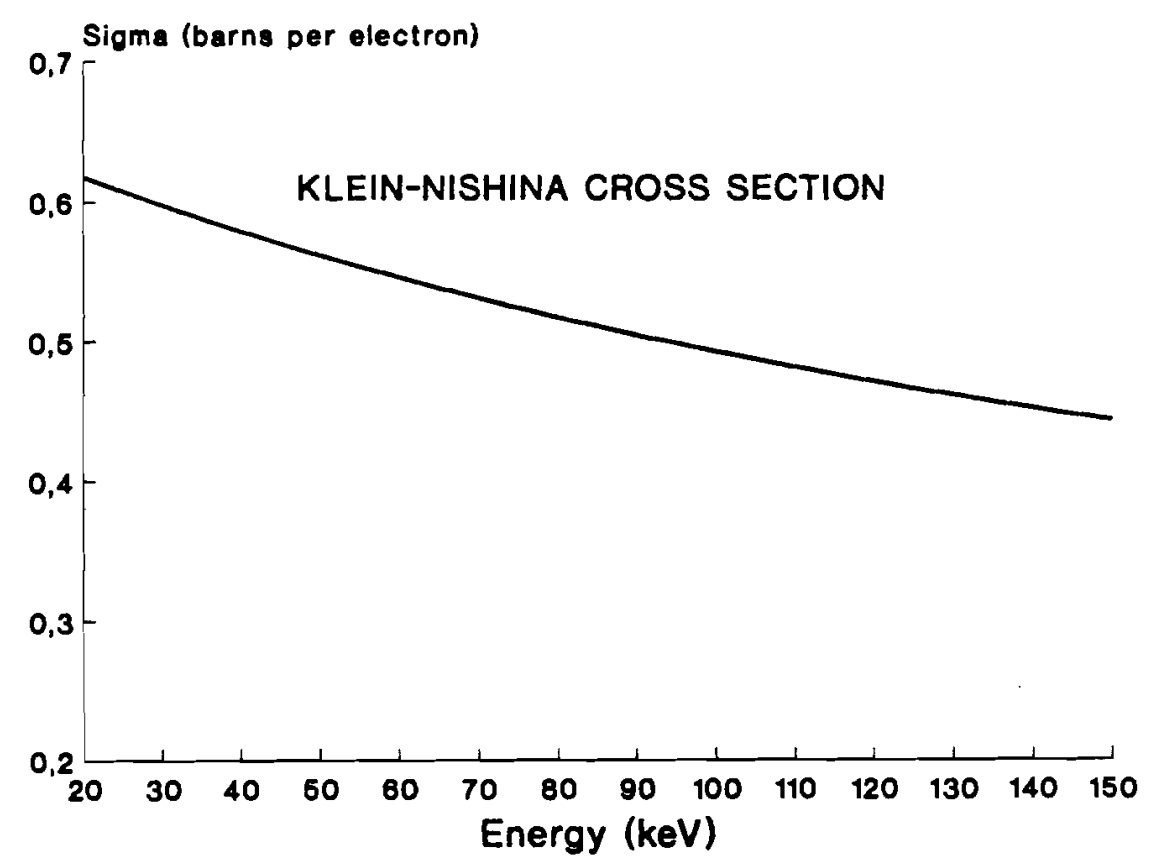

Figure 2.8: Variation of the Klein-Nishina cross section over the diagnostic energy range. 
The Klein-Nishina function is graphed for the diagnostic energy range in figure 2.8. The function is slowly-varying over the energy range of interest, and there is clearly a great deal of scope for fitting a much simpler function to it. Using least squares fitting to polynomial functions, it was found that a third degree polynomial would match the data with a maximum error of $0.05 \%$ over the range from 20 to $150 \mathrm{keV}$.

Table 2.2: Accuracies of Fits to the Klein-Nishina Cross Section.

\begin{tabular}{||c|c|c|c|}
\hline Parameters & $\begin{array}{c}\text { polynomial } \\
\text { (least } \\
\text { squares) }\end{array}$ & $\begin{array}{l}\text { polynomial } \\
\text { (minimax) }\end{array}$ & $\begin{array}{c}\text { rational } \\
\text { function }\end{array}$ \\
\hline 3 & $\begin{array}{l}0.37 \% \\
0.05 \% \\
0.006 \%\end{array}$ & $\begin{array}{l}0.25 \% \\
0.03 \% \\
0.003 \%\end{array}$ \\
\hline
\end{tabular}

Details of the curve fitting techniques used in the present work are presented in appendix B. An alternative method for fitting polynomial functions to a set of data is the minimax technique [61], which does not use all the data as does the least squares technique, but involves selection of the points at which the fitting function is matched to the data. This method produces the polynomial fit which minimises the maximum error over a given range for a polynomial of a given degree. The number of points at which the polynomial is fitted is the same as the number of coefficients in the polynomial. Details of how these points are selected are given in appendix $B$, but the process is illustrated in figure 2.9, which shows the values at which 3,4 and 5 point polynomial fits should be made in the range 20 to $150 \mathrm{keV}$.

The accuracies of minimax fits to the Klein-Nishina function are given in table 2.2. Acceptable accuracy would still require the use of a cubic polynomial fit and the use of four parameters. However the appearance of the curve in figure 2.8 suggests that simpler fits ought to be possible. A further possible choice of fitting function is a rational function, that is, a ratio of polynomials [61]:

$$
y(x)=\frac{a_{0}+a_{1} \cdot x+a_{2} \cdot x^{2}+\ldots}{b_{0}+b_{1} \cdot x+b_{2} \cdot x^{2}+\ldots}
$$

Using continued fraction fitting techniques, which are discussed in section 2.10 and in appendix B, it is found that the following three parameter rational function matches the Klein-Nishina function over the range of interest with an accuracy of better than $\pm 0.008 \%$ : 


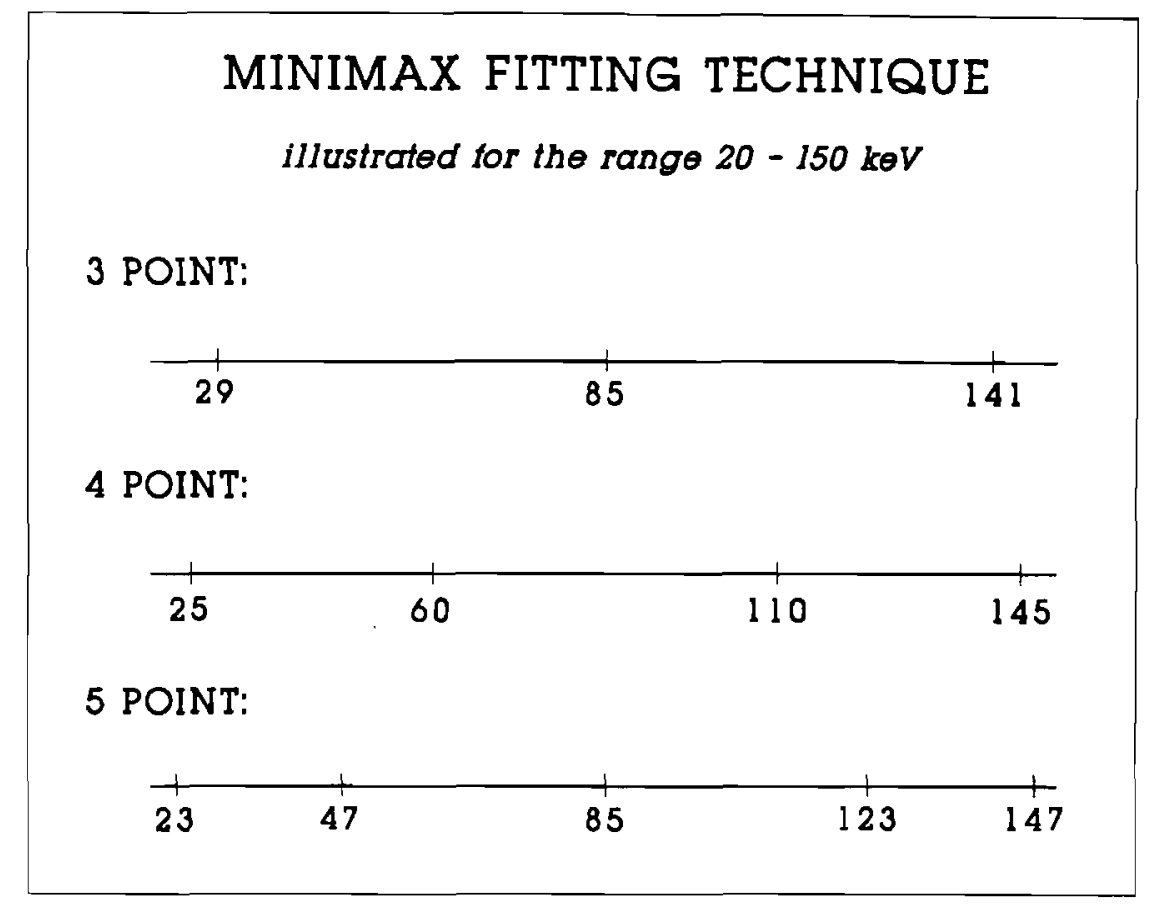

Figure 2.9: The minimax fitting technique.

This technique involves selecting the points at which a polynomial is fitted to a given set of data over a particular range, in this case $20-150 \mathrm{keV}$. The polynomial so fitted will have the least possible maximum deviation from the given data values over this range. This differs from the more common least squares technique which minimizes the rootmean-square deviation from the data.

$$
\sigma_{\mathrm{KN}}(\mathrm{E})=\frac{132.2303+0.1502041 \times \mathrm{E}}{198.8485+\mathrm{E}}
$$

As the accuracy of the above expression exceeds the level necessary for the present purpose there is scope for rounding the coefficients. This was achieved by rounding the coefficient on the bottom line to 200 and the first one on the top line to 133 and then determining the optimum value of the remaining coefficient using a generalised curve fitting program (Bevington's CURFIT [62] - see appendix B). The recommended formula for the computation of the Klein-Nishina function over the energy range 20 $150 \mathrm{keV}$ is therefore [66]:

$$
\sigma_{\mathrm{KN}}(E)=\frac{133+0.1482 \times E}{200+E}
$$


which has a maximum error of $0.024 \%$ over this range. This expression involves only two multiplications or divisions and two additions, no transcendental functions, and only three very simple constants! Some authors restrict their attention to the energy range from 50 to $100 \mathrm{keV}$ [65] as this encompasses the range of effective energies of C.T. scanners. In this case it is possible to produce an even simpler rational function expression:

$$
\sigma_{\mathrm{KN}}(\mathrm{E})=\frac{200.76}{307.86+\mathrm{E}}
$$

which matches the Klein-Nishina function over the range $50-100 \mathrm{keV}$ with an accuracy of $0.1 \%$.

\subsection{Simple Empirical Formulae for Calculating Attenuation Coefficients.}

Weber \& Van den Berge in 1969 [64] proposed an equation of the form:

$$
\left[\frac{\mu}{\rho}\right]=\mathrm{n}_{\mathrm{g}} \cdot\left[\sigma_{\mathrm{KN}}+\mathrm{a} \cdot \frac{\mathrm{z}^{\mathrm{b}}}{\mathrm{E}^{\mathrm{C}}}+\mathrm{d} \cdot \frac{\mathrm{z}^{\epsilon}}{\mathrm{E}^{\mathrm{f}}}\right] \times 10^{-24}
$$

Where the symbols are as defined previously, and the constants $\mathrm{a}, \mathrm{b}, \mathrm{c}, \mathrm{d}, \epsilon$, and $\mathrm{f}$ are empirically adjusted to give best fit to the various components of the total mass attenuation coefficient. Equation (2.14) represents an extension of the equation describing the attenuation due to Compton collisions between photons and free electrons, which is simply the product of the mass electron density, $\mathrm{n}_{\mathbf{g}}$, and the Klein-Nishina cross section. The second term within the brackets is an empirical fit to tabulated values of the photoelectric cross section, and the third term is an error term to account for coherent scatter and the neglect of effect of electron binding effects on the Compton scatter cross section.

Electron densities of elements may be computed using equation (2.6). To compute the attenuation coefficients of mixtures of elements (including compounds) use may be made of the mixture rule, equation (2.7), or it is possible to regard the mixture/compound as a single entity. In the last case $n_{g}$ is now the weighted sum of the electron densities of the constituents:

$$
n_{g}=\sum_{i} w_{i} \cdot n_{g}(i)
$$

where $w_{i}$ is the proportion by weight of the $\mathrm{i}^{\text {th }}$ element. The mass attenuation coefficient of the mixture may now calculated from equation (2.14) provided that "effective" atomic 
number of the mixture is known. As the equation (2.14) is not linear in $\mathrm{Z}$ it is necessary to define two "effective" atomic numbers $\mathrm{z}^{*}$, and $\mathrm{Z}$ ' which respectively replace the first and second occurrences of $\mathrm{z}$ in equation (2.14):

$$
\begin{aligned}
& z^{*}=\left[\Sigma w_{i} \cdot z_{i} b\right]^{1 / b} \\
& z^{\prime}=\left[\Sigma w_{i} \cdot z_{i} \epsilon\right]^{1 / \epsilon}
\end{aligned}
$$

where the constants $b$ and $\epsilon$ are as in equation (2.14). In figure $\overline{2.10(a)}$ attenuation coefficients for oxygen calculated using the Weber equation are compared with the data of Hubbell (1969) [49] over the diagnostic energy range. Comparisons for a number of other elements indicate that the Weber equation is accurate to within $5 \%$ for $1 \leq \mathrm{Z} \leq 20$ and for $30 \leq \mathrm{E} \leq 150$. Accuracy of this order may be sufficient for some purposes, but is certainly not adequate for analysis of C.T. data. In 1975 McCullough [16] proposed different coefficients for Weber's equation, optimised for the elements of principal interest in medical work $(\mathrm{H}, \mathrm{C}, \mathrm{N}, \mathrm{O})$. Figure 2.10(b) compares calculated attenuation coefficients for the elements up to $\mathrm{Z}=20$, at $60 \mathrm{keV}$, with the data of Hubbell (1969). It can be seen that the equation of McCullough fits the data well for atomic numbers below 13, but for $\mathrm{Z}>13$ the fit is very poor. From figure 2.10(a) it can be seen that the McCullough equation gives a good fit for oxygen only at energies above $50 \mathrm{keV}$.

\section{Table 2.3: Parameters Used in Empirical Expressions for the Linear Attenuation} Coefficient (equation 2.14).

\begin{tabular}{||l|r|c|c|c|c|c||}
\hline Author & a & b & c & d & $\epsilon$ & $f$ \\
\hline Mccullough & 9.8 & 3.8 & 3.2 & 1.25 & 2.0 & 1.9 \\
Weber & 17.7 & 3.4 & 3.1 & 1.12 & 1.7 & 1.7 \\
\hline
\end{tabular}

Several other authors have produced formulae of this type. Some omit the third term, the second term then being fitted to the difference between the Klein-Nishina cross section and the total cross section. The accuracy suffers accordingly. Henson [65] has extended this approach to give closer fits over the diagnostic energy range. In his formula separate terms are fitted to both the coherent scatter cross section and the correction to the Compton scatter cross section to account for binding effects: 


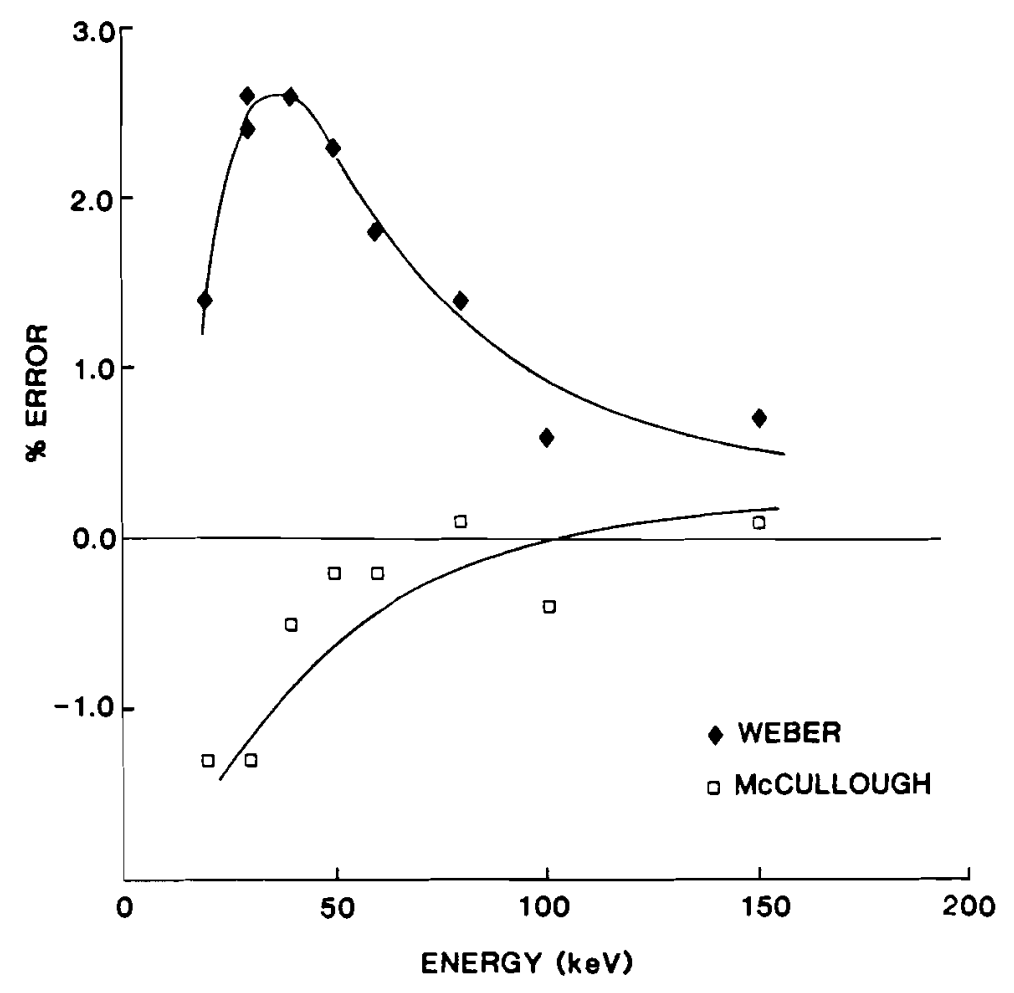

(a) Errors for oxygen.

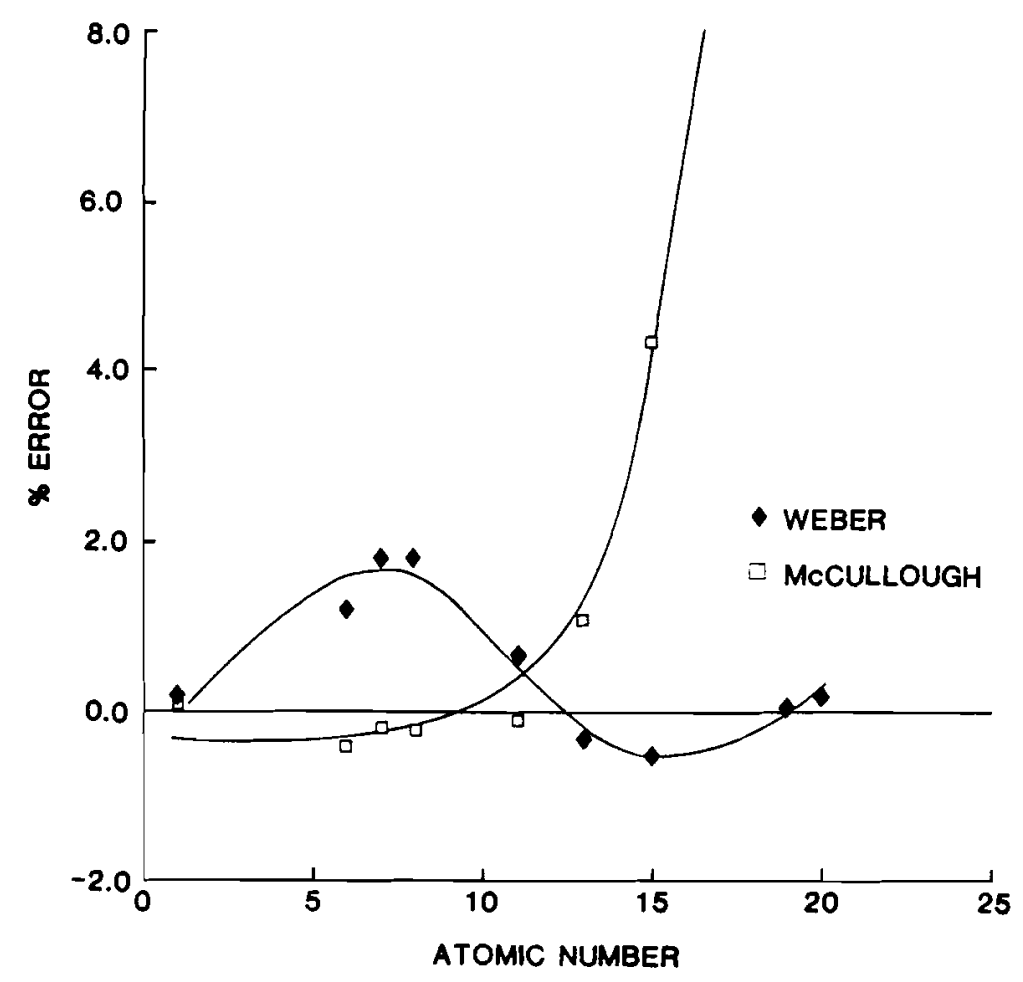

(b) Errors for a number of elements at $60 \mathrm{keV}$.

Figure 2.10: Accuracy of the Equation of Weber \& van Den Berge [64].

Errors are plotted with respect to the tabulated coefficients of Hubbell [49] when using equation (2.14) with the constants proposed by Weber \& Van Den Berge [64] and McCullough [16]. 


$$
\left[\frac{\mu}{\rho}\right]=F\left[\mathrm{Z}\left[\sigma_{\mathrm{KN}}(E)+\mathrm{a} \cdot E^{\mathrm{b}}+\mathrm{c} \cdot \mathrm{E}^{\mathrm{d}}\right]+\epsilon \cdot \log (E)+\mathrm{h}\right]
$$

where $\mathrm{F}=\mathrm{N}_{\mathrm{A}} / \mathrm{A} \times 10^{-24}$, and the other symbols are as defined previously. The term involving parameters $\mathrm{a}$ and $\mathrm{b}$ is fitted to the photoelectric cross section, and the term in $\mathrm{c}$ and $d$ is fitted to the coherent scatter cross section. The expression $[\epsilon \cdot \log (E)+h]$ is fitted to the difference between the Klein-Nishina cross section and the true Compton cross section. In the Henson formula no attempt is made to find an expression for the atomic number dependence of the attenuation coefficient, the seven parameters for each of the first thirty elements being listed separately. Henson's fits are optimised for the energy range $50-100 \mathrm{keV}$, and the claim is made that the maximum error over this range is $0.55 \%$, with most calculated values agreeing to within $0.2 \%$ with those tabulated by Veigele [57].

With the exception of that of Henson, the empirical formulae so far quoted rely on the ability to express the attenuation coefficient in terms of separate functions of both energy and atomic number, that is:

$$
\mu(Z, E)=F(Z) \cdot G(E)
$$

Jackson \& Hawkes [50] assert that such a factorisation is not possible and that it is this fact that limits the accuracy of simple empirical formulae. This being so, it follows that the accuracy of simple expressions for the attenuation coefficient may possibly be improved by allowing the exponents for photon energy to be functions of atomic number. This approach has been followed by Henson, but in view of the fact that in the energy range of interest the attenuation is largely Compton it would seem that better fits are possible, perhaps with fewer constants. In the present work an attempt has been made to fit the attenuation coefficient to an expression of the form:

$$
\frac{\mu}{\rho}=\mathrm{n}_{\mathrm{g}} \cdot\left[\sigma_{\mathrm{KN}}+\mathrm{a} \cdot \mathrm{E}^{\mathrm{b}}\right] \times 10^{-24}
$$

where the 'constants' $\mathrm{a}$ and $\mathrm{b}$ are determined separately for each element. The expressions were fitted to the data of Hubbell (1982) [58]. Data files containing the attenuation coefficients for each element in $1 \mathrm{keV}$ steps were first created by 8 point continued fraction interpolation (see section 2.10). Optimum values for a and b over the energy range from 50 to $100 \mathrm{keV}$ were then determined using a generalised curve fitting program based on Bevington's CURFIT [62]. The values obtained, which are graphed in figure 2.11, are listed in appendix $C$. It is of interest to note that the value of the exponent, $b$, varies only slightly for elements above $Z=14$, a fact which will be 
referred to again in section 3.4 in the context of algorithms for dual energy computed tomography.

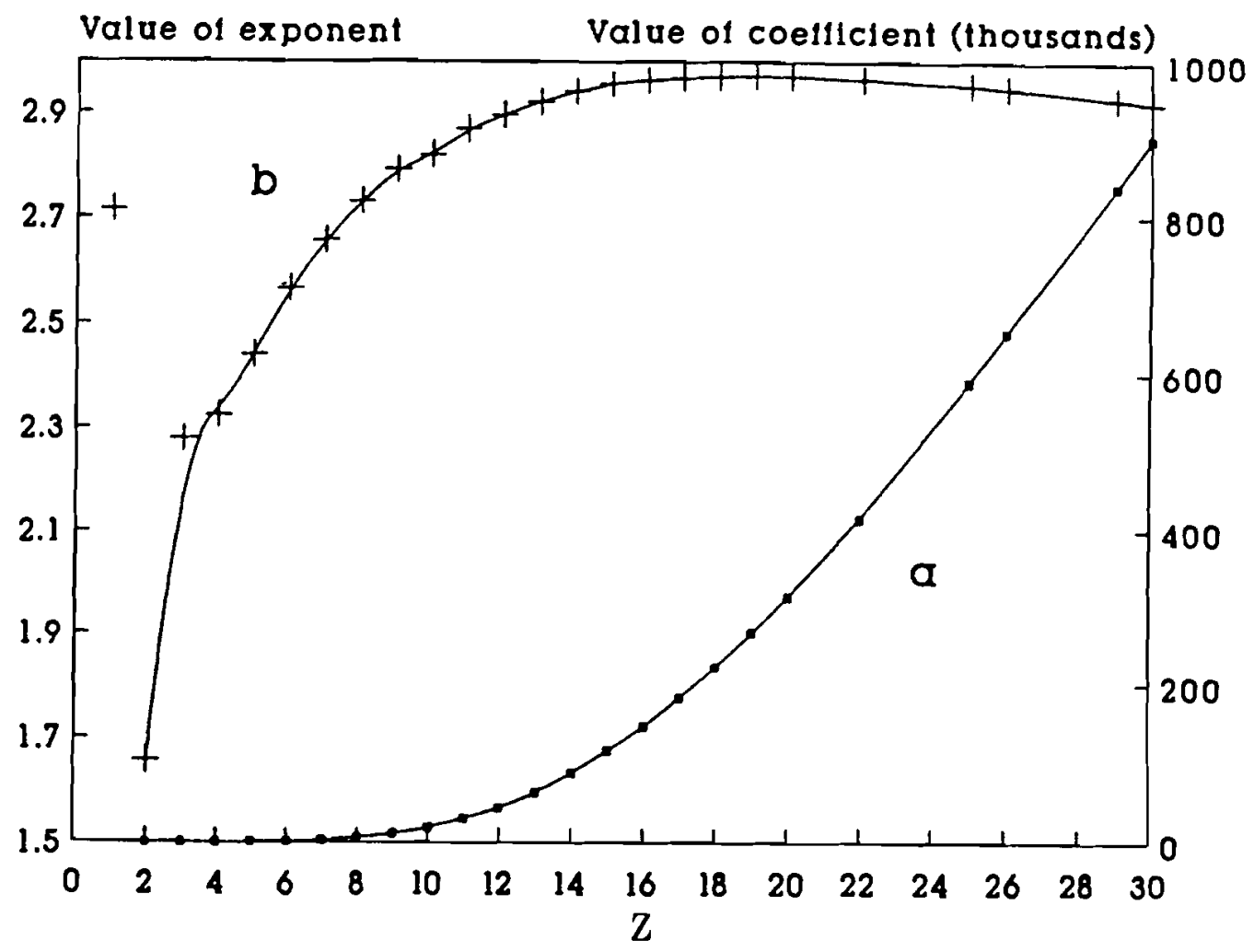

Figure 2.11: Values for the Two Parameter Fit to the Attenuation Coefficient $(50-100 \mathrm{keV})$.

The accuracy of the method was tested by computing the attenuation coefficients in $1 \mathrm{keV}$ steps and comparing with the values stored in the data files. Values calculated using equation (2.19) with the constants listed in appendix $\mathrm{C}$ agree to within $0.15 \%$ with values interpolated from the data of Hubbell [58] for $\mathrm{H}, \mathrm{C}, \mathrm{N}$ and $\mathrm{O}$. For the other elements up to $Z=30$ listed by Hubbell the agreement is within $0.35 \%$. The accuracy of the method is a little better than that of Henson, and yet it requires only three constants per element (constants $\mathrm{a}$ and $\mathrm{b}$ plus the electron density, $\mathrm{n}_{\mathrm{g}}$ ), as opposed to seven in the case of the latter.

\subsection{A Semi-Empirical Formula for the Attenuation Coefficient.}

Jackson \& Hawkes [50, 68] have reviewed theoretical formulae for calculating attenuation coefficients with the object of producing simplified formulae which are somewhat more accurate than the simple formulae currently used. The approach taken is semi-empirical, that is, formulae are derived from theory, but with some constants adjusted for agreement with experimental data. 


\subsubsection{Coherent Scatter.}

The coherent scatter cross section is expressed in terms of the cross section of a standard element, via an energy transformation. For soft tissues oxygen is a good choice for the standard element, since they are composed mainly of water. The cross section for the standard element is stored as an empirical fit.

$$
\begin{aligned}
\sigma \operatorname{coh}(Z, E) & =\left[\frac{Z}{Z^{\prime}}\right]^{2} \cdot \sigma \operatorname{coh}\left(Z^{\prime}, E^{\prime}\right) \\
E^{\prime} & =\left[\frac{Z}{Z^{\prime}}\right]^{1 / 3} \cdot E \\
Z^{\prime} & =8 .
\end{aligned}
$$

\subsubsection{Compton Scatter.}

Jackson and Hawkes found that the correction to the Klein-Nishina formula to account for binding effects can also expressed in terms of the coherent cross section for a standard element:

$$
\sigma^{\operatorname{Com}}(Z, E)=Z \cdot \sigma_{K N}(E)-z^{-\frac{3}{2}} \cdot \sigma^{\operatorname{coh}}(Z, E)
$$

\subsubsection{Photoelectric Effect.}

Jackson \& Hawkes [50] found that the ratio of the total photoelectric cross section to that due to the 1s electrons only was constant for a given element. This constant $U(Z)$ is tabulated for the first 54 elements [50]. As a starting point for calculating the cross section due to the $1 \mathrm{~s}$ electrons they take the Born approximation:

$$
\sigma_{1 s^{B A}}=4 \cdot \sqrt{ } 2 \cdot z^{5} \cdot \alpha^{4} \cdot\left[\frac{m_{e} C^{2}}{E}\right]^{7 / 2} \cdot \phi_{0}
$$

where $\phi_{0}$ is the cross section for Thomson scattering, and $\alpha$ is the fine structure constant ( $\left.=\mathrm{e}^{2} / \mathrm{hc}\right)$. The cross section for Thomson scattering is given by:

$$
\phi_{0}=\frac{8}{3} \cdot \pi \cdot\left[\frac{\mathrm{e}^{2}}{\mathrm{~m}_{\mathrm{e}^{\mathrm{c}^{2}}}}\right]^{2}
$$


A better approximation to the cross section due to the $1 \mathrm{~s}$ electrons is obtained from the Stobbe formula. Here the wave functions are assumed to be hydrogen-like, but it is still based on the assumptions of an electric dipole approximation with a non-relativistic treatment.

$\sigma_{1 s} S t=4 \cdot \sqrt{ } 2 \cdot z^{5} \cdot \alpha^{4} \cdot\left[\frac{m_{e}{ }^{2}}{E}\right]^{7 / 2} \cdot \phi_{0} \cdot 2 \pi\left[\frac{E_{K}}{E}\right]^{\frac{1}{2}} \cdot f\left(n_{1}\right)$

where $E_{K}$ is the theoretical $K$ shell binding energy given by:

$$
E_{K}=\frac{1}{2}(Z . \alpha)^{2}\left(m_{e} C^{2}\right)
$$

$\mathrm{n}_{1}$ and $\mathrm{f}\left(\mathrm{n}_{1}\right)$ are defined as follows:

$$
\begin{aligned}
n_{1} & =\left[\frac{E_{K}}{E-E_{K}}\right]^{\frac{1}{2}} \\
f\left(n_{1}\right) & =\frac{\exp \left[-4 n_{1} \cot ^{-1}\left(n_{1}\right)\right]}{1-\exp \left(-2 \pi n_{1}\right)}
\end{aligned}
$$

The cross section for the photoelectric effect is then:

$$
\sigma_{\mathrm{PE}}=\sigma_{1 \mathrm{~s}} \mathrm{St} \cdot U(\mathrm{Z}) \cdot \mathrm{g}(\mathrm{E})
$$

where $U(Z)$ is the ratio of the total photoelectric cross section to that due to the $1 \mathrm{~s}$ electrons, as discussed previously, and the function $g(E)$ is incorporated to allow for relativistic effects and is defined as:

$$
\begin{aligned}
g(E) & =1+0.143 B^{2}+1.667 B^{8} \\
B & =1-\frac{m_{e} c^{4}}{\left(E-E_{K}+m_{e} C^{2}\right)^{2}}
\end{aligned}
$$

\subsubsection{Total Cross Section.}

The total atomic cross section is the sum of the cross section due to the photoelectric effect and the cross section due to scatter:

$$
\sigma_{\text {total }}=\sigma_{\mathrm{PE}}+\sigma_{\text {scatter }}
$$


The photoelectric cross section is given by equation (2.28), and the scatter cross sections may be combined to give:

$$
\sigma_{\text {scatter }}(Z, E)=Z \cdot \sigma_{K N}(E)+\left(1-Z^{-\frac{1}{2}}\right) \cdot\left(Z / Z^{\prime}\right)^{2} \cdot \sigma^{\operatorname{coh}}\left(Z^{\prime}, E^{\prime}\right)
$$

A program XATTEN has been written to evaluate the expressions of Jackson \& Hawkes and is listed in appendix C.

\subsubsection{Accuracy of the Jackson \& Hawkes Formulae.}

Jackson \& Hawkes tabulate the ratio of the attenuation coefficient calculated using their formula to published coefficients for selected elements up to $Z=53$ (iodine). Their figures show a maximum error of $0.7 \%$ which occurs in the value for sodium at $30 \mathrm{keV}$. The maximum error quoted for those elements listed with $Z \leq 10$ over the energy range $30 \leq \mathrm{E}(\mathrm{keV}) \leq 150$ is $0.3 \%$. A simple program has been written in BASIC which calculates the attenuation coefficient using the formulae of Jackson \& Hawkes and compares the results with stored "best" theoretical values as discussed in section 2.4. Comparisons with the theoretical base have been made for all elements up to $Z=35$ (bromine) over the energy range $30-150 \mathrm{keV}$, at the energy values for which attenuation coefficients are listed in the published tabulations. For $Z \leq 10$ the errors are less than $0.5 \%$ with the exceptions of $\mathrm{He}$ and $\mathrm{Li}$ at the lower extreme of the energy range where the error is $-0.9 \%$. For the other elements tested the accuracy is also better than $0.5 \%$, except at $30 \mathrm{keV}$ for the following elements (errors in brackets): $\mathrm{Ne}(-0.8 \%)$, $\mathrm{Na}(-0.7 \%), \mathrm{Mg}(-0.6 \%)$ and $\mathrm{Al}(-0.55 \%)$.

\subsection{Accurate Interpolation of Attenuation Coefficient Data.}

The Jackson \& Hawkes formulae match the attenuation coefficient data of the theoretical base with an accuracy $(<0.5 \%)$ which is sufficient for many purposes. However the formulae are not adequate for those occasions when greater accuracy is required, as in the present work, or when it is desired to interpolate other coefficients such as those of Hubbell [58] or Veigele [57]. The type of interpolation to be used depends on the degree of accuracy required. To establish the accuracy of various types of interpolation it is first necessary to be able to determine the "true" values at points intermediate to those tabulated. This was done by finding two very different techniques which could be expected to perform this function well, and comparing the results obtained. The first of these was continued fraction interpolation [61], in which a single expression was fitted to the seven points tabulated between 30 and $150 \mathrm{keV}$. The second technique was based on the formulae of Jackson \& Hawkes. The theoretical data to which the Jackson \& Hawkes expressions are fitted was stored in a data file, and the 
difference between the value calculated by the expressions and that stored in the data file was used to compute a correction factor for each tabulated point. At intermediate points the correction factor was obtained by linear interpolation between the correction factors obtained at the two nearest tabulated points. Comparison of the values computed by the two techniques for oxygen (computed in $1 \mathrm{keV}$ steps from $30 \mathrm{keV}$ up to $150 \mathrm{keV}$ ) showed agreement to within $0.01 \%$. Reference values in the present work have been obtained by continued fraction interpolation since it is applicable to all tabulations and is more convenient to use. Eight point continued fraction interpolation is used when it is necessary to extend the range down to $20 \mathrm{keV}$, and ten point interpolation takes in the values tabulated at 15 and $10 \mathrm{keV}$ as well.

A continued fraction is an expression of the form [61]:

$$
F(x)=q_{0}+\frac{\left(x-x_{1}\right)}{q_{1}+\frac{\left(x-x_{2}\right)}{q_{2}+\frac{\left(x-x_{3}\right)}{q_{3}+\frac{\left(x-x_{4}\right)}{q_{4}}}}}
$$

The expression may be extended to higher terms if desired. Continued fraction expressions may be fitted to data in the same sense as polynomial expressions are commonly used to express relationships in experimental data, or as approximations to more complicated functions. However continued fraction expressions are often more suitable than polynomials in that often fewer terms are necessary to model given data to a particular level of accuracy. Constants $\mathrm{q}_{0} \ldots . \mathrm{q} 44$ are parameters whose values are adjusted so that the continued fraction expression matches the data values at each of (in this case) four points whose values of the independent variable are $x_{1} \ldots x_{4} . \quad$ In this respect a continued fraction fit differs from a polynomial fit in that the continued fraction expression is an exact fit at a small number of widely spaced points whereas a polynomial fit would normally be a least squares fit over a larger number of data points covering the same range. The need to store the values $\mathrm{x}_{1} \ldots . \mathrm{x}_{4}$ as well as the parameters $\mathrm{q}_{0} \ldots . \mathrm{q}_{4}$ is not a serious disadvantage in this case since the same ' $x$ ' values (energies) may be used for all elements to be fitted and it is only necessary to store the ' $q$ ' values separately for each element. Equation (2.33) may be evaluated as follows:

$$
F(x)=q_{0}+\left(x-x_{1}\right) /\left(q_{1}+\left(x-x_{2}\right) /\left(q_{2}+\left(x-x_{3}\right) /\left(q_{3}+\left(x-x_{4}\right) / q_{4}\right)\right)\right)
$$

Although the expression appears somewhat complicated it is actually no more difficult to evaluate than a polynomial, but involving the division operation rather than multiplication. In BASIC the above expression may be evaluated as follows: 


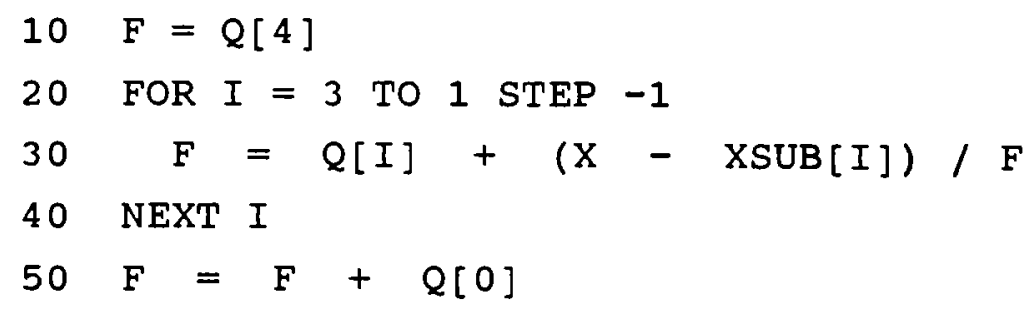

Where ' $\mathrm{F}$ ' is the value of the continued fraction expression, ' $\mathrm{X}$ ' is the independent variable, and arrays $Q$ and XSUB store the ' $Q$ ' and ' $x$ ' values respectively. Evaluation of the expression starts by evaluating the fraction of which $\mathrm{Q}_{4}$ is the denominator, adds the result to $\mathrm{Q}_{3}$, divides $\left(\mathrm{x}-\mathrm{x}_{3}\right)$ by this value, and so on. The power of this interpolation method can be seen from the fact that a seven point continued fraction interpolation gives virtually perfect interpolation for oxygen whereas the accuracy of a seven point (sixth degree) polynomial fit is $1 \%$. Where a smaller energy range is required, an acceptable fit may be achieved with fewer parameters. Over the range $50-100 \mathrm{keV}$, the attenuation coefficient has in the present work been fitted to a five point continued fraction expression. The fitting program computes the parameters $\mathrm{q}_{0}$ through to $\mathrm{q}_{4}$ for each element and then compares the values output by the 5 point fitting expression with those of the 7 point expression discussed previously at $1 \mathrm{keV}$ intervals over the range $50-100$ $\mathrm{keV}$. A problem with continued fraction interpolation is a tendency to give very large erroneous values over small ranges of abscissae ("spikes"), but this may be avoided by careful selection of the abscissae at which the fit takes place. The strategy adopted here was to start with the Tchebyshev points [61] for the range 50 to 100 (that is, the zeroes of the $\mathrm{n}^{\text {th }}$ order Tchebyshev polynomial over this range, where $\mathrm{n}$ is the number of points at which the fit is to take place), and fit expressions for the first 35 elements at these points. These expressions were then evaluated at $1 \mathrm{keV}$ intervals over the whole range, and any erroneous points noted. The fitting points were then adjusted slightly and the process was repeated. Eventually a set of fitting points were found which gave correct interpolation for the whole 35 elements. The energy values obtained were: 50, 58, 66, 71 and 98 $\mathrm{keV}$. The Tchebyshev polynomial is described in more detail in appendix B. The values of the parameters obtained, $\mathrm{q}_{0} \ldots \mathrm{q}_{4}$, are given in appendix $\mathrm{C}$. The accuracy has been found to be better than $0.1 \%$ compared to the theoretical base for the first 35 elements. Thus there is a five-fold improvement in accuracy compared with Henson over a comparable atomic number range, achieved with six parameters per element, compared with his seven!

A program MCOEF has been written in FORTRAN IV which computes the attenuation coefficient of any compound made up of the first thirty elements by eight point continued fraction interpolation over the energy range 20 to $150 \mathrm{keV}$. This program has been translated into FORTRAN 77 operating in an MSDOS environment and is listed in appendix $C$. 


\subsection{Calculation of X-ray Spectra.}

Interpretation of C.T. measurements frequently makes use of the concept of an effective energy of the spectrum of a C.T. scanner. As stated previously, this approach is only valid for a small range of atomic numbers. In practice this means atomic numbers close to that of oxygen. Interpretation of C.T. measurements of samples containing elements of higher atomic numbers may require a more subtle approach involving a knowledge of the X-ray spectrum produced by the scanner. There are three possible ways in which the spectrum for a C.T. scanner may be obtained:

(i) Direct measurement [70].

(ii) Calculation from measurements of transmission of the beam through some standard material $[71,72]$.

(iii) Calculation from published semi-empirical equations, using manufacturer's data on the $\mathrm{X}$-ray tube.

Direct measurement involves the use of an X-ray detector followed by a pulse height analyser. Problems are experienced in obtaining a sufficiently low photon flux to prevent excessive dead-time. Scanner geometry limits the maximum distance between tube and detector, and the minimum tube current on the G.E. 8800 is $20 \mathrm{~mA}$. Possible reduction of flux using a lead pinhole collimator would involve difficult corrections for scatter. Approach (ii) seems very promising, but relies on accurate attenuation data for (usually) aluminium and requires the use of aluminium of high purity. The Laplace transformation method of obtaining the spectrum from the transmission measurements is very sensitive to errors in the original measurements. Thus approach (iii) has been used in this work, with transmission measurements being used as a check on the answer obtained.

A computer program (XSPECT) for the calculation of X-ray spectra has been written following the work of Birch \& Marshall [51, 69]. This program calculates the ideal Bremsstrahlung and characteristic spectra, corrected for attenuation within the target material. Correction is also made for inherent filtration within the tube, and it is possible to compensate for further filtration by any material. The program is listed in appendix D.

\subsubsection{Calculation of the Bremsstrahlung Spectrum.}

The differential intensity at a given photon energy $E$ arising from an electron travelling a distance $d x$ is given by: 


$$
d I(E)=Q \cdot\left[\frac{N_{A}}{A}\right] \cdot \rho \cdot d x
$$

where $\mathrm{N}_{\mathrm{A}}=$ Avogadro's number, $\mathrm{A}=$ atomic weight of the target material, $\mathrm{q}=$ density, $Q=X$-ray intensity per unit energy interval per incident electron flux per atom. The total continuous spectrum is obtained by integrating along the electron path. By substitution of variables, this is equivalent to integrating from the maximum electron energy (the energy of the electrons at the surface of the target, which equals the maximum possible photon energy [73]) down to the energy of the emitted photons [69]. Lower electron energies corresponding to greater depths need not be considered since the photon energy can never be greater than the energy of the electron undergoing the Bremsstrahlung collision.

$$
d I(E)=\frac{e \cdot N_{A}}{A} \cdot \int_{T=T_{0}}^{T=E} Q \cdot\left[\frac{d T}{d x}\right]^{-1} \cdot d T
$$

Here $\mathrm{T}$ is the kinetic energy of the electron in $\mathrm{keV}, \mathrm{T}_{0}$ being the energy of the incident electrons. Birch \& Marshall have obtained empirical values for the integrand in the previous equation by fitting to measured spectra. The relationship is expressed as a function of $z=E / T$, where $E$ is the photon energy:

$$
Q^{\prime} \equiv Q \cdot\left[\frac{d T}{d x}\right]^{-1}=c_{0}+c_{1} z+c_{2} z^{2}+c_{3} z^{3}+c_{4} z^{4}
$$

where the constants are given as: $c_{0}=0.503, c_{1}=-0.94597, c_{2}=0.1553$, $c_{3}=1.1632$ and $c_{4}=-0.6818$. The value of $Q^{\prime}$ is scaled for a $1 \mathrm{~mm}^{2}$ area at a distance of $0.75 \mathrm{~m}$ from the target. The expression for I must be modified to allow for attenuation of the X-ray beam within the target, which means that the depth within the target at which the photon is generated must be determined. Since the integration is performed with respect to electron energy, a relationship is needed between the distance the electron has travelled and its remaining kinetic energy. This is given by the Thomson-Whiddington relationship:

$$
T=\left[T_{0}^{2}-e \cdot c \cdot x\right]^{\frac{3}{2}}
$$

' $\mathrm{C}$ ' in equation (2.38) is the Thomson-Whiddington constant. Birch \& Marshall tabulate "best" values for the Thomson-Whiddington constant as follows: 
Table 2.4: Values of the Thomson-Whiddington Constant.

\begin{tabular}{|c|c|}
\hline $\begin{array}{c}\text { Incident electron } \\
\text { energy }(\mathrm{keV})\end{array}$ & \multicolumn{1}{|c|}{$\begin{array}{c}\mathrm{c} \\
\left(\mathrm{keV}^{2} \mathrm{~cm}^{2} \mathrm{~g}^{-1}\right)\end{array}$} \\
\hline 25 & $0.39 \times 10^{6}$ \\
50 & $0.54 \times 10^{6}$ \\
75 & $0.625 \times 10^{6}$ \\
100 & $0.70 \times 10^{6}$ \\
150 & $0.84 \times 10^{6}$ \\
\hline
\end{tabular}

The program computes $\mathrm{C}$ via a continued fraction fit to the above data. The depth at which the photon is generated is then given by:

$$
x=\frac{T_{0}^{2}-T^{2}}{\rho \cdot C}
$$

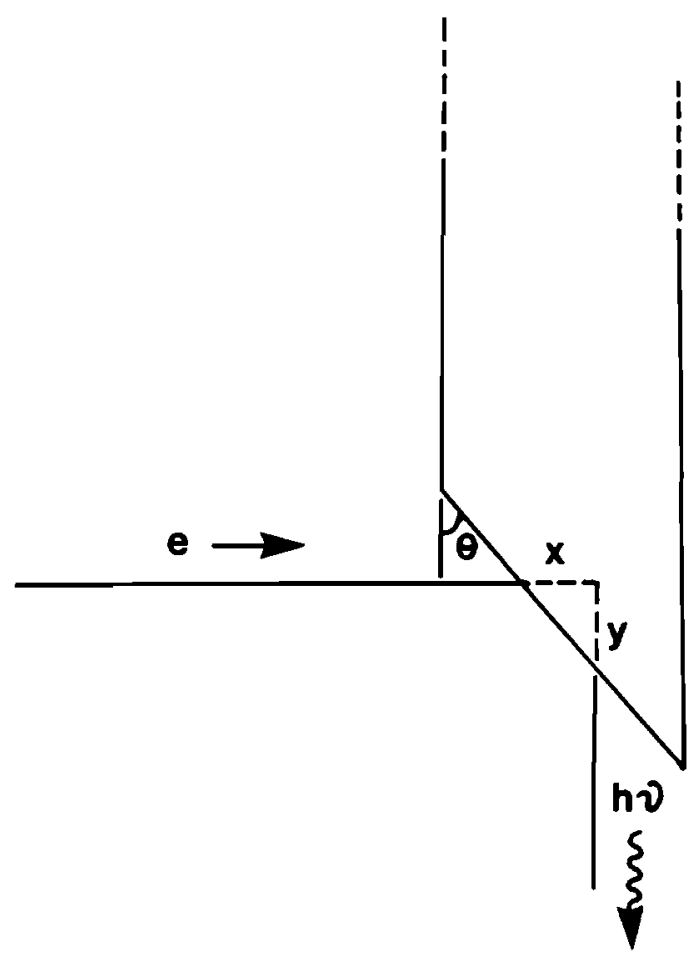

Figure 2.12: Geometry of the X-ray Target.

The above is not drawn to scale. It can be shown from equation (2.38) that the range of $120 \mathrm{keV}$ electrons in a tungsten target is $0.01 \mathrm{~mm}$ ! 
The distance the photon must travel through the target is then (see figure 2.12):

$$
\mathrm{Y}=\frac{\mathrm{T}_{0}{ }^{2}-\mathrm{T}^{2}}{\rho . \mathrm{C}} \cdot \cot \theta
$$

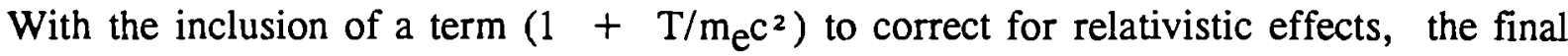
expression for the continuous spectrum is:

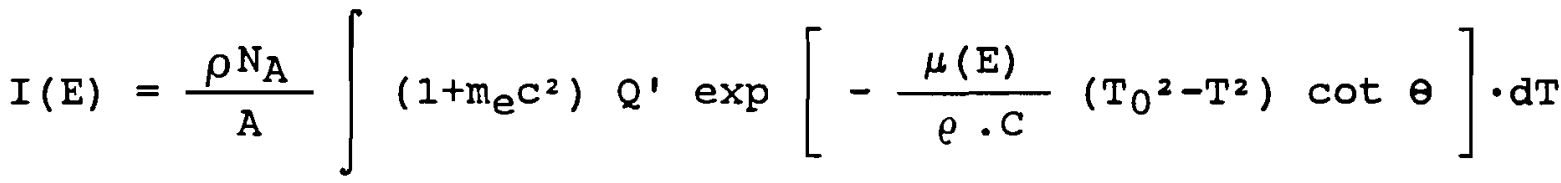

The program evaluates the integrand in $1 \mathrm{keV}$ steps, the integral then being obtained using Gregory's formula [74]. Gregory's formula has been used since it is comparable to Simpson's rule both in terms of simplicity and accuracy, but does not require the number of points to be even.

\subsubsection{Calculation of the Characteristic Spectrum.}

Birch \& Marshall [69] included characteristic radiation in their program by making use of published data on the relative intensities of the tungsten characteristic peaks, and using a scale factor chosen to match calculated and measured spectra at $140 \mathrm{kVp}$. The intensities at other voltages are obtained using the equation:

$$
I_{C h}=\text { scale factor } x\left[\frac{E}{E_{K}}-1\right]^{1.63}
$$

Here $E_{K}$ is the $\mathrm{K}$-shell binding energy of tungsten. Birch \& Marshall do not appear to correct the characteristic peaks for target attenuation. In this work correction for target attenuation is applied, following Green \& Cosslett [75]. Here it is assumed that the average depth of production of the characteristic photons is a quarter of the range of the electrons in the target, the range being obtained from the Thomson-Whiddington relationship by setting $T=0$ and solving for $x$. In the present work the scale factor in equation (2.42) has been chosen so that the intensity of the $K_{\alpha 1}$ line matches the value given in Birch \& Marshall [51] at $140 \mathrm{kVp}$. Allowance for target attenuation in this work means that the relative intensities here will not necessarily match those of Birch \& Marshall, except at $140 \mathrm{kVp}$. Relative intensities in this work were therefore chosen to agree with Birch \& Marshall at $140 \mathrm{kVp}$. 
Table 2.5: Assumed Relative Intensities of $\mathbf{K}$ Characteristic Lines for Tungsten.

\begin{tabular}{|l|c|c|}
\hline Line & $\begin{array}{r}\text { Energy } \\
(\mathrm{keV})\end{array}$ & $\begin{array}{c}\text { Relative } \\
\text { Intensity }\end{array}$ \\
\hline $\mathrm{K}_{\alpha 1}$ & 59.32 & 100.0 \\
$\mathrm{~K}_{\alpha 2}$ & 57.99 & 55.9 \\
$\mathrm{~K}_{\beta 1,3,5}$ & 67.15 & 38.2 \\
$\mathrm{~K}_{\beta 2,4}$ & 69.13 & 10.4 \\
\hline
\end{tabular}

The final expression for the characteristic peaks is:

$I_{C h}(E)=$ scale $x$ rel intens $x\left[\frac{E}{E_{K}}-1\right] \times \exp \left[-\frac{\frac{1}{4} \mu T_{0}{ }^{2}}{\rho \cdot C} \cot \theta\right]$ $(2.43)$

\subsubsection{Correction for Inherent Filtration.}

The X-ray spectrum will undergo filtration by any tube materials traversed. This will include the glass envelope enclosing the tube insert, the insulating oil surrounding the insert, and the window material covering the exit port of the tube housing. Such unavoidable filtration is referred to as inherent filtration, and is specified as the equivalent distance of aluminium which would give the same attenuation at a particular kilovoltage. In this program the attenuation of aluminium is estimated by a continued fraction fit to the attenuation coefficient data tabulated by McMaster [56]. The data of McMaster has been used in preference to more recent data since this is the data used by Birch \& Marshall in obtaining their empirically fitted spectra, and thus is most likely to yield accurate spectra when used with the semi-empirical formulae quoted above.

\subsubsection{Additional Filtration.}

The effects of additional filtration (eg shaped filter in a C.T. scanner: see section 1.2.3) may be allowed for using a subroutine (AFILT) which is a simple modification of program MCOEF which calculates the attenuation coefficient of a compound, as discussed in section 2.10. Attenuation coefficients are obtained by 10 point continued fraction interpolation of the data of Hubbell (1982) [58]. 


\subsubsection{Accuracy of the Program.}

Spectra calculated using this program at $50 \mathrm{kV}$ and $150 \mathrm{kV}$ are compared with computed spectra of Birch \& Marshall [51] in figure 2.13. These spectra are normalised for a total photon count of 100000 . In both cases the continuous spectra agree very closely except at the peak, where XSPECT predicts a lower value than is given in Birch \& Marshall. However it can be seen from spectra published by Birch \& Marshall [69] (figure 2.14) that their program overestimates the peak intensity in both cases.

While the shapes of the spectra computed by XSPECT agreed well with the published spectra, the total intensity did not. This is of little consequence for the present work, but for the sake of completeness this problem has been addressed. Careful checking of the equations used has not revealed any cause for the discrepancy, and so an empirical solution has been sought. A correction factor has been determined which depends solely on the peak kilovoltage. This factor has been fitted to a quadratic:

factor $=2.45 \times 10^{-3}-\left(1.4106 \times 10^{-5}\right) \times(\mathrm{kV})+\left(4.1721 \times 10^{-8}\right) \times(\mathrm{kV})^{2}$

With this correction applied the total intensity of the spectra match those of Birch \& Marshall to within $3 \%$. 


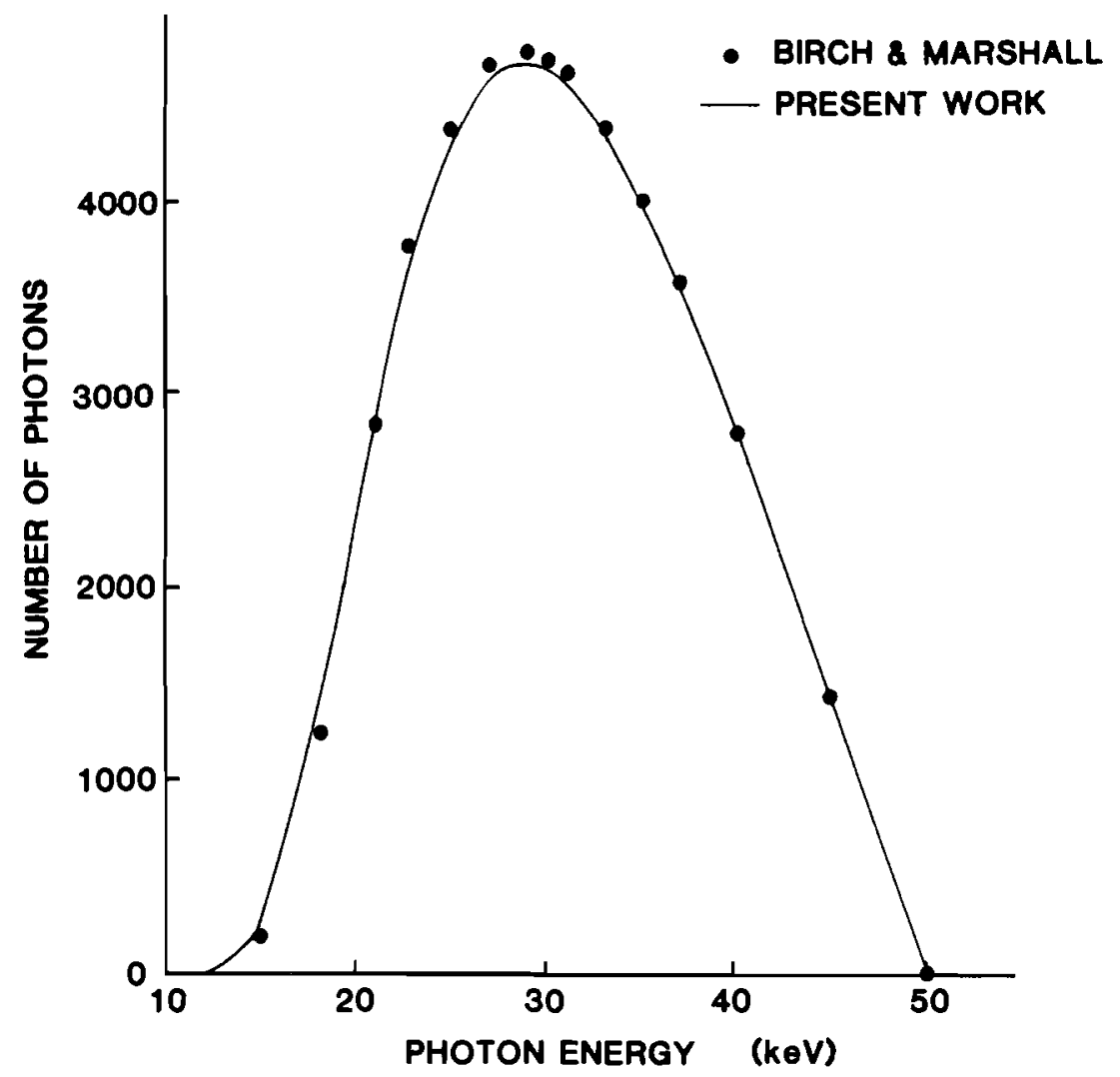

$50 \mathrm{kV}$

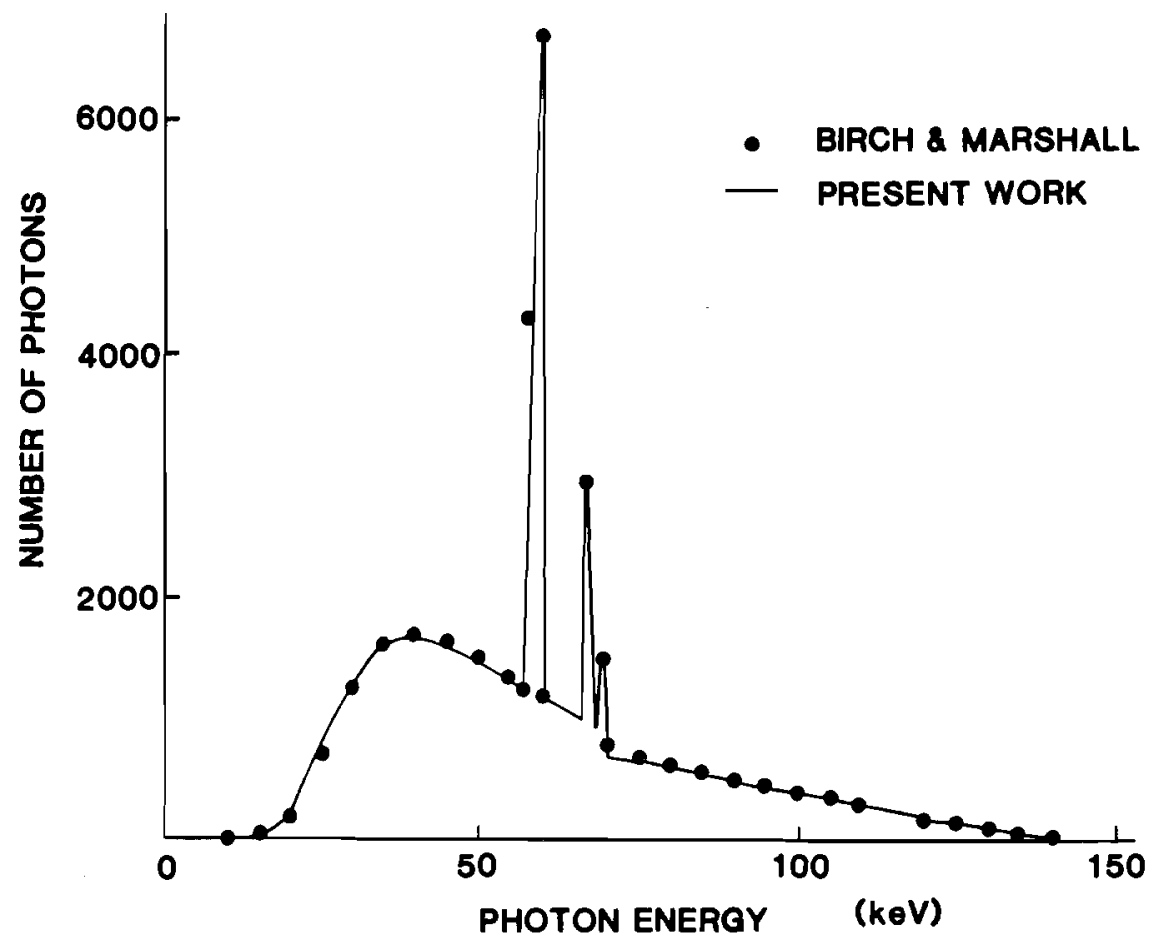

$140 \mathrm{kV}$

Figure 2.13: Accuracy of Computed Spectra.

Comparison of spectra computed using program XSPECT with a published spectrum of Birch \& Marshall [51]. The first spectrum is for a constant potential generator set to $50 \mathrm{kV}, 10$ degree target angle and total filtration equivalent to $2.0 \mathrm{~mm}$ of aluminium. The second spectrum is for a constant potential generator set to $140 \mathrm{kV}, 17$ degree target angle and total filtration equivalent to $2.5 \mathrm{~mm}$ of aluminium. 


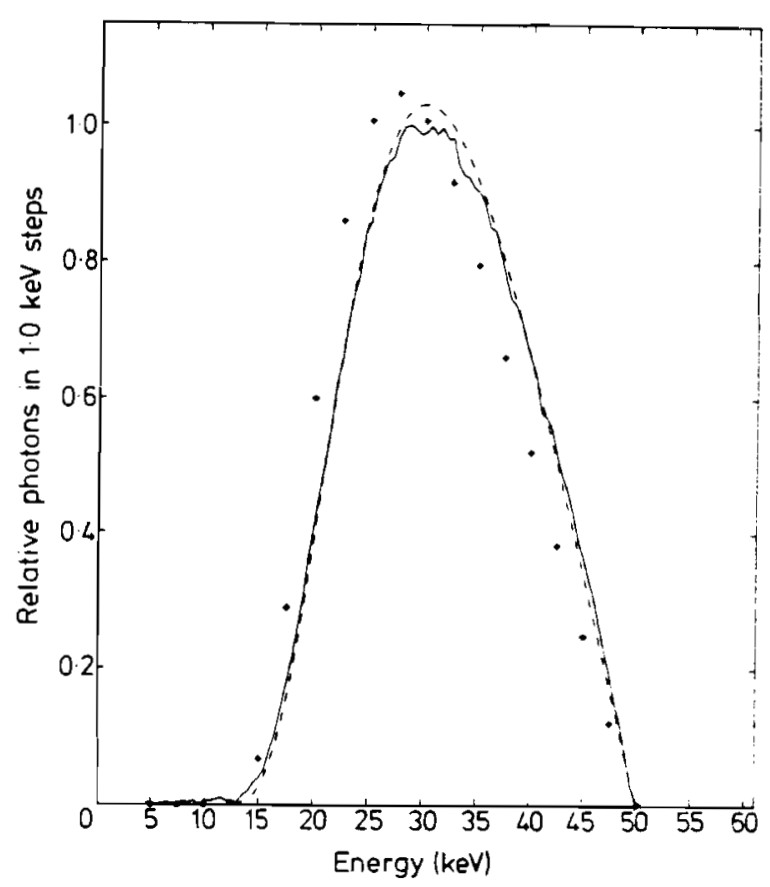

$50 \mathrm{kV}$

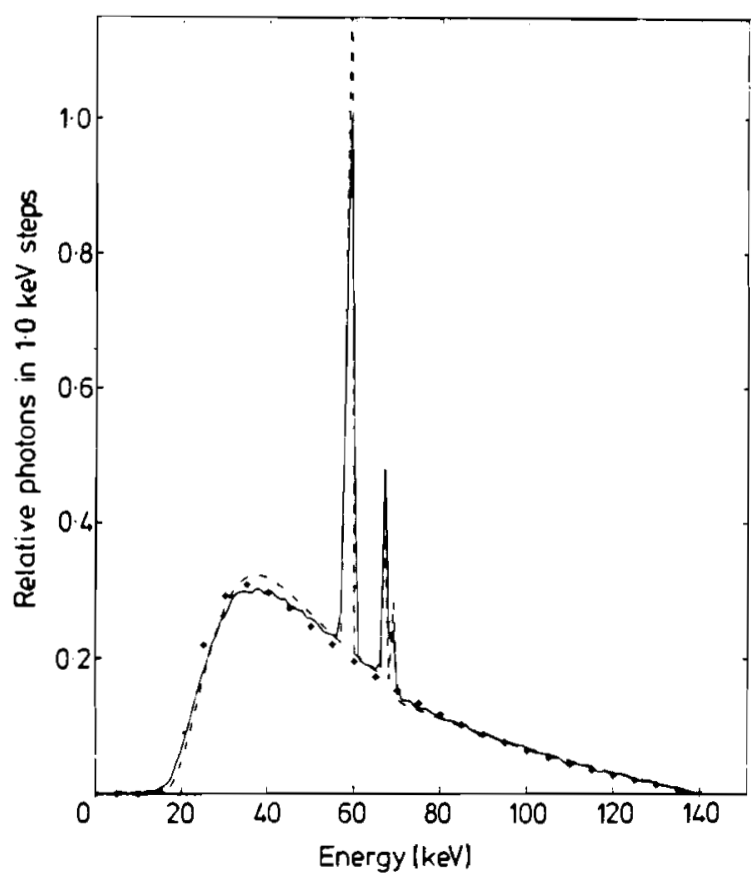

$140 \mathrm{kV}$

Figure 2.14: Comparison of Measured and Computed Spectra: Birch and Marshall.

Figures taken from Birch \& Marshall [69] comparing their computed spectra with their measurements taken using a Ge (Li) detector. Their program overestimates the intensity at the peak of the continuous spectrum. 


\subsection{Estimation of the spectrum of the Wellington Hospital C.T. scanner.}

The Wellington Hospital G.E. CT/T 8800 scanner has a Maxiray 100-CT X-ray tube. The following specifications [76] are relevant to the calculation of the X-ray spectrum:

Target angle:

Anode material:

Inherent filtration:

Insert:

Housing:

Added filtration:

\section{7 degrees.}

$90 \%$ tungsten, $10 \%$ rhenium.

$0.8 \mathrm{~mm} \mathrm{Al}$ equivalent at $150 \mathrm{kVp}$

$0.4 \mathrm{~mm} \mathrm{Al}$ equivalent at $150 \mathrm{kVp}$

$1.5 \mathrm{~mm} \mathrm{Al}$ equivalent at $150 \mathrm{kVp}$

(fixed filter in collimator adaptor).

The spectra of Birch \& Marshall assume a pure tungsten target, although most $\mathrm{X}$-ray tube manufacturers add $10 \%$ rhenium to prevent "crazing" of the target surface [77]. Since the atomic number of rhenium is only one higher than tungsten the effect on the spectrum is expected to be minimal.

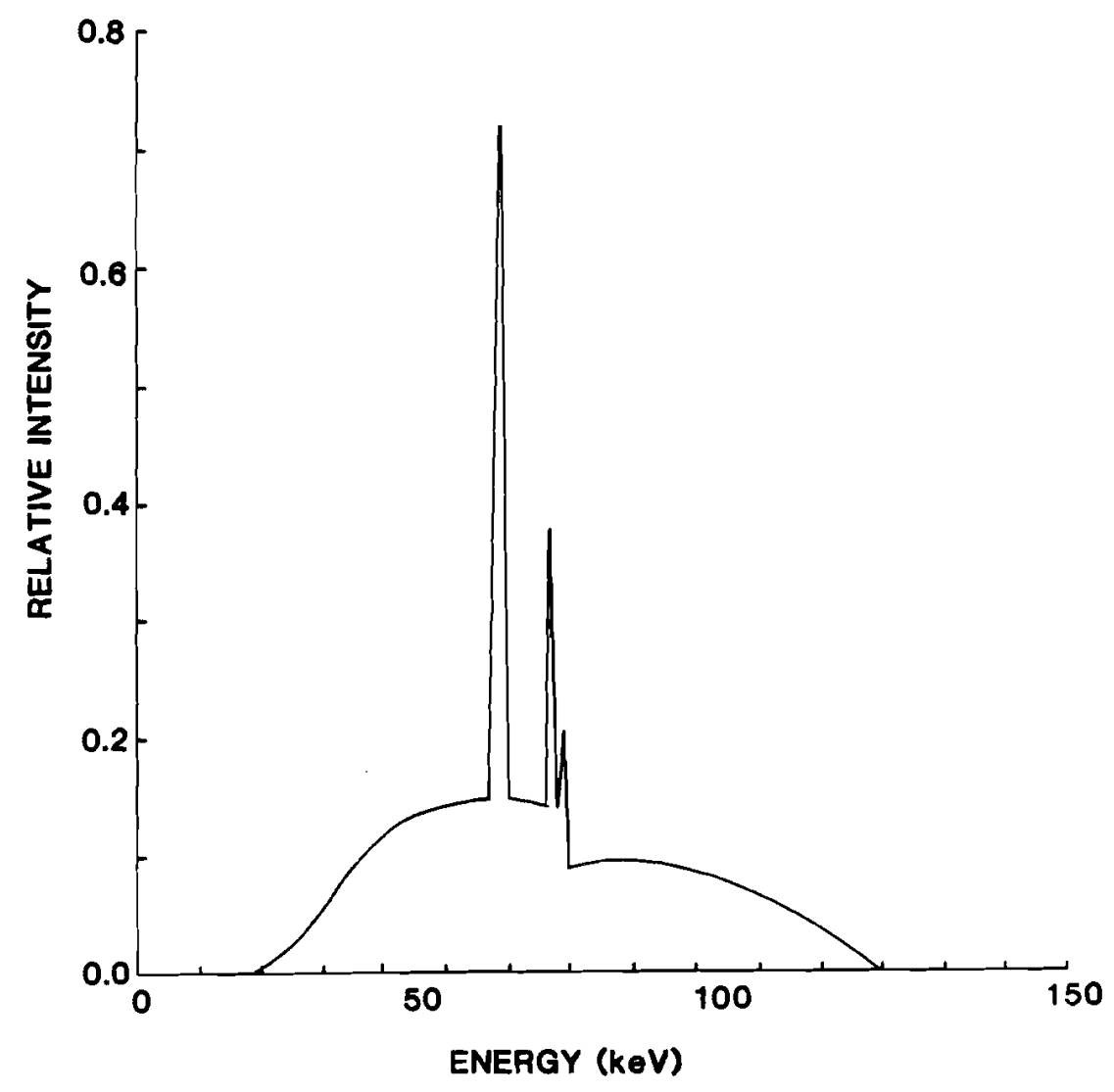

Figure 2.15: Computed Spectrum of the Wellington Hospital C.T. Scanner at $120 \mathrm{kVp}$. 
The X-ray spectrum predicted for the C.T. scanner is plotted in figure 2.15 , and a printout is given in appendix E. An attempt has been made to verify this spectrum by determining whether it accurately predicts the fractional transmission of the beam through varying thicknesses of aluminium. To this end aluminium collars were obtained to fit over a thimble ionisation chamber. The chamber was placed in the isocentre of the scanner, and five scans were performed at each of $0 \mathrm{~mm}, 4 \mathrm{~mm}, 6 \mathrm{~mm}, 8 \mathrm{~mm}$ and 10 $\mathrm{mm}$ thicknesses of aluminium collar. These scans were performed using a system troubleshooting program called TABTEST, so that the scans could be performed without the shaped (bow-tie) filter. The results are summarised in table 2.6.

Table 2.6: Comparison of Measured \& Predicted Transmission Ratios.

\begin{tabular}{||c|c|c|}
\hline $\begin{array}{c}\text { Thickness of Al } \\
\text { collar (mm). }\end{array}$ & $\begin{array}{l}\text { Fraction of beam } \\
\text { intensity transmitted }\end{array}$ & $\begin{array}{c}\text { Fraction } \\
\text { predicted }\end{array}$ \\
\hline $4 \mathrm{~mm}$ & $0.69 \pm 0.01$ & 0.72 \\
$6 \mathrm{~mm}$ & $0.60 \pm 0.01$ & 0.62 \\
$8 \mathrm{~mm}$ & $0.52 \pm 0.01$ & 0.54 \\
$10 \mathrm{~mm}$ & $0.45 \pm 0.01$ & 0.47 \\
\hline
\end{tabular}

It can be seen that the predicted transmission ratios lie just outside the range of measurement uncertainty. However in view of the known limitations of the technique the degree of agreement may be regarded as satisfactory. The limitations result from the departure from narrow beam geometry, since scatter produced in the aluminium collar may reach the detector, and the presence of high atomic number impurities within the aluminium [78].

Comparison of the computed spectrum of the Wellington scanner (figure 2.15) with the typical C.T. spectrum taken from Birch \& Marshall (figure 2.3) shows the two to be quite similar except just above the tungsten $\mathrm{K}$ edge at $69.5 \mathrm{keV}$ where the discontinuity in the continuum is some three times greater. This is a consequence of the small target angle $\left(7^{\circ}\right)$ of the tube used in the G.E. 8800 , whereas the spectrum computed by Birch \& Marshall assumes a $15^{\circ}$ target angle.

\subsection{Reprise.}

The X-ray attenuation coefficient is of central importance in the interpretation of the pixel values reported by a C.T. scanner. Three processes contribute to the total attenuation coefficient in the diagnostic energy range (20 to $150 \mathrm{keV})$, and for elements 
with $Z \leq 20$ there is a transition from photoelectric dominant attenuation in the lower portion of the energy range, with strong energy dependence, to Compton dominated attenuation with very weak energy dependence in the higher portion of the energy range. The third attenuation process, coherent scatter, never contributes more than $10 \%$ of the total attenuation but must still be included in calculations. There are differences in the values of the attenuation coefficients listed in published tables which would appear to be large enough to affect the interpretation of C.T. numbers, but it is shown in the present work that C.T. number measurements on samples whose effective atomic numbers are close to that of water correlated equally well with attenuation coefficients interpolated from three different sets of tables representing recent examples of purely experimental, purely theoretical and hybrid tabulations. Since the C.T. number uses water as a reference material it is clear that it is the ratio of the attenuation coefficient of each element of interest to that of water which is important. Such an exercise has been shown to greatly reduce the effects of differences in tables around $\mathrm{Z}=8$, but not for higher atomic number elements. As the elements with higher atomic numbers usually contribute only a small proportion of the total attenuation of human tissues the inaccuracies introduced are generally not of significance except in those cases in which attenuation measurements are used to determine the concentrations of materials composed of these heavier elements, as in the case of bone mineral measurement which is discussed in chapter four.

The diagnostic energy range is only a small portion of the total range of energies covered by tables of attenuation coefficients. The tables list only a limited number of values in the energy range of interest in the present work, and yet the nature of the attenuation curves makes interpolation very difficult. There have been several attempts to produce simple expressions, ideally with few constants, which reproduce tabulated attenuation coefficients with acceptable accuracy. All of these require the computation of the KleinNishina cross section, which is generally computed from moderately complicated formulae which involve unacceptably long computations when large amounts of data are involved. Continued fraction techniques have been used in the present work to derive an extremely simple expression which matches the Klein-Nishina function over the diagnostic energy range with an accuracy which exceeds the requirements. It is shown that simple empirical expressions for the total attenuation coefficient cannot meet the accuracy requirements unless a smaller energy range is considered and some constants are listed separately for each element. In the present work a very simple expression (equation (2.19)) has been found which matches tabulated attenuation coefficients over the range from 50 to $100 \mathrm{keV}$ with errors of less than $0.35 \%$. This expression requires only three constants to be known separately for each element, of which one is simply derived from the atomic number and atomic weight (see equation 2.6). Accuracy of $0.1 \%$ may be achieved over the same restricted energy range with a five parameter continued fraction expression, equation 2.33 . It has been shown that virtually perfect interpolation over the 
full diagnostic energy range can be achieved with an eight parameter continued fraction expression. Software has been devised for performing this task and is further discussed in appendix $\mathrm{C}$.

In certain circumstances it is not sufficient to consider the polychromatic X-ray spectrum of a C.T. scanner to be equivalent to a monochromatic beam of a certain 'effective' energy, in which case it is necessary to be able to compute the spectrum. A program has been developed which computes the spectrum from the expressions of Birch \& Marshall [69]. This program incorporates a subroutine which permits the effects of filtration by various materials to be studied, and has been used to compute the spectrum for the Wellington hospital C.T. scanner. 


\section{CHAPTER THREE: DUAL ENERGY COMPUTED TOMOGRAPHY}

\subsection{Limitations of Single Energy Computed Tomography.}

The question naturally arises whether the physical property measured by computed tomography scanners, the C.T. number, is of diagnostic value in its own right other than by simply determining the shade of grey to be used to represent the pixels having a particular C.T. number. While it is possible to say for example that in general C.T. numbers in the range 0 to - 100 represent fatty tissues, it is not possible to define with precision C.T. number ranges for the different types of tissue since the measured C.T. number depends to some extent on the characteristics of the scanner used to make the measurement. Even different scanners of the same manufacturer and model can report different C.T. numbers for the same object [79]. Differences in calibration between scanners exist because not all have the calibration factor, $\mathrm{K}$ (equation 2.3 ), set to exactly 1000; and because quite large differences in X-ray beam quality are found. Peak kilovoltages used in single energy C.T. range from $110 \mathrm{kVp}$ to as much as $140 \mathrm{kVp}$, a range large enough to have a pronounced effect on the measured $C . T$. numbers of tissues whose effective atomic numbers differ from that of water [80, 81], and large differences in filtration also exist. Values taken on a single, well calibrated C.T. scanner may also vary with time due to changes in beam quality resulting from the deposition of tungsten on the glass envelope of the tube insert as the tube ages.

A further limitation on the usefulness of the Hounsfield number results from its definition in terms of the linear attenuation coefficient, which in turn depends on the density and effective atomic number of the tissue as well as on the quality of the X-ray beam used to make the measurement. The measurement is thus ambiguous in the sense that changes in attenuation due to changes in chemical composition may be masked by compensating changes in density. The linear attenuation coefficient cannot in any sense be regarded as a fundamental property of tissue, and it is found that it does not correlate well with many pathologies. For example, differential diagnosis of most types of brain tumour cannot be made on the basis of measured C.T. numbers. A given type of tumour may have a higher or lower C.T. number than the surrounding normal tissue, or there may be no change in C.T. number at all. Some parts of a tumour may have higher attenuation while others exhibit lower attenuation than the surrounding tissue [82]. As another example, it is found that the C.T. number correlates well with mineral concentration in bone [83], but the mineral concentration estimated from single energy C.T. measurements may be in error by as much as $30 \%$ due to variations in the composition of the bone marrow [84]. This problem may be alleviated by concurrently measuring the marrow density using dual energy techniques, as is discussed in chapter 
four.

Dual energy C.T. techniques have been developed in an attempt to measure more clinically relevant parameters such as effective atomic number. An important test of the suitability of such techniques is that the values obtained should be scanner independent: calibration methods should be established so that the value obtained should be the same regardless of which scanner is used to make the measurements.

\subsection{Theoretical Basis of Dual Energy Computed Tomography.}

Although the linear attenuation coefficient is of limited value in characterising tissue, it depends on two tissue properties which are of more fundamental significance: mass density and atomic number. The variation of the attenuation coefficient with energy is independent of the density and depends on the atomic number alone. If the linear attenuation coefficient of a material is measured at two different X-ray energies, the difference in attenuation coefficient is indicative of the effective atomic number of that material. This possibility was mentioned by Hounsfield [8], and was first applied by Rutherford et al. [85]. They expressed the linear attenuation coefficient in a manner similar to that of Weber and van den Berge [64]:

$\mu(E)=20.64 N . Z^{4.62} E^{-3.28}+\sigma_{K N}(E) N . Z+2.8 N . Z^{2.86} E^{-2.02}$

where $E$ is the effective energy of the $X$-ray beam, $Z$ is the effective atomic number of the absorbing material and $\mathrm{N}$ is the number of atoms per unit volume. The constants were optimized for oxygen, so that equation 3.1 may be expected to be accurate for most soft tissues. Taking the ratio of the linear attenuation coefficients at different energies removes the dependence on $\mathrm{N}$, leaving an equation expressed in terms of $\mathrm{Z}$ alone:

$$
\begin{aligned}
\mu\left(E_{1}\right) & =20.64 \mathrm{Z}^{4.62} \mathrm{E}_{1}-3.28+\sigma_{\mathrm{KN}}\left(\mathrm{E}_{1}\right) \mathrm{z}+2.8 \mathrm{z}^{2.86} \mathrm{E}_{1}-2.02 \\
\mu\left(\mathrm{E}_{2}\right) & =20.64 \mathrm{Z}^{4.62} \mathrm{E}_{2}-3.28+\sigma_{\mathrm{KN}}\left(\mathrm{E}_{2}\right) \mathrm{z}+2.8 \mathrm{z}^{2.86} \mathrm{E}_{2}-2.02
\end{aligned}
$$

Iterative techniques may be used to solve equation 3.2 for $\mathrm{Z}$, and $\mathrm{N}$ may then be obtained by back substitution into equation 3.1 at one of the two energies. The product N.Z is the volume electron density, that is, the number of electrons per unit volume.

Rutherford et al obtained X-ray beams of differing effective energies by taking two images at different kilovoltage settings: $140 \mathrm{kVp}$ and $100 \mathrm{kVp}$. The effective energies were $74 \mathrm{keV}$ and $62 \mathrm{keV}$ respectively. They estimate the precision in the measurement of 
electron density when these energies are used to be of the order of $0.5 \%$, and that of effective atomic number to be about $3 \%$. They note that if it were possible to use energies of 40 and $80 \mathrm{keV}$ the uncertainty in $\mathrm{Z}$ could be reduced to better than $0.25 \%$ [86]. Problems noted with the technique were possible patient movement between the two scans, and incorrect results due to the presence of beam hardening artefacts (see section 1.3.1) on the images.

\subsection{Equipment Modifications for Dual Energy Computed Tomography.}

Successful application of the dual energy technique requires the ability to measure the transmission of two X-ray beams of differing energies either simultaneously or in rapid succession. Simultaneous acquisition of both the high and low energy images has the advantage of minimizing the effects of patient movement, and can be achieved at a single kVp by using "split" detectors. Split detectors are comprised of two sections, the one nearest the patient acting as a filter so that the one further away receives a higher energy beam. One such approach was to use a xenon gas detector where each electrode was split into two sections. The electrode sections at the front of the detector would respond principally to the lower energy photons within the beam, and those at the rear would be responding to a higher average energy of radiation. A system using such a detector is said to be capable of $2 \%$ accuracy in measuring electron density and an accuracy of $4 \%$ in the measurement of effective atomic number [87]. A similar approach involves the use of a split scintillation crystal detector where a thin $\mathrm{CaF}_{2}$ crystal is placed above a thicker $\mathrm{NaI}$ crystal. Each crystal is viewed by a photomultiplier crystal mounted at the side. A modified first generation scanner using a detector of this type has been claimed to give superior energy separation to that obtained with the dual $\mathrm{kVp}$ technique [88].

A disadvantage of the split detector technique is that the noise is increased when compared to a single energy image since fewer photons are available to form each of the dual energy images. The reduced image quality for conventional C.T. imaging precludes the use of this technique except perhaps in a very few research institutions where a scanner may be dedicated to dual energy applications alone. To overcome this problem some workers have experimented with the dual filter technique. The first attempt along these lines [89] was a modification of a second generation C.T. scanner so that every second detector was covered with a thin foil of tin. The detectors so modified receive an X-ray beam of higher quality (effective energy) and thus a simultaneous dual energy scan is obtained. This modification is relatively easy to implement, and it is also easy to restore the instrument to its original condition. However the high and low energy images must now be obtained with only half as many detectors, with consequent increase in noise and loss of high contrast resolution. The radiation absorbed in the filters means a certain 
amount of "wasted" dose to the patient. This last objection may be overcome by the use of a pre-patient filter [90], where the filter assembly is included in the tube/collimator assembly. Such an approach is probably only viable with a second generation scanner where the number of detectors is relatively small (less than thirty) since the filter must be accurately aligned with the detector assembly. The pre-patient filter would be more difficult to assemble and disassemble that the pre-detector filter described previously.

All of the above modifications require manipulation of the detector readings prior to reconstruction. For example the pre-detector split filter technique requires two complete sets of raw data to be produced, a complete set of unfiltered data being obtained by interpolation to estimate the values missing due the use of alternate detectors for filtered readings. In most cases it is possible to then use the manufacturer's software to reconstruct images from the adjusted readings.

C.T. scanners are now available with dual energy available as an option. The most promising technique is to use a pulsed high tension supply where the pulses alternate between the selected high and low kilovoltage settings, permitting simultaneous acquisition of the high and low energy images [91].

\subsection{Algorithms for Dual Energy Computed Tomography.}

\subsubsection{Limitations of the Rutherford Algorithm.}

The algorithm of Rutherford et al (equation 3.2) assumes in its derivation that the attenuation undergone by a polychromatic X-ray beam traversing a material is the same as that undergone by an equivalent monochromatic beam. The energy of this monochromatic beam is the "effective" energy of the polychromatic beam. However the concept of an effective energy only has meaning for a particular material, although the equivalence remains approximately true for a narrow range of atomic numbers centred on the effective atomic number of the reference material. An effective energy defined in respect of a reference material whose effective atomic number is in the range of soft tissues (6 - 8) will be of little value in the estimation of the attenuation of an X-ray beam in bone $(Z=13)$ [92]. To illustrate this fact, the effective energy of the G.E. 8800 scanner operated at $120 \mathrm{kVp}$ has been calculated for a range of atomic numbers. As a first step, the exit spectrum has been calculated (using program XSPECT) by assuming that the patient is equivalent to $25 \mathrm{~cm}$ of water. This corresponds to the conditions under which the scanner is calibrated for head scans. The exit spectrum, normalised to a total intensity of unity, is plotted in figure 3.1 . 


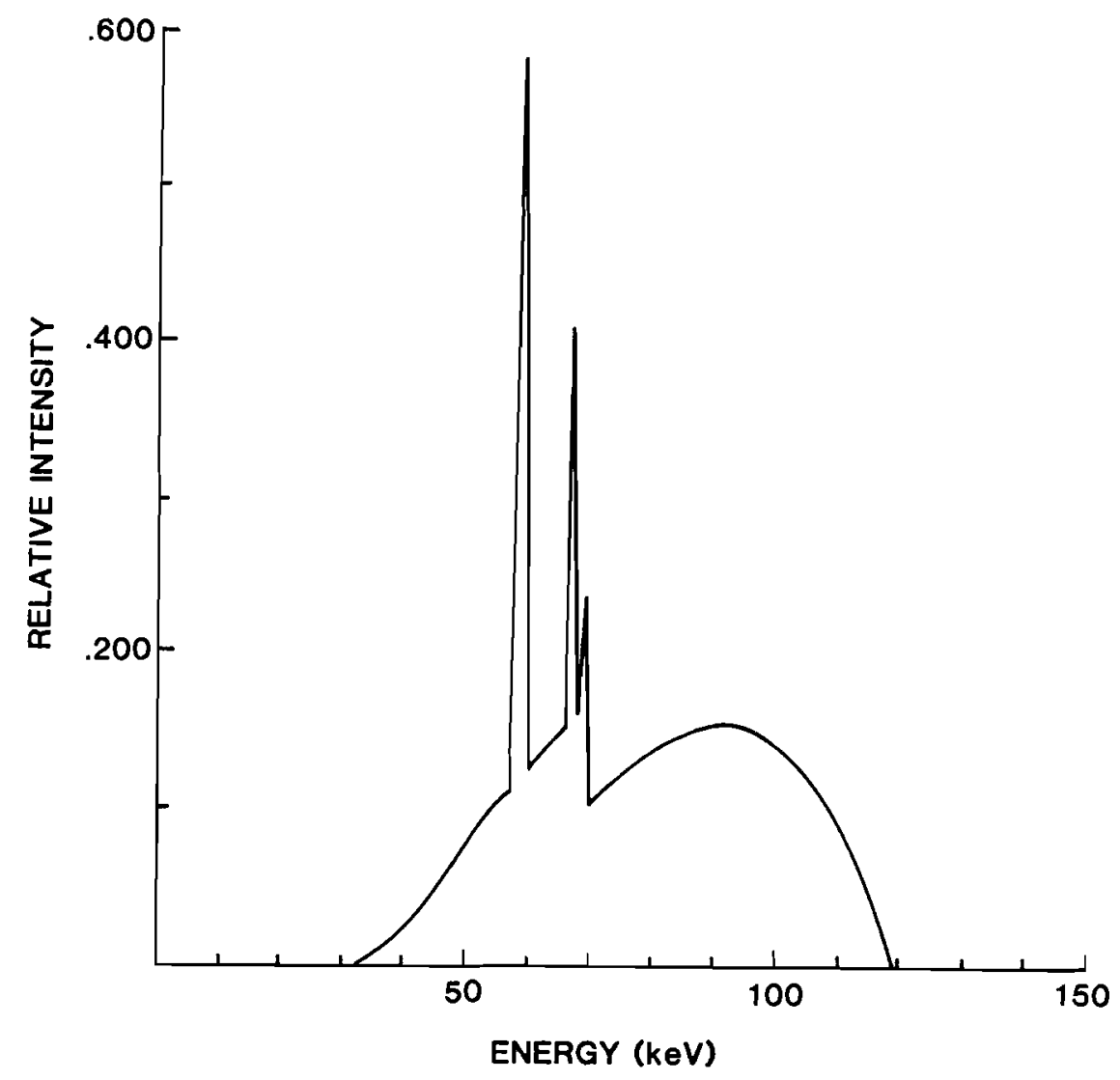

Figure 3.1: Exit Spectrum of the G.E. 8800 at $120 \mathrm{kVp}$.

The effect of the patient has been simulated by filtration by $25 \mathrm{~cm}$ of water.

The effective attenuation coefficient, for a given element, for the exit spectrum is given by:

$$
\mu_{\text {eff }}=\quad \int_{10 \mathrm{keV}}^{120 \mathrm{keV}} \mathrm{S}(E) \cdot \mu(E) \cdot \mathrm{dE}
$$

where $S(E)$ is the normalised intensity spectrum. The effective energy of the spectrum is the monochromatic energy at which the mass attenuation coefficient of the particular element is equal to the effective mass attenuation coefficient calculated using equation 3.3. The integration was performed numerically by summation at $1 \mathrm{keV}$ steps, using "best" theoretical attenuation coefficients, as defined in section 2.4. The calculated effective energies ranged from $77.2 \mathrm{keV}$ for hydrogen through $73.1 \mathrm{keV}$ for nitrogen to $68.8 \mathrm{keV}$ for calcium, and are plotted in figure 3.2. Unfortunately the slope is greatest at about $Z=7$, so that the difference between the effective energies for nitrogen and its two neighbours on the periodic table is of the order of $1 \mathrm{keV}$. 


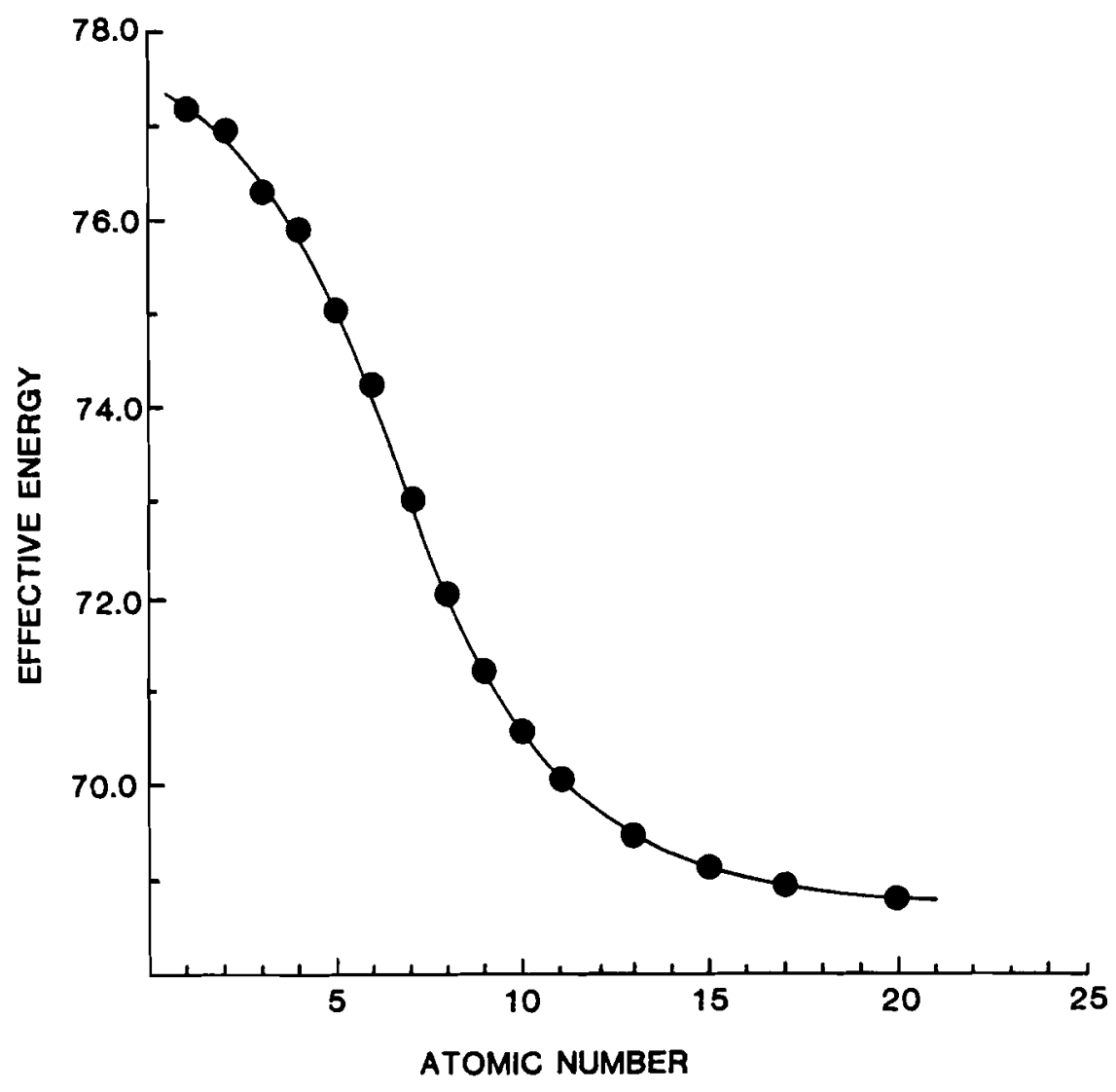

Figure 3.2: Effective Energy of the G.E. 8800 as a Function of Atomic Number.

The effective energy of a scanner is normally calculated for materials whose effective atomic number is about 7, corresponding to-an effective energy of $73.1 \mathrm{keV}$. This is quite different to the effective energy for bone $(Z=13)$ of $69.5 \mathrm{keV}$.

Since the effective energy of an X-ray beam depends on the effective atomic number of the absorbing material, it follows that the effective atomic number determined from C.T. measurements will depend on the X-ray spectra used to make the measurement. The dual energy parameters will not be independent of the scanner used to make the measurements, and so the method is unsuitable for general application.

A further limitation of the Rutherford algorithm is its computational complexity, since it involves an iterative solution to a moderately involved equation. Any calculation involving of the order of 250000 pixels must be kept relatively simple if reasonable computational times are to be achieved. Some simplification of the expression may be achieved by the use of a calibration curve in which effective atomic number is plotted as a function of the ratio of attenuation coefficients at the two energies [93]. This curve may be stored digitally as a "look-up" table. 


\subsubsection{The Algorithm of Brooks.}

The formalism of Brooks [94, 95] may be derived as follows. The total linear attenuation coefficient is factorised into an ideal Compton (Klein-Nishina) component and another factor which represents all other effects: photoelectric effect, coherent scatter and corrections to the Compton cross section to account for electron binding effects.

$$
\mu(E)=\mu_{c}(E)+\mu_{p}(E)
$$

"Compton" and "photoelectric" Hounsfield numbers are then defined as follows:

$$
\begin{aligned}
& \mathrm{H}_{\mathrm{C}}=1000 \cdot\left[\frac{\mu_{\mathrm{c}}}{\mu_{\mathrm{CW}}}-1\right] \\
& \mathrm{H}_{\mathrm{p}}=1000 \cdot\left[\frac{\mu_{\mathrm{p}}}{\mu_{\mathrm{pw}}}-1\right]
\end{aligned}
$$

where $\mu_{\mathrm{cw}}$ and $\mu_{\mathrm{pw}}$ are the "Compton" and "photoelectric" attenuation coefficients for water. Given the above definitions, and the assumption that all materials have the same energy dependence for the "photoelectric" component of the attenuation coefficient (the energy dependence of the Compton cross section being given by the Klein-Nishina expression for all materials), the conventional Hounsfield number may be related to the "Compton" and "photoelectric" Hounsfield numbers as follows:

$$
H=\frac{\mathrm{H}_{\mathrm{C}}+\mathrm{B} \cdot \mathrm{H}_{\mathrm{p}}}{1+B}
$$

where the factor $B$ is the ratio of the "photoelectric" component of water to the "Compton" component of water, as measured with the spectrum of a particular scanner at a particular kilovoltage. The "Compton" and "photoelectric" Hounsfield numbers may be calculated from the above equation if the measurement is performed at two different energies, so that two equations are obtained in the two unknowns.

The dependence of the "Compton" and "photoelectric" Hounsfield numbers on the scanner used to make the measurements has been estimated by computer simulation for a number of elements up to $Z=30$. The two parameters were calculated using equation 3.6 but using mass attenuation coefficients rather than Hounsfield numbers, which is equivalent to an assumption of a density of $1 \mathrm{~g} / \mathrm{cc}$. The effect of using different scanners was simulated by calculating the parameters firstly at (monochromatic) energies of 55 and $73 \mathrm{keV}$, and secondly at energies of 65 and $80 \mathrm{keV}$. The first energy pair (55 and $73 \mathrm{keV}$ ) was selected as representing the lowest values reported in both the low (70 - 
$110 \mathrm{kVp})$ and high $(120-140 \mathrm{kVp})$ kilovoltage ranges. The second energy pair represents the highest values reported. The results of the simulations are plotted in figure 3.3. It can be seen that the parameters are not independent of the energies used to make the measurement, and that the differences due to the measurement conditions are large compared with the expected differences due to pathology [94]. This machine dependence almost certainly results from the assumption of a common energy dependence of the photoelectric component of the attenuation coefficient for all materials. Reference to figure 2.11 shows that the exponent ' $b$ ' in equation (2.19), which represents the energy dependence of the non-Klein-Nishina portion of the total linear attenuation coefficient, is relatively constant only for elements with atomic numbers greater than about 13 . The value of the exponent depends quite strongly on atomic number in the region of principal interest here: from carbon through to oxygen.

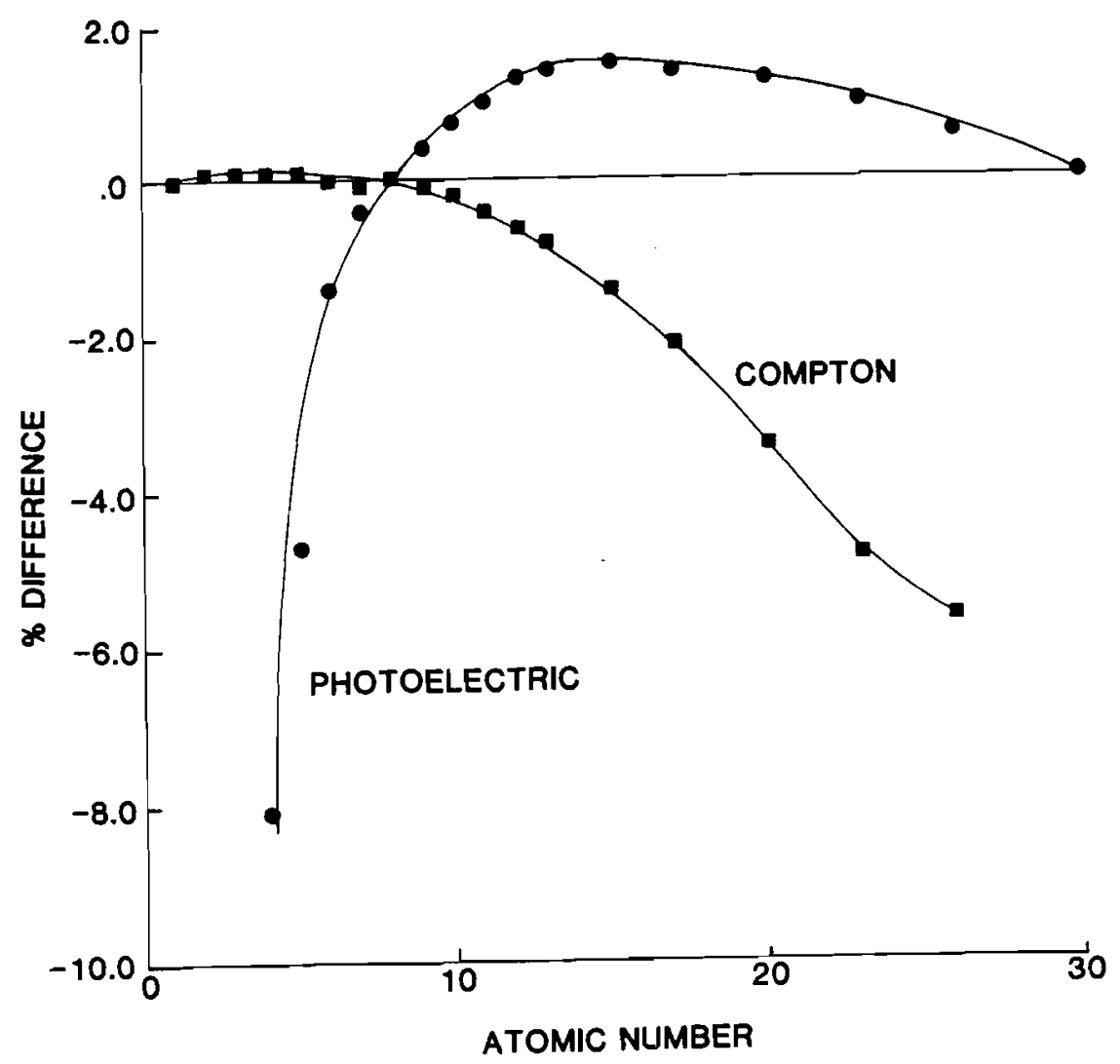

Figure 3.3: Energy Dependence of the Brooks Parameters.

The quantity plotted is the percentage difference between values calculated at monochromatic energies of 55 and $73 \mathrm{keV}$, compared with values calculated at energies of 65 and $80 \mathrm{keV}$. 


\subsubsection{The Algorithm of Alvarez \& Macovski (PREDECT*).}

This technique is applied to the raw transmission readings prior to image reconstruction [99]. The attenuation coefficient is viewed as a linear combination of simpler functions of energy:

$$
\mu(E)=a_{1} \cdot f_{1}(E)+a_{2} \cdot f_{2}(E)+\ldots+a_{N} \cdot f_{N}(E)
$$

The values of the constants may in principle be obtained by repeating the measurement at $\mathrm{N}$ energies and solving the system of $\mathrm{N}$ simultaneous equations. When measurements are made at two energies the linear attenuation coefficient is expressed as a linear combination of two basis functions. In their earlier work [96, 97] the attenuation coefficient was factorised into Klein-Nishina and non-Klein-Nishina components as follows:

$$
\mu(E)=a_{1} \cdot \frac{1}{E^{3}}+a_{2} \cdot \sigma_{K N}(E)
$$

where $a_{1}$ is a simple function of atomic number and $a_{2}$ is a simple function of electron density. The calculated line integrals of electron density and effective atomic number are then used to reconstruct images from these variables. This approach is limited by the fact that these functions do not give a good approximation to the linear attenuation coefficient (see chapter two). In subsequent work [98, 99] a different set of basis functions is used, the linear attenuation coefficients of perspex and aluminium. The effective atomic number of perspex approximates to that of soft tissues while that of aluminium is similar to the effective atomic number of bone. The transmission reading of each detector at the two energies is used to express the total attenuation along each ray as equivalent to attenuation by certain lengths of aluminium and perspex. This approach has the advantage that calibration can be achieved directly against phantoms composed of these materials.

A major advantage of applying the dual energy algorithm to the raw (unreconstructed) data is that the technique avoids some of the problems due to polychromaticity of the X-ray beam. Incomplete correction for beam hardening produces well known artefacts such as the Hounsfield dark space on single energy images. When two such single energy images are manipulated to produce a dual energy image, these errors can in some circumstances lead to unrealistic values of such quantities as effective atomic number. The use of calibration curves of X-ray transmission versus thicknesses of perspex and aluminium can potentially lead to a large reduction in polychromatic artefacts [99]. 
Computer simulations have been performed to assess the scanner dependence of the algorithm of Alvarez and Macovski. Since the focus of the present work has been on post reconstruction techniques, for reasons discussed in section 3.5.2, a modified algorithm has been devised. The algorithm has the same form as equation 3.8 , but the functions of energy are now the mass attenuation coefficients of perspex and aluminium.

$$
\left[\frac{\mu}{\rho}\right](E)=a_{1} \cdot\left[\frac{\mu}{\rho}\right]_{\mathrm{Al}}(\mathrm{E})+\mathrm{a}_{2} \cdot\left[\frac{\mu}{\rho}\right]_{\text {Perspex }}(\mathrm{E})
$$

The parameters $\mathrm{a}_{1}$ and $\mathrm{a}_{2}$ were calculated at two different pairs of energies, $55 / 73 \mathrm{keV}$ and $65 / 80 \mathrm{keV}$, as was done in the previous section, and the results are plotted in figure 3.4. Comparison with figure 3.3 shows that the Alvarez parameters are much less sensitive to the energies used in measuring them than are the Brooks parameters. In figure 3.5 the energy sensitivity of the aluminium component is shown to depend on the choice of the low atomic number basis material. Surprisingly the parameter a has the same energy dependence for each of the three materials tested. *

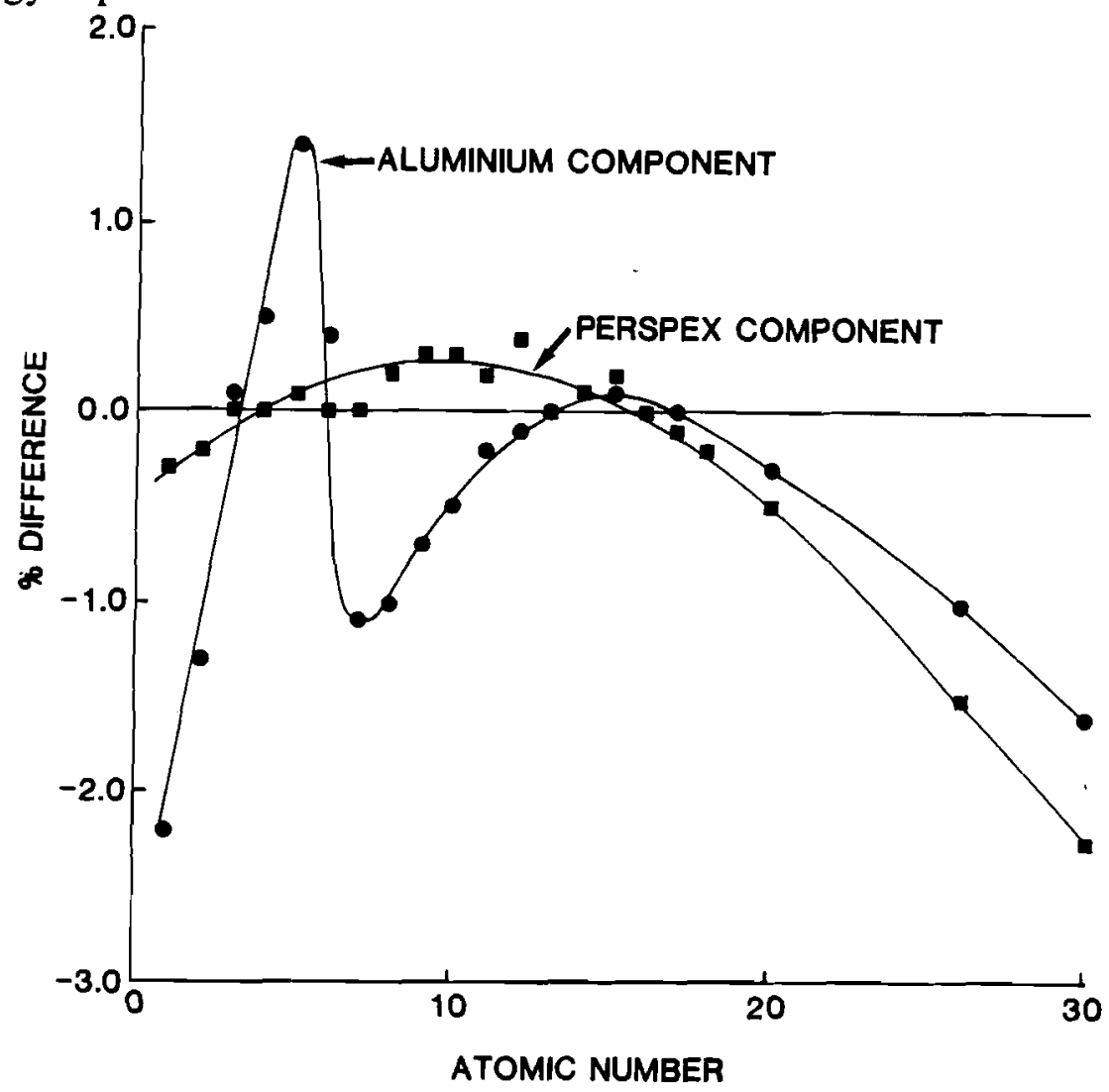

Figure 3.4: Energy Dependence of the Alvarez Parameters.

The quantity plotted is the percentage difference between values calculated at monochromatic energies of 55 and $73 \mathrm{keV}$, compared with values calculated at energies of 65 and $80 \mathrm{keV}$. 


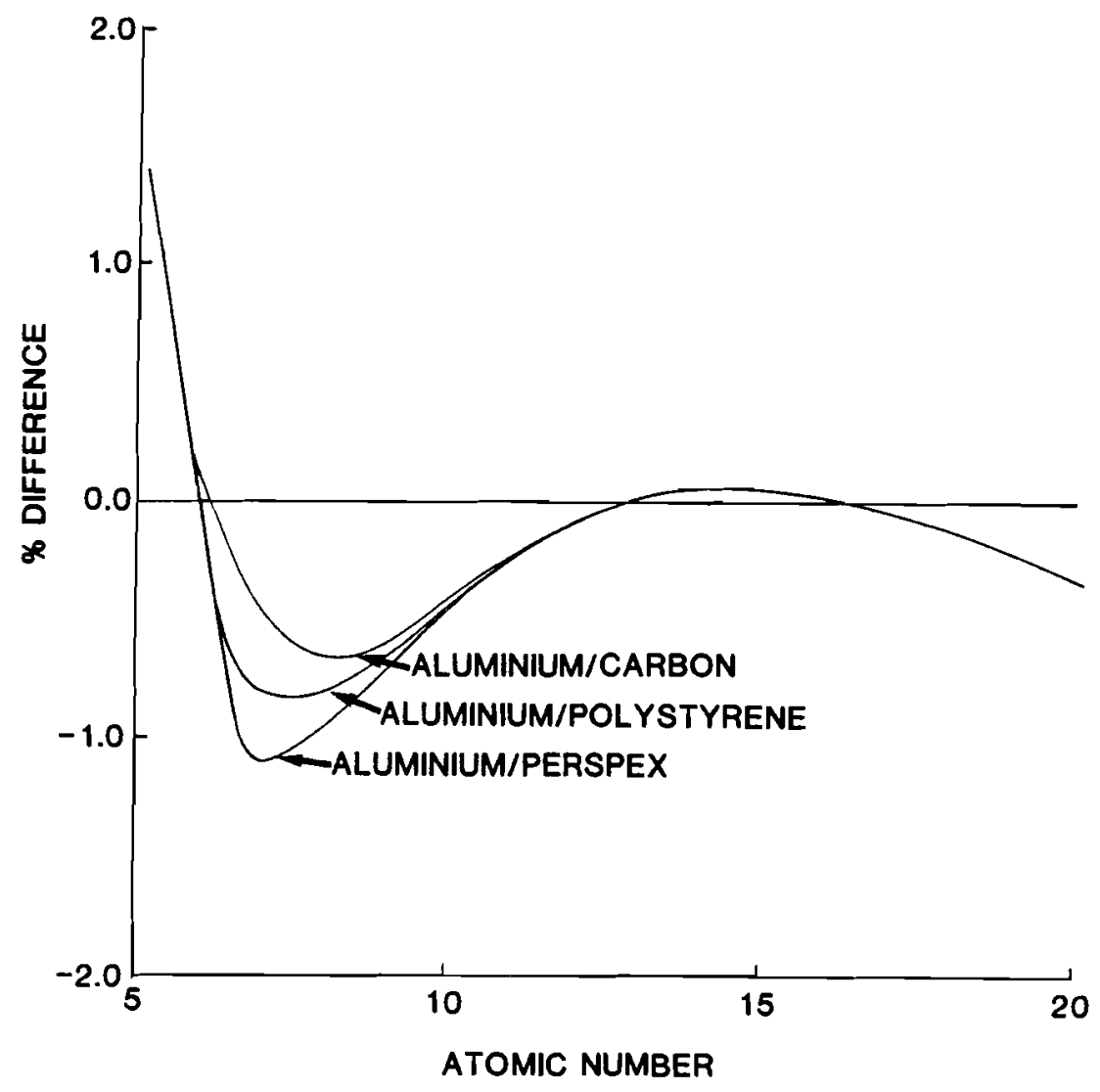

Figure 3.5: Energy Dependence of the Alvarez Parameters Using Different Basis Materials.

The quantity plotted is the percentage difference between values of the aluminium component calculated at monochromatic energies of 55 and $73 \mathrm{keV}$, compared with values calculated at energies of 65 and $80 \mathrm{keV}$. The energy dependence is lowest over the range of atomic numbers which are of most interest in the medical field when carbon is used as the low atomic number basis material. The energy dependence of the plastic component is essentially the same for the three materials tested.

\subsubsection{The Work of Christ.}

Christ [100] has proposed an alternative pre-reconstruction technique in which the beam hardening correction is applied analytically. The linear attenuation coefficient is parametrised as:

$$
\mu(E)=\rho \cdot \mathrm{n}_{\mathrm{g}}\left\{\mathrm{Z}^{\mathrm{n}} \cdot \sigma_{\mathrm{p}^{\prime}}(\mathrm{E})+\sigma_{\mathrm{S}^{\prime}}(\mathrm{E})\right\}
$$

where $\mathrm{n}_{\mathrm{g}}$ is the mass electron density. The exponent $\mathrm{n}$ is selected for optimal fit over the atomic number range of from 4 to 13 , and was given a value of 3.6. The term $\sigma_{\mathrm{p}}$ ' is the cross section, per electron, due to the photoelectric effect; and the expression $\sigma_{\mathrm{S}}{ }^{\prime}$ represents the energy dependence of both scatter processes. When the effect of polychromaticity is taken into account the total attenuation coefficient becomes: 


$$
\mu=\rho \cdot \mathrm{n}_{\mathrm{g}} \mathrm{z}^{\mathrm{n}} \frac{\int_{0}^{E_{\mathrm{m}}} \sigma_{\mathrm{p}^{\prime}(E) \cdot I_{0} \cdot d E}}{\int_{0}^{E_{\mathrm{m}}} I_{0} \cdot d E}+\rho \cdot \mathrm{n}_{\mathrm{g}} \frac{\int_{0}^{E_{m}} \sigma_{s^{\prime}(E) \cdot I_{0} \cdot d E}}{\int_{0}^{E_{m}} I_{0} \cdot d E}
$$

where the integrations are performed over the range of energies present in the incident Xray spectrum. When the transmission measurements are performed at two kilovoltages there are two such equations:

$$
\begin{aligned}
& \mu_{1}=\rho \cdot n_{g}\left(z^{n} \cdot p_{1}+s_{1}\right) \\
& \mu_{2}=\rho \cdot n_{g}\left(z^{n} \cdot p_{2}+s_{2}\right)
\end{aligned}
$$

where the integral for the photoelectric effect (per electron) is represented by $p$ and the integral for scatter is represented by $s$. These equations are then solved for effective atomic number and volume electron density as follows:

$$
\begin{aligned}
\mathrm{z}_{\text {eff }} & =\left[\frac{\mu_{1} \mathrm{~s}_{2}-\mu_{2} \mathrm{~s}_{1}}{\mu_{2} \mathrm{~s}_{1}-\mu_{1} \mathrm{~s}_{2}}\right]^{1 / \mathrm{n}} \\
\mathrm{D}_{\mathrm{V}} \equiv \rho \cdot \mathrm{n}_{\mathrm{g}} & =\frac{\mu_{2} \mathrm{p}_{1}-\mu_{1} \mathrm{p}_{2}}{\mathrm{p}_{1} \mathrm{~s}_{2}-\mathrm{p}_{2} \mathrm{~s}_{1}}
\end{aligned}
$$

The previous equations assume that the shape of the $\mathrm{X}$-ray energy spectrum remains constant as it passes through the patient. Each transmission measurement corresponds to an average attenuation along the ray:

$$
\mu_{\mathrm{av}}=\frac{1}{\mathrm{x}^{\prime}} \int_{0}^{\mathrm{x}^{\prime}} \frac{\int_{0}^{\mathrm{E}_{\mathrm{m}}} \mu(E) \cdot \mathrm{I}_{\mathrm{x}}(E) \cdot d E}{\int_{0}^{E_{m}} I_{x}(E) \cdot d E} \cdot d x
$$

where $\mathbf{x}^{\prime}$ is the length of the ray. In this method the measured average attenuation coefficients (equation 3.14) at the two kilovoltages are used in equations (3.13) to produce a first estimate of the volume electron density and the effective atomic number. These values are then used to estimate the value of the attenuation coefficient via:

$$
\mu^{\prime}(E)=\frac{D_{V^{\prime}} \cdot\left\{\sigma_{p}\left(E, Z^{\prime}\right)+\sigma_{S}\left(E, Z^{\prime}\right)\right\}}{Z^{\prime}}
$$


where $\mu^{\prime}(E), \quad D_{V}^{\prime}$ and $Z^{\prime}$ are the first estimates of the monochromatic attenuation coefficient, volume electron density and effective atomic number respectively. The estimated attenuation coefficient is then used to correct for hardening of the incident beam:

$$
\mu_{\mathrm{av}}^{\prime}=\frac{1}{\mathrm{x}^{\prime}} \text { In } \frac{\int_{0}^{E_{\mathrm{m}} I_{0}(E) \cdot d E}}{\int_{0}^{E_{\mathrm{m}}} I_{0}(E) \cdot \exp \left(-\mu^{\prime}(E) \cdot x^{\prime}\right) \cdot d E}
$$

The measured average attenuation coefficient is then replaced by a better estimate, which is its original value less the difference between its original value and the corrected value from equation (3.16). Several iterations are required. This technique requires that the path length for each ray $\left(x^{\prime}\right)$ be known, along with the incident spectra at both kilovoltages.

In common with the techniques of Rutherford et al and that of Brooks, the parametrisation of the attenuation coefficient used is too crude to yield results of optimal accuracy. Equation 3.10 neglects the effect of atomic number on the coherent scatter cross section, and assumes that the photoelectric cross section can be factorised into separate energy- and atomic number dependent functions. This is not a sufficiently accurate approximation even over the narrow range of atomic numbers considered: see sections 2.8 and 3.4.1. These objections could perhaps be overcome by replacing equation 3.10 with an improved expression, but even with the simple expression used by Christ a very large amount of computing is required. The approach of Alvarez \& Macovski would be equally successful in removing polychromatic errors using empirical rather than analytical means.

\subsubsection{The Algorithm of Hawkes \& Jackson.}

The approach taken by Hawkes and Jackson [102] is similar to that of Alvarez \& Macovski in that the attenuation coefficients of two materials are used as basis functions, the attenuation coefficient of any material being expressed as a combination of the attenuation coefficients of those two materials. It differs in that it is applied to the reconstructed images at two kilovoltages, not to the raw data from which the images are reconstructed. Representing the mass attenuation coefficient of a material as a linear combination of the mass attenuation coefficients of the two reference materials: 
$\left[\frac{\mu}{\rho}\right](E)=a_{W} \cdot\left[\frac{\mu}{\rho}\right]_{W}(E)+a_{C} \cdot\left[\frac{\mu}{\rho}\right]_{C}$

where the subscripts $\mathrm{w}$ and $\mathrm{c}$ refer to the reference materials, in this case water and calcium chloride. The mass attenuation coefficient of $\mathrm{CaCl}_{2}$ is estimated from C.T. measurements of a weak aqueous solution ( $5 \%$ by weight) of calcium chloride to minimize errors due to the beam hardening effect. For compatibility with the Hounsfield number, the equation must be rewritten so that the linear attenuation coefficient of the tissue is the subject of the equation:

$$
\mu(E)=\rho_{\mathrm{W}} \cdot\left[\mathrm{m}_{\mathrm{W}}\left[\frac{\mu}{\rho}\right]_{\mathrm{W}}(\mathrm{E})+\mathrm{m}_{\mathrm{C}}\left[\frac{\mu}{\rho}\right]_{\mathrm{C}}\right. \text { (E) }
$$

where the coefficients $m_{W}$ and $m_{C}$ are related to the coefficients $a_{W}$ and $a_{C}$ as follows:

$$
\begin{aligned}
& \mathrm{m}_{\mathrm{W}}=\left[\frac{\rho}{\rho_{\mathrm{W}}}\right] \cdot \mathrm{a}_{\mathrm{W}} \\
& \mathrm{m}_{\mathrm{C}}=\left[\frac{\rho}{\rho_{\mathrm{W}}}\right] \cdot \mathrm{a}_{\mathrm{C}}
\end{aligned}
$$

When expressed in terms of the Hounsfield number, the equation becomes:

$$
H=1000\left(m_{W}-1\right)+m_{C} \cdot 1000 \cdot\left[\frac{\mu}{\rho}\right]_{C} \cdot\left[\frac{\mu}{\rho}\right]_{W}^{-1}
$$

The ratio of the mass attenuation coefficients of the two reference materials may be estimated from measurements on phantoms containing the reference materials. The use of chemical solutions as the reference materials is a significant advantage over the use of aluminium and plastic in that they can be easily reproduced with accuracy anywhere in the world. Commercially available aluminium may contain variable amounts of high atomic number impurities [78], and commercial perspex may vary considerably in both density and composition. 
The energy dependence of the Hawkes parameters have been estimated in exactly the same way as for the Alvarez parameters, using equation 3.17 for the purposes of simulation. It can be seen from figure 3.6 that the use of water and calcium chloride as the basis materials significantly reduces the energy (and hence scanner) dependence of the parameters.

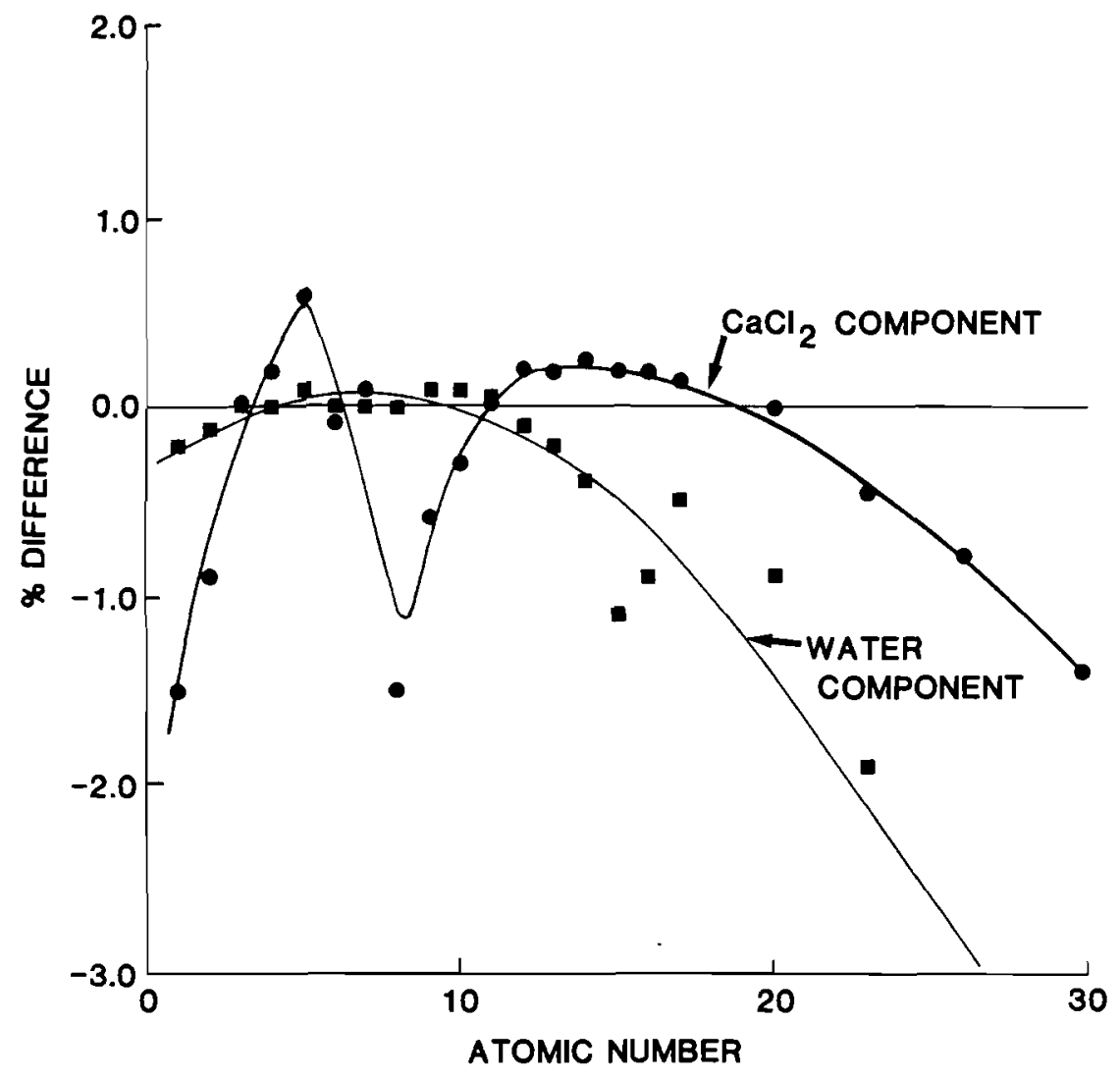

Figure 3.6: Energy Dependence of the Hawkes Parameters.

The quantity plotted is the percentage difference between values of the coefficients $a_{1}$ and $a_{2}$ (equation 3.17) calculated at monochromatic energies of 55 and $73 \mathrm{keV}$, compared with values calculated at energies of 65 and $80 \mathrm{keV}$.

\subsection{A Method for Obtaining Dual Energy C.T. Scans Using the G.E. 8800 C.T. Scanner.}

\subsubsection{Equipment Considerations.}

The G.E. 8800 is designed for single energy applications with a fixed kilovoltage of $120 \mathrm{kV}$. It is maintained under a service contract whose conditions forbid modification of the equipment in any material way. Furthermore the clinical workload dictates that the additional time taken by dual energy C.T. scans must be kept to a minimum, and that the quality of the single energy scans must not be compromised by the addition of dual energy 
capability. This last factor rules out the split filter technique, and the split detector technique would require drastic changes to the scanner which would be to the detriment of its single energy performance. The technique in which the high tension voltage rapidly alternates between two fixed kilovoltage levels has great appeal since the two images are acquired simultaneously, however the degree of equipment modification required is obviously excessive. The only feasible method of obtaining dual energy scans with the Wellington Hospital C.T. scanner is therefore by taking sequential scans at differing kilovoltages. We are fortunate in that the X-ray generator used in the C.T. scanner is a modified version of a standard G.E. diagnostic generator, which has provision for the setting of kilovoltage in $1 \mathrm{kVp}$ steps. Interlocks are fitted which prevent exposure unless the kilovoltage is set to $120 \mathrm{kVp}$, so the only modification required has been the addition of a link on the back of the kilovoltage selector switch identifying other kilovoltages as being permissible. This modification was performed by a G.E. service engineer.

The choice of the two kilovoltages to be used was also clear cut in our case. The maximum rating for the high tension cables is $120 \mathrm{kV}$. While higher kilovoltages can be used, it is possible that the life of the cables could be reduced causing complications in respect of the service contract. Published work [103] suggests that the lower energy should be of the order of $40 \mathrm{keV}$. Limitations on the filament current on the X-ray tube used (100-CT) dictate a minimum applied kilovoltage of $80 \mathrm{kVp}$, corresponding to an effective energy somewhat greater than the optimum.

In scanners designed for multiple $\mathrm{kV}$ operation a separate calibration is performed at each kilovoltage, the calibration data for each permitted kilovoltage being stored separately in the computer system. The G.E. 8800 does not have this facility: there is a single file called WCALIF which stores the calibration data, however a way to 'work around' this problem has been developed in the present work. Calibration data at $80 \mathrm{kVp}$ may be obtained and stored by exiting the scanning program (called MONITOR), saving the $120 \mathrm{kVp}$ calibration data by renaming file WCALIF to some other name (120KV), and creating another file called WCALIF with the same characteristics as the original. The scanning program is then invoked and is used to acquire calibration data in the usual way, but with the generator kilovoltage set to $80 \mathrm{kVp}$. To revert to $120 \mathrm{kVp}$ operation it is necessary to exit the scanning program, rename WCALIF (to 80KVP) and rename file $120 \mathrm{KV}$ to WCALIF, and restart the scanning program.

Low kilovoltage scans were originally obtained by exiting the MONITOR program, renaming the $80 \mathrm{kV}$ calibration file (80KVP) to WCALIF, reinvoking MONITOR and scanning at $80 \mathrm{kVp}$. Since the start up routine of MONITOR takes $3-4$ minutes this process is time consuming and problems were experienced with patient movement between scans. To avoid the need to exit and re-enter MONITOR during an examination, work was undertaken to see if scans taken at $80 \mathrm{kV}$ with the $120 \mathrm{kV}$ calibration could be 
subsequently reprocessed to give a correctly calibrated image. This seemed feasible since it is possible to store the raw data from which the images are reconstructed on magnetic tape. The raw data can be reloaded into the computer system and further reconstructions made (possibly using special algorithms: see section 1.3) at any time. The requirement was to find some way of undoing the $120 \mathrm{kVp}$ calibration on the raw data, and of imposing the appropriate calibration procedure at $80 \mathrm{kVp}$ using the data stored in file $80 \mathrm{KVP}$. Fortunately it was found that the only processing undergone by the raw data at the time of scanning was correction for the differing gains and offsets of the detector elements and amplifiers. This 'electronic' calibration is independent of the tube kilovoltage. Subsequent investigation showed that the appropriate part of the calibration data in WCALIF is stored at the beginning of the file containing the raw data for each image (SLICEFXX, where XX ranges from 00 to 47 ) at the time the image is taken. The calibration data is specific to each possible slice width $(10,5$ or $1.5 \mathrm{~mm})$ and to each calibration size $(25 \mathrm{~cm}, 35 \mathrm{~cm}$ or $42 \mathrm{~cm})$. The locations of the various calibration data sets within the WCALIF file have been determined, and are shown in table 3.1. Correct calibration of the $80 \mathrm{kV}$ slices can therefore be obtained by inserting the correct calibration data into the first 24 blocks of the raw data file prior to reconstruction. Software has been written to perform this task on a routine basis. I am grateful to $\mathrm{Mr}$ Adam Zyskowski for assistance in writing this software.

Table 3.1: Location of Calibration Data Within WCALIF.

\begin{tabular}{||r|l|l|r||}
\hline Blocks & Filter & Phantom & Slice width \\
\hline \hline $9-72$ & Head & Air & $1.5 \mathrm{~mm}$ \\
$73-96$ & Head & Small & $1.5 \mathrm{~mm}$ \\
$145-168$ & Head & Air & $5.0 \mathrm{~mm}$ \\
$169-192$ & Head & Small & $5.0 \mathrm{~mm}$ \\
$241-264$ & Head & Air & $10.0 \mathrm{~mm}$ \\
$265-288$ & Head & Large & $10.0 \mathrm{~mm}$ \\
$385-408$ & Body & Medium & $1.5 \mathrm{~mm}$ \\
$409-432$ & Body & Large & $1.5 \mathrm{~mm}$ \\
$433-456$ & Body & Air & $1.5 \mathrm{~mm}$ \\
$481-504$ & Body & Medium & $5.0 \mathrm{~mm}$ \\
$505-528$ & Body & Large & $5.0 \mathrm{~mm}$ \\
$529-552$ & Body & Air & $5.0 \mathrm{~mm}$ \\
$577-600$ & Body & Medium & $10.0 \mathrm{~mm}$ \\
$601-624$ & Body & Large & $10.0 \mathrm{~mm}$ \\
$625-648$ & Body & Air & $10.0 \mathrm{~mm}$ \\
\hline
\end{tabular}




\subsubsection{Choice of Algorithm.}

The most basic choice was to decide whether to perform the dual energy calculations prior to reconstruction, or afterwards. Pre-reconstruction techniques have the advantage of reducing or even eliminating image artefacts due to polychromaticity of the measuring beam, but do so at the expense of increased complexity and consequent longer processing times. The approach taken in the present work has been to prove the technique using post reconstruction techniques, with the option of reverting to the pre-reconstruction technique at a later stage if a particular application is adversely affected by beam hardening errors. Confining attention to post reconstruction algorithms, those of Rutherford et al (section 3.2) and Brooks et al (section 3.4.2) are based on approximate parametrisations of the Xray attenuation coefficient which have been shown in the present work to produce parameters which vary according to the X-ray energies used to make the measurement. A post reconstruction technique based on the pre-reconstruction technique of Alvarez et al has been shown (section 3.4.3) to produce parameters which are less sensitive to differences in the measurement energies, and the algorithm of Hawkes \& Jackson provides parameters which exhibit the least energy sensitivity and which would therefore be expected to depend least on the scanner used to make the measurement. The technique of Hawkes and Jackson uses liquids as standards which would be expected to be more reproducible at different locations than would be samples of aluminium and plastic (see section 3.4.5). The algorithm of Hawkes \& Jackson is therefore to be preferred for general dual energy computed tomography.

\subsection{Imaging Performance Tests.}

In view of the fact that the G.E. 8800 scanner is intended to operate at $120 \mathrm{kVp}$ only it is necessary to establish that the imaging performance at lower kilovoltages is satisfactory. This is especially necessary since the beam hardening correction algorithm applied to the detector readings (see section 1.3) will be optimised for $120 \mathrm{kVp}$ and would be expected to be significantly in error at other kilovoltages. It is possible that errors from this source may be compensated for by the calibration procedure, in which the property reconstructed is not the absolute attenuation coefficient but the difference in attenuation coefficient between the object scanned and the calibration phantom. The most fundamental imaging test is to ensure that the image of a uniform phantom produces a uniform image without undue change in quality between the centre of the image and the periphery. Scans were performed on a $25 \mathrm{~cm}$ diameter water phantom at both $70 \mathrm{kVp}$ and $120 \mathrm{kVp}$ in the HEAD mode of the scanner. The tube current was $500 \mathrm{~mA}, \mathrm{X}$-ray pulse width was $4 \mathrm{~ms}$ and the slow scan speed was selected (576 pulses per image). These measurements were made before we were advised of the $80 \mathrm{kVp}$ minimum limit when using the X-ray generator with the 100-CT tube. Measurements of average C.T. 
numbers were made in $2 \mathrm{~cm} \times 2 \mathrm{~cm}$ squares in the centre and at four positions at the periphery of each image. The peripheral readings were centred $10 \mathrm{~cm}$ above and below the centre of the image, and $8 \mathrm{~cm}$ to the left and right of centre. The means and standard deviations at each location were averaged for two images at each of the three slice widths available, and are quoted in table 3.2.

Table 3.2: Uniformity and Noise at $80 \mathrm{kVp}$ and $120 \mathrm{kVp}$.

\begin{tabular}{|c|c|c|c|}
\hline \multirow[b]{2}{*}{ SLICE WIDTH } & \multicolumn{3}{|c|}{ LOCATION } \\
\hline & $\begin{array}{c}\text { centre } \\
(0,0 \mathrm{~cm}) \\
\text { mean } \quad \text { S.D. }\end{array}$ & $\begin{array}{l}\text { horizontal } \\
( \pm 8 \mathrm{~cm}, 0) \\
\text { mean S.D. }\end{array}$ & $\begin{array}{c}\text { vertical } \\
(0, \pm 10 \mathrm{~cm}) \\
\text { mean } \quad \text { S.D. }\end{array}$ \\
\hline
\end{tabular}

$70 \mathrm{kVp}$

\begin{tabular}{|rllllll|}
\hline $10.0 \mathrm{~mm}$ & 1.1 & 11.2 & 1.7 & 14.0 & 3.5 & 17.5 \\
$5.0 \mathrm{~mm}$ & 1.1 & 17.1 & 0.9 & 21.9 & 3.2 & 27.3 \\
$1.5 \mathrm{~mm}$ & 3.5 & 36.7 & 0.4 & 50.6 & 6.3 & 63.4 \\
\hline
\end{tabular}

\section{$120 \mathrm{kVp}$}

\begin{tabular}{|rrrrrrr|}
\hline $10.0 \mathrm{~mm}$ & -1.1 & 3.4 & -2.8 & 4.2 & -5.4 & 4.8 \\
$5.0 \mathrm{~mm}$ & -0.3 & 5.1 & -2.0 & 6.5 & -3.7 & 7.3 \\
$1.5 \mathrm{~mm}$ & -1.4 & 9.1 & -1.2 & 10.7 & -2.7 & 12.3 \\
\hline
\end{tabular}

Changes in mean C.T. number with position are minimal at both kilovoltages, but there is marked increase in noise (standard deviation) at $70 \mathrm{kVp}$. However increased noise is to be expected at the lower kilovoltage since there is decreased tube output, and there is also lower transmission of the $\mathrm{X}$-rays through the water phantom. Using program XSPECT, it has been found that the output at $120 \mathrm{kVp}$ is 4.4 times greater than at $70 \mathrm{kVp}$ and that the fractional transmission of the beam through $25 \mathrm{~cm}$ of water is 2.7 times larger for the $120 \mathrm{kVp}$ spectrum than for the $70 \mathrm{kVp}$ spectrum. The expected increase in noise can be approximated to within a few percent [104] by assuming that the noise is inversely proportional to the square root of the energy flux reaching the detector. The increase in noise is of the expected magnitude except at the periphery in the $1.5 \mathrm{~mm}$ slices. 
Having established that the uniformity and noise are as good as can be expected given the lower energy flux, it is necessary to establish that there is good linearity between measured C.T. numbers and calculated linear attenuation coefficients. This was done for measurements on the linearity section of the A.A.P.M. phantom using the analysis proposed by Millner et al [60], and the results are quoted in table 3.3.

Table 3.3: Results of Linearity Tests at $80 \mathrm{kVp}$ and $120 \mathrm{kVp}$.

\begin{tabular}{|c|c|c|c|c|}
\hline $\begin{array}{c}\text { Kilovoltage } \\
(\mathrm{kVp})\end{array}$ & $\begin{array}{c}E_{\text {eff }} \\
(\mathrm{keV})\end{array}$ & \multicolumn{2}{|c|}{$\mathrm{K}$} & $\mu_{\mathrm{W}}$ \\
(see equation 2.3) & $\mathrm{r}$ \\
\hline 80 & 59.65 & $\begin{array}{l}1097.6 \\
1001.5\end{array}$ & $\begin{array}{l}0.2057 \\
0.1916\end{array}$ & $\begin{array}{l}0.99919 \\
0.99887\end{array}$ \\
\hline
\end{tabular}

The scans were performed at $500 \mathrm{~mA}, 4 \mathrm{~ms}$ pulse width and 576 pulses per image. The INFANT $(25 \mathrm{~cm})$ calibration mode was used since this most closely matches the size of the A.A.P.M. phantom (22 cm diameter). The linearity at $80 \mathrm{kVp}$, as assessed by the linear correlation coefficient, $r$, is marginally better than at $120 \mathrm{kVp}$. The parameter $\mu_{\mathrm{W}}$ for $80 \mathrm{kVp}$ matches the attenuation coefficient for water at the quoted effective energy. A slight disadvantage is that the scale factor $K$ is 1100 rather than the usual 1000. To assess the effectiveness of the beam hardening correction the above measurements were repeated with a bone simulating ring ( $\left(\frac{1}{4} "\right.$ teflon) surrounding the phantom. The effective energy at $120 \mathrm{kVp}$ increased by $1 \mathrm{keV}$ while the $80 \mathrm{kVp}$ effective energy increased by $2 \mathrm{keV}$. As an additional check on the beam hardening correction measurements were repeated on the same phantom using both the medium body $(35 \mathrm{~cm})$ and large body $(42 \mathrm{~cm})$ field sizes. This is an effective test on the beam hardening correction since in the case of the large body calibration the phantom under test is just a fraction over half the size of the phantom used for calibration. Two slices were taken at each field size and the measurements for the two images were averaged. The results are given in table 3.4. The measured effective energies lie within a range of $0.4 \mathrm{keV}$ at $80 \mathrm{kVp}$, and within a range of $1.3 \mathrm{keV}$ at $120 \mathrm{kVp}$. Also listed are the average C.T. numbers for each of the plastic inserts: polyethylene (PE), polystyrene (PS), polycarbonate (PC), nylon (N) and acrylic (AC). At $80 \mathrm{kVp}$ the greatest difference between the measured C.T. numbers for any one insert at different field sizes is 4.4 Hounsfield units $(0.44 \%$ of the attenuation coefficient of water), whereas the corresponding figure for $120 \mathrm{kVp}$ is 4.3 Hounsfield units. It is clear that the calibration procedure is compensating for the incorrect linearisation of the detector readings at 80 $\mathrm{kVp}$, and that the overall effect is that, for this phantom, beam hardening errors at 80 $\mathrm{kVp}$ are no worse than at $120 \mathrm{kVp}$. These tests have been performed with materials whose effective atomic numbers are close to that of water, and may not truly reflect performance with high atomic number materials such as bone mineral, a problem which 
Table 3.4: Effect of Increased Field Size.

\begin{tabular}{||c|c|c|c|c|c|c||}
\hline \multirow{2}{*}{ Field size } & \multirow{2}{*}{$\begin{array}{c}\text { Eeff } \\
(\mathrm{keV})\end{array}$} & \multicolumn{6}{|c|}{ Measured Hounsfield Numbers } \\
\cline { 3 - 7 } & & PE & PS & PC & N & AC \\
\hline
\end{tabular}

$80 \mathrm{kVp}$

\begin{tabular}{|l|l|l|l|l|l|l|}
\hline $25 \mathrm{~cm}$ & 59.7 & -106.0 & -54.3 & 92.9 & 93.2 & 122.6 \\
$35 \mathrm{~cm}$ & 59.3 & -110.0 & -57.9 & 91.6 & 88.8 & 122.5 \\
$42 \mathrm{~cm}$ & 59.7 & -109.0 & -56.0 & 92.6 & 90.3 & 122.8 \\
\hline
\end{tabular}

$120 \mathrm{kVp}$

\begin{tabular}{l|l|l|l|l|l|l}
$25 \mathrm{~cm}$ & 70.5 & -72.6 & -27.3 & 104.4 & 105.4 & 131.0 \\
$35 \mathrm{~cm}$ & 70.2 & -73.4 & -26.6 & 106.8 & 105.9 & 132.8 \\
$42 \mathrm{~cm}$ & 69.2 & -73.6 & -27.9 & 105.8 & 101.6 & 133.5 \\
\hline
\end{tabular}

is addressed in the next chapter.

Other imaging parameters such as resolution and sensitivity profile (slice width) are not expected to be affected by the high tension voltage but have been included for completeness. High contrast resolution is best expressed in terms of the modulation transfer function of the system $[46,105]$. System resolution is usually measured using a bar phantom, from which it is possible to obtain the square wave response of the system. Coltman [106] has published a formula which permits the calculation of the sinusoidal response, $R(f)$, (ie the M.T.F.) to be calculated from the square wave response $r(f)$ :

$R(f)=\frac{\pi}{4} \cdot\left[r(f)+\frac{r(3 f)}{3}-\frac{r(5 f)}{5}+\frac{r(7 f)}{7}+\frac{r(11 f)}{11}-\frac{r(13 f)}{13} \cdots\right]$

In the above it would appear that there ought to be a term in $9 \mathrm{f}$, but it is clear from the original reference that there is not. Obtaining the square wave response from the C.T. images of bar phantoms is a tedious process, but fortunately a method has been published which permits the M.T.F. to be calculated directly from the measurement of standard deviations of the bar patterns [107]. Such measurements are easily performed on C.T. images, and I am grateful to John Poletti [108] for drawing my attention to this method. A computer program (MTF) has been written for the computation of the M.T.F. from 
measurements of standard deviations taken from images of the G.E. bar phantom. This program, which is listed in appendix E, has been found to give M.T.F. curves which agree well with ones calculated using Coltman's formula (equation 3.21). Details of the calculations are clearly set out in reference [108]. M.T.F. curves at $80 \mathrm{kVp}$ and $120 \mathrm{kVp}$, calculated using program MTF, are plotted in figure 3.7. The images from which these curves were computed were reconstructed using the standard convolution filter. There is no significant difference except perhaps at spatial frequencies greater than 4.5 line pairs per centimeter, however the apparent lower M.T.F. at these frequencies is almost certainly due to increased noise on the $80 \mathrm{kVp}$ image. The limiting resolution as determined by the visibility of the individual bar patterns was found to be 4.9 line pairs per centimeter in both cases, corresponding to a resolution of $1 \mathrm{~mm}$.

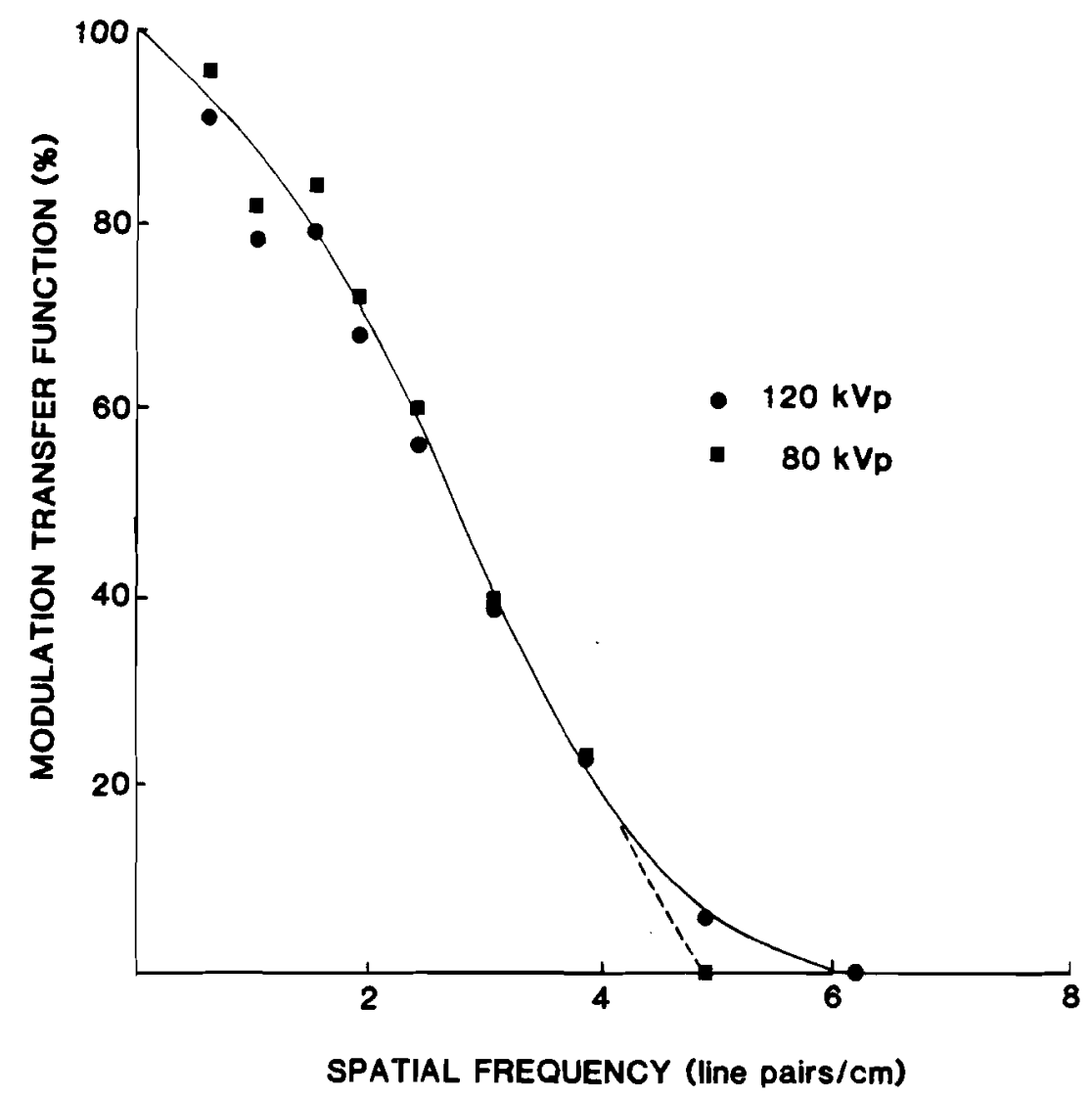

Figure 3.7: Modulation Transfer Function Curves at $120 \mathrm{kVp}$ and $80 \mathrm{kVp}$.

Effective slice width (sensitivity profile) was estimated using the A.A.P.M. phantom [109]. The "slice width" section of this phantom contains three thin strips of aluminium inclined at 45 degrees to the long axis of the phantom. The width of the images of these strips as viewed on an axial section is a measure of the effective slice width. Measurements were made for each nominal slice width setting for both $80 \mathrm{kVp}$ and 120 $\mathrm{kVp}$. Other technique factors were: $500 \mathrm{~mA}, 576$ pulses, INFANT $(25 \mathrm{~cm})$ calibration. 
Table 3.5: Effective Slice Widths.

\begin{tabular}{|c|c|c|c|c|}
\hline \multirow{2}{*}{$\begin{array}{l}\text { Peak } \\
\text { Kilovoltage }\end{array}$} & \multirow{2}{*}{$\begin{array}{l}\text { Nominal } \\
\text { slice width }\end{array}$} & \multicolumn{3}{|c|}{$\begin{array}{c}\text { FWHM of Sensitivity Profile } \\
\text { (mm) }\end{array}$} \\
\hline & & right & middle & left \\
\hline $80 \mathrm{kVp}$ & $10.0 \mathrm{~mm}$ & 10.3 & 9.9 & 10.4 \\
\hline $80 \mathrm{kVp}$ & $5.0 \mathrm{~mm}$ & 5.5 & 5.3 & 5.5 \\
\hline $80 \mathrm{kVp}$ & $1.5 \mathrm{~mm}$ & 2.6 & 2.5 & 2.6 \\
\hline $120 \mathrm{kVp}$ & $10.0 \mathrm{~mm}$ & 10.4 & 10.1 & 10.4 \\
\hline $120 \mathrm{kVp}$ & $5.0 \mathrm{~mm}$ & 5.5 & 5.4 & 5.5 \\
\hline $120 \mathrm{kVp}$ & $1.5 \mathrm{~mm}$ & 2.7 & 2.6 & 2.7 \\
\hline
\end{tabular}

There is no significant difference in slice width at $80 \mathrm{kVp}$ as compared with the 120 $\mathrm{kVp}$ images. The measured full width at half maximum (F.W.H.M.) of the sensitivity profiles at $1.5 \mathrm{~mm}$ exceeds the nominal slice width by a considerable margin, however the error is the same at both kilovoltages. The measurements at the nominal $1.5 \mathrm{~mm}$ slice width compare favourably with measurements taken at installation of the scanner: the scanner simply does not meet specification in this respect.

Sensitivity to image artefact formation has been tested using the A.A.P.M. phantom. The appropriate section contains a centrally located aluminium pin $6 \mathrm{~mm}$ in diameter [109], providing a test of the artefact sensitivity of the scanner [110]. Images of this section taken at $80 \mathrm{kVp}$ and $120 \mathrm{kVp}$ are displayed in figure 3.8. The images were taken using the INFANT calibration, $500 \mathrm{~mA}, 576$ pulses and $10 \mathrm{~mm}$ slice width. The display settings used are selected to highlight any irregularities in the image: the selected window width (see section 1.4) of 20 is a quarter of the smallest width used for the routine display of clinical images at Wellington Hospital. The degree of artefact is minimal in both cases, but is perhaps less visible at $80 \mathrm{kVp}$ due to the increased noise resulting from the lower tube output at $80 \mathrm{kVp}$. 


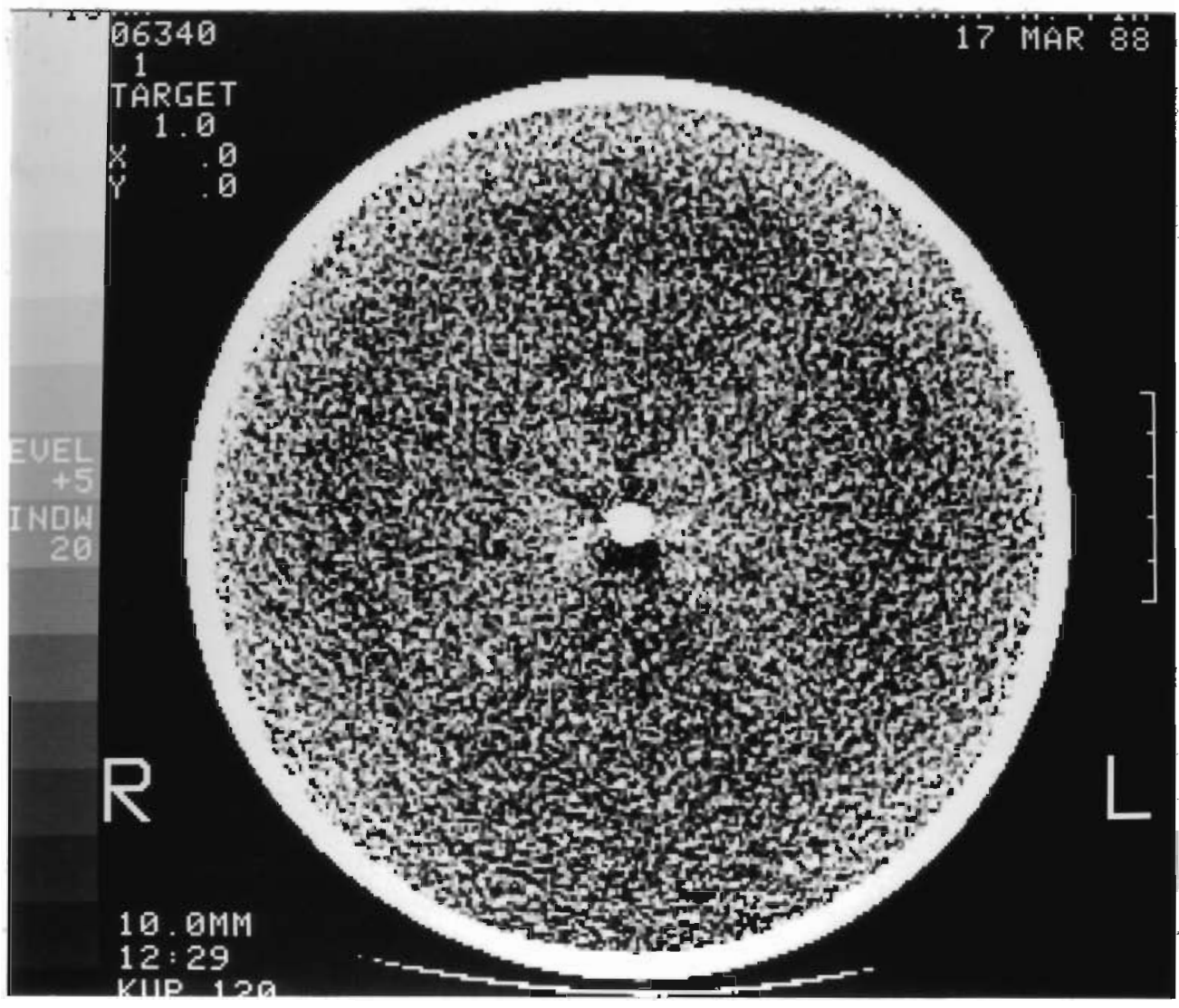

\section{$80 \mathrm{kVp}$}

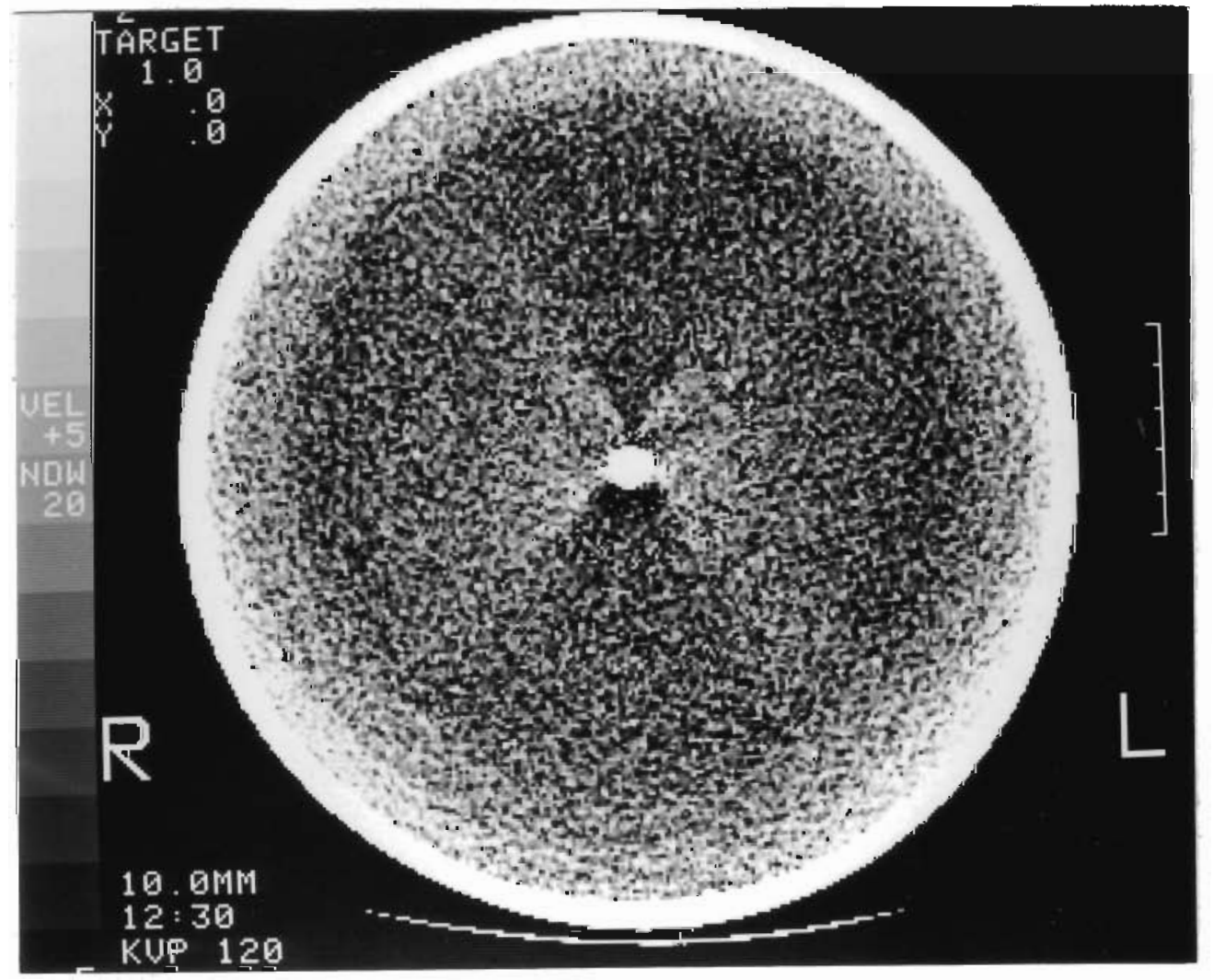

120 kVp

Figure 3.8: Sensitivity to Artefact Formation at $120 \mathrm{kVp}$ and $80 \mathrm{kVp}$. 


\subsection{Reprise.}

The introduction of the C.T. scanner resulted in images whose characteristics in terms of shades of grey could be quantified in a physically meaningful way since each shade represents a specific range of C.T. numbers. Attempts to make use of these measurements in diagnosis were hampered by a lack of standardisation between scanners, and by the fact that they are derived from linear X-ray attenuation coefficients which are not important physical properties of tissue, per se. However the linear attenuation coefficient itself depends on the energy of the beam used to make the measurement, which is to a certain extent changeable by the operator of the scanner, and the density and 'effective' atomic number of the tissues scanned. Density would seem to be an important parameter in its own right, and effective atomic number could perhaps be useful as an index of chemical composition. Since changes in density would cause equal changes in C.T. number, regardless of the energy of the measuring beam, it is clear that any difference in C.T. number between images taken at different energies reflects the 'effective' atomic number of the tissue, and that the signal common to both energies is a measure of tissue density. This is the basic principle behind dual energy computed tomography, which aims to measure clinically-relevant parameters in a way which is independent of the scanner performing the measurements.

The simplest way of obtaining dual energy C.T. images is to perform sequential scans, one at a higher kilovoltage and one at a lower kilovoltage. However problems could be experienced due to patient movement between scans, particularly with the earlier C.T. scanners which require longer scanning times. Attempts have been made to obtain simultaneous dual energy images by splitting the detectors into two sections, the one nearest to the source acting as a filter so the section furthest from the source receives a 'harder' or more energetic beam. Such a technique is not feasible in Wellington since it would require virtually complete rebuilding of the detector (value $\$ 100000$ ) and the sacrifice of image quality for ordinary diagnostic work performed at a single energy. Another alternative is to apply selective further filtration to half of the X-ray beam so that half the detectors receive a harder beam than the others. This is also not feasible on the Wellington scanner since it would again compromise the standard imaging performance at a single energy. Although it would be possible to remove this filter for normal scanning, it would not be practical to keep fitting and removing such a filter because with a 523 element detector it would require a very accurate (and therefore time consuming) alignment procedure. Dual energy scans at Wellington are therefore performed by the sequential scanning method. Software has been written to align the two single energy images prior to dual energy processing, but in practice this has not been needed.

The original dual energy algorithm of Rutherford et al [85] is based on a simple empirical expression relating the linear attenuation coefficient to photon energy and the 
atomic number of the absorbing medium which was shown in the previous chapter to be of very limited accuracy. Difficulties also arise because the measurement is not made with a monochromatic beam. Computer simulations have shown that the 'effective' energy of the beam of the G.E. 8800 scanner varies significantly with the atomic number of the absorbing material, and that the slope of this curve is greatest in the important region around $Z=8$. A number of other algorithms have been suggested. The algorithm of Brooks is based on an overly simplistic parametrisation of the attenuation coefficient and has been shown in the present work to produce measures which are strongly dependent on the X-ray beam quality and which would therefore depend on the properties of the scanner used to make the measurements. The algorithm of Alvarez \& Macovski is intended to be applied to the raw data prior to reconstruction of the image. Such a technique has the advantage of reducing errors due to beam hardening, but its application in Wellington would require considerable extra work in determining just how the raw data is stored. In the present work the suitability of a similar algorithm applied to the reconstructed images has been studied. This algorithm uses the mass attenuation coefficients of perspex and aluminium as 'basis' functions, so that the attenuation coefficient of any element may be expressed as a linear combination of these two functions. The present author has found this to be an accurate parametrisation of the attenuation coefficient, and computer simulations in the present work have shown that the parameters produced by this algorithm, the perspex and aluminium components, to have reduced sensitivity to the exact conditions of measurement. The algorithm of Christ has the advantage of explicitly addressing the problems associated with beam hardening, but is of a complexity which would result in unacceptably long computation times when applied to images. It also uses a parametrisation of the attenuation coefficient which is unrealistic. The algorithm of Jackson \& Hawkes is similar in concept to that of Alvarez \& Macovski but is explicitly intended for post reconstruction processing and is therefore better suited to our needs.

The G.E. 8800 scanner is intended for use at a single kilovoltage. It has been easy to modify the X-ray generator so that operation at other kilovoltages is possible, but the storage and use of calibration files for other kilovoltages has not been so straightforward. It has been found that the calibration data to be used in reconstructing each image is stored at the beginning of each raw data file. Dual energy scans may be obtained more efficiently if the $120 \mathrm{kVp}$ calibration file is current, even when the $80 \mathrm{kVp}$ scans are taken. The locations of the different types of calibration data (pertaining to different slice widths and field sizes) within the calibration file WCALIF have been determined, and software has been written to permit the substitution of appropriate calibration data taken at $80 \mathrm{kVp}$ into the raw data files for the low energy images after the examination is complete. 
Imaging performance tests have shown that the image quality at $80 \mathrm{kVp}$ is as good as at $120 \mathrm{kVp}$ except in respect of image noise and beam hardening compensation. The degradation in noise is of the amount to be expected based on the reduced tube output and the reduced transmission of the softer beam, and would be similar to that of other scanners operated at this kilovoltage. The tests described in this chapter show that the beam hardening compensation is not as effective when a bone simulating ring is used, but is equally effective when the ratio of the size of the water equivalent phantom to that of the calibration phantom is varied by a factor of almost two. 


\section{CHAPTER FOUR: C.T. SPINE MINERAL DENSITOMETRY}

\subsection{The Clinical Need for Bone Mineral Measurement.}

The mineral content of human bones decreases significantly from the fifth decade of life onwards. In some individuals the rate of decrease is more marked than usual, and in extreme cases the bones are weakened to the point where they are unable to bear their normal load and a fracture occurs, commonly in the lumbar vertebrae or in the hip (femur) $[111,112]$. Fractures of the lumbar vertebrae are more common since these can occur with a smaller degree of demineralisation than is required to cause a fracture in the femur [113]. The seriousness of such fractures may be judged from the fact that some 10 to $20 \%$ of elderly women who fracture their hip do not survive longer than six months [113]. Earlier bone mineral measurement techniques could not measure the mineral content of the lumbar spine, and gave only a crude measurement in the femur [114]. The majority of the earlier methods measure the mineralisation in either the hand or forearm, and it is possible to biopsy the bone tissue in the iliac crest of the pelvis. However it has been noted that the correlation between the mineral content at these sites and in the lumbar spine is often poor [115].

The body of a vertebra consists of a densely mineralised outer shell (the cortex) which is typically $1-2 \mathrm{~mm}$ thick, surrounding the central cancellous region which is composed of a matrix of thin mineralised plates called trabeculae whose interspaces are filled with varying proportions of red (blood forming) and yellow (fatty) marrow. At age 20 the percentage of red marrow is typically $60 \%$, but the red marrow is progressively replaced by yellow marrow so that at age 60 the composition of marrow is commonly $40 \%$ red and $60 \%$ yellow [116]. The variation in composition of vertebral bone with age is plotted in figure 4.1. The rate of mineral turnover in the trabeculae is some eight times that in the cortex [117], so that the effects of demineralisation are more severe, and noted at an earlier stage, in the cancellous region. This is especially significant since most of the structural strength of the vertebral body is derived from the cancellous region, rather than the cortex.

Bone mineral measurements are necessary for the following groups of patients: those suffering from conditions of which osteoporosis is a complication, perimenopausal women and those who are otherwise at risk of osteoporosis [118]. Osteoporosis is a sideeffect of several medical conditions, including hyperparathyroidism (excess activity of the parathyroid gland), renal osteodystrophy (caused by excessive removal of calcium salts from the blood by the kidney), over production of corticosteroids by the adrenal glands (caused by problems in the glands themselves or by incorrect chemical signals caused by 


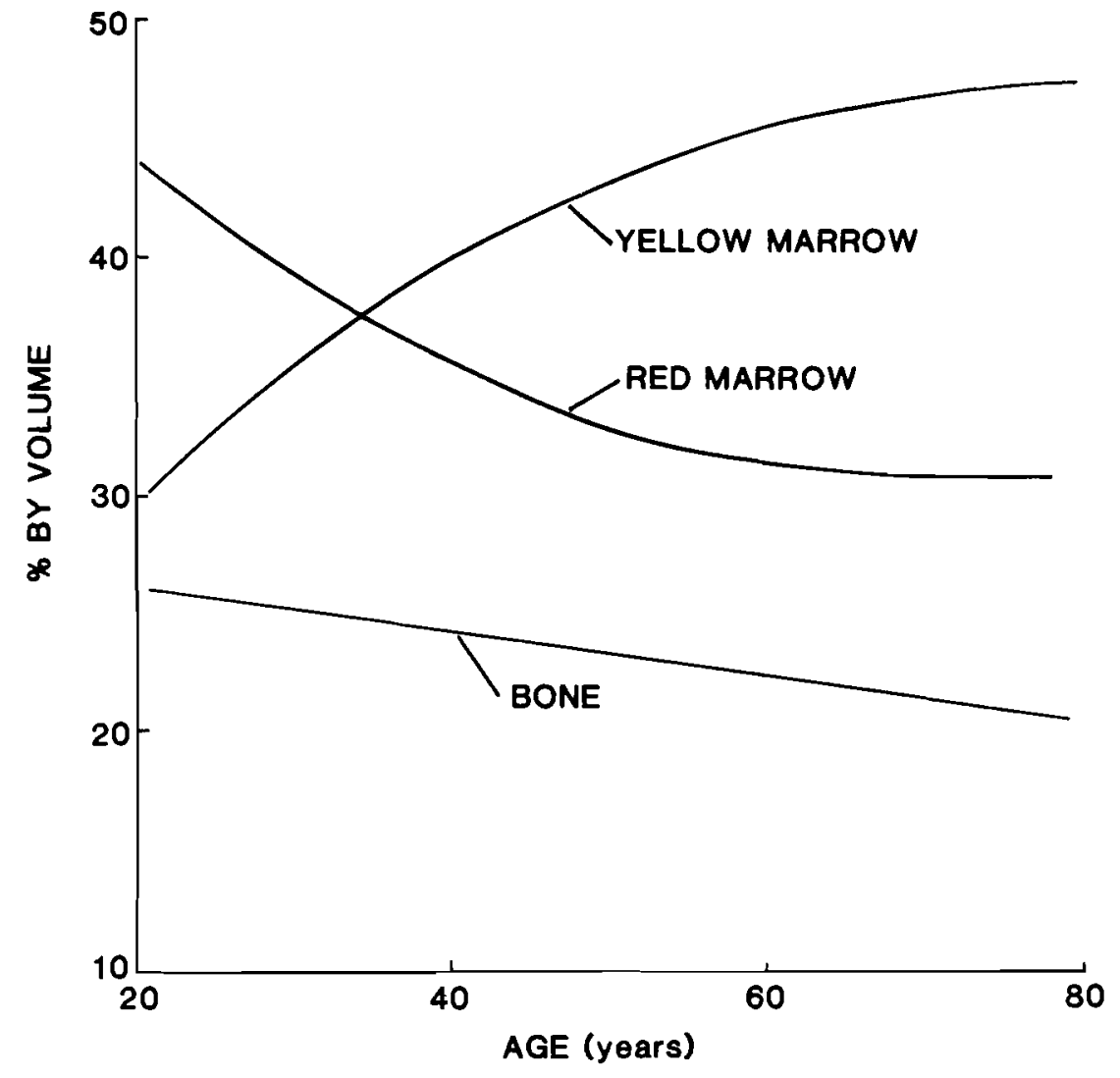

Figure 4.1: Variation of the Composition of Vertebral Bone with Age.

From regressions published by Dunnill et al. [116]. The measurements were of the whole vertebral body, including the cortex but not including the pedicles or any of the other processes located posterior to the vertebral body.
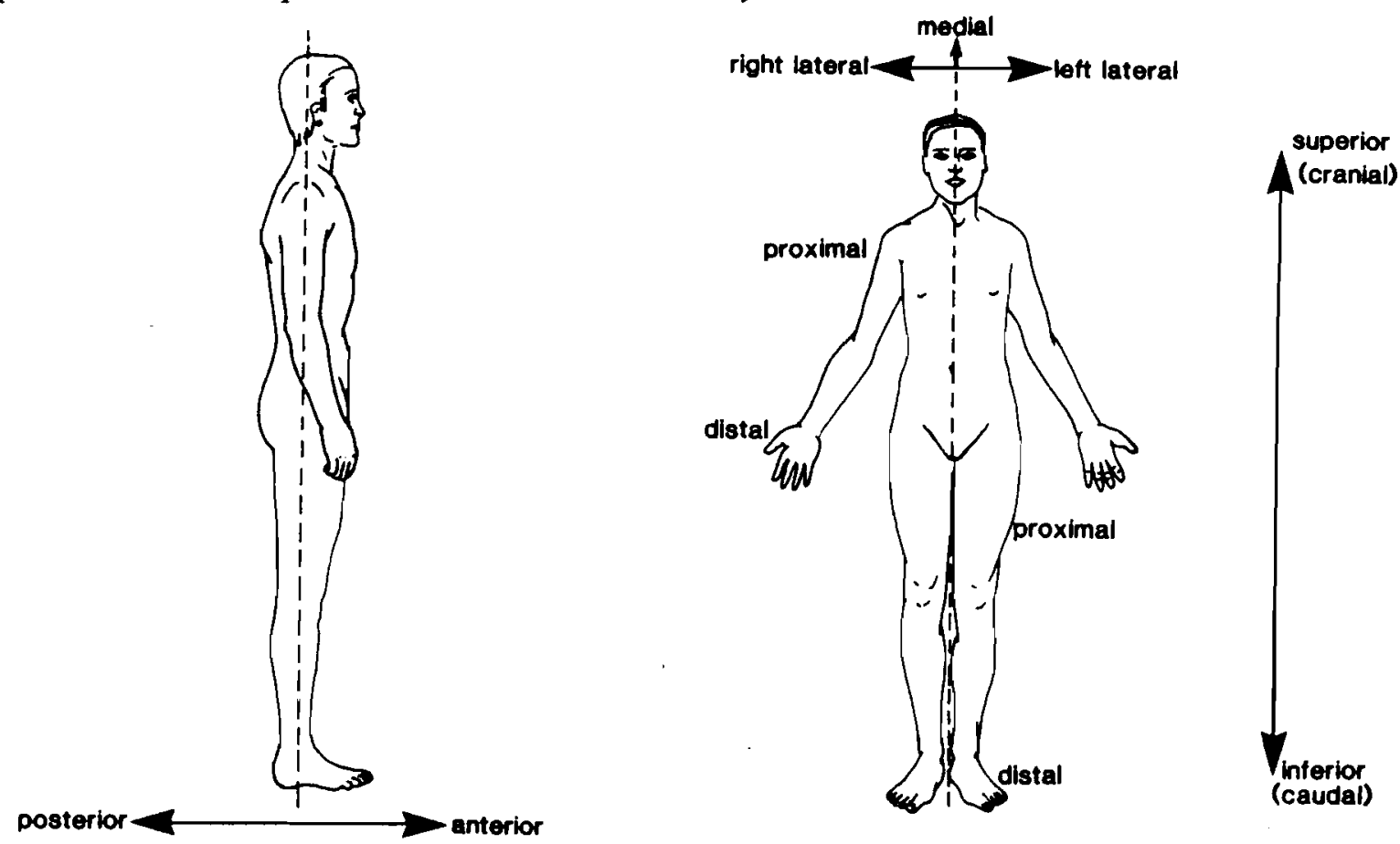

Figure 4.2: Reference Frame for the Human Body.

In limbs (and some other structures) the end closest to the body is called the proximal end, the other end being referred to as distal. 
problems in the pituitary gland). Also included in this first group are those who develop osteoporosis following periods of immobilisation and those who require long term corticosteroid treatment for conditions such as asthma. It is important to monitor the bones of such patients so that any loss of bone mineral is treated before fractures occur. The largest group of people who may require mineral measurements are women who are at or about the age of menopause. Since women generally have a lower mineral content than men, and are subject to more rapid loss of mineral in the first few years after the menopause, they are more likely to develop spontaneous fractures. This loss can be arrested by hormone replacement therapy, but such treatment cannot be applied to all post-menopausal women due to concern about possible side effects. It is therefore desirable to identify those women whose bone mineral content is below average at menopause and is therefore likely to subsequently drop to levels at which fractures are probable, and treat this 'at risk' group only. Finally, several clinical 'risk factors' have been identified which predispose towards osteoporosis. These factors include: being female, of European or Asian racial origin, menopausal, elderly, presence of atraumatic fracture, inactivity, petite frame, family history of osteoporosis, lowcalcium diet and a history of alcohol or tobacco consumption. People who have several of these risk factors are more likely to have osteoporosis, but the presence of these risk factors alone does not justify treatment and measurements are required to establish a diagnosis of osteoporosis. Once treatment for osteoporosis is under way it is necessary to repeat the bone mineral measurements at intervals to establish the effectiveness of treatment. Unfortunately the expected changes can be very small, sometimes less than $1 \%$ in a year, and the question arises as to whether a reported change from a follow-up measurement truly reflects a change in the patient's condition or is simply due to measurement uncertainties. The reproducibility or precision of a technique is assessed from repeated measurements on the same subject, and is usually expressed as a coefficient of variation which is the standard deviation divided by the mean ( $x 100$ if quoted as a percentage). It is estimated that a measurement technique with a precision of $2 \%$ can detect a $3.6 \%$ change in mineral content with a $90 \%$ level of confidence [118].

Disease processes giving rise to depletion of bone mineral content are collectively called osteopaenia [111]. The most common subgrouping is osteoporosis, where the mineral forming process proceeds in the normal fashion but at a lesser rate than the bone mineral depletion process so than there is a net loss of bone mineral content over time. Osteoporosis is distinguished from osteomalacia where the mineral forming process is disturbed: the organic matrix on which the mineral is laid down forms as per usual, but fails to mineralise. The two conditions require different treatments [111]. 


\subsection{Bone Mineral Measurement Techniques.}

There have been a number of recent reviews of techniques for the measurement of bone mineral content [118 - 123]. The best known of these methods are listed in table 4.1.

Table 4.1: Bone Measurement Techniques.

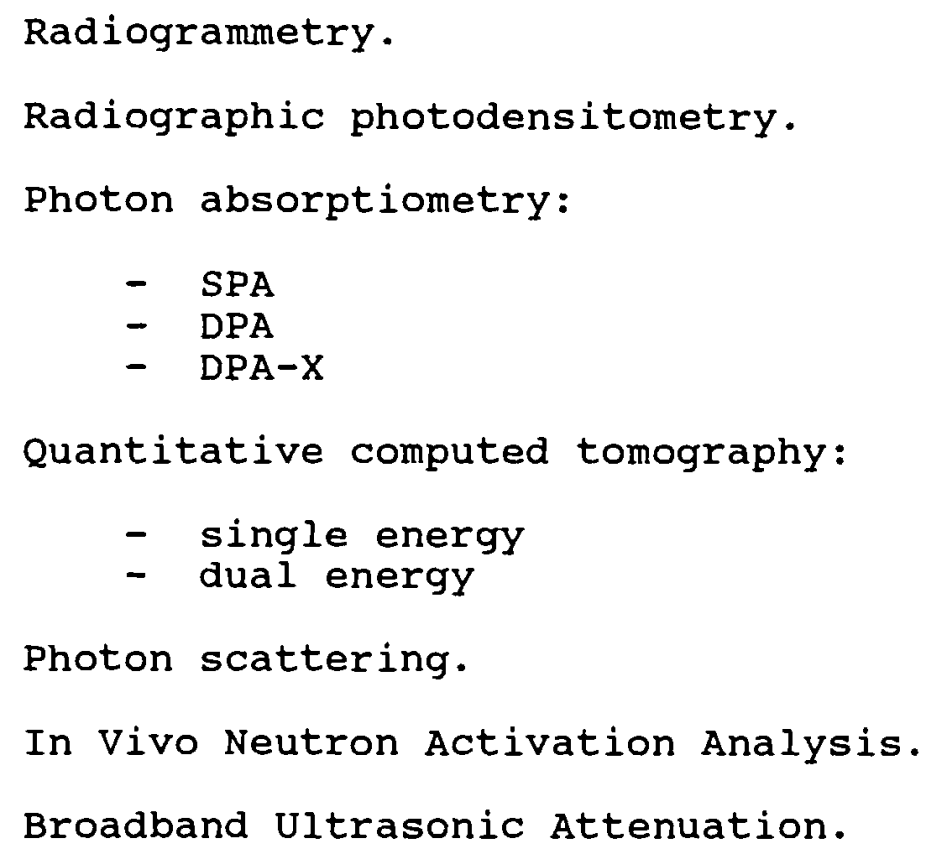

While qualitative observations of ordinary X-ray films can identify patients with severe osteoporosis, methods of greater sensitivity are required since the mineral loss must be of the order of $40 \%$ before changes are visible on films [123]. However techniques have been developed which increase the sensitivity by quantifying the appearances of X-ray films. The simplest of these involves applying a grading scheme to the appearance of the trabeculae on a plain X-ray film of the upper femur. The grading so obtained is called a Singh Index $[124,125]$ and has been found to correlate with the presence of crush fractures in the vertebrae. Radiogrammetry [126] involves taking measurements of the thickness of the bone cortex on X-ray films of the metacarpal bones in the hand, with measurement accuracies of $0.1 \mathrm{~mm}$ being required. The accuracy of the results varies according to the skill of the person making the measurements, the best figure being reported is that of Horsman [127] who could detect changes in cortical width of $1.5 \%$ with a $95 \%$ level of confidence. Radiogrammetry has been used to quantify differences in bones between different ethnic groups, between the sexes and to monitor the loss in females after the menopause [119]. However the overlap between the measurements on the normal groups and those suffering from osteoporosis is too large for 
the technique to be of great value in individual cases [113]. This is because the technique measures the thickness of the cortex only, not its porosity, and because the greatest changes are found in the trabeculae which have a greater surface to volume ratio. An alternative approach is radiographic photodensitometry [128] in which a densitometer measures the optical density of a bone image on an X-ray film. Best isolation of bone from soft tissue occurs in the fingers where the thickness of the flesh is thinnest. As the density of the film is dependent on several technique factors such as peak kilovoltage, voltage waveform, filtration, imaging geometry and film factors it is necessary to calibrate against a step wedge (of a material of similar effective atomic number to that of bone mineral) which is imaged on the same film. An alloy of aluminium has been found which closely matches bone mineral. This technique has an accuracy similar to that of radiogrammetry, and its clinical efficacy suffers from the same drawbacks [122].

Single photon absorptiometry (SPA) [129] is an extension of the radiographic densitometry technique. It is most commonly applied to the bones of the arm, and is based on measurements of the transmission of gamma rays from a radioactive source (usually 125 I, gamma energy $27 \mathrm{keV}$ ) through the bone being studied. The source is coupled to a scintillation crystal / photomultiplier detector on the other side of the body part being measured, and the complete source / detector assembly is scanned in a raster pattern over the area of interest. The effect of soft tissue overlying the bone is partially compensated for by immersing the limb in a water bath so the total thickness is constant. The mineral content is estimated by comparing the transmission of the beam through the arm with that through the same thickness of water. The measurement is most frequently taken at a position on the shaft of the radius where the thickness is relatively constant so that slight differences in positioning on subsequent examinations will not compromise comparisons made between the two measurements. However measurements at this position, where there is very little trabecular bone, are of little value in predicting the state of bones such as the vertebrae and the proximal femur which are largely trabecular and are at a greater risk of fracture [130]. Some workers have taken their measurements nearer the distal end of the radius where the trabecular content can be as high as $25 \%$ or more, however the thickness of the radius at this point is a strong function of the position along its length and care is required to overcome reproducibility problems due to positioning errors. The precision of single photon absorptiometry of the radius is quoted as a little more than $1 \%$ at the normal measurement position [122, 131]. As well as being applied to the bones of the forearm and hand, this technique has also been applied to the femur and the calcaneus (heel bone) [122].

The single photon technique can be applied to the limbs since in these areas the composition of the soft tissues is relatively constant, and it is possible to use a water bath to maintain a constant path length. It is not practical to use a water bath technique for measurements in the trunk, so in this case it is necessary to use a dual energy technique 
called dual photon absorptiometry (DPA) in which the measurement at a second energy permits the computation of the mineral equivalent in $\mathrm{mg} / \mathrm{cm}^{2}$ independent of the thickness. Until recently the radiation source on such instruments has been a radioisotope, generally ${ }^{153} \mathrm{Gd}$ which emits photons of $44 \mathrm{keV}$ and $100 \mathrm{keV}$. Such scanners can take measurements in the two high risk areas, the lower spine and the hip, and software packages are available which also permit the estimation of the total body calcium content. Accuracy and precision are in the range of from $2 \%$ to $4 \%$ [113], depending on the site of the measurement, and on which absorptiometer is used [121]. The performance of the dual photon absorptiometer has been greatly improved over the past few years by the replacement of the radioisotope source by a conventional X-ray tube and generator [132]. Such instruments are variously denoted DPA-X or simply DPX. The examination time is substantially reduced and the precision errors are decreased to as little as $1 \%$. There has been a rather vigorous discussion in the literature over the relative merits of this technique and the next one to be discussed, quantitative computed tomography. Further description of the DPA technique will be presented in section 4.8 , in which comparisons between the two techniques are made.

Quantitative computed tomography is applied principally to the cancellous bone tissue in the vertebrae (in which case it is also referred to as C.T. spine mineral densitometry), and involves comparison of C.T. number readings taken in this area with C.T. number readings of a material being used as a standard for calibration purposes. Since the C.T. scanner produces a cross sectional image it is not necessary to use dual energy techniques to remove the effects of overlying soft tissues, but the use of dual energy techniques removes the dependence of the mineral reading on changes in marrow composition. Single energy QCT is discussed in the next section, and dual energy QCT is discussed in section 4.4 .

There are two major types of photon scattering techniques, the first based on measurements of Compton scattered photons and the second based on the ratio of coherently scattered photons to Compton scattered photons. A gamma ray source is used permitting the distinction of coherently scattered photons from Compton scattered photons since the former will have the same energy as those emitted by the source (see section 2.3). In both cases the measurement volume is defined by the intersection of the beam, which is determined by the source collimator, and the acceptance volume of the detector, which is defined by the detector collimator. In the purely Compton technique multiple measurements are made at different angles so that the effect of overlying bone and soft tissue may be removed. With the ratio method this is not necessary since the ratio of the two types of scatter is directly indicative of the density in the measurement volume [133]. Photon scattering techniques have been applied in both the calcaneus and the distal radius, sites where there is little overlying soft tissue. The precision of the purely Compton technique is of the order of 1 to $2 \%$, while the ratio method when tested on calcaneus 
bones taken from cadavers was found to have an accuracy of $5 \%$ and a precision of $3 \%$ [122].

In vivo neutron activation analysis (IVNAA) is most commonly applied to the whole body, which is first irradiated with neutrons which cause some of the atoms of the body to become radioactive. The body is then viewed by a number of NaI scintillation detectors which collect a spectrum of characteristic gamma rays, permitting elemental analyses to be made. The reaction most often monitored is ${ }^{48} \mathrm{Ca}(\mathrm{n},)^{49} \mathrm{Ca}$, although some workers are using equipment which is able to detect the prompt $\left(10^{-15_{S}}\right.$ !) gamma radiation from the ${ }^{40} \mathrm{Ca}(\mathrm{n},)^{41} \mathrm{Ca}$ reaction, the motivation for doing so being that the ${ }^{40} \mathrm{Ca}$ isotope is much more abundant in the body than ${ }^{48} \mathrm{Ca}[134,135]$. Although this technique administers a higher dose than the other methods described in this section (of the order of $50 \mathrm{mSv}$ whole body) it is regarded as the standard against which other methods are compared, having an accuracy of about $5 \%$ and a precision of $2-3 \%$ [122].

Broadband ultrasonic attenuation (BUA) is a technique which is applied to the calcaneus. The heel is placed in a water bath between an ultrasonic transmitter and receiver. During the course of the measurement, which takes 5 seconds, the frequency of the ultrasound beam is increased from $200 \mathrm{kHz}$ to $1 \mathrm{MHz}$. The slope of the linear portion of the curve of attenuation versus frequency, expressed in $\mathrm{dB} / \mathrm{MHz}$, is found to be indicative of both the mineral content and its structure [122]. The technique has a precision of between $1 \%$ and $5 \%$, and the measurements have been found to correlate with crush fractures of the spine at least as well as DPA of the spine [136].

\subsection{Single Energy C.T. Bone Mineral Measurement.}

The C.T. scanner would seem to be ideally suited to the measurement of bone mineral content since it is an imaging technique, permitting exact localisation of the tissue to be measured, and the Hounsfield number is strongly related to the linear attenuation coefficient of the tissue. Since the advent of the whole body scanner in the mid 1970's it has been possible to image any bone in the body. Some of the earliest work in this field was performed by Ruegsegger and his co-workers [137] who used a homemade C.T. scanner to perform bone mineral measurements in the forearm. Their scanner used a 125 I source instead of an X-ray tube so that the attenuation was dominated by the higher atomic number elements found in bone mineral. Early work using a conventional C.T. scanner was done by Reich et al [138] who examined thirty one patients both by C.T. and using single photon absorptiometry of the radius. Moderate correlation was found $(r=0.72$ ), but considerably better correlation was found between C.T. measurements on excised human bones and an atomic absorption assay on the same bones $(r=0.97$, 
Taking the composition of bone mineral to be that of calcium-poor hydroxyapatite, $\mathrm{Ca} 9\left(\mathrm{H}_{3} \mathrm{O}\right)_{2}\left(\mathrm{PO}_{4}\right)_{6}(\mathrm{OH})_{2}[141]$, and using the attenuation coefficients tabulated by Hubbell [58], it has been found by the present author that the mass attenuation coefficients of the two materials are within $0.5 \%$ of each other over the energy range 40 $80 \mathrm{keV}$. Referencing the C.T. measurement of the bone mineral to that of the standard solution permits direct comparison of readings taken on different scanners. In the single energy technique of Genant and his co-workers [142] the cancellous region of the vertebra is modelled by a two component mixture. Bone mineral is represented by dipotassium hydrogen phosphate, and the other tissues by water. Changes in Hounsfield number are considered to be solely due to changes in mineral concentration, which is determined according to the following formalism. The derivation starts with a form of the mixture rule:

$$
\mu=\frac{c_{b}}{\rho_{\mathrm{b}}} \cdot \mu_{\mathrm{b}}+\frac{c_{\mathrm{s}}}{\rho_{\mathrm{s}}} \cdot \mu_{\mathrm{s}}
$$

where $c_{b}$ and $c_{s}$ are the concentrations of bone mineral and soft tissues respectively (grams per cc), $\rho_{\mathrm{b}}$ and $\rho_{\mathrm{S}}$ are the densities, and $\mu_{\mathrm{b}}$ and $\mu_{\mathrm{S}}$ are the linear attenuation coefficients of the individual components. The quantity $\left(c_{i} / \rho_{i}\right)$ is equal to the proportion of the $i^{\text {th }}$ element by volume. Since the volume fractions of the two elements must sum to unity, the volume fraction of marrow may be substituted for in terms of the volume fraction of mineral. The solution of the above equation is:

$$
c_{b}=\frac{H-H_{S}}{H_{b}-H_{s}} \cdot \rho_{b}
$$

where the linear attenuation coefficients have been converted to Hounsfield units $(H)$. This formalism only holds good when the equivalent mineral concentration is less than that of the standard solution (usually $100 \mathrm{mg} / \mathrm{ml}$ ), since a higher concentration would necessarily imply (equation 4.1) a negative soft tissue concentration. In later work Genant et al. [143] propose a slightly modified version of the same formula:

$$
c_{b}=\frac{H-H_{S}}{H_{b}-H_{S}} \cdot c_{k}
$$

where $c_{k}$ is the concentration of the dipotassium hydrogen phosphate solution used as the standard. The accuracy of the mineral concentration determined by the above formula will be limited by the extent to which the simplified tissue model represents the bone tissue being measured. One such limitation of this model is the variable composition of the soft tissue component, due to the replacement of red marrow with yellow (fatty) marrow with increasing age. In phantom studies where the effect of varying fat concentration was simulated by adding up to $40 \%$ alcohol to a potassium phosphate solution, the estimate of mineral content was found to vary by as much as $50 \%$ (Genant 
and Boyd [140]), although it was suggested that the maximum error in patient measurements due to this cause would be about $30 \%$.

\subsection{Dual Energy Spine Mineral Densitometry.}

The single energy technique estimates the mineral content of cancellous bone from a single measurement taken in the cancellous region. It can be seen in figure 4.4 that the trabeculae typically only occupy $12 \%$ of the total volume in this region. Although the trabculae have a greater density than marrow, and the effective atomic number of bone mineral is some two times greater, the proportion of the total attenuation coefficient of cancellous tissue which is due to bone mineral per se is only a little more than $25 \%$. This means for example that a $1 \%$ change in the attenuation coefficient of the marrow will be misreported by the single energy technique as a $3 \%$ change in the mineral content! Dual energy techniques greatly reduce errors due to this cause.

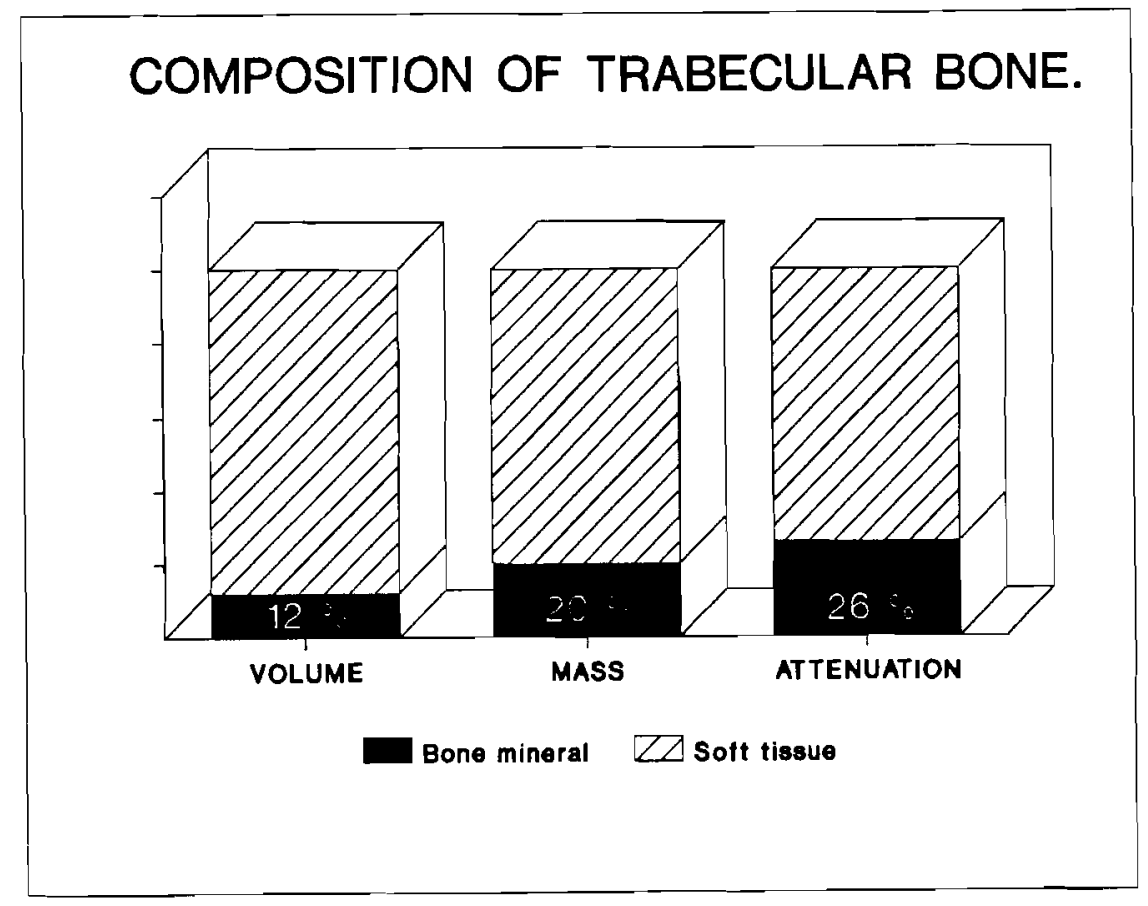

Figure 4.4: Mineral Content of Cancellous Bone.

Single energy techniques rely on a measurement of the total linear attenuation coefficient but the parameter of interest, the bone mineral, contributes only $26 \%$ of this 'signal'. Modified from [120].

Dual energy techniques for the measurement of bone mineral rely on the stronger energy dependence of the attenuation coefficients of the higher atomic number elements in 
bone, compared with those in soft tissues, a fact which is illustrated in figure 4.5 . If the attenuation of the mixture is measured at two energies, then the difference in attenuation at the two energies will be predominantly due to the mineral content.

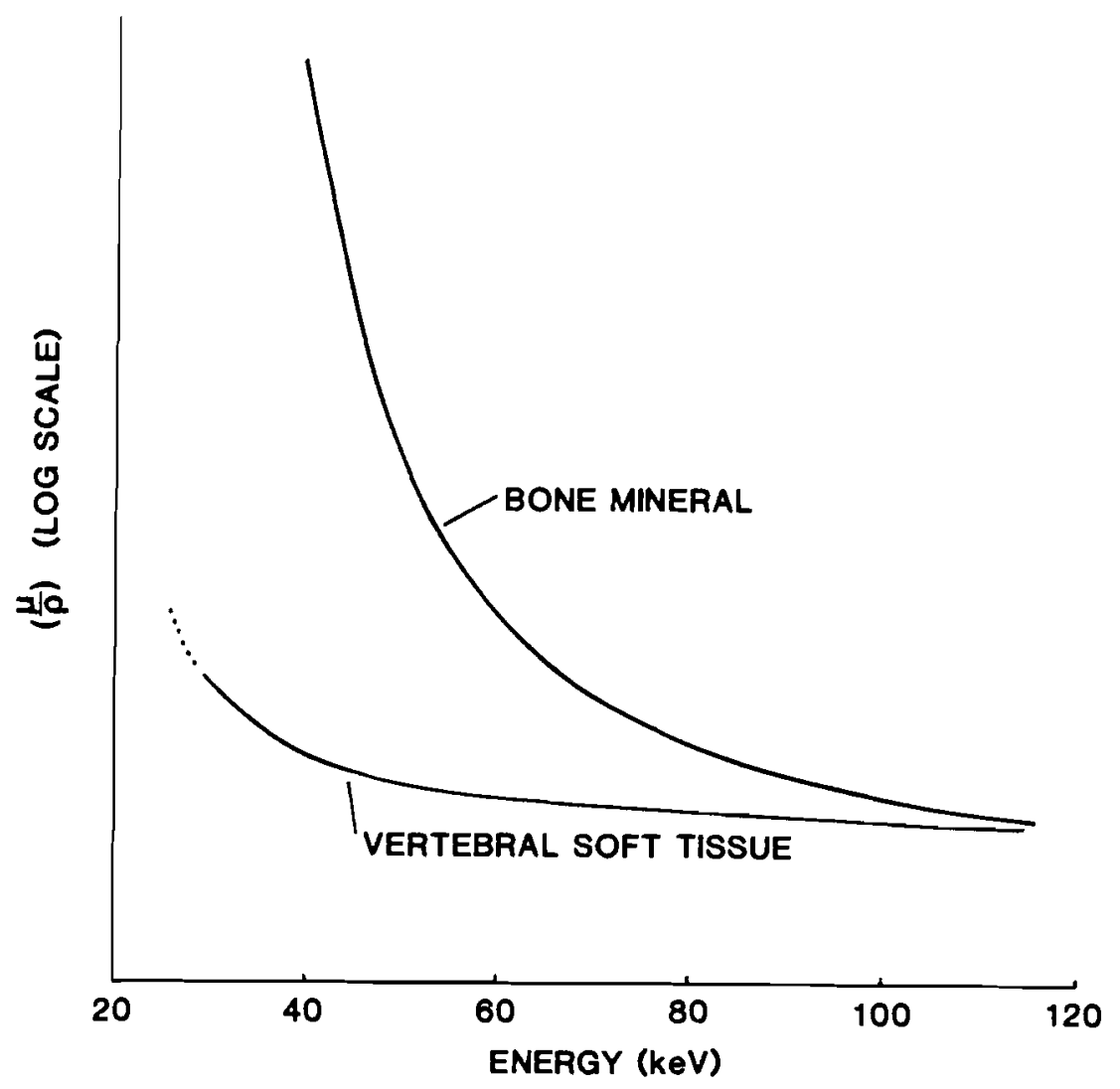

Figure 4.5: Attenuation Coefficients of Bone Mineral and Marrow.

If a C.T. measurement of cancellous bone is repeated at a lower energy, the difference between the two measurements is indicative of the mineral content and is only weakly dependent on the marrow component.

Several different formulae have been proposed for calculating the mineral concentration from the difference in attenuation at two energies. The dual energy technique of Genant et al. [140] uses a three component model for cancellous bone. Mineral and soft tissue are modelled as before, but the effect of increased yellow marrow content is allowed for by including an ethanol solution (60\% ethanol by volume in water) in the tissue model to simulate fat. The derivation starts from the mixture rule with three components:

$$
\mu=\frac{c_{b}}{\rho_{b}} \mu_{b}+\frac{c_{s}}{\rho_{s}} \mu_{s}+\frac{c_{f}}{\rho_{f}} \mu_{f}
$$


where the subscript $f$ denotes fat. In terms of Hounsfield units this equation becomes:

$$
H-H_{S}=\frac{c_{b}}{\rho_{b}}\left[H_{b}-H_{S}\right]+\frac{c_{f}}{\rho_{f}}\left[H_{f}-H_{S}\right]
$$

This equation cannot be solved as it stands since there are two unknowns: the concentrations of bone mineral and fat (ethanol). The problem may be overcome by repeating the C.T. measurement at a different kilovoltage, yielding two equations similar to equation 4.5 which may then be solved simultaneously for the two unknowns. In practice the above approach does not seem to produce a true measure of bone mineral concentration. Although its derivation appears to be valid, the tissue model on which it is based is expressed in terms of linear attenuation coefficients whereas the equivalence between bone mineral and $\mathrm{K}_{2} \mathrm{HPO}_{4}$ is in respect of the mass attenuation coefficient.

An alternative approach is that of Laval-Jeantet et al. [147] in which the Hounsfield number of vertebral bone is regarded as being linearly dependent on both mineral and fat concentrations:

$$
\mathrm{H}-\mathrm{H}_{\mathrm{S}}=\mathrm{a} \cdot \mathrm{B}+\mathrm{b}+\alpha \cdot \mathrm{F}+\beta
$$

Here $B$ represents the concentration of bone mineral, expressed as an equivalent concentration of $\mathrm{K}_{2} \mathrm{HPO}_{4}$ solution, and $\mathrm{F}$ is the equivalent concentration of fat. Again, fat is modelled by an ethanol solution. The constants $a$ and $b$ are obtained by linear regression of the measured Hounsfield numbers of standard solutions of $\mathrm{K}_{2} \mathrm{HPO}_{4}$ against their concentrations in grams per litre. The constants $\alpha$ and $\beta$ are similarly obtained by reference to the fat simulating solution.

\subsection{The Effect of Varying Marrow Composition.}

Genant and Boyd [140] simulated the effect on the mineral measurement of varying the fat content of the marrow by adding ethanol to a solution of dipotassium hydrogen phosphate. The effect on the single energy estimate of bone mineral concentration was shown to be large, but it is hard to relate such errors to the likely errors when measuring the bone mineral content of humans. Estimates in the literature of the likely errors due to fat vary from $20 \%$ to as much as $65 \%$ in patients with severe osteoporosis [145, 146, 147, 148]. Clinical studies using both dual- and single energy C.T. have shown that the single energy method overestimates the mineral loss following radiotherapy by a factor of two due to greatly increased fat content [149], and that patients with a history of anorexia nervosa have a significantly greater fat content in the marrow [150]. On the other hand the fat content is reported to remain the same in the Cushing syndrome [150] and actually decreases in Gaucher disease [151] so that in this case the single energy technique may 
slightly underestimate the degree of mineral loss. These exceptions aside, in general there is an increase in intravertebral fat in osteopaenia so that single energy techniques overestimate the degree of mineral loss [150]. A method has been proposed whereby single energy mineral measurements are corrected for fat using empirical correction factors based on regressions of fat content versus age [152]. However the range of fat values at any one age is large, even in patients not suffering from conditions which affect the vertebral fat content [153], so it is clear that this method will be of limited accuracy.

In the present work it has been possible to estimate the magnitude of these effects in the clinical situation by performing computer simulations which take into account the effect of varying density as well as composition. In addition to providing an independent estimate of the errors due to fat, the simulations provide a means of assessing the susceptibility of different algorithms to errors from this cause. The following tissue model was assumed for vertebral bone. The volume fraction of the trabeculae was taken to be $10 \%$, the remaining $90 \%$ of the volume being filled with varying proportions of red and yellow marrow. This would be representative of either an elderly patient or a mildly osteoporotic younger patient. The elemental compositions and densities of these three components were taken to be the average values quoted in I.C.R.P. Reference Man [117], and are listed in table 4.2. The attenuation coefficient of vertebral bone was obtained by interpolation of attenuation coefficients of the elements [58], via the mixture rule:

$$
\left[\frac{\mu}{\rho}\right]_{\text {tot }}=w_{t b} \cdot\left[\frac{\mu}{\rho}\right]_{t b}+w_{r m} \cdot\left[\frac{\mu}{\rho}\right]_{r m}+w_{Y m} \cdot\left[\frac{\mu}{\rho}\right]_{y m}
$$

where $w_{t b}, w_{r m}$, and $w_{y m}$ are the proportions by weight of trabeculae, red marrow and yellow marrow respectively.

Table 4.2: Assumed Composition and Density of Bone Components.

\begin{tabular}{|c|c|c|c|c|c|c|c|c|}
\hline \multirow[t]{2}{*}{ COMPONENT } & \multicolumn{2}{|c|}{$\begin{array}{l}\text { DENSITY } \\
(g / C C)\end{array}$} & \multicolumn{6}{|c|}{$\begin{array}{l}\text { ELEMENTAL COMPOSITION } \\
\text { (fractions by weight) }\end{array}$} \\
\hline & mean & range & $\mathrm{H}$ & $\mathrm{c}$ & $\mathrm{N}$ & 0 & $\mathbf{P}$ & $\mathrm{Ca}$ \\
\hline Trabeculae & 1.92 & & 0.051 & 0.133 & 0.046 & 0.441 & 0.108 & 0.221 \\
\hline Red marrow & 1.03 & \pm 38 & 0.104 & 0.431 & 0.033 & 0.431 & & \\
\hline Yellow marrow & 0.98 & \pm 58 & 0.115 & 0.643 & 0.007 & 0.230 & & \\
\hline Bone mineral & & & 0.008 & & & 0.450 & 0.190 & 0.360 \\
\hline
\end{tabular}


Where the proportions of the components are expressed in terms of volume the proportion by weight was obtained using:

$$
w_{i}=v_{i} \cdot \frac{\rho_{i}}{\rho_{\text {tot }}}
$$

where $v_{i}$ is the volume fraction of the $i^{\text {th }}$ component. The linear attenuation coefficient is obtained by multiplying the mass attenuation coefficient by the density of the mixture, which in turn is given by:

$$
\rho_{\text {tot }}=\Sigma v_{i} \cdot \rho_{i}
$$

The Hounsfield number was then obtained using the usual relationship (equation 2.3). The scale factor $\mathrm{K}$ and the attenuation coefficient of water used in the simulations (listed in table 4.3) are typical values for the G.E. 8800 scanner. The effective energy values quoted are not appropriate for bone mineral due to its high atomic number relative to that of water (see section 3.4). The effective mass attenuation coefficients quoted for $\mathrm{K}_{2} \mathrm{HPO}_{4}$ were obtained from measured C.T. values of dilute solutions ( $\leq 100 \mathrm{~g} / 1$ ), using the listed values of $\mathrm{K}$ and $\mu_{\mathrm{W}}$. The mineral concentration, as would be estimated from C.T. measurements, was then obtained using equation 4.3 (single energy) or equation 4.6 (dual energy). The values of the constants used in equation 4.6 were typical values for the Wellington Hospital scanner, and are listed in table 4.4 .

Table 4.3: C.T. Parameters Assumed in the Simulations.

\begin{tabular}{|c|c|c|c|c|}
\hline $\begin{array}{c}\text { peak } \\
\text { kilovoltage }\end{array}$ & $\begin{array}{c}\text { effective } \\
\text { energy } \\
(\mathrm{keV})\end{array}$ & $\mathrm{k}$ & $\begin{array}{c}\mu_{\mathrm{W}} \\
\left(\mathrm{cm}^{-1}\right)\end{array}$ & $\frac{\mu}{\left(\mathrm{cm}^{2} / \mathrm{g}\right)}$ \\
\hline 120 & 76.5 & 996.7 & 0.1867 & 0.3340 \\
80 & 61.5 & 1096.6 & 0.2031 & 0.4447 \\
\hline
\end{tabular}

Table 4.4: Regression Constants Used with Equation 4.6.

\begin{tabular}{|c|c|c|c|}
\hline $\begin{array}{c}\text { peak } \\
\text { kilovoltage }\end{array}$ & $\mathrm{a}$ & $\alpha$ & $\mathrm{b}+\mathrm{B}$ \\
\hline 120 & 1.4889 & -1.0851 & 0.4151 \\
80 & 2.1053 & -1.1703 & 0.4817 \\
\hline
\end{tabular}


The effect of variable marrow composition on the single energy mineral estimation is shown in figure 4.6, where the effect is seen of varying the composition of marrow from $10 \%$ red marrow to $90 \%$ red marrow by volume. The single energy $c_{b}(120 \mathrm{kV})$ values vary about the mean value (50\% yellow marrow) by $\pm 11 \%$. That is, a $1 \%$ change from the mean marrow composition will cause a $0.25 \%$ change in the estimated mineral content. Given the labile nature of bone marrow [154], this has serious implications for the clinical application of single energy C.T. bone mineral measurement. There is only a slight improvement at $80 \mathrm{kV}$, where the mineral estimate varies by $\pm 10 \%$ of the mean value over the range of composition plotted.

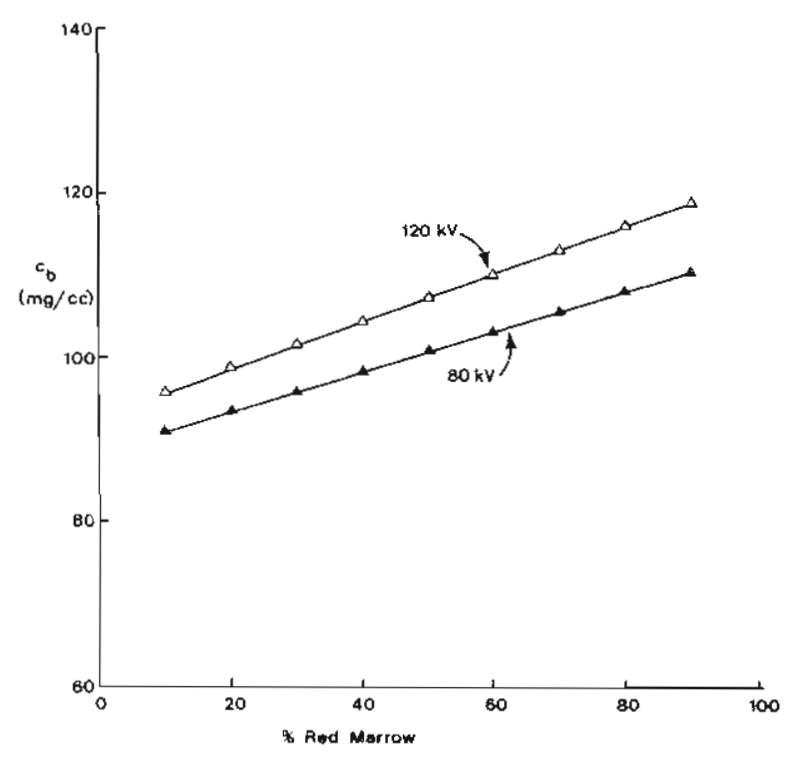

Effect of Varying Composition

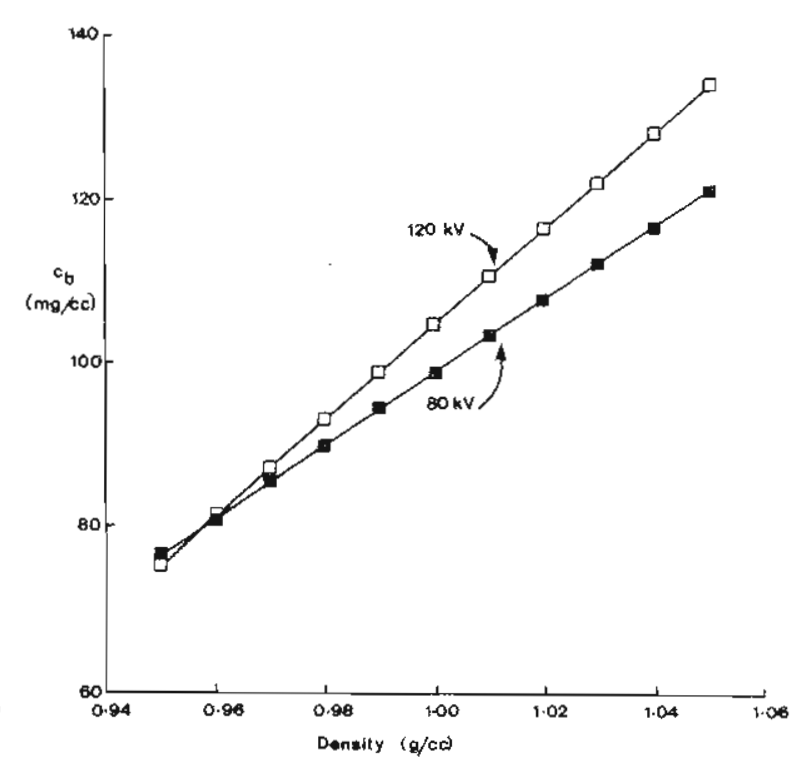

Effect of Varying Density

Figure 4.6: Effect on Single Energy Mineral Estimate of varying Marrow Composition and Density.

Since the mass attenuation coefficients of red and yellow marrow differ by only $0.1 \%$ at $73 \mathrm{keV}$, the changes observed must be due principally to the decrease in density as the proportion of yellow marrow increases. Indeed, the change in linear attenuation coefficient of yellow marrow over its density range $( \pm 5 \%)$ is greater than the difference in linear attenuation coefficient between average yellow marrow and average red marrow. The importance of density changes is also shown in figure 4.6 where the right hand graph gives the results of a simulation exercise where only the density of the marrow was changed, the elemental composition of the marrow being held constant at $50 \%$ yellow marrow by weight. Here we see $\mathrm{a} \pm 28 \%$ variation about the mean value of $\mathrm{cb}$ $(120 \mathrm{kV})$ with changes in density of $\pm 5 \%$. Again there is a slight improvement at $80 \mathrm{kVp}$ where the variation of $c_{b}$ over the same density range is $\pm 23 \%$. These figures suggest that for normal and mildly-osteoporotic patients the maximum error due to variations in marrow fat is of the order of $25-30 \%$, which is in agreement with the 
value suggested by Genant [140].

When the mineral content is estimated using the dual energy formula of LavalJeantet et al (equation 4.6) there is much lower sensitivity to changes within the marrow compartment. Variations in composition may cause inaccuracies in the mineral determination of the order of $\pm 5 \%$ (figure 4.7), that is, half the variation found in the case of the single energy simulations. In a simulation in which the marrow density alone is varied an even more dramatic improvement is seen; variation of marrow density over the same range as used for the single energy simulations causes the estimated mineral content to vary by no more than $\pm 1 \%$. The relatively low sensitivity to variations in marrow density is somewhat surprising since equation 4.6 does not include density as a variable. However it is clear from figure 4.8 that variations in density are interpreted as changes in 'fat' content $(F)$, to the extent that in some cases the estimated fat content is negative.

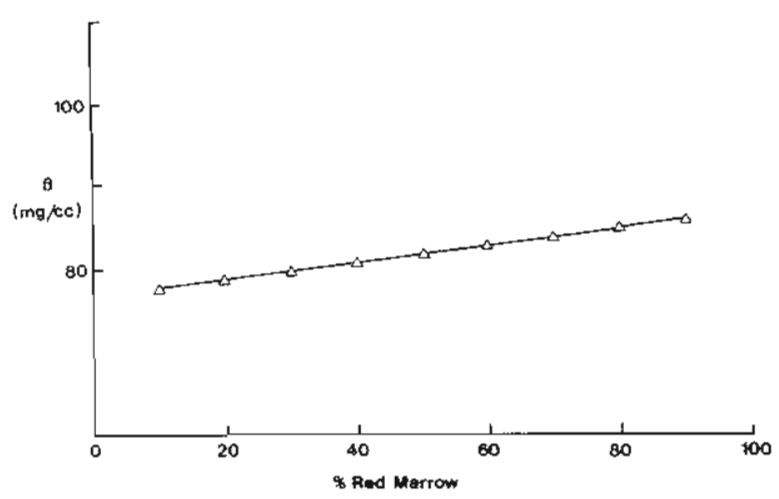

Effect of Varying Composition

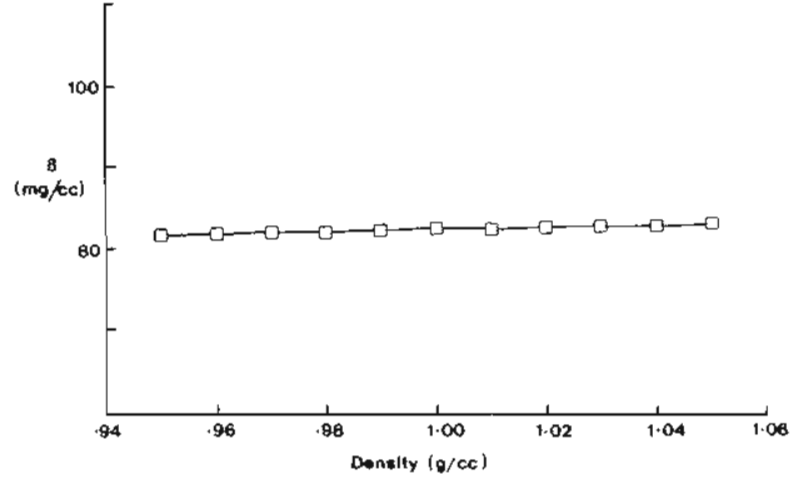

Effect of Varying Density

Figure 4.7: Simulated Effect on the Dual Energy Mineral Estimate of Varying Marrow Composition and Density.

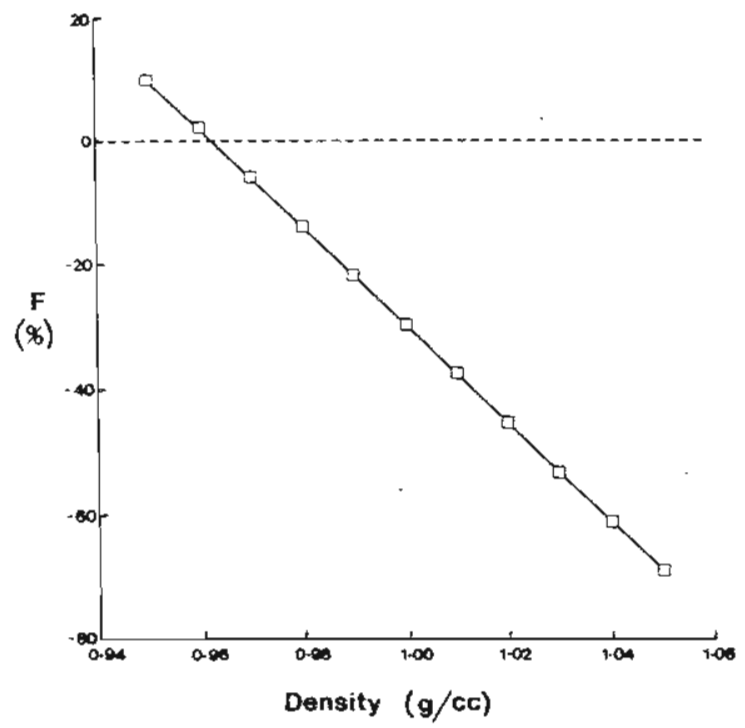

Figure 4.8: Simulated Effect on the Estimate of Fat Concentration of Varying Density. 


\subsection{An Alternative Dual Energy Formula.}

It has been shown that the dual energy method provides an estimate of the bone mineral content which is much less sensitive to changes within the marrow component. However the second parameter, F, is not a true measure of the fat content. Another approach is required if two useful quantities are to be obtained from the dual energy technique. The tissue model of the cancellous tissue used by Genant et al [142] is expressed in terms of linear attenuation coefficients. Since the linear attenuation coefficient of a material is a function of composition (effective atomic number) and density, this implies that both the composition and density of each component of the tissue model (mineral, red marrow, yellow marrow) do not vary. However it can be seen in table 4.2 that the densities of these components do vary. Although the range of densities appear to be rather small when expressed as a percentage of the mean, it has been shown in the previous section that the effect on the estimated mineral content is large. The effect of variable density may be reduced by expressing the tissue model in terms of mass attenuation coefficients which depend only on the composition of the materials involved, and not their density. For a two component model composed of bone mineral and marrow the appropriate form of the mixture rule is [49]:

$$
\left[\frac{\mu}{\rho}\right]_{\text {tot }}=\mathrm{w}_{\mathrm{b}}\left[\frac{\mu}{\rho}\right]_{\mathrm{b}}+\mathrm{w}_{\mathrm{m}}\left[\frac{\mu}{\rho}\right]_{\mathrm{m}}
$$

where $w_{b}$ and $w_{s}$ are the proportions by weight of bone mineral and soft tissue respectively. Soft tissue in this context is defined as tissue other than bone mineral. The collagen matrix upon which the bone mineral is deposited is included in the soft tissue component. Since the proportions by weight must sum to unity, this equation may be rearranged as follows:

$$
\mu_{\text {tot }}=\rho \cdot \mathrm{wb}_{\mathrm{b}}\left[\left[\frac{\mu}{\rho}\right]_{\mathrm{b}}-\left[\frac{\mu}{\rho}\right]_{\mathrm{m}}\right]+\left[\frac{\mu}{\rho}\right]_{\mathrm{m}}
$$

The quantity $\rho \cdot \mathrm{w}_{\mathrm{b}}$ is the concentration of bone mineral. The two unknowns, bone density and mineral concentration, may both be determined by performing the C.T. measurement at two energies. The linear attenuation coefficient may be obtained from the Hounsfield number using equation 2.3. The mass attenuation coefficient of marrow is obtained from the elemental composition adopted in I.C.R.P. Reference Man, using the attenuation coefficients tabulated by Hubbell [58]. Marrow was assumed to consist of $50 \%$ red marrow and $50 \%$ yellow marrow by weight. The mass attenuation coefficient 
of bone mineral, as measured by the X-ray beam of the C.T. scanner, is calculated from C.T. measurements of a $\mathrm{K}_{2} \mathrm{HPO}_{4}$ solution.

The proposed algorithm (equation 4.11) was subjected to the same tests for sensitivity to changes in the marrow as were the existing formulae (equations 4.3 and 4.6), the results of which are summarised in table 4.5. Of the three algorithms tested, the Wellington algorithm shows the least sensitivity to changes in marrow density, which is to be expected since the Wellington algorithm is the only one which explicitly includes density in the formula. The variation in the mineral figure computed from the Wellington formula varies by $\pm 3.5 \%$ as the ratio of red to yellow marrow varies from $10 \%$ red / $90 \%$ yellow to $90 \%$ red and $10 \%$ yellow. This compares well with $\pm 5 \%$ for the Laval-Jeantet dual energy formula (equation 4.6), and $\pm 10 \%$ for the single energy formula (equation 4.3). Since equation 4.10 neglects variations in the proportions of red and yellow marrow it may appear strange that the mineral concentration estimated from equation 4.11 is less sensitive to such changes than are the other dual energy formulae which make special provision for this effect. This serves to emphasise the fact that the difference in linear attenuation coefficient between the two types of marrow is predominantly due to the differences in density, not elemental composition.

Table 4.5: Results of Computer Simulations.

\begin{tabular}{||l|rr||}
\hline \multicolumn{1}{|c|}{ ALGORITHM } & \multicolumn{2}{c|}{ VARIABLE } \\
& Composition & Density \\
\hline Genant (single energy) & $\pm 10 \%$ & $\pm 28 \%$ \\
Laval-Jeantet (dual energy) & $\pm 5 \%$ & $\pm 1 \%$ \\
Wellington (dual energy) & $\pm 3.5 \%$ & $\pm 0 \%$ \\
\hline
\end{tabular}

The table shows the variation of the mineral parameter calculated using the three algorithms as the density of the marrow is varied by $\pm 5 \%$ and as the composition is varied from $90 \%$ red marrow, $10 \%$ yellow marrow through to $10 \%$ red and $90 \%$ yellow.

It can be seen from the above derivations that the proposed formula gives a direct measure of bone mineral concentration in grams of bone mineral per millilitre of bone tissue. Because the tissue model is expressed in terms of mass attenuation coefficients the $\mathrm{K}_{2} \mathrm{HPO}_{4}$ solution is used solely to estimate the mass attenuation coefficient of bone mineral. It is not necessary to express the mineral concentration as an 'equivalent' concentration of $\mathrm{K}_{2} \mathrm{HPO}_{4}$ solution, as is the case with the parameters $c_{b}$ and $\mathrm{B}$. However there is a strong relationship between the mineral content as estimated using the proposed formula, and the quantity B (equation 4.6). From dual energy C.T. studies of 
23 lumbar vertebrae, in vivo, the following relationship is found between the two quantities:

$$
\begin{aligned}
c_{\min } & =0.86 \times \mathrm{B}+24 \\
(\mathrm{r} & =0.99992, \mathrm{n}=23)
\end{aligned}
$$

where $C_{\min }\left(=\rho \cdot w_{b}\right)$ is the mineral concentration obtained using equation 4.11.

A number of papers [146, 155 - 159] have appeared since this work was carried out outlining a variety of different algorithms for estimating bone mineral content from dual energy C.T. scans. These have all been based on tissue models expressed in terms of linear attenuation coefficients, and are of greater complexity than that suggested in the present work (equation 4.11). A recent review of dual energy algorithms [160] has concluded that accurate mineral figures are obtained when using the formulae of Goodsitt [156] or Nickoloff [159], but it was noted that there was strong correlation between values computed using Goodsitt's formula and those computed using the formalism of Laval-Jeantet et al [144].

\subsection{A Spine Mineral Measurement Protocol for Wellington Hospital.}

Scanning protocols for the examination as it is performed at Wellington Hospital are listed in table 4.6. After the patient is positioned on the scanner table a planning scan ('scout view') is taken which produces an image comparable to a lateral plain film (see figure 4.3). This image is used to determine the settings necessary to obtain axial images through the centres of the required vertebrae, with the gantry tilted so that the image planes are at right angles to the long axis of each vertebra. Accurate location of the images is necessary to obtain optimum reproducibility of the measurements for serial studies [161], so this task is performed either by the physicist or the senior radiographer. Two axial slices are taken through each of the second, third and fourth lumbar vertebrae, the first slice at $80 \mathrm{kVp}$ and the second at $120 \mathrm{kVp}$. The raw data for the $80 \mathrm{kVp}$ slices are then corrected for the nonstandard kilovoltage, as discussed in section 3.5, and magnified images are reconstructed from the data taken at both kilovoltages. C.T. number readings are taken of the cancellous region of each vertebra using a box shaped cursor set to the largest possible size consistent with avoiding cortex on the top and the two sides, and avoiding the area of lowered attenuation posteriorly. Use of an elliptical cursor would permit the measurement of a larger tissue volume given the above constraints [162], but the G.E. software only permits the use of this cursor in the 'dynamic scan' mode. Dynamic scanning on the G.E. 8800 scanner involves rapid braking of the tube and detectors at the end of each scan, accompanied by loud noises which can be disturbing to the patient. Dynamic scans are therefore not used for this examination due to the possible incidence of patient movement between images. 
Table 4.6: Wellington Densitometry Technique.

\begin{tabular}{|c|}
\hline 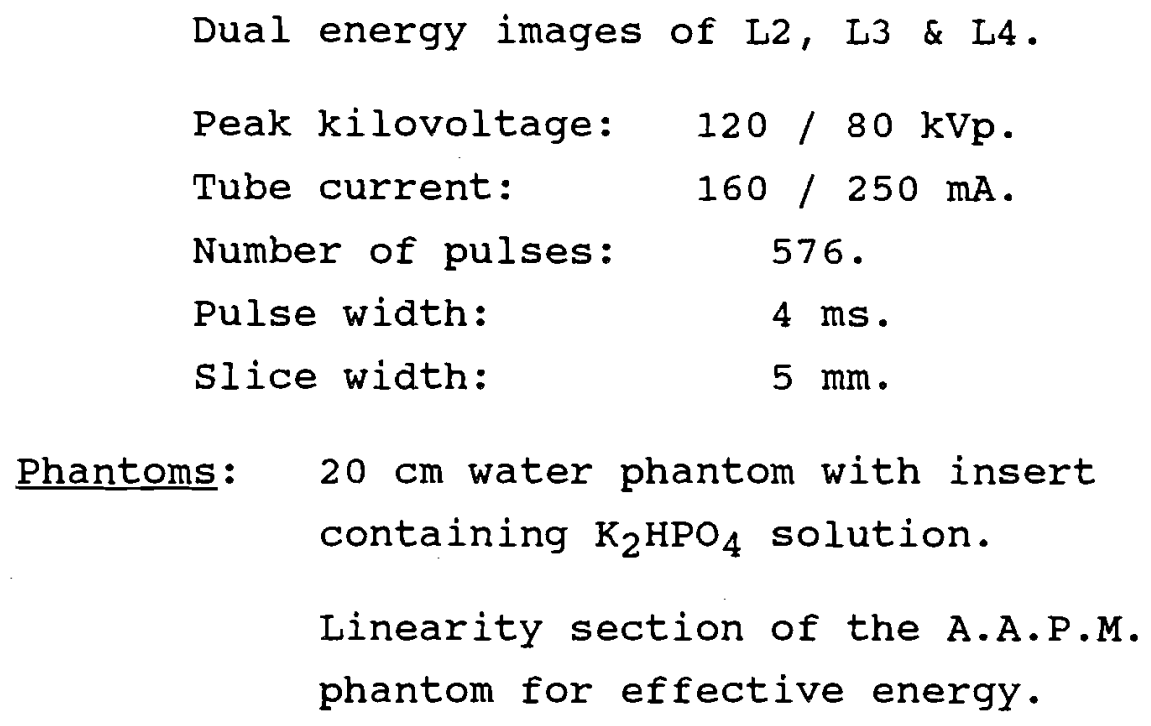 \\
\hline
\end{tabular}

Quantitative applications of computed tomography require more scrupulous attention to radiographic technique than routine studies. The reconstructed C.T. numbers are most accurate when the size of the patient cross section closely matches the size of the phantom against which the scanner is calibrated, and will not be accurate if more than a minimal amount of tissue lies outside the area reconstructed since the attenuation due to such tissue will be attributed to tissue within the reconstruction circle. With the G.E. 8800 scanner it is necessary to decide whether to use the $35 \mathrm{~cm}$ field of view or the $42 \mathrm{~cm}$ field of view when scanning the abdomen, a task which sounds simple but which in fact requires considerable skill considering that a patient who will just fit into the $35 \mathrm{~cm}$ field of view will only do so if correctly centred in the gantry aperture. A further point to be considered is that the table height must be set so that the top surface of the patient lies within the field of view, but must not be so low that the table impinges on the reference area at the edge of the beam at the times during the scanning movement when the tube and detectors are horizontally opposed.

Calculation of bone mineral concentration requires C.T. measurements of a solution of $\mathrm{K}_{2} \mathrm{HPO}_{4}$. The technique of Genant and Cann [163] uses a crescent shaped phantom placed underneath the patient's back. This phantom, which is made of perspex, contains cylindrical cavities containing $\mathrm{K}_{2} \mathrm{HPO}_{4}$ solutions of varying strengths. A similar phantom has been made at Wellington Hospital, but it has been found that this phantom is not well imaged by the G.E. 8800 scanner, particularly if there is an appreciable air gap between the phantom and the patient. Even without an air gap, it has been found that the C.T. number of the perspex at a distance of $7.5 \mathrm{~cm}$ from the middle of the phantom is some 15 Hounsfield units lower than at the middle. Measurements 
taken under the same conditions using a polyethylene phantom of the same dimensions indicate a C.T. number difference of 6 Hounsfield units which is an improvement, but the C.T. number measurements are still compromised by any appreciable air gap between the phantom and the patient. Similar problems have been reported in the literature $[160,164]$. The Wellington protocol does not make use of a crescent phantom and relies instead on measurements of a $100 \mathrm{~g} / 1$ solution of $\mathrm{K}_{2} \mathrm{HPO}_{4}$ contained within a $32 \mathrm{~mm}$ diameter polypropylene test tube which in turn is located inside a water phantom. The water phantom was some $20 \mathrm{~cm}$ in diameter, which is too small to be strictly representative of the abdomen, but which was the largest size available when this technique was under development. Images of this phantom are taken either immediately before or after scanning a series of patients. The 'linearity' section of the A.A.P.M. phantom is also scanned at this time to obtain the effective energies and constants $K$ and $\mu_{\mathrm{W}}$ (equation 2.3) at the two kilovoltages.

The use of the crescent phantom is said to have the advantage of making possible compensation for scan to scan fluctuations in scanner calibration caused by such effects as variations in peak kilovoltage [165]. However such effects on the G.E. 8800 scanner are minor. Analysis of 43 readings of the mean C.T. number of the $\mathrm{K}_{2} \mathrm{HPO}_{4}$ standard solution taken at intervals over a period of a year gave a coefficient of variation (standard deviation divided by the mean) of $1.1 \%$ at $120 \mathrm{kVp}$ and $1.4 \%$ at $80 \mathrm{kVp}$. Short term fluctuations would be expected to be considerably less than this. A further possible reason for using a crescent phantom is if the measured C.T. numbers are inaccurate. Since the crescent phantom is close to the area being measured it would be expected that the errors in the spine measurement and in the measurement of the standard solution would be comparable and would tend to cancel. Possible causes of such errors are variations in size of the patient [166], errors in patient positioning where the table intercepts the reference area at the edges of the beam leading to a shading artefact [167], and incorrect selection of the imaging field size where the patient section is not completely within the field of view leading to falsely large C.T. numbers. The last two causes of errors can be avoided by the use of proper scanning techniques. The effect of variations in patient size can be corrected empirically [166], although such correction is not performed at Wellington Hospital due to the large amount of additional work involved. The results of tests presented in section 3.6 suggest that variations in phantom size have only a minor effect on the accuracy of C.T. number measurements. To estimate the magnitude of possible errors due to patient thickness an oval phantom of axis lengths 30 $\mathrm{cm} \times 20 \mathrm{~cm}$ was constructed with a test tube containing a $100 \mathrm{~g} / 1$ solution of $\mathrm{K}_{2} \mathrm{HPO}_{4}$ placed at a position representative of the spine. The C.T. number difference between this solution and that in the $20 \mathrm{~cm}$ circular phantom was 12 Hounsfield units at $120 \mathrm{kVp}$ and 17 Hounsfield units at $80 \mathrm{kVp}$. These differences are of comparable magnitude to the differences found at different locations in a perspex crescent phantom (see above), and would give rise to a $8 \%$ error in the single energy mineral estimate obtained using 
equation 4.3 and a $13 \%$ error in the dual energy mineral estimate, Cmin, obtained using equation 4.11. These differences are larger than would be expected from the tests described in section 3.6, showing that the beam hardening correction software of the C.T. scanner functions best for circular objects.

The effect of varying thickness of cortex was simulated by placing aluminium collars of different thicknesses over the test tube containing $\mathrm{K}_{2} \mathrm{HPO}_{4}$ solution in the $20 \mathrm{~cm}$ diameter circular water phantom. These indicated a shift in measured C.T. number of the solution of approximately 5 Hounsfield units per $1 \mathrm{~mm}$ thickness of aluminium. Given that the average thickness of cortex is about $1.5 \mathrm{~mm}$, it can be seen that the thickness of cortex must vary by a very large percentage to have a significant effect on the measured C.T. number of the cancellous region contained within the cortex.

A series of programs (written in FORTRAN 77) has been written for automatic analysis of the C.T. readings. These are listed in appendix F. Processing of the data starts with the keying in of the phantom readings using program LCORR which is invoked by the macro command PHANTOMS. This program then computes the effective energies at $80 \mathrm{kVp}$ and $120 \mathrm{kVp}$, along with the parameters $\mathrm{K}$ and $\mu_{\mathrm{W}}$, using the method of Millner et al. [60]. To obtain the effective energies, the correlation coefficient (see section 2.5) is first computed at energies of 56,60 and $64 \mathrm{keV}$ for the $80 \mathrm{kVp}$ images and 67,71 and $75 \mathrm{keV}$ for the $120 \mathrm{kVp}$ images. The data for each kilovoltage are fitted to a parabola from which the energy of greatest correlation is estimated. Since the assumption of a parabolic relationship between energy and correlation coefficient will only be valid at the turning point, an improved estimate is then obtained by parabolic fit at the previous estimate of the effective energy and at points at $1 \mathrm{keV}$ each side of it. This program also computes the effective mass attenuation coefficient of $\mathrm{K}_{2} \mathrm{HPO}_{4}$ from the measured C.T. number of the standard solution, and computes the mass attenuation coefficient of vertebral soft tissue (as defined in section 4.6) at the effective energies via a previously computed continued fraction fit. The computed parameters are stored in a file called SERIES.XXX where XXX is a three digit number.

The patient readings are then keyed in using the command PATIENTS which invokes the program PATS which inputs patient name, sex and date of birth as well as the readings themselves. This information is appended to file SERIES.XXX. Both single and dual energy mineral estimates are computed (using equations 4.3 and 4.11 respectively) by program AWMIN which is invoked by the command SUMS, the results being printed out and also stored in file CSERIES.XXX. Program SPINEMIN (invoked by the command REPORT) prints a graph of the normal range of the single energy mineral estimate as a function of age, with the readings from each patient marked on it. The data from the patients used as controls in the anorexia nervosa study (see section 5.2) has been used to derive a normal range for females as measured using the Wellington protocol. Males 
form only a small proportion of the patients scanned at Wellington Hospital and no local control studies have been performed at this stage. For males the normal data published by Genant et al. [162] is used. The program also produces a draft examination report for each patient.

Tests of the reproducibility of the technique have been performed using the oval shaped water phantom described previously. The phantom was scanned seven times using the protocol of appendix F, making a total of 21 axial images at each of $80 \mathrm{kVp}$ and $120 \mathrm{kVp}$. Gantry angles of 15,0 and -15 degrees were used for 'L2', 'L3' and 'L4' respectively to simulate patient scanning conditions. From readings of the C.T. number of the $\mathrm{K}_{2} \mathrm{HPO}_{4}$ solution were calculated the single energy mineral estimate (equation 4.3) at each kilovoltage, and the density and mineral concentration (equation 4.11). The coefficients of variation (standard deviation divided by the mean) for the total series of 21 slices at each kilovoltage are given in table 4.7. These figures may be used to derive an expected precision for patient examinations in which the values for three vertebrae are averaged. The standard error of the mean values for the three 'vertebrae' of the 'patients' is given by the coefficient of variation divided by $\sqrt{3}$.

Table 4.7: Reproducibility of Mineral Estimates in vitro.

\begin{tabular}{||c|c|c|c|c||}
\hline \hline Parameter & $\begin{array}{c}\mathrm{Cb}_{\mathrm{b}} \\
(120 \mathrm{kVp})\end{array}$ & $\begin{array}{c}\mathrm{Cb}_{\mathrm{b}} \\
(80 \mathrm{kVp})\end{array}$ & Rho & $\mathrm{C}_{\text {min }}$ \\
\hline $\mathrm{C.V}$. & $1.1 \%$ & $1.9 \%$ & $0.8 \%$ & $6.0 \%$ \\
\hline $\begin{array}{c}\text { std. error } \\
\text { of means. }\end{array}$ & $0.6 \%$ & $1.1 \%$ & $0.5 \%$ & $3.5 \%$ \\
\hline
\end{tabular}

The single energy figures are comparable with a coefficient of variation figure of $1.5 \%$ for individual excised vertebrae in a torso shaped phantom published by Cann and Genant [168]. Genant [123] quotes a coefficient of variation of 3 to $5 \%$ for the dual energy mineral parameter in healthy young patients (mean values for 3 vertebrae). Reproducibility of the Wellington technique for humans has been estimated by scanning two volunteer patients each on five separate occasions. The first was a female who was found to have severe osteoporosis with a value of $c_{b}(68 \mathrm{~g} / 1$ at $80 \mathrm{kV})$ which was well below the level at which there is increased risk of crush fracture $[123,162,169]$. The second was a male in his forties whose mineral content was found to be in the normal range. The coefficients of variation for the four parameters measured are given in table 4.8 . 
Table 4.8: Reproducibility of Mineral Estimates in vivo.

\begin{tabular}{||l|c|c|c|c||}
\hline \hline Parameter & $\begin{array}{c}\mathrm{C}_{\mathrm{b}} \\
(120 \mathrm{kVp})\end{array}$ & $\begin{array}{c}\mathrm{C}_{\mathrm{b}} \\
(80 \mathrm{kVp})\end{array}$ & Rho & $\mathrm{C}_{\text {min }}$ \\
\hline $\begin{array}{l}\text { Osteoporotic } \\
\text { volunteer }\end{array}$ & $8 \%$ & $11 \%$ & $3 \%$ & $27 \%$ \\
\hline $\begin{array}{l}\text { Normal } \\
\text { Volunteer }\end{array}$ & $2 \%$ & $1 \%$ & $7 \%$ \\
\hline
\end{tabular}

The precision is many times worse in the measurements of the osteoporotic volunteer than in those of the normal volunteer, a phenomenon also noted by other authors [113]. The precision obtained in measurements of the normal volunteer is comparable with the figures cited above from the work of Genant and his co-workers.

Absolute accuracy is of lesser importance than precision since the latter parameter determines whether or not the technique is capable of measuring the often small changes in mineral content in response to treatment, while accuracy is necessary to determine the relationship between value obtained and the average value for people of the same age and sex. The effect of systematic errors is removed if the normal range is measured for each measurement system, although errors due to random effects such as variable fat content will remain. Laval-Jeantet et al [144] scanned 42 excised lumbar vertebrae in a phantom, and then compared the C.T. measurements with the ashed weights for the vertebral tissue scanned. They obtained correlation coefficients of 0.74 (females) and 0.82 (males) for the single energy measurement (computed using equation (4.3)). Correlation coefficients of 0.93 (females) and 0.96 (males) were obtained for their dual energy measurements using equation (4.6). These figures would also apply to measurements computed by the Wellington formula (equation 4.11) since there is extremely good correlation between the two mineral parameters (equation 4.12). Burgess et al [153] also reported good correlation $(r=0.95)$ between a dual energy mineral estimation and a chemical determination of bone mineral on a sample of 50 excised vertebrae.

\subsection{Comparison Between C.T. Spine Mineral Densitometry and Dual Photon Absorptiometry.}

Although there is general agreement that current bone mineral measurement techniques are now sufficiently sensitive to assess the mineral content of individual patients, rather than simply determining differences between groups of patients (eg preand post-menopausal women), it has been noted that the literature contains conflicting 
results and that there is considerable controversy over the relative merits of competing measurement systems in various clinical situations [123]. It is agreed that measurements of cancellous bone are more sensitive to changes in the patient's condition than those of purely cortical bone, and it is further agreed that measurements are best taken at the site of clinical interest. For example, measurements taken in the radius or metacarpal bones show only moderate correlation with DPA or C.T. measurements in the lumbar spine [180]. However in the case of the lumbar spine there is considerable debate over the relative merits of quantitative computed tomography and DPA. Since a C.T. scanner is some five to twenty times more expensive than a DPA machine, there is no question of purchasing a C.T. scanner for the sole purpose of performing spine mineral measurements [122]. Indeed the annual service contract price for the G.E. 8800 scanner is approximately the same as the purchase price for a DPA instrument! However since C.T. scanners are now widespead (as of the time of writing there were at least 18 C.T. scanners in New Zealand), it makes sense to make use of any excess capacity for bone mineral measurements, all other factors being equal. The important clinical factors are accuracy, precision, sensitivity and patient dose $[113,122,118]$.

Both C.T. and DPA are imaging techniques, permitting precise identification of the measurement area, but the image obtained by DPA is a projection image which necessarily involves simultaneous measurement of both cortical and cancellous bone while C.T. produces tomographic images permitting the measurement to be restricted to cancellous bone tissue where the rate of mineral turnover is some eight times greater [141]. The importance of the cancellous bone can be inferred from the fact that the compressive strength of the vertebral body decreases by only $10 \%$ when the cortex is removed [170]. However, some clinical studies suggest that the sensitivity of QCT is only some 1.5 to 2 times greater than for DPA [118]. Jones et al [171] noted the importance of obtaining readings of purely cancellous bone in diagnosing early bone loss after the menopause, since marked decrease in the cancellous bone tissue occurs before there is any discernible mineral loss in the cortex.

Table 4.9: Comparison of QCT with DPA.

\begin{tabular}{||l|r|r|r||}
\hline & precision & accuracy & sensitivity \\
\hline DPA & $1-3 \%$ & $4-6 \%$ & $2 \times$ \\
DPA-X & $1-2 \%$ & $3-5 \%$ & $2 \times$ \\
QCT (single energy) & $1-2 \frac{8}{6}$ & $5-20 \%$ & $3-4 \times$ \\
QCT (dual energy) & $3-5 \%$ & $2-4 \%$ & $3-4 \times$ \\
\hline
\end{tabular}

Figures taken from [118] and [123]. Sensitivity figures are expressed on a scale relative to $S P A=1$. 
Single energy C.T. is prone to errors caused by variable fat content in the marrow, as discussed in section 4.5. The maximum error due to this cause in most patients is of the order of $30 \%$. However the DPA technique is also subject to errors caused by fat, in this case fat lying outside the vertebra. The software of most DPA instruments uses readings immediately adjacent to the vertebral body to compensate for this effect, but it has been shown that there can be a median error due to this cause of $8 \%$ [172]. Assuming gaussian statistics, this would imply that in $95 \%$ of cases the error is less than $23 \%$, a figure which is comparable with the error in the C.T. technique due to fat! The use of dual energy C.T. techniques virtually eliminates errors due to fat, at the expense of reduced precision.

DPA is susceptible to errors due to mineral being present in structures which are in the path of the beam, but do not contribute to the strength of the vertebra. Examples include osteophytes (boney outgrowths from the vertebral body), and calcification of the aorta, which lies just anterior to the vertebral body. Also the presence of vertebral processes posterior to the vertebral body can cause repositioning errors for follow-up studies. Some of these problems can be averted by the use of special patient cradles which permit the use of lateral rather than antero-posterior views, making it possible to exclude the aorta and the vertebral processes from the measurement area. However the precision for the lateral technique is quoted as $2 \%$, some two times greater than can be achieved using the AP technique when using modern DPA instruments.

DPA can be applied to many sites in the body, but is principally used for measurements in the lumbar spine and femur and for estimations of the total body calcium content. On the other hand most C.T. work is only applied to the lumbar spine. If a machine is to be purchased solely for the purpose of making bone measurements, this flexibility would suggest the purchase of a DPA-X instrument. On the other hand the increased sensitivity of QCT would suggest that this is the instrument of choice for performing measurements in the lumbar spine, particularly if follow-up measurements may be necessary since the value of DPA for serial studies is questionable [173]. C.T. has been shown to be a better predictor of vertebral crush fracture risk than any other available densitometric method [174].

The dose from QCT is unquestionably much greater than for DPA or DPA-X. However it will be seen in the next section that the dose from QCT remains small in comparision with other common X-ray procedures. 


\subsection{Patient Dose.}

Most medical procedures carry some degree of risk, and X-ray procedures are no exception. The International Commission for Radiological Protection [175] requires that irradiation of humans should not take place unless it is shown that the benefits accruing outweigh the risks. With the low levels of radiation occurring in diagnostic procedures the only risks to be considered are increased risk of cancer in the exposed individual and increased risk of hereditary defects being passed onto the descendants of the exposed person. In unusual circumstances there can be concerns about exposure of the lens of the eye leading to the eventual formation of cataracts, but this is a threshold effect which will not occur if the dose is kept below $150 \mathrm{mSv}$ per annum. As C.T. of the lumbar spine does not involve direct irradiation of the lens this effect is not relevant to the present discussion. Absorbed dose is defined as the amount of energy (in joules) absorbed per kilogram of tissue. Dose equivalent is derived from the absorbed dose by multiplying by a factor which takes into account the biological effectiveness of the type of radiation involved. This factor is unity for X-rays. A dose index called the effective dose equivalent [175] is defined in which the doses to various organs are multiplied by organspecific multiplying factors which take into account the susceptibility to radiation induced carcinogenic and genetic effects. Pye et al [176] have computed the effective dose equivalent for a DPA examination and obtained a figure of $0.6 \mu \mathrm{Sv}$ for males and $6 \mu \mathrm{Sv}$ for females, the larger figure arising from the possibility of direct irradiation of the ovaries.

A similar computation has been performed for the C.T. spine mineral densitometry examination in Wellington. The exposure factors for the examination, which are given in table 4.6, were selected to be comparable to those recommended by Cann [165]. The levels recommended were the lowest possible consistent with maintaining the imprecision due to reduced X-ray exposure down to less than $30 \%$ of the total inaccuracy. Doses from the Wellington G.E. 8800 scanner have been measured by the National Radiation Laboratory [177], whose figures have been used to provide an estimate of the dose at 120 $\mathrm{kV}$. Program XSPECT has been used to calculate the corresponding doses at $80 \mathrm{kV}$, details of the calculations being given in appendix G. The effective dose equivalent due to the axial slices is estimated to be $31 \mu \mathrm{Sv}$. The computation of the effective dose equivalent due to the planning scan is difficult because the variability of the anatomical position of the ovaries is such that the ovaries will be in the direct beam in some patients and not in others. Taking a conservative approach and assuming that the ovaries are in the direct beam, the effective dose equivalent due to the planning scan will be $65 \mu \mathrm{Sv}$ in females and $26 \mu \mathrm{Sv}$ in males. The effective dose equivalent for females for the entire examination is therefore approximately $96 \mu \mathrm{Sv}$. This compares well with a figure quoted by Tothill [122] for a single energy examination of $120 \mu \mathrm{Sv}$. The Wellington figure is 
lower because three vertebrae are scanned, not four; the slice width in Wellington is $5 \mathrm{~mm}$, not $10 \mathrm{~mm}$; and because the $X$-ray output at $80 \mathrm{kV}$ is very much smaller. In figure 4.9 the effective dose equivalent due to the Wellington densitometry examination is compared with effective dose equivalents for some other X-ray examinations.

\section{EFFECTIVE DOSE EQUIVALENTS \\ for some diagnostic examinations}

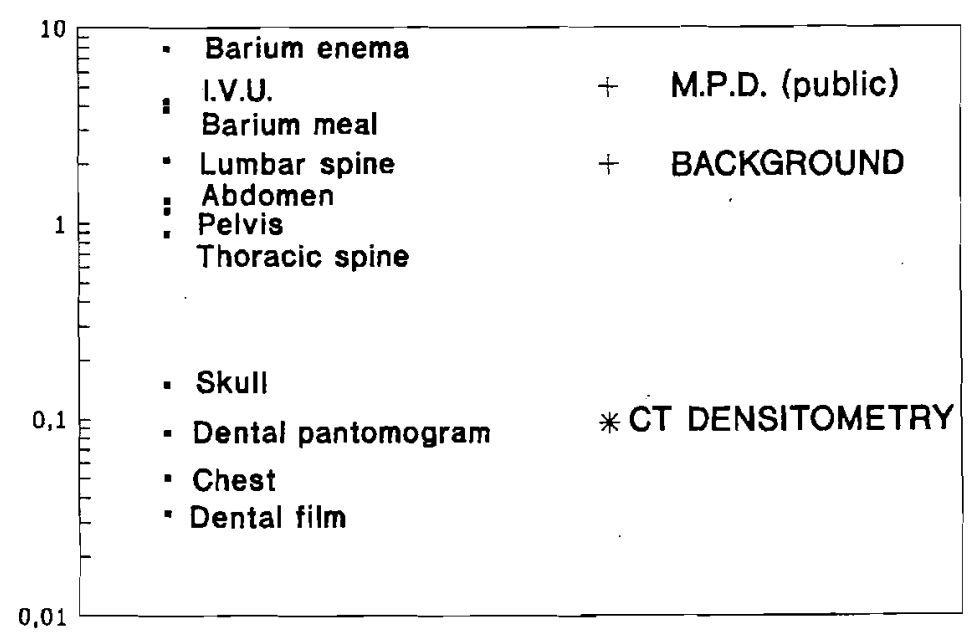

Figure 4.9: Effective Dose Equivalents for Some Diagnostic Examinations.

Dose figures (in $\mathrm{mSv}$ ) are from Wall [178]. The effective dose equivalent due to the Wellington densitometry examination is comparable with other low dose examinations such as dental X-rays and chest films, and is equal to about 18 days' background radiation. Also shown on the diagram are the average annual dose due to background radiation in New Zealand, and the current maximum permissible annual dose to a member of the public. The M.P.D. for occupationally-exposed persons is some ten times greater.

The E.D.E. of the Wellington C.T. spine mineral densitometry examination equates to about $5 \%$ of the average annual dose due to background radiation. This is small compared with the fluctuations in background dose from one location to another. Epidemiological studies have failed to show increased cancer rates in areas with higher natural background levels [179].

\subsection{Reprise}

Bone measurement methods are required firstly to identify those persons in need of treatment for low bone mass, and secondly to monitor the response to such treatment. The first task requires the measurement technique to be accurate, whereas precision is 
perhaps more important in the case of follow-up examinations so that apparent changes can definitely be attributed to changes in the patient's condition rather than random fluctuations. However these two parameters alone do not completely describe a measurement system because a measurement which is accurate and precise, but which is perhaps taken at a site where there is very little change regardless of the condition of the patient, will be of little value. It is therefore necessary to consider the sensitivity of each technique. Best sensitivity occurs when the measurement is taken in an area of cancellous bone tissue since the rate of bone turnover in the trabeculae is many times greater than in the cortex. Even when there is moderate correlation between the mineral content of different bones ( $r=0.8$, for example), the mineral content in the one will still not be a particularly good predictor of the mineral content in the other so that it is usually best to make the mineral measurement in the bone of clinical interest. In the case of the lumbar spine the two currently-available measurement techniques are dual photon absorptiometry (DPA) and quantitative computed tomography. Quantitative computed tomography has the greater sensitivity since it is applied to cancellous tissue alone whereas DPA measures a combination of cortical and cancellous bone. The two techniques are of similar accuracy and precision, and both are susceptible to fairly large errors due to variable fat content. Dual energy C.T. can all but eliminate such errors, but at the expense of reduced precision so that some authors have suggested that dual energy C.T. should be used for initial examinations but that single energy is more appropriate for subsequent ones. However others state that it is important to use the dual energy technique on follow ups since it is important not to mis-report changes in fat content as changes in mineral concentration.

While most workers seem to be content to use the single energy formula of Genant and his co-workers (equation 4.3), there are a large number of different equations for the computation of mineral content from dual energy C.T. scans, some of them of considerable complexity. The derivations of two of these are described in the present work, and simulations have been performed to assess the sensitivity of the reported mineral content to changes in the fat content of the marrow. While the single energy figure can be in error by as much as $30 \%$ for patients with normal or low-normal mineral content, this error is greatly reduced when using the dual energy algorithm of LavalJeantet et al. The derivation of the Laval-Jeantet formula is purely empirical and gives an index of the mineral content rather than a direct measurement of it, and the fat parameter given by this formula has been shown in the present work to be not a true measure of the fat content. Other authors have since confirmed this, although it has been shown that the mineral content reported by the Laval-Jeantet formula correlates well with the corresponding figure given by the formula which is currently regarded as being the best.

The present author has derived a dual energy formula whose derivation starts from a somewhat simpler tissue model than is generally used, and which is expressed in terms of 
mass attenuation coefficients and not linear attenuation coefficients. The Wellington formula deals explicitly with the density of the marrow and ignores changes in chemical composition, an approach which can be justified since the mass attenuation coefficients of red and yellow marrow differ by as little as $0.1 \%$. Apparent differences due to fat express themselves primarily as changes in density. The computer simulations have shown that the Wellington formula shows the least sensitivity to changes in marrow composition and density, a fact which may be attributed to its derivation in terms of mass attenuation coefficients. Computations based on any tissue model will only be accurate in so far as the model truly reflects the tissue being studied. The use of a model expressed in terms of linear attenuation coefficients involves more assumptions since it requires a knowledge of both the chemical composition (effective atomic number) and the density of each component, whereas the use of mass attenuation coefficients only involves assumptions about composition. Further, since the mass attenuation coefficient of $\mathrm{K}_{2} \mathrm{HPO}_{4}$ closely matches the mass attenuation coefficient of bone mineral, the Wellington formula theoretically gives the true mineral concentration rather than simply an index which correlates with it. However it has been found that there is a systematic error in the values reported by the Wellington protocol since measurements of the $\mathrm{K}_{2} \mathrm{HPO}_{4}$ solution are made using a circular phantom which has subsequently been found to not truly represent the approximately elliptical shape of the abdomen. It is estimated that the errors due to this cause are $8 \%$ for single energy and $13 \%$ for the dual energy mineral measurement. Since a normal range has been established separately for the Wellington system these systematic errors do not affect the interpretation of patient measurements.

The Wellington dual energy mineral values have been found to correlate almost perfectly with the mineral parameter computed using the Laval-Jeantet formula, which in turn has been found to correlate well with ash weights of excised vertebrae. Phantom studies in the present work have indicated that the precision of the Wellington system is 2 $\%$ for single energy and $6 \%$ for the dual energy mineral measurement. Serial studies on a non-osteoporotic male gave figures of $2 \%$ and $7 \%$ respectively, figures which are in satisfactory agreement with other reported values. The patient doses in Wellington are comparable with those of other C.T. densitometry systems, but are many times greater than for DPA. However the dose is still at the lower end of the range of doses for diagnostic X-ray examinations and is small compared with the magnitudes of fluctuations in the level of the natural background radiation. The increase in cancer risk, if any, will perhaps be of the order of 1 in 100,000. Such a cancer would perhaps express itself some 20 years after exposure, during which period there is in New Zealand a 1 in 250 risk of being killed on the road. 


\section{CHAPTER FIVE: CLINICAL APPLICATIONS OF QUANTITATIVE C.T.}

\subsection{Computation of Electron Densities.*}

While much radiotherapy treatment planning in the past has been based on the assumption that the attenuation properties of the human body are the same as those of water, accurate planning of radiotherapy treatments requires a more detailed knowledge of the electron densities (electrons per $\mathrm{cc}$ ) at the site to be treated and in the overlying tissues. Knowledge of electron densities is particularly important when using electron therapies, as is now possible with the recently-installed CLINAC 2100 accelerator at Wellington Hospital. There have been a number of papers published on the use of C.T. images to compute electron densities [180 - 182]. A commonly quoted formula for computing electron densities from measured C.T. numbers is:

$$
r_{e} / r_{e}(w)=R_{\sigma} \cdot\left[1+\frac{H}{1000}\right]
$$

where $r_{e}$ is the tissue electron density (electrons per $\left.c c\right), r_{e}(w)$ is the volume electron density of water, $H$ is the measured C.T. number and $R_{\sigma}$ is the ratio of the cross section per electron of the tissue to that of water. A common approximation is to use set $\mathrm{R}_{\sigma}$ to unity for soft tissues and to use another value for all bone tissue. Relationships such as this ignore differences in calibration between C.T. scanners, and the determination of $\mathrm{R}_{\sigma}$ for tissues whose composition is not precisely known is difficult. In the present work the formalism of chapter two has been used to develop a very simple, yet precise, methodology. The linear attenuation coefficient is obtained from the measured C.T. number using equation 2.3, which requires that the constants $\mathrm{K}$ and $\mu_{\mathrm{W}}$ must first have been determined by a method such as that of Millner et al [60]. The electron density may then be obtained from [64]:

$$
\mu(\rho, \mathrm{Z}, \mathrm{E})=\rho \cdot \mathrm{n}_{\mathrm{g}} \cdot \sigma_{\mathrm{e}}(\mathrm{Z}, \mathrm{E}) \times 10^{-24}
$$

where $\mu$ is the linear X-ray attenuation coefficient in $\mathrm{cm}^{-1}, \rho$ is the density $(\mathrm{g} / \mathrm{cc}), \mathrm{n}_{\mathrm{g}}$ is the mass electron density (electrons per gram) and $\sigma_{\mathrm{e}}$ is the electronic cross section in barns per electron. The volume electron density (electrons per cc) is simply the product of the mass electron density and the physical density and is given by:

* I am grateful to Tony Johnson for suggesting this project and for providing several references. Tony also provided the tissue-mimicking materials, and many helpful discussions. 


$$
\rho \cdot \mathrm{n}_{\mathrm{g}}(\mathrm{Z})=\frac{\mu(\rho, \mathrm{Z}, \mathrm{E})}{\sigma_{\mathrm{e}}(\mathrm{Z}, \mathrm{E})} \times 10^{24}
$$

The quantity $\sigma_{\mathrm{e}}(\mathrm{Z}, \mathrm{E})$ may be determined for elements by dividing the atomic cross section (obtained from tables) by the atomic number. The electronic cross section for tissue can be obtained in a similar way, provided that the effective atomic number of the tissue is known, by interpolating between the values of the two nearest elements. If the effective atomic number is not known then it is necessary to use an average figure for the type of tissue involved, or to determine the effective atomic number using dual energy C.T. From equation (5.2) it is clear that if one takes the ratio of the linear attenuation coefficients at two different energies then the densities will cancel and the ratio will depend on the effective photon energies and the effective atomic numbers only:

$$
\frac{\mu(\rho, \mathrm{Z}, 71 \mathrm{keV})}{\mu(\rho, \mathrm{Z}, 59 \mathrm{keV})}=\frac{\sigma_{e}(\mathrm{Z}, 71 \mathrm{keV})}{\sigma_{e}(\mathrm{Z}, 59 \mathrm{keV})}
$$

Since the effective energies are known it is possible in principle to solve equation (5.3) for the effective atomic number, even although it may not be solvable by analytical means. Latchaw et al. [93] developed an empirical technique for solving a similar equation as part of their work on the differential diagnosis of brain tumours from the rates at which they take up high atomic number contrast agents. They plotted a 'calibration curve' of the ratio of linear attenuation coefficients at two energies versus atomic number. Such a curve is displayed in figure 5.1 (next page).

When the atomic number is an integer the electronic cross section may be obtained by dividing the atomic cross section by the atomic number, as previously described, or otherwise a second 'calibration curve', this time of $\sigma_{\mathrm{e}}(\mathrm{Z}, \mathrm{E})$ versus $\mathrm{Z}$, may be used to obtain the electronic cross section at a particular energy. An example of a curve of this type is given in figure 5.2 (overleaf).

The volume electron density may now be calculated from equation (5.2). Unless it is particularly desired to calculate the effective atomic number, the previous two steps could be combined into one using an empirical relationship between $\sigma_{\mathrm{e}}(\mathrm{Z}, \mathrm{E})$ and the ratio of attenuation coefficients at two energies. This relationship, which is plotted in figure 5.3, is a weak function of atomic number which preliminary investigations suggest can be fitted to a three parameter continued fraction expression over the atomic number range from 5 to 9 with an accuracy of better than $0.2 \%$. At energies of $59 \mathrm{keV}$ and 71 $\mathrm{keV}$ the relationship is as follows:

$$
\sigma_{e}(z, 71 \mathrm{keV})=\frac{0.2383+0.0476 \times R}{R-0.4333}
$$




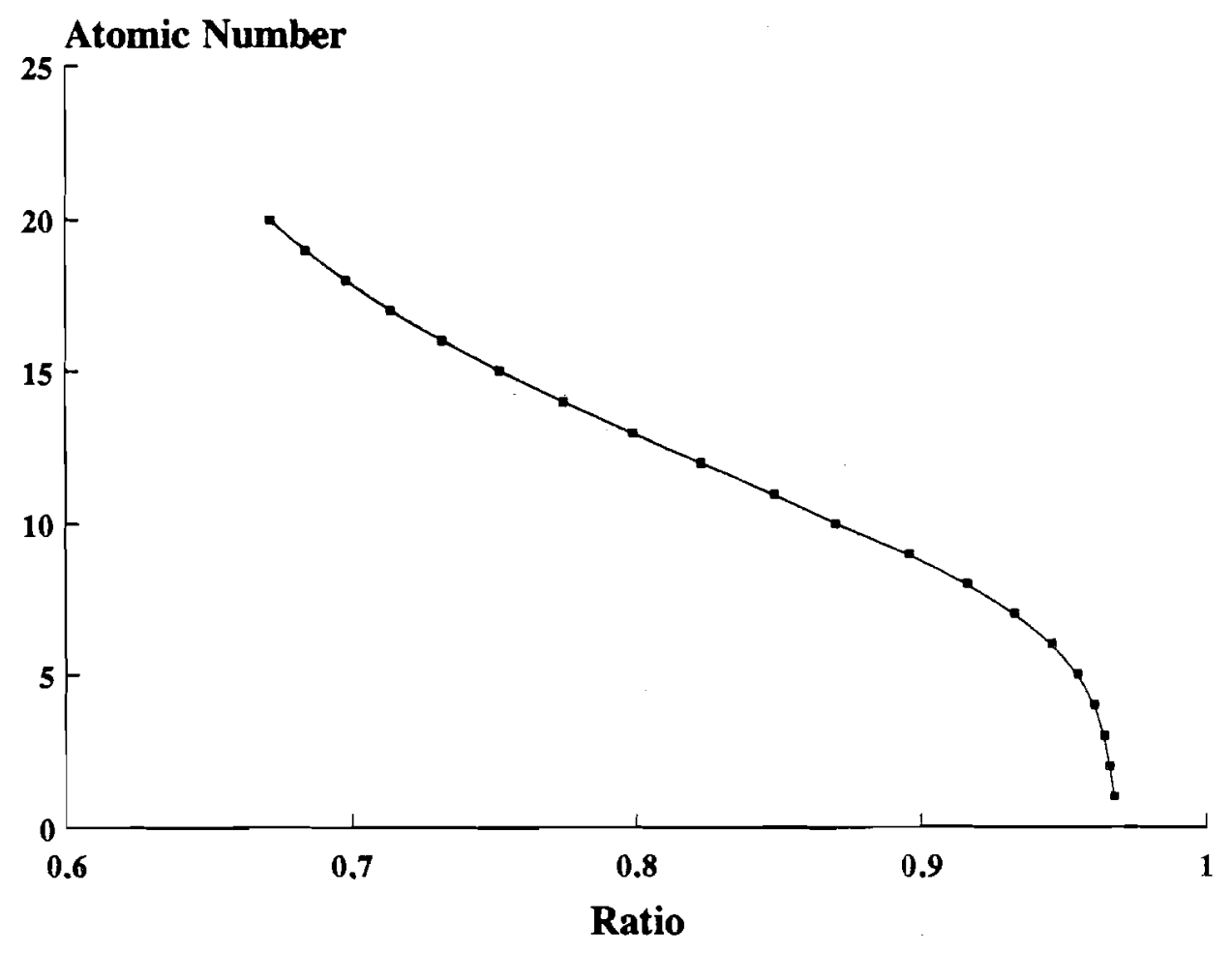

Figure 5.1: Calibration Curve of Atomic Number Versus Ratio of Attenuation Coefficients at Two Energies.

Plotted for photon energies of $71 \mathrm{keV}$ and $59 \mathrm{keV}$, corresponding to kilovoltage settings of $120 \mathrm{kVp}$ and $80 \mathrm{kVp}$ on the G.E. 8800 scanner. The attenuation coefficients are taken from Hubbell [58].

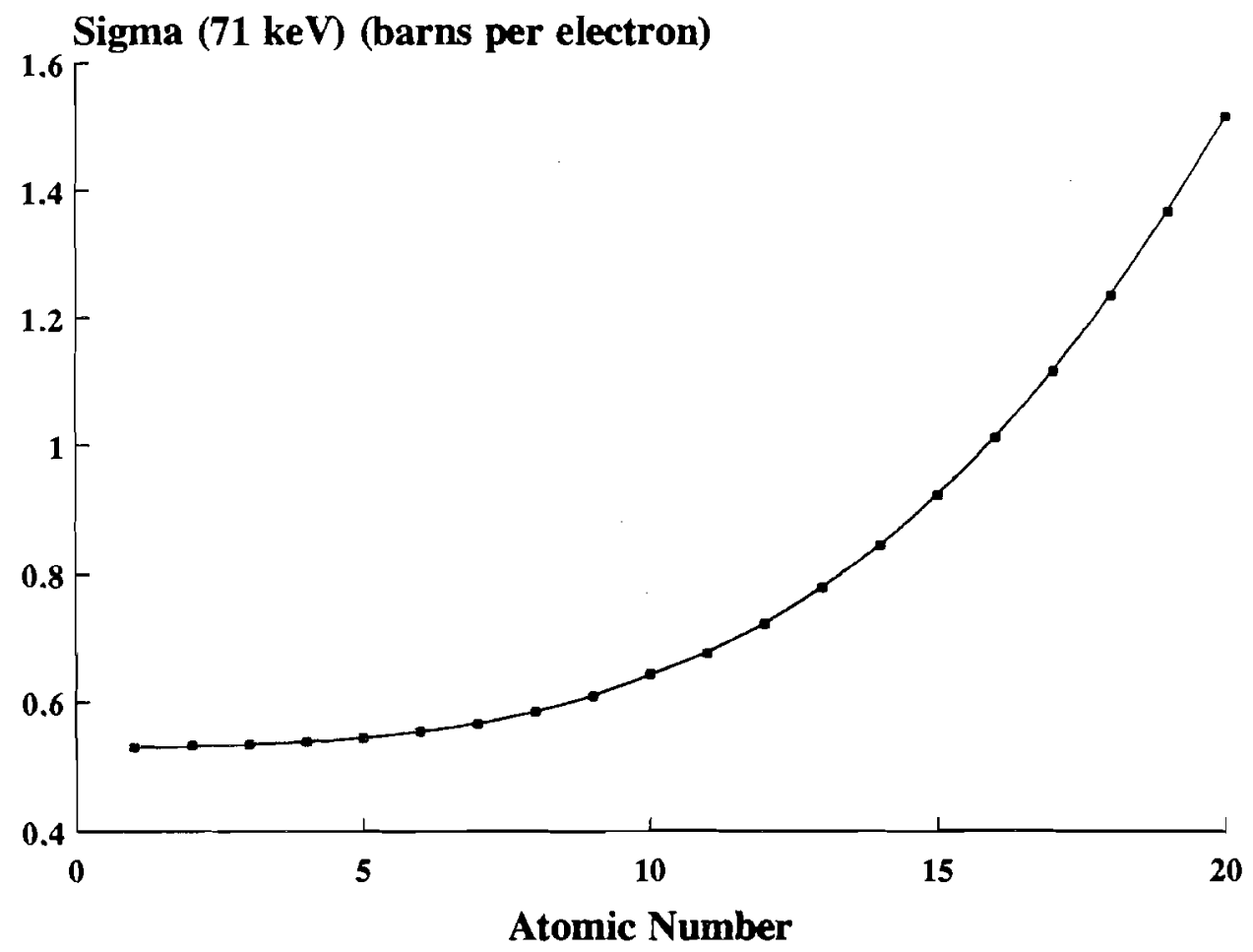

Figure 5.2: Calibration Curve of Electronic Cross Section Versus Atomic Number.

Plotted for a photon energy of $71 \mathrm{keV}$. The attenuation coefficient data is taken from Hubbell [58]. 


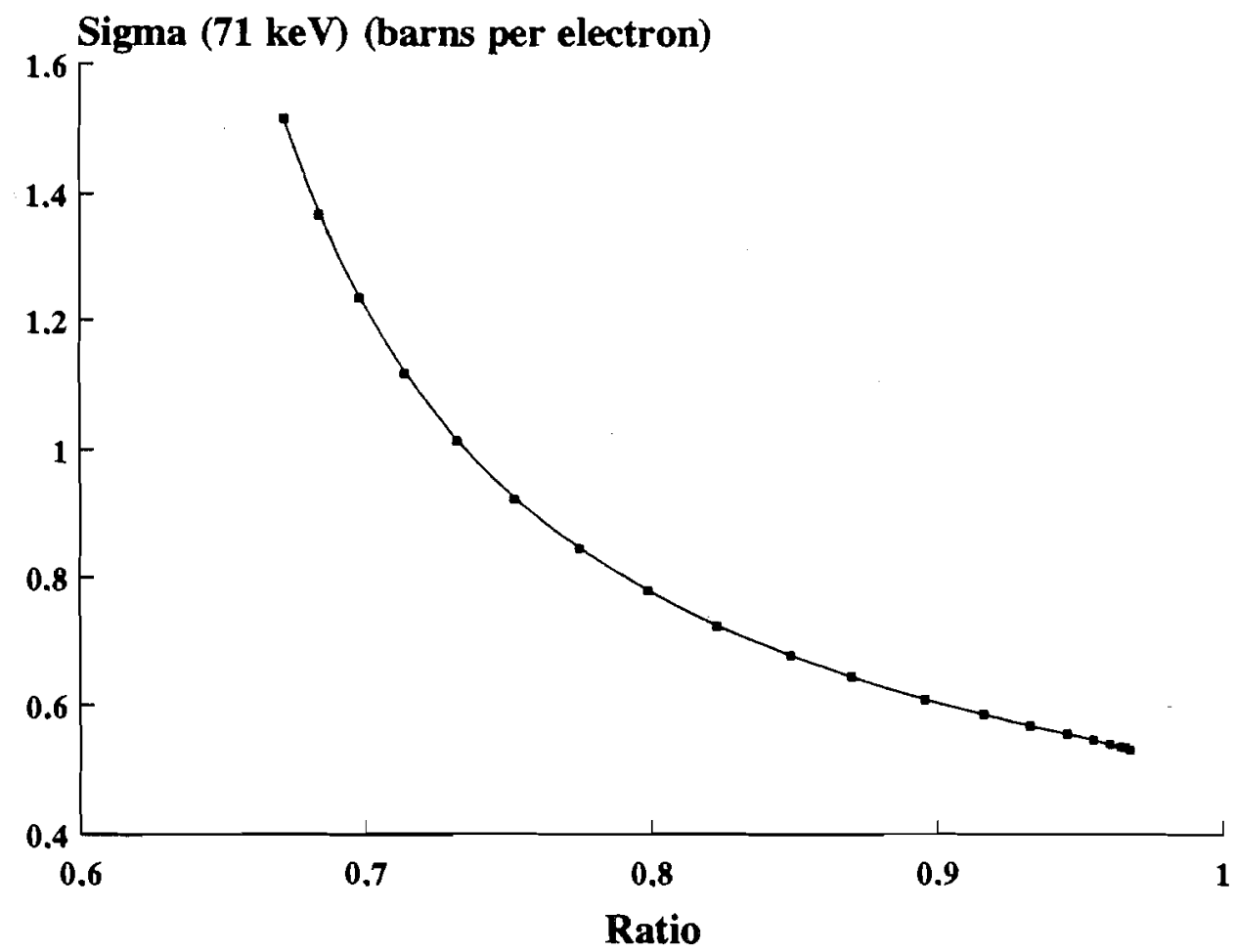

Figure 5.3: Calibration Curve of Electronic Cross Section Versus Ratio of Attenuation Coefficients.

Combines the calibrations of the previous two graphs into a single graph. The photon energies are 59 and $71 \mathrm{keV}$, and the attenuation data is that of Hubbell [58].

In equation (5.5) $\mathrm{R}$ is the ratio of the attenuation coefficients at the two energies. To verify the proposed method, the calibration of the C.T. scanner was first established at $120 \mathrm{kVp}$ and $80 \mathrm{kVp}$ by scanning the linearity section of the A.A.P.M. phantom twice at each energy, and averaging the results. The values obtained were:

Table 5.1: C.T. Measurements of the AAPM Linearity Inserts.

\begin{tabular}{||l|r|r|}
\hline \multirow{2}{*}{ Material } & \multicolumn{2}{|c|}{ Measured C.T. Numbers } \\
\cline { 2 - 3 } & $80 \mathrm{kVp}$ & $120 \mathrm{kVp}$ \\
\hline Polyethylene & -111.94 & -74.31 \\
Polystyrene & -59.64 & -27.81 \\
Water & 0.00 & 0.00 \\
Nylon & 89.88 & 103.17 \\
Lexan & 89.49 & 105.28 \\
Plexiglass & 122.74 & 131.64 \\
\hline
\end{tabular}

The effective energies at the two peak kilovoltages, along with the calibration constants (equation 2.3), were calculated from the above figures using the method of 
Millner et al. [60] (see section 2.5). Using linear attenuation coefficients interpolated from the data of Hubbell [58], the following values were obtained:

Table 5.2: Effective Energies and Regression Parameters.

\begin{tabular}{||c|c|c|c|c||}
\hline \hline $\mathrm{kVp}$ & $\begin{array}{c}\text { Effective } \\
\text { Energy }\end{array}$ & $\mathrm{K}$ & $\mu$ & $\mathrm{r}$ \\
\hline 120 & 71.2 & 1011.7 & 0.19125 & 0.99928 \\
80 & 58.8 & 1119.1 & 0.20743 & 0.99930 \\
\hline
\end{tabular}

As a check on the method, the above parameters were used to calculate the linear attenuation coefficients of the materials contained in the linearity section of the A.A.P.M. phantom from the measured C.T. numbers listed in table 5.1. The values obtained were then compared with values calculated from the published compositions and densities of these materials [60], which are listed in table 5.3.

Table 5.3: Published Compositions and Densities of the AAPM Linearity Inserts.

\begin{tabular}{||l|l|l|c||}
\hline Material & Abbrev. & Formula & $\begin{array}{r}\text { Density } \\
(\mathrm{g} / \mathrm{CC})\end{array}$ \\
\hline Polyethylene & $\mathrm{PE}$ & $\mathrm{C}_{2} \mathrm{H}_{4}$ & 0.94 \\
Polystyrene & $\mathrm{PS}$ & $\mathrm{C}_{8} \mathrm{H}_{8}$ & 1.05 \\
Nylon & $\mathrm{N}$ & $\mathrm{C}_{6} \mathrm{H}_{11} \mathrm{NO}$ & 1.15 \\
Lexan & $\mathrm{L}$ & $\mathrm{C}_{16} \mathrm{H}_{14} \mathrm{O}$ & 1.20 \\
Plexiglass & $\mathrm{P}$ & $\mathrm{C}_{5} \mathrm{H}_{8} \mathrm{O}_{2}$ & 1.19 \\
\hline
\end{tabular}

In table 5.4 the linear attenuation coefficients which were calculated from the C.T. measurements are compared with those computed from the compositions and densities using tables of attenuation coefficients [58]: 
Table 5.4: Measured and Calculated Attenuation Coefficients of the AAPM Inserts.

\begin{tabular}{|c|c|c|c|}
\hline Insert & $\begin{array}{l}\mu \text { (from CT) } \\
\text { measurements }\end{array}$ & $\begin{array}{c}\mu \\
\text { (from tables) }\end{array}$ & Difference \\
\hline
\end{tabular}

$120 \mathrm{kVp}$

\begin{tabular}{|l|l|r|r|}
\hline PE & 0.1772 & 0.1765 & $0.40 \%$ \\
PS & 0.1860 & 0.1868 & $-0.43 \%$ \\
N & 0.2108 & 0.2102 & $0.29 \%$ \\
L & 0.2112 & 0.2118 & $-0.28 \%$ \\
P & 0.2161 & 0.2158 & $0.14 \%$ \\
\hline
\end{tabular}

\section{$80 \mathrm{kVp}$}

\begin{tabular}{|l|l|r|r|}
\hline PE & 0.1867 & 0.1861 & $0.32 \%$ \\
PS & 0.1940 & 0.1973 & $-0.46 \%$ \\
N & 0.2241 & 0.2233 & $0.36 \%$ \\
L & 0.2240 & 0.2244 & $-0.28 \%$ \\
P & 0.2302 & 0.2303 & $-0.04 \%$ \\
\hline
\end{tabular}

From the relatively strong correlation between the errors at $71.2 \mathrm{keV}$ and at 58.8 $\mathrm{keV}$ it appears that the differences between the values calculated from C.T. numbers and those calculated from tables could be largely due to uncertainties in the published densities which are quoted to three significant figures only, and could therefore be in error by as much as $0.5 \%$. Effective atomic numbers of the A.A.P.M. inserts were calculated using the calibration method illustrated in figure 5.1. Values were calculated both from the C.T. measurements and from the values listed in tables of attenuation coefficients at the same energies. 
Table 5.5: Measured and Computed Effective Atomic Numbers of the AAPM Inserts.

\begin{tabular}{||c|c|c|}
\hline \multirow{2}{*}{ Material } & \multicolumn{2}{|c|}{ Effective atomic number } \\
\cline { 2 - 3 } & C.T. & tables \\
\hline PE & 5.631 & 5.703 \\
PS & 5.837 & 5.863 \\
N & 6.394 & 6.339 \\
L & 6.213 & 6.128 \\
P & 6.543 & 6.671 \\
\hline
\end{tabular}

Electron densities were calculated from the C.T. measurements using the relationship illustrated in figure 5.3 to obtain the electronic cross section, and then using equation (5.3) to obtain the volume electron density (electrons per cc).

Table 5.6: Measured and Computed Electron Densities for the AAPM Inserts.

\begin{tabular}{||c|c|c|c||}
\hline \multirow{2}{*}{ Material } & \multicolumn{2}{|c|}{ Electron density $\left(x 10^{23}\right)$} & \multirow{2}{*}{ Difference } \\
\cline { 2 - 3 } & from C.T. & from tables & \\
\hline & 3.225 & 3.229 & $-0.12 \%$ \\
PS & 3.373 & 3.400 & $-0.19 \%$ \\
N & 3.779 & 3.795 & $-0.42 \%$ \\
L & 3.801 & 3.836 & $-0.91 \%$ \\
P & 3.861 & 3.866 & $-0.13 \%$ \\
\hline
\end{tabular}

Other materials were scanned after the A.A.P.M. phantom, the results being tabulated below. With the exception of the Humanoid soft tissue substitute, the materials were all contained within a $20 \mathrm{~cm}$ diameter water phantom. Linear attenuation coefficients were calculated using equation 2.3 and the calibration figures obtained from scans of the A.A.P.M. phantom. 
Table 5.7: C.T. Measurements of Tissue Analogue Materials.

\begin{tabular}{||l|r|r|r|r||}
\hline \multirow{2}{*}{ Material } & \multicolumn{2}{|c|}{ C.T. numbers } & \multicolumn{2}{c|}{ Attenuation coeffs. } \\
\cline { 2 - 5 } & $120 \mathrm{kVp}$ & $80 \mathrm{kVp}$ & $120 \mathrm{kVp}$ & $80 \mathrm{kVp}$ \\
\hline \multirow{2}{*}{ AP6 } & -115.84 & -145.23 & 0.1692 & 0.1797 \\
LN10 & -733.57 & -798.05 & 0.0526 & 0.0595 \\
IB1 & 262.38 & 356.88 & 0.2409 & 0.2736 \\
HUMANOID ST & -21.48 & -29.96 & 0.1872 & 0.2019 \\
\hline
\end{tabular}

The above linear attenuation coefficients were then used to calculate effective atomic numbers for these materials, the values so obtained being compared with ones which were calculated from the known compositions and densities using tabulated attenuation coefficients. The two sets of results are compared in table 5.8. The compositions of materials AP6 (representing of adipose tissue) and IB1 (inner or cancellous bone) were obtained from [183]. The composition of LN10 (lung mimicking material) was taken to be: C (60.5\%), H (8.38\%), N (1.68\%), O (17.28\%), Mg (11.17\%), Si (0.84\%), Cl $(0.15 \%)$.

Table 5.8: Computed and Measured Effective Atomic Numbers for Tissue Analogues.

\begin{tabular}{||c|c|c||}
\hline \multirow{2}{*}{ Material } & \multicolumn{2}{|c|}{ Effective atomic number } \\
\cline { 2 - 3 } & C.T. & tables \\
\hline AP6 & 6.5860 & 6.5600 \\
LN10 & 9.5020 & 7.7630 \\
IB3 & 9.6270 & 9.7900 \\
ST & 7.3380 & \\
\hline
\end{tabular}

'ST' = Humanoid soft tissue equivalent.

The values are approximately correct except for the lung simulating material LN10, where the calculated atomic number is in error by about 2 units. The electron densities were calculated from the C.T. measurements using the same protocol as previously, and are compared with values calculated from the known compositions as follows: 
Table 5.9: Electron Densities of Tissue Analogue Materials.

\begin{tabular}{||c|c|c|c|}
\hline \multirow{2}{*}{ Material } & \multicolumn{2}{|c|}{ Electron density (x 1023) } & \multirow{2}{*}{$\begin{array}{c}\text { Difference } \\
(\%)\end{array}$} \\
\cline { 2 - 3 } & from C.T. & from tables & 1.0 \\
AP6 & 3.022 & 2.992 & -9.8 \\
LN10 & 0.845 & 0.937 & -2.7 \\
IB1 & 3.843 & 3.742 & \\
ST & 3.274 & & \\
\hline
\end{tabular}

The agreement is satisfactory except for material LN10. Investigations have shown the source of the error to be the estimated attenuation coefficient at $80 \mathrm{kVp}$, and this error appears to be caused by the use of a nonstandard relationship between C.T. numbers and attenuation coefficients. Instead of using equation (2.3), the software of the G.E. 8800 scanner is quoted as using the following relationship [142]:

$$
\mathrm{H}=\frac{1000}{\mu_{\mathrm{W} 1}} \cdot\left[\mu-\mu_{\mathrm{W} 2}\right]
$$

where $\mu_{\mathrm{w} 1}$ is quoted as being 0.193 . However it is known that this factor has changed since reference [142] was published. Comparison with regular quality assurance measurements suggests a value of 0.190 . This value is fixed and is unaffected by changes in C.T. calibration (including changes in $\mathrm{kVp}$ ). The appropriate relationship between the measured C.T. number and the linear attenuation coefficient is therefore:

$$
\mathrm{H}=5263.16 \cdot\left[\mu-\mu_{\mathrm{W} 2}\right]
$$

where $\mu_{\mathrm{w} 2}$ is found to be $0.1915 \mathrm{~cm}^{-1}$ at $120 \mathrm{kVp}$ and $0.2083 \mathrm{~cm}^{-1}$ at $80 \mathrm{kVp}$. The calculated effective atomic numbers and electron densities then become:

Table 5.10: Electron Densities of Tissue Analogues.

\begin{tabular}{||c|c|c|c||}
\hline \multirow{2}{*}{ Material } & \multicolumn{2}{|c||}{ Electron density $\left(\times 10^{23}\right)$} & \multirow{2}{*}{$\begin{array}{c}\text { Difference } \\
\left(\frac{8}{6}\right)\end{array}$} \\
\cline { 2 - 3 } & from C.T. & from tables & 1.4 \\
AP6 & 3.035 & 2.992 & -3.8 \\
LN10 & 0.901 & 0.937 & -2.7 \\
IB1 & 3.843 & 3.742 & \\
ST & 3.278 & & \\
\hline
\end{tabular}


The agreement is now satisfactory. The effective atomic number for LN10 becomes 7.72, and that for IB1 is now 9.73. The values for the other materials are not significantly different as their C.T. numbers lie in the range for which equation (2.3) is sufficiently accurate. The errors caused by using equation (2.3) arise because when this equation is fitted to C.T. measurements both the slope and the intercept are adjusted for best fit, whereas the C.T. scanner software appears to use equation (5.7) and fits this equation to the data by adjusting the intercept only. These measurements have detected this problem because the attenuation coefficient of LN10 is only $30 \%$ of that of water, which is used as the reference material. For the other materials listed above, including IB3 which represents cancellous bone tissue, the error in the calculated linear attenuation coefficient arising from the use of equation (2.3) is less than $1 \%$.

Measurements were also made of the solid bone substitute SB3. These figures were not included with those above since this material was not imaged well, and because the effective energies calculated from the A.A.P.M. inserts would not strictly apply for bone. However the values calculated from the C.T. measurements are not drastically in error: the calculated effective atomic number of SB3 was 12.1 and the volume electron density was $6.1 \times 10^{23}$ electrons per cc. The electron density calculated from C.T. measurements is $7.7 \%$ higher than the value calculated from its composition and density.

The effective atomic number of most soft tissues lies in the range between 6 and 8 . Since the cross section per electron at atomic number 8 is only $5.6 \%$ greater than that at atomic number 6 , it would seem feasible to perform only a single energy examination for soft tissues and assume some average figure for the cross section per electron when using equation (5.3). Assuming an average atomic number of 7 (electronic cross section 0.5657 barns per electron), the following electron densities are obtained from the $120 \mathrm{kVp}$ C.T. measurements alone: 
Table 5.11: Electron Densities Calculated From Single Energy Measurements.

\begin{tabular}{||l|c|c||}
\hline Material & $\begin{array}{c}\text { Electron } \\
\text { density } \\
\left(x 10^{23}\right)\end{array}$ & $\begin{array}{c}\text { Error } \\
(\%)\end{array}$ \\
\hline PE & 3.14 & -2.9 \\
PS & 3.29 & -3.2 \\
N & 3.73 & -1.7 \\
L & 3.74 & -2.5 \\
P & 3.83 & -1.0 \\
AP6 & 3.00 & 0.1 \\
LN10 & 0.92 & -1.7 \\
IB1 & 4.27 & 14.0 \\
SB3 & 8.20 & 43.8 \\
ST & 3.31 & 1.1 \\
\hline
\end{tabular}

Apart from the bone materials, the errors are somewhat less than $5 \%$. Computer programs have been written to perform many of the calculations described in this section, copies of which are available on request from the author.

The measurements described in this section suggest that dual energy C.T. techniques may be used to calculate electron densities to within $5 \%$ for soft tissues, to within $3 \%$ for cancellous bone and to within $8 \%$ for cortical bone. Single energy C.T. techniques have comparable accuracy for soft tissues but in their present form do not give sufficient accuracy for bone.

\subsection{Normal Range for Bone Mineral Measurements.}

Some 34 volunteer women were scanned to determine the normal range for the mineral content of New Zealand females in the age range 20 to 40 years. This group was to be used as controls for the anorexia nervosa study described in the next section. One subject was removed from the control group as she was part Polynesian and would therefore be expected to have a somewhat higher mineral content than the others. A further two were removed from the control group as they were subsequently found to have had some history of eating disorders. The average age of the remaining 31 controls was 30.9 years, with a standard deviation of 5.8 years. The ages of the controls covered the full range from 20 to 40 years. The subjects were scanned according to the protocol of 
appendix F, and estimates of the mineral concentration Cmin and density RHO were calculated from equation (4.11) using the software listed in appendix G. Single energy mineral estimates, $\mathrm{Cb} 80$ and $\mathrm{Cb120}$, were calculated from measurements made at 80 and $120 \mathrm{kVp}$ using equation (4.3). The figures for the three vertebrae were averaged to give a single figure for each subject. Histograms illustrating the distributions of Cmin and RHO are given in figure 5.4, and those of $\mathrm{Cb} 80$ and $\mathrm{Cb} 120$ are given in figure 5.5. The distribution of Cmin is somewhat skewed to the right, but a chi-squared test showed that deviations of this magnitude or more from a Gaussian distribution with the same mean and standard deviation can be expected in some $75 \%$ of cases. The distributions of $\mathrm{Cb} 80$ and $\mathrm{Cb} 120$ are also skewed to the right, the degree of skew being greater in the distribution of $\mathrm{Cb80}$. A chi-squared test on the distribution of $\mathrm{Cb} 80$ showed that deviations of equal or greater magnitude can be expected in samples of this size drawn from the parent population in $82 \%$ of cases, assuming again that the parent population is Gaussian. It is clear that the sample sizes are not sufficient to draw conclusions on the types of distributions from which they are drawn, and so non-parametric or distribution free statistical methods have been used in this chapter to confirm results obtained by conventional statistical means. A discussion of the statistical methods used in the present work is given in appendix I. The parameters of the distributions of the bone measurements are given in table 5.12.

Table 5.12: Distribution Parameters for Bone Measurements.

\begin{tabular}{||c|c|c|c||}
\hline $\begin{array}{c}\text { Bone } \\
\text { Measurement }\end{array}$ & Mean & $\begin{array}{c}\text { Std Error } \\
\text { of Mean }\end{array}$ & $\begin{array}{c}\text { Standard } \\
\text { Deviation }\end{array}$ \\
\hline Cb80 & 148.5 & 4.3 & 24.0 \\
Cb120 & 147.5 & 4.8 & 26.7 \\
Cmin & 196.2 & 5.0 & 28.0 \\
RHO & 1.092 & 0.007 & 0.041 \\
\hline
\end{tabular}

There is no significant difference between the two single energy means, even although the low energy estimate, $\mathrm{Cb} 80$, is more sensitive to the mineral content per se; and the high energy measurement, $\mathrm{Cb120}$, has greater sensitivity to changes in total density. The mean value for RHO compares well with a value of $1.08 \mathrm{~g} / \mathrm{cc}$ given for cancellous bone in I.C.R.P. Reference Man [116]. The standard deviation of Cmin is comparable with those of the single energy mineral estimates, a somewhat surprising result since its calculation involves the difference between high and low energy measurements and the proportional error would be expected to increase due to the 'small differences between large numbers' syndrome. 

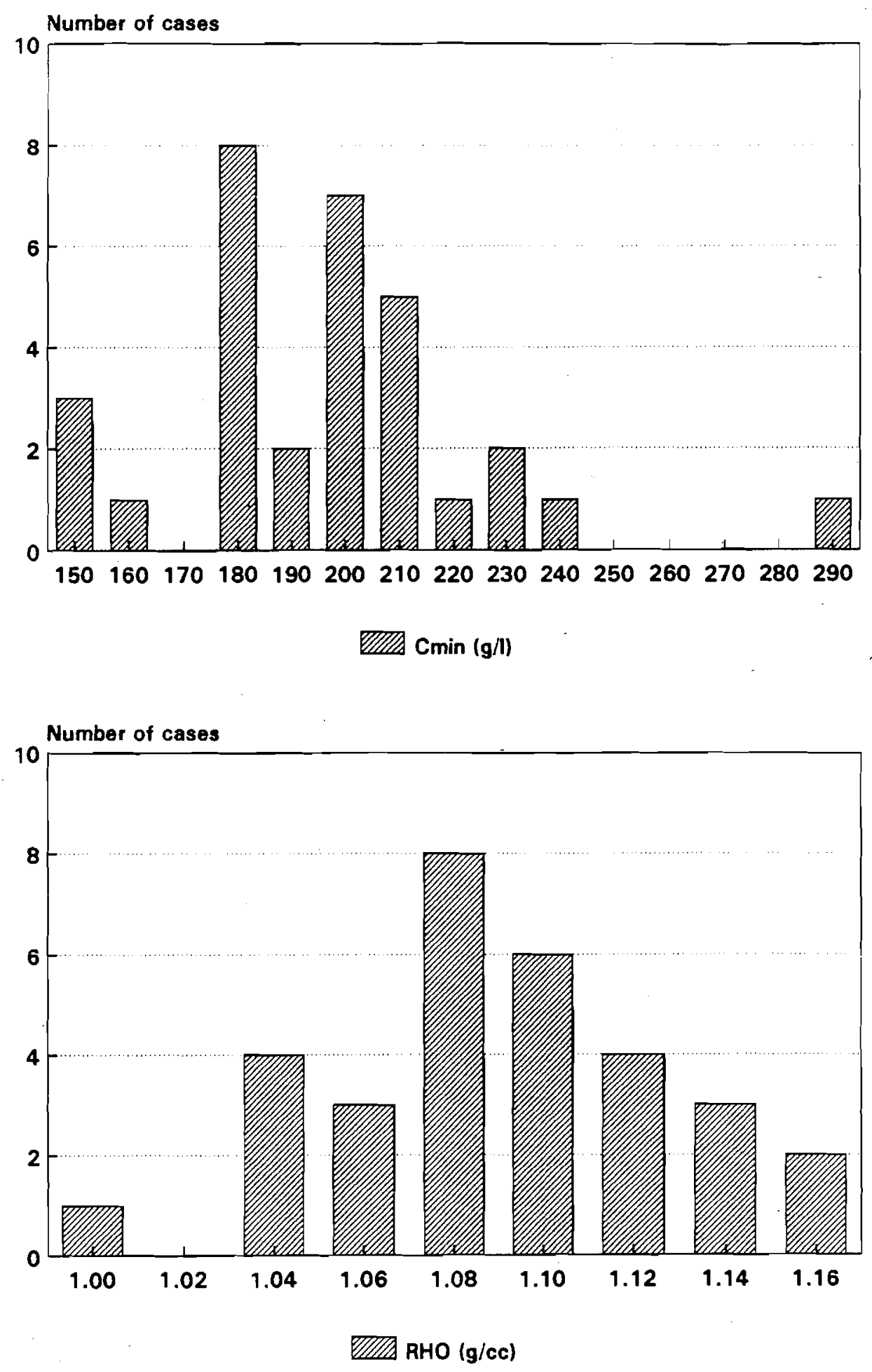

Figure 5.4: Distributions of the Dual Energy Measurements of the Control Group. The figures plotted are the means for the three vertebrae scanned on each subject. 

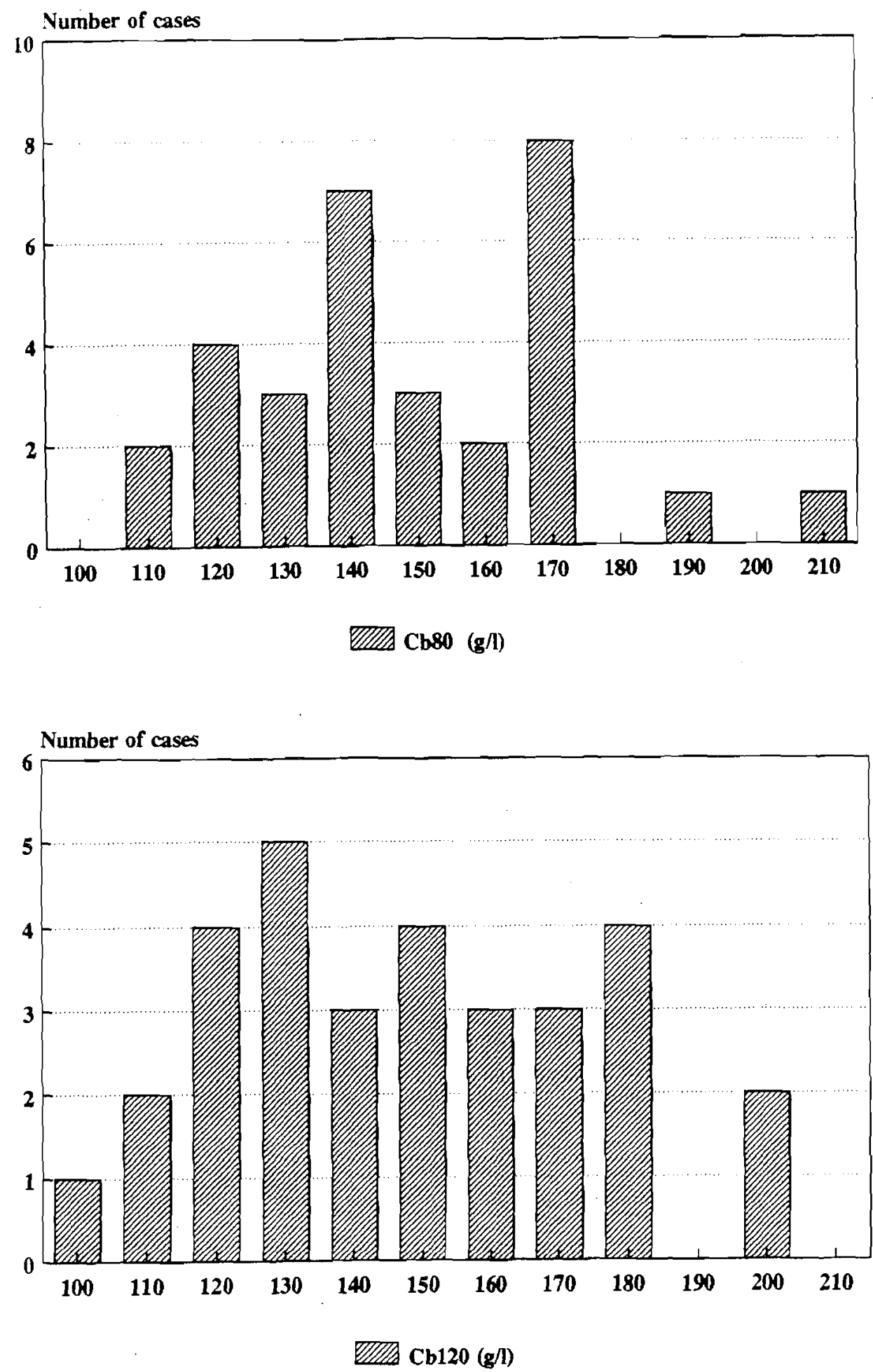

Figure 5.5: Distributions of the Single Energy Measurements of the Control Group. The figures plotted are the means for the three vertebrae scanned on each subject.

A number of authors have published graphs of the variation of mean bone mineral content with age for the normal population as measured by single energy computed tomography. The populations studied are from San Francisco (Genant $[184,185])$, New York (Firooznia [186]), Sydney (Marrel1 [187]) and Cardiff (Compston [188]). The data 
from these studies is displayed in figure 5.6, with the mean value of $\mathrm{Cb} 80$ for the Wellington control group superimposed. The Wellington value is close to that reported by the Sydney group, and is in reasonable agreement with the values of Compston and to a slightly lesser extent with the values measured in New York by Firooznia et al.

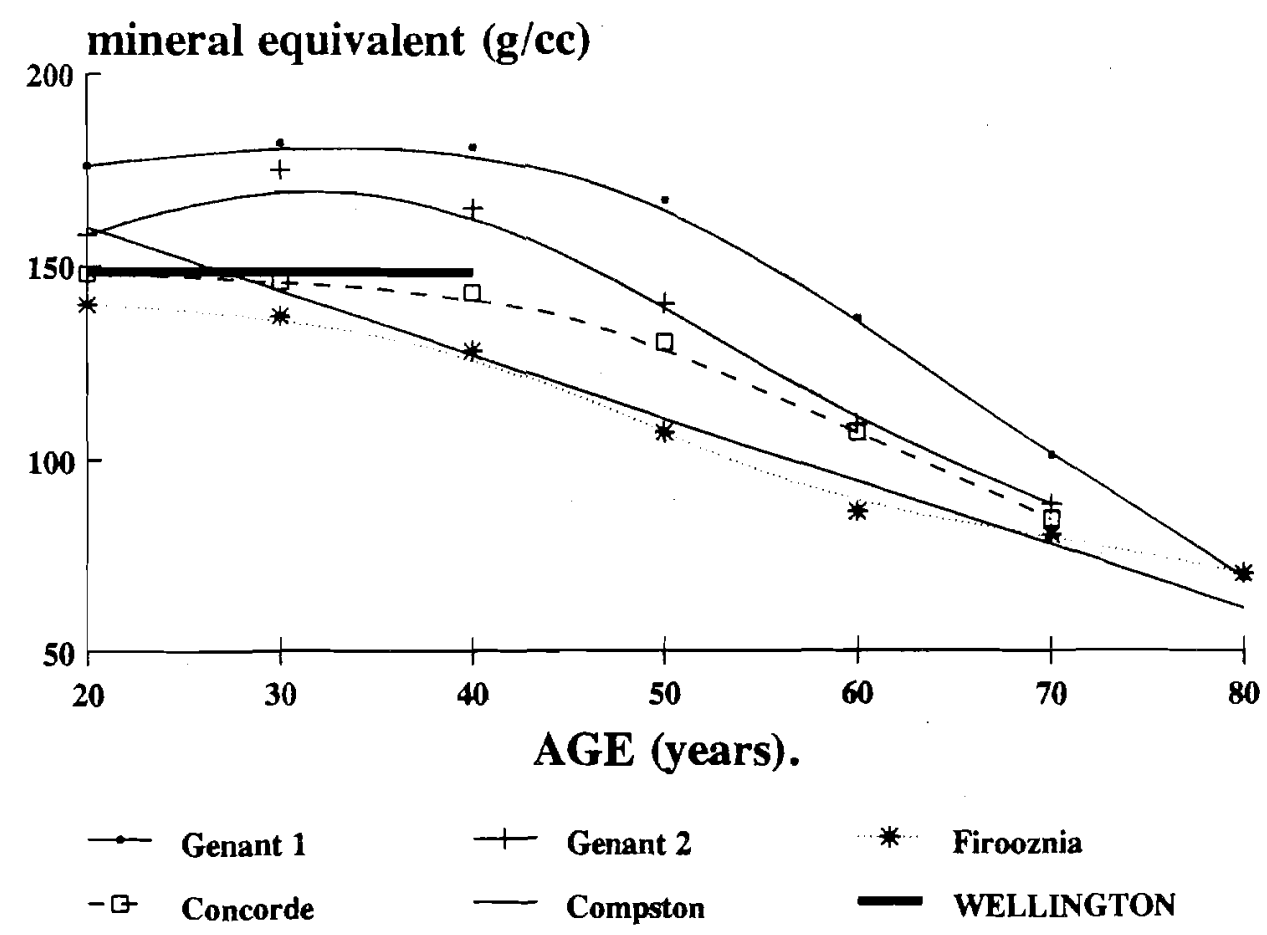

Figure 5.6: Published Single Energy C.T. Measurements of the Normal Population.

The standard deviations for the single energy measurements in Wellington are lower than the figures for California (33) and New York (29), but somewhat greater than the value reported by the Sydney group (18) [185 - 187]. However the Sydney figure was computed for a sample of only 15 subjects between 20 and 40 years of age and is not significantly different from the Wellington figure when allowance is made for sampling uncertainties.

The degree of correlation between the mineral parameters has been computed by the Spearman rank test (see appendix I) and is presented in table 5.13. 
Table 5.13: Correlation Between Bone Measurements for Controls. Spearman Rank Correlation Coefficients.

\begin{tabular}{||l|l|l|r||}
\hline & Cb120 & Cmin & RHO \\
\hline $\operatorname{Cb80}$ & 0.98 & 0.45 & 0.73 \\
$\operatorname{Cb} 120$ & & 0.34 & 0.80 \\
$\operatorname{Cmin}$ & & & -0.20 \\
\hline
\end{tabular}

Probabilities that the Correlations are Due to Chance.

\begin{tabular}{|l|l|l|l||}
\hline & Cb120 & Cmin & RHO \\
\hline & & & \\
$\operatorname{Cb80}$ & $<10^{-7}$ & 0.01 & $10^{-4}$ \\
$\operatorname{Cb} 120$ & & 0.06 & $10^{-5}$ \\
$\operatorname{Cmin}$ & & & 0.27 \\
\hline
\end{tabular}

There is high correlation between the two single energy mineral measurements, suggesting that the exact peak kilovoltage used has little effect on the value obtained in a single energy examination. The low energy mineral estimate, $\mathrm{Cb} 80$, has stronger atomic number dependence due to increased photoelectric effect at the lower energy and therefore correlates more strongly with the dual energy mineral estimate than does $\mathrm{Cb} 120$. On the other hand there is stronger correlation between $\mathrm{Cb} 120$ and RHO than there is between $\mathrm{Cb} 80$ and $\mathrm{RHO}$. The apparent negative correlation between $\mathrm{Cmin}$ and $\mathrm{RHO}$ is not statistically significant. The moderate degree of correlation between each of $\mathrm{Cb} 80$ and $\mathrm{Cb} 120$ and the dual energy mineral measurement, $\mathrm{Cmin}$, may seem surprising since the single energy mineral figures are often felt to be a measure of the mineral concentration in the same sense as Cmin, but with less accuracy. This situation is addressed in section 4.4. The single energy measurements are effectively scaled versions of the linear attenuation coefficients of the cancellous tissue. In figure 4.4 (page 107) it can be seen that the bone mineral typically contributes only $26 \%$ to the total linear attenuation coefficient of cancellous bone tissue: any change in the remaining $74 \%$ will be falsely reported by the single energy technique as changes in the mineral component. The fact that the correlation between the single and dual energy mineral estimates is only moderate confirms that changes in the marrow component can confuse the interpretation of single energy measurements.

Bone mineral content is known to decrease with age, although the main losses occur after the age of forty. Density can also be expected to decrease due to the decrease in mineralisation and the conversion of red marrow to yellow marrow with aging (figure 4.1). Correlations between bone measurements and age are given in table 5.14. 
Table 5.14: Correlations Between Bone Measurements and Age.

\begin{tabular}{|l|c|c||}
\hline $\begin{array}{l}\text { Bone } \\
\text { Measurement }\end{array}$ & $r_{\mathbf{s}}$ & $\mathrm{p}$ \\
\hline Cb80 & -0.30 & 0.10 \\
$\operatorname{Cb} 20$ & -0.34 & 0.06 \\
Cmin & -0.15 & 0.41 \\
RHO & -0.23 & 0.21 \\
\hline
\end{tabular}

Although all correlations were negative, those of the dual energy parameters Cmin and RHO are not statistically significant while those of $\mathrm{Cb} 80$ and $\mathrm{Cb} 120$ are possibly significant but do not achieve the $5 \%$ level. Linear regression gives rates of decrease of $1.3 \mathrm{~g} / 1$ per year for $\mathrm{Cb} 80$ and $1.6 \mathrm{~g} / 1$ per year for $\mathrm{Cb} 120$. The standard errors of the regression parameters are $\pm 0.7 \mathrm{~g} / 1$ and $\pm 0.8 \mathrm{~g} / 1$ respectively. Data published by Compston [188] correspond to an annual rate of loss of $1.8 \mathrm{~g} / 1$ per year for single energy measurements taken at $120 \mathrm{kVp}$.

In reviewing the bone measurements it has been very noticeable that not all vertebrae in the same subject give the same measurements. The differences can be quite small in some subjects and very large in others, implying that in some cases mineral loss is uniform while in others the loss may occur principally in only one of the three vertebrae measured. An alternative explanation could be that the pattern of demineralisation in each vertebra is patchy, and that the differences observed between vertebrae could represent sampling variations determined by the precise location of the measurement volume with respect to the locations of areas of demineralisation within each vertebra. This effect has been quantified in the present work by calculating the coefficients of variation of the various bone measurements for each subject. Uniform mineral loss would result in a small coefficient of variation being measured whereas uneven demineralisation would be characterised by a larger coefficient of variation for that subject. The distributions of the coefficients of variation of Cmin and RHO for the control group are given in figure 5.7, and the corresponding distributions for $\mathrm{Cb} 80$ and $\mathrm{Cb} 120$ are given in figure 5.8. Average coefficients of variation for the whole group are given in table 5.15. These values may be used to estimate the likely error due to random fluctuations of the mean value of the three vertebrae measured on each subject. The standard error of the mean of three values may be obtained from the coefficient of variation by dividing the C.V. by the square root of 3 . These are also listed in table 5.15 . 


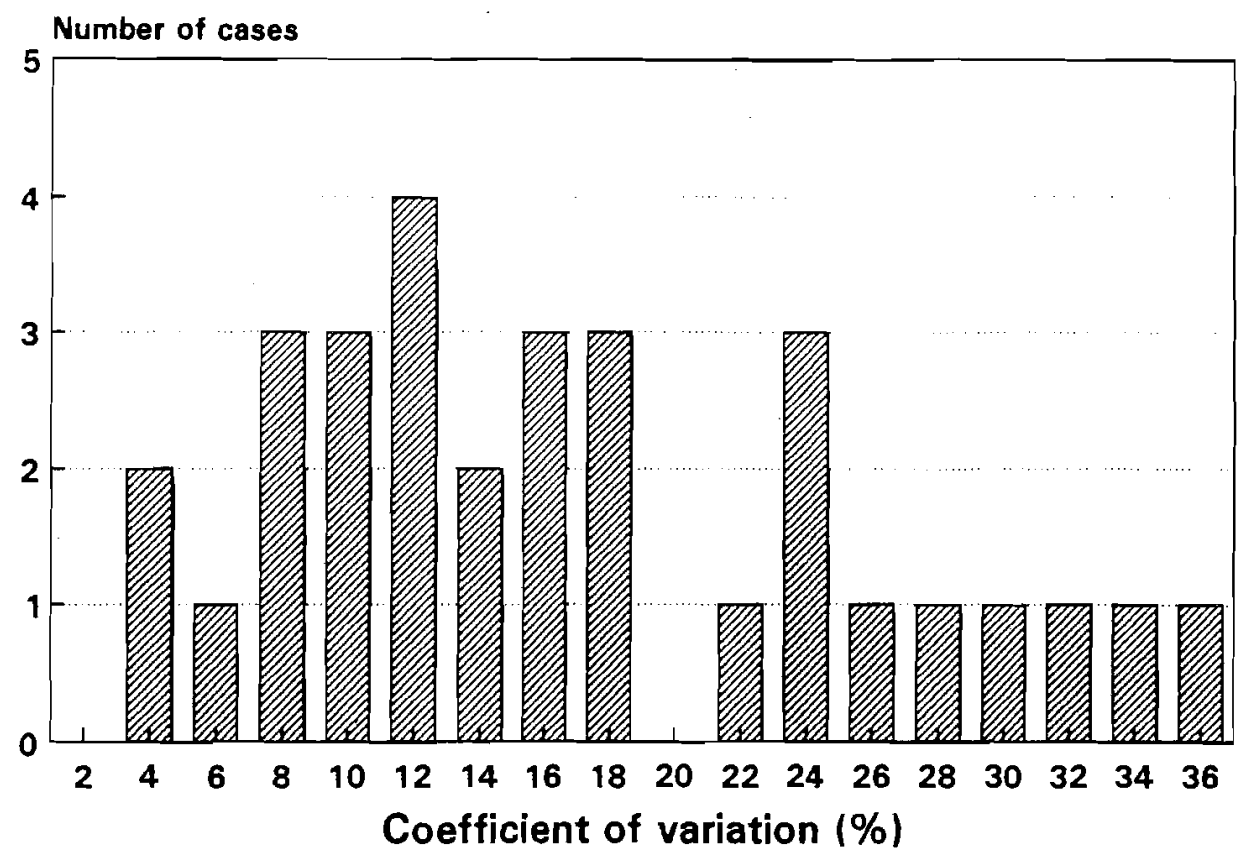

प10 $C_{\text {min }}$

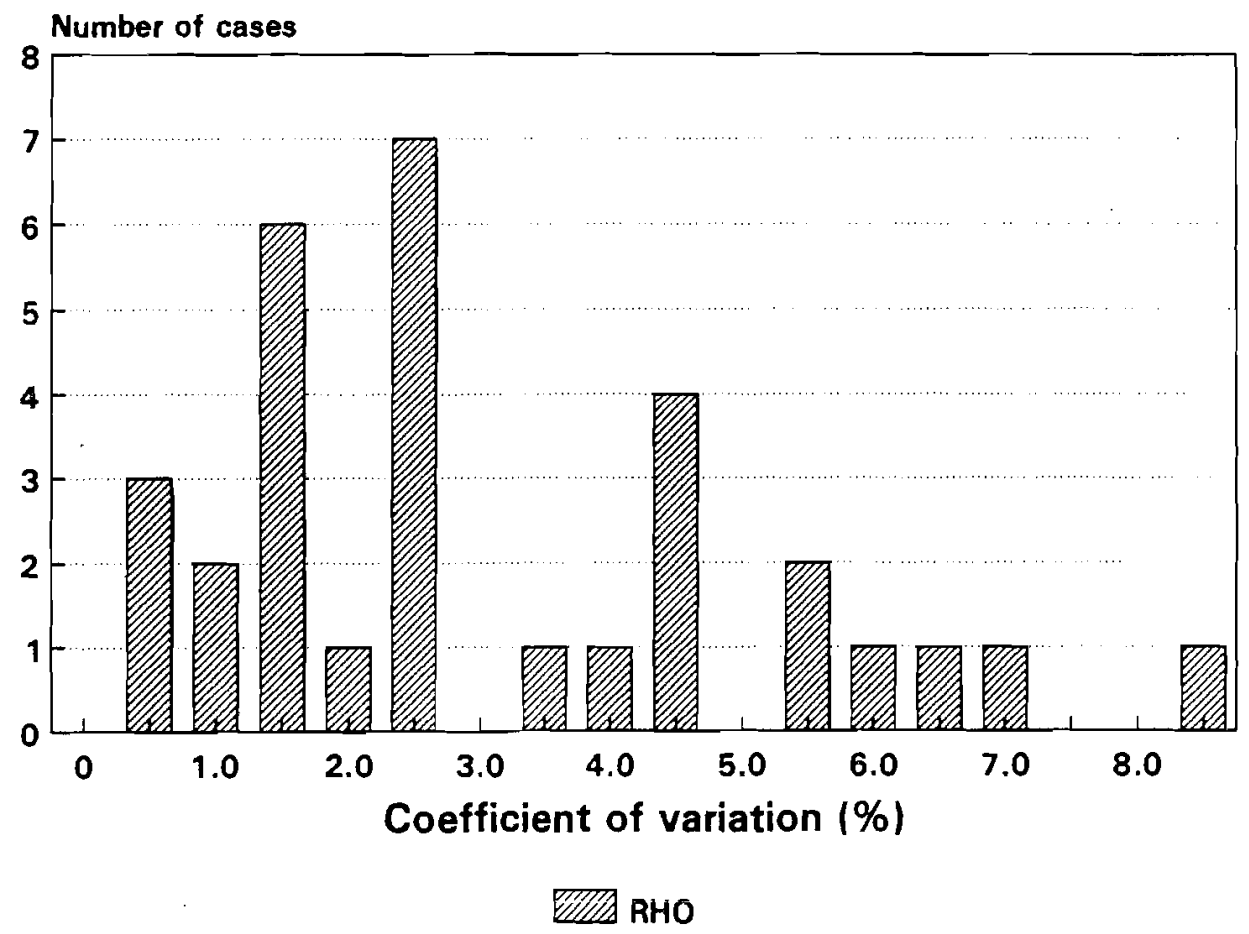

Figure 5.7: Distributions of the Coefficients of Variation of the Dual Energy Measurements of the Control Group.

The figures plotted are the coefficients of variation for the three vertebrae scanned on each subject. 

Table 5.15: Coefficients of Variation of Bone Measurements.

\begin{tabular}{||l|c|c||}
\hline $\begin{array}{l}\text { Bone } \\
\text { Measurement }\end{array}$ & $\begin{array}{c}\text { Average } \\
\text { Coefficient } \\
\text { of variation } \\
\left(\frac{\circ}{6}\right)\end{array}$ & $\begin{array}{c}\text { Std error } \\
\text { of mean } \\
(\%)\end{array}$ \\
\hline Cb80 & 5.1 & 3.0 \\
Cb120 & 5.0 & 2.9 \\
Cmin & 17.0 & 9.8 \\
RHO & 3.1 & 1.8 \\
\hline
\end{tabular}

Although the percentage C.V. is lowest for the parameter RHO, it should be noted that the normal range for this parameter is also small. Attempts have been made to correlate the C.V.'s of the bone measurements with the age of the subject, and with the bone measurements themselves. An increase in C.V. with aging would indicate that the distribution of bone mineral in the spinal column becomes less uniform with aging. Any tendency for localised mineral loss would manifest itself as a negative correlation between the C.V. and the bone measurement. Spearman rank correlation coefficients of the C.V.'s with age are given in table 5.16.

Table 5.16: Correlations of C.V.'s with Age for the Control Group.

\begin{tabular}{||c|c|c||}
\hline C.V. of ... & $\mathrm{r}_{\mathbf{S}}$ & $\mathrm{p}$ \\
\hline Cb80 & 0.01 & 0.95 \\
Cb120 & -0.21 & 0.26 \\
Cmin & -0.40 & 0.03 \\
RHO & -0.48 & 0.01 \\
\hline
\end{tabular}

There are significant negative correlations between the coefficients of variation of Cmin and age, and between the coefficients of variation of RHO and age, suggesting that the vertebrae become more uniform in mineral distribution and density as the subject approaches the age of 40 . 
Table 5.17: Correlations of C.V.'s with Mineral Measurements.

\begin{tabular}{|l|r|l|}
\hline c.v. of $\ldots$ & $r_{\mathbf{s}}$ & $\mathrm{p}$ \\
\hline Cb80 & 0.00 & 0.98 \\
Cb120 & -0.08 & 0.68 \\
Cmin & 0.43 & 0.02 \\
RHO & -0.01 & 0.95 \\
\hline
\end{tabular}

The only significant correlation is between the coefficient of variation of Cmin and the average value of $\mathrm{Cmin}$ for the three vertebrae measured on each subject, suggesting that the pattern of normal bone mineral loss occurs in such a way as to make the distribution of mineral more uniform.

Thus far the analysis has been on the means of readings for the three vertebrae measured on each subject. It is reasonable to ask if there are significant differences between the measurements made on the individual vertebrae. For example, it is known that the cross sectional area of L4 is greater than that of L2 or L3, presumably because it must support a slightly larger weight. A similar comment could perhaps apply to the mineral content. Mean values for each of the three vertebrae for the 31 controls are presented in table 5.18 .

There is no significant difference between the means for each of the three vertebrae on the basis of the single energy mineral estimates, $\mathrm{Cb} 80$ and $\mathrm{Cb} 120$. On the basis of the Cmin figures there is no significant difference between the means for L3 and L4, but the average figure for L2 is $14 \%$ lower than that of L3, and $11 \%$ lower than the mean value for L4. A Student's $t$ test gave probabilities of 0.02 and 0.01 respectively that these differences were due to chance. This result was checked using non parametric statistics. A Wilcoxon test (see appendix I) gave $Z$ values of -2.27 and -2.46 respectively, corresponding to the same probabilities as above. The mean RHO value for L2 was $3 \%$ higher than the mean value for L3, with probability values of 0.02 (Student) and 0.04 (Wilcoxon). There are no other significant differences between the mean RHO values of the different vertebrae.

To facilitate the design of a phantom to be used for the scanning of excised vertebrae (to be discussed in chapter six), measurements of abdominal cross sections have been taken from the C.T. films of the control group. Measurements have also been taken to determine the position of the vertebral bodies within the abdomen. At the time this survey was carried out the films of only 25 of the control group were available. The films are of cross sections through the abdomen at the level of the mid-point of the vertebral body (see figure 4.3). Since the spine mineral densitometry protocol requires 
Table 5.18: Mean Bone Measurements for $L 2, L 3 \&$ LA for Controls.

\begin{tabular}{|l|l|l|l|}
\hline Vertebra & Mean & S.D. & $\begin{array}{l}\text { std error } \\
\text { of mean }\end{array}$ \\
\hline
\end{tabular}

\section{Cb80}

\begin{tabular}{|l|l|l|l|}
\hline L2 & 146.84 & 23.27 & 4.18 \\
L3 & 148.39 & 24.87 & 4.47 \\
L4 & 151.05 & 26.39 & 4.74 \\
\hline
\end{tabular}

\section{Cb120}

\begin{tabular}{|l|l|l|l|}
\hline L2 & 150.06 & 27.88 & 5.01 \\
L3 & 144.75 & 24.61 & 4.42 \\
L4 & 149.38 & 29.11 & 5.23 \\
\hline
\end{tabular}

Cmin

\begin{tabular}{|l|l|l|l|}
\hline L2 & 178.06 & 33.73 & 6.06 \\
L3 & 206.23 & 52.12 & 9.36 \\
L4 & 200.71 & 34.48 & 6.19 \\
\hline
\end{tabular}

RHO

\begin{tabular}{|l|l|l|l|}
\hline L2 & 1.11 & 0.05 & 0.01 \\
L3 & 1.08 & 0.05 & 0.01 \\
L4 & 1.09 & 0.05 & 0.01 \\
\hline
\end{tabular}

gantry tilt to be applied so that the imaged plane is at right angles to the long axis of the vertebral body, the cross section imaged is not the same as one which is taken at right angles to the long axis of the body. However since the maximum possible amount of gantry tilt is $\pm 15^{\circ}$ the maximum degree of elongation of the antero-posterior (AP) dimension will be about $3 \%$. Figure 5.9 shows a typical series of cross sections at the levels of the three vertebrae, and figure 5.10 shows the locations of the measurements taken from the films.

It can be seen from figure 5.9 that the AP dimension of the abdomen decreases steadily from the position of $\mathrm{L} 2$ through to the position of $\mathrm{L} 4$, and that the converse is true for the lateral dimension: the body is at its broadest at the position of L4. The measurements, which are quoted in table 5.19, confirm this trend. 

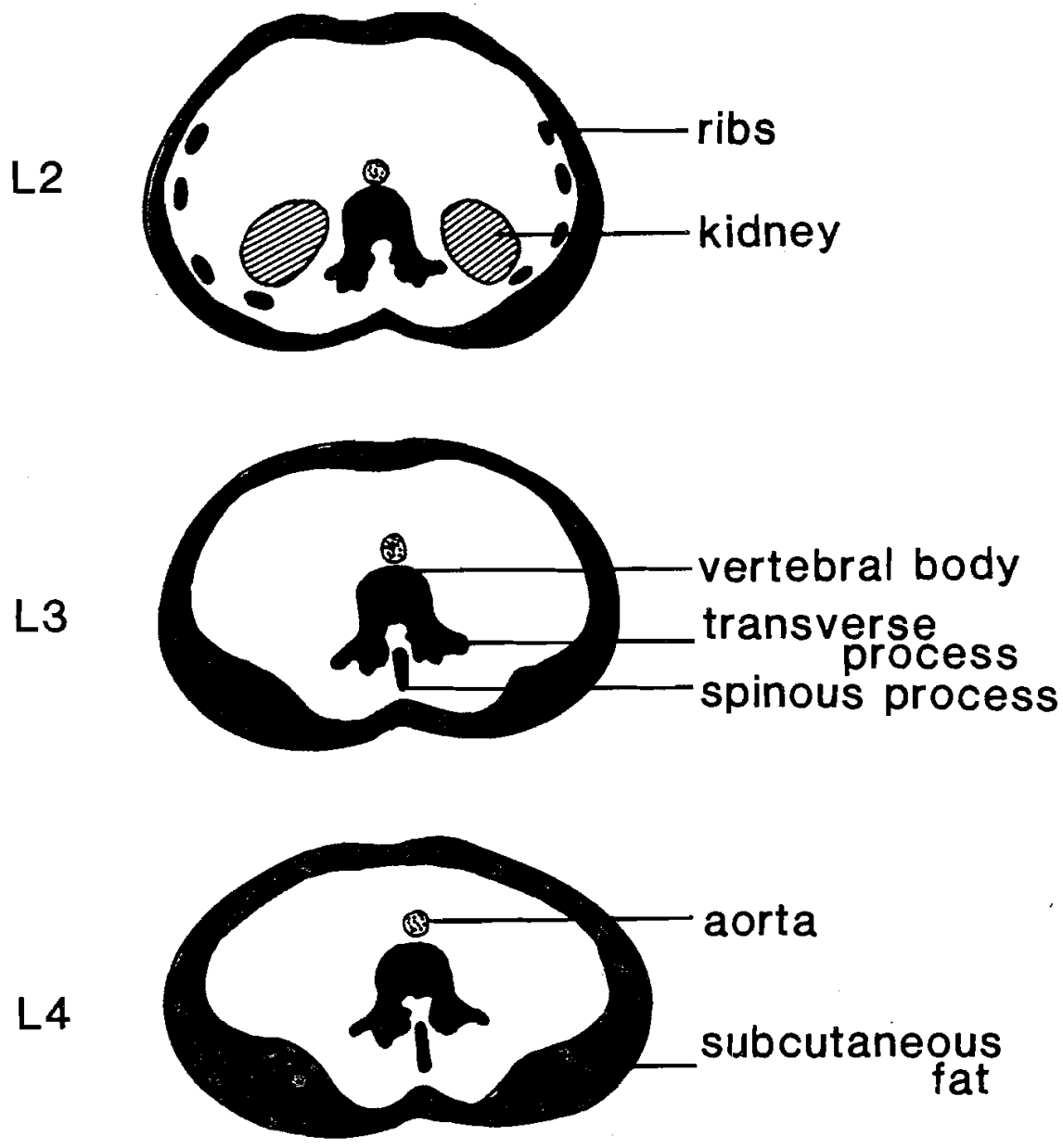

$30 \mathrm{~cm}$

Figure 5.9: Typical Abdominal Cross Sections at the Levels of L2, L3 \& LA.
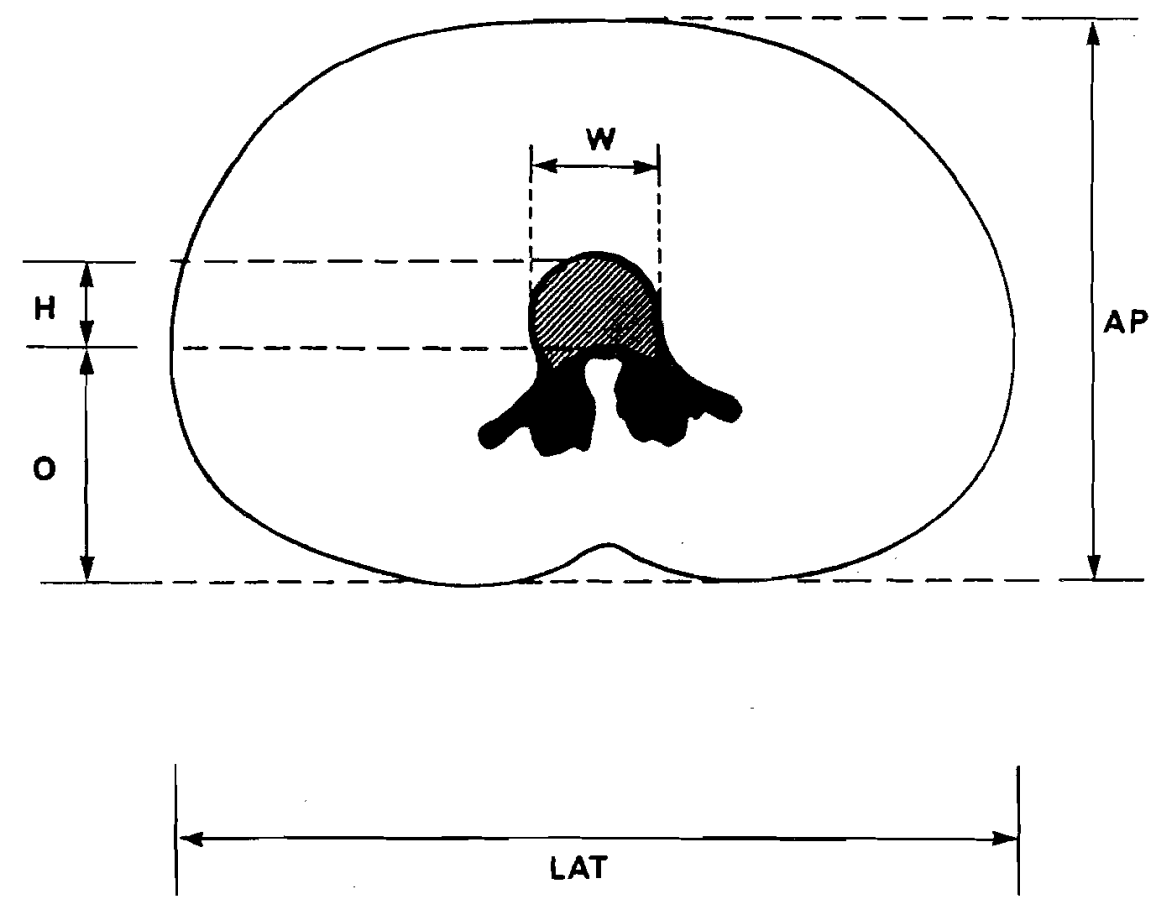

Figure 5.10: Locations of Measurements Taken From Films. 
Table 5.19: Measurements of the Abdominal Cross Section.

\begin{tabular}{||c|cc|cc||}
\hline \multirow{2}{*}{} & \multicolumn{2}{|c|}{ AP } & \multicolumn{2}{c|}{ LATERAL } \\
\cline { 2 - 5 } & $\begin{array}{c}\text { mean } \\
(\mathrm{cm})\end{array}$ & $\begin{array}{c}\text { SD } \\
(\mathrm{cm})\end{array}$ & $\begin{array}{c}\text { mean } \\
(\mathrm{cm})\end{array}$ & $\begin{array}{c}\text { SD } \\
(\mathrm{cm})\end{array}$ \\
\hline L2 & 19.2 & 1.6 & 27.1 & 2.0 \\
L3 & 17.7 & 1.6 & 27.6 & 2.5 \\
L4 & 17.5 & 1.8 & 29.5 & 2.7 \\
\hline
\end{tabular}

The measurements of the location and size of the vertebral body are given in table 5.20. The average position of L4 is some $8 \mathrm{~mm}$ further from the posterior margin than is the average position of L2, and there are small but significant increases in both the lateral and AP dimensions of the vertebral bodies going from L2 through to L4. The average lateral dimension of L4 is some $7 \%$ greater than that of L2, and the average AP dimension of L4 is $10 \%$ greater than that of L2.

Table 5.20: Measurements of the Size and Position of the Vertebral Body.

\begin{tabular}{||c|cc|cc|cc|}
\hline & \multicolumn{2}{|c|}{ Offset $(\mathrm{O})$} & \multicolumn{2}{|c|}{ Lateral $(\mathrm{W})$} & \multicolumn{2}{|c|}{ AP $(\mathrm{H})$} \\
\cline { 2 - 7 } & $\begin{array}{c}\text { mean } \\
(\mathrm{Cm})\end{array}$ & $\begin{array}{c}\mathrm{SD} \\
(\mathrm{Cm})\end{array}$ & $\begin{array}{c}\text { mean } \\
(\mathrm{cm})\end{array}$ & $\begin{array}{c}\text { SD } \\
(\mathrm{cm})\end{array}$ & $\begin{array}{c}\text { mean } \\
(\mathrm{cm})\end{array}$ & $\begin{array}{c}\text { SD } \\
(\mathrm{cm})\end{array}$ \\
\hline L2 & 6.7 & 0.8 & 2.8 & 0.3 & 3.6 & 0.3 \\
L3 & 7.2 & 0.9 & 2.9 & 0.3 & 3.7 & 0.4 \\
L4 & 7.5 & 1.0 & 3.0 & 0.3 & 4.0 & 0.3 \\
\hline
\end{tabular}

The letters $O, W$ and $H$ refer to the distances marked on figure 5.10.

\subsection{Patients with a History of Anorexia Nervosa.}

Sixty eight patients were examined by dual energy C.T. spine mineral densitometry. These were taken from a group of 110 consecutive attenders at the eating disorders clinic of Wellington Hospital [189]. Only non-Polynesian people were included in the group of 110 , and those under 20 years of age were excluded due to possible effects of bone growth while those over 40 were excluded due to possible post-menopausal bone loss. A total of 41 patients were either unavailable, or refused to take part in the survey, or were excluded as they had other medical disorders which could have affected bone mineral content. Due to equipment failure a satisfactory dual energy examination was not obtained on a further patient. The average age of the patient group was 27.5 years, with a standard deviation of 5.8 years. The average age of the patient group was 3.4 years 
younger than that of the control group.

Scanning procedures and calculations were performed in exactly the same manner as for the control group. The distributions for the dual energy parameters, Cmin and RHO, are given in figure 5.11, and the distributions of the single energy mineral estimates are given in figure 5.12. The distribution of Cmin appears Gaussian, with a chi-square value of 8.9 (eight degrees of freedom), corresponding to a probability of $35 \%$ that deviations of this magnitude or larger may occur with samples of this size drawn from a parent population whose distribution is Gaussian. The distribution of RHO also appears Gaussian, but with large random variations superimposed. A chi-squared test yielded a probability of $1 \%$, suggesting a very poor fit. However the graph does not resemble any of the other common statistical distributions. The distributions of the two single energy parameters appear normal, with chi-square probabilities of approximately $71 \%$ $(\mathrm{Cb} 80)$ and $88 \%(\mathrm{Cb} 120)$.

The parameters for the distributions of the four bone measurements are given in table 5.21.

Table 5.21: Bone Measurements of Anorexics.

\begin{tabular}{||l|c|c|c|}
\hline $\begin{array}{c}\text { Bone } \\
\text { Measurement }\end{array}$ & Mean & $\begin{array}{c}\text { Std Error } \\
\text { of Mean }\end{array}$ & $\begin{array}{c}\text { Standard } \\
\text { Deviation }\end{array}$ \\
\hline Cb80 & 121.4 & 3.1 & 25.8 \\
Cb120 & 121.4 & 3.3 & 27.2 \\
Cmin & 159.3 & 4.6 & 38.1 \\
RHO & 1.085 & 0.005 & 0.038 \\
\hline
\end{tabular}

Again there is clearly no significant difference between the two single energy means. There are no significant differences between the standard deviations of the single energy measurements of the anorexic group and those of the controls (see table 5.12), a somewhat surprising result since the anorexic group includes some people who are currently anorexic and some who are not and would therefore be expected to be a less homogeneous group. There was also no significant difference between the standard deviations of the parameter RHO for the patient group and for the controls, but the standard deviation of $\mathrm{Cmin}$ for the patient group was significantly larger than that for the controls (Student, $\mathrm{p}=0.05$ ). The differences between the means of the bone measurements on the anorexic group and those of the controls are listed in table 5.22. The significance of the observed differences has been investigated using both the Student $t$ test and the Wilcoxon test, with the probabilities that the observed differences are due to chance also being listed in table 5.22 . 

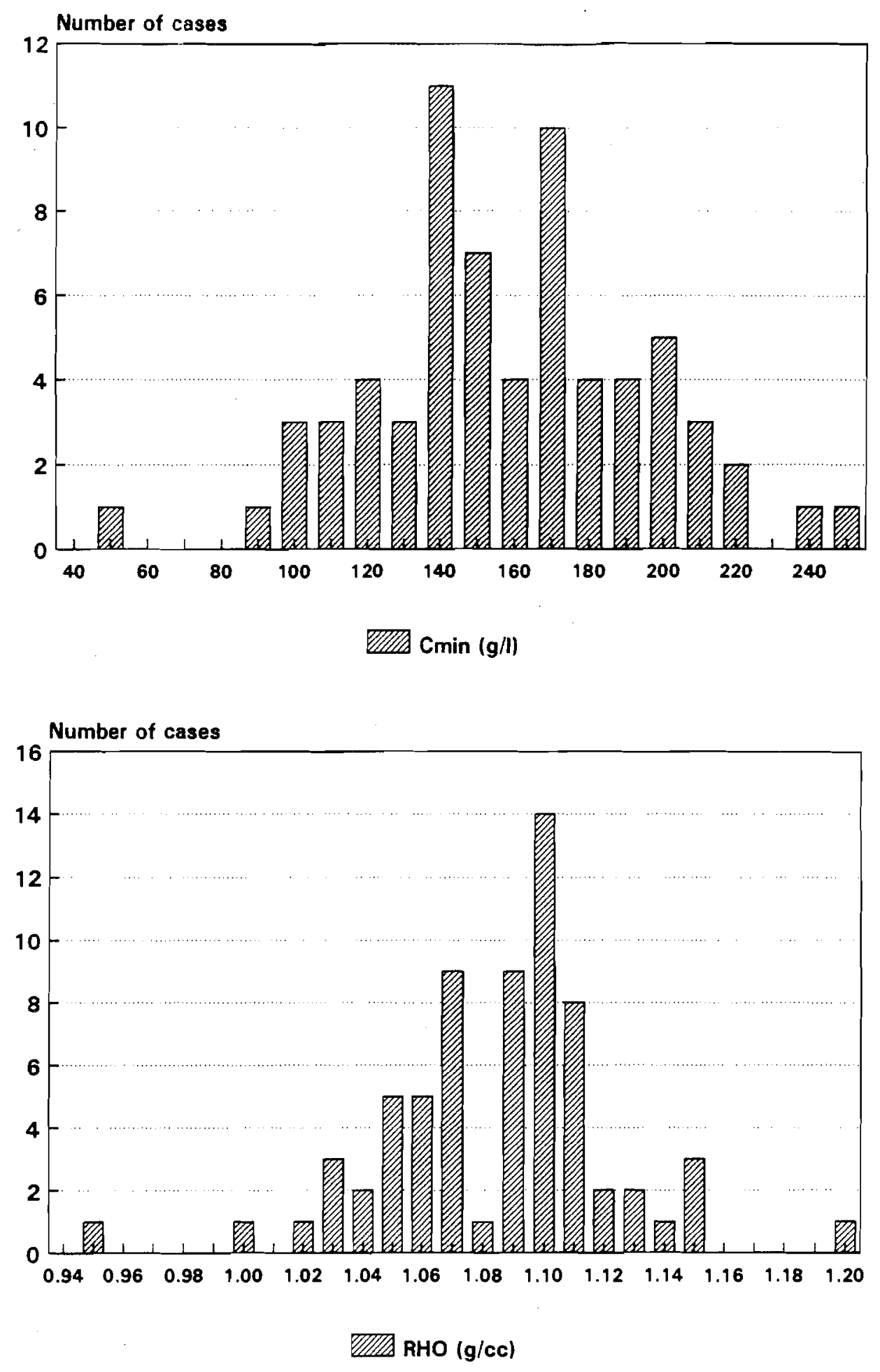

Figure 5.11: Distributions of the Dual Energy Measurements of the Anorexics.

The figures plotted are the means for the three vertebrae scanned on each patient. 

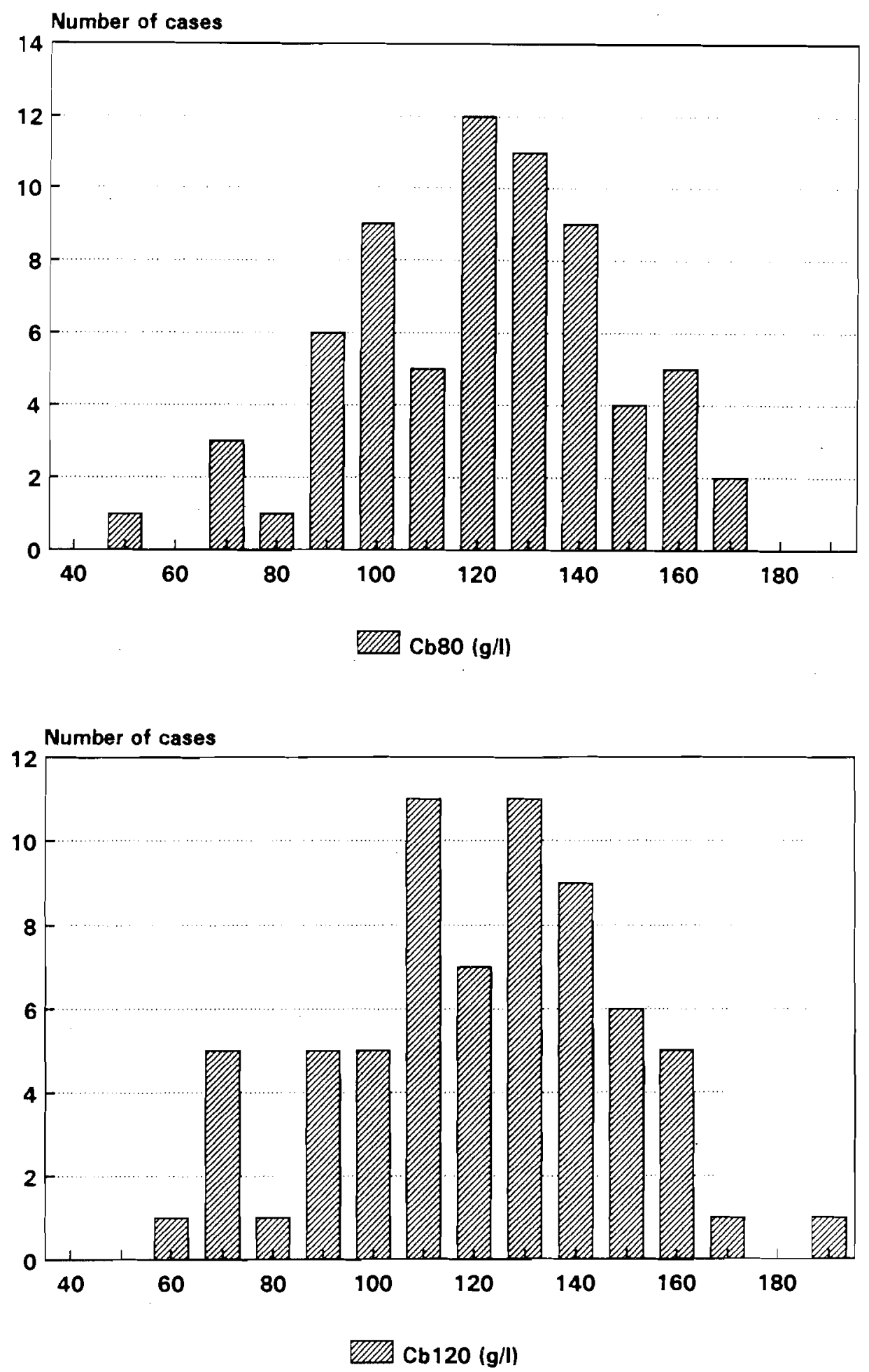

Figure 5.12: Distributions of the Single Energy Measurements of the Anorexics. The figures plotted are the means for the three vertebrae scanned on each patient. 
Table 5.22: Differences Between Anorexic Group and Controls.

\begin{tabular}{||c|c|c|c||}
\hline \multirow{2}{*}{ Parameter } & \multirow{2}{*}{$\begin{array}{c}\text { Difference } \\
\left(\frac{\circ}{8}\right)\end{array}$} & Student & Wilcoxon \\
\cline { 3 - 4 } & $-18.3 \%$ & $2 \times 10^{-6}$ & $2 \times 10^{-5}$ \\
Cb80 & $-17.7 \%$ & $2 \times 10^{-5}$ & $1 \times 10^{-4}$ \\
Cb120 & $-18.8 \%$ & $5 \times 10^{-7}$ & $3 \times 10^{-6}$ \\
Cmin & $-0.6 \%$ & 0.42 & 0.56 \\
RHO & $-0.5 \%$ & \\
\hline
\end{tabular}

There is a consistent decrease of approximately $18 \%$ in each of $\mathrm{Cb} 80, \mathrm{Cb} 120$ and Cmin between the patient and control groups. The agreement between the single energy measurements and the dual energy mineral estimate is particularly important because the single energy measurement is very sensitive to changes taking place in the bone marrow. Such changes have been observed in anorexics [150], whose intravertebral fat content was found to be substantially greater than in the normal population, and it is therefore important to know whether the changes observed with the single energy C.T. technique genuinely involve a loss of bone mineral or whether such changes are predominantly or even solely due to changes in the density and composition of the marrow. The above figures confirm that the loss of bone mass involves both the marrow and mineral components. The apparent decrease in RHO is not statistically significant. While the differences in mineral measurements between the two groups are rated as highly significant by both the Student and Wilcoxon tests, the probabilities given by the Wilcoxon test are consistently higher, suggesting that the departure from true Gaussian distributions is affecting the values obtained by Student's $t$ test.

Table 5.23: Correlation Between Bone Measurements for Anorexics.

Spearman Rank Correlation Coefficients.

\begin{tabular}{||l|l|l|r|}
\hline \hline & Cb120 & Cmin & RHO \\
\hline Cb80 & 0.97 & 0.70 & 0.48 \\
Cb120 & & 0.56 & 0.62 \\
Cmin & & & -0.21 \\
\hline
\end{tabular}

Probabilities that the Correlations are Due to Chance.

\begin{tabular}{||l|c|c|c||}
\hline & $\operatorname{Cb} 120$ & Cmin & RHO \\
\hline Cb80 & $<10^{-7}$ & $<10^{-7}$ & $10^{-4}$ \\
$\operatorname{cb} 120$ & & $4 \times 10^{-6}$ & $4 \times 10^{-7}$ \\
Cmin & & & 0.09 \\
\hline
\end{tabular}


In comparing the degrees of correlation between the bone measurements made on the anorexics group with those between the measurements made on the control group (tables 5.13 and 5.23), it can be seen that again there is strong correlation between the two single energy measurements, $\mathrm{Cb} 80$ and $\mathrm{Cb} 120$. However in the anorexic group there is stronger correlation between each of the single energy measurements and Cmin, and weaker correlation between the single energy parameters and RHO. Again there is an apparent negative correlation between the dual energy mineral estimate, $\mathrm{Cmin}$, and the dual energy density estimate, RHO. The probability of this arising by chance is $9 \%$, leading to the conclusion that it is possibly significant. Linear regression yielded similar correlation coefficients to those listed in table 5.23.

In comparing the control and anorexic groups it is necessary to consider the likely effects of the age difference between the two groups. In the previous section it was found that there was no significant correlation between the dual energy measurements and age, and that there was a possibly significant negative correlation between each of the single energy parameters and age. Since the patient group is somewhat younger than the control group, the anorexia patients would in the absence of other factors be expected to have a somewhat higher average single energy mineral concentration. Using the linear regression parameter for $\mathrm{Cb} 120$ quoted in the previous section, it is calculated that the average figure for $\mathrm{Cb} 120$ for a group taken from the normal population whose average age was the same as that of the anorexics would be some $5 \mathrm{mg} / \mathrm{cc}$ higher than for the control group. In fact the average value of $\mathrm{Cb} 120$ for the anorexic group is some $26 \mathrm{mg} / \mathrm{cc}$ lower than that of the control group. Precise quantification of the effects of the different age structures of the two groups is not possible due to the uncertainties involved, but it is clear that the age difference between the two groups is such that this study is likely to slightly underestimate any difference due to anorexia nervosa.

Correlations between bone measurements and age for the anorexic group are listed in table 5.24.

Table 5.24: Correlations Between Bone Measurements and Age for Anorexics.

\begin{tabular}{|l|c|c|}
\hline $\begin{array}{l}\text { Bone } \\
\text { Measurement }\end{array}$ & $r_{\mathbf{s}}$ & $p$ \\
\hline Cb80 & -0.26 & 0.04 \\
Cb120 & -0.32 & 0.01 \\
Cmin & -0.05 & 0.68 \\
RHO & -0.28 & 0.02 \\
\hline
\end{tabular}


Again there is no significant correlation between $\mathrm{Cmin}$ and age. There are weak negative correlations between the other three measurements and age which are all significant at the $5 \%$ level. The rates of decrease (standard error of the regression parameter in brackets) obtained by linear regression are Cb80: $-1.1 \mathrm{~g} / 1$ per year $( \pm 0.5 \mathrm{~g} / 1) ; \quad \mathrm{Cb} 120$ : $-1.3 \mathrm{~g} / 1$ per year $( \pm 0.6 \mathrm{~g} / \mathrm{l}) ;$ and RHO: $-1.4 \mathrm{~g} / 1$ per year $( \pm 0.8 \mathrm{~g} / 1)$. Due to the large uncertainties in these rates it is not possible to make meaningful comparisons between the rates of loss of bone in this group and the corresponding rates in the control group.

Distributions of the coefficients of variation of the dual energy mineral measurements are given in figure 5.13, and those of the single energy bone measurements are given in figure 5.14. All four distributions are skewed to the right. The average coefficient of variation for measurements of three vertebrae on each patient are listed in table 5.25. This figure, divided by the square root of 3 , may be used to give an estimate of the standard error of the mean of three measurements on a patient, and is also listed in table 5.25.

Table 5.25: Average Coefficients of Variation of Bone Measurements for Anorexics.

\begin{tabular}{||l|c|c||}
\hline $\begin{array}{l}\text { Bone } \\
\text { Measurement }\end{array}$ & $\begin{array}{c}\text { Average } \\
\text { Coefficient } \\
\text { of variation } \\
(\%)\end{array}$ & $\begin{array}{c}\text { Std error } \\
\text { of mean } \\
(\%)\end{array}$ \\
\hline Cb80 & 6.9 & 4.0 \\
Cb120 & 6.6 & 3.8 \\
Cmin & 18.6 & 10.8 \\
RHO & 2.6 & 1.5 \\
\hline
\end{tabular}

The differences between this group and the controls is small, the only statistically significant difference being between the average coefficients of variation of $\mathrm{Cb} 120$ of the two groups (Wilcoxon, $p=0.04$ ), the coefficient of variation of the anorexic group being slightly larger. 


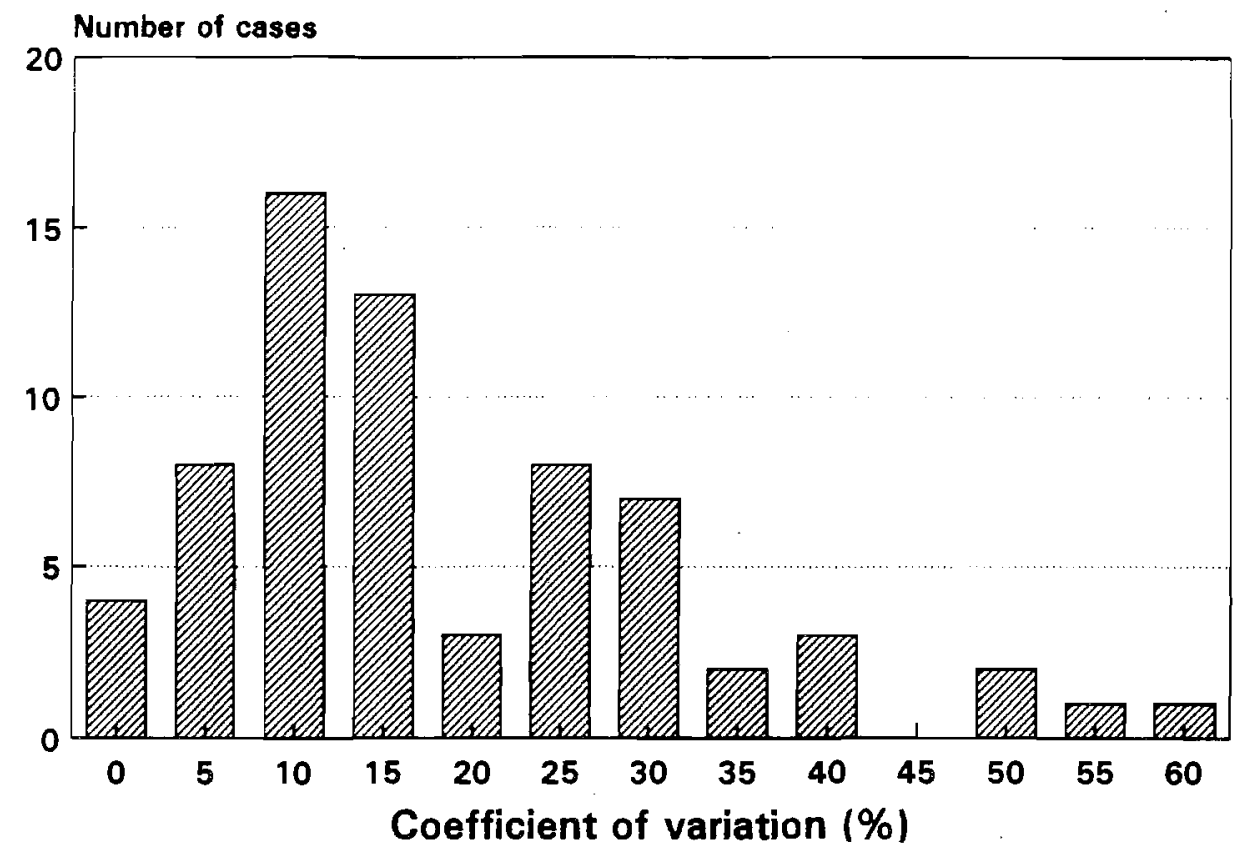

VIITS Cmin

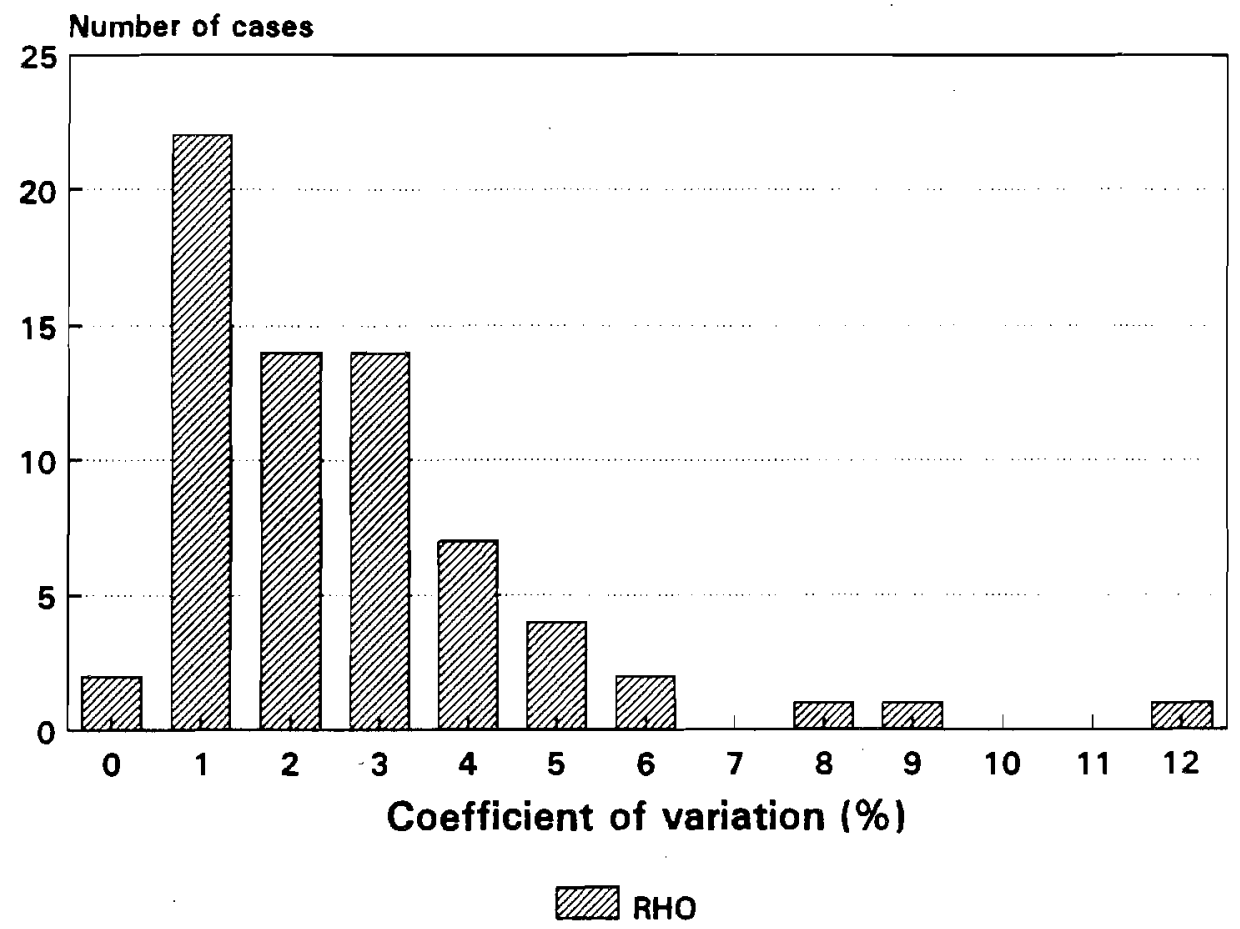

Figure 5.13: Distributions of the Coefficients of Variation of the Dual Energy Measurements of the Anorexic Group.

The figures plotted are the coefficients of variation for the three vertebrae scanned on each patient. 


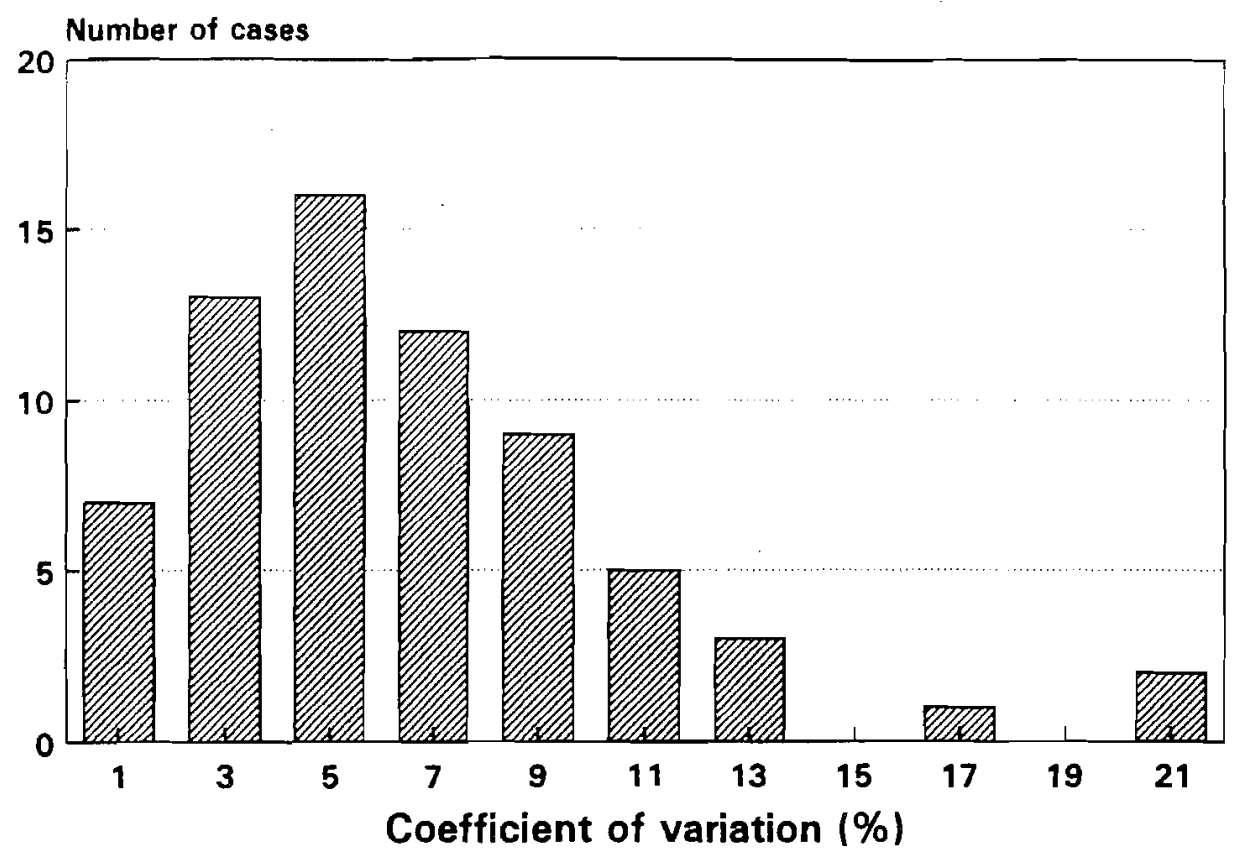

WIIT $\mathrm{Cb} 80$

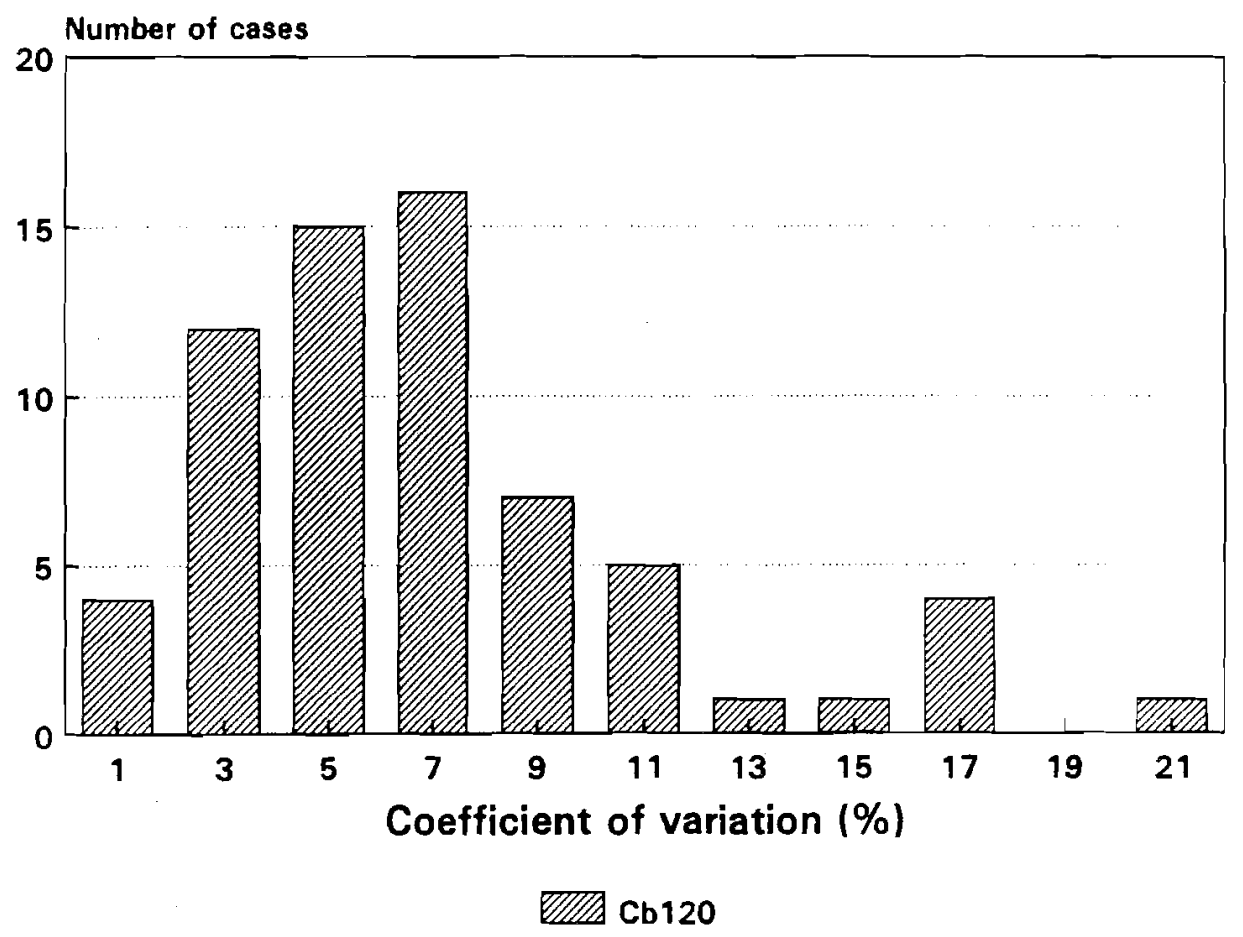

Figure 5.14: Distributions of the Coefficients of Variation of the Single Energy Measurements of the Anorexic Group.

The figures plotted are the coefficients of variation for the three vertebrae scanned on each patient. 
Table 5.26: Correlations of C.V.'s with Age for Anorexics.

\begin{tabular}{||l|l|l|}
\hline C.V. of $\ldots$ & $r_{\mathbf{S}}$ & $p$ \\
\hline Cb80 & 0.04 & 0.77 \\
Cb120 & 0.28 & 0.02 \\
Cmin & 0.26 & 0.03 \\
RHO & 0.27 & 0.03 \\
\hline
\end{tabular}

There are significant positive correlations between patient age and the coefficients of variation of each of $\mathrm{Cb} 120, \mathrm{Cmin}$ and $\mathrm{RHO}$. Age correlations for the controls (section 5.2) showed no significant correlation for the single energy parameters, but negative correlation between the coefficients of variation of the dual energy measurements and age. The positive correlations for the patient group suggest that the loss of bone in anorexia nervosa is not evenly distributed between L2, L3 and L4. This effect could also be due to any possible recovery not occurring equally in the three vertebrae.

Table 5.27: Correlations of C.V.'s with Mineral Measurements.

\begin{tabular}{||c|c|c||}
\hline C.V. of $\cdots$ & $r_{\mathbf{S}}$ & $p$ \\
\hline Cb80 & -0.41 & 0.0009 \\
Cb120 & -0.45 & 0.0002 \\
Cmin & -0.32 & 0.0008 \\
RHO & -0.10 & 0.40 \\
\hline
\end{tabular}

There are highly significant negative correlations between the mean values of $\mathrm{Cb} 80$, $\mathrm{Cb} 120$ and $\mathrm{Cmin}$ for each patient and the coefficients of variation for the three vertebrae. As the mineral content becomes less, the variability increases, supporting the suggestion that there is uneven loss of bone mineral in anorexia nervosa. 
Table 5.28: Mean Bone Measurements for $L 2, L 3 \& L A$ for anorexics.

\begin{tabular}{|c|c|c|c|}
\hline Vertebra & Mean & S.D. & $\begin{array}{l}\text { std error } \\
\text { of mean }\end{array}$ \\
\hline
\end{tabular}

Cb80

\begin{tabular}{|l|l|l|l|}
\hline L2 & 123.05 & 25.93 & 3.14 \\
L3 & 118.23 & 29.07 & 3.53 \\
L4 & 123.18 & 29.01 & 3.52 \\
\hline
\end{tabular}

Cb120

\begin{tabular}{|l|l|l|l|}
\hline L2 & 123.05 & 25.93 & 3.14 \\
L3 & 119.03 & 26.70 & 3.24 \\
L4 & 122.20 & 27.51 & 3.34 \\
\hline
\end{tabular}

\section{Cmin}

\begin{tabular}{|l|l|l|l|}
\hline L2 & 161.99 & 42.66 & 5.17 \\
L3 & 159.54 & 53.07 & 6.44 \\
L4 & 156.38 & 46.91 & 5.69 \\
\hline
\end{tabular}

RHO

\begin{tabular}{|l|l|l|l|}
\hline L2 & 1.09 & 0.04 & 0.01 \\
L3 & 1.08 & 0.06 & 0.01 \\
L4 & 1.09 & 0.05 & 0.01 \\
\hline
\end{tabular}

Inspection of the above shows that there are no significant differences between the vertebrae in respect of any of the four bone measurements. Application of Student's $t$ test to those readings above which show the greatest apparent differences has confirmed this finding. While the large coefficients of variation of the readings for the three vertebrae in each patient imply that bone is not lost evenly from each of the vertebrae measured, the fact that there are no significant differences between the mean values above suggests that the vertebra which loses most mineral is not the same for all patients. 


\subsection{Correlation Between Bone Measurements and Clinical Parameters.}

In the previous two sections correlations have been made between the various bone measurements for the control group (section 5.2), and for the group composed of patients treated for anorexia nervosa (section 5.3). In the control group there was an apparent decrease in each of the four bone measurements (Cb80, Cb120, Cmin and RHO) as the age of the patient increased, but these trends were not statistically significant. In the anorexia group significant negative correlations were found between age and the two single energy bone measurements, and also between age and RHO. The correlations with age observed in the patient group presumably reflect the duration of the condition, a hypothesis which may be tested by performing correlations with variables which more directly reflect the clinical state of the patient group. The results of correlations between the single energy bone measurement, $\mathrm{Cb80}$, and the various clinical parameters recorded on the group of patients studied in Wellington have already been published [189]. However the interpretation of single energy C.T. measurements in anorexia nervosa is complicated by the fact that single energy measurements are highly sensitive to changes in the marrow component of the cancellous tissue (see section 4.4). Such changes have been reported in patients suffering from anorexia nervosa [190, 191], and other studies using dual energy computed tomography have reported increased marrow fat in anorexic patients [150].

The clinical variables pertaining to both the patient and control groups are listed in table 5.29. Additional variables pertaining to diet and menstrual history were determined for the patient group alone and are discussed later. The clinical information and the bone measurements were stored on an IBM-compatible PC and analysed using software described in appendix $\mathrm{I}$.

The first group of variables listed are the bone measurements which are to be correlated with the others which reflect the conditions of the patients and controls. The other single energy measurement, $\mathrm{Cb} 120$, has not been included in the analysis of the present section as it is vary strongly correlated with Cb80 (see sections 5.2 \& 5.3) and would add nothing of value to the analysis. The group following age reflect the height and weight of the subjects at the time of the C.T. examination. Since these two variables are not independent, use is also made of the body mass index (BMI) in which the weight is normalised to the height of the patient by dividing the weight (in kilograms) by the height (in meters) squared. The next group of variables are ones which might affect bone but which are not specifically related to anorexia nervosa: the number of children born to the subjects, whether or not there is a family history of osteoporosis, and the number of bone fractures the subject has had in her lifetime. The fourth group of variables are concerned with the amount of calcium in the diet, both at the time of examination and in 
Table 5.29: Clinical Variables for Both Patients and Controls.

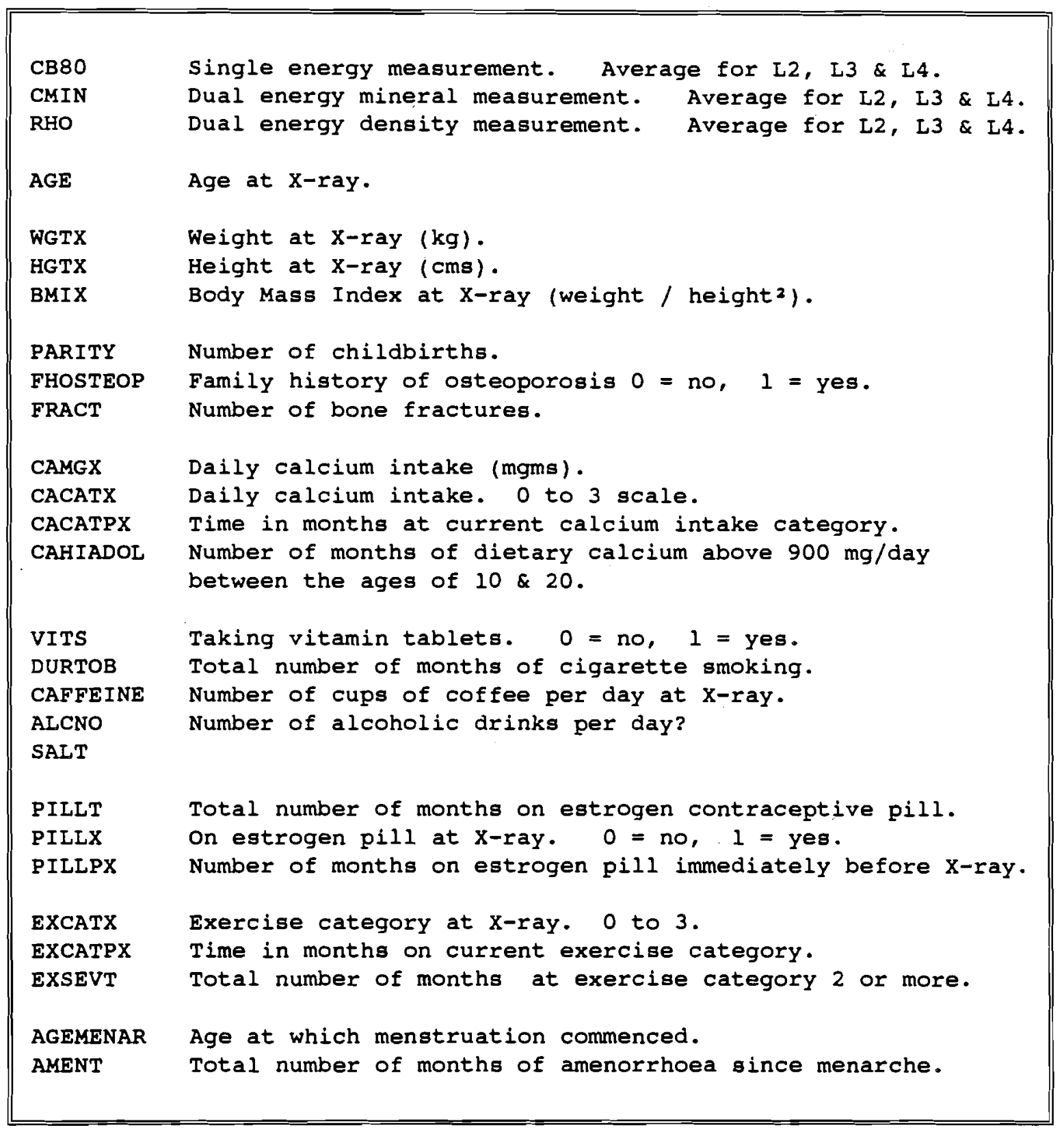

adolescence. The next group comprises dietary factors other than calcium intake, and is followed by a group concerned with the use of estrogen-based contraceptive pills. Estrogen therapy is used to treat post-menopausal osteoporosis, and it is possible that estrogen could help prevent osteoporosis in younger women. The next group is concerned with the amount of exercise both at time of examination and in the past. Exercise has been shown in other studies to build up bone mass and could perhaps inhibit the loss of bone due to anorexia nervosa [192]. The final group is concerned with the age at which menstruation commenced (menarche), and with the total number of months since menarche when menstruation did not occur (apart from pregnancy). 
In appendix $\mathrm{J}$ distribution parameters for the above variables are tabulated separately for the patient and control groups. The parameters given are mean, standard deviation, moment coefficient of skew; and maximum, minimum and median values. In table 5.30 the means for the patient and control group are given, along with the Wilcoxon probabilities that the apparent differences between the two means could have been due to chance. Where the probability is less than $5 \%$ the percentage difference between the two means is listed. For those variables for which percentages are not an appropriate means of expressing differences (for example, when the variable categorises a quantity such as exercise on an arbitrary scale) other means of describing the difference are used. Differences are quoted in brackets when the probability is greater than $5 \%$ but less than or equal to $10 \%$.

Table 5.30: Comparison between Controls and Anorexics.

\begin{tabular}{|c|c|c|c|c|}
\hline Variable & $\begin{array}{r}\text { Mean } \\
\text { Controls }\end{array}$ & $\begin{array}{l}\text { alues } \\
\text { Anorexics }\end{array}$ & Difference & $\mathrm{p}$ \\
\hline $\begin{array}{l}\text { CB80 } \\
\text { CMIN } \\
\text { RHO }\end{array}$ & $\begin{array}{l}148.5 \\
196.2 \\
1.092\end{array}$ & $\begin{array}{l}121.4 \\
159.3 \\
1.085\end{array}$ & $\begin{array}{l}-18.2 \% \\
-18.8 \%\end{array}$ & $\begin{array}{ll}2 & E-5 \\
3 & E-6 \\
0.56\end{array}$ \\
\hline $\begin{array}{l}\text { WGTX } \\
\text { HGTX } \\
\text { BMIX }\end{array}$ & $\begin{array}{r}63.0 \\
165.5 \\
22.8\end{array}$ & $\begin{array}{r}51.7 \\
164.2 \\
19.2\end{array}$ & $\begin{array}{l}-17.9 \% \\
-15.8 \frac{\%}{6}\end{array}$ & $\begin{array}{cc}<1 & E-7 \\
0.38 \\
1 & E-6\end{array}$ \\
\hline $\begin{array}{l}\text { PARITY } \\
\text { FHOSTEOP } \\
\text { FRACT }\end{array}$ & $\begin{array}{l}1.3 \\
0.1 \\
0.5\end{array}$ & $\begin{array}{l}0.4 \\
0.1 \\
0.3\end{array}$ & $-69.2 \%$ & $\begin{array}{l}1 \quad E-4 \\
0.56 \\
0.29\end{array}$ \\
\hline $\begin{array}{l}\text { CAMGX } \\
\text { CACATX } \\
\text { CAHIADOL }\end{array}$ & $\begin{array}{r}669.4 \\
1.5 \\
43.5\end{array}$ & $\begin{array}{r}640.1 \\
1.3 \\
32.6\end{array}$ & & $\begin{array}{l}0.42 \\
0.32 \\
0.38\end{array}$ \\
\hline $\begin{array}{l}\text { VITS } \\
\text { DURTOB } \\
\text { CAFFEINE } \\
\text { ALCNO } \\
\text { SALT }\end{array}$ & $\begin{array}{r}0.2 \\
38.1 \\
5.5 \\
0.4 \\
1.8\end{array}$ & $\begin{array}{r}0.4 \\
56.3 \\
4.9 \\
0.4 \\
1.4\end{array}$ & $\begin{array}{c}+ \\
(+47.8 \%)\end{array}$ & $\begin{array}{l}0.03 \\
0.10 \\
0.68 \\
0.86 \\
0.01\end{array}$ \\
\hline $\begin{array}{l}\text { PILLT } \\
\text { PILLX } \\
\text { PILLPX }\end{array}$ & $\begin{array}{r}30.3 \\
0.2 \\
3.4\end{array}$ & $\begin{array}{r}21.0 \\
0.2 \\
7.6\end{array}$ & & $\begin{array}{l}0.27 \\
0.94 \\
0.84\end{array}$ \\
\hline $\begin{array}{l}\text { EXCATX } \\
\text { EXSEVT }\end{array}$ & $\begin{array}{r}1.1 \\
40.6\end{array}$ & $\begin{array}{r}1.4 \\
68.1\end{array}$ & $\begin{array}{c}+ \\
+67.7 \%\end{array}$ & $\begin{array}{l}0.04 \\
0.03\end{array}$ \\
\hline $\begin{array}{l}\text { AGEMENAR } \\
\text { AMENT }\end{array}$ & $\begin{array}{r}12.6 \\
1.6\end{array}$ & $\begin{array}{l}13.3 \\
51.2\end{array}$ & $\begin{array}{c}(+9 \text { months }) \\
+++\end{array}$ & $\begin{array}{l}0.06 \\
<1 \quad E-7\end{array}$ \\
\hline
\end{tabular}


As discussed in section 5.3, the mean values of $\mathrm{Cb} 80$ and $\mathrm{Cmin}$ are some $18 \%$ lower in the patient group than in the controls. The average weight of the patient group is also $18 \%$ lower, and the BMI of the patient group is $16 \%$ lower than that of the control group. Other significant differences found were that the anorexic patients had fewer children, were more likely to use vitamin pills, had a lower salt intake, took more exercise at the time of the $\mathrm{X}$-ray examination and had on average maintained a high exercise regime for a much longer period of time than had the controls, and had predictably had a much greater incidence of amenorrhoea. The age at which the anorexic group started menstruating was some nine months later than that for the control group, a finding which is to be expected since significant numbers of the patient group were dieting in their early teens, but the probability that the difference in age at menarche between the two groups was due to chance alone is a little greater than $5 \%$. The patient group have a greater history of tobacco use, but this could be a chance finding and may be unrelated to anorexia nervosa.

The difference in calcium intake (CAMGX) between the two groups is perhaps less than would be expected. It has been found that the average calcium intake for those members of the patient group who were not menstruating at the time of the C.T. examination (604 mg/day) was not greatly less than that for those who were menstruating at that time $(672 \mathrm{mg} /$ day). However it should be noted that there are a significant number of the patient group who have very low daily calcium intakes (down to 63 $\mathrm{mg} /$ day) and this is presumably counterbalanced by those who take calcium supplements and therefore have very high calcium intakes. The calcium intake for the members of the control group varied from 242 to $1071 \mathrm{mg} /$ day, while the range for the patient group was from 63 to $1600 \mathrm{mg} /$ day. In comparing those members of the patient group who were menstruating at X-ray with the other members of this group who were not, it was found that the menstruating group exercised more, but that the difference was small (of the same order as that between the patient and control groups), and was not statistically significant (Wilcoxon, $p=0.13$ ). The difference in average weights between the two subgroups of the patient group (46.5 kg for the amenorrheic subgroup, $56.2 \mathrm{~kg}$ for those menstruating at time of examination) was comparable with the difference between the patient group and the controls, although the average weight of the members of the patient group who were menstruating was still lower than that of the controls (56.2 kg compared with $63.0 \mathrm{~kg}, \mathrm{p}<0.01$ ).

Variables specific to the anorexic group are outlined in table 5.31. The first group of variables is concerned with the menstrual history, the second group being specific to the related condition bulimia nervosa, culminating in a variable, ANBULT, which reflects the total time in months during which the patient suffered from either of these two conditions. The next group of variables reflect the durations of periods of low weight, both in total and immediately prior to the C.T. examination, and the final group is 
composed of those variables which did not fit into the previous groups: total duration of low calcium intake, number of hospital admissions, and a final variable which is the clinician's assessment of the likely state of the patient's bones based on the clinical information.

\section{Table 5.31: Clinical Variables for Anorexia Nervosa.}

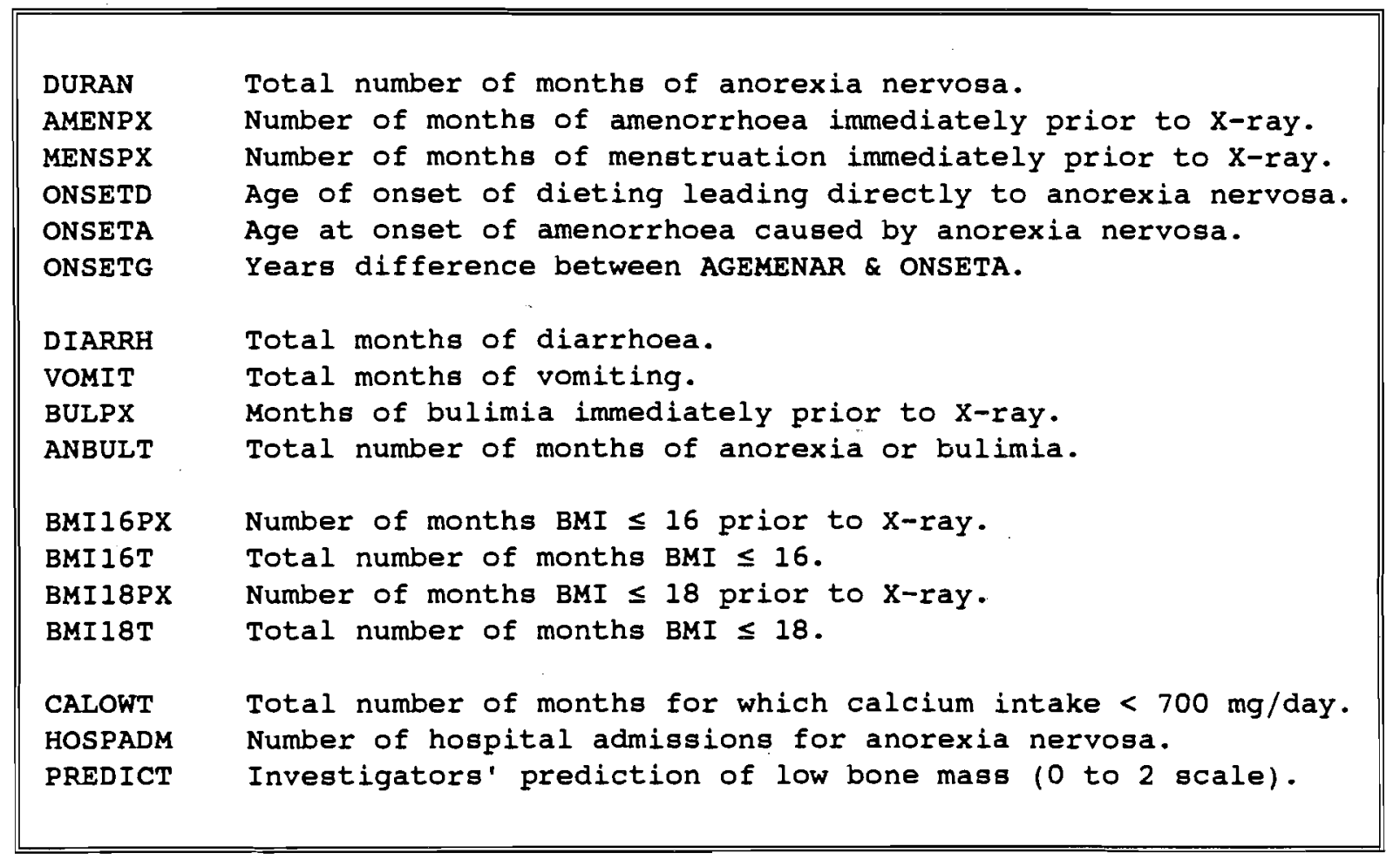

Table 5.32: Distributions of Clinical Variables for Anorexia Nervosa.

\begin{tabular}{|l|rrrrr||}
\hline & Sean & S.D. & Minimum & Maximum & Median \\
& & & & & \\
& & & & & \\
DURAN & 58.0 & 52.5 & 12 & 228 & 36 \\
AMENPX & 22.8 & 47.3 & 0 & 228 & 0 \\
MENSPX & 37.5 & 54.8 & 0 & 264 & 1 \\
ONSETD & 18.3 & 4.3 & 12 & 31 & 17 \\
ONSETA & 18.8 & 4.6 & 12 & 31 & 17 \\
ONSETG & 5.6 & 4.5 & 0 & 17 & 5 \\
& & & & & \\
DIARRH & 25.5 & 49.3 & 0 & 276 & 0 \\
VOMIT & 20.1 & 40.8 & 0 & 228 & 0 \\
BULPX & 20.7 & 43.9 & 0 & 192 & 0 \\
ANBULT & 78.9 & 59.2 & 12 & 228 & 60 \\
BMI16PX & 9.5 & 27.7 & 0 & 144 & 0 \\
BMI16T & 26.6 & 44.4 & 0 & 216 & 9 \\
BMI18PX & 15.5 & 32.0 & 0 & 144 & 0 \\
BMI18T & 55.3 & 60.6 & 0 & 276 & 36 \\
CALOWT & 86.8 & 72.3 & 0 & 312 & 72 \\
HOSPADM & 1.2 & 2.1 & 0 & 12 & 1 \\
PREDICT & 1.0 & 0.9 & 0 & 2 & 1 \\
& & & & & \\
\hline
\end{tabular}


The distribution of the variable DURAN, which is illustrated in figure 5.15, has a strong positive skew. While most patients had only a few years' history of anorexia nervosa (median value $=3$ years), a small number had much larger total numbers of years of anorexia. Two patients had 19 years' history of anorexia nervosa. Thirty two of the 68 patients were not menstruating at the time of examination. The median duration of amenorrhoea for this subgroup in the period prior to X-ray was 30 months, but again the distribution is highly skewed with a small number of patients having not menstruated for up to nineteen years prior to X-ray. Of the thirty six members of the patient group who were menstruating regularly at the time of X-ray, the number of months of menstruation in the period immediately prior to $\mathrm{X}$-ray was also very variable with a positively skewed distribution. The median length of menstruation was $51 / 2$ years for this subgroup, the maximum duration of regular menstruation being 22 years. Of the total patient group, some two thirds had their first onset of anorexia nervosa while between the ages of ten and twenty, but again the distribution is skewed with a small number of patients first suffering from anorexia at about thirty years of age.

\section{Total duration of anorexia nervosa.}

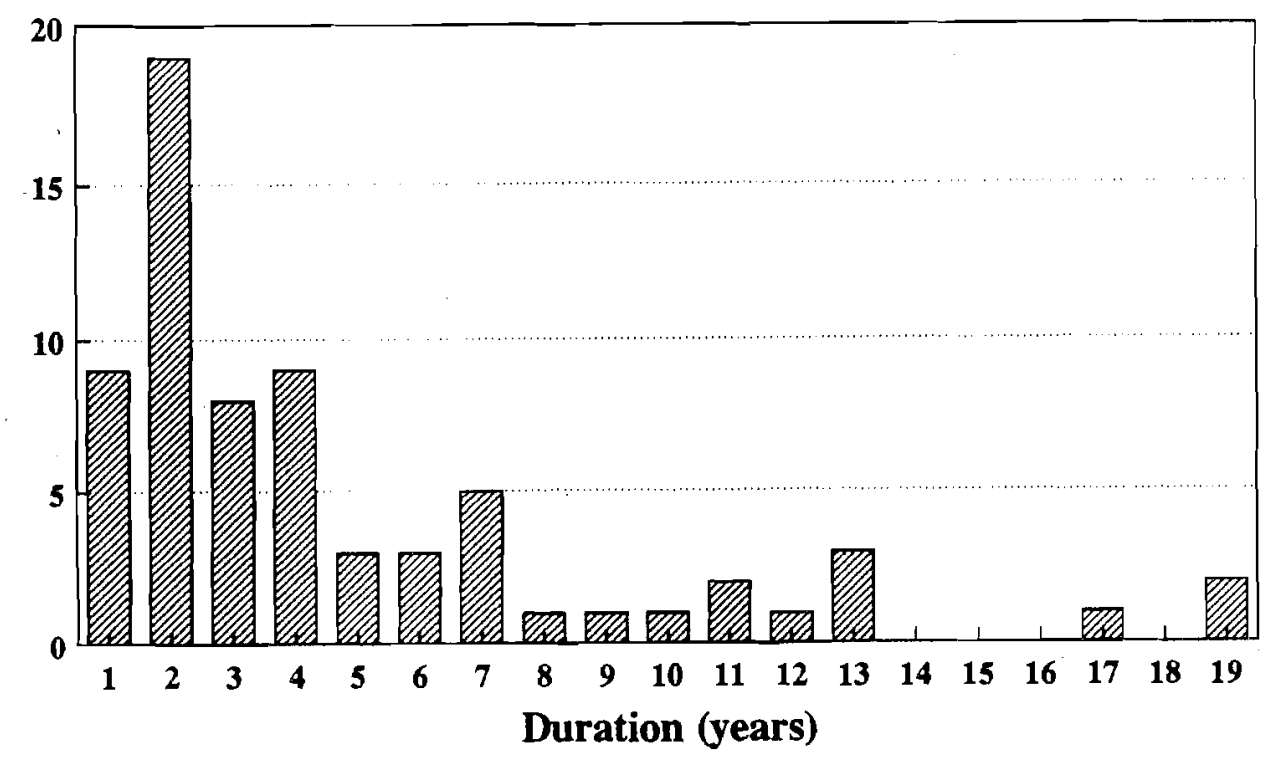

WIIB DURAN

\section{Figure 5.15: Distribution of the Total Duration of Anorexia Nervosa.}

Bulimia nervosa is characterised by periods of low food intake followed by binge eating. To limit the absorption of food the subjects may resort to emetics so that the food is regurgitated before it can be absorbed, or to the use of laxatives so that the absorption of nutrients in the bowel is reduced. Approximately half the patient group had used laxatives, the median period of use for this subgroup being $2 \frac{1 / 2}{2}$ years. A slightly 
smaller number (29) had induced vomiting; of those who had the median total length of time for which emetics had been used was again $2 \frac{1}{2}$ years. In both cases the distributions were strongly skewed with the maximum duration of diarrhoea being 23 years and the maximum duration of vomiting being a little less at 19 years. Nineteen patients were exhibiting the symptoms of bulimia nervosa at the time of examination, the median duration prior to $\mathrm{X}$-ray for this subgroup being five years and the maximum duration noted was sixteen years. The median value for bulimia prior to $\mathrm{X}$-ray is about twice the median value for the duration of anorexia immediately prior to X-ray. The distribution of the total duration of either anorexia nervosa or bulimia nervosa (ANBULT) is illustrated in figure 5.16. The median duration is 5 years, and again there is a strong positive skew.

\section{Total duration of anorexia or bulimia}

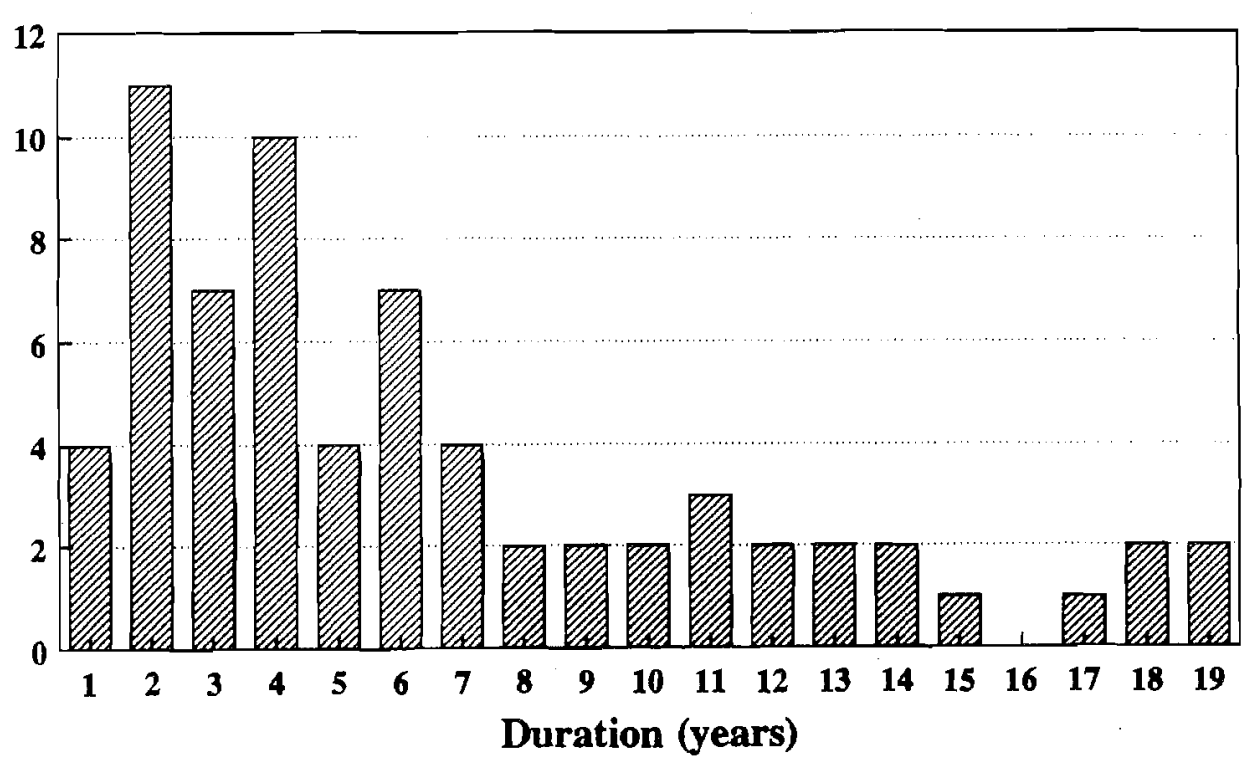

WIIS ANBULT

\section{Figure 5.16: Distribution of the Total Duration of Either Anorexia Nervosa or Bulimia Nervosa.}

Twenty five patients had BMI's of 18 or less at the time of examination (median duration at this level in the period prior to X-ray $=2$ years), and half of these patients in fact had BMI's of less than or equal to 16 with a median duration at this level of 3 years. Three quarters of the patient group had some history of BMI values less than or equal to 16 (median total duration 2 years), and virtually all of them (66 patients) had some history of BMI values less than or equal to 18 (median total duration 2.5 years). Five out of every six patients had some history of low calcium intake (CALOWT), median duration for the whole patient group being 6 years. Slightly over half of the patients had 
been admitted to hospital for anorexia nervosa (36 patients), most of these had had only one or two admissions but one patient had had ten admissions and another had had twelve. The investigators' predictions were that 24 members of the patient group were likely to be osteoporotic, a further 18 could be osteoporotic (but with lower probability), and that the remaining 26 were unlikely to be osteoporotic. Table 5.33 compares the mean values of bone measurements of the three groups.

Table 5.33: Comparison of Clinicians' Prediction with Bone Measurements.

\begin{tabular}{|l|ccc|}
\hline $\begin{array}{l}\text { Prediction } \\
\text { of osteoporosis }\end{array}$ & Cb80 & Cmin & Rho \\
\hline Likely & 106.0 & 149.0 & 1.067 \\
Possible & 120.2 & 164.4 & 1.076 \\
Unlikely & 136.5 & 165.2 & 1.107 \\
Probability & $1 \times 10^{-3}$ & 0.16 & $3 \times 10^{-5}$ \\
\hline
\end{tabular}

The probabilities quoted in the table are the Wilcoxon probabilities that the differences between the 'likely' and the 'unlikely' groups are due to chance. The prediction relates most strongly to the density of the bone, and least strongly to the mineral concentration per se.

The results for correlations between the clinical variables and the bone measurements for the control group are given in table 5.34. The only correlations which were significant at the $5 \%$ level were a negative correlation between $\mathrm{Cb} 80$ and height, a positive correlation between body mass index and RHO, and a negative correlation between the number of past fractures and RHO. There is no obvious explanation for the correlation between $\mathrm{Cb80}$ and height. The correlation between BMI and RHO seems reasonable, but it is clear that this correlation is not due to increased mineral content. The negative correlation between number of fractures and RHO also seems reasonable, but again this is not due to increased mineral content. This finding is in agreement with Rao et al. [158] who stated that the fracture risk is better related to total bone density than to mineral concentration per se. It is not yet known why this should be so, but a possible hypothesis is that as the density of the marrow decreases with the conversion of red marrow to yellow marrow, the soft tissue component may become more compressible. The stresses due to weight bearing may then be less uniformly distributed throughout the vertebral body, and fractures may occur at points where the stresses are the greatest. 
Table 5.34: Clinical Correlations for the Control Group.

\begin{tabular}{|c|c|c|c|c|c|c|}
\hline \multirow{2}{*}{$\begin{array}{l}\text { Clinical } \\
\text { variable }\end{array}$} & \multicolumn{2}{|c|}{$\mathrm{Cb} 80$} & \multicolumn{2}{|c|}{ Cmin } & \multicolumn{2}{|c|}{ RHO } \\
\hline & $r_{\mathbf{S}}$ & $p$ & $r_{s}$ & $p$ & $r_{\mathbf{s}}$ & $p$ \\
\hline WGTX & * & * & * & * & * & * \\
\hline $\begin{array}{l}\text { HGTX } \\
\text { BMIX }\end{array}$ & $\begin{array}{r}-0.35 \\
10.33\end{array}$ & $\begin{array}{l}0.05 \\
0.07)\end{array}$ & $\begin{array}{r}(-0.21 \\
(0.06\end{array}$ & $\begin{array}{l}0.25) \\
0.73)\end{array}$ & $\begin{array}{r}(-0.23 \\
0.40\end{array}$ & $\begin{array}{l}0.22) \\
0.03\end{array}$ \\
\hline PARITY & * & * & * & * & * & * \\
\hline FHOSTEOP & * & * & * & * & * & * \\
\hline FRACT & $(-0.31$ & $0.09)$ & $(0.00$ & $0.99)$ & -0.37 & 0.04 \\
\hline VITS & * & * & * & * & * & * \\
\hline DURTOB & * & * & * & * & * & * \\
\hline CAFFEINE & * & * & * & * & * & * \\
\hline ALCNO & * & * & * & * & * & * \\
\hline SALT & * & * & * & * & * & * \\
\hline PILLT & * & * & * & * & * & * \\
\hline PILLX & * & * & * & * & * & * \\
\hline PILLPX & * & * & * & * & * & * \\
\hline EXCATX & * & * & * & * & * & * \\
\hline EXSEVT & * & * & $\star$ & $\star$ & * & * \\
\hline CAMGX & * & * & * & * & * & * \\
\hline CACATX & * & * & * & * & * & * \\
\hline CAHIADOL & * & * & * & * & * & * \\
\hline AGEMENAR & * & * & * & * & * & * \\
\hline AMENT & * & * & * & * & * & * \\
\hline
\end{tabular}

* = not significant.

The results of correlations between the clinical variables of the patient group and their bone measurements are given in table 5.35. Correlation coefficients and probabilities for variables whose correlation probabilities are less than 0.05 for all three bone parameters are not quoted separately. Where one or more of the correlations have $p$ $\leq 0.05$, the figures for all three are given so that comparisons may be made. As was the case with the controls, none of the purely dietary variables (VITS, DURTOB, CAFFEINE, ALCNO or SALT), nor any of the parameters concerning the use of the contraceptive pill, correlated with the bone parameters. Accordingly these variables have not been listed in table 5.35. 
Table 5.35: Correlations for Anorexics.

\begin{tabular}{|c|c|c|c|c|c|c|}
\hline \multirow{2}{*}{$\begin{array}{l}\text { Clinical } \\
\text { variable }\end{array}$} & \multicolumn{2}{|c|}{$\mathrm{Cb} 80$} & \multicolumn{2}{|c|}{ Cmin } & \multicolumn{2}{|c|}{ RHO } \\
\hline & $r_{s}$ & $\mathrm{p}$ & $r_{s}$ & $p$ & $r_{\mathbf{s}}$ & $p$ \\
\hline $\begin{array}{l}\text { WGTX } \\
\text { HGTX }\end{array}$ & $\begin{array}{c}0.29 \\
\star\end{array}$ & $\begin{array}{c}0.02 \\
*\end{array}$ & $-\underset{*}{0.02}$ & $\begin{array}{c}0.90 \\
*\end{array}$ & 0.43 & 4 E-4 \\
\hline BMIX & 0.35 & 0.004 & -0.02 & 0.90 & 0.51 & $2 E-5$ \\
\hline PARITY & * & * & * & * & * & * \\
\hline FHOSTEOP & * & * & * & * & * & * \\
\hline FRACT & * & * & * & * & * & * \\
\hline $\begin{array}{l}\text { EXCATX } \\
\text { EXSEVT }\end{array}$ & 0.08 & $\underset{*}{0.51}$ & $\underset{*}{0.25}$ & 0.04 & $-\underset{*}{0.22}$ & $\begin{array}{l}0.07 \\
*\end{array}$ \\
\hline AMENT & -0.36 & 0.003 & -0.15 & 0.23 & -0.36 & 0.003 \\
\hline CAMGX & * & * & * & * & * & * \\
\hline CACATX & * & * & * & * & * & * \\
\hline CAHIADOL & 0.25 & 0.04 & 0.23 & 0.05 & 0.01 & 0.96 \\
\hline DURAN & -0.45 & $2 E-4$ & -0.13 & 0.28 & -0.48 & $8 E-5$ \\
\hline AMENPX & -0.24 & 0.05 & 0.09 & 0.46 & -0.42 & $5 \quad E-4$ \\
\hline MENSPX & 0.32 & 0.01 & 0.12 & 0.34 & 0.26 & 0.04 \\
\hline ONSETD & -0.10 & 0.43 & 0.12 & 0.32 & -0.29 & 0.02 \\
\hline ONSETA & -0.04 & 0.74 & 0.15 & 0.22 & -0.24 & 0.05 \\
\hline ONSETG & * & * & * & * & * & * \\
\hline DIARRH & * & * & * & * & * & * \\
\hline VOMIT & * & * & * & * & * & * \\
\hline BULPX & * & * & * & * & * & * \\
\hline ANBULT & -0.40 & 0.001 & -0.26 & 0.03 & -0.25 & 0.04 \\
\hline BMI 16PX & -0.03 & 0.81 & 0.24 & 0.05 & -0.32 & 0.01 \\
\hline BMI16T & -0.29 & 0.02 & -0.05 & 0.68 & -0.29 & 0.02 \\
\hline BMI 18PX & -0.17 & 0.16 & 0.11 & 0.38 & -0.39 & 0.002 \\
\hline BMI18T & -0.30 & 0.01 & -0.07 & 0.59 & -0.31 & 0.01 \\
\hline CALOWT & * & * & * & * & * & * \\
\hline HOSPADM & * & * & $\star$ & * & * & * \\
\hline PREDICT & 0.47 & $1 E-4$ & 0.21 & 0.09 & 0.39 & 0.002 \\
\hline
\end{tabular}

* = not significant.

There is a significant positive correlation between $\mathrm{Cb80}$ and weight, but this is clearly due to changes in RHO as there does not appear to be correlation between weight and Cmin. These correlations are a little stronger when expressed in terms of the body mass index (BMIX), but again there is no correlation evident between this parameter and Cmin. A feature of the single energy analysis [189] was a failure to demonstrate any correlation between the single energy bone measurement and exercise. It can be seen from table 5.35 why this is so: there is a significant positive correlation between Cmin 
and exercise, but there is also a negative correlation between RHO and exercise which approaches, but does not reach, the $5 \%$ level of significance. The single energy measurement does not detect the increase in mineral due to exercise because this is counterbalanced by a decrease in marrow density. Although there is a weak negative correlation between $\mathrm{Cmin}$ and $\mathrm{RHO}$ both for the controls and for the patients $\left(r_{S} \approx-0.2\right)$ this does not account for the trends observed in respect of exercise: to do so would require $r_{S} \approx-1$. There are significant positive correlations between the duration of high calcium intake during adolescence and both $\mathrm{Cb} 80$ and $\mathrm{Cmin}$. This parameter does not correlate with RHO, which is a reasonable finding because of the three bone measurements RHO is the least sensitive to changes in bone mineral.

The variable DURAN, which is the total length of time for which each patient had suffered anorexia nervosa, correlated strongly with both $\mathrm{Cb} 80$ and $\mathrm{Cmin}$. However it did not correlate significantly with Cmin, a somewhat unexpected finding since there is a significant difference in the average level of Cmin between the patient group and the controls. Perhaps any possible relationship between Cmin and DURAN is masked by other relationships (eg between Cmin and exercise) or by the reduced precision of the variable Cmin. The length of amenorrhoea immediately prior to $\mathrm{X}$-ray appears to correlate significantly with both $\mathrm{Cb} 80$ and RHO; however when the correlation is restricted to those patients who are currently not menstruating no such relationship is found except perhaps for RHO $\left(r_{S}=-0.3, p=0.09\right)$. The 'correlations' found in the whole group are merely reflecting the differences between the subgroup who are currently menstruating and the other subgroup who are not. A similar comment applies to the variable MENSPX which is a measure of the length of normal menstruation prior to $\mathrm{X}$ ray: there are significant correlations between this variable and both $\mathrm{Cb} 80$ and $\mathrm{RHO}$ when the patient group is treated as a single entity, but significant correlations are not found when the analysis is restricted to those patients who are currently menstruating. There are significant correlations between the age at first onset of dieting and RHO, and between age at first onset of anorexia nervosa and RHO.

All three bone measurements correlated significantly with the total duration of either anorexia nervosa or bulimia nervosa (ANBULT: see figure 5.17). The correlation coefficient for the relationship between ANBULT and RHO was half that for the relationship between the total duration of anorexia nervosa (DURAN) and RHO ( $r_{S}=0.5$ and 0.25 respectively), suggesting that anorexia nervosa may be a more significant influence on cancellous tissue density than bulimia nervosa. On the other hand there is a significant relationship between ANBULT and Cmin $\left(r_{S}=0.26\right)$, but there was only a weak and not statistically significant relationship between DURAN and Cmin. It is possible that this is a consequence of the reduced precision of $\mathrm{Cmin}$ : the values of ANBULT are somewhat greater than the values of DURAN, and it is possible that this increased amount of 'signal' is sufficient to counteract the increased 'noise' associated 
with Cmin. However the difference between the mean values of DURAN and ANBULT is not particularly large (the mean of ANBULT is $36 \%$ greater), and it may be that bulimia nervosa has a stronger effect on the mineral content than anorexia nervosa.
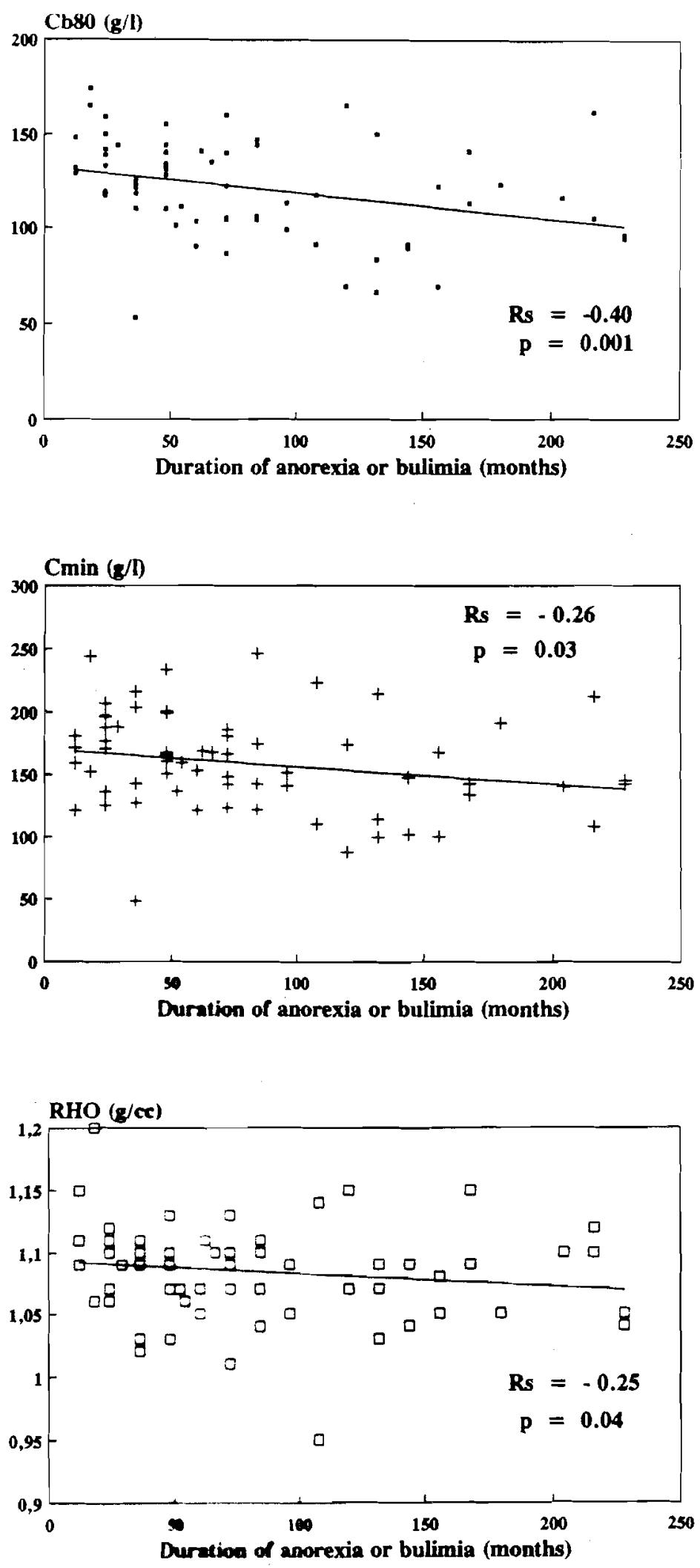

Figure 5.17: Correlations of Bone Measurements with ANBULT. 
Although there were apparent correlations between the duration of low weight immediately prior to X-ray and some of the bone measurements, these did not remain when the analysis was restricted to only those patients who were of low weight at X-ray. Total duration of low weight correlated moderately with $\mathrm{Cb} 80$ and RHO, but there was no evidence of a correlation between total length of low BMI and Cmin.

Finally, in table 5.36 comparisons are made between the bone measurements on those patients who are currently menstruating and those who are not currently menstruating. The subgroup who are currently menstruating have significantly larger average values of $\mathrm{Cb} 80$ and $\mathrm{RHO}$, but the difference in Cmin is small and not statistically significant. In section 5.3 it was shown that the difference in Cmin between the total patient group and the controls was of the same percentage as the difference in $\mathrm{Cb} 80$, suggesting that the differences between the two groups detected by single energy C.T. were reflecting true loss of bone mineral and was not due to reduced density of the marrow alone. The absence of a significant difference between the $\mathrm{Cmin}$ values for the menstruating / not menstruating subgroups of the patients suggests that there is little or no recovery of mineral in the cancellous bone tissue once anorexia nervosa ceases.

Table 5.36: Comparison Between Two Patient Groups with a History of Anorexia Nervosa.

\begin{tabular}{|l|ccc||}
\hline & \multicolumn{4}{|c|}{ Mean values of $\ldots$} & \\
& Cb80 & Cmin & RHO \\
\hline Not menstruating & 113.2 & 157.3 & 1.071 \\
Currently menstruating & 128.7 & 161.0 & 1.097 \\
Wilcoxon probabilities & 0.03 & 0.95 & 0.003 \\
\hline
\end{tabular}

In Hay et al [189] the patient group was divided into three subgroups according to the degree of recovery from anorexia nervosa, following the classification scheme of Morgan \& Russell [193]. Mean values of the bone measurements of the three groups are given in table 5.37, along with the Wilcoxon probabilities that the differences between the 'good' and 'poor' groups are due to chance. 
Table 5.37: Comparison of Bone Measurements Between Different Recovery Groups.

\begin{tabular}{||l|ccc||}
\hline $\begin{array}{l}\text { Recovery } \\
\text { Category }\end{array}$ & Cb80 & Cmin & RHO \\
\hline Good & 137.2 & 165.6 & 1.109 \\
Intermediate & 117.9 & 156.3 & 1.081 \\
Poor & 111.5 & 156.6 & 1.069 \\
Probability & $1 \times 10^{-3}$ & 0.43 & $5 \times 10^{-5}$ \\
\hline
\end{tabular}

There are highly significant differences between the 'good' and 'poor' recovery categories in respect of $\mathrm{Cb80}$ and $\mathrm{RHO}$. There were also significant differences between the 'good' and 'intermediate' groups in respect of Cb80 $(\mathrm{p}=0.01)$, and in respect of RHO ( $p=0.01$ ). The small difference between the average values of $\mathrm{Cmin}$ for the 'good' and 'poor' groups was not statistically significant. It is clear that changes taking place in the marrow due to anorexia nervosa are to some extent reversible, however the above tests have failed to establish any recovery of the mineral component and it would appear that if such recovery does occur its extent is small. Treasure et al [194] found that a group of 25 recovered anorexics had the same spine mineral density (as measured with a dual photon absorptiometer) as did a group of 20 age-matched controls, from which they concluded that the bone changes due to anorexia nervosa were reversible. It is possible that their readings have been affected by differences in the amount of extra-vertebral fat in the two groups (see [172]), but assuming that their finding is correct it would appear that the recovery is principally due to increases in cortical bone which has only a minor effect on the strength of the vertebral body.

\subsection{Reprise.}

The formalism presented in chapter two has been used to develop a very simple, yet precise, method for computing electron densities from dual energy C.T. images. The method uses an empirical relationship between the ratio of linear attenuation coefficients at two different energies and the effective atomic number of a material. Since an empirical relationship can also be determined between effective atomic number and the cross section per electron, it is possible to combine these two relationships to give a direct empirical relationship between the ratio of attenuation coefficients and the electronic cross section. The volume electron density, which is the parameter required for treatment planning purposes, may then be computed from equation (5.3). This dual energy technique is shown to yield the volume electron density with an accuracy of $5 \%$ for soft tissues, of $3 \%$ for cancellous bone and $8 \%$ for cortical bone. The use of single energy techniques 
involves the assumption of a single value for the electronic cross section for all soft tissues, and does not appear to compromise the accuracy significantly. Some authors extend the single energy technique to encompass tissues containing bone mineral by identifying all tissues with C.T. numbers greater than a certain value (eg 100) as bone and effectively assuming a single cross section per electron for bone tissue. However cancellous bone contains a large proportion of soft tissue and its effective atomic number of 9.5 is closer to that of soft tissue (7.5) than that of bone mineral (approximately 13), and it seems unlikely that the assumption of a single value for the electronic cross section for all bone tissue will give sufficiently accurate results. It is recommended that dual energy techniques be used for treatment planning in those situations where the treatment beam must traverse bone tissue.

Spine mineral measurements on some 31 volunteer women between the ages of 20 and 40 have been used as a control group for a study of the effects of anorexia nervosa on bone mass, and have been used to provide a range of normal values against which to compare clinical measurements made with the Wellington Hospital C.T. scanner. The mean value for single energy measurements made on this group is of similar magnitude to values reported by workers in Australia, the United Kingdom and in New York. The mean value for the cancellous tissue density $(1.092 \mathrm{~g} / \mathrm{cc})$ is comparable to a mean value for excised cancellous tissue measurements reported by the ICRP [117] of $1.08 \mathrm{~g} / \mathrm{cc}$. The mean values of the two single energy measurements, $\mathrm{Cb} 80$ and $\mathrm{Cb} 120$, for the patients with a history of anorexia nervosa $(\mathrm{N}=68$ ) were $18 \%$ lower than were the comparable figures for the controls. However these figures alone do not prove that bone mineral is lost in anorexia nervosa because such changes could have been due solely to changes occurring within the marrow component of the vertebral body. However the mean value of the dual energy mineral measurement, Cmin, was some $19 \%$ lower in the patient group than in the controls confirming that the change detected by the single energy technique genuinely involves both components, and that the loss of mineral is of the same magnitude as the reduction indicated by the single energy measurements. Although there were apparent negative correlations between the bone measurements of the control group and the ages of the subjects, these correlations were not statistically significant. In the patient group there were significant negative correlations between the two single energy measurements and age, and between cancellous tissue density and age.

The standard deviations of the two single energy measurements on the controls were slightly less than that of the dual energy mineral measurement on the same group. The standard deviation of the dual energy measurement of cancellous tissue density, RHO, was some $50 \%$ greater in absolute terms, but very much smaller when expressed as a percentage of the mean value. The standard deviations of the bone measurements of the patients with a history of anorexia nervosa were comparable except in the case of Cmin. The standard deviation of $\mathrm{Cmin}$ for the patient group was $50 \%$ greater than the 
comparable figure for the controls.

A striking feature of the patient measurements has been that while the values for adjacent vertebrae may be very similar in some patients, there have been a number of patients in which large differences have been observed between measurements in neighbouring vertebrae. This effect has been quantitated by computing the coefficient of variation (standard deviation divided by the mean, expressed as a percentage) of the values for the three vertebrae measured in each patient. The coefficient of variation for the single energy measurements was typically $5 \%$ for the controls and $7 \%$ for the patient group. The average coefficient of variation for RHO was $3 \%$ for both groups, but the average value for the coefficient of variation of $\mathrm{Cmin}$ was much greater at $17 \%$ for the controls and $19 \%$ for the patient group, suggesting that the bone mineral is not evenly distributed. However it would appear that the distribution normally becomes more uniform with age since there was a significant negative correlation between the coefficient of variation of $\mathrm{Cmin}$ and age for the controls. This is not the case for the patients: there is a positive correlation between the coefficients of variation (CV's) of $\mathrm{Cb} 120, \mathrm{Cmin}$ and RHO with age, implying that the bone loss is not uniform. The existence of negative correlations between the CV's of $\mathrm{Cb} 80, \mathrm{Cb} 120$ and $\mathrm{Cmin}$ and the bone measurements themselves is also suggestive of localised rather than uniform bone loss. This being so, the question arises as to whether the loss tends to be greater in one particular vertebra in all cases, eg L2. Comparisons of bone measurements in L2, L3 and L4 do not support this contention.

Previous studies have established a link between anorexia nervosa and low bone mass. In the present study attempts have been made to determine the effects of a number of relevant variables which characterise the severity of the disease such as body mass index, duration of amenorrhoea, duration of vomiting and diarrhoea, and duration of low calcium intake both at time of examination and in the bone-forming years of adolescence. Also included have been a number of factors which might tend to counteract any bone loss due to anorexia nervosa: use of estrogen based contraceptive pills, and exercise. The results of correlations between the clinical variables and the single energy measurement $\mathrm{Cb} 80$ have already been published [189]. The present author has extended the analysis to include the dual energy measurements $\mathrm{Cmin}$ and RHO as well. The strongest correlations found in the present work were between body mass index (BMI) at the time of examination and the cancellous tissue density $\left(r_{S}=0.4\right.$ for the controls and $r_{S}=0.5$ for the patient group). Correlations of this magnitude may be regarded as strong in the present context where a relatively large number of variables is involved. In the patient group RHO correlated almost as strongly with the total duration of anorexia $\left(r_{S}=-0.48, p=10^{-4}\right)$, a fact which is unsurprising since body mass index and duration of anorexia nervosa are themselves well correlated $\left(r_{s}=-0.63, p=3 x\right.$ $10^{-7}$ ). Cb80 correlated almost as well with duration of anorexia, but there was only 
weak and non-significant correlation between this variable and Cmin. However this does not mean that bone mineral is not lost in anorexia nervosa: the mean value of Cmin for the whole patient group shows the same decrease with respect to the controls as was indicated by the single energy measurements. A more likely explanation is either that the lower precision associated with $\mathrm{Cmin}$ is masking the effect of anorexia, or that other clinical variables such as exercise are having a counterbalancing effect. When the total duration of both anorexia and bulimia nervosa is considered, significant negative correlations are found with $\mathrm{Cb} 80, \mathrm{Cmin}$ and RHO.

The single energy analysis did not show a significant relationship between exercise and bone mass. It is clear in the present work that this was because a significant positive correlation between $\mathrm{Cmin}$ and exercise at the time of $\mathrm{X}$-ray was masked by a negative correlation between RHO and exercise $\left(r_{s}=-0.22, p=0.07\right)$. There has been no evidence in the present work of a protective effect from the use of estrogen contraceptive pills, but the duration of high calcium intake in adolescence gave significant positive correlations with $\mathrm{Cb} 80$ and $\mathrm{Cmin}$.

The work of Treasure et al [194], based on DPA measurements in the femur and lumbar spine, has suggested that bone loss due to anorexia nervosa is reversible. This conclusion was based on the fact that their group of recovered anorexics had greater bone mass than those who were amenorrheic at the time of examination. However although the recovered group in that study had the same median duration of amenorrhoea as the amenorrheic group, the duration of illness in the recovered group was significantly less than in the other group and it may be this factor, rather than reversal of bone loss, which is the cause of the difference in bone mass between the two groups. In the present work it was found that those patients in the 'good' recovery category had significantly higher Cb80 values than those on the 'poor' recovery categories. The same finding was noted in respect of the RHO values, but in the case of Cmin there was only a small and not significant difference between the patients in the two categories. The findings in respect of $\mathrm{Cb80}$ and RHO suggest that some recovery may take place, although the present study has the same problem as that of Treasure in that the patients in the 'poor' recovery category had shorter illness durations than those in the 'good' recovery category. However the findings of the present study in respect of Cmin show no evidence of recovery of lost bone mineral following anorexia nervosa. 


\section{CHAPTER SIX: CORRELATION OF C.T. IMAGES WITH BONE STRUCTURE.}

\subsection{Introduction.}

Although computed tomography is a very sensitive technique for monitoring changes in the degree of mineralisation of the spine [174], it is nonetheless clear that single energy C.T. can only identify groups of patients who are at increased risk of fracture: it cannot predict which individuals will suffer fractures and which will not. There is a large degree of overlap between the single energy measurements of patients who have not suffered fractures and those who have [164]. While dual energy mineral measurements, being more sensitive to changes in the mineral and relatively insensitive to concurrent changes in the status of the marrow, may prove to be better predictors of fracture, studies have so far failed to demonstrate this [120]. If the mineral concentration is not a good predictor of fracture then it is possible that its arrangement within the vertebral body is an important factor. A vertebra with a lesser amount of bone mineral which is arranged in a structurally effective way may be stronger than one with more mineral arranged less effectively. When viewing patient films it is noticeable that the texture of the image in the cancellous region of the vertebral body is quite variable. In some patients the appearance is quite smooth; in others the appearance is grainy with relatively large radiolucent (dark) areas which presumably indicate voids in the trabecular structure. The aim of the work described in this chapter has been to correlate the appearance of the C.T. images with the structural parameters of the bones being imaged. To this end a phantom has been designed to approximate the shape of the human abdomen, with a removable platform to support excised human vertebrae at a position representative of that of the spine in living humans. The platform contains a marker which is visible on the C.T. images and permits the vertebrae to be subsequently sectioned with a rotating diamond saw blade in the same positions as the C.T. images. These sections can then be imaged using a scanning electron microscope to determine bone structure. However it has first been necessary to perform a number of preliminary investigations to confirm the feasibility of the technique prior to obtaining ethical approval to work with human tissue.

In the next section the structure of human cancellous tissue is described, along with the processes which are concerned with the remodelling of the trabeculae throughout adult life. The following section describes semi-quantitative attempts to describe the texture of the C.T. images using a grading scheme, relating the findings to other bone measurements. Current C.T. techniques use the mean C.T. number in the cancellous region of the vertebral body to estimate mineral concentration. All C.T. scanners also report the standard deviation of the pixel values in the region of interest. In section 6.4 
formed from collagen fibres which are aligned parallel with each other. This is in contradistinction to woven bone in which the collagen fibres have quite random orientations. Woven bone is formed after fractures, and is eventually replaced by lamellar bone following remodelling.

Bone remodelling is a process by which in adult humans bone tissue is renewed to prevent the accumulation of fatigue damage [198]. The process involves the erosion of old bone tissue (bone resorption) followed by the formation of new bone substance. Net bone gain or loss over time depends on the balance between the amount of bone material lost in the resorption process and the amount replaced in the bone formation process. Up to $20 \%$ of free bone surfaces are undergoing remodelling at any one time. In the cortex the bone remodelling unit is the Haversian system (also called the secondary osteon [196]). In humans a completed secondary osteon forms a cylinder of lamellar bone about 200 to $250 \mu \mathrm{m}$ in diameter. There is a central canal, typically $50 \mu \mathrm{m}$ in diameter, which contains blood vessels and nerves. The corresponding bone structural unit in trabecular bone is called the trabecular osteon and is a crescent-shaped structure on the surface of the trabecula, approximately $600 \mu \mathrm{m}$ long and $60 \mu \mathrm{m}$ deep at the centre. The formation of the trabecular osteon, which takes place over four to eight months, is described below.

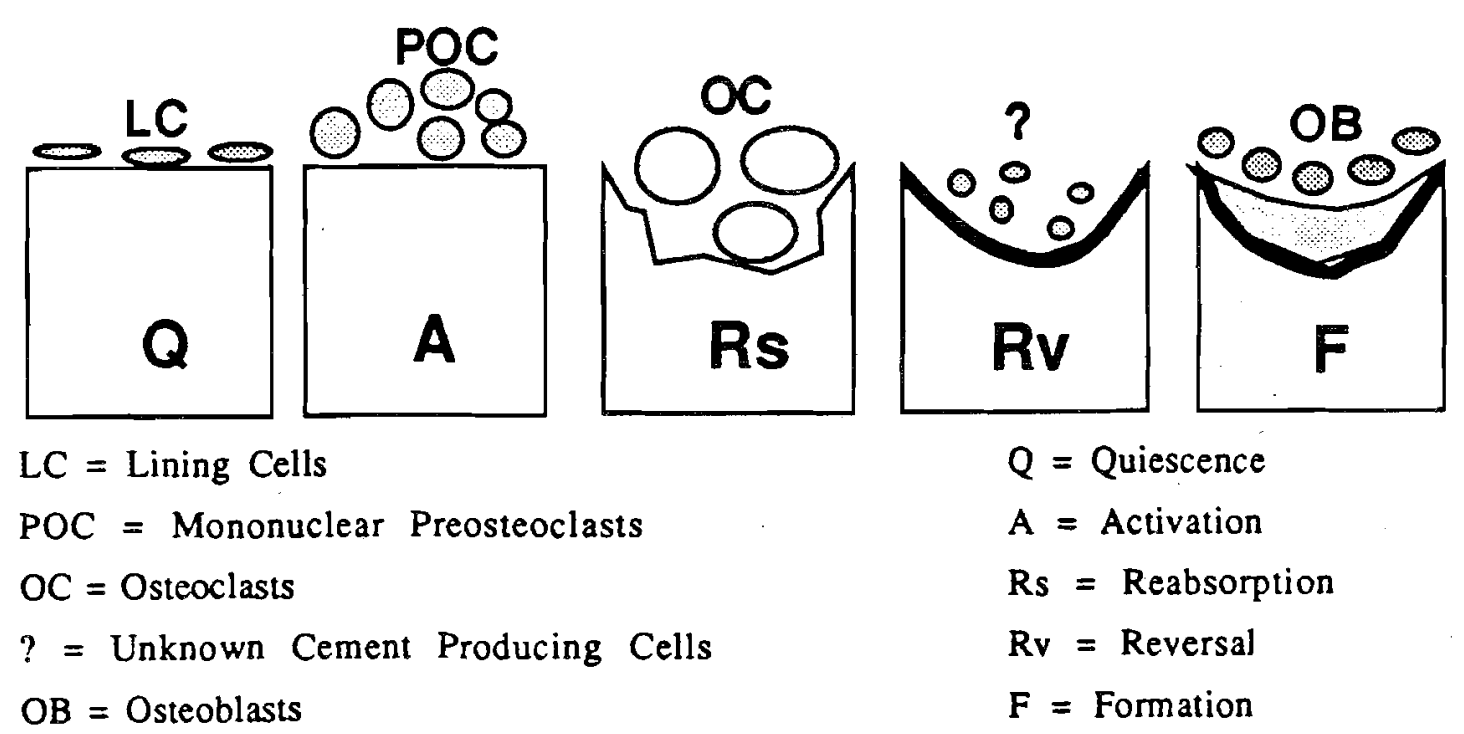

Figure 6.3: Bone Remodelling Sequence.

In its quiescent state the surface of the trabecula is protected by lining cells. In the activation stage cells called preosteoclasts penetrate the layer of lining cells and then undergo fusion into osteoclasts which in the resorption stage erode a cavity called a Howship's lacuna. In the reversal stage cells of unknown origin smooth off the surface of the Howship's lacuna and lay down a cement line on which the osteoblasts lay down the new bone in the formation stage. The cycle is completed when lining cells cover the surface of the new bone. Reprinted from Golder [199], with permission. 
vertebrae. The $120 \mathrm{kV}$ images were selected for classification in preference to the $80 \mathrm{kV}$ images since the photon flux was much greater at the higher kilovoltage and the image exhibited less quantum mottle. Any grainy appearance due to either the ' $\mathrm{Y}$ ' structure or the stellate pattern was not permitted to contribute to the granularity grading. A difficulty encountered was that not all films had been taken at the same window and level settings and some films were noticeably darker than others. Efforts were made to grade the films independently of any differences due to this effect. The distribution of the granularity grading for the full sample is illustrated in figure 6.7. The distributions of the ' $\mathrm{Y}$ ' and stellate gradings are given in figures 6.8 and 6.9 respectively.

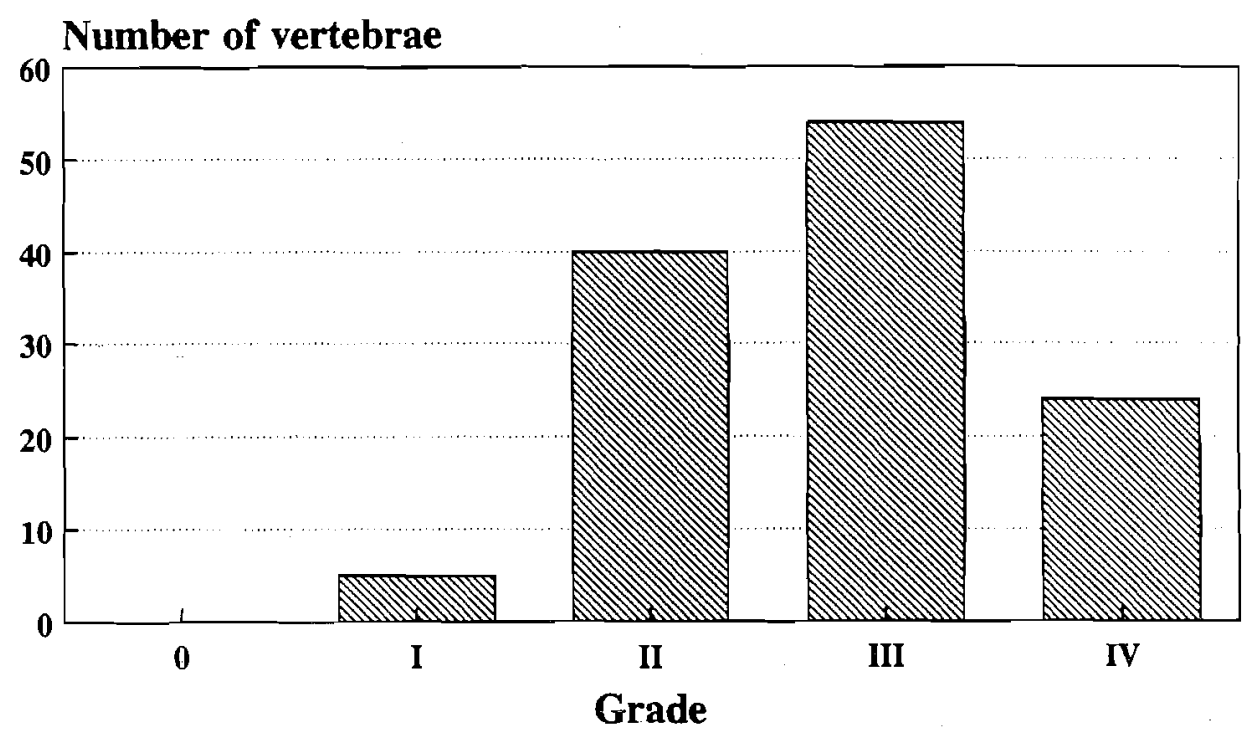

GRANULARITY

Figure 6.7: Distribution of Granularity Classifications.

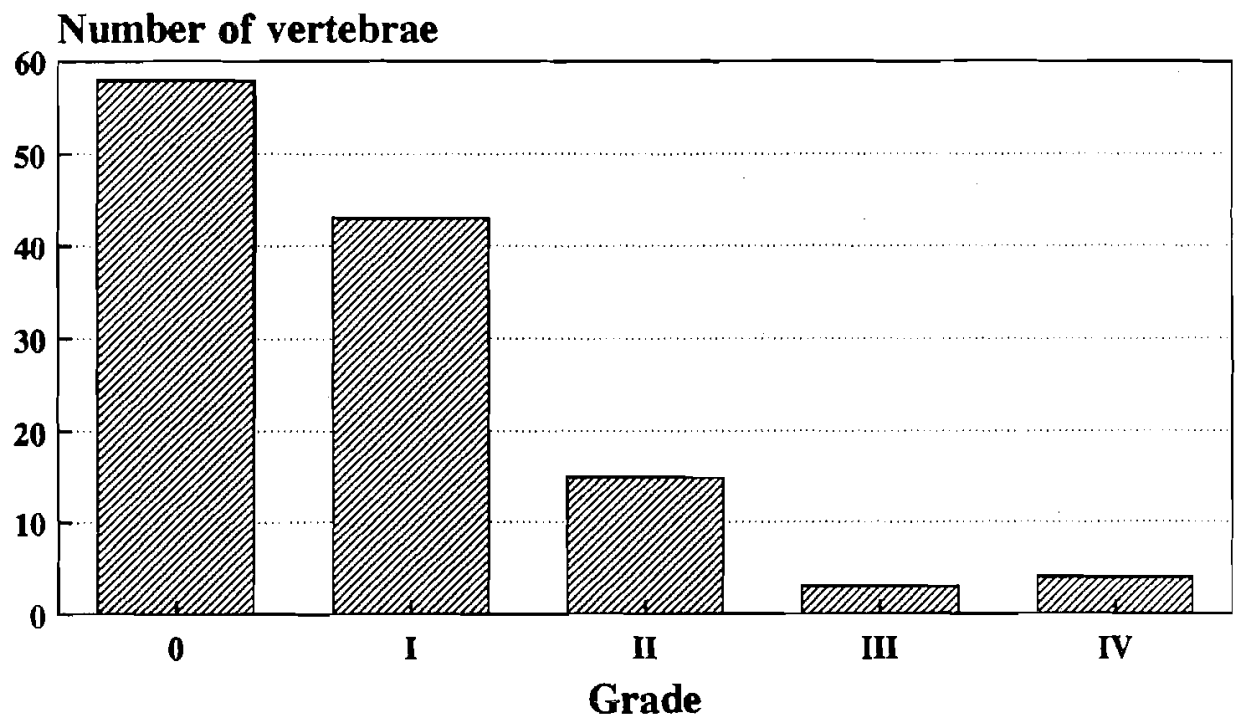

WIIT 'Y' STRUCTURE

Figure 6.8: Distribution of ' $Y$ ' Structure Classifications. 


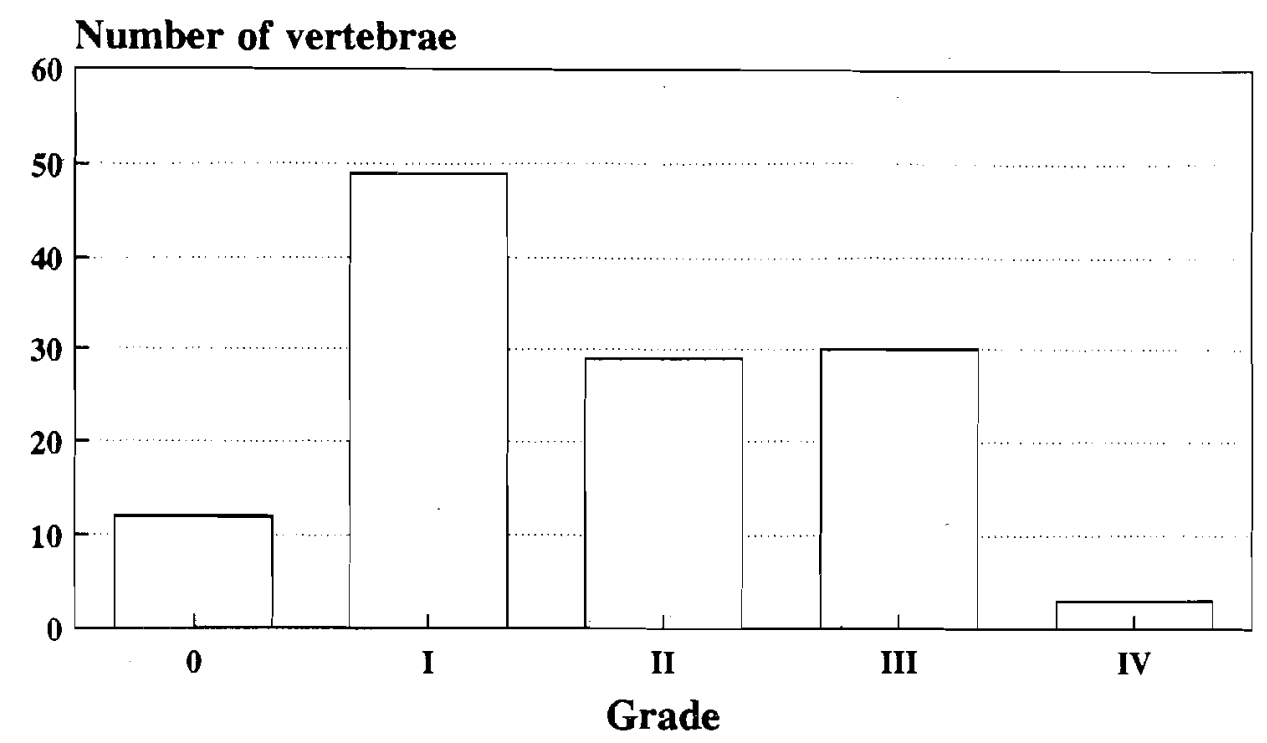

STELLATE PATTERN

Figure 6.9: Distribution of Stellate Pattern Classifications.

The median grading for granularity for all vertebrae was grade III, ' $Y$ ' structure was much less prevalent with a median grading of $\mathrm{I}$, and the median grading for stellate pattern was grade II. Wilcoxon tests did not show any significant differences in ' $\mathrm{Y}$ ' or stellate structure between L2, L3 and L4. There is a trend of increasing granularity going down the spine from L2 through to LA but this could be due to chance: a Wilcoxon test applied to the granularity readings of $\mathrm{L} 2$ and $\mathrm{L} 4$ yielded a $\mathrm{p}$ value of 0.10 . A separate but related issue is whether the morphological parameters are characteristic of the patient or whether they relate only to the vertebra measured. Correlations between the values of the morphological variables for the three vertebrae are listed in table 6.1, which also gives the probabilities that the observed correlations are due to chance.

Table 6.1: Correlations Between Morphological Variables on Different Vertebrae.

\begin{tabular}{|l|l|l|}
\hline Granularity & 'Y' structure & stellate pattern \\
\hline
\end{tabular}

\section{Spearman Rank Correlation Coefficients}

\begin{tabular}{|ccc|ccc|ccc|}
\hline & G3 & G4 & & Y3 & Y4 & & S3 & S4 \\
G2 & 0.80 & 0.66 & Y2 & 0.43 & 0.76 & s2 & 0.50 & 0.24 \\
G3 & & 0.73 & Y3 & & 0.40 & S3 & 0.56 \\
\hline
\end{tabular}

\section{Probabilities}

\begin{tabular}{|c|c|c|c|c|c|c|c|c|}
\hline & G3 & G4 & & Y3 & Y4 & & s3 & s4 \\
\hline G2 & $<10^{-6}$ & $<10^{-4}$ & Y2 & $<10^{-2}$ & $<10^{-6}$ & s2 & $<10^{-2}$ & 0.14 \\
\hline G3 & & $<10^{-5}$ & Y3 & & 0.01 & s3 & & $<10^{-3}$ \\
\hline
\end{tabular}


There are highly significant correlations between the granularity gradings of L2, L3 and L4 suggesting that granularity is a variable whose significance is not confined to the vertebra on which it is assessed. There were lesser correlations between the ' $Y$ ' structure gradings of the different vertebrae, but the correlations were still highly significant. A similar comment applies to the stellate gradings. The strength of these correlations indicates that these gradings are reflecting real characteristics of the bones being examined. To determine whether the morphological variables are truly independent, and not simply different measures of the same underlying phenomenon, correlations were made between the three variables for each of the three vertebrae.

Table 6.2: Correlations Between Morphological Variables for Each Vertebra.

\begin{tabular}{|l|l|l|}
\hline L2 & L3 & L4 \\
\hline
\end{tabular}

Spearman Rank Correlation Coefficients

\begin{tabular}{|ccc|ccc|ccc|}
\hline & Y2 & S2 & & Y3 & S3 & & Y4 & S4 \\
G2 & 0.09 & 0.40 & G3 & -0.03 & 0.36 & G4 & 0.22 & 0.46 \\
Y2 & & 0.44 & Y3 & & 0.13 & Y4 & & 0.29 \\
\hline
\end{tabular}

Probabilities

\begin{tabular}{|ccc|ccc|ccc|}
\hline & Y2 & S2 & & Y3 & S3 & & Y4 & S4 \\
G2 & 0.55 & 0.01 & G3 & 0.87 & 0.02 & G4 & 0.16 & $<0.01$ \\
$Y 2$ & & $<0.01$ & Y3 & & 0.40 & $Y 4$ & & 0.07 \\
\hline
\end{tabular}

Granularity and ' $\mathrm{Y}$ ' pattern are clearly either unrelated or very weakly related. There is a highly significant relationship between granularity and the stellate pattern, but the correlation coefficients are not so high as to suggest that they are in reality the same variable. There appears to be a weak relationship between ' $\mathrm{Y}$ ' pattern and the stellate pattern, although the correlation was significant only for the readings of L2.

The degree of correlation between the morphological variables and the bone measurements has been determined, the results being given in table 6.3. There is a tendency for increased granularity to be associated with lower values of $\mathrm{Cb} 120$, perhaps indicating that the thinning of the bones is patchy, but the correlation is weak and only achieves the $5 \%$ level of significance in the case of L2. Since the correlations between granularity and Cmin are very weak it would seem that the previous correlations with $\mathrm{Cb} 120$ are more likely to be caused by fluctuations in density rather than fluctuations in the bone mineral itself. The ' $\mathrm{Y}$ ' pattern is associated with higher values of $\mathrm{Cb} 120$ 
Table 6.3: Correlations Between Morphological Variables and Bone Measurements.

\begin{tabular}{|l|llllllllll|}
\hline & G2 & Y2 & S2 & G3 & Y3 & S3 & G4 & Y4 & S4 \\
\hline
\end{tabular}

Spearman Rank Correlation Coefficients

\begin{tabular}{|l|rrrrrrrrrr|}
\hline Cb120 & -0.33 & 0.61 & 0.10 & -0.25 & 0.09 & 0.35 & -0.20 & 0.36 & 0.18 \\
RHO & -0.33 & 0.39 & 0.13 & -0.26 & -0.07 & 0.09 & -0.22 & 0.29 & -0.11 \\
Cmin & -0.16 & 0.58 & 0.14 & -0.06 & 0.19 & 0.47 & -0.02 & 0.32 & 0.39 \\
120SD & 0.11 & 0.28 & 0.44 & 0.17 & 0.12 & 0.25 & 0.18 & 0.10 & -0.08 \\
\hline
\end{tabular}

\section{Probabilities}

\begin{tabular}{|l|rrrrrrrrr|}
\hline $\mathrm{Cb} 120$ & 0.04 & $10^{-4}$ & 0.52 & 0.11 & 0.58 & 0.03 & 0.20 & 0.02 & 0.25 \\
$\mathrm{RHO}$ & 0.03 & 0.01 & 0.43 & 0.10 & 0.67 & 0.58 & 0.16 & 0.07 & 0.48 \\
$\mathrm{Cmin}$ & 0.31 & $<10^{-3}$ & 0.37 & 0.70 & 0.24 & $<0.01$ & 0.91 & 0.04 & 0.01 \\
$\mathrm{120SD}$ & 0.50 & 0.08 & $<0.01$ & 0.27 & 0.45 & 0.12 & 0.26 & 0.51 & 0.60 \\
\hline
\end{tabular}

(significant correlations in the cases of L2 and L4) but again the correlation coefficients are not large, and the probabilities are at the threshold of statistical significance. In the cases of L2 and L4, greater visibility of the ' $Y$ ' pattern appears to be associated with both mineral content and total cancellous tissue density, with mineral content perhaps being the more important factor. The presence of the stellate pattern is weakly correlated in the positive sense with Cmin. With the exception of the stellate pattern for L2 (S2), the pixel standard deviation at $120 \mathrm{kV}$ (denoted 120SD in the above table) did not correlate with the morphological variables for any of the individual vertebrae. However it was noted that the standard deviation readings increased going down the spine. The differences between the standard deviations of L3 and L2 were not statistically significant, but those between L4 and L3 (Wilcoxon $p=0.01$ ) and between L4 and L2 ( $p=0.003$ ) were definitely significant.

As a by product of the above exercise, the bone readings stored in the computer have been used to determine whether any simple empirical relationship exists between the two single energy measurements, $\mathrm{Cb} 80$ and $\mathrm{Cb} 120$, and each of the dual energy measurements Cmin and RHO. Program MULTREG (see appendix I) was used to perform multiple regression between each dual energy measurement and the two single energy ones. For the equation below, in which $\mathrm{Z}$ may represent either $\mathrm{Cmin}$ or $\mathrm{RHO}$, the regression parameters listed in table 6.4 were obtained.

$$
z=a_{0}+a_{1} \cdot C b 120+a_{2} \cdot C b 80
$$


Table 6.4: Multiple Regression Parameters.

\begin{tabular}{||cccccc||}
\hline z & Vertebra & $\mathrm{a}_{0}$ & $\mathrm{a}_{1}$ & $\mathrm{a}_{2}$ & Rmult \\
\hline Cmin & L2 & 29.4 & -3.2 & 4.3 & 0.982 \\
Cmin & L3 & 28.2 & -3.7 & 4.8 & 0.979 \\
Cmin & L4 & 27.3 & -3.6 & 4.7 & 0.976 \\
RHO & L2 & 1.007 & 0.0040 & -0.0034 & 0.967 \\
RHO & L3 & 1.008 & 0.0046 & -0.0040 & 0.961 \\
RHO & L4 & 1.009 & 0.0043 & -0.0037 & 0.960 \\
\hline \hline
\end{tabular}

In the above Rmult is the multiple linear regression coefficient. Equations of the form of (6.1) can be used to compute the dual energy measurements from the single energy ones with reasonable accuracy, but the use of equation (4.11) is still to be preferred. The values listed in table 6.4 do however show why the uncertainties associated with the dual energy mineral measurement, Cmin, are relatively large: it is obtained from the difference between the two single energy measurements. The small offset value, $a_{0}$, and the fact that the scaling factors $a_{1}$ and $a_{2}$ are of comparable magnitude, constitute near optimal conditions for the propagation of uncertainties in $\mathrm{Cb} 80$ and $\mathrm{Cb} 120$ through to uncertainties in the computed value of $\mathrm{Cmin}$.

\subsection{Pixel distributions.}

Histograms of C.T. numbers have been successfully used to estimate the percentage of fat in sheep [202]. Software developed for this purpose has been written in Christchurch for the G.E. 8800 and has been made available to us. The program has been modified to suit the needs of the present work. The changes are described in appendix $K$, the major modification being the addition of a subroutine to compute the distribution parameters; namely mean, standard deviation, moment coefficient of skew and moment coefficient of kurtosis. An example of a histogram of the C.T. numbers in the cancellous region is given in figure 6.10. 
Table 6.5: Single Energy Distributions for Various Patient Groups.

\begin{tabular}{|c|c|c|c|c|c|c|c|c|}
\hline \multirow{2}{*}{ I.D. } & \multicolumn{4}{|c|}{ CB80 } & \multicolumn{4}{|c|}{ CB120 } \\
\hline & mean & SD & skew & kurt & mean & SD & skew & kurt \\
\hline & & & & 'Norm & & & & \\
\hline A & 118 & 24 & -.3 & 3.4 & 119 & 23 & -.3 & 3.4 \\
\hline B & 132 & 21 & -.3 & 3.0 & 136 & 21 & & 3.0 \\
\hline C & 113 & 25 & -.2 & 3.3 & 115 & 26 & .1 & 3.4 \\
\hline D & 92 & 25 & .2 & 3.1 & 94 & 24 & .0 & 3.2 \\
\hline Avrg. & 114 & 24 & -0.2 & 3.2 & 116 & 24 & -0.1 & 3.3 \\
\hline
\end{tabular}

Anorexia

\begin{tabular}{|lrrrrrrrr|}
\hline E1 & 80 & 22 & -.4 & 3.4 & 78 & 24 & -.4 & 3.3 \\
E2 & 86 & 21 & -.4 & 3.3 & 92 & 21 & -.5 & 3.4 \\
F1 & 66 & 25 & .0 & 3.4 & 65 & 26 & -.0 & 3.2 \\
F2 & 86 & 26 & .2 & 3.3 & 83 & 24 & .2 & 3.5 \\
G & 141 & 29 & .0 & 3.0 & 143 & 29 & .0 & 2.8 \\
Avrg. & 92 & 25 & 0.1 & 3.3 & 92 & 25 & 0.1 & 3.2 \\
\hline
\end{tabular}

Glucocorticoids

\begin{tabular}{|lrrrrrrrr|}
\hline H & 108 & 24 & -.3 & 3.2 & 107 & 21 & -.2 & 3.2 \\
I & 11 & 23 & .1 & 3.3 & 7 & 20 & .1 & 3.3 \\
J1 & 80 & 38 & .1 & 3.1 & 71 & 28 & -.0 & 3.5 \\
J2 & 78 & 33 & -.1 & 3.0 & 82 & 25 & .1 & 2.9 \\
K & 87 & 28 & .3 & 3.1 & 96 & 31 & .2 & 2.9 \\
Avrg. & 73 & 29 & 0.0 & 3.1 & 73 & 25 & 0.0 & 3.2 \\
\hline
\end{tabular}

Osteoporosis

\begin{tabular}{|lrrrrrrrr|}
\hline $\mathrm{L}$ & 60 & 25 & -.1 & 2.9 & 62 & 23 & .0 & 3.0 \\
$\mathrm{M}$ & 95 & 22 & -.1 & 3.1 & 92 & 21 & .0 & 2.9 \\
$\mathrm{~N}$ & 22 & 20 & .3 & 2.9 & 26 & 19 & .2 & 2.8 \\
$\mathrm{O}$ & 60 & 25 & -.1 & 3.0 & 54 & 24 & .1 & 2.8 \\
Avrg. & 59 & 23 & 0.0 & 3.0 & 59 & 22 & 0.1 & 2.9 \\
\hline
\end{tabular}

Pituitary

\begin{tabular}{|lllrlllll|}
\hline $\mathrm{P}$ & 93 & 29 & .1 & 2.7 & 95 & 28 & .0 & 2.7 \\
$\mathrm{Q}$ & 77 & 29 & -.0 & 2.9 & 82 & 26 & .1 & 2.9 \\
Avrg. & 85 & 29 & 0.1 & 2.8 & 89 & 27 & 0.1 & 2.8 \\
\hline
\end{tabular}


Table 6.6: Dual Energy Distributions for Various Patient Groups.

\begin{tabular}{|c|c|c|c|c|c|c|c|c|}
\hline \multirow{2}{*}{ I.D. } & \multicolumn{4}{|c|}{ RHO } & \multicolumn{4}{|c|}{ Cmin } \\
\hline & mean & SD & skew & kurt & mean & SD & skew & kurt \\
\hline . & & & & 'Norm & & & & \\
\hline A & 1.094 & 63 & -.0 & 2.8 & 147 & 65 & -.0 & 3.0 \\
\hline B & 1.097 & 51 & -.0 & 3.2 & 166 & 52 & -.1 & 3.0 \\
\hline C & 1.099 & 67 & .3 & 3.2 & 168 & 63 & -.1 & 2.9 \\
\hline D & 1.082 & 67 & -.1 & 3.0 & 115 & 68 & .1 & 3.0 \\
\hline Avrg. & 1.093 & 0.062 & 0 & 3.1 & 149 & 62 & 0 & 3.0 \\
\hline
\end{tabular}

Anorexia

\begin{tabular}{|cccccrrrr|}
\hline E1 & 1.073 & 48 & -.0 & 2.9 & 103 & 45 & -.1 & 3.1 \\
E2 & 1.085 & 50 & -.1 & 2.9 & 103 & 51 & .0 & 3.0 \\
F1 & 1.051 & 55 & .0 & 3.1 & 96 & 56 & -.0 & 2.9 \\
F2 & 1.060 & 73 & .0 & 3.3 & 122 & 80 & .0 & 3.1 \\
G & 1.125 & 58 & .1 & 2.9 & 157 & 60 & -.0 & 2.8 \\
Avrg. & 1.079 & 0.058 & 0 & 3.0 & 116 & 58 & 0 & 3.0 \\
\hline
\end{tabular}

Glucocorticoids

\begin{tabular}{|llrrrrrrr|}
\hline $\mathrm{H}$ & 1.084 & 68 & .1 & 3.0 & 139 & 74 & .1 & 3.0 \\
$\mathrm{I}$ & 0.958 & 71 & .0 & 3.0 & 97 & 74 & -.0 & 3.0 \\
$\mathrm{~J} 1$ & 1.029 & 117 & -.0 & 3.1 & 137 & 133 & .0 & 3.0 \\
$\mathrm{~J} 2$ & 1.080 & 104 & -.0 & 2.7 & 95 & 117 & .0 & 2.7 \\
$\mathrm{~K}$ & 1.106 & 67 & .1 & 3.0 & 90 & 56 & .1 & 3.3 \\
Avrg. & 1.051 & 0.085 & 0 & 3.0 & 112 & 91 & 0 & 3.0 \\
\hline
\end{tabular}

Osteoporosis

\begin{tabular}{|cccccrrrr|}
\hline $\mathrm{L}$ & 1.063 & 59 & -.0 & 3.0 & 79 & 63 & .0 & 2.9 \\
$\mathrm{M}$ & 1.07 & 72 & .0 & 3.2 & 189 & 74 & .1 & 3.3 \\
$\mathrm{~N}$ & 1.024 & 46 & .0 & 3.0 & 53 & 47 & -.0 & 2.9 \\
$\mathrm{O}$ & 1.027 & 69 & .0 & 2.8 & 108 & 75 & -.0 & 2.9 \\
Avrg. & 1.030 & 0.062 & 0 & 3.0 & 107 & 65 & 0 & 3.0 \\
\hline
\end{tabular}

Pituitary

\begin{tabular}{|cccrrrrrr|}
\hline $\mathrm{P}$ & 1.085 & 77 & .2 & 2.9 & 114 & 78 & -.0 & 2.9 \\
$\mathrm{Q}$ & 1.079 & 97 & -.0 & 2.8 & 92 & 100 & .0 & 2.8 \\
Avrg. & 1.082 & 0.087 & 0.1 & 2.9 & 103 & 89 & 0 & 2.9 \\
\hline
\end{tabular}

6.6. The patient group described as 'normal' consists of patients who were referred for examination on suspicion of having low bone mass, but whose results were found to be in 
the normal range. Other groups studied were ones with a history of anorexia nervosa, patients who were suffering from hypopituitarism, patients with osteoporosis, and patients who had had prolonged treatment with glucocorticoids for conditions such as asthma. Each number quoted in the tables is an average over the three vertebrae scanned on each patient.

There was no evidence of a bimodal distribution in any of the patients, despite the fact that some had very low bone mass, nor was there any suggestion that any of the distributions departed significantly from the Gaussian distribution. Apart from the means, which in any case are available without the necessity to determine histograms of pixel values, there appear to be no obvious differences between the distribution parameters of the different patient groups in respect of the single energy measurements. None of the single energy distributions exhibited a marked degree of skew, and the average standard deviation for each patient group was consistently at a level of approximately $25 \mathrm{~g} / 1$. All single energy distributions were approximately mesokurtic. In the case of the dual energy distributions there is also very little skew or departure from the degree of kurtosis found in the Gaussian distribution (moment coefficient of kurtosis = 3).

\subsection{Pilot Study on Sheep Bones.}

The aim of the present chapter has been to correlate the C.T. appearances of the vertebral body with its trabecular structure as determined by scanning electron microscopy (SEM). This involves scanning excised vertebrae in a phantom whose shape approximates that of the human abdomen, obtaining sections of the tissue scanned by C.T., and examining the sections using the SEM. This exercise was first carried out using sheep bones so that optimal techniques could be determined and the credibility of the proposed method could be established prior to ethical application to work with human tissues. The work described in the present section has been carried out in collaboration with the Department of Physiology and Anatomy, Massey University, who have been interested in the bones of sheep in their own right.

The dimensions of the C.T. phantom were based on the measurements taken from the C.T. films of the normal volunteer patients, as described in section 5.2. For ease of construction the mean measurements obtained were rounded to a lateral dimension of $30 \mathrm{~cm}$ and a vertical (AP) dimension of $20 \mathrm{~cm}$. The phantom was fabricated from a section of tubular perspex $20 \mathrm{~cm}$ in diameter. The tube was cut in half longitudinally and $10 \mathrm{~cm}$ sections of flat persex was inserted top and bottom between the two semicircular sections to give the correct lateral dimension. A removable section was placed in the bottom straight section with columns attached to support a removable base plate to 
pressurised water jet, and then to soak in approximately $1 \mathrm{M} \mathrm{NaOH}$ solution at $40{ }^{\circ} \mathrm{C}$ for 1 minute to remove any remaining soft tissues. The sections were then rinsed in water and finally soaked in acetone to speed up drying. The sections were then sputter-coated with gold and scanned in the electron microscope. The SEM images were stored in a SUN workstation and analysed using SEMPER software, version six.

A total of nine sheep were studied, three emaciated sheep and six which were normal. The sections imaged by C.T. were the mid sections of the fourth cervical vertebra, the third and eleventh thoracic vertebrae, and the second and fifth lumbar vertebrae. A further section was taken of L2 mid-way between the mid section and the superior (cranial) end plate. The results of the C.T. measurements have been reported by Golder [199]. Significant differences were found between the emaciated and normal sheep in respect of the single energy readings and in respect of the parameter RHO. Although the average values of $\mathrm{Cmin}$ for the normals were slightly higher than those for the emaciated sheep, the differences were not statistically significant. In table 6.7 comparison is made between the averages of the bone measurements taken in the middle of L2 and L5 for the normal sheep and average values for L2, L3 and L4 in humans:

Table 6.7: Comparison of Bone Measurements in Sheep and Humans.

\begin{tabular}{|c|c|c|c|c|}
\hline & & \multicolumn{2}{|c|}{ SHEEP } & \multirow{2}{*}{ HUMANS } \\
\hline & & emaciated & normal & \\
\hline $\mathrm{Cb} 120$ & $(g / 1)$ & 244 & 297 & 148 \\
\hline Cmin & $(g / 1)$ & 296 & 314 & 196 \\
\hline RHO & $(g / c c)$ & 1.20 & 1.25 & 1.09 \\
\hline
\end{tabular}

Even the emaciated sheep have substantially higher values of $\mathrm{Cb} 120, \mathrm{Cmin}$ and RHO than normal humans. When the SEM images were analysed using SEMPER it was found that the average percentage by volume of trabeculae (TBV) in the cancellous region of the sheep vertebral bodies was $32 \%$, which is substantially higher than in humans whose TBV is typically $15 \%$ [204]. The appearance of the SEM images of cancellous tissue in the sheep showed the trabeculae to be thicker in proportion to the size of the marrow spaces than in published SEM images of human cancellous bone [205]. In the sheep a higher proportion of the trabeculae were in the form of plates, rather than rods, than was the case in the humans. Unfortunately the C.T. images of the sheep vertebrae did not exhibit the textures due to structure that were seen in the images of human cancellous tissue, and so it was not possible to correlate the C.T. appearance with the structure visible in SEM images. The lack of structural texture was presumably due to the small size of the vertebral bodies and their marrow spaces when compared to humans, 
and to their much greater degree of mineralisation so that even in the emaciated sheep the loss of mineral had not progressed to the point where noticeable voids were visible on the C.T. images.

Linear regression between trabecular bone volume (TBV) and the C.T. bone measurements yielded the following correlation coefficients: $\mathrm{Cb} 120(0.87), \mathrm{Cmin}(0.81)$ and $\mathrm{RHO}(0.74)$. It is perhaps surprising that $\mathrm{Cb} 120$ correlated slightly better than $\mathrm{Cmin}$ since the purpose of the dual energy technique is to improve accuracy. However Cmin has much reduced precision when compared with $\mathrm{Cb} 120$, and is based on assumptions about the compositions of bone mineral and marrow which may not apply to sheep. Also the higher percentage of trabecular bone volume in sheep means that the single energy measurement in sheep will be much less sensitive to errors due to varying marrow composition than is the case in humans. A TBV of $32 \%$ would in humans imply that the cancellous tissue has $45 \%$ by weight of bone mineral, and the percentage of the linear attenuation coefficient due to mineral would be about $55 \%$. This is twice the comparable figure for normal humans (see figure 4.4, page 107).

\subsection{Measurements on Human Vertebrae.}

Bone mineral measurements must have the best possible reproducibility or precision if they are to be sensitive to the small changes caused by treatment. The measurements are traditionally made in the middle of the vertebral body, but this is often the site of greatest inhomogeneity. Figure 6.12 shows a photograph of a sagittal section of three sheep vertebrae. It can be seen that the greatest voids occur in the middle. If a C.T. bone mineral densitometry examination were performed on vertebrae such as these the figures measured would depend on the exact location of the measurement: an offset of a few millimeters could result in either the inclusion or exclusion of one or more voids. This being so, it would perhaps be advantageous not to perform the measurement in the middle of the vertebral body. In the present section an attempt has been made to determine whether the measurement precision is greater at sites halfway between the middle of the vertebral body and the superior and inferior endplates. Comparison has also been made between the precision of $10 \mathrm{~mm}$ and $5 \mathrm{~mm}$ thick slices taken in the middle of the vertebral body. 
Table 6.8: Effect of Slice Thickness on Reproducibility.

\begin{tabular}{|c|c|c|c|c|c|c|c|c|c|c|}
\hline \multirow{2}{*}{ Location } & \multicolumn{3}{|c|}{$\mathrm{Cb} 120$} & \multicolumn{3}{|c|}{$\mathrm{Cb} 80$} & \multicolumn{2}{|c|}{ RHO } & \multicolumn{2}{|c|}{ Cmin } \\
\hline & mean & c.v. & & mean & c.v. & & mean & C.v. & mean & c.v. \\
\hline $\mathrm{L} 2-10 \mathrm{~mm}$ & 93.8 & $0.39=$ & 8 & 86.0 & $0.75 \approx$ & 8 & 1.099 & 0.218 & 91.8 & $2.92 \%$ \\
\hline $\mathrm{L} 2-5 \mathrm{~mm}$ & 96.0 & $0.92 \div$ & 8 & 88.2 & 1.519 & 8 & 1.100 & $0.01 \&$ & 94.2 & $3.13 \div$ \\
\hline $\mathrm{L3}-10 \mathrm{~mm}$ & 81.1 & 1.169 & 8 & 74.3 & 1.90 & 8 & 1.086 & $0.31 \%$ & 84.0 & $5.19 ?$ \\
\hline L3 - $5 \mathrm{~mm}$ & 78.6 & 0.669 & 8 & 74.0 & $1.41 \%$ & 8 & 1.076 & $0.26 q$ & 91.6 & $3.98 \%$ \\
\hline $\mathrm{L} 4-10 \mathrm{~mm}$ & 84.1 & $1.46 \%$ & 8 & 77.3 & 1.629 & 8 & 1.088 & $0.67 \%$ & 86.8 & $8.52 \%$ \\
\hline $\mathrm{L} 4-5 \mathrm{~mm}$ & 83.8 & $2.63 \%$ & 8 & 77.1 & 1.199 & 8 & 1.087 & $0.63 q$ & 87.2 & $5.09 ?$ \\
\hline
\end{tabular}

Visual inspection of the above data does not show any tendency for the $10 \mathrm{~mm}$ means to be either greater or less than the means of the $5 \mathrm{~mm}$ measurements, nor does there appear to be any tendency for the $10 \mathrm{~mm}$ data to be more consistent. Wilcoxon tests failed to detect any significant differences between the two slice thicknesses in respect of either means or coefficients of variation.

In table 6.9 comparisons are made between bone measurements obtained at the midpoint of the vertebral body and those made half-way between the mid-point and either the top of bottom end plate. The slice thickness was $5 \mathrm{~mm}$ in all cases.

Table 6.9: Efrect of Slice Location on Reproducibility.

\begin{tabular}{|c|c|c|c|c|c|c|c|c|c|c|c|c|}
\hline \multirow{2}{*}{ Location } & \multicolumn{2}{|c|}{$\mathrm{Cb} 120$} & & \multicolumn{3}{|c|}{$\mathrm{Cb} 80$} & \multicolumn{3}{|c|}{ RHO } & \multicolumn{3}{|c|}{ Cmin } \\
\hline & mean & C.V. & & mean & C.V. & & mean & C.V. & & mean & C.V. & \\
\hline L2 middle & 96.0 & 0.92 & $\checkmark$ & 88.2 & 1.51 & 8 & 1.100 & $0.01 \%$ & 8 & 94.2 & 3.13 & 8 \\
\hline L3 middle & 78.6 & 0.66 & 1 & 74.0 & 1.41 & 8 & 1.076 & $0.26 q$ & 8 & 91.6 & 3.98 & 8 \\
\hline L2 upper & 91.1 & 1.82 & $\checkmark$ & 83.6 & 1.66 & 8 & 1.096 & 0.26 & 8 & 90.2 & 2.13 & 8 \\
\hline L2 bottom & 94.5 & 1.27 & 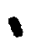 & 87.4 & 1.82 & 8 & 1.097 & 0.42 & 8 & 95.6 & 5.52 & q \\
\hline L3 upper & 82.2 & 1.47 & $\checkmark$ & 75.7 & 0.27 & 8 & 1.086 & 0.46 & 8 & 85.8 & 4.15 & $\%$ \\
\hline I3 bottom & 85.0 & 1.13 & 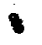 & 78.6 & 1.51 & 8 & 1.087 & $0.42 q$ & 8 & 89.4 & 5.63 & 8 \\
\hline
\end{tabular}

Contrary to expectations, the above figures show a tendency for the coefficients of variation to be lower at the middle of the vertebral body, although the sample sizes are not large enough to establish the significance of this. There is no apparent trend in the means. 
The C.T. bone measurements on the first human were well above average for an elderly woman. It is quite possible that bone loss had not progressed to the point where voids develop, in which case the postulated lower variability in mineral content away from the middle of the vertebral body would not apply. In the first subject contiguous $1.5 \mathrm{~mm}$ slices were taken through the length of L2 only. As time was limited only single energy images were taken, at $80 \mathrm{kVp}$. The second human subject was a man in his fifties with no evidence of diseases affecting bones. In this case contiguous dual energy images were obtained through the lengths of L3 and L4.

In figure 6.13 graphs of the variation of Cb80 along the length of the vertebra are given for L2 of the first human subject and L3 and L4 for the second human subject. Also plotted is the standard deviation of the C.T. numbers in each contiguous slice. Increased inhomogeneity in a slice would cause an increase in the standard deviation figure for that slice. Away from the vertebral endplates the distribution of $\mathrm{Cb} 80$ is quite uniform throughout the length of the vertebra. However there is a small but noticeable increase in the standard deviations in the middle of the vertebral body in the case of human 1, and a very pronounced increase in both vertebrae of human 2. Althotgh the ' $\mathrm{Y}$ ' pattern was evident in some images taken near the middle of the vertebrae of human 2 , the portion of the curve showing increased standard deviations extended well beyond the region where the ' $\mathrm{Y}$ ' pattern was visible. This implies that the inhomogeneity was not solely due to the venous sinuses. In human 1 only a trace of ' $Y$ ' pattern was visible, and only in the image at $11 \mathrm{~mm}$ (top graph). In figure 6.14 the variation along the length of the vertebral body of the two dual energy measurements is displayed, with $\mathrm{Cb} 80$ included for comparison. 


\section{HUMAN 1, L2}

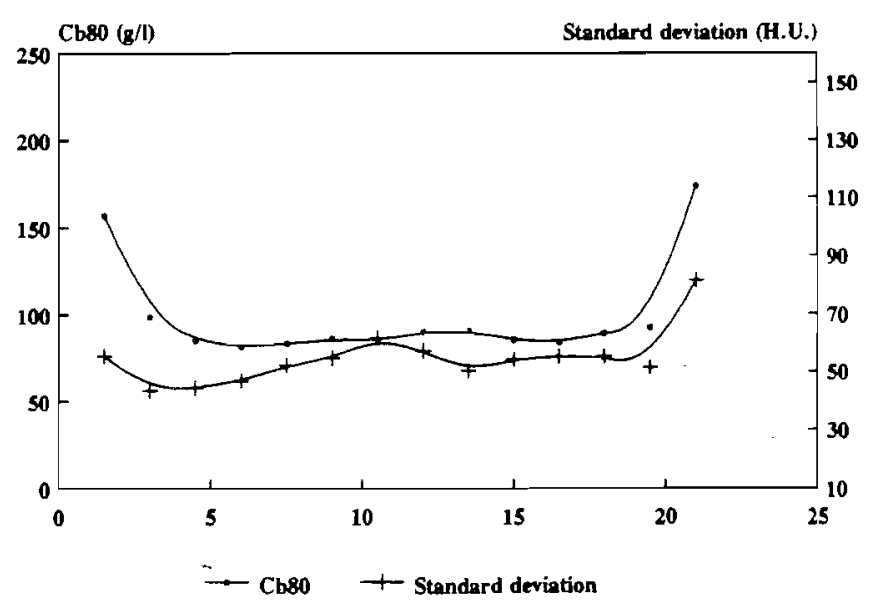

Human 2, L3

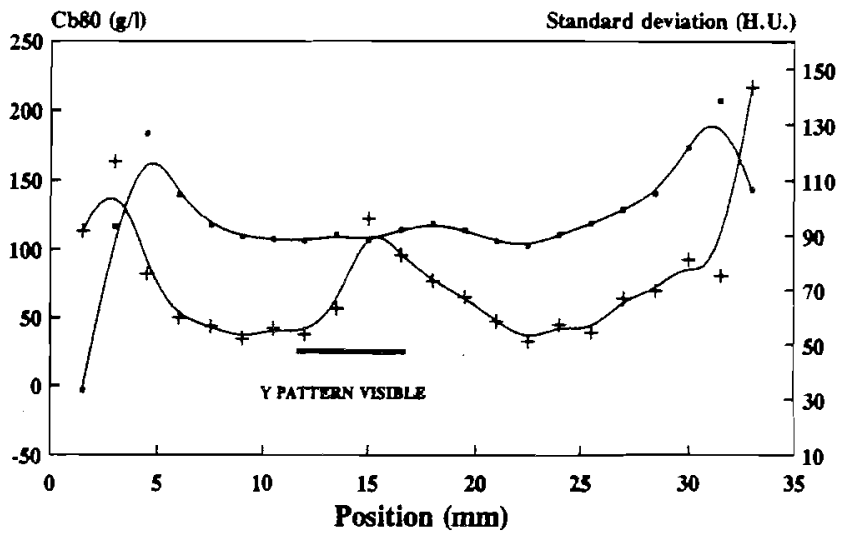

$\rightarrow$ Cb8O + Standard deriation

\section{HUMAN 2, L4}

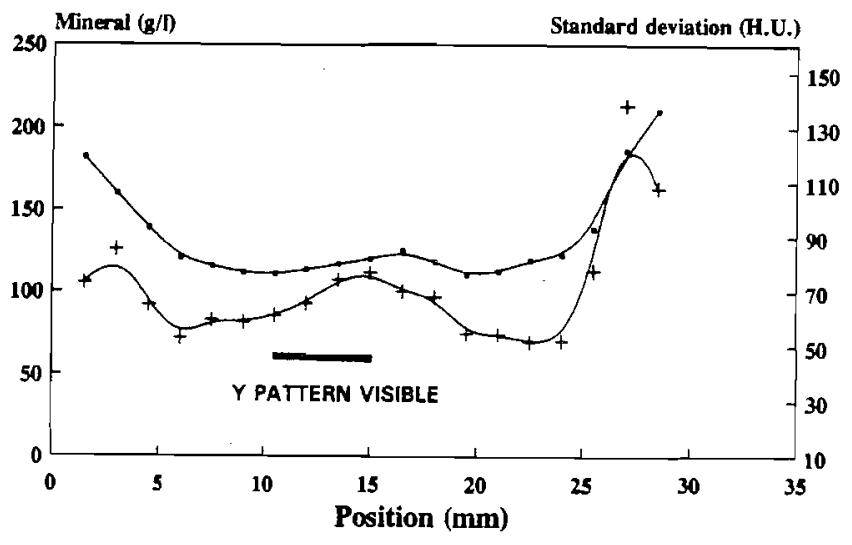

$\rightarrow$ Cb80 + Standard deviation

Figure 6.13: Variation of Single Energy Measurement Along the Long Axis of the Vertebra. 

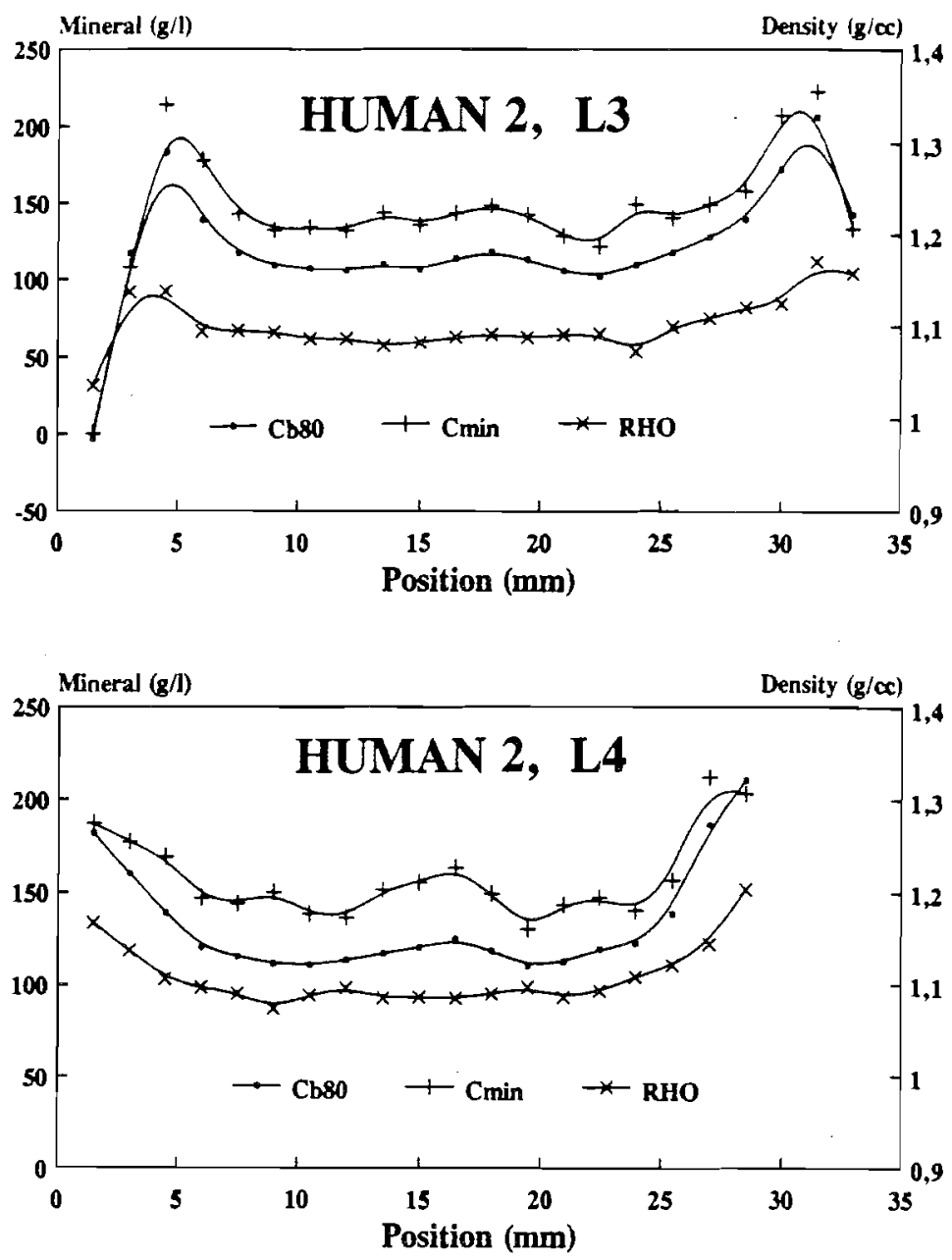

Figure 6.14: Variation of Dual Energy Measurements Along the Long Axis of the Vertebra.

The curves for $\mathrm{Cb} 80$ and $\mathrm{RHO}$ show little variation along the length of the vertebrae, apart from at the endplates, but the Cmin curves show increased variability which is in part due to the reduced precision of Cmin. However the similarity of the Cmin curves for the two vertebrae suggests that, at least in this subject, there are real fluctuations in Cmin along the length of the vertebral body. However the fact that the mineral increases in the middle suggests that in this case the inhomogeneity is due either to islands of increased mineralisation in the middle or to the presence of voids on either side of the central region.

\subsection{Reprise.}

If the predictive value of C.T. bone measurements is to be improved it may be necessary to consider the structural arrangement of the bone mineral as well as its 
quantity. Differences in the texture of the cancellous region of the vertebral body are apparent on C.T. images which must reflect gross features of the underlying trabecular structure. The systematic classifications of these appearances discussed in section 6.3 have shown that, while some differences exist between the appearances of different vertebrae on the same patient, the correlations between the classifications of the three vertebrae scanned on each patient suggest that the classifications have a more global significance and do not relate solely to the vertebrae imaged. There was a tendency for the granularity of the image to increase as Cb120 decreased, although the correlation was relatively weak and was only statistically significant in the case of L2.

Attempts have been made to determine whether pixel distributions provide a useful means of quantifying the appearance of the C.T. images. Studies have been made of the pixel distributions of the parameters $\mathrm{Cb80}, \mathrm{Cb} 120$, $\mathrm{RHO}$ and $\mathrm{Cmin}$ on seventeen different patients suffering from a range of different conditions. The distributions were all Gaussian, and there were no trends observed in standard deviations or the moment coefficients of skew and kurtosis which would suggest that these contained useful structural information. The use of pixel distributions does not at this stage seem to be a promising method for quantifying the texture visible on C.T. images, although it is possible that the proportion of pixels below a yet to be determined threshold value may be a useful parameter. If so, this value may be computed from the mean and standard deviation since the distributions are approximately Gaussian.

Although the ultimate aim is to correlate the C.T. appearance of the vertebral body with its structure as determined by SEM, a necessary step towards gaining ethical approval to work with human tissue has been to prove the technique using sheep vertebrae. While this exercise has been valuable in developing the required techniques, it has not been possible to correlate the C.T. appearances of the sheep vertebrae with parameters derived from SEM images describing the structure. This was because the textures observable in images of humans were not present on the sheep images, a finding which may be attributed to the fact that the sheep vertebral bodies are much smaller than in humans and the structure present is below the limit of resolution of the C.T. scanner. Also the mineral content of the sheep was much higher than that of humans so that the potential for the formation of voids was much more limited.

While ethical approval has been granted to work with vertebrae for a total of six patients in the first instance, to date the vertebrae from only two subjects have been made available to us. The vertebrae from the first subject have been used to determine whether better reproducibility of the C.T. measurements is obtained away from the mid-plane of the vertebral body, and whether the use of thicker slices improves reproducibility. Neither factor was found to improve reproducibility in the first human subject, but it is clear that this exercise will have to be repeated on a number of subjects since the mineral 
measurements on the first subject were at the lower end of the normal range for females under forty (the subject in her eighties!) and the images showed only a small degree of granularity, indicating that the mineral loss in this case was relatively uniform. Reproducibility studies were not performed for human two as the time available for scanning this patient was used to obtain contiguous thin dual energy images along the full length of the two vertebrae. However these measurements showed increased inhomogeneity in the middle of the vertebra which was only partly due to the venous sinuses which give rise to the ' $\mathrm{Y}$ ' pattern on the images.

When the C.T. scanning of the vertebrae of the first series of subjects is complete, images of the specimens will be analysed using SEMPER. The taking of contiguous thin C.T. images will permit analysis in three planes using image reformatting software. Changes in structure noted will be related to the ages of the subjects, and also to the number of crush fractures (if any) visible on plain X-ray films of the spine. The use of macro photography for imaging the vertebral specimens is being investigated as this will permit the entire vertebral section to be included in one image.

At this stage pixel histograms do not seem to be a promising method for assessing the differences in texture visible on C.T. images of the cancellous tissue. An alternative method for texture quantification which will be investigated is to use Fourier techniques to determine the amplitudes of the Fourier spectrum over a range of spatial frequencies. A possible simple method for doing this, made possible by the central section theorem (see section 1.3 and appendix $\mathrm{A}$ ), is outlined in figure 6.15. The Fourier spectrum of the images of the sections obtained either by photography or SEM will be computed using SEMPER. The presence of voids in the structure will be indicated by increased amplitudes at the low frequency end of the spectrum. 


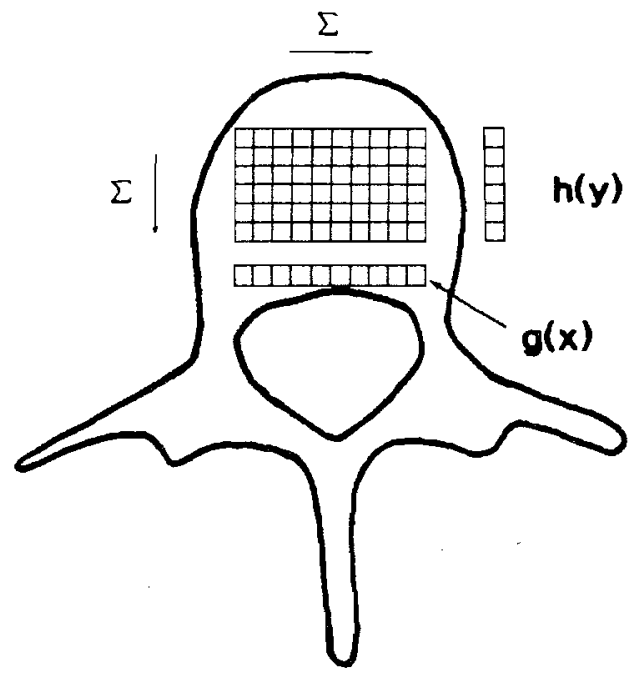

REAL SPACE

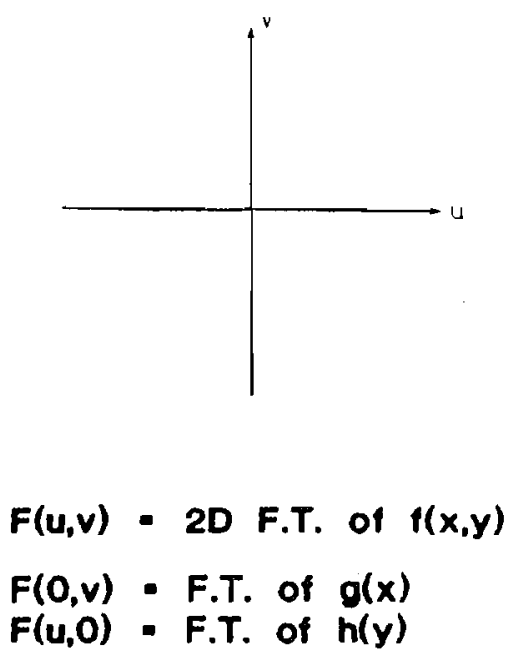

FREQUENCY SPACE

Figure 6.15: Simple Method for Obtaining Fourier Spectrum from C.T. Images.

The method involves summing the pixel values for each row and column in the area of interest. The amplitudes of the Fourier spectrum in the horizontal direction may be obtained (Central Section Theorem) by one dimensional Fourier transformation of the function $h(y)$. The amplitudes in the vertical direction may be obtained by $1 D$ Fourier transformation of $g(x)$.

As of the time of writing, Wellington Hospital is in the process of purchasing a replacement C.T. scanner. The high contrast resolution of the new scanner is expected to be better than that of the present scanner by a factor of three, which will greatly enhance the visibility of any gross trabecular structure on the images. 


\section{CHAPTER SEVEN: CONCLUSION}

In the interests of brevity only the most salient points are mentioned again in the conclusion. Fuller summaries are included at the end of each chapter. The production of a C.T. image involves obtaining X-ray transmission data, the use of mathematical algorithms to reconstruct images from this data, and displaying the images on a screen or producing a permanent copy of the image on film. Subject to the limitations discussed in chapter one, the reconstructed image is related to the pattern of $\mathrm{X}$-ray attenuation within the patient. Quantitative interpretation of C.T. images requires the ability to compute attenuation coefficients, a process which is made difficult by the lack of simple, accurate formulae to compute attenuation coefficients of materials from their composition and density. This difficulty could be circumvented by the use of tables, but it is shown in the present work that there are significant differences between the values of the various published tabulations. The definition of the Hounsfield unit in terms of the ratio of the linear attenuation coefficient of the material of interest to that of water reduces the impact of differences between tables for soft tissues whose effective atomic numbers are close to that of water, but the residual differences are still of importance for higher atomic number materials such as bone. A further problem with tables of attenuation coefficients is that values are listed at only a small number of energies within the diagnostic energy range, and interpolation is made difficult by the transition from strong energy dependence at the bottom of this range due to predominantly photoelectric attenuation to weak energy dependence at the top end of the range where attenuation is mostly due to Compton scatter. In the present work numerical techniques have been used to produce a very simple expression which matches tabulated attenuation coefficients over the range from 50 to $100 \mathrm{keV}$ with errors of less than $0.35 \%$. This expression requires only three constants to be known separately for each element, of which one is simply derived from the atomic number and atomic weight. Accuracy of $0.1 \%$ may be achieved over the same restricted energy range with a five parameter continued fraction expression. It has been shown that virtually perfect interpolation over the full diagnostic energy range can be achieved with an eight parameter continued fraction expression. Software has been devised for performing this task. In certain circumstances it is not sufficient to consider the polychromatic X-ray spectrum of a C.T. scanner to be equivalent to a monochromatic beam of a certain 'effective' energy, in which case it is necessary to be able to compute the spectrum. A program has been developed which computes the spectrum from the expressions of Birch \& Marshall [69].

The introduction of the C.T. scanner resulted in images whose characteristics in terms of shades of grey could be quantified in a physically meaningful way since each shade represents a specific range of C.T. numbers. Attempts to make use of these 
measurements in diagnosis were hampered by a lack of standardisation between scanners, and by the fact that they are derived from linear X-ray attenuation coefficients which have little clinical significance. However the linear attenuation coefficient itself depends on the energy of the beam used to make the measurement, which is to a certain extent changeable by the operator of the scanner, and the density and 'effective' atomic number of the tissues scanned. Density would seem to be an important parameter in its own right, and effective atomic number could perhaps be useful as an index of chemical composition. Since changes in density would cause equal changes in C.T. number, regardless of the energy of the measuring beam, it is clear that any difference in C.T. number between images taken at different energies reflects the 'effective' atomic number of the tissue, and that the signal common to both energies is a measure of tissue density. This is the basic principle behind dual energy computed tomography, which aims to measure clinically-relevant parameters in a way which is independent of the scanner used in making the measurements.

Methods of obtaining dual energy images are discussed in chapter three. The simplest way of obtaining dual energy C.T. images is to perform sequential scans,- one at a higher kilovoltage and one at a lower kilovoltage. Problems could arise due to patient movement between scans, and different scanning methods have been developed to avoid these. However none of the improved scanning methods were suitable for implementation on the Wellington G.E. 8800 scanner. In practice the sequential scanning method does not seem to have caused problems as the amount of movement between scans is normally only of the order of a millimeter or so. Available algorithms for producing images of parameters such as density and effective atomic number from two images taken at different energies are also discussed in chapter three. For general dual energy work the most appropriate algorithm is that of Hawkes and Jackson [102]. The G.E. 8800 scanner is designed for operation at $120 \mathrm{kVp}$ only. In the present work methods have been devised to store calibration data appropriate for $80 \mathrm{kVp}$ operation, and to recalibrate $80 \mathrm{kVp}$ images prior to reconstruction using the standard system software. However it has not been possible to alter the system beam hardening correction, which is optimised for $120 \mathrm{kVp}$ operation. While the recalibration procedure removes some of the errors due to this cause, imaging tests have shown that in some circumstances the $80 \mathrm{kVp}$ images are more prone to beam hardening artefacts than those taken at $120 \mathrm{kVp}$. With the exception of image noise, which is an inherent physical limitation, the other imaging parameters were found to be essentially the same at both kilovoltages.

Single energy computed tomography and dual photon absorptiometry (DPA) are the most widely-used techniques for measurement of the mineral content of the lumbar spine. Quantitative computed tomography has the greater sensitivity since it is applied to cancellous tissue alone whereas DPA measures a combination of cortical and cancellous bone. The dose due to computed tomography is much greater than that due to DPA, but 
is still small when compared with the doses due to may other diagnostic X-ray examinations. The two techniques are of similar accuracy and precision, and both are susceptible to fairly large errors due to variable fat content. Dual energy C.T. can all but eliminate such errors, but at the expense of reduced precision. While most workers seem to be content to use the single energy formula of Genant and his co-workers (equation 4.3), there are a large number of different equations for the computation of mineral content from dual energy C.T. scans, some of them of considerable complexity. The derivations of two of these are described in chapter four, and simulations have been performed to assess the sensitivity of the reported mineral content to changes in the fat content of the marrow. While the single energy figure can be in error by as much as 30 $\%$ for patients with normal or low-normal mineral content, this error is greatly reduced when using the dual energy algorithm of Laval-Jeantet et al. The derivation of the LavalJeantet formula is purely empirical and gives an index of the mineral content rather than a direct measurement of it, and the fat parameter given by this formula has been shown in the present work to be not a true measure of the fat content. Other authors have since confirmed this.

The present author has derived a dual energy formula whose derivation starts from a somewhat simpler tissue model than is generally used, and which is expressed in terms of mass attenuation coefficients and not linear attenuation coefficients. The Wellington formula deals explicitly with the density of the marrow and ignores changes in its chemical composition, an approach which can be justified since the mass attenuation coefficients of red and yellow marrow differ by as little as $0.1 \%$. Apparent differences due to fat express themselves primarily as changes in density. The computer simulations have shown that the Wellington formula shows the least sensitivity to changes in marrow composition and density, a fact which may be attributed to its derivation in terms of mass attenuation coefficients. Computations based on any tissue model will only be accurate in so far as the model truly reflects the tissue being studied. The use of a model expressed in terms of linear attenuation coefficients involves more assumptions since it requires a knowledge of both the chemical composition (effective atomic number) and the density of each component, whereas the use of mass attenuation coefficients only involves assumptions about composition. Further, since the mass attenuation coefficient of $\mathrm{K}_{2} \mathrm{HPO}_{4}$ closely matches the mass attenuation coefficient of bone mineral, the Wellington formula theoretically gives the true mineral concentration rather than simply an index which correlates with it. However it has been found that there is a systematic error in the values reported by the Wellington protocol since measurements of the $\mathrm{K}_{2} \mathrm{HPO}_{4}$ solution are made using a circular phantom which has subsequently been found to not truly represent the approximately elliptical shape of the abdomen. It is estimated that the errors due to this cause are $8 \%$ for single energy and $13 \%$ for the dual energy mineral measurement. Since a normal range has been established separately for the Wellington system these systematic errors do not affect the interpretation of patient 
measurements.

The Wellington dual energy mineral values have been found to correlate almost perfectly with the mineral parameter computed using the Laval-Jeantet formula, which in turn has been found to correlate well with ash weights of excised vertebrae. Phantom studies in the present work have indicated that the precision of the Wellington system is $2 \%$ for single energy and $6 \%$ for the dual energy mineral measurement. Serial studies on a non-osteoporotic male gave figures of $2 \%$ and $7 \%$ respectively, figures which are in satisfactory agreement with other reported values.

The formalism presented in chapter two has been used to develop a very simple, yet precise, method for computing electron densities from dual energy C.T. images. The method uses an empirical relationship between the ratio of linear attenuation coefficients at two different energies and the effective atomic number of a material. Since an empirical relationship can also be determined between effective atomic number and the cross section per electron, it is possible to combine these two relationships to give a direct empirical relationship between the ratio of attenuation coefficients and the electronic cross section. The volume electron density, which is the parameter required for treatment planning purposes, may then be computed from equation (5.3). This dual energy technique is shown to yield the volume electron density with an accuracy of $5 \%$ for soft tissues, of $3 \%$ for cancellous bone and $8 \%$ for cortical bone. The use of single energy techniques involves the assumption of a single value for the electronic cross section for all soft tissues, and does not appear to compromise the accuracy significantly. It is recommended that dual energy techniques be used for treatment planning in those situations where the treatment beam must traverse bone tissue.

Spine mineral measurements on some 31 volunteer women between the ages of 20 and 40 have been used as a control group for a study of the effects of anorexia nervosa on bone mass, and have been used to provide a range of normal values against which to compare clinical measurements made with the Wellington Hospital C.T. scanner. The mean value for single energy measurements made on this group is of similar magnitude to values reported by workers in Australia, the United Kingdom and in New York. The mean value for the cancellous tissue density $(1.092 \mathrm{~g} / \mathrm{cc})$ is comparable to a mean value for excised cancellous tissue measurements reported by the ICRP [117] of $1.08 \mathrm{~g} / \mathrm{cc}$. The mean values of the two single energy measurements, $\mathrm{Cb} 80$ and $\mathrm{Cb} 120$, for the patients with a history of anorexia nervosa $(\mathrm{N}=68$ ) were $18 \%$ lower than were the comparable figures for the controls. However these figures alone do not prove that bone mineral is lost in anorexia nervosa because such changes could have been due solely to changes occurring within the marrow component of the vertebral body. However the mean value of the dual energy mineral measurement, Cmin, was some $19 \%$ lower in the patient group than in the controls confirming that the change detected by the single energy 
technique genuinely involves both components, and that the loss of mineral is of the same magnitude as the reduction indicated by the single energy measurements.

Previous studies have established a link between anorexia nervosa and low bone mass. In the present study attempts have been made to determine the effects of a number of relevant variables which characterise the severity of the disease such as body mass index, duration of amenorrhoea, duration of vomiting and diarrhoea, and duration of low calcium intake both at time of examination and in the bone-forming years of adolescence. Also included have been a number of factors which might tend to counteract any bone loss due to anorexia nervosa: use of estrogen based contraceptive pills, and exercise. The results of correlations between the clinical variables and the single energy measurement $\mathrm{Cb} 80$ have already been published [189]. The present author has extended the analysis to include the dual energy measurements Cmin and RHO as well. The strongest correlations found in the present work were between body mass index (BMI) at the time of examination and the cancellous tissue density $\left(r_{S}=0.4\right.$ for the controls and $\mathrm{r}_{\mathrm{S}}=0.5$ for the patient group). Correlations of this magnitude may be regarded as strong in the present context where a relatively large number of variables is involved. In the patient group RHO correlated almost as strongly with the total duration of anorexia $\left(r_{S}=-0.48, p=10^{-4}\right)$, a fact which is unsurprising since body mass index and duration of anorexia nervosa are themselves well correlated $\left(r_{S}=-0.63, p=3 x\right.$ $10^{-7}$ ). Cb80 correlated almost as well with duration of anorexia, but there was only weak and non-significant correlation between this variable and $\mathrm{Cmin}$. However this does not mean that bone mineral is not lost in anorexia nervosa: the mean value of Cmin for the whole patient group shows the same decrease with respect to the controls as was indicated by the single energy measurements. A more likely explanation is either that the lower precision associated with $\mathrm{Cmin}$ is masking the effect of anorexia, or that other clinical variables such as exercise are having a counterbalancing effect. When the total duration of both anorexia and bulimia nervosa is considered, significant negative correlations are found with $\mathrm{Cb} 80, \mathrm{Cmin}$ and RHO.

The single energy analysis did not show a significant relationship between exercise and bone mass. It is clear in the present work that this was because a significant positive correlation between $\mathrm{Cmin}$ and exercise at the time of $\mathrm{X}$-ray was masked by a negative correlation between RHO and exercise $\left(r_{s}=-0.22, p=0.07\right)$. There has been no evidence in the present work of a protective effect from the use of estrogen contraceptive pills, but the duration of high calcium intake in adolescence gave significant positive correlations with $\mathrm{Cb} 80$ and $\mathrm{Cmin}$.

The work of Treasure et al [194], based on DPA measurements in the femur and lumbar spine, has suggested that bone loss due to anorexia nervosa is reversible. This conclusion was based on the fact that their group of recovered anorexics had greater bone 
mass than those who were amenorrheic at the time of examination. However although the recovered group in that study had the same median duration of amenorrhoea as the amenorrheic group, the duration of illness in the recovered group was significantly less than in the other group and it may be this factor, rather than reversal of bone loss, which is the cause of the difference in bone mass between the two groups. In the present work it was found that those patients in the 'good' recovery category had significantly higher $\mathrm{Cb} 80$ values than those on the 'poor' recovery categories. The same finding was noted in respect of the RHO values, but in the case of Cmin there was only a small and not significant difference between the patients in the two categories. The findings in respect of Cb80 and RHO suggest that some recovery may take place, although the present study has the same problem as that of Treasure in that the patients in the 'poor' recovery category had longer illness durations than those in the 'good' recovery category. However the findings of the present study in respect of Cmin show no evidence of recovery of lost bone mineral following anorexia nervosa.

If the predictive value of C.T. bone measurements is to be improved it may be necessary to consider the structural arrangement of the bone mineral as well as its quantity. Differences in the texture of the cancellous region of the vertebral body are apparent on C.T. images which must reflect gross features of the underlying trabecular structure. The systematic classifications of these appearances discussed in section 6.3 have shown that, while some differences exist between the appearances of different vertebrae on the same patient, the correlations between the classifications of the three vertebrae scanned on each patient suggest that the classifications have a more global significance and do not relate solely to the vertebrae imaged. There was a tendency for the granularity of the image to increase as $\mathrm{Cb} 120$ decreased, although the correlation was relatively weak and was only statistically significant in the case of L2. Attempts have been made to determine whether pixel distributions provide a useful means of quantifying the appearance of the C.T. images. Studies have been made of the pixel distributions of the parameters $\mathrm{Cb80}, \mathrm{Cb} 120, \mathrm{RHO}$ and $\mathrm{Cmin}$ on seventeen different patients suffering from a range of different conditions. The distributions were all Gaussian, and there were no trends observed in standard deviations or the moment coefficients of skew and kurtosis which would suggest that these contained useful structural information. Pixel distributions would appear to have limited replicational utility in this regard.

Although the ultimate aim is to correlate the C.T. appearance of the vertebral body with its structure as determined by SEM, a necessary step towards gaining ethical approval to work with human tissue has been to prove the technique using sheep vertebrae. While this exercise has been valuable in developing the required techniques, it has not been possible to correlate the C.T. appearances of the sheep vertebrae with parameters derived from SEM images describing the structure. This was because the textures observable in images of humans were not present on the sheep images. 
While ethical approval has been granted to work with vertebrae for a total of six patients in the first instance, to date the vertebrae from only two subjects have been made available to us. Repeated scanning of the vertebrae of the first human subject has shown no improvement in reproducibility when the slice width was increased from $5 \mathrm{~mm}$ to 10 $\mathrm{mm}$, or when the readings are taken in the upper or lower portions of the vertebral body rather than in the middle. However the appearances of the images on this subject, and the standard deviation readings, suggest that in this particular subject the relatively small amount of bone loss which had occurred was uniformly distributed. This exercise will have to be repeated on several subjects before any conclusions may be reached.

As of the time of writing, Wellington Hospital is in the process of purchasing a replacement C.T. scanner. The high contrast resolution of the new scanner is expected to be better than that of the present scanner by a factor of three, which will greatly enhance the visibility of any gross trabecular structure on the images. 


\section{APPENDIX A: Proof of the Central Section Theorem.}
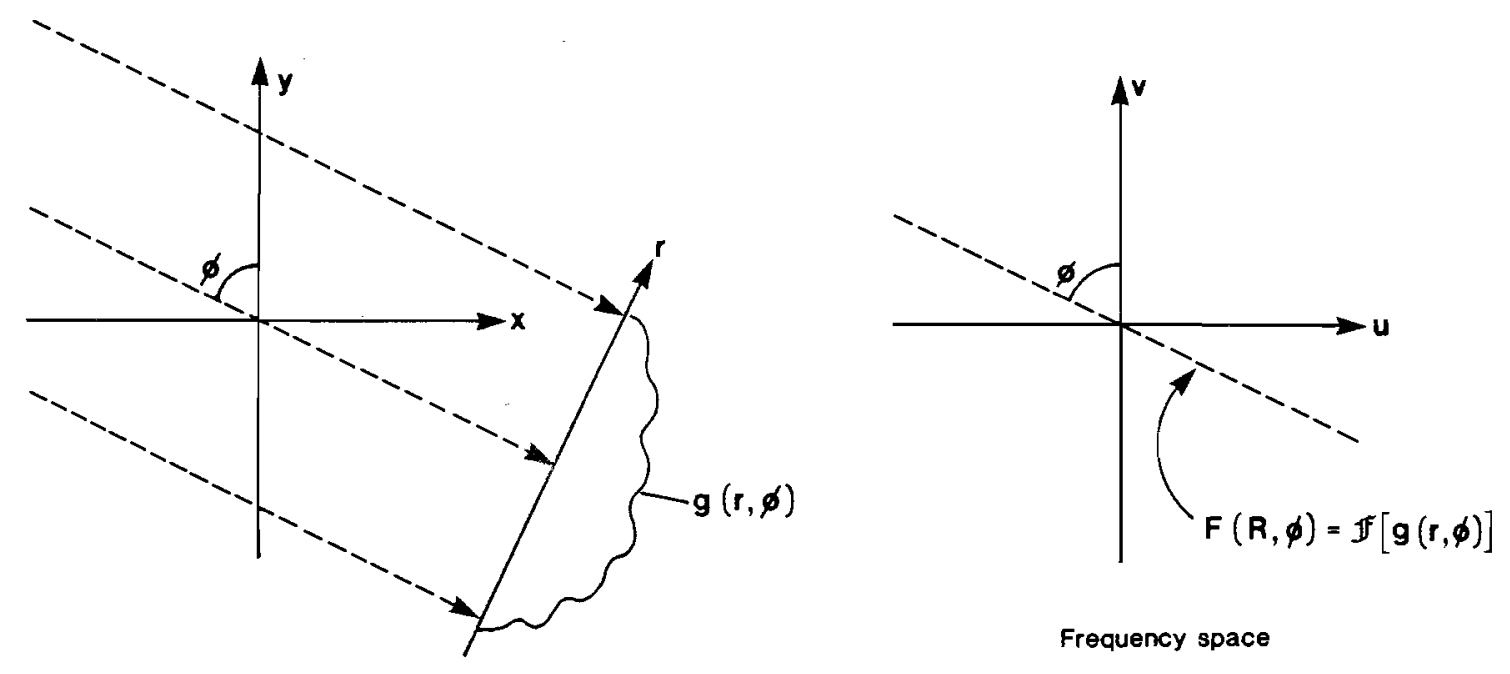

Real space

Figure A.1: The Central Section Theorem.

The central section theorem states that the values of the two dimensional Fourier transform, $F(u, v)$, of a function $f(x, y)$ may be obtained by one dimensional Fourier transformation of individual projections of $f(x, y)$. The values of $F(u, v)$ along a line at angle $\phi$ in frequency space (see figure A.1) are equal to the one dimensional Fourier transform of the projection $g(r, \phi)$ taken at the same angle.

The values of the projection $\mathrm{g}(\mathrm{r}, \phi)$ at a fixed angle $\phi$ are given by the following integral:

$$
g(r, \phi)=\iint f(x, y) \delta(x \cos \phi+y \sin \phi-r) \cdot d x \cdot d y
$$

In the above the effect of the Kronecker delta function is to permit summation of the function $f(x, y)$ only along the line through the origin at distance $r$ as defined in figure 1.12 (page 19). It must be shown how this function $\mathrm{g}(\mathrm{r}, \phi)$ is related to the function $\mathrm{F}(\mathrm{R}, \phi)$, where $R$ and $\phi$ are the polar co-ordinates in frequency space. By definition, $F(u, v)$ is obtained by Fourier transformation [26] as follows:

$$
F(u, v)=\iint f(x, y) \exp (-i 2 \pi(u x+v y)) \cdot d x \cdot d y
$$

To express the Fourier transform function in polar coordinates it is necessary to substitute $\mathrm{u}=\mathrm{R} \cdot \cos \phi, \mathrm{v}=\mathrm{R} \cdot \sin \phi$ and we obtain:

$$
F(R, \phi)=\iint f(x, y) \exp (-i 2 \pi R(x \cos \phi+y \sin \phi)) \cdot d x \cdot d y
$$


It is helpful to regard this integration as being performed sequentially at all possible distances from the origin $r$ :

$F(R, \phi)=\iiint f(x, y) \delta(x \cos \phi+y \sin \phi-r) \exp (-i 2 \pi R r) \cdot d x \cdot d y \cdot d r$

where again the delta function selects only those points along line $\mathrm{r}$ (figure 1.11), and the complete integral is obtained by integrating over all possible values of $r$. Rearranging the terms in equation A.4:

$F(R, \phi)=\int\left[\iint f(x, y) \delta(x \cos \phi+y \sin \phi-r) \cdot d x \cdot d y\right] \exp (-i 2 \pi R r) \cdot d r$

it can be seen by comparison with equation A. 1 that:

$$
F(R, \phi)=\int g(r, \phi) \exp (-i 2 \pi R r) \cdot d r
$$

The integral on the right hand side corresponds to the definition of the one dimensional Fourier transform of the function $g(r, \phi)$. Thus the values of $F(R, \phi)$ along a line at angle $\phi$ are equal to the one dimensional Fourier transform of $g(r, \phi)$ at the same angle, and this is the proposition which was to be proved. 


\section{APPENDIX B: Curve Fitting Techniques.}

\section{Polynomial Interpolation.}

Traditionally, polynomial interpolation involves the use of either the Newtonian or Lagrangian form of the interpolating polynomial [74]. A program (NEWTPOLY) which uses the Newtonian form is included in the listings in this appendix. This type of interpolation is used when the number of data points equals the number of degrees of freedom of the fitting function, in this case the degree of the equation plus one. When there are more data points than this least squares techniques may be used, in which case the root-mean-square error is a minimum. An alternative technique is the minimax technique, which minimises the maximum absolute error. This technique involves selecting the points at which the polynomial is matched to the data. A minimax fit is obtained when the Tchebyshev abscissae are the points at which the fit occurs [61]. These points may be computed using program TCHEB, which is also listed in this appendix.

\section{Continued Fraction Interpolation.}

In many circumstances data can be matched more effectively when the fitting function is a continued fraction, rather than a polynomial. A continued fraction is a function of the form:

$$
F(x)=q_{0}+\frac{\left(x-x_{1}\right)}{q_{1}+\frac{\left(x-x_{2}\right)}{q_{2}+\frac{\left(x-x_{3}\right)}{q_{3}+\frac{\left(x-x_{4}\right)}{q_{4}}}}}
$$

The expression may be extended to higher terms if desired. Equation (B.1) may be evaluated as follows:

$$
F(x)=q_{0}+\left(x-x_{1}\right) /\left(q_{1}+\left(x-x_{2}\right) /\left(q_{2}+\left(x-x_{3}\right) /\left(q_{3}+\left(x-x_{4}\right) / q_{4}\right)\right)\right)
$$

Alternatively, any continued fraction may be expressed as a rational function:

$$
y(x)=\frac{a_{0}+a_{1} \cdot x+a_{2} \cdot x^{2}+\ldots}{b_{0}+b_{1} \cdot x+b_{2} \cdot x^{2}+\cdots}
$$


Program INTCF, which is listed in this appendix, fits a continued fraction expression to any given data and then converts the continued fraction to a rational function for ease of use.

General Curve Fitting Program

Bevington [62] has published a generalised curve-fitting program, CURFIT, which will fit data to any given function. Its use requires the fitting function to be compiled as a separate subroutine. The present author has written a FORTRAN 77 version of this program. It is not listed here, but is available on request. All software in these appendices is written in FORTRAN 77 for an MS-DOS operating system, except the spine mineral software described in appendix $G$ which runs under a Data General AOS operating system.

\section{Listings}

Listings start on the next page. The programs listed are:

$\begin{array}{lll}\text { NEWTPOLY } & \text { Interpolation via Newton's polynomial } & \text { page } 216 \\ \text { TCHEB } & \text { Tchebyshev points for a range } & \text { page } 219 \\ \text { INTCF } & \text { Interpolation by continued fractions } & \text { page } 220\end{array}$


Program NEWTPOLY: Interpolation via Newton's interpolating polynomial.

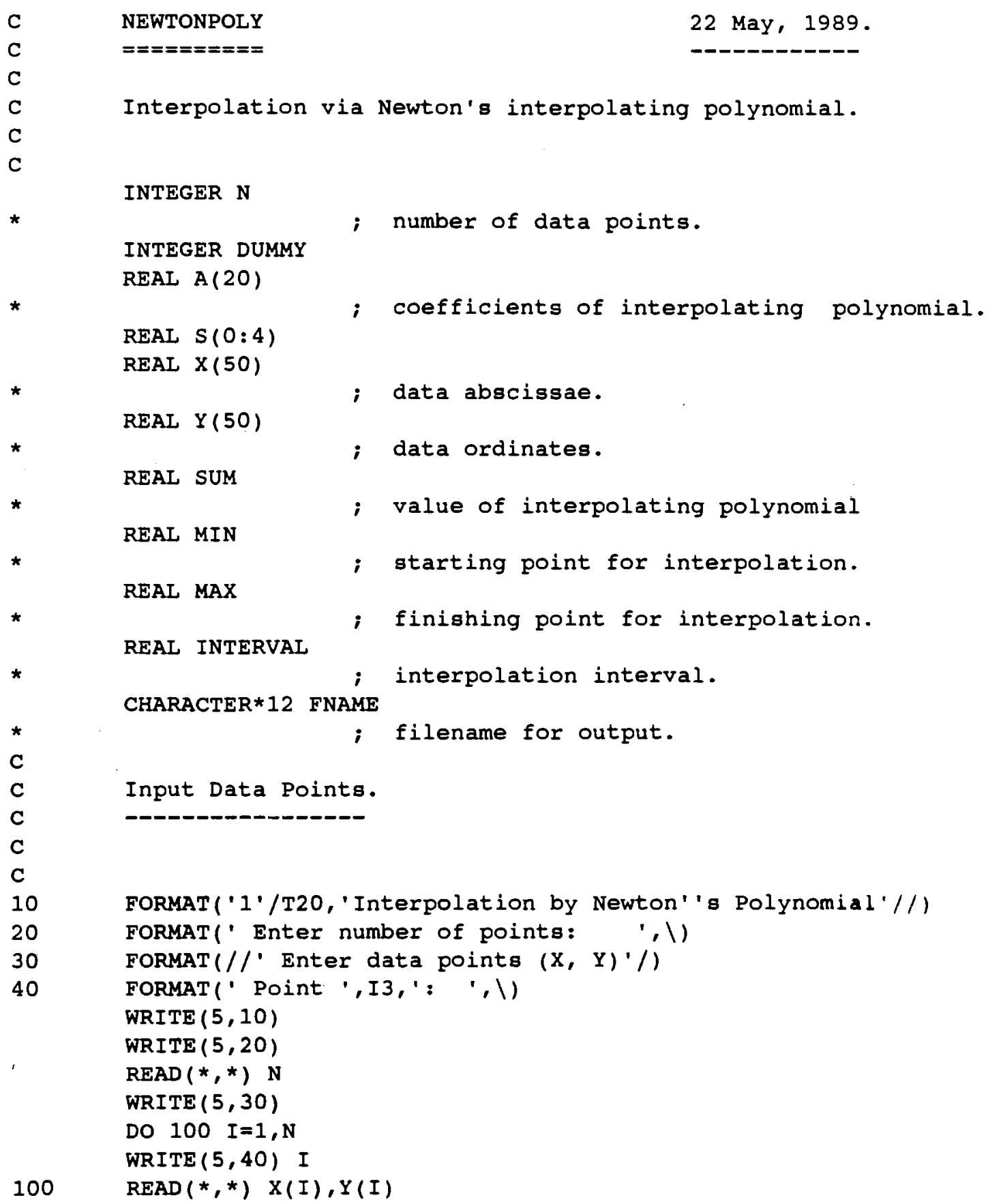


C
C
C
C
C

C Calculate Coefficients.

C
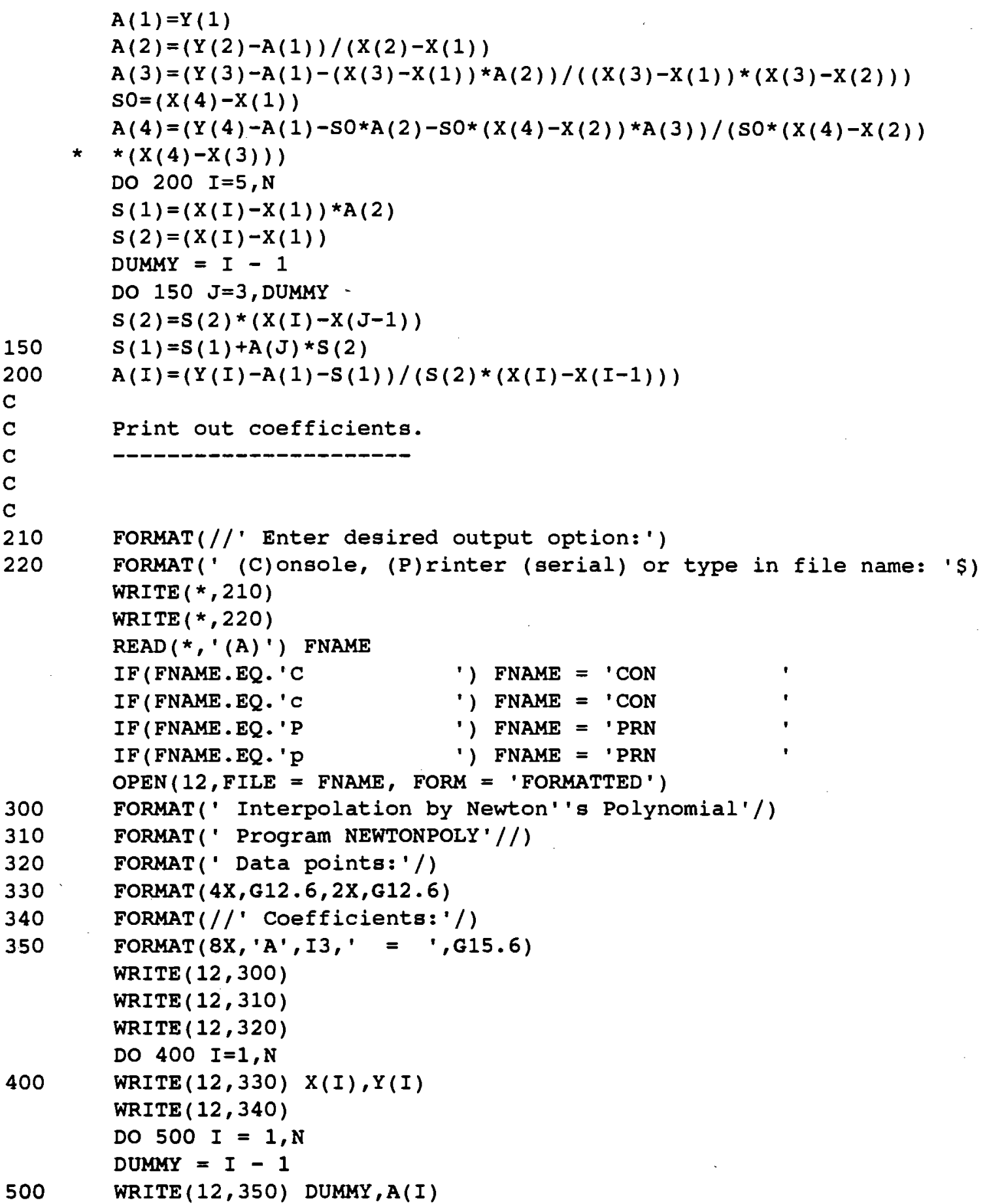


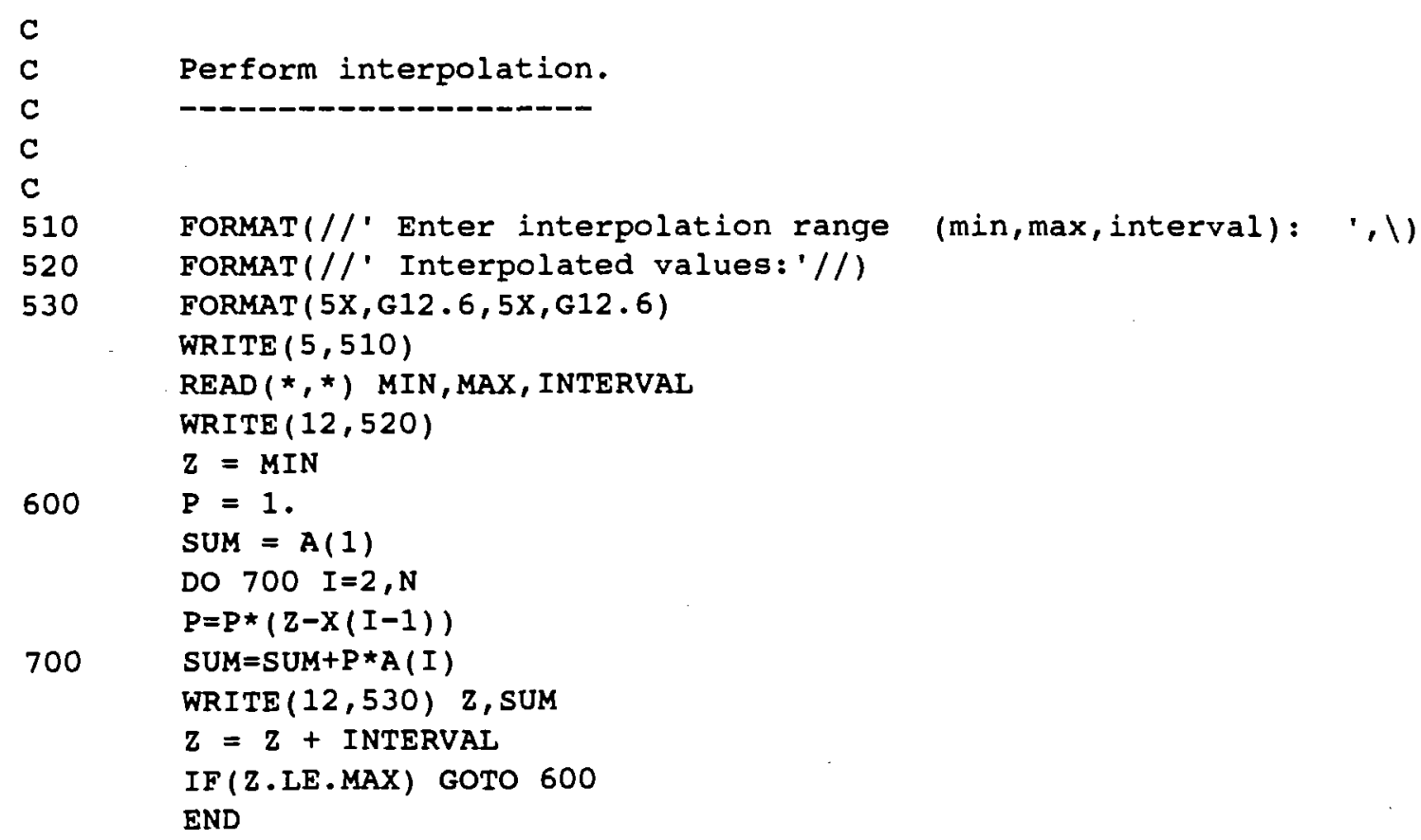


Program TCHEB: Calculates the Tchebyshev points for a range.

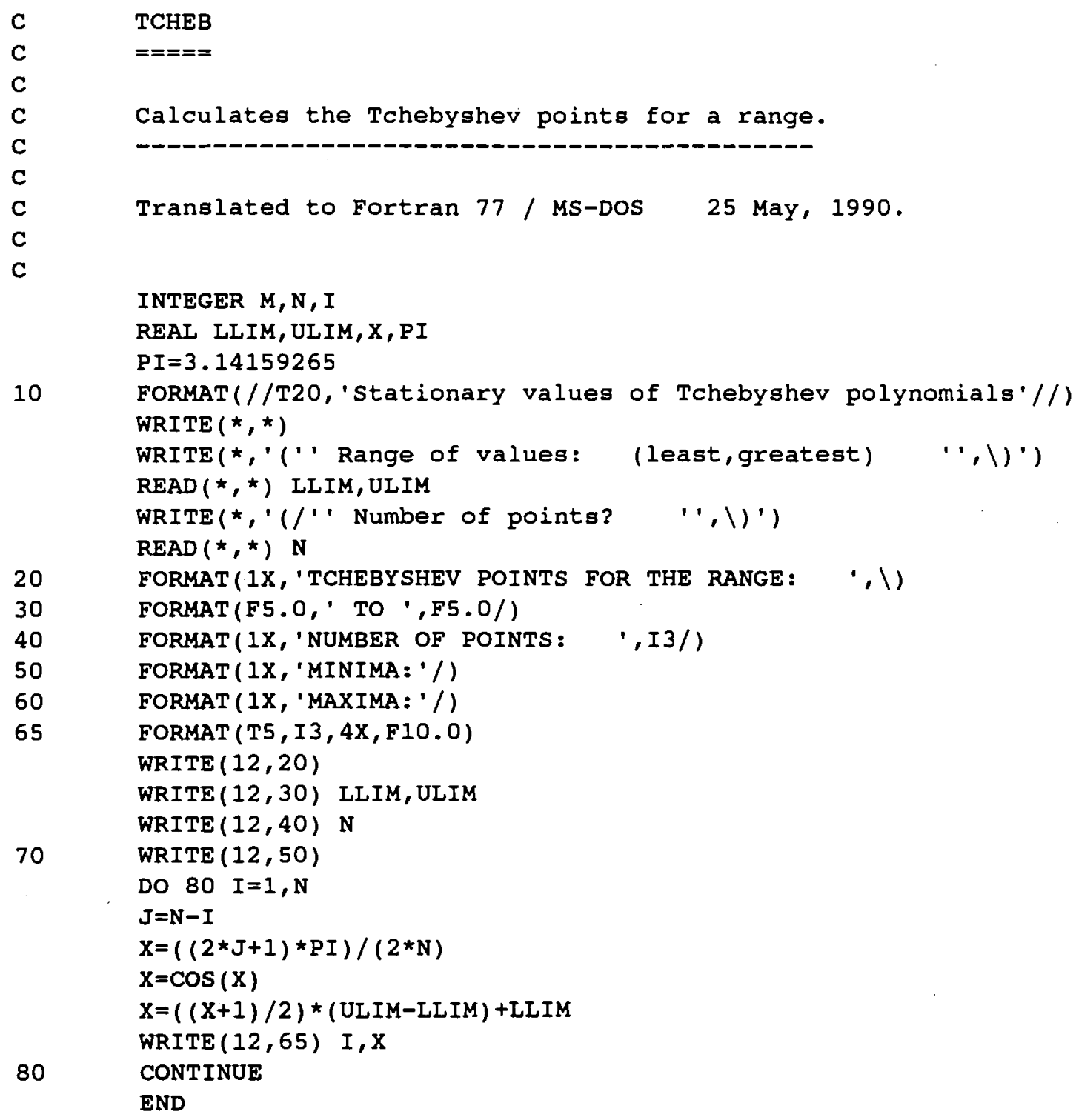


Program INTCF: Interpolation by continued fractions.

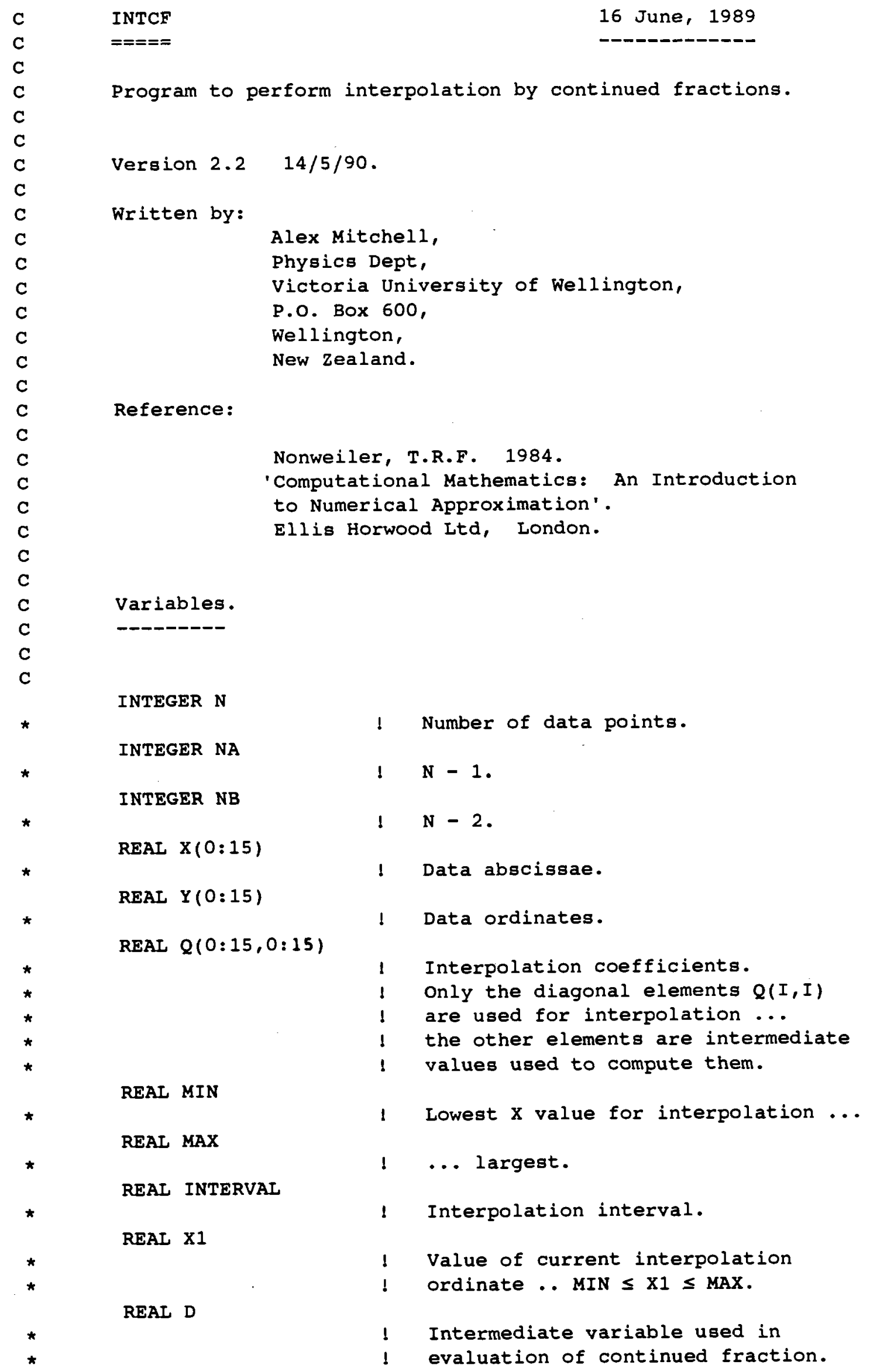


REAL $\operatorname{AR}(0: 1,0: 15)$

$\star$
$\star$
$\star$
$\star$

$\star$

$\star$
$\star$
$\star$
$\star$

$\star$
$C$
$C$
$C$
$C$
$C$
10
11
12
13
14
15
16
17
18
19
20
30
40

INTEGER DCOEF

\section{INTEGER DRF}

INTEGER NEWRANGE

INTEGER IOUT

CHARACTER*12 FILENAME

Array used in computing rational function.

$! \quad Y{ }^{\prime}=$ print coefficients.

! 'Y ' = compute rational function.

'Y ' = input new interpolation range.

! output device:

' $S$ ' = screen (default).

'P ' = printer.

'F ' = disk file.

! Name of disk file for output.

Input data points.

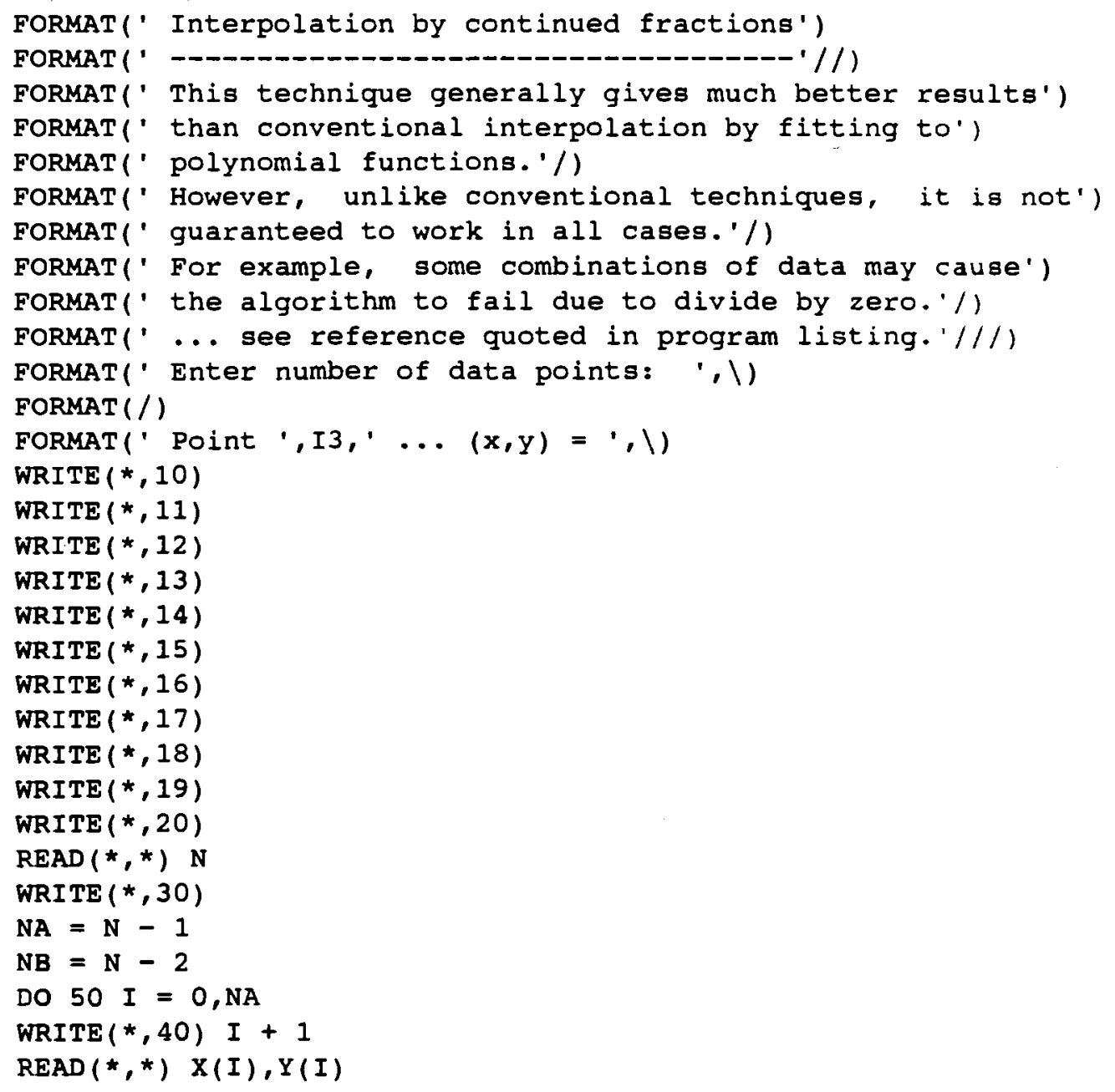


C

C

Compute coefficients.

C

C

60

DO $60 I=0, N A$

$Q(I, 0)=Y(I)$

DO $70 \mathrm{~K}=0$, NB

DO $70 \mathrm{~J}=\mathrm{K}$, NA

70

$Q(J+1, K+1)=(X(J+1)-X(K)) /(Q(J+1, K)-Q(K, K))$

$\mathrm{C}$

Print input data.

C

-

FORMAT (//, Output to [S]creen, (P)rinter or (F)ile? ' $/$ )

FORMAT (T8, 'INTERPOLATION BY CONTINUED FRACTIONS'//)

FORMAT(' Program INTCF.FOR'//)

FORMAT (' Input data: '/)

FORMAT (T18, 'I', T24, 'X(I) ', T43, 'Y(I)'/)

FORMAT (T17, I2, G15.6, T37, G15.6)

WRITE $(*, 72)$

$\operatorname{READ}\left(*, \cdot(\mathrm{A} 2)^{\prime}\right)$ ) IOUT

IF(IOUT.EQ.' $P$ '.OR.IOUT.EQ.' $p$ ') THEN $\operatorname{OPEN}(1, F I L E=$ 'PRN')

ELSEIF (IOUT.EQ.'F' 'OR. IOUT.EQ.' $f$ ') THEN

WRITE $(*, '("$ Enter file name: "'l)')

READ (*,' (A12)') FILENAME

$\operatorname{OPEN}(1$, FILE = FILENAME)

ELSE

ENDIF

$\operatorname{OPEN}\left(1, \operatorname{FILE}={ }^{\prime} \operatorname{CON}{ }^{\prime}\right)$

WRITE $(1,80)$

WRITE $(1,85)$

WRITE $(1,90)$

WRITE $(1,95)$

DO $110 I=0$, NA

110

$\operatorname{WRITE}(1,100) I, X(I), Y(I)$

C

C

Print coefficients.

C

C

C

FORMAT (//' Print coefficients? $\mathrm{Y}$ or $[\mathrm{N}]\},, \mathrm{l}$ ) FORMAT (A2)

FORMAT (//' Interpolation coefficients: '//)

FORMAT (T29, ' $Q^{\prime}, I 2,^{\prime}='$, T38,G14.7)
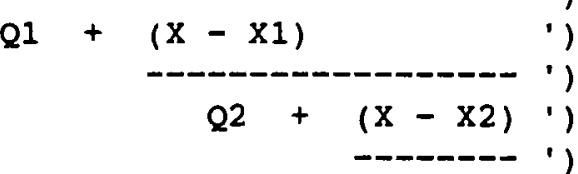

Q3 $/ / 1$ 


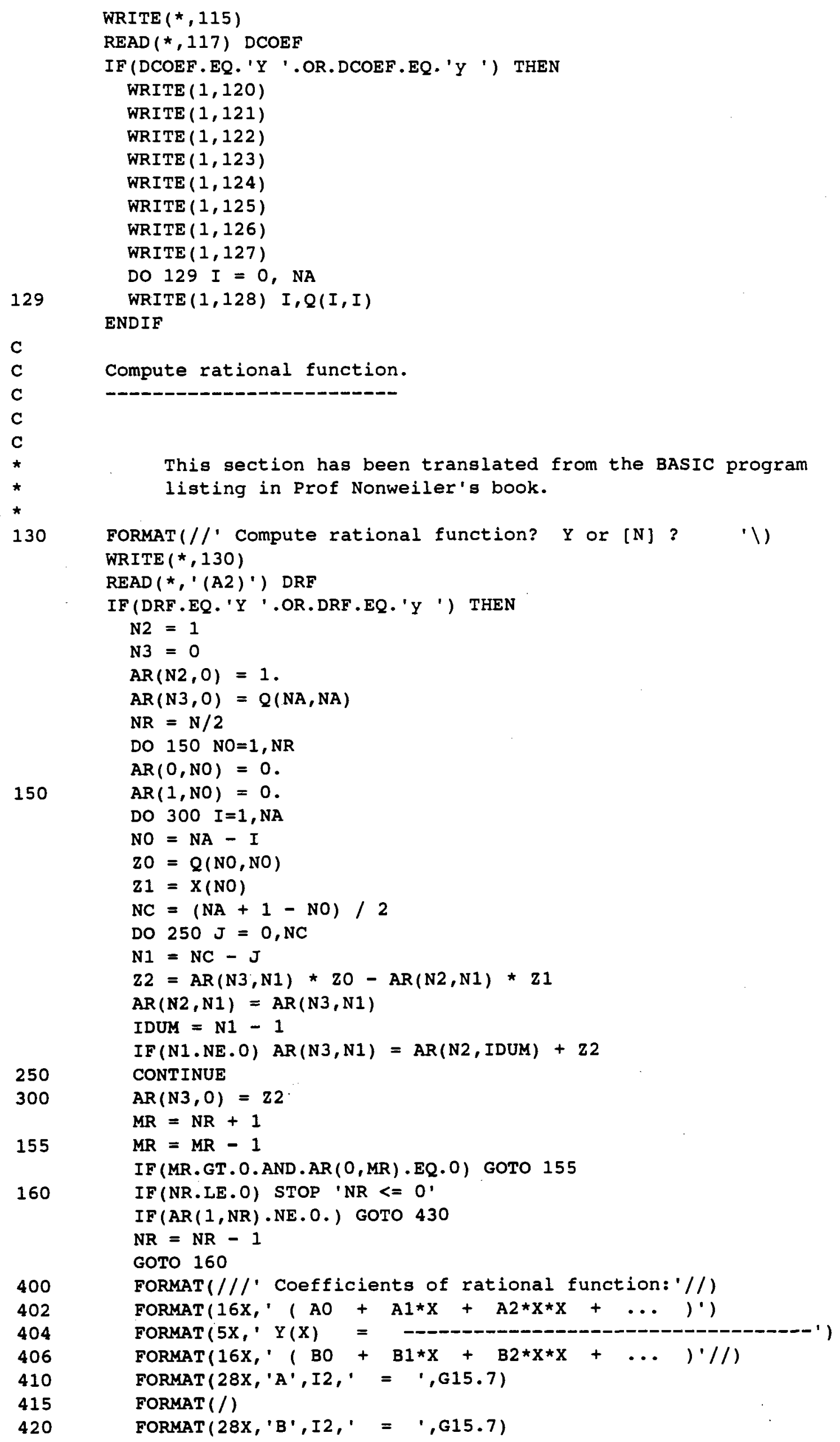




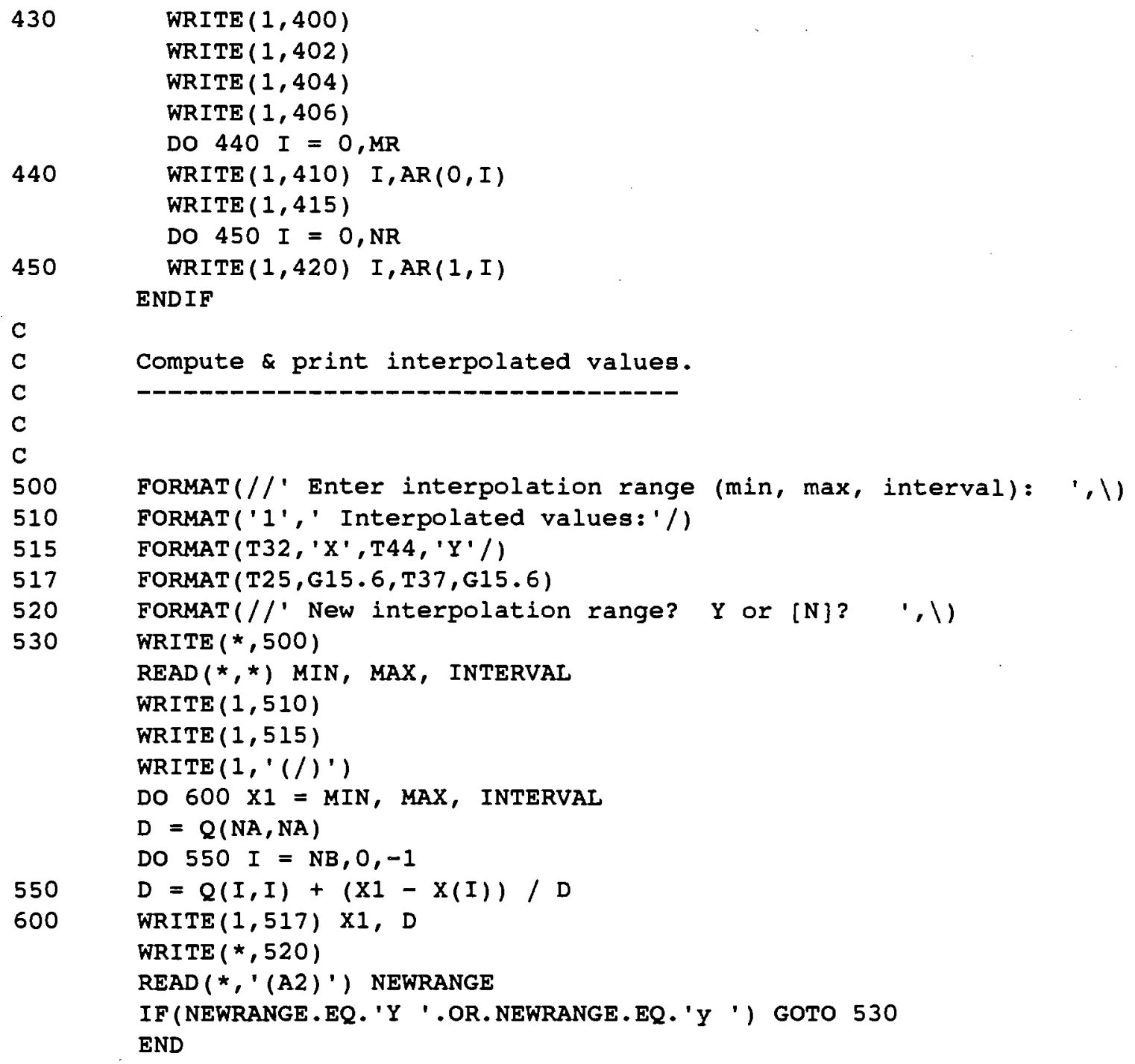




\section{APPENDIX C: Computation of X-ray attenuation Coefficients.}

\section{C.1: Program XATTEN.}

Program XATTEN calculates the atomic cross section, and mass and linear attenuation coefficients of elements using the equations of Jackson \& Hawkes. For a description of the method see section 2.7. Also included here are the contents of the two data files used by this program, UZ.DAT and ATOM.DAT. Table C. 1 lists values U(Z) as stored in file UZ.DAT. The values are taken from reference [52], except for $\mathrm{Z}>54$ for which the values listed are extrapolations.

Table C.1: Values of $\mathrm{U}(\mathrm{Z})$.

\begin{tabular}{||l|cccccc||}
\hline \hline & $1-9$ & $10-19$ & $20-29$ & $30-39$ & $40-49$ & $50-59$ \\
& & & & & \\
\hline & & 0.9901 & 1.0484 & 1.0791 & 1.1089 & 1.1389 \\
0 & 0.5000 & 0.9979 & 1.0520 & 1.0822 & 1.1120 & 1.1417 \\
1 & 0.7360 & 1.0075 & 1.0566 & 1.0854 & 1.1151 & 1.1445 \\
3 & 0.8694 & 1.0133 & 1.0591 & 1.0882 & 1.1182 & 1.1471 \\
4 & 0.9197 & 1.0199 & 1.0614 & 1.0912 & 1.1209 & 1.1511 \\
5 & 0.9457 & 1.0249 & 1.0653 & 1.0931 & 1.1235 & 1.1561 \\
6 & 0.9615 & 1.0303 & 1.0682 & 1.0970 & 1.1260 & 1.1592 \\
7 & 0.9722 & 1.0348 & 1.0710 & 1.1001 & 1.1297 & 1.1601 \\
8 & 0.9798 & 1.0390 & 1.0739 & 1.1003 & 1.1331 & 1.1625 \\
9 & 0.9856 & 1.0436 & 1.0758 & 1.1062 & 1.1361 & 1.1650 \\
\hline
\end{tabular}


Table C.1: Values Stored in ATOM.DAT.

\begin{tabular}{|c|c|c|c|c|c|c|}
\hline $\mathrm{Z}$ & \multicolumn{2}{|c|}{ Element } & Atomic wt & Density & M.P. & B.P. \\
\hline 1 & $\mathrm{H}$ & Hydrogen & 1.00794 & .0899 & -259.3400 & -252.8000 \\
\hline 2 & $\mathrm{He}$ & Helium & 4.00260 & .1785 & -272.2000 & -268.9000 \\
\hline 3 & Li & Lithium & 6.94100 & .5340 & 180.5400 & 1342.0000 \\
\hline 4 & $\mathrm{Be}$ & Beryllium & 9.01218 & 1.8500 & 1278.0000 & 2970.0000 \\
\hline 5 & B & Boron & 10.81000 & 2.3400 & 2300.0000 & 2550.0000 \\
\hline 6 & C & Carbon & 12.01100 & 3.5100 & .0000 & .0000 \\
\hline 7 & $\mathrm{~N}$ & Nitrogen & 14.00670 & 1.2506 & -209.8600 & -195.8000 \\
\hline 8 & 0 & oxygen & 15.99940 & 1.4290 & -218.4000 & -182.9620 \\
\hline 9 & $F$ & Fluorine & 18.99840 & 1.6900 & -219.6200 & -188.1400 \\
\hline 10 & $\mathrm{Ne}$ & Neon & 20.17900 & .9002 & -248.6700 & -245.9000 \\
\hline 11 & $\mathrm{Na}$ & Sodium & 22.98977 & .9700 & 97.8100 & 882.9000 \\
\hline 12 & Mg & Magnesium & 24.30500 & 1.7400 & 648.8000 & 1107.0000 \\
\hline 13 & Al & Aluminium & 26.98154 & 2.7020 & 660.3700 & 2467.0000 \\
\hline 14 & $\mathrm{Si}$ & silicon & 28.08550 & 2.3300 & 1410.0000 & 2355.0000 \\
\hline 15 & $P$ & Phosphorus & 30.97380 & 1.8200 & 44.1000 & 280.0000 \\
\hline 16 & $\mathbf{S}$ & Sulfur & 32.06400 & 2.0700 & 112.8000 & 444.6740 \\
\hline 17 & $\mathrm{Cl}$ & Chlorine & 35.45300 & 3.2140 & -100.9000 & -34.6000 \\
\hline 18 & Ar & Argon & 39.94800 & 1.7840 & -189.2000 & -185.7000 \\
\hline 19 & $\mathrm{~K}$ & Potassium & 39.09830 & .8600 & 63.2500 & 760.0000 \\
\hline 20 & $\mathrm{Ca}$ & Calcium & 40.08000 & 1.5400 & 839.0000 & 1484.0000 \\
\hline 21 & Sc & Scandium & 44.95590 & 2.9890 & 1541.0000 & 2836.0000 \\
\hline 22 & $\mathrm{Ti}$ & Titanium & 47.88000 & 4.5000 & 1660.0000 & 3287.0000 \\
\hline 23 & $\mathrm{~V}$ & Vanadium & 50.94150 & 5.9600 & 1890.0000 & 3380.0000 \\
\hline 24 & $\mathrm{Cr}$ & Chromium & 51.99600 & 7.2000 & 1857.0000 & 2672.0000 \\
\hline 25 & Mn & Manganese & 54.93800 & 7.2000 & 1244.0000 & 1962.0000 \\
\hline 26 & $\mathrm{Fe}$ & Iron & 55.84700 & 7.8600 & 1535.0000 & 2750.0000 \\
\hline 27 & Co & Cobalt & 58.93320 & 8.9000 & 1495.0000 & 2870.0000 \\
\hline 28 & $\mathrm{Ni}$ & Nickel & 58.69000 & 8.9000 & 1455.0000 & 2730.0000 \\
\hline 29 & $\mathrm{Cu}$ & Copper & 63.54600 & 8.9200 & 1083.4000 & 2567.0000 \\
\hline 30 & $\mathrm{Zn}$ & Zinc & 65.38000 & 7.1400 & 419.5800 & 907.0000 \\
\hline 31 & $\mathbf{G a}$ & Gallium & 69.72000 & 5.9040 & 29.7800 & 2403.0000 \\
\hline 32 & $\mathrm{Ge}$ & Germanium & 72.59000 & 5.3500 & 937.4000 & 2830.0000 \\
\hline 33 & As & Arsenic & 74.92160 & 5.7270 & 817.0000 & 613.0000 \\
\hline 34 & $\mathrm{Se}$ & Selenium & 78.96000 & 4.8100 & 217.0000 & 684.0000 \\
\hline 35 & $\mathrm{Br}$ & Bromine & 79.90400 & 2.9280 & -7.2000 & 58.7800 \\
\hline 36 & $\mathrm{Kr}$ & Krypton & 83.80000 & 3.7360 & -156.6000 & -152.3000 \\
\hline 37 & $\mathrm{Rb}$ & Rubidium & 85.46780 & 1.5320 & 38.8900 & 686.0000 \\
\hline 38 & $\mathrm{sr}$ & strontium & 87.62000 & 2.6000 & 769.0000 & 1384.0000 \\
\hline 39 & $Y$ & Yttrium & 88.90590 & 4.4690 & 1522.0000 & 3338.0000 \\
\hline 40 & $\mathrm{Zr}$ & Zirconium & 91.22000 & 6.4900 & 1852.0000 & 4377.0000 \\
\hline 41 & $\mathrm{Nb}$ & Niobium & 92.90640 & 8.5700 & 2468.0000 & 5127.0000 \\
\hline 42 & Mo & Molybdenum & 95.94000 & 10.2000 & 2610.0000 & 5560.0000 \\
\hline 43 & $\mathrm{Tc}$ & Technetium & 98.00000 & .0000 & 2172.0000 & 4877.0000 \\
\hline 44 & Ru & Ruthenium & 101.07000 & 12.3000 & 2310.0000 & 3900.0000 \\
\hline 45 & $\mathrm{Rh}$ & Rhodium & 102.90550 & 12.4000 & 1966.0000 & 3727.0000 \\
\hline 46 & $\mathrm{Pd}$ & Palladium & 106.42000 & 12.0200 & 1554.0000 & 2970.0000 \\
\hline 47 & $\mathrm{Ag}$ & Silver & 107.86820 & 10.5000 & 961.9300 & 2212.0000 \\
\hline 48 & $c d$ & Cadmium & 112.41000 & 8.6420 & 320.9000 & 765.0000 \\
\hline 49 & In & Indium & 114.82000 & 7.3000 & 156.6100 & 2080.0000 \\
\hline 50 & $\mathrm{sn}$ & Tin & 118.69000 & 5.7500 & 231.9681 & 2270.0000 \\
\hline
\end{tabular}




\begin{tabular}{|c|c|c|c|c|c|c|}
\hline $\mathbf{Z}$ & \multicolumn{2}{|c|}{ Element } & Atomic wt & Density & M.P. & B.P. \\
\hline 51 & $\mathrm{Sb}$ & Antimony & 121.75000 & 6.6840 & 630.5000 & 1750.0000 \\
\hline 52 & $\mathrm{Te}$ & Tellurium & 127.60000 & 6.2500 & 452.0000 & 1390.0000 \\
\hline 53 & $I$ & Iodine & 126.90450 & 4.9300 & 113.5000 & 184.3500 \\
\hline 54 & $\mathrm{Xe}$ & Xenon & 131.29000 & 5.8870 & -111.9000 & -107.1000 \\
\hline 55 & Cs & Cesium & 132.90540 & 1.8785 & 28.4000 & 669.3000 \\
\hline 56 & $\mathrm{Ba}$ & Barium & 137.33000 & 3.5100 & 725.0000 & 1640.0000 \\
\hline 57 & La & Lanthanum & 138.90550 & 6.1460 & 921.0000 & 3464.0000 \\
\hline 58 & $\mathrm{Ce}$ & Cerium & 140.12000 & 6.6890 & 798.0000 & 3443.0000 \\
\hline 59 & Pr & Praseodymium & 140.90770 & 6.7730 & .0000 & .0000 \\
\hline 60 & $\mathrm{Nd}$ & Neodymium & 144.24000 & 7.0080 & 1021.0000 & 3074.0000 \\
\hline 61 & $\mathrm{Pm}$ & Promethium & 145.00000 & .0000 & 1042.0000 & 3000.0000 \\
\hline 62 & $\mathrm{Sm}$ & Samarium & 150.36000 & 7.5200 & 1074.0000 & 1794.0000 \\
\hline 63 & Eu & Europium & 151.96000 & 5.2440 & 822.0000 & 1527.0000 \\
\hline 64 & Gd & Gadolinium & 157.25000 & .0000 & 1313.0000 & 3273.0000 \\
\hline 65 & $\mathrm{~Tb}$ & Terbium & 158.92540 & 8.2300 & 1356.0000 & 3230.0000 \\
\hline 66 & Dy & Dysprosium & 162.50000 & 8.5510 & 1412.0000 & 2567.0000 \\
\hline 67 & Ho & Holmium & 164.93040 & 8.9750 & 1474.0000 & 2700.0000 \\
\hline 68 & Er & Erbium & 167.26000 & 9.0660 & 1529.0000 & 2868.0000 \\
\hline 69 & $\mathrm{Tm}$ & Thulium & 168.93420 & 9.3210 & 1545.0000 & 1950.0000 \\
\hline 70 & $\mathrm{Yb}$ & Ytterbium & 173.04000 & 6.9600 & 819.0000 & 1196.0000 \\
\hline 71 & $\mathrm{Lu}$ & Lutetium & 174.96700 & 9.8410 & 1663.0000 & 3402.0000 \\
\hline 72 & $\mathrm{Hf}$ & Hafnium & 178.49000 & 13.3100 & 2227.0000 & 4602.0000 \\
\hline 73 & $\mathrm{Ta}$ & Tantalum & 180.94790 & 16.6000 & 2996.0000 & 5425.0000 \\
\hline 74 & $\mathrm{~W}$ & Tungsten & 183.85000 & 19.3500 & 3410.0000 & 5660.0000 \\
\hline 75 & $\operatorname{Re}$ & Rhenium & 186.20700 & 20.5300 & 3180.0000 & 5627.0000 \\
\hline 76 & Os & Osmium & 190.20000 & 22.4800 & 2700.0000 & 5300.0000 \\
\hline 77 & Ir & Iridium & 192.22000 & 22.4210 & 2410.0000 & 4130.0000 \\
\hline 78 & Pt & Platinum & 195.08000 & 21.4500 & 1772.0000 & 3827.0000 \\
\hline 79 & $\mathrm{Au}$ & Gold & 196.96650 & 19.3100 & 1064.4300 & 2808.0000 \\
\hline 80 & $\mathrm{Hg}$ & Mercury & 200.59000 & 13.5462 & -38.8700 & 356.5800 \\
\hline 81 & $\mathrm{Tl}$ & Thallium & 204.38300 & 11.8500 & 303.5000 & 1457.0000 \\
\hline 82 & $\mathrm{~Pb}$ & Lead & 207.20000 & 11.3437 & 327.5020 & 1740.0000 \\
\hline 83 & $\mathrm{Bi}$ & Bismuth & 208.98040 & 9.8000 & 271.3000 & 1560.0000 \\
\hline 84 & Po & Polonium & 209.00000 & 9.4000 & 254.0000 & 962.0000 \\
\hline 85 & At & Astatine & 210.00000 & .0000 & 302.0000 & 337.0000 \\
\hline 86 & $\mathrm{Rn}$ & Radon & 222.00000 & 9.7300 & -71.0000 & -61.8000 \\
\hline 87 & Fr & Francium & 223.00000 & .0000 & 27.0000 & 677.0000 \\
\hline 88 & $\mathrm{Ra}$ & Radium & 226.02540 & 5.0000 & 700.0000 & 1140.0000 \\
\hline 89 & Ac & Actinium & 227.02780 & .0000 & 1050.0000 & 3200.0000 \\
\hline 90 & $\mathrm{Th}$ & Thorium & 232.03810 & 11.7000 & .0000 & .0000 \\
\hline 91 & $\mathrm{~Pa}$ & Protactinium & 231.03590 & 15.3700 & 1600.0000 & .0000 \\
\hline 92 & $\mathrm{U}$ & Uranium & 238.02890 & 19.0500 & 1132.3000 & 3818.0000 \\
\hline 93 & Np & Neptunium & 237.04820 & 20.4500 & 630.0000 & .0000 \\
\hline 94 & $\mathrm{Pu}$ & Plutonium & 242.05900 & 19.8400 & 641.0000 & 3232.0000 \\
\hline 95 & Am & Americium & 243.00000 & .0000 & 994.0000 & 2607.0000 \\
\hline 96 & $\mathrm{Cm}$ & Curium & 247.00000 & .0000 & 1340.0000 & .0000 \\
\hline 97 & $\mathrm{Bk}$ & Berkelium & 247.00000 & .0000 & .0000 & .0000 \\
\hline 98 & Cf & Californium & 251.00000 & .0000 & .0000 & .0000 \\
\hline 99 & Es & Einsteinium & 252.00000 & .0000 & .0000 & .0000 \\
\hline 100 & $\mathrm{Fm}$ & Fermium & 257.00000 & .0000 & .0000 & .0000 \\
\hline 101 & Md & Mendelevium & 258.00000 & .0000 & .0000 & .0000 \\
\hline 102 & No & Nobelium & 259.00000 & .0000 & .0000 & .0000 \\
\hline 103 & Lw & Lawrencium & 260.00000 & .0000 & .0000 & .0000 \\
\hline
\end{tabular}




\section{Program XATTEN: Attenuation Coefficients of Elements.}

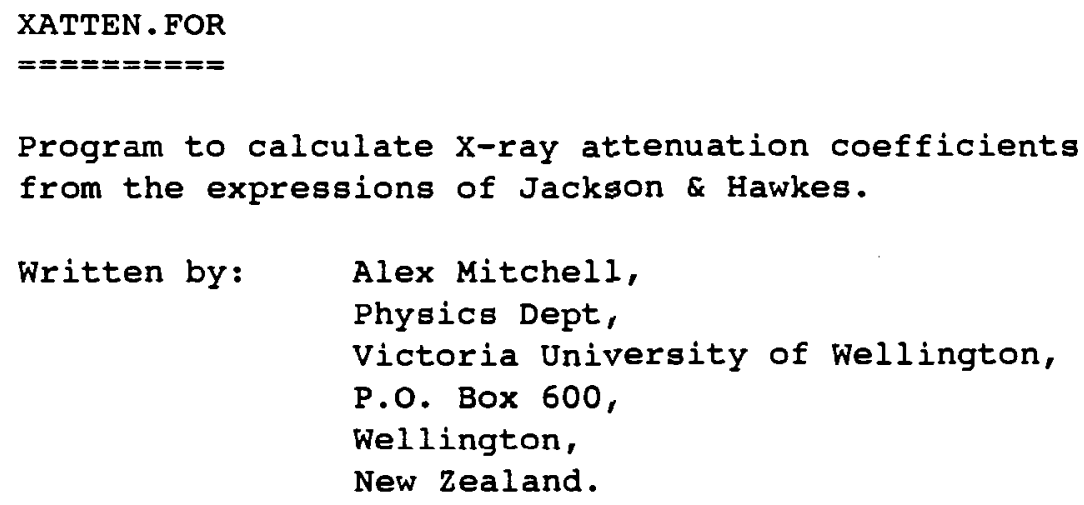


REAL COHERENT

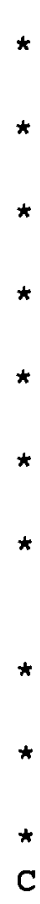

REAL SIGMAPE

REAL COMPTON

REAL SIGMATOT

REAL MUMASS

REAL MULIN

Linear attenuation coefficient.

PARAMETER ( ALPHA $=7.2973506 \mathrm{E}-3$ )

PARAMETER ( $\mathrm{PI}=3.14159265)$

PARAMETER ( $\mathrm{mc2}=511.0034$ )

PARAMETER ( PHI $=0.6652448)$

Rest mass energy of the electron.

PARAMETER ( AVOGADRO $=6.02252 \mathrm{E} 23$ )

Avogadro's number.

DATA $X / 15,20,30,40,50,60,80,100,150,200,300 /$

DATA A/3.948, $-3.78501,-4.78898,75.2795,1.17671,-84.6219$,

$+-0.363487,2044.22,0.0287639,3.24746 \mathrm{E} 6,-1.87777 \mathrm{E}-5 /$

C

10

20

30

40

50

60

70

80

90

100

C

C

C

C

FORMAT (/' XATTEN: Program to compute attenuation coefficients')
FORMAT( FORMAT(" These formulae give values which are close to')

FORMAT(" 'best" theoretical values. Physical constants for') FORMAT(' the elements are taken from the 1990 edition of the')

FORMAT(' Handbook of Chemistry \& Physics. The density value')

FORMAT(" used for each element was that of the most common ')

FORMAT(' isotope.'//)

FORMAT(' Enter atomic number: ', I)

FORMAT (/' Enter energy range (min,max, interval): ', l)

WRITE $(*, 10)$

WRITE $(*, 20)$

WRITE $(*, 30)$

WRITE $(*, 40)$

WRITE $(*, 50)$

WRITE $(*, 60)$

WRITE $(*, 70)$

WRITE $(*, 80)$

105 WRITE $(*, 90)$

$\operatorname{READ}(*, *) 2$

$\mathrm{RZ}=\mathrm{FLOAT}(\mathrm{Z})$

IF(z.GT.60) WRITE(*,*) ' $z$ must be less than 61'

IF (Z.GT.60) GOTO 105

Read data files.

$\operatorname{OPEN}(1, F I L E=' U Z \cdot D A T ')$

DO $120 \mathrm{IZ}=1,60$

$\operatorname{READ}(1, *) U(I Z)$

$\operatorname{OPEN}(2, \mathrm{FILE}=$ 'ATOM.DAT', ACCESS = 'DIRECT', RECL $=57$,

+ FORM = 'FORMATTED')

FORMAT (A2, 1X, A12, 1X, I3 , 1X, F9.5, 1X, F7 . 4, 1X, F9.4 , 1X, F9.4)

FORMAT $\left(/ 1 \mathrm{X}, \mathrm{A},{ }^{\prime}\right.$ Atomic wt $=$ ',F8.4,' Density $=$ ', F8.4,' $\mathrm{g} / \mathrm{CC}{ }^{\prime}$ )

$\operatorname{READ}(2,130, \mathrm{REC}=$ Z) SYMBOL, NAME , I, AWT , DENSITY , MP , BP

WRITE $(*, 140)$ NAME, AWT, DENSITY 
C
C
C
C
C

Input Energy Range.

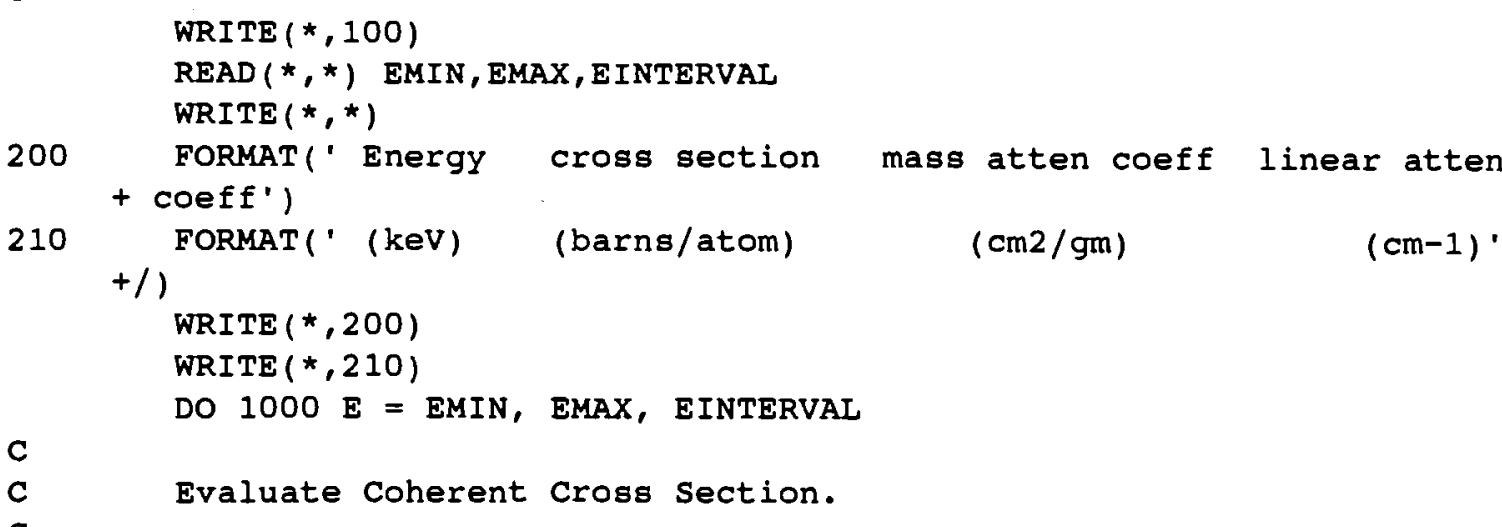




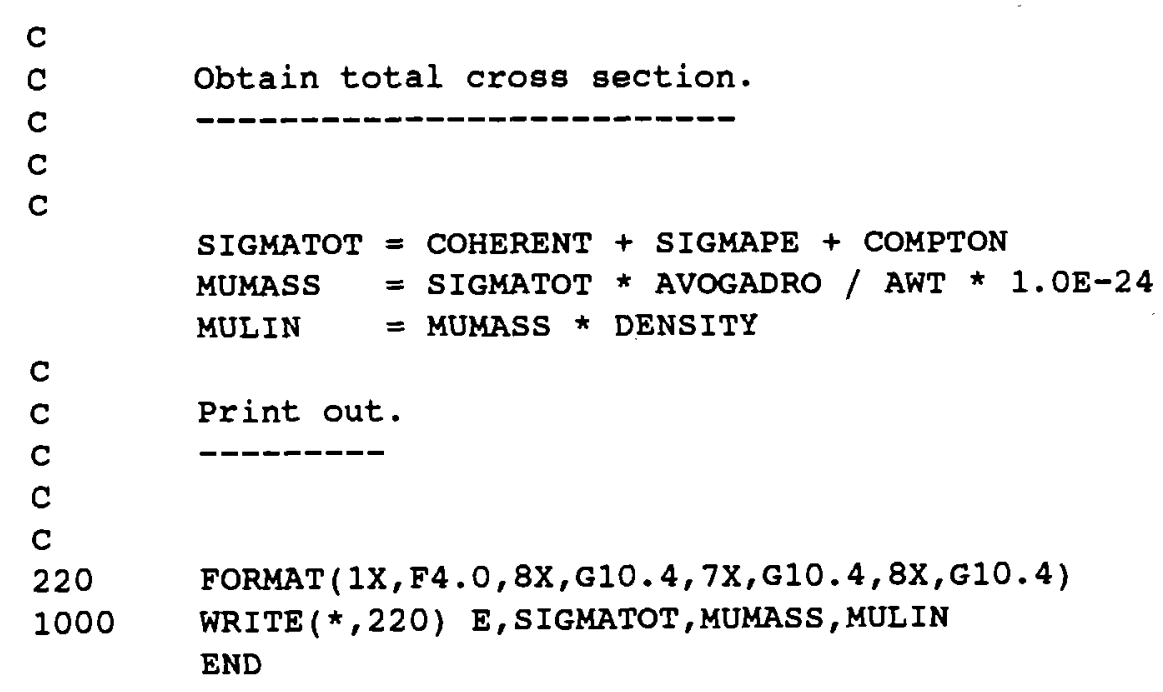




\section{C.2: Program MCOEF: Attenuation Coefficients of Compounds.}

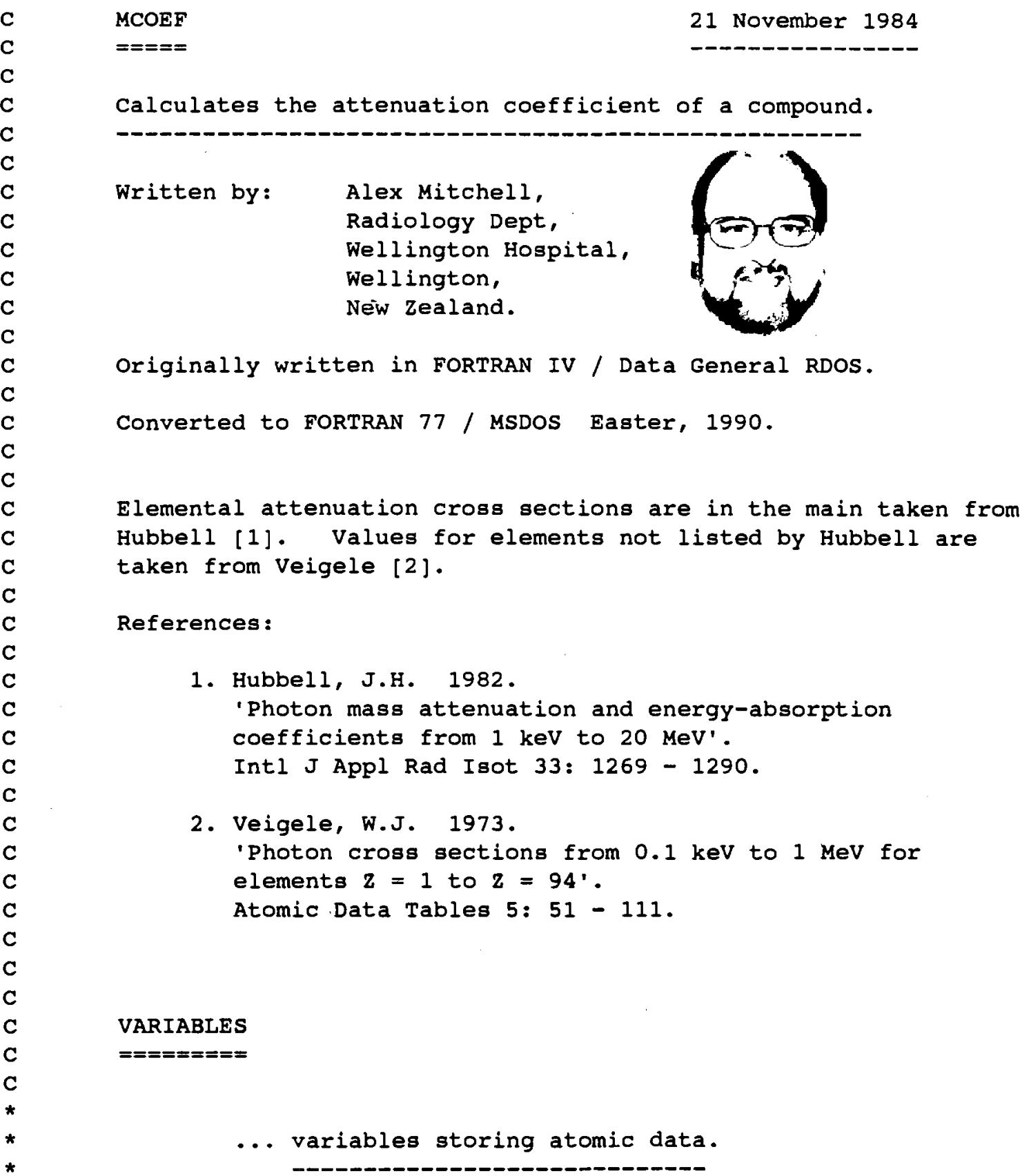

REAL AVOGADRO

INTEGER $\mathrm{Z}$

Avogadro's number.

REAL MWT

Atomic number.

INTEGER*2 SYMBOL (75)

REAL AWT (75)

REAL DENSITY (75)

REAL RDENSITY

Molecular weight.

Array of element symbols.

Array of element atomic weights.

Array of element densities.

Density of compound. 


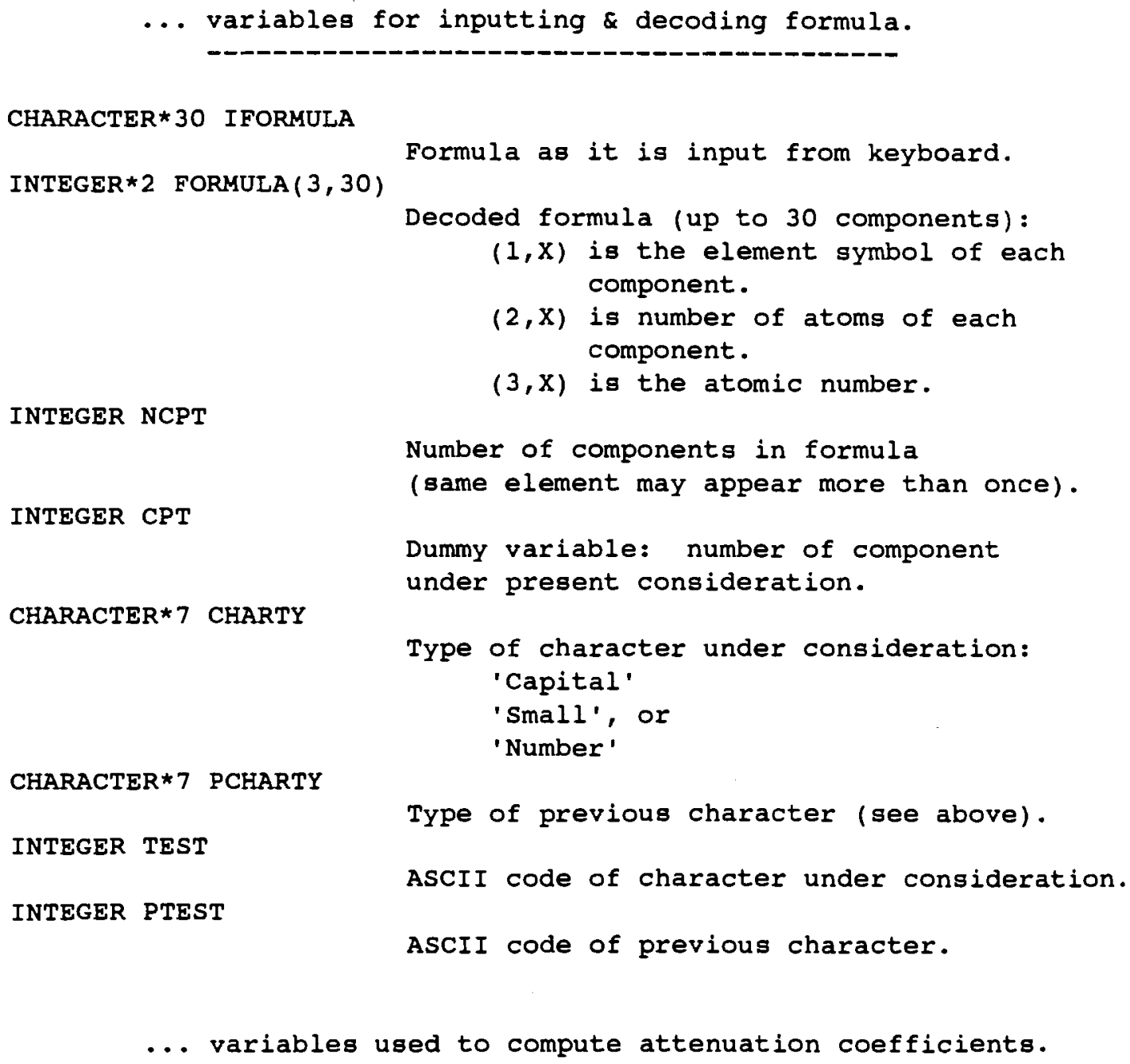

Photon energy. 


$*$
$*$
$*$
$*$
$\star$
$*$
$*$
$\star$
$*$
$*$
C
C
C
C

\section{... variables for program control.}

\section{INTEGER DEVICE}

INTEGER DUMMY

INTEGER DCHANGE

INTEGER DPRINT

INTEGER DSTORE

Initialise constants.
Unit number of current output device. ... $3=$ printer, $4=$ disk file.

Dummy variable for use in DO loops.

Confirmation that formula is correct. ' $Y$ ' means formula is to be changed

'Y ' means values are to be printed.

'Y ' means store values in a file.

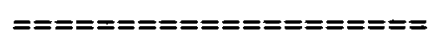

DATA $x / 10.0,15.0,20.0,30.0,40.0,50.0,60.0,80.0,100.0,150.0 /$ DPRINT $=$ '

AVOGADRO $=6.02252 \mathrm{E} 23$

$\operatorname{OPEN}(1, \operatorname{FILE}=$ 'ATOM.DAT', ACCESS = 'DIRECT', RECL = 57,

+ FORM $=$ 'FORMATTED', MODE = 'READ')

$\operatorname{OPEN}(2, \mathrm{FILE}=$ 'H82COEF.DAT', ACCESS = 'DIRECT', RECL = 13,

+ FORM $=$ 'FORMATTED', MODE = 'READ')

FORMAT (A2 , 1X, A12, 1X, I3, 1X, F9.5, 1X, F7.4)

DO $302 \mathrm{Z}=1,75$

$\operatorname{READ}(1,301, \operatorname{REC}=\mathrm{Z}) \operatorname{SYMBOL}(\mathrm{Z}), \operatorname{NAME}, \mathrm{I}, \operatorname{AWT}(\mathrm{Z}), \operatorname{DENSITY}(Z)$

INPUT FORMULA IN ASCII AND DECODE

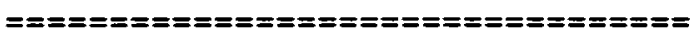

The formula is entered as it would be written, but without subscripts for the numbers, eg Na2HPO4.

Brackets are not permitted. Write $\mathrm{Ca}(\mathrm{OH}) 2$ as $\mathrm{CaO} 2 \mathrm{H} 2$.

The program uses the presence of a space to signify the end of the formula. A capital is recognised as signifying the beginning of a new element symbol.

The program decodes the formula by examining the input on a character by character basis, with separate routines to handle capital letters, small letters, and numbers.

FORMAT (' 1 //T22, 'ATTENUATION COEFFICIENTS OF A COMPOUND'//) FORMAT(' The formula is entered as it would be written, but') FORMAT(' without subscripts for the numbers, eg Na2HPO4.'/) FORMAT(' Brackets are not permitted. Write $\mathrm{Ca}(\mathrm{OH}) 2$ as $\mathrm{CaO} 2 \mathrm{H} 2$. '/) FORMAT(' Element symbols may be used more than once in the') FORMAT(' formula, eg C2H5OH'/) FORMAT (/, Enter formula (eg NaCl): ' FORMAT (A30) WRITE $(*, 10)$ WRITE $(*, 12)$ WRITE (*, 14) WRITE (*, 16) WRITE (*, 18) WRITE ( *, 19) WRITE $(*, 20)$ 


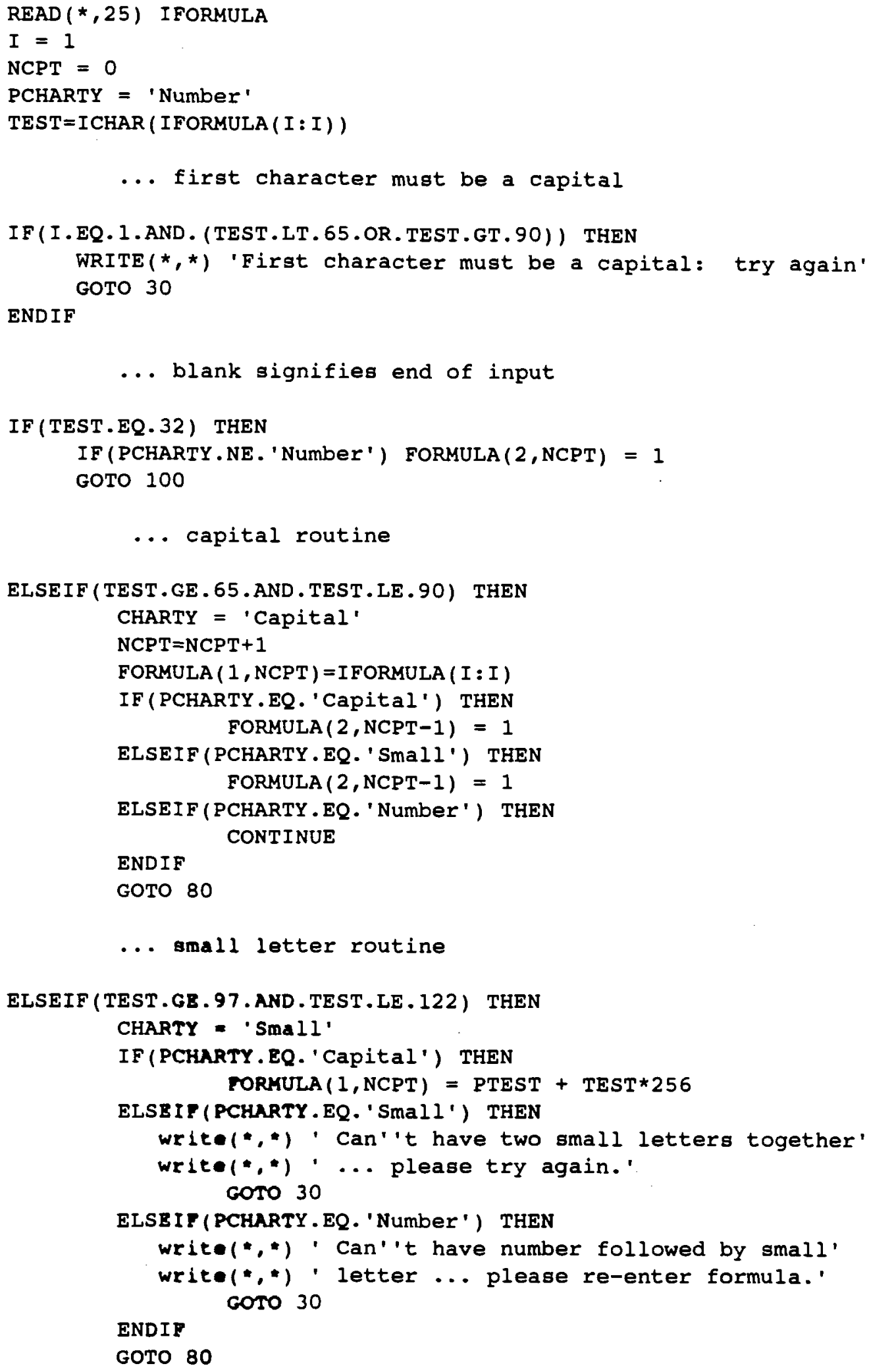




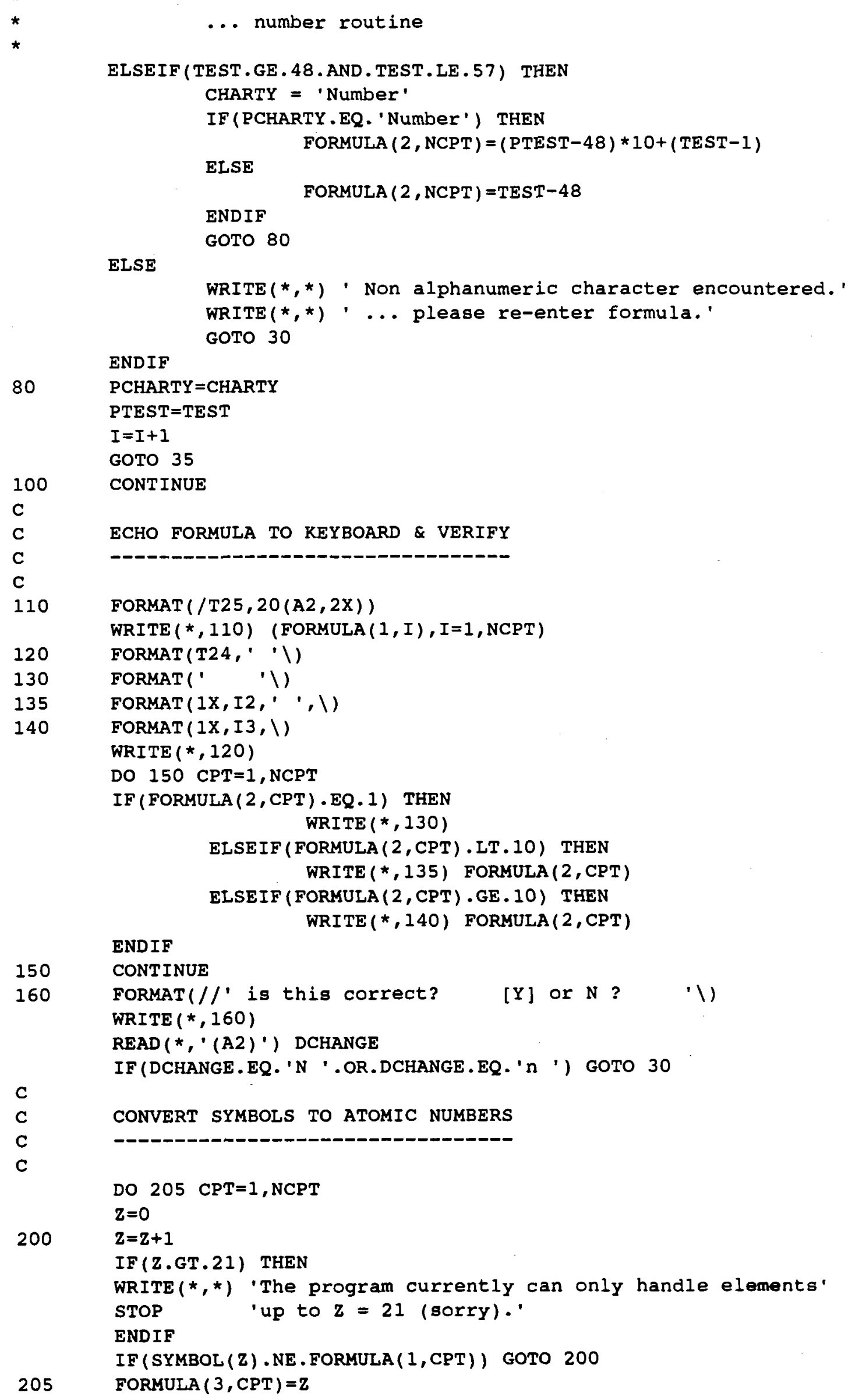




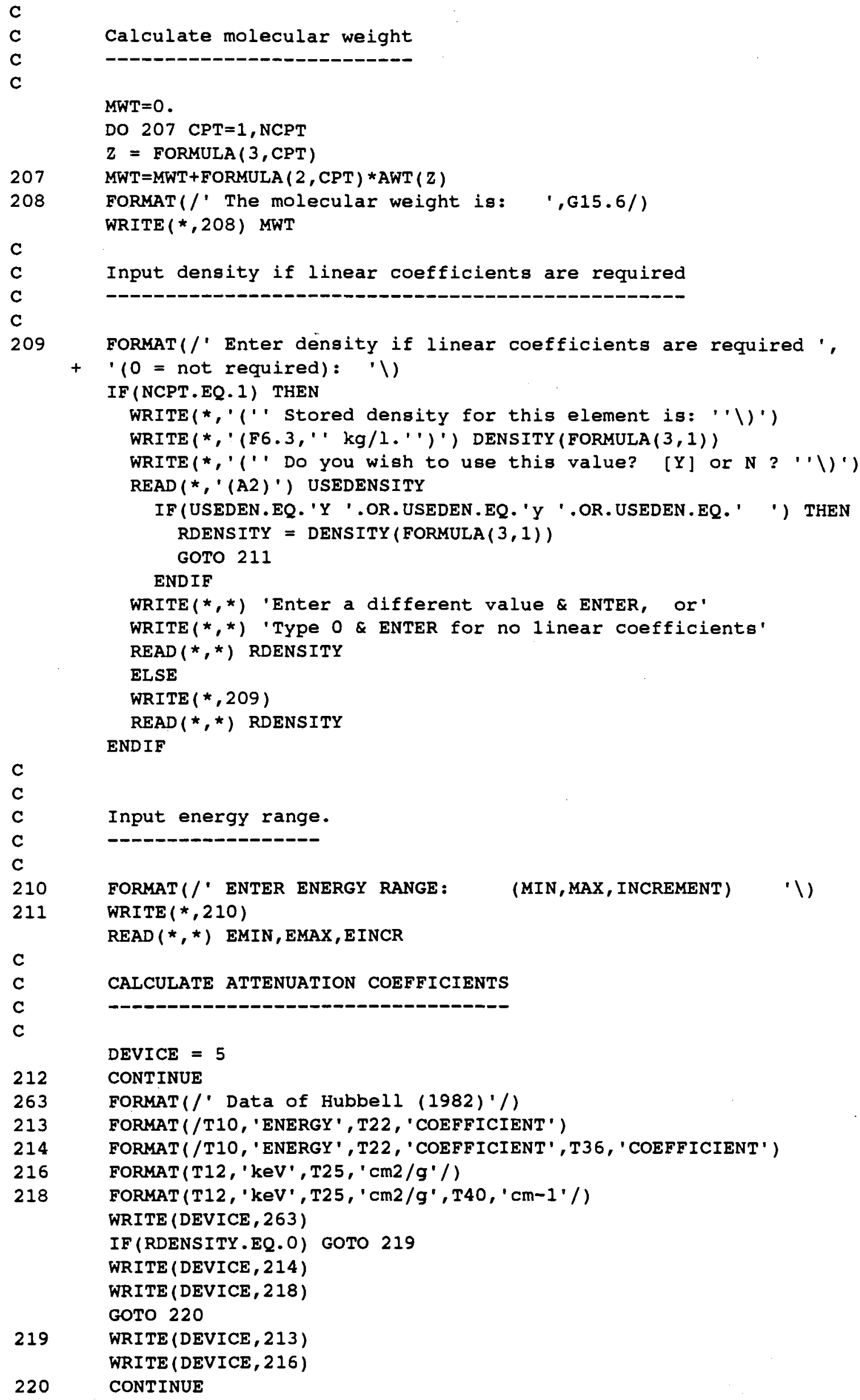

Input energy range.

FORMAT (/' ENTER ENERGY RANGE: (MIN,MAX, INCREMENT) ' ) WRITE $(*, 210)$ READ (*, *) EMIN, EMAX, EINCR

CALCULATE ATTENUATION COEFFICIENTS 
C
C
C
C
C

1000
$C$
$C$
$C$
$C$
$C$

N2 $=8$

$E=E M I N$

1010 TATTEN=0.

DO $230 \mathrm{CPT}=1$, NCPT

$Z=F O R M U L A(3, C P T)$

$\mathrm{D}=\mathrm{Q}(\mathrm{CPT}, 10)$

DO $1020 I=1, N 2$

DUMMY $=10-I$

$1020 \quad D=Q(C P T, D U M M Y)+(E-X(D U M M Y)) / D$

$A T T E N=Q(C P T, 1)+(E-X(1)) / D$

ATTEN $=$ ATTEN $/($ AVOGADRO $/$ AWT $(Z) * 1 . E-24)$

TATTEN=TATTEN+FORMULA $(2$, CPT $)$ *ATTEN

230

CONTINUE

ATTEN $=$ TATTEN * AVOGADRO $/ \mathrm{MWT} * 1 \mathrm{E}-24$

LATTEN $=$ ATTEN $*$ RDENSITY

240 FORMAT (T11, F6.2, T23, G11.4, T38, G11.4)

C 250 FORMAT(T11, F6.2,T23,G11.4,T38,G11.4)

IF (RDENSITY.EQ.0) WRITE (DEVICE, 240) E,ATTEN

IF (RDENSITY.NE.0) WRITE(DEVICE, 240) E, ATTEN, LATTEN $E=E+E I N C R$

IF (E.LE.EMAX) GOTO 1010 
PRINT OUT

-ーーーーーー-

IF (DPRINT.EQ.'T') GOTO 310

IF (DPRINT.EQ.'S ') STOP

260

FORMAT (/' Print the above values? Y or [N] ? '

261

FORMAT (T30, 'ATTENUATION COEFFICIENTS //)

FORMAT(' COMPOUND:')

WRITE $(*, 260)$

READ (*,'(A2)') DPRINT

IF (DPRINT.NE.'Y ' AND.DPRINT.NE.'Y') GOTO 310

DEVICE $=3$

WRITE $(*, *)$

$\operatorname{WRITE}(*, *)$ ' Enter filename for printer ...'

WRITE $(*, *)$, ... PRN for serial printer, or'

WRITE $(*, *)$ ' ... COMI for parallel printer'

$\operatorname{WRITE}(*, *)$

$\operatorname{WRITE}(3,261)$

WRITE $(3,272)$

275 FORMAT (/T25,20(A2,2X))

WRITE $(3,275)$ (FORMULA $(1, I), I=1, \operatorname{NCPT}$ )

280

285

290

292

295

ENDIF

FORMAT (T24,' ' $)$

FORMAT("

')

FORMAT $(1 \mathrm{X}, \mathrm{I2}, '$ ', $)$

FORMAT $(1 \mathrm{X}, \mathrm{I3}, \backslash)$

WRITE $(3,280)$

DO $295 \mathrm{CPT}=1$, NCPT

IF (FORMULA (2, CPT) . EQ.1) THEN

$\operatorname{WRITE}(3,130)$

ELSEIF (FORMULA (2, CPT). LT. 10) THEN

WRITE $(3,135)$ FORMULA $(2$, CPT $)$

ELSEIF (FORMULA ( 2, CPT) . GE. 10) THEN

WRITE $(3,140)$ FORMULA $(2$, CPT $)$

297

CONTINUE

FORMAT (/)

298 FORMAT (/' DENSITY: ', T12,G15.6/)

IF (RDENSITY.EQ.0) WRITE $(3,297)$

IF (RDENSITY.NE.0) WRITE $(3,298)$ RDENSITY

DPRINT $=$ ' $T$ '

GOTO 212

C

Store values in a file

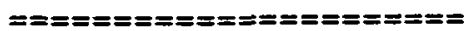

C

C

FORMAT (/' Store values for this compound? Y or [N] ? '

310 WRITE $(*, 300)$

$\operatorname{READ}(*, '(A 2)$ ') DSTORE

IF (DSTORE. NE. ' Y ' .AND.DSTORE.NE.' Y ') STOP

DPRINT $=$ 'S '

DEVICE $=4$

GOTO 212

END 


\section{C.3: Coefficients for Simple Empirical Formula.}

The following coefficients are for the two parameter fit to the attenuation coefficient described in section 2.8 .

$\begin{array}{rc}1 & -7.685 \\ 2 & 3.803 \\ 3 & 72.18 \\ 4 & 170.8 \\ 5 & 489 . \\ 5 & 1340 \\ 7 & 3046 \\ 8 & 6209 \\ 9 & 11560 \\ 10 & 18990 \\ 11 & 30190 \\ 12 & 44600 \\ 13 & 53920 \\ 14 & 88260 \\ 15 & 117100 \\ 16 & 147600 \\ 17 & 183300 \\ 18 & 222000 \\ 19 & 266800 \\ 20 & 312800 \\ 22 & 415600 \\ 25 & 589600 \\ 26 & 652300 \\ 29 & 838300 \\ 30 & 901200\end{array}$

2. 714

1.657

2. 279

2.324

2. 440

2.567

2.657

2.730

2.791

2.820

2.869

2.896

2.922

2.942

2. 957

2. 963

2.968

2.970

2. 972

2. 971

2.967

2. 954

2. 949

2.928

2. 921 


\section{C.4: Interpolation Coefficients for Five Parameter Fit.}

The following coefficients are to be used for computing attenuation coefficients of the elements using 5 point continued fraction interpolation. See section 2.10.

00

01

$$
\begin{aligned}
& 3.35525 E-01 \\
& 1.703 \mid 2 E-01 \\
& 1.48890 E-01 \\
& 1.55426 E-01 \\
& 1.6050 \mid E-01 \\
& 1.8709 \mid E-01 \\
& 1.97967 E-01
\end{aligned}
$$

$2.13265 E-01$

$2.214|0 \mathrm{E}-0|$

$2.57903 \mathrm{E}-01$

$2.80383 E \sim 01$

$3.29 ! 28 E-0 !$

$3.68179 E-01$

$4.38533 E-0$

4.91677E-O

$5.84952 E-0.1$

$6.48354 E-01$

7. 01254 E-OI

$8.67844 E=01$

1. $0.1948 E+00$

$1.08722 E+00$

$1.21303 E+00$

I. $34758 E+00$

$1.550 .16 \mathrm{E}+00$

$1.71386 E * 00$

$1.95804 E+00$

$2.14379 E+\infty$

$2.47332 E+00$

$2.61364 E \neq 00$

$2.89351 E+00$

$3.076 / 3 E+00$

$3.33624 \mathrm{E}+00$

$3.63489 E+00$

$3.864 .52 \mathrm{E}+00$

$4.26375 E+00$
$-1.04647 E+03$

$-1.91074 E+03$

$-.1 .96334 \mathrm{E}+03$

$-.1 .59633 E+03$

$-1.19115 E+03$

$-8.16792 E+02$

$-5.88555 E+02$

$-4.21747 E+02$

$-3.21818 E+02$

$-2.25929 E+02$

$-.1 .75807 \mathrm{E}+02$

$-1.30367 E+02$

$-1.04094 E+02$

$-7.97362 \mathrm{E}+01$

$-6.60415 E+01$

$-5.22949 E+01$

$-4.49586 E+01$

$-3.99740 E+01$

$-3.128 .77 E+01$

$-2.59509 E+01$

$-2.38269 E+01$

$-2.09914 E+01$

$-1.36360 E+01$

$-1.60171 \mathrm{E}+01$

$-1.43548 E * 01$

$-1.24713 E+01$

$-1.13 .186 E+01$

$-9.76525 E+00$

$-9.20543 E \neq 00$

$-8.291 .39 E+00$

$-7.7804 .0 E \neq 00$

$-7.16190 \mathrm{E}+00$

$-6.56611 E+00$

$-6.17 .149 E+00$

$-5.59147 E+00$
Q2

$-2.50248 E-01$

$-9.61550 E-02$

$-6.83334 E-02$

$-5.69581 E-02$

$-5.48903 \mathrm{E}-02$

$-6.16096 E-02$

-7.20876 E- 02

$-8.99192 E-02$

$-1.09096 E-0.1$

$-1.47363 E-01$

$-1.82904 E-01$

-2.4078 I.E-0.1

$-2.96700 E-O 1$

$-3.82793 E-01$

$-4.5852 .1 E-01$

$-5.76238 E-01$

$-6.6789 \mid E-01$

$-7.49407 E-01$

$-9.56121 E-01$

$-.1 .15213 E+00$

$-1.25449 E+00$

$-1.42442 \mathrm{E}+00$

$-1.60631 E+00$

$-1.87127 E+00$

$-2.09205 E+00$

$-2.41250 E+00$

$-2.66115 E+00$

$-3.09191 E+00$

$-3.28675 E+00$

$-3.658 .70 E+00$

$-3.90688 E+00$

$-4.25527 E+\infty 0$

$-4.65250 E+00$

$-4.96268 E+00$

$-5.49123 E+00$
Q3

Q4

$4.87738 E+03$

$-2.1 .1662 E+03$

$-2.14 .993 E+03$

$-2.25011 E+03$

$-2.64467 E+03$

$-3.22278 E+0.3$

$-4.47822 E+0.3$

$-7.46052 E+03$

$-3.56355 E+04$

$1.04336 E+04$

$4.01811 \mathrm{E}+03$

$2.18687 E+03$

$1.47318 E+03$

i. $00.995 E^{\prime}+03$

$7.75567 E+02$

$5.80639 E+02$

$4.79294 \mathrm{E}+02$

$4.13497 E+02$

$3.16259 E+02$

$2.57307 E+02$

$2.33162 \mathrm{E}+02$

$2.03220 E+02$

$1.78438 \mathrm{E}+02$

$1.52184 E+02$

$1.3510 .1 E+02$

I. $16565 E+02$

J. $.05686 E+02$

$9.06257 E+01$

$8.51283 E+01$

$7.62867 E+0$.

$7.14750 E+.01$

$6.55655 E+01$

$6.00277 E+01$

b. $63091 E+01$

$5.09260 E+01$
$.4 .93013 E-03$

$-2.71105 E-02$

$-2.04713 E-02$

$.4 .20452 \mathrm{E}-02$

$-7.50592 \mathrm{E}-02$

$-2.30891 E+00$

$6.89083 \mathrm{E}-02$

i. $70260 \mathrm{E}-02$

$1.12795 E-03$

$-4.76888 E-\infty 6$

$0.10410 E-03$

1.76724E-02

3. $27790 E-02$

$5.32991 \mathrm{E}-02$

7. 54084E-02

$1.05829 \mathrm{E}-01$

1.32698E-0I.

$1.57568 \mathrm{E}-01$

2.1.0349E-O1

$2.63347 E-01$

$2.93549 \mathrm{E}-\mathrm{OI}$

3. $42085 E-01$

$3.92101 \mathrm{E}-0$

4.03407E-OI

$5.28087 E-0$

$6.17656 E-01$

$6.83934 \mathrm{E}-0$

7.99948E-01

8.5724|E-OI

$9.59672 E-01$

1. $03005 E+00$

$1.12987 E+00$

$1.23853 E+\infty 0$

$1.32947 E+00$

$1.47793 \mathrm{E}+04$ 


\section{APPENDIX D: Computation of X-ray Spectra.}

\section{D.1: Program XSPECT.}

Note: The listing for the subroutine AFILT has not been included as it is similar to program MCOEF which is listed in appendix $C$. The listing is available from the author if required.

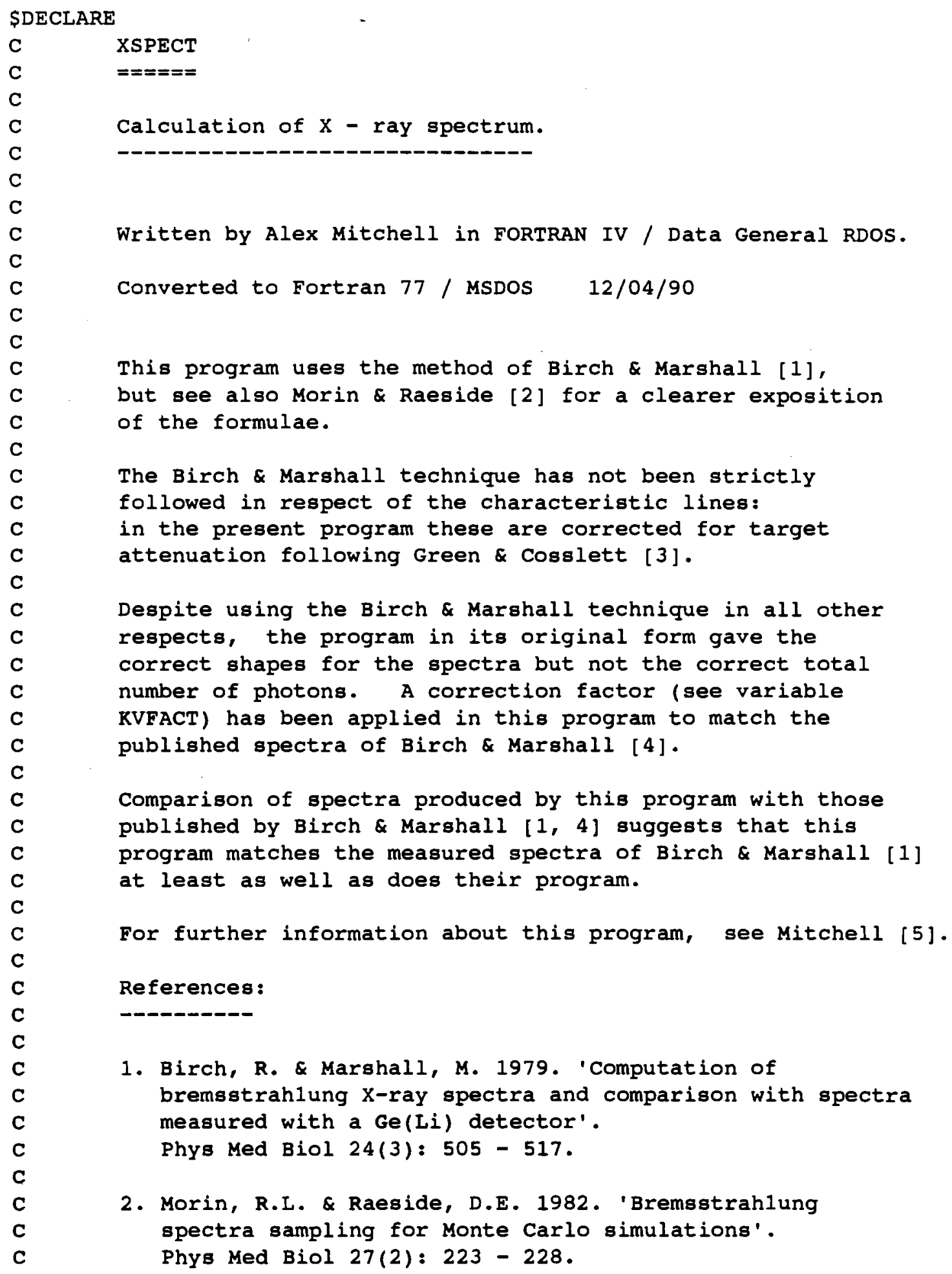


c

C

C

C

C

C

C

C

C

C

C

C

C

C

C

C

C

C

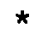

*

*

*

*
3. Green M. \& Cosslett, V.E. 1968. 'Measurements of $\mathrm{K}, \mathrm{L}$ and $M$ shell $X$-ray production efficiencies'.

Brit J Appl Phys (J Phys D) 1: 425 - 436.

4. Birch, R.; Marshall, M.; Ardran, G.M. 1979. 'Catalogue of spectral data for diagnostic X-rays'.

Hospital Physicists' Association, London.

5. Mitchell, A.W. 1985. 'A program for the calculation of X-ray spectra'.

Radiology Department report, Wellington Hospital,

Wellington, New Zealand.

\section{VARIABLES}

$=======$

REAL SPECT (150)

REAL TSPECT

REAL PSPECT (150)

REAL INTENSITY(150)

REAL DINTENSITY

REAL EMAX

REAL T

REAL THETA

REAL NA

REAL A

REAL RHO

REAL SCALE

REAL KVFACT

REAL MU

REAL MULX (7)

REAL MULQ(7)

REAL MUHX(4)

REAL MUHQ(4)
Array to store spectrum in $1 \mathrm{kV}$ steps. Sum of energy spectrum.

Photon spectrum.

Integral intensity of the $\mathrm{X}$ - ray beam. Intensity per unit energy interval.

Maximum photon energy (= kVp)

Kinetic energy of electron in target.

Target angle.

Avogadro's number.

Target atomic weight.

Target density.

scaling factor $=$ RHO * NA / A / 1.32

Factor depending on $\mathrm{kVp}$ which adjusts total intensity for agreement with Birch \& Marshall.

Target mass attenuation coefficient $(\mathrm{cm} 2 / \mathrm{g})$.

C.F. coefficients for MU (low energy).

C.F. coefficients for MU (low energy).

C.F. coefficients for $M U$ (high energy).

C.F. coefficients for MU (high energy). 
REAL Q

REAL QB (0:4)

REAL QC (0:4)

REAL $Q Q(0: 4)$

REAL $\mathrm{z}$

REAL C

REAL CX (5)

REAL $C Q(5)$

VARIABLES (continued)

REAL TFILT

REAL TFACT

REAL MUFX (10)

REAL MUFQ (10)

REAL NPHOTONS

INTEGER DPHOTON

INTEGER DNORM

INTEGER DADD

INTEGER DSTORE

INTEGER IFILT

INTEGER* 2 FORMULA $(3,30)$

INTEGER * 2 CFORMULA $(15,2,30)$

INTEGER NCPT

INTEGER CCPT(30)

REAL THICK

REAL CTHICK(15)
Intensity per unit energy interval ...

Coefficients for calculation of $Q$.

Coefficients for calculation of $Q$.

Recurrence variables used in evaluation of $Q$.

$E / T$, parameter for evaluation of $Q$.

Thomson - Whiddington constant

Coefficients for calculation of $\mathrm{C}$

Coefficients for calculation of $\mathrm{C}$

Thickness of tube filtration $(\mathrm{mm})$.

TFILT/10.*2.7 (TFILT (mm) * density)

C.F. coefficients for calculation of MU (filter

C.F. coefficients for calculation of MU (filter Total number of photons in beam.

'I ' = intensity spectrum, else photon spectru

' $N$ ' = not normalised, else normalised.

'Y' = apply additional filtration.

'Y' = store spectrum in a file.

number of passes through AFILT.

Composition of filtration material as

reported by subroutine AFILT.

store for up to 15 filtration materials.

Number of elements in filtration material as reported by subroutine AFILT.

Store for number of elements in up to 15 filtration materials.

Thickness of filtration material as reported by subroutine AFILT.

Store for thicknesses of up to 15

filtration materials. 
REAL RDENSITY

REAL CDENSITY (15)

Density of filtration material as reported by subroutine AFILT.

Store for densities of up to 15

filtration materials.

REAL ARG

INTEGER CPT

INTEGER IEMAX

INTEGER DUMMY, IE, IT, I

Argument for exponential function.

INTEGER IPRMAX, IPRINT

REAL E, RSCALE, PC58, PC67, PC69, CHARF , E2, RMEAN, AVPHOT

INITIALISE VARIABLES

Dummy variable for component number within a compound.

Maximum photon energy.

C

C

- - - - - - - - - - - - - - -

C.F. coefficients for calculation of Thomson - Whiddington constant.

DATA CX/25.0,50.0,75.0,100.0,150.0/

DATA CQ/0.39,0.1666667E3,0.5423075E0,0.2049551E3,0.1058986E2/

C

C.F. coefficients for mass attenuation coefficient of tungsten.

Taken from Veigele (1973).

$10-69.53 \mathrm{keV}$.

DATA MULX/15.0,20.0,30.0,40.0,50.0,60.0,69.53/

DATA MULQ/0.1448E3, $-0.638569 \mathrm{E}-1,-0.1700613 \mathrm{E} 3$,

$+0.1524997 \mathrm{E} 1,0.2745761 \mathrm{E} 2,-0.2221333 \mathrm{E} 2,-0.2906854 \mathrm{E} 1 /$

C

$69.53-150 \mathrm{keV}$.

DATA MUHX/69.53,80.0,100.0,150.0/

DATA MUHQ/0.11663E2,-0.2905106E1,-0.1490775E2,0.4630377E2/

C

C

C.F. coefficients for mass attenuation coefficient of aluminium.

Taken from Mc Master (1969) via H.P.A. catalogue of spectra.

C

DATA MUFX/10.0,15.0,20.0,30.0,40.0,50.0,60.0,80.0,100.0,150.0/

DATA MUFQ/0.2637E2, $-0.2705923 \mathrm{E0},-0.3037891 \mathrm{E2}, 0.6265691 \mathrm{E} 1$,

C

$+0.4425465 E 1,-0.2041978 E 3,-0.3425229 E 0,0.2986811 E 4$,

$+0.1265102 \mathrm{E}-1,-0.6597586 \mathrm{E} 4 /$

C

$A=183.85$

$\mathrm{RHO}=19.3$

$\mathrm{NA}=6.02252 \mathrm{E} 23$

SCALE $=$ RHO $* \mathrm{NA} / \mathrm{A} / 1.32$

TSPECT $=0.0$

DO $5 I=1,150$

$\operatorname{SPECT}(I)=0$. 


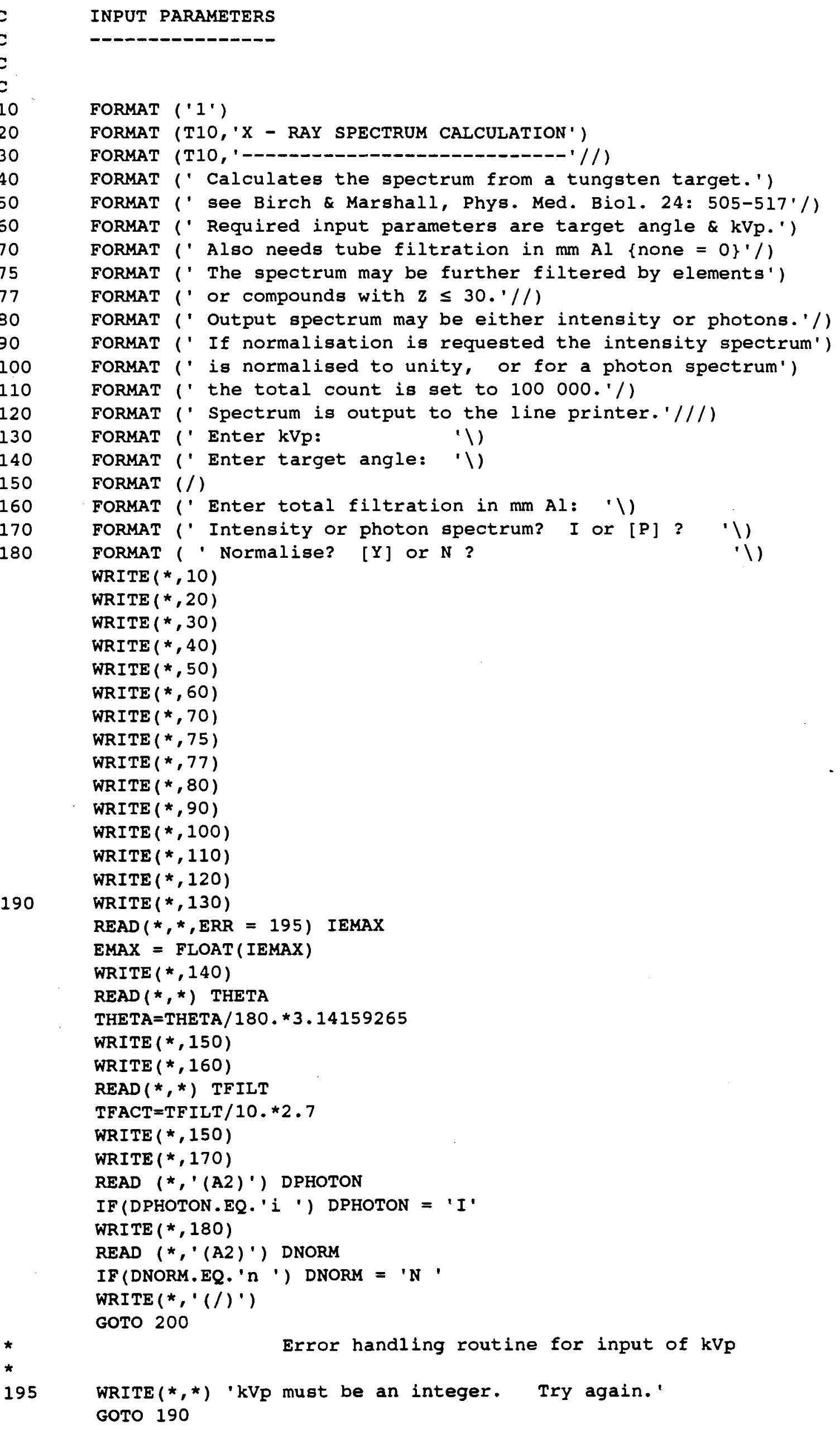


C

C

C

C

C

200

210

*

C

C

C

C

C

C

C

C

C

C

C

C

C

C

C

C

DO 650 IE $=10$, IEMAX

*

$$
E=\text { FLOAT (IE) }
$$$$
\text { DO } 550 \text { IT }=\text { IE, IEMAX }
$$

$T=\operatorname{FLOAT}(I T)$

C

C

C

C

C

C

C

C

C

$\mathrm{C}=\mathrm{CQ}(5)$

DO $210 \quad I=1,3$

DUMMY $=5-I$

CQ (1) + (EMAX-CX (1))/C

$\operatorname{WRITE}(*, *) \quad \mathrm{C}=$ ', C

SCALE = SCALE $*$ KVFACT

COMPUTE SPECTRUM

= = = = = = = = = = = = =

$\mathrm{Z}=\mathrm{E} / \mathrm{T}$

$Q=Q * 1 . O E-14$

CALCULATE THOMSON - WHIDDINGTON CONSTANT

C $=$ Q $($ DUMMY $)+($ EMAX $-C X(D U M M Y)) / C$

ADJUST SCALE FACTOR ACCORDING TO KVP

KVFACT $=(0.24541 \mathrm{E}-2-0.14106 \mathrm{E}-4 *$ EMAX $+0.41721 \mathrm{E}-7$ *EMAX $*$ EMAX $)$

Calculate bremsstrahlung spectrum.

Calculate spectrum in $1 \mathrm{keV}$ steps.

Integrate from current energy up to maximum energy.

Bvaluate $Q$.

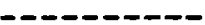

$Q=0.503-0.94597 * Z+0.1553 * Z * Z+1.1632 * Z * Z * Z-0.6818 * Z * Z * Z * Z$

Apply relativistic correction.

DINTENSITY $=Q *(1 .+T / 511.006)$ 
C

C

C

C

C

IF (IE.GT.69) GOTO 520

$M U=M U L Q(7)$

DO $510 \quad I=1,5$

DUMMY $=7-I$

$510 \quad M U=M U L Q$ (DUMMY) $+(E-M U L X$ (DUMMY) ) / MU

$M U=M U L Q(1)+(E-M U L X(1)) / M U$

GOTO 540

$520 \quad M U=M U H Q(4)$

DO $530 \quad I=1,2$

DUMMY $=4-I$

$530 \mathrm{MU}=$ MUHQ (DUMMY) $+(E-M U H X$ (DUMMY) $) / M U$

$M U=M U H Q(1)+(E-M U H X(1)) / M U$

540 CONTINUE

$A R G=-(M U *(E M A X * E M A X-T * T)) /(C * T A N(T H E T A))$

DINTENSITY $=$ DINTENSITY *EXP (ARG)

Summation.

C

C

C

C

C

C

550 INTENSITY (IT) $=$ DINTENSITY

DO 600 IT $=$ IE, IEMAX

600 SPECT (IE) = SPECT (IE) +INTENSITY (IT)

SPECT (IE) $=\operatorname{SPECT}($ IE $)-(5 . / 8$.) * (INTENSITY (IE)

$+\quad+$ INTENSITY (IEMAX))

$\operatorname{SPECT}($ IE $)=\operatorname{SPECT}($ IE $)+(1 . / 6$.$) * ($ INTENSITY $($ IE +1$)$

+ +INTENSITY (IEMAX-1))

SPECT (IE) $=\operatorname{SPECT}($ IE $)-(1 . / 24) *.($ INTENSITY $($ IE +2$)$

+ +INTENSITY (IEMAX-2))

IF (IE.EQ. IEMAX-1) SPECT (IE) $=(\operatorname{INTENSITY~(IE)+INTENSITY~}(\operatorname{IEMAX})) / 2$.

IF (IE.EQ. IEMAX) SPECT (IE) $=$ INTENSITY (IE) $/ 14$.

$\operatorname{SPECT}(I E)=\operatorname{SPECT}(I E) *$ SCALE

TSPECT $=$ TSPECT + SPECT $($ IE $)$

610 FORMAT (' Energy $=1, F 5.0, \cdot \mathrm{keV}$, Intensity $=1, E 8.3$ )

615 FORMAT(' 2A')

$\operatorname{WRITE}(*, 610)$ E, SPECT (IE)

650

WRITE $(*, 615)$ 
$\mathrm{C}$
$\mathrm{C}$
$\mathrm{C}$
$\mathrm{C}$
$\mathrm{C}$ C
C
C Add characteristic spectrum.

IF (EMAX.LT.69.53) GOTO 700

TSPECT $=T S P E C T-S P E C T(58)-S P E C T(59)-\operatorname{SPECT}(67)-\operatorname{SPECT}(69)$ RSCALE $=0.25$

WRITE $(*, *)$

PC58 $=55.9$

$\mathrm{PC} 67=38.2$

PC69 $=10.4$

CHARF $=6.73336 E-15 *((\operatorname{EMAX} / 69.53-1) * * 1.63)$.$* SCALE$

E2 $=$ EMAX * EMAX

$\mathrm{T}=57.99$

$\mathrm{MU}=4.03716$

$\operatorname{SPECT}(58)=\operatorname{SPECT}(58)+P C 58 * \operatorname{CHARF} * \operatorname{EXP}(-(\operatorname{RSCALE} * M U *(E 2)) /$

/ (C*TAN (THETA)))

$\mathrm{T}=59.32$

$\mathrm{MU}=3.81083$

$\operatorname{SPECT}(59)=\operatorname{SPECT}(59)+100$. *CHARF*EXP $(-(\operatorname{RSCALE} * M U *(E 2)) /$

/ (C*TAN (THETA)) )

$\mathrm{T}=67.15$

$\mathrm{MU}=2.78097$

$\operatorname{SPECT}(67)=\operatorname{SPECT}(67)+\mathrm{PC} 67 * \mathrm{CHARF}^{*} \operatorname{EXP}(-(\operatorname{RSCALE} * M U *(E 2)) /$

$/(C * \operatorname{TAN}($ THETA $)))$

$\mathrm{T}=69.13$

$\mathrm{MU}=2.58269$

$\operatorname{SPECT}(69)=\operatorname{SPECT}(69)+\operatorname{PC} 69 * \mathrm{CHARF}^{\star} \operatorname{EXP}(-(\operatorname{RSCALE}$ *MU * $(E 2)) /$

/ (C*TAN (THETA)) )

TSPECT =TSPECT+SPECT ( 58) +SPECT (59) +SPECT (67)+SPECT (69)

C

C

C

Filter intensity spectrum.

C

C

$700 \quad \operatorname{IEMAX}=\operatorname{IFIX}(\operatorname{EMAX}+0.1)$

TSPECT $=0.0$

DO 720 IE $=10$, IEMAX

$E=F L O A T$ (IE)

$M U=M U F Q(10)$

DO $710 \quad I=1,8$

DUMMY $=10-I$

$710 \quad M U=M U F Q$ (DUMMY) $+(E-M U F X(D U M M Y)) / M U$

$M U=M U F Q(1)+(E-M U F X(1)) / M U$

$\operatorname{SPECT}(I E)=S P E C T(I E) \star \operatorname{EXP}(-M U * T F A C T)$

720

TSPECT $=$ TSPECT + SPECT $(I E)$ 


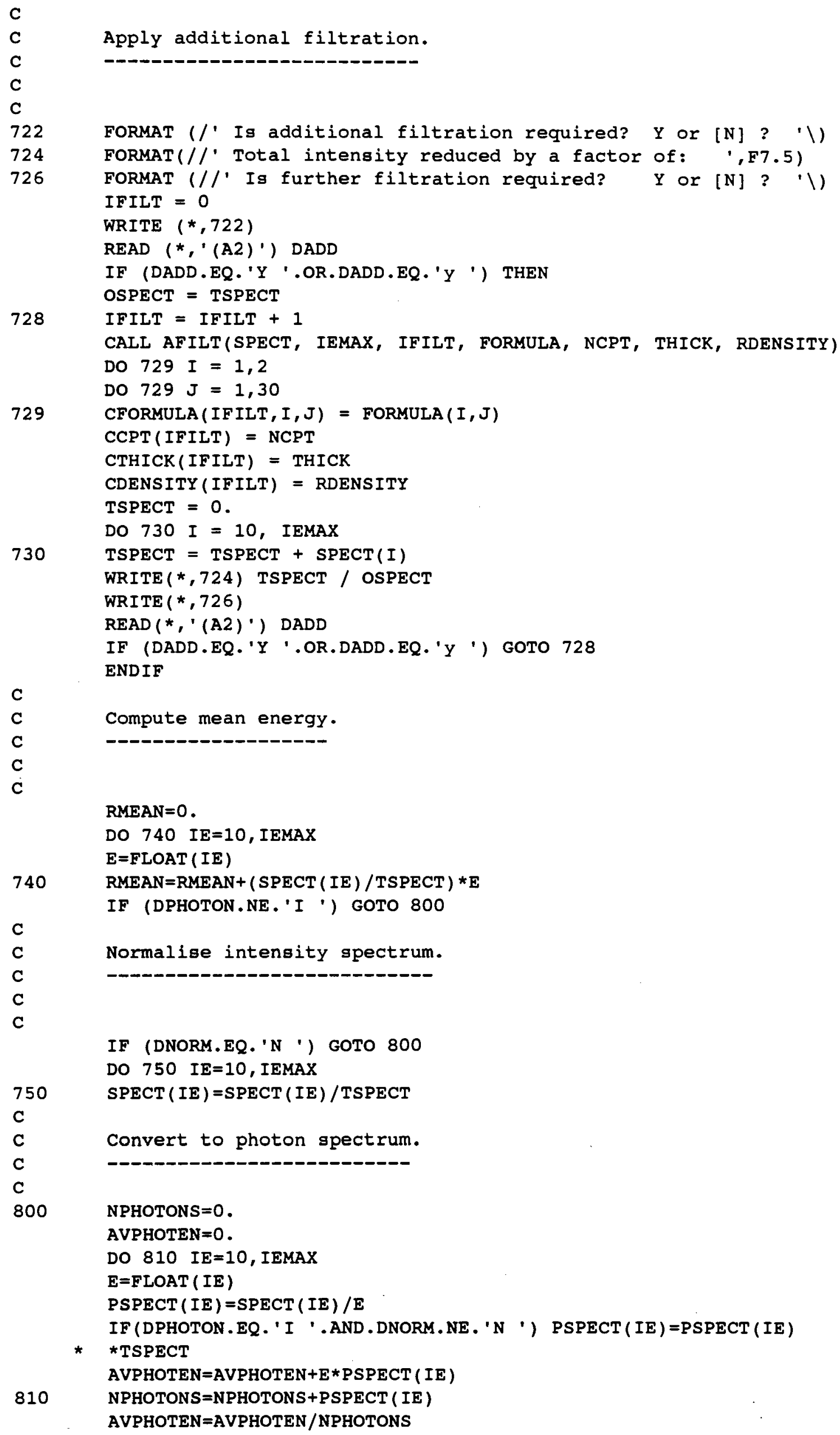


Normalise photon spectrum.

C

IF (DNORM.EQ.'N ') GOTO 1000

DO 910 IE=10, IEMAX

910 PSPECT (IE) $=$ PSPECT (IE) /NPHOTONS $* 100000$.

C

PRINT SPECTRUM

CONTINUE

THETA $=T H E T A * 180 . / 3.14159265$

... convert angle back to degrees.

IF (DPHOTON.EQ. ' I ') GOTO 1125

DO 1005 IE $=10$, IEMAX

$\operatorname{SPECT}(I E)=\operatorname{PSPECT}(I E)$

FORMAT (T25, 'COMPUTED PHOTON SPECTRUM'/)

FORMAT (T25,' COMPUTED INTENSITY SPECTRUM'/)

FORMAT (' Tungsten target, angle $=$ ',F5.1,' degrees. ')

FORMAT ('Tube voltage: ',F5.1,' kV (DC).')

FORMAT (' Tube filtration: ',F5.1,' mm Al.'/)

FORMAT ('Additional filtration: /)

FORMAT $(T 5,20(A 2,2 X))$

FORMAT $(T 4, \cdot$ ' ' $)$

FORMAT (' '

FORMAT $(1 X, 12, \cdot, \backslash)$

FORMAT $(1 X, 13,1)$

FORMAT $\left(/ 17 \mathrm{X},{ }^{\prime}\right.$ (Density $='$, F5.2, ${ }^{\prime}$ Thickness $\left.\left.=', F 5.2,{ }^{\prime} \mathrm{cm}.\right) ' /\right)$

FORMAT (" spectrum normalised to 100000 counts.")

FORMAT (' spectrum normalised to unity. '/)

FORMAT (' Total energy: ',E10.4, )

FORMAT (' Mean energy:',13X,F5.1,' keV.')

FORMAT (' Number of photons: ',E10.4\)

FORMAT (' Av. photon energy: ',F8.1,' keV.'/)

FORMAT (' Energy ' $)$

FORMAT $\left(5 \mathrm{X}, 0^{\prime}, 13 \mathrm{X}, \mathrm{\prime}^{\prime}, 13 \mathrm{X}, \mathrm{\prime}^{\prime}, 13 \mathrm{x}, \mathrm{\prime}^{\prime}, 13 \mathrm{x}, \mathrm{\prime}^{\prime} \mathrm{\prime}^{\prime}\right)$

FORMAT (' (keV) ' ()

FORMAT $\left(5 X, 5^{\prime}, 13 \mathrm{X}, 6^{\prime}, 13 \mathrm{X}, 7^{\prime}, 13 \mathrm{X}, 8^{\prime}, 13 \mathrm{X}, 9^{\prime}\right)$

FORMAT $(/ I 3, \cdot-\cdot, I 3)$

FORMAT $(5 X, F 9.01)$

FORMAT $(5 X, E 9.4 \backslash)$

IF (DPHOTON.NE.'I ') WRITE $(12,1010)$

IF (DPHOTON.EQ.' I ') WRITE $(12,1020)$

WRITE $(12,1030)$ THETA

WRITE $(12,1040)$ EMAX

WRITE $(12,1045)$ TFILT

IF (IFILT.NE.0) THEN

WRITE $(12,1047)$

DO $1150 \mathrm{~K}=1$, IFILT

WRITE $(12,1050)$ (CFORMULA $(K, 1, I), I=1, \operatorname{CCPT}(K)$ )

WRITE $(12,1052)$

DO $1140 \mathrm{CPT}=1, \mathrm{CCPT}(\mathrm{K})$

IF (CFORMULA (K, 2, CPT) . EQ. 1 ) THEN

WRITE $(12,1054)$

ELSEIF (CFORMULA ( $K, 2, C P T)$. LT. 10) THEN

WRITE $(12,1056)$ CFORMULA $(K, 2, C P T)$

ELSEIF (CFORMULA ( $K, 2$, CPT) . GE. 10) THEN

WRITE $(12,1058)$ CFORMULA $(K, 2, C P T)$ 


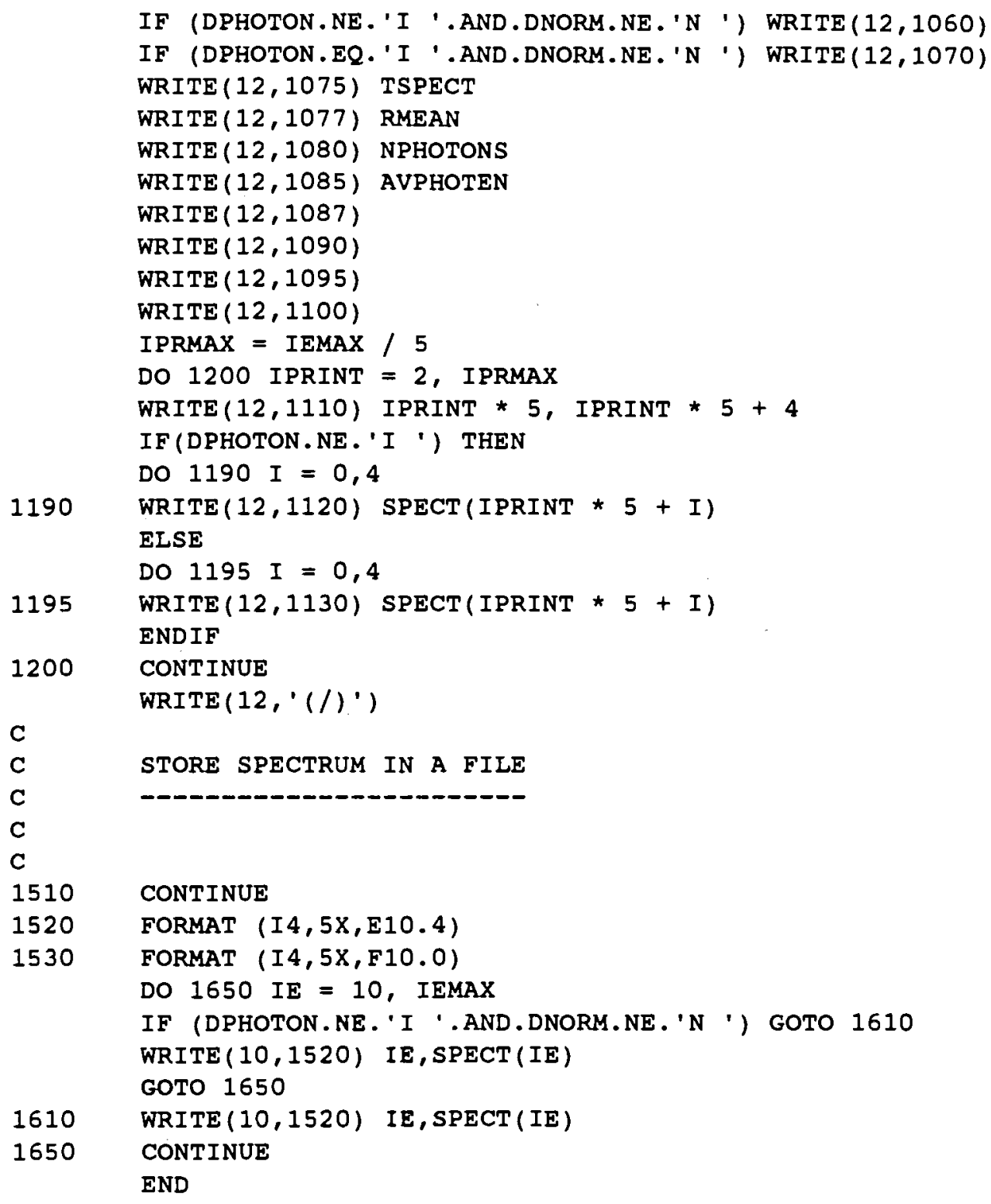


D.2: The Intensity Spectrum of the G.E. 8800 Scanner at $120 \mathrm{kVp}$.

Spectrum normalised to unity.

Total energy: $\quad 0.3239 \mathrm{E} 9$

Mean energy:

$65.1 \mathrm{keV}$.

Number of photons: 0.5577E 7 Av. photon energy:

$58.1 \mathrm{keV}$.

$10.0000 \mathrm{E} 0$

$20.0000 \mathrm{E} 0$

$3 \quad 0.0000 \mathrm{E} 0$

4

5

6

7

8

9

10

11

12

13

14

15
$0.0000 \mathrm{EO}$

$0.0000 \mathrm{EO}$

$0.0000 E 0$

$0.0000 E 0$

$0.0000 E 0$

$0.0000 E 0$

$0.3740 \mathrm{E}-11$

$0.5636 \mathrm{E}-9$

$0.1996 \mathrm{E}-7$

$0.2691 E-6$

$0.1881 \mathrm{E}-5$

$0.8324 \mathrm{E}-5$

$0.2662 \mathrm{E}-4$

$0.6724 E-4$

$0.1425 \mathrm{E}-3$

$0.2645 \mathrm{E}-3$

$0.4435 \mathrm{E}-3$

$0.6865 \mathrm{E}-3$

$0.9975 E-3$

$0.1377 \mathrm{E}-2$

$0.1824 \mathrm{E}-2$

$0.2332 \mathrm{E}-2$

$0.2895 E-2$

$0.3505 \mathrm{E}-2$

$0.4152 \mathrm{E}-2$

$0.4827 \mathrm{E}-2$

$0.5518 \mathrm{E}-2$

$0.6216 \mathrm{E}-2$

$0.6911 \mathrm{E}-2$

$0.7596 \mathrm{E}-2$

$0.8262 \mathrm{E}-2$

$0.8905 E-2$

$0.9518 \mathrm{E}-2$

$0.1010 \mathrm{E}-1$

$0.1065 \mathrm{E}-1$

$0.1116 \mathrm{E}-1$

$0.1163 E-1$

$0.1207 \mathrm{E}-1$

$0.1246 \mathrm{E}-1$

$0.1283 E-1$

$0.1316 \mathrm{E}-1$

$0.1345 \mathrm{E}-1$

$0.1371 \mathrm{E}-1$

$0.1395 \mathrm{E}-1$

$0.1415 \mathrm{E}-1$

$0.1433 \mathrm{E}-1$

$0.1448 \mathrm{E}-1$
51

52

53

54

55

56

57

58

59

60

61

62

63

64

65

66

67

68

69

70

71

72

73

74

75

76

77

78

79

80

81

82

83

84

85

86

87

88

89

90

91

92

93

94

95

96

97

98

99

100
$0.1461 \mathrm{E}-1$

$0.1472 \mathrm{E}-1$

$0.1481 \mathrm{E}-1$

$0.1487 \mathrm{E}-1$

$0.1493 \mathrm{E}-1$

$0.1496 \mathrm{E}-1$

$0.1498 \mathrm{E}-1$

$0.4609 \mathrm{E}-1$

$0.7139 \mathrm{E}-1$

$0.1495 \mathrm{E}-1$

$0.1492 \mathrm{E}-1$

$0.1488 \mathrm{E}-1$

$0.1482 \mathrm{E}-1$

$0.1476 \mathrm{E}-1$

$0.1469 \mathrm{E}-1$

$0.1460 \mathrm{E}-1$

$0.3763 \mathrm{E}-1$

$0.1441 \mathrm{E}-1$

$0.2069 \mathrm{E}-1$

$0.9039 \mathrm{E}-2$

$0.9148 \mathrm{E}-2$

$0.9246 \mathrm{E}-2$

$0.9331 \mathrm{E}-2$

$0.9404 \mathrm{E}-2$

$0.9465 \mathrm{E}-2$

$0.9514 \mathrm{E}-2$

$0.9551 \mathrm{E}-2$

$0.9574 \mathrm{E}-2$

$0.9586 \mathrm{E}-2$

$0.9584 E-2$

$0.9570 \mathrm{E}-2$

$0.9544 \mathrm{E}-2$

$0.9505 \mathrm{E}-2$

$0.9453 \mathrm{E}-2$

$0.9388 E-2$

$0.9311 \mathrm{E}-2$

$0.9222 \mathrm{E}-2$

$0.9120 \mathrm{E}-2$

$0.9006 \mathrm{E}-2$

$0.8879 E-2$

$0.8740 \mathrm{E}-2$

$0.8589 \mathrm{E}-2$

$0.8426 \mathrm{E}-2$

$0.8250 \mathrm{E}-2$

$0.8063 \mathrm{E}-2$

$0.7864 \mathrm{E}-2$

$0.7654 \mathrm{E}-2$

$0.7431 \mathrm{E}-2$

$0.7198 \mathrm{E}-2$

$0.6953 \mathrm{E}-2$
101

$0.6698 \mathrm{E}-2$

$1020.6431 \mathrm{E}-2$

$103 \quad 0.6154 \mathrm{E}-2$

$1040.5866 \mathrm{E}-2$

$1050.5568 \mathrm{E}-2$

$106 \quad 0.5259 \mathrm{E}-2$

$107 \quad 0.4941 \mathrm{E}-2$

$108 \quad 0.4613 \mathrm{E}-2$

$1090.4276 \mathrm{E}-2$

$110 \quad 0.3929 \mathrm{E}-2$

$1110.3573 \mathrm{E}-2$

$1120.3208 \mathrm{E}-2$

$1130.2834 \mathrm{E}-2$

$1140.2453 \mathrm{E}-2$

$1150.2063 \mathrm{E}-2$

$1160.1665 \mathrm{E}-2$

$1170.1259 \mathrm{E}-2$

$118 \quad 0.8465 \mathrm{E}-3$

$1190.4266 \mathrm{E}-3$

$120 \quad 0.3071 \mathrm{E}-4$ 


\section{APPENDIX E: Computation of the Modulation Transfer Function.}

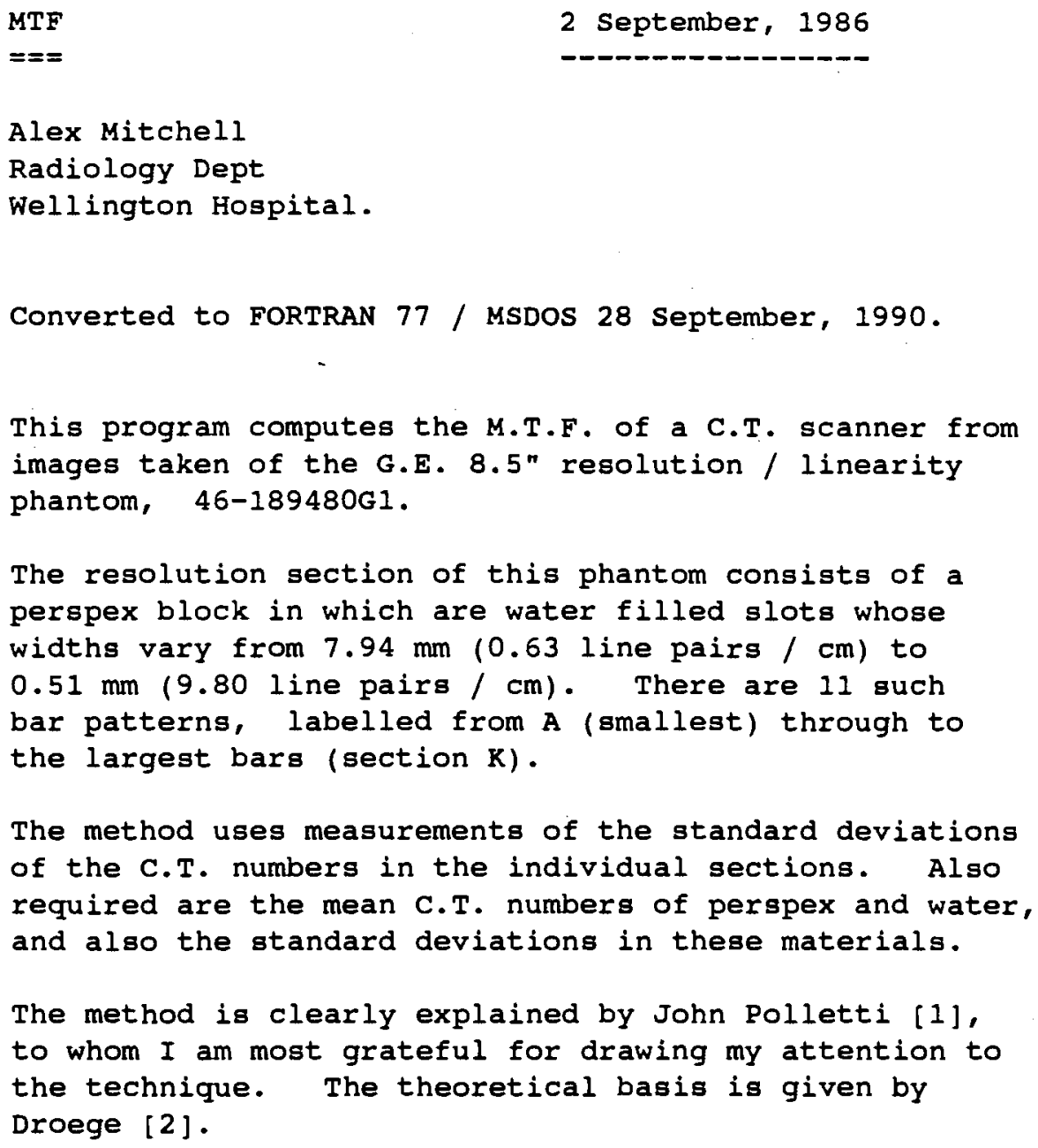


REAL HPERSPEX

$\star$

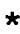

REAL HWATER

REAL NPERSPEX

REAL NWATER

REAL NSQ

REAL FREQ(11)

REAL M(11)

REAL HFREQ (10)

REAL HM(11)

CHARACTER ${ }^{1}$ CFREQ
C.T. number of perspex.

C.T. number of water.

noise (S.D.) in perspex.

noise in water.

Mean square noise.

Spatial frequencies of bars (GE phantom).

Response at each frequency.

Frequencies of required harmonics.

Interpolated response at harmonics.

Section identification

REAL SCALE, MSO, Q $(15,15), \mathrm{X1}, \mathrm{D}$

INTEGER $N, N 1, N 2$, DUMMY

DATA FREQ/0.63,1.05,1.54,1.93,2.43,3.07,3.85,4.9,6.17,7.81,9.8/

DATA HFREQ/1.89,3.15,4.41,4.62,5.25,5.79,7.29,7.35,7.70,9.21/

INPUT READINGS

FORMAT('1'/T25, 'M.T.F. Calculation from variances'//)

FORMAT (/' Enter mean C.T. numbers:'/)

FORMAT(' Perspex: ',l)

FORMAT(' Water: ', l)

FORMAT(/' Enter standard deviations:'/)

FORMAT(' Perspex: ',l)

FORMAT(' Water: ', I)

FORMAT (/' Enter standard deviations of bar sections: '/)

FORMAT(' section ',I3,' ', I)

WRITE $(5,10)$

WRITE $(5,20)$

WRITE $(5,30)$

$\operatorname{READ}(*, *)$ HPERSPEX

$\operatorname{WRITE}(5,40)$

READ (*, *) HWATER

WRITE $(5,50)$

WRITE $(5,60)$

$\operatorname{READ}(*, *)$ NPERSPEX

WRITE $(5,70)$

$\operatorname{READ}(*, *)$ NWATER

MO $=($ HPERSPEX - HWATER $) / 2$.

SCALE $=1.11072 /$ MO

NSQ $=($ NPERSPEX NPERSPEX + NWATER $*$ NWATER $) / 2$.

WRITE $(5,80)$

DO 150 IFREQ $=1,11$

JFREQ = 12 - IFREQ

CFREQ $=\operatorname{CHAR}($ JFREQ+64)

WRITE $(5, '('$ section ' 'A1,' ': ' ', ') CFREQ

$\operatorname{READ}(*, *) \quad M(I F R E Q)$

$M S Q=M($ IFREQ $) * M($ IFREQ $)$

$I F(M S Q \cdot L E . N S Q) \quad M(I F R E Q)=0$.

IF (MSQ.LE.NSQ) GOTO 150

$M($ IFREQ $)=\operatorname{SQRT}(M($ IFREQ $) * M($ IFREQ $)-N S Q)$

CONTINUE 


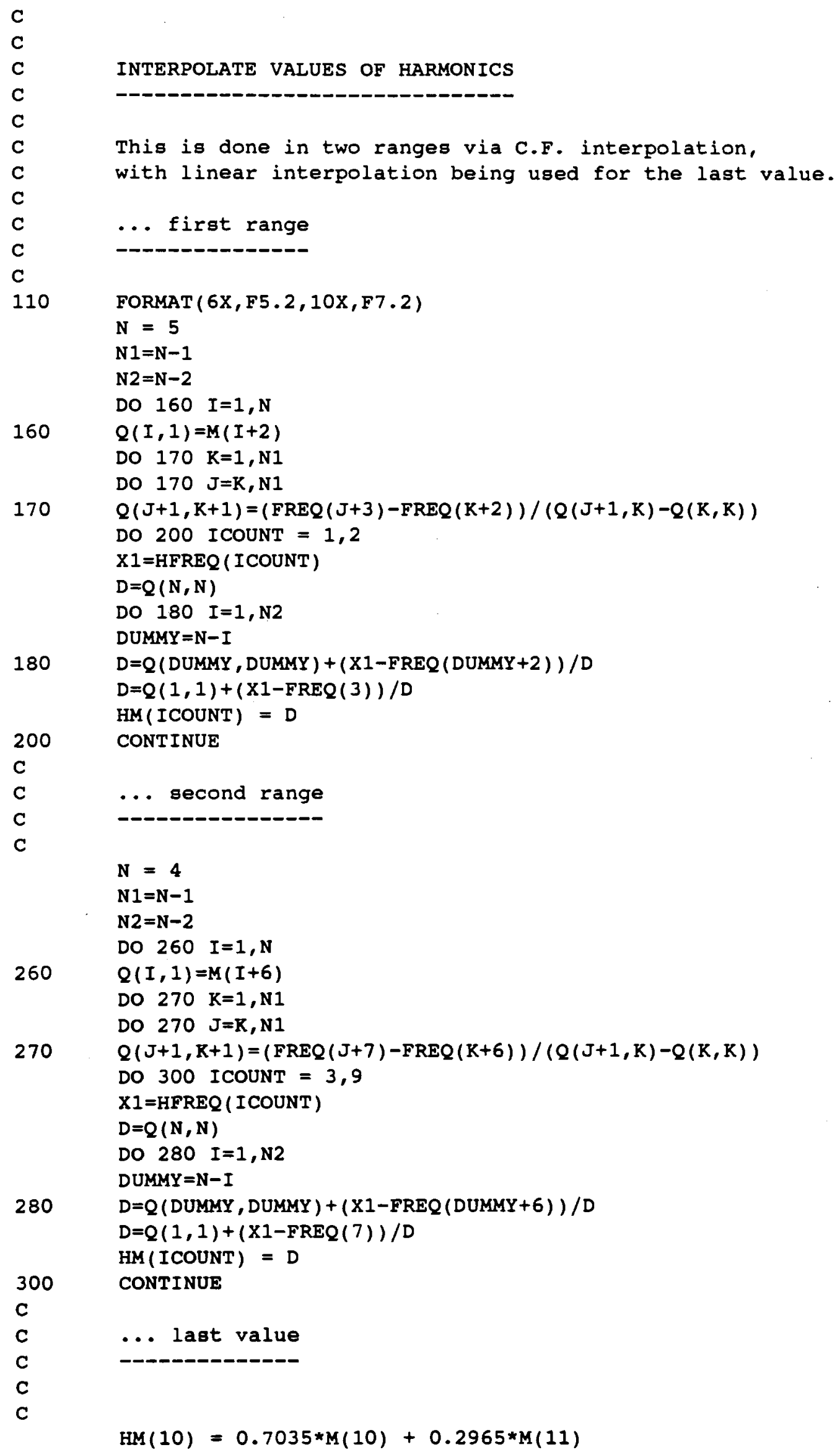




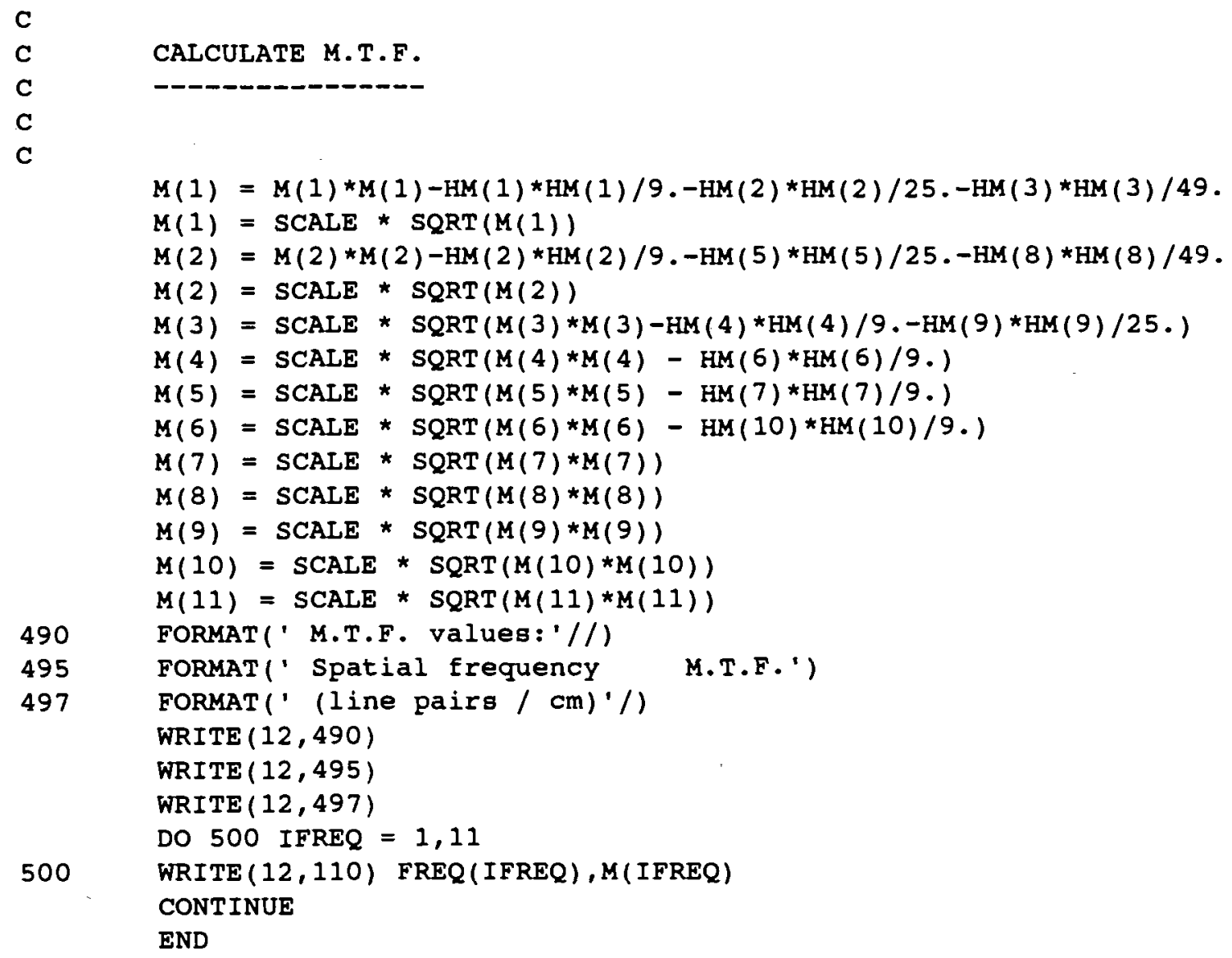




\section{APPENDIX F: Wellington Protocol for Spine Mineral Densitometry.}

\section{Scan calibration solutions in W.P.H. phantom}

1. Secure phantom to mounting bracket as is used with calibration phantoms.

2. Centre the phantom using the laser alignment lights

3. Enter scan information. Use standard default file LSMIN which sets the following:

$\begin{array}{ll}\text { prospective target: } & \text { NO } \\ \text { calibration: } & \text { BODY } \\ \text { head/feet first: } & \text { FEET } \\ \text { position: } & \text { SUPINE } \\ \text { views per slice: } & 576 \\ \text { landmark: } & \text { IC }\end{array}$

4. Key in patient name and I.D. exactly as follows:
patient I.D.:
PHANTOM
patient name:
W.P.H.

5. Axial slices:

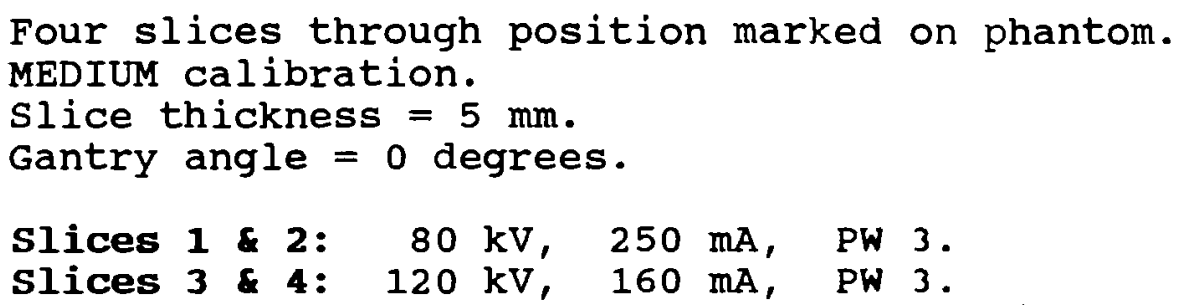

II. Scan A.A.P.M. linearity phantom at $80 \& 120 \mathrm{kV}$

1. Position phantom on gantry end of C.T. table. The phantom is placed directly on the table: remove black cushion.

2. Centre the phantom using the laser alignment lights

3. Enter scan information. This must be done by using option 2 (rescan) and for the run number type in ' $M R$ '.

4. Change the patient name to: A.A.P.M.

Do not change any other details

5. Axial slices:

Four slices through position marked on phantom. MEDIUM calibration.

Slice thickness $=5 \mathrm{~mm}$.

Gantry angle $=0$ degrees.

Slices $1 \& 2: \quad 80 \mathrm{kV}, 250 \mathrm{~mA}, \mathrm{PW} 3$.

slices $3 \& 4: 120 \mathrm{kV}, 160 \mathrm{~mA}, \mathrm{PW} 3$. 


\section{Patient Scanning.}

1. Position patient on table (feet towards gantry) with pillow under knees.

Tuck sheets etc in tight around patient to avoid oversize.

2. Centre as follows:

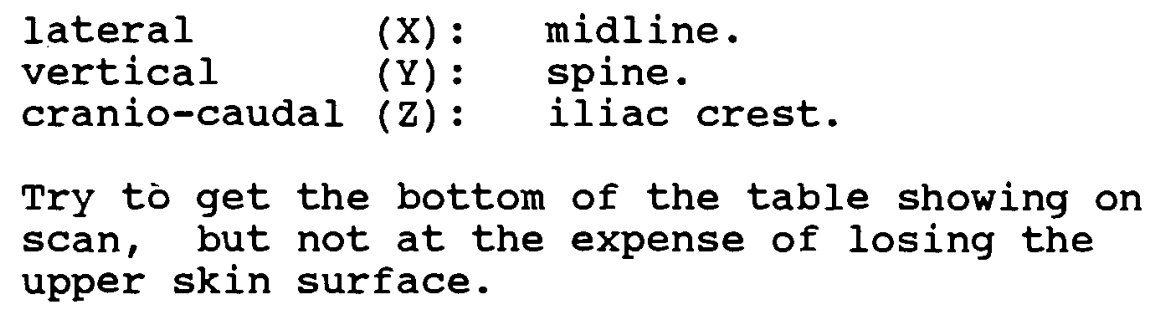

3. Enter scan information. Use standard default file LSMIN which sets the following:

$\begin{array}{ll}\text { prospective target: } & \text { NO } \\ \text { calibration: } & \text { BODY } \\ \text { head/feet first: } & \text { FEET } \\ \text { position: } & \text { SUPINE } \\ \text { views per slice: } & 576 \\ \text { landmark: } & \text { IC }\end{array}$

4. Scout view L1 - L5:

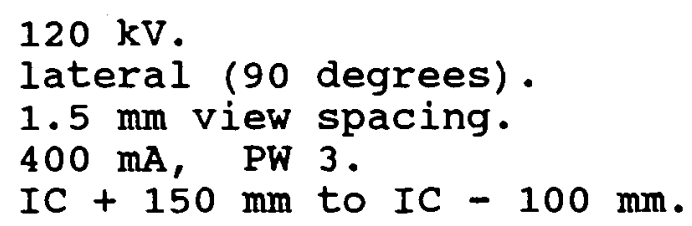

5. Axial slices:

Two slices $(120 / 80 \mathrm{kV})$ through the centres of $\mathrm{L} 2$, L3 and L4.

Select MEDIUM or LARGE calibration. MEDIUM is preferred, but the entire body section must be within the reconstruction circle.

Slice thickness $=5 \mathrm{~mm}$.

$80 \mathrm{kV}: 250 \mathrm{~mA}, \mathrm{PW} 3$.

$120 \mathrm{kV}: 160 \mathrm{~mA}$, PW 3 .

Tilt gantry perpendicular to axis of each vertebra. 
IV. Processing.

1. RECAL $80 \mathrm{kV}$ slices.

2. TARGET all slices:

$\begin{array}{ll}\text { algorithm: } & \text { SOFT } \\ \text { centre: } & (0,0) \\ \text { magnification: } & \mathrm{x} 2 \\ \text { oversize: } & \text { NO }\end{array}$

3. Obtain C.T. readings of the 3 vertebrae.

4. Record readings on film.

5. Store C.T. runs on floppy.

6. Record phantom readings.

7. Store phantom readings in the computer using command PHANTOMS.

8. Store patient readings in the computer using command PATIENTS.

9. Compute mineral content using command SUMS.

10. Produce patient graphs \& reports using command REPORT. 


\section{APPENDIX G: Spine mineral software.}

Program AWMIN, which performs the calculations, is listed here. The programs which store the phantom and patient readings, and the program which produces the reports, are available from the author on request. Unlike the other software discribed in the present work, which runs in an MS-DOS environment, the spine mineral software is written in FORTRAN 77 for the Data General AOS operating system.

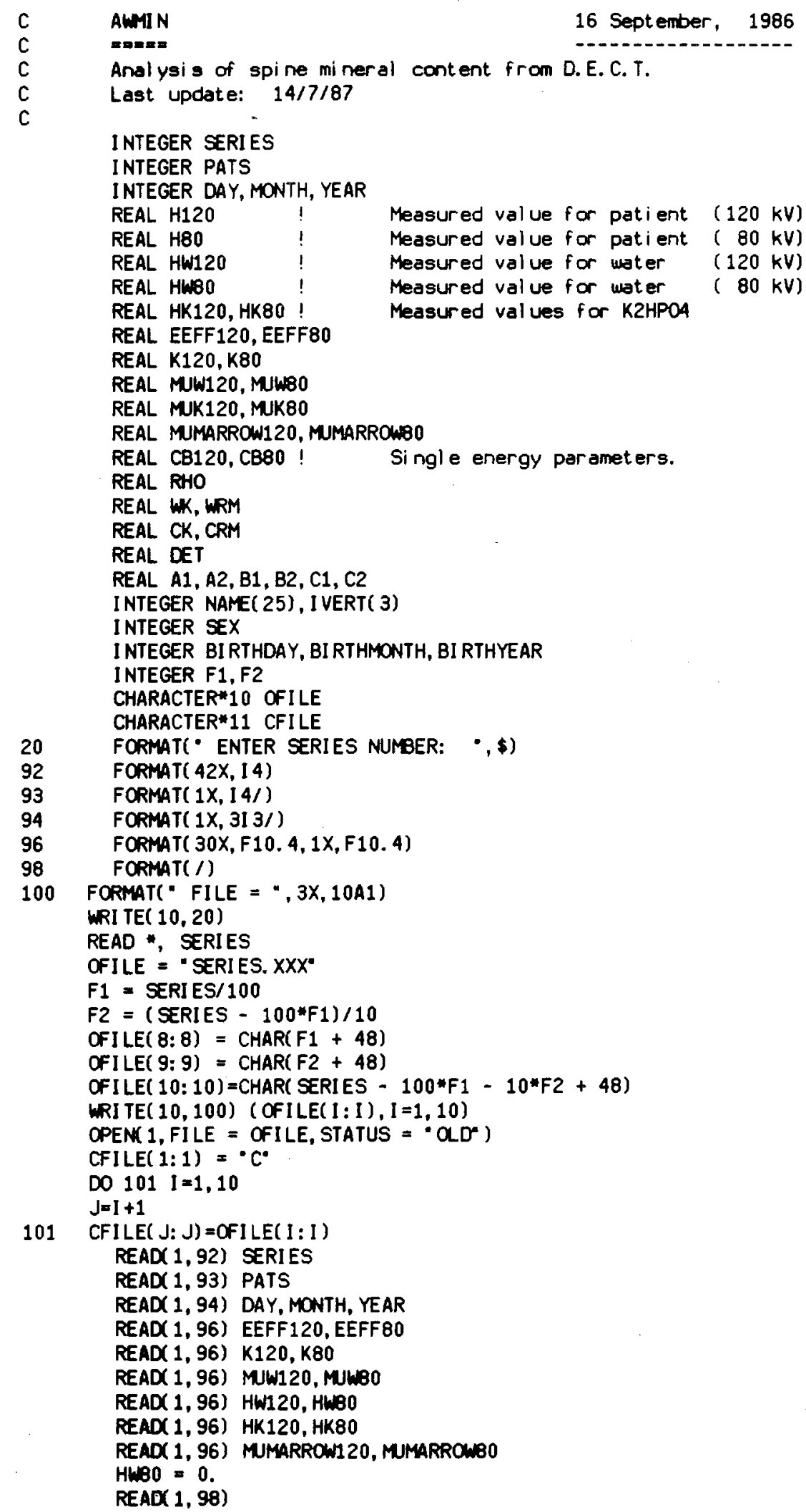




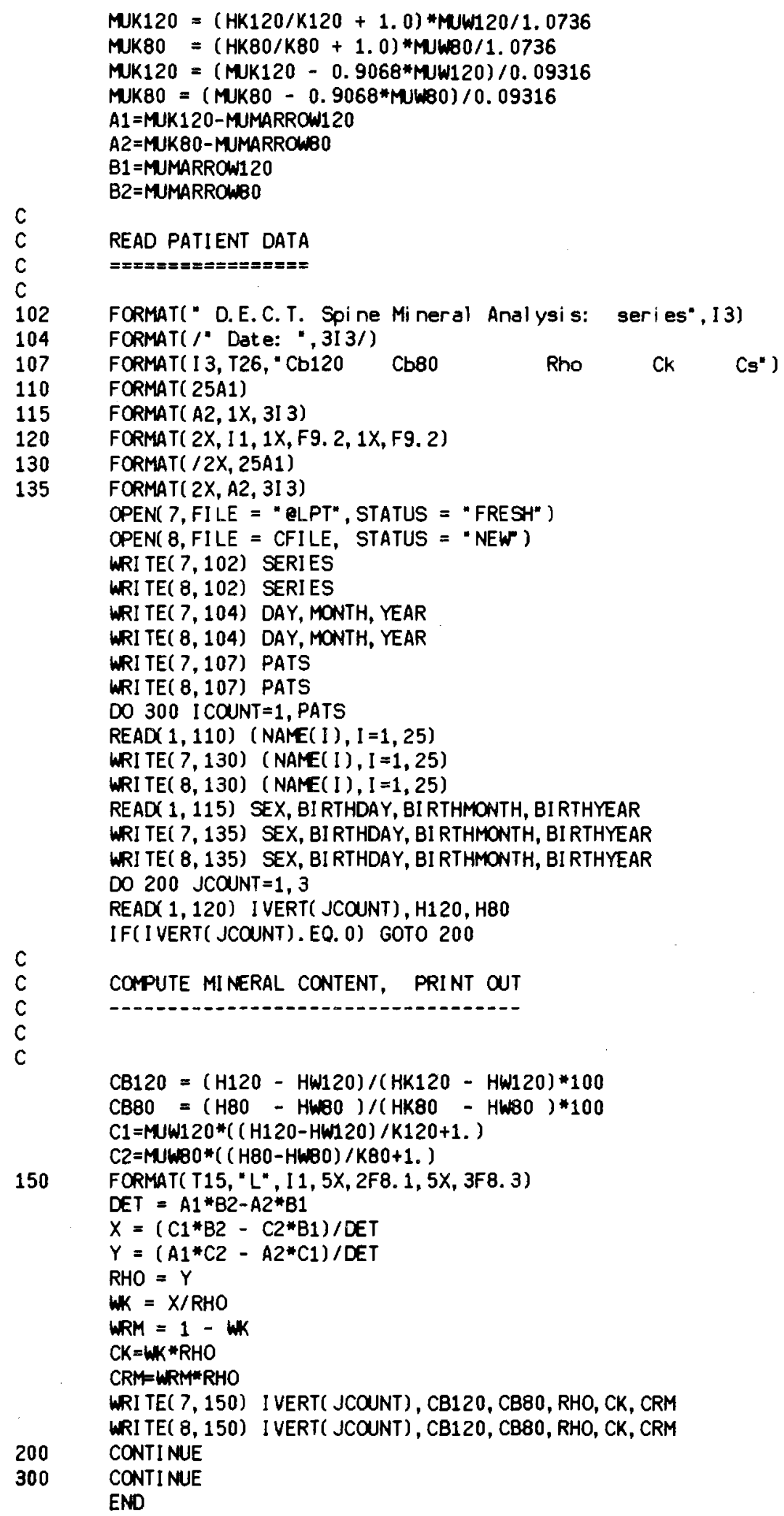




\section{APPENDIX H: Dose Due to C.T. Spine Mineral Densitometry.}

Measurements taken on the Wellington Hospital C.T. scanner by the National Radiation Laboratory [177] yielded an average dose in their body-sized phantom of $3392 \mu \mathrm{Sv}$ at $120 \mathrm{kV}, 80 \mathrm{~mA}$ and $4 \mathrm{~ms}$ pulse width. Thus the average dose at $160 \mathrm{~mA}$ as used for the $120 \mathrm{kV}$ slices in the densitometry examination will be $6784 \mu \mathrm{Sv}$. Program XSPECT has been used to show that the X-ray output of the G.E. 8800 scanner at $80 \mathrm{kV}$ is three times lower than at $120 \mathrm{kV}$, all other factors being the same. The program has also been used to show that the proportion of the beam passing through 20 $\mathrm{cm}$ of water (representative of the thinnest portion of the abdomen) is about $0.4 \%$ at $120 \mathrm{kV}$ and $0.2 \%$ at $80 \mathrm{kV}$, so it can be assumed, to a good degree of approximation, that the dose at $80 \mathrm{kV}$ will be in direct proportion to the $\mathrm{X}$-ray output at that kilovoltage. The dose at $80 \mathrm{kV}$ is therefore, making allowance for the increased $\mathrm{mA}$ at $80 \mathrm{kV}$ :

$$
\begin{aligned}
\text { Dose at } 80 \mathrm{kV} & =6784 \mu \mathrm{SV} \times \frac{1}{3} \times \frac{250}{160} \\
& =3533 \mu \mathrm{Sv} .
\end{aligned}
$$

The dose to the exposed area at the level of each vertebral body is therefore 10317 $\mu \mathrm{Sv}$ for the scans at the two kilovoltages. Pye et al [176], in computing the effective dose equivalent for the DPA examination, determined that the use of the organ multiplying factors for organs such as the red bone marrow, bone surfaces was not warranted since only a small portion of these organs was irradiated. This is even more true of the C.T. technique since most of the dose is concentrated in three transverse sections each some $5 \mathrm{~mm}$ thick. In the case of C.T. there is no direct exposure of the ovaries from the axial slices, so that the only weighting factor to be used in computing the effective dose equivalent is the factor of 0.3 which is applied to organs other than the 5 which explicitly listing by the I.C.R.P. [175]. The proportion of the body irradiated may be estimated by assuming an average body weight of $70 \mathrm{~kg}$, and estimating the weight of tissue exposed as follows. Measurements from patient films have shown (see section 5.2) that the female abdomen is approximately elliptical in shape with a major axis of $30 \mathrm{~cm}$ and a minor axis of $20 \mathrm{~cm}$. At a slice thickness of $5 \mathrm{~mm}$ this corresponds to a volume of $236 \mathrm{cc}$, or a weight of $236 \mathrm{~g}$ assuming a density of $1 \mathrm{~g} / \mathrm{cc}$. The effective dose equivalent due to the three sets of axial slices is therefore:

$$
\text { E.D.E. }=10317 \times \frac{708}{70,000} \times 0.3 \mu \mathrm{Sv}
$$

The N.R.L. measurements [177] for scout views correspond to an average dose of $154 \mu \mathrm{Sv}$ at $400 \mathrm{~mA}$. This dose is applied to a volume whose cross sectional area is as above, but whose length is some $250 \mathrm{~mm}$, corresponding to a total weight of exposed 
tissue of $11.8 \mathrm{~kg}$. As discussed in the text, the position of the ovaries is variable [206] so that there will be direct exposure of the ovaries of some women during the scout scan, but not others. Assuming that the ovaries are exposed, and using their multiplying factor of 0.25 , the effective dose equivalent for scout views on females is therefore:

$$
\text { E.D.E. }=154 \mu \mathrm{Sv} \times \frac{11.8}{70}+154 \mu \mathrm{Sv} \times 0.25
$$

The E.D.E. is therefore $65 \mu \mathrm{Sv}$ for females and $26 \mu \mathrm{Sv}$ for males, giving effective dose equivalents for the whole examination as follows:

Table G.1: Effective Dose Equivalents for the Wellington Densitometry Examination.

\begin{tabular}{|c|c|}
\hline Male & $57 \mu \mathrm{Sv}$ \\
\hline Female & $96 \mu \mathrm{Sv}$ \\
\hline
\end{tabular}




\section{APPENDIX I: Statistical Techniques.}

This appendix is in three sections. In the first section a discussion of nonparametric statistical techniques has been included as these are likely to be less familiar to physicists than more common techniques such as Student's $t$ test and linear regression. The next section consists of notes on the various computer programs developed to carry out the analyses required in this thesis, and the listings of these programs are given in the final section.

\section{I.1: Nonparametric Statistics.}

Standard statistical tests frequently involve mathematical assumptions which are not always explicit. For example, Student's $t$ test for significant differences between means assumes that the two variables being compared are normally distributed. The test may give reasonable answers in cases where the departure from the normal distribution is not great [140], but a point can be reached where the errors introduced are too large and other methods are required. Standard tests may compare two groups by computing statistical parameters such as means or standard deviations for each group, and then examining the differences between the corresponding parameters for the two groups. Nonparametric tests avoid the use of such parameters since their use frequently involves assumptions about the types of distribution involved. It is for this reason that nonparametric tests are also referred to as distribution-free methods. The two tests described in this section are the Wilcoxon test for significant difference between two sets of data, and the Spearman rank correlation test.

\section{Wilcoxon Test.}

The nonparametric method used in place of Student's $t$ test for significant differences between two groups is the Wilcoxon test [140]. This test involves combining the two groups and then listing the combined group in rank order. The lowest value is given rank 1, the next lowest value 2 , and so on. The Wilcoxon parameter $T_{1}$ is the sum of the ranks of the members of the first group in the combined grouping. If there is no difference in the magnitudes of the two groups then the expectation value of $T_{1}$ is [140]:

$$
\overline{\mathrm{T}}_{1}=0.5 \times \mathrm{n}_{1}\left(\mathrm{n}_{1}+\mathrm{n}_{2}+1\right)
$$


where $n_{1}$ and $n_{2}$ are the numbers of elements in each group. The variance of the distribution of $T_{1}$ is given by:

$$
\operatorname{var}\left(T_{1}\right)=n_{1} n_{2}\left(n_{1}+n_{2}+1\right) / 12
$$

in the absence of ties. If two or more elements have the same value then each element of the tied group is given a ranking equal to the mid-rank of the group.

The variance of $T_{1}$ in the presence of ties is given by:

$$
\begin{aligned}
& \operatorname{var}\left(T_{1}\right)=\frac{n_{1} n_{2}}{12\left(n_{1}+n_{2}\right)\left(n_{1}+n_{2}-1\right)} \\
& \text { - }\left[\left(n_{1}+n_{2}\right)^{3}-n_{1}-n_{2}-\Sigma\left(t^{3}-t\right)\right]
\end{aligned}
$$

where the summation is performed over all tied groups, and $t$ is the number of elements in each tied group. If the values of $n_{1}$ and $n_{2}$ are greater than about 8 [143] a parameter $z$ may be defined which has the standard normal distribution:

$$
z=\frac{T_{1}-\bar{T}_{1}}{\sqrt{ } \operatorname{var}\left(T_{1}\right)}
$$

\section{Spearman Rank Correlation Test.}

The Spearman test may be used in place of linear regression to test the degree of correlation between two variables. By comparing rankings it avoids the assumption of a linear relationship between the two variables, but it yields no information such as regression parameters. Given a set of $n$ paired observations $\left(x_{i}, y_{i}\right)$, the test starts by ranking the observations according to the values of $x_{i}$ and separately in accordance with the values of $y_{i}$. A value $D_{i}$ is defined as the difference between the ranking of $x_{i}$ with respect to the other ' $x$ ' values and the ranking of $y_{i}$ with respect to the other ' $y$ ' values. If:

$$
D_{i}=\operatorname{rank}\left(x_{i}\right)-\operatorname{rank}\left(y_{i}\right)
$$

then:

$$
r_{s}=1-\frac{6 \sum_{i=1}^{n} D_{i}^{2}}{n\left(n^{2}-1\right)}
$$


Spearman's rank correlation coefficient is 0 if there is no correlation between the two variables, 1 if there is perfect direct correlation, and - 1 if there is perfect agreement in the inverse order of rankings. A variable, $z$, is defined as follows:

$$
z=r_{s} \cdot(n-1)^{\frac{1}{2}}
$$

This variable is normally distributed with a mean value of zero and a standard deviation of one, and may be used to provide an estimate of the probability that the observed correlation is due to chance.

\section{I.2: Computer programs.}

Computer programs have been written to store sets of data, to compute distribution parameters such as mean and standard deviation, to divide sets of data into groups so that histograms may be plotted, to test for significant differences between two sets of data and to estimate the degree of correlation between two sets of data. A further program has been written to perform linear regression between a dependent variable and two independent variables. All of these programs can accept data from the keyboard (in which case the user is asked if the data is to be copied onto the disk) or from disk files, and at the end the user is asked if the printout of the results is to appear on the screen or be sent to a printer or a disk file.

\section{Program DIST.}

Computes distribution parameters for a set of data. The quantities computed are mean, standard deviation, moment coefficient of skew, moment coefficient of kurtosis, minimum value, maximum value and median value. The mean and standard deviation are computed in the normal fashion:

$$
\begin{aligned}
\bar{x} & =\frac{1}{N} \sum_{i=1}^{N} x_{i} \\
s^{2} & =\frac{1}{N-1} \sum_{i=1}^{N}\left(x_{i}-\bar{x}\right)^{2} \\
& =\frac{1}{N-1} \sum_{i=1}^{N} x_{i}{ }^{2}-N \cdot \bar{x}^{2}
\end{aligned}
$$

Skew is quantified in terms of the moment coefficient of skew, denoted a3, as follows:

$$
a_{3}=m_{3} /\left(m_{2}\right)^{3 / 2}
$$


where the moments $\mathrm{m}_{\mathrm{r}}$ are calculated from:

$$
m_{r}=\frac{1}{N} \cdot i \stackrel{N}{=}_{1}\left(x_{i}-\bar{x}\right)^{r}
$$

Kurtosis is the degree of peakedness of a distribution when compared to a normal distribution of the same mean and standard deviation. The moment coefficient of kurtosis, $a_{4}$, is defined by:

$$
a_{4}=m_{4} / m_{2}^{2}
$$

The normal distribution has $a_{4}=3$, so that a distribution with $a_{4}>3$ is more sharply peaked (leptokurtic) and one with $\mathrm{A}_{4}<3$ is less sharply peaked (platykurtic) than a comparable normal distribution. The median is obtained by listing the data in order of increasing value, the value quoted being that of the data point whose ranking is half that of the maximum value. This is achieved by a 'bubble sorting' routine, where the program starts at one end of the array of data values and compares the value of each data point with that of its neighbour. If it is desired to have the lowest value at the beginning of the array then if the value of the first element is greater than that of the second the program interchanges these two values. The program then examines points two and three, interchanging them if necessary, and so on until the other end of the array is reached. This single pass through the array will have increased the tendency for the data to be in order of increasing value. Perfect ordering is obtained by repeating this process several times until each data value is less than that of its successor, a state which is reached when a complete pass through the data array occurs without any interchanges taking place.

\section{Program HIST.}

This program has the same data entry routine as the previous program. Its purpose is to simplify the production of histograms from a set of data. The program first determines the maximum and minimum values of the data set, and then asks the user for the value to be used for the mid-point of the first cell of the histogram, and for the cell width. The program then determines the number of cells required and counts the number of data points whose values lie in each cell. The output from the program gives the boundaries of each cell, its midpoint, and the number of values lying in that cell. This information may be displayed on the screen, or printed out, but more commonly will be stored in a disk file which may be used to pass the information to a graphics program such as Harvard Graphics or DrawPerfect. 


\section{Program WILCOXON.}

Following input of the two sets of data, which may be either from the keyboard or from disk files, the program combines the two sets into a single larger group which it then arranges in order of increasing value using a bubble sorting routine (see notes on program DIST above). The Wilcoxon parameter $T_{1}$ is the sum of the rankings of the members of the first set of data within the combined group. The program computes $\mathrm{T}_{1}$ and then outputs its value, along with the range of possible values for this quantity given the numbers of elements in the two samples. For example if the first group has 2 elements and the second set has 3 elements then if the members of the first group all have values smaller than all the members of the second set the value of $\mathrm{T}_{1}$ will be $1+2=3$. Conversely if the values of the first set are all greater than those of the second set the $T_{1}$ value will be $4+5=9$. The program also outputs the expectation value of $T_{1}$ and the standard deviation of the Wilcoxon distribution for the sample sizes, the parameter $\mathrm{z}$ (see equation I.4) and the probability that the difference between the observed value of $T_{1}$ and the mean of the Wilcoxon distribution for the two sample sizes could have arisen due to chance.

It has been established that the program correctly computes the values of $T_{1}$ and $z$ by checking it against an example (number 13.3) quoted in Armitage [140]. The probability that the value of $z$ could have been due to chance is obtained from the area under the tail of the normal distribution curve between $z$ and infinity, and is computed from a polynomial approximation [144]:

$$
p(z)=f(z) \cdot\left(b_{1} \cdot t+b_{2} \cdot t^{2}+b_{3} \cdot t^{3}+b_{4} \cdot t^{4}+b_{5} \cdot t^{5}\right)
$$

where:

$$
\begin{aligned}
f(z) & =\frac{1}{(2 \pi)^{\frac{1}{2}}} e^{-\left(z^{2} / 2\right)} \\
t & =\frac{1}{1+r z}
\end{aligned}
$$

and:

$$
\begin{aligned}
& r=0.2316419 \\
& b_{1}=-0.31938153 \\
& b_{2}=-0.356563782 \\
& b_{3}=-1.781477937 \\
& b_{4}=-1.821255978 \\
& b_{5}=1.330274429
\end{aligned}
$$

Equation (1.12) gives the (one-sided) probability that values of $\mathrm{z}$ equal to or greater than the given value will occur. The normal probabilities computed have been checked against a card programmable calculator (H.P. 65) with a program (Stat 1-10A) which uses the same algorithm. 


\section{Program CORREL.}

The program ranks the two data sets individually using a bubble sort routine and uses the differences in ranking between corresponding elements in the two sets to compute the Spearman rank correlation coefficient, $r_{S}$, using equation (I.5). The value of $r_{S}$ output by the program has been checked by computing the values of an example given in the manual for a statistics package produced for the H.P. 65 calculator [\#\#]. The $\mathrm{z}$ value, calculated from equation (I.6), is also output along with the probability calculated according to the normal distribution in the same manner as described for the previous program. In addition, the program computes the linear regression correlation coefficient, $r$, and the calculated regression constants an and $a_{1}$ defined by:

$$
y=a_{0}+a_{1} x
$$

\section{Program MULTREG.}

Performs linear regression between a single dependent variable and two independent variables. For the regression equation:

$$
z=a_{0}+a_{1} x+a_{2} y
$$

the regression parameters are computed as follows:

$$
\begin{aligned}
& a_{2}=\frac{A-B}{\left[n \Sigma x_{i}^{2}-\left(\Sigma x_{i}\right)^{2}\right]\left[n \Sigma y_{i}^{2}-\left(\Sigma y_{i}\right)^{2}\right]-\left[n \Sigma x_{i} Y_{i}-\left(\Sigma x_{i}\right)\left(\Sigma y_{i}\right)\right]^{2}} \\
& A=\left[n \Sigma x_{i}^{2}-\left(\Sigma x_{i}\right)^{2}\right] \cdot\left[n \Sigma y_{i} z_{i}-\left(\Sigma y_{i}\right)\left(\Sigma z_{i}\right)\right] \\
& B=\left[n \Sigma x_{i} Y_{i}-\left(\Sigma x_{i}\right)\left(\Sigma y_{i}\right)\right] \cdot\left[n \Sigma x_{i} z_{i}-\left(\Sigma x_{i}\right)\left(\Sigma z_{i}\right)\right] \\
& a_{1}=\frac{\left[n \Sigma x_{i} z_{i}-\left(\Sigma x_{i}\right)\left(\Sigma z_{i}\right)\right]-a_{2}\left[n \Sigma x_{i} Y_{i}-\left(\Sigma x_{i}\right)\left(\Sigma y_{i}\right)\right]}{n \Sigma x_{i}{ }^{2}-\left(\Sigma x_{i}\right)^{2}} \\
& a_{0}=\frac{1}{n}\left[\Sigma z_{i}-a_{2} \Sigma y_{i}-a_{1} \Sigma x_{i}\right]
\end{aligned}
$$

Computation of the linear regression coefficients first requires the variances of the individual variables, $s_{x}{ }^{2}, s_{y}^{2}$ and $s_{z}^{2}$, to be calculated via: 


$$
\begin{aligned}
& s_{x}^{2}=\frac{1}{n-1}\left[\Sigma x_{i}{ }^{2}-\frac{1}{n}\left(\Sigma x_{i}\right)\left(\Sigma x_{i}\right)\right] \\
& s_{y}^{2}=\frac{1}{n-1}\left[\Sigma y_{i}^{2}-\frac{1}{n}\left(\Sigma y_{i}\right)\left(\Sigma y_{i}\right)\right] \\
& s z^{2}=\frac{1}{n-1}\left[\Sigma z_{i}{ }^{2}-\frac{1}{n}\left(\Sigma z_{i}\right)\left(\Sigma z_{i}\right)\right]
\end{aligned}
$$

and secondly requires the covariances $s_{x z}{ }^{2}, s_{y z}{ }^{2}$ and $s_{x y}{ }^{2}$ to be computed as follows:

$$
\begin{aligned}
& s_{X z^{2}}=\frac{1}{n-1}\left[\Sigma x_{i} z_{i}-\frac{1}{n}\left(\Sigma x_{i}\right)\left(\Sigma z_{i}\right)\right] \\
& s_{Y z^{2}}=\frac{1}{n-1}\left[\Sigma y_{i} z_{i}-\frac{1}{n}\left(\Sigma y_{i}\right)\left(\Sigma z_{i}\right)\right] \\
& s_{X Y}{ }^{2}=\frac{1}{n-1}\left[\Sigma x_{i Y}-\frac{1}{n}\left(\Sigma x_{i}\right)\left(\Sigma y_{i}\right)\right]
\end{aligned}
$$

The linear regression coefficients between pairs of variables are then given by:

$$
\begin{aligned}
R_{X z} & =\frac{s_{X z}{ }^{2}}{s_{X} s_{z}} \\
R_{y z} & =\frac{s_{y z}{ }^{2}}{s_{y} s_{z}} \\
R_{X Y} & =\frac{s_{X y}}{s_{X} s_{y}}
\end{aligned}
$$

And the multiple linear regression coefficient is then:

$$
R_{m u l t}=\left[a_{1} \frac{s_{x}}{s_{z}} R_{x z}+a_{2} \frac{s_{y}}{s_{z}} R_{y z}\right]^{\frac{1}{2}}
$$




\section{I.3: Program Listings.}

\section{Program DIST: Computation of Distribution Parameters.}

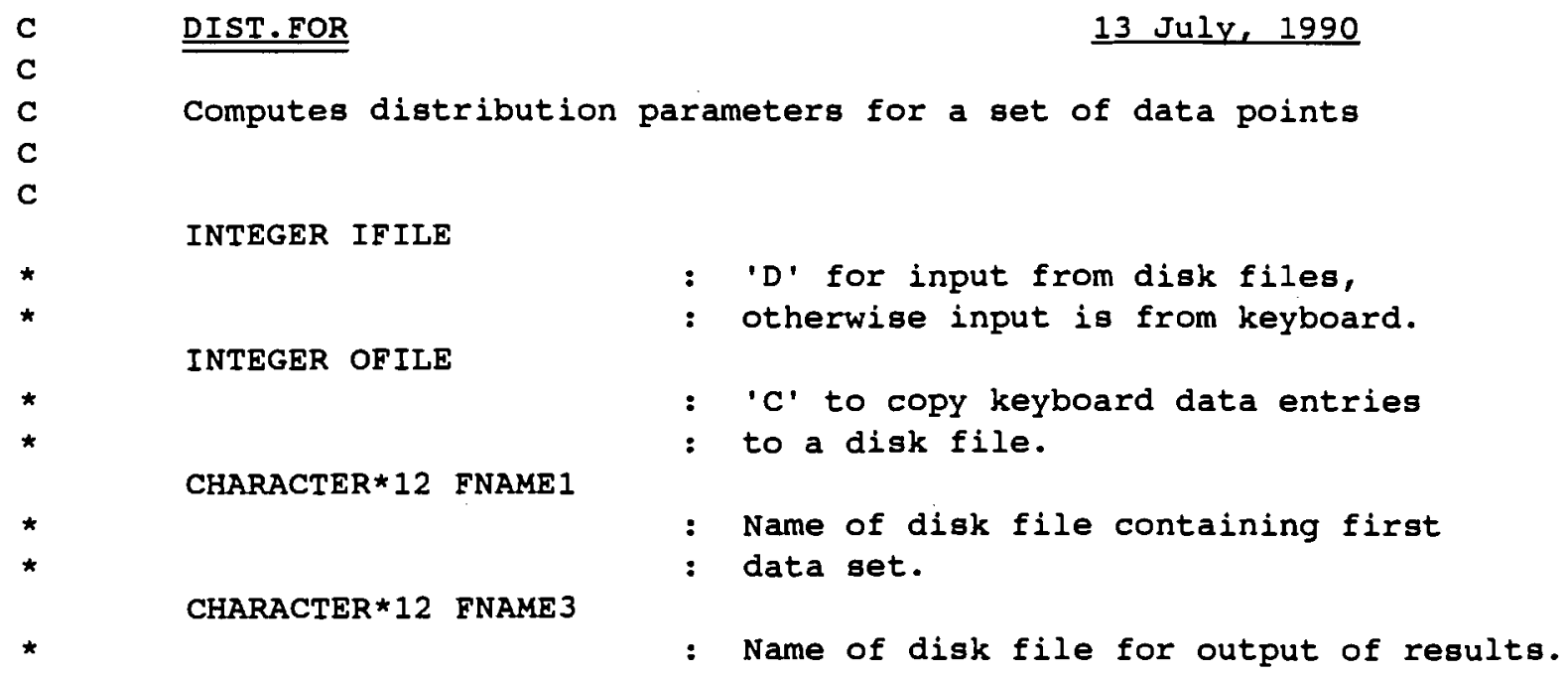

C 


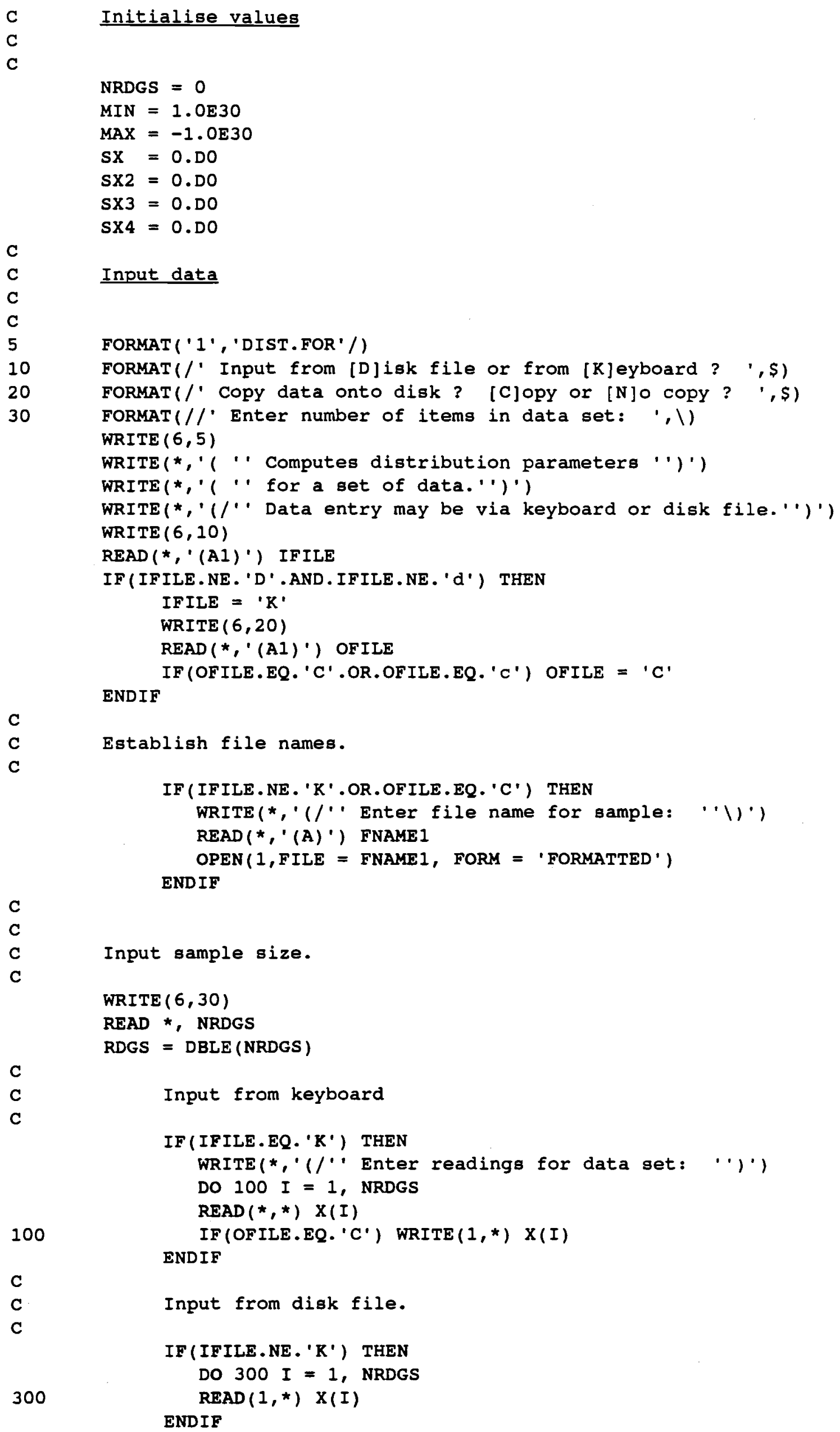




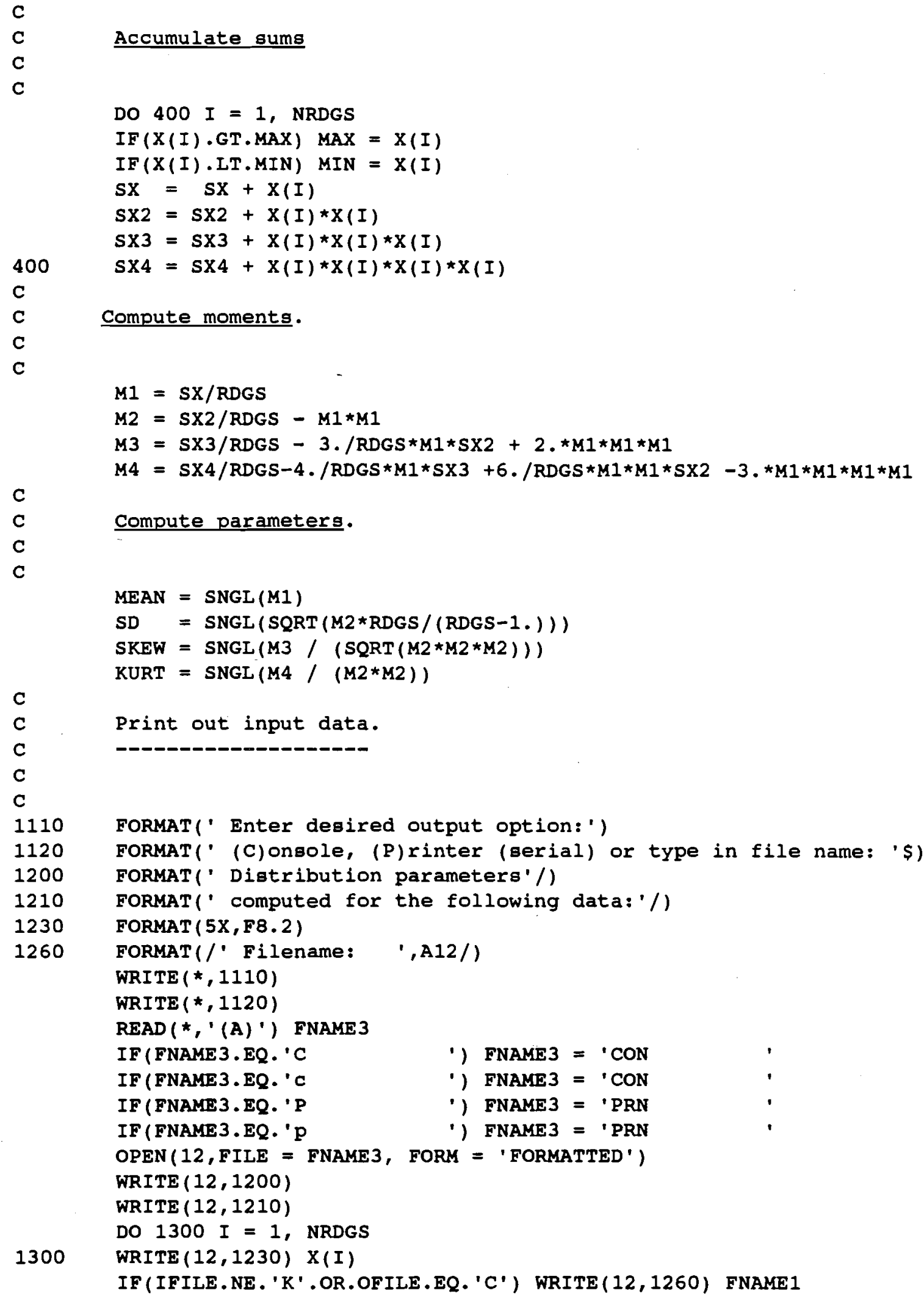




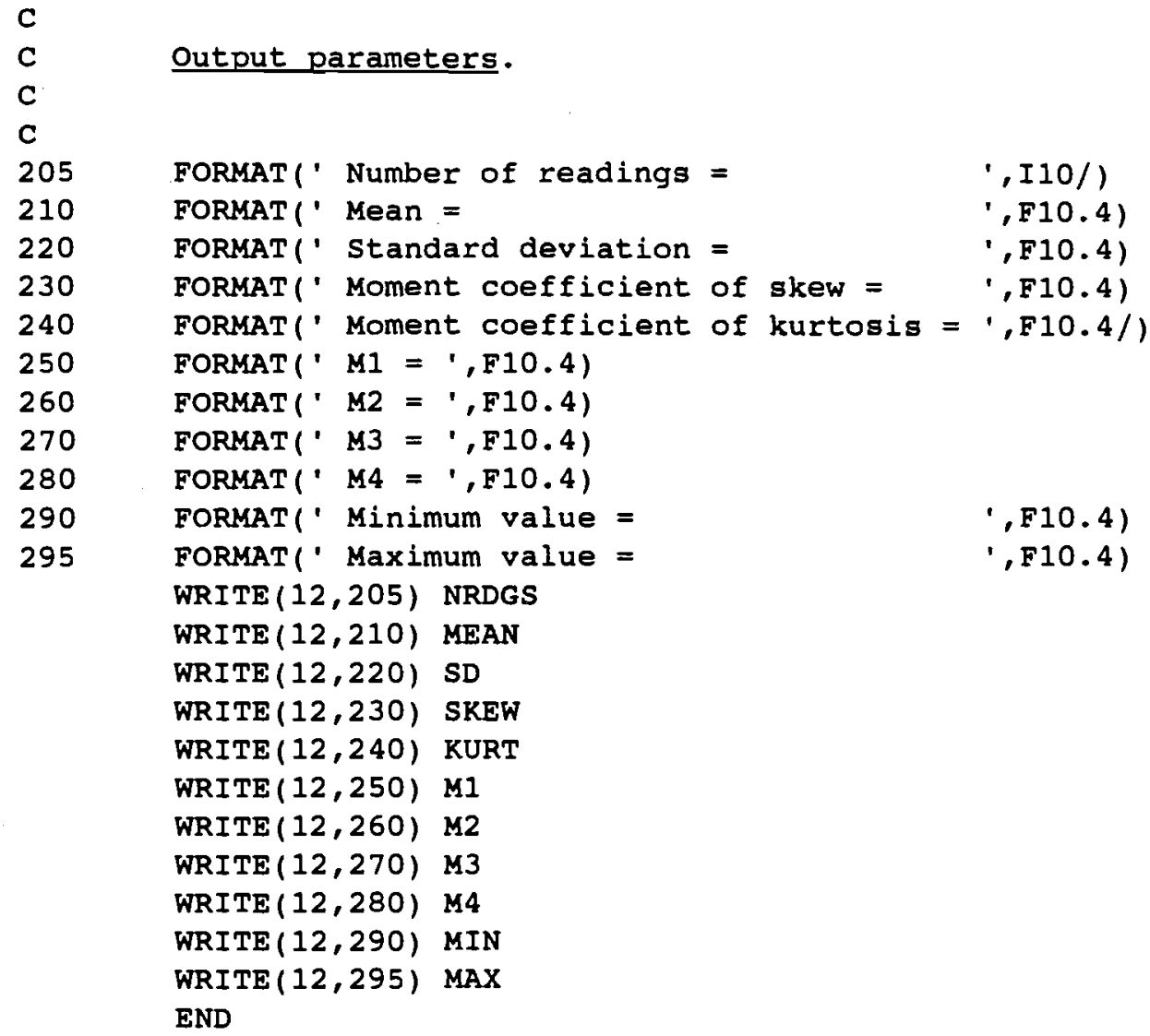




\section{Program HIST: Computation of Histogram Cell Values.}

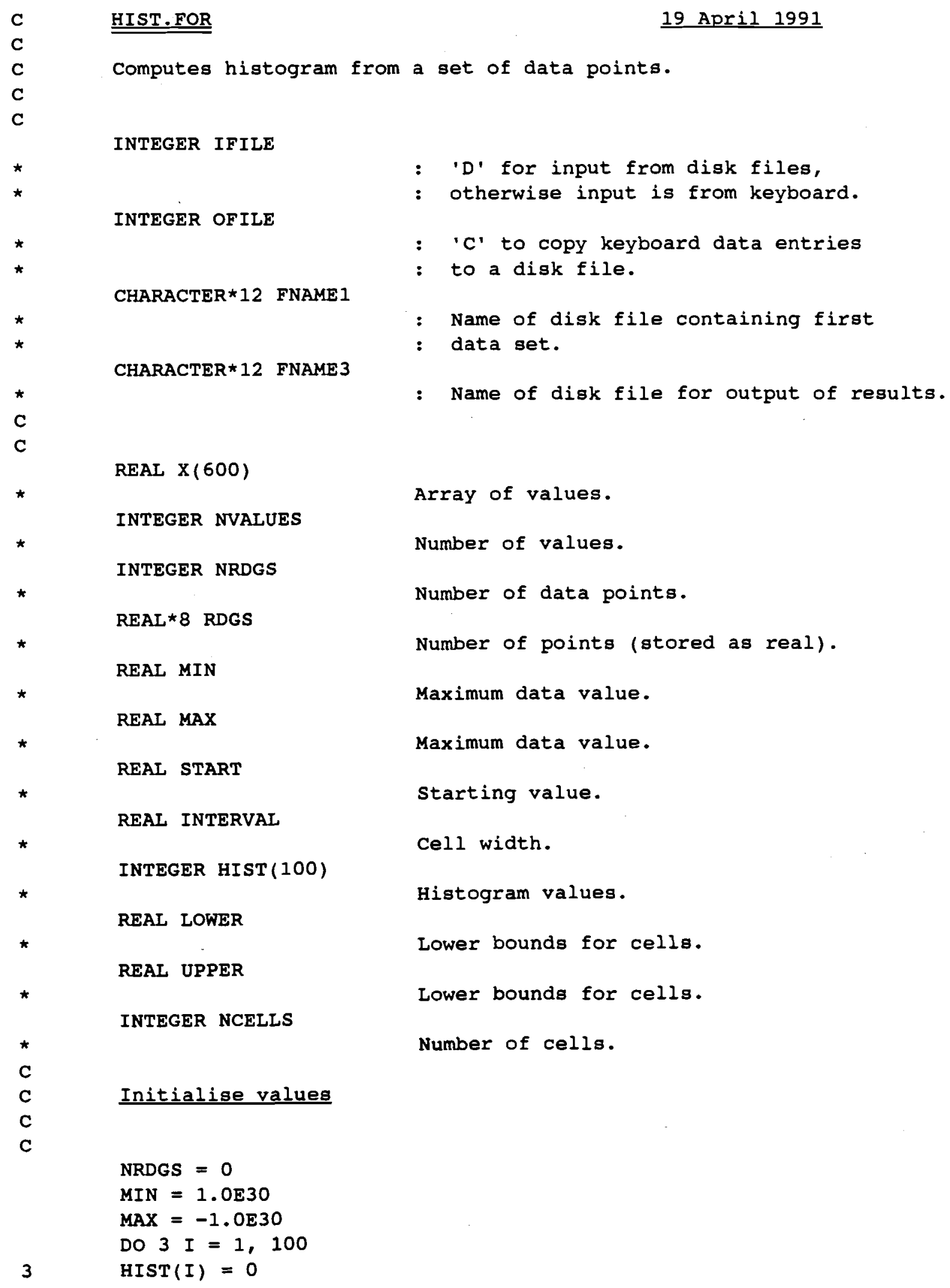

C : Name of disk file containing first 
C

C

C

C

5

C

C

C

C

10

20

30

C

C

C

C

C

C

C

100

C

C

C

\section{Input data}

FORMAT (' 1', 'HIST.FOR' /)

WRITE $(6,5)$

WRITE(*,' ( 'C Computes histogram values ' ')')

WRITE (*,' (' for a set of data.' ')')

WRITE $(*, '(/$, ' Data entry may be via keyboard or disk file.' ')')

Input data.

FORMAT(/' Input from [D] isk file or from [K] eyboard? ', \$)

FORMAT (/' Copy data onto disk? [C]Opy or [N]O copy ? ', \$)

FORMAT (//, Enter number of items in data set: ', 1 )

WRITE $(6,10)$

READ (*,' (A1)') IFILE

IF ( IFILE. NE. 'D' . AND. IFILE.NE. ' $d$ ') THEN

IFILE = ' $\mathrm{K}$ '

WRITE $(6,20)$

$\operatorname{READ}(*, '(A 1)$ ') OFILE

IF (OFILE.EQ. 'C'.OR.OFILE.EQ. ' $C$ ') OFILE = 'C'

ENDIF

Establish file names.

IF (IFILE.NE . ' $K$ ' . OR. OFILE.EQ. ' $C$ ') THEN

WRITE (*,' (/'" Enter file name for sample: ' $/)$ ')

READ (*,' (A)') FNAME 1

$\operatorname{OPEN}(1, F I L E=$ FNAME 1, FORM $=$ 'FORMATTED' $)$ ENDIF

Input sample size.

WRITE $(6,30)$

READ * , NRDGS

RDGS = DBLE (NRDGS)

Input from keyboard

IF ( IFILE . EQ. 'R') THEN

WRITL(", (/,' Enter readings for data set: ' )')

DO $100 I=1$, NRDGS

$\operatorname{READ}(*, *) X(I)$ ENDIF

IF (OFILE.EQ. 'C') WRITE $(1, *) X(I)$

Input from disk file.

IF (IFILE.NE. ' $\mathrm{K}$ ') THEN

DO $300 I=1$, NRDGS

$\operatorname{READ}(1, \star) \quad X(I)$

ENDIF 
C
C
C
C
C
C

Compute range of values.

500

510

520

530

540

DO $500 I=1$, NRDGS

$I F(X(I) \cdot L T \cdot M I N) M I N=X(I)$

$I F(X(I) \cdot G T \cdot M A X) \quad M A X=X(I)$

CONTINUE

FORMAT(' Minimum value is: ',G10.4)

FORMAT(' Maximum value is: ', G10.4)

FORMAT(/' Enter starting value: 'l)

FORMAT( 'Enter interval: '

WRITE $(*, 510)$ MIN

WRITE $(*, 520)$ MAX

WRITE $(*, 530)$

$\operatorname{READ}(*, *)$ START

$\operatorname{WRITE}(*, 540)$

$\operatorname{READ}(*, *)$ INTERVAL

START = START - INTERVAL / 2 .

NCELLS $=0$

VALUE = START

550 NCELLS $=$ NCELLS +1

VALUE = VALUE + INTERVAL

IF (VALUE.LE.MAX) GOTO 550

C

C

Compute histogram.

C

C

C

C

DO $600 I=1$, NRDGS

ICELL $=(X(I)-$ START $) /$ INTERVAL + 1

$600 \operatorname{HIST}($ ICELI) $=\operatorname{HIST}($ ICELL) +1

c

Print out input data.

C

C

C

1110 FORMAT(' Enter desired output option:')

1120 FORMAT(' (C)onsole, (P)rinter (serial) or type in flle name: '\$)

1200 FORMAT(" Distribution parameters'/)

1210 FORMAT(' computed for the following data: '/)

1230 FORMAT (5X,F8.2)

1260 FORMAT (/' Filename: ',A12/)

WRITE $(*, 1110)$

WRITE $(*, 1120)$

READ (*,' (A) ') FNAME3

IF (FNAME 3 . EQ. ' C

IF (FNAME 3. EQ. ' C

IF (FNAME 3 . EQ. ' P

IF (FNAME 3.EQ. 'P

') FNAME3 $=$ CON

') FNAME3 $=$ ' CON

') FNAME3 $=$ 'PRN

') FNAME3 = 'PRN

OPEN $(12$, FILE = FNAME3, FORM = 'FORMATTED' $)$

WRITE $(12,1200)$

WRITE $(12,1210)$

DO $1300 I=1$, NRDGS

1300 WRITE $(12,1230) \times(I)$

IF (IFILE.NE. 'K' .OR.OFILE.EQ.'C') WRITE $(12,1260)$ FNAME1 


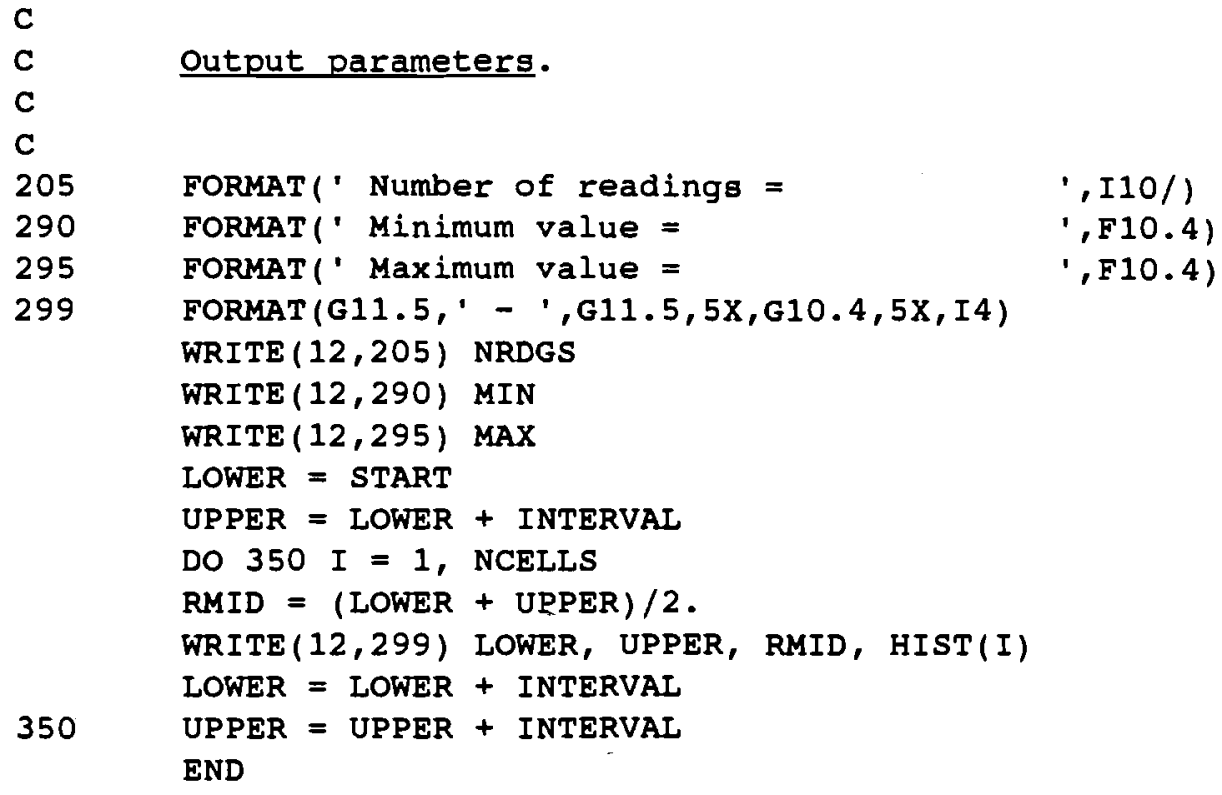




\section{Program WILCOXON: Nonparametric Test for Significant Differences.}

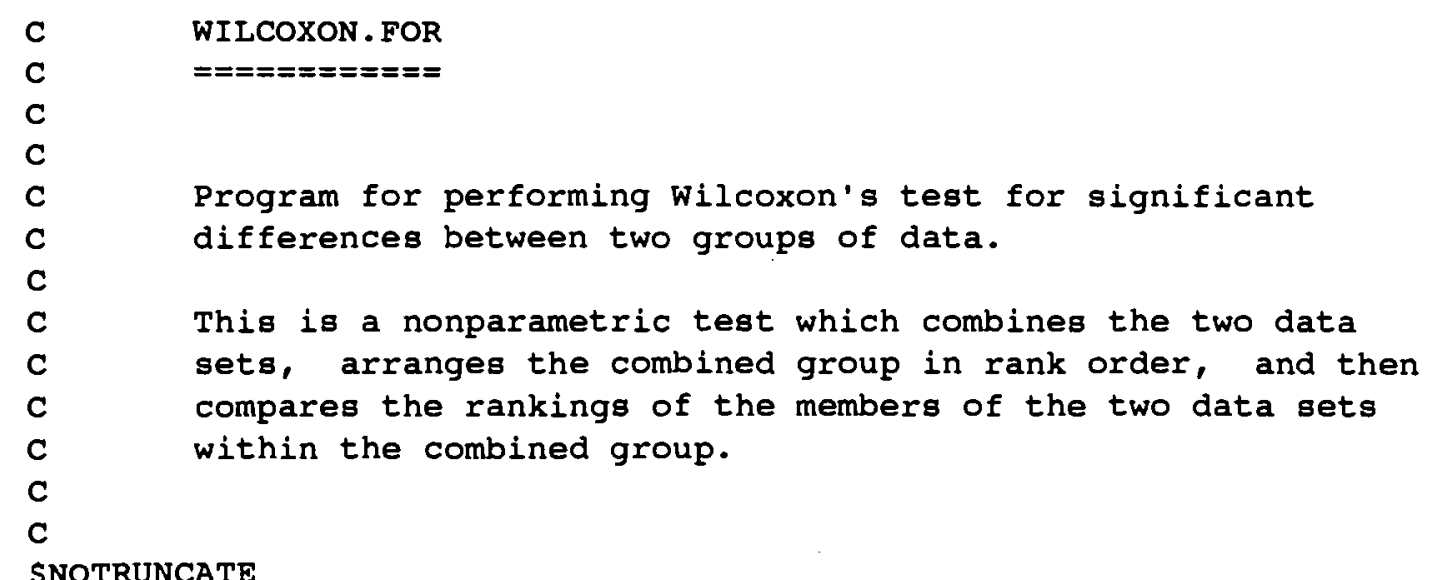

INTEGER IFILE

INTEGER OFILE

: 'D' for input from disk files,

: otherwise input is from keyboard.

REAL SAMPLE1(100)

REAL SAMPLE2 (100)

: Arrays to store data values.

REAL RANK1

REAL RANK2

: Sum of ranks of sample 1 (= T1).

: Sum of ranks of sample 2 .

REAL COMBSAMPLE $(2,200)$

INTEGER NSAMPLE 1

INTEGER NSAMPLE2

INTEGER * 4 NTOT

INTEGER FLIPS

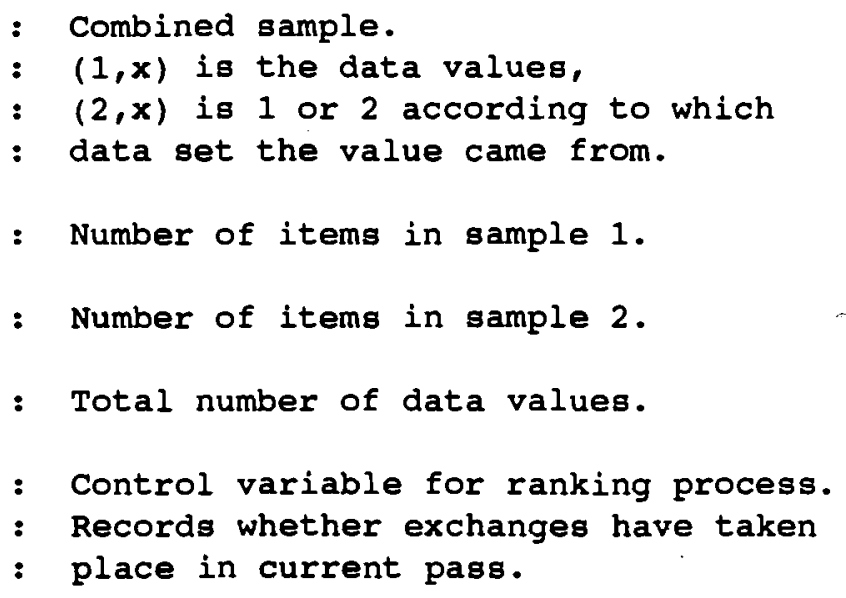


REAL TEMP

\section{INTEGER INITRANK}

INTEGER NTIED

REAL AVRANK

REAL TIESUM

REAL LBOUND

REAL UBOUND

REAL WILMEAN

REAL WILSD

REAL CHECKSD

REAL $\mathbf{Z}$
: Temporary store for flips.

: Rank of the first member of a tied group.

: Number of tied ranks in a group.

: Average rank of a tied group.

: Sum of $t 3$ - $t$ for calculating variance.

: Lowest possible value for $\mathrm{T} 1$.

: Highest possible value for $T 1$.

: Mean of Wilcoxon distribution for present

: sample sizes.

: SQRT of Wilcoxon variance.

: S.D. ignoring ties.

: Normal variate calculated from

: T1, WILMEAN \& WILSD.

Variables for the computation of $p$.

REAL *8 DZ

: Double precision version of $\mathrm{z}$.

$\operatorname{REAL} \star 8 \quad Q$

$\operatorname{REAL} * 8 \quad B(5)$

$R E A L * 8 T$

REAL *8 R

REAL* $8 \mathrm{~S}$

: One sided area under tail of normal

: distribution.

: Coefficients of polynomial fit.

Initialise constants

$R=0.2316419$

$S=0.39894228$

$B(1)=0.31938153$

$B(2)=-0.356563782$

$B(3)=1.781477937$

$B(4)=-1.821255978$

$B(5)=1.330274429$

C

C

FORMAT (' 1 ', ' WILCOXON.FOR'/)

WRITE $(6,5)$

WRITE (*,' (/" Nonparametric test for significant' ')')

WRITE $(*, '(\cdot$ differences between two data sets.' ')')

WRITE (*, ' (/, Data entry may be via keyboard or disk files.' ')') 
C

C

C

C

C

c

c

C

C

c

C

C

C

100

C

C

c

Input data.

FORMAT (/" Input from [D]isk files or from [K] eyboard ? ',\$) FORMAT (/" Copy data onto disk ? [C]Opy or [N]O copy ? ', FORMAT(//' Enter number of items in sample 1: ', /) FORMAT(, Enter number of items in sample 2: ', ) WRITE $(6,10)$

$\operatorname{READ}\left(*, '(A 1)^{\prime}\right)$ IFILE

IF (IFILE.NE. 'D' .AND. IFILE.NE. ' $d$ ') THEN

IFILE $=$ ' $\mathrm{K}$ '

WRITE $(6,20)$

$\operatorname{READ}(*, '(A 1)$ ') OFILE ENDIF

IF (OFILE.EQ. ' $\mathrm{C}$ '. OR.OFILE.EQ. ' $\mathrm{C}$ ') OFILE $={ }^{\prime} \mathrm{C}$ '

Establish file names.

IF (IFILE.NE . ' $R$ ' . OR . OFILE.EQ. ' $C$ ') THEN

WRITE(*,' (/' Enter file name for sample 1: " $\left./)^{\prime}\right)$

READ (*,' (A)') FNAME1

OPEN $(1$, FILE = FNAME 1, FORM = 'FORMATTED')

WRITE(*,' (' Enter file name for sample 2: ' $\backslash)$ ')

$\operatorname{READ}\left(*,{ }^{\prime}(\mathrm{A})\right.$ ') FNAME2 ENDIF

$\operatorname{OPEN}(2, F I L E=$ FNAME2, FORM $=$ 'FORMATTED')

Input sample sizes.

WRITE $(6,30)$

$\operatorname{READ}(*, *)$ NSAMPLE 1

WRITE $(6,35)$

$\operatorname{READ}(*, *)$ NSAMPLE 2

NTOT = NSAMPLE1 + NSAMPLE2

Input from keyboard

IF (IFILE. EQ.' $\mathbf{K}$ ') THEN

WRITE $(*, \cdot(/ \cdots$ Enter readings for data set $1:$ ' ')') DO $100 I=1$, NSAMPLE 1

READ (*, ) SAMPLE1 (I)

IF (OFILE.EQ. 'C') WRITE(1,*) SAMPLE I(I)

WRITE(*,'(/, Enter readings for data set $2:$ ' ')')

DO $200 I=1$, NSAMPLE2

READ (*, ) SAMPLE2 (I) ENDIF

IF (OFILE.EQ. 'C') WRITE $\left(2,{ }^{*}\right)$ SAMPLE2 (I)

Input from disk file.

IF (IFILE.NE. ' $R$ ') THEN

DO $300 I=1$, NSAMPLE 1

$\operatorname{READ}(1, \star)$ SAMPLE1 (I)

DO $400 I=1$, NSAMPLE2

$\operatorname{READ}(2, *)$ SAMPLE2 (I)

ENDIF 
C
C
C
C
C

\section{Produce combined sample}

DO $700 I=1$, NSAMPLE 1

COMBSAMPLE $(2, I)=1$

700 COMBSAMPLE $(1, I)=\operatorname{SAMPLE} 1(I)$

DO $710 I=($ NSAMPLE $1+1)$, NTOT

COMBSAMPLE $(2, I)=2$

710 COMBSAMPLE $(1, I)=\operatorname{SAMPLE2}(I$ - NSAMPLE1)

C

Place combined sample in rank order

C

C

$750 \quad$ FLIPS $=0$

DO $800 I=1$, (NTOT - 1)

IF (COMBSAMPLE $(1, I) \cdot$ LE. COMBSAMPLE $(1, I+1)$ ) GOTO 800

FLIPS $=1$

TEMP $=$ COMBSAMPLE $(1, I)$

$\operatorname{COMBSAMPLE}(1, I)=\operatorname{COMBSAMPLE}(1, I+1)$

COMBSAMPLE $(1, I+1)=$ TEMP

TEMP = COMBSAMPLE $(2, I)$

$\operatorname{COMBSAMPLE}(2, I)=\operatorname{COMBSAMPLE}(2, I+1)$

COMBSAMPLE $(2, I+1)=$ TEMP

800 CONTINUE

IF(FLIPS.EQ.O) GOTO 850

GOTO 750

850 CONTINUE

860 FORMAT (I3, F7.1,2X,F7.1)

DO $870 I=1$, NTOT

$\operatorname{WRITE}(6,860) I, \operatorname{COMBSAMPLE}(1, I), \operatorname{COMBSAMPLE}(2, I)$

870 CONTINUE

C

Form rank sums

C

C

C

TIESUM $=0$.

NTIED $=0$

RANR1 $=0$.

RANR2 $=0$.

COMBSAMPLE $(1, N T O T+1)=\operatorname{COMBSAMPLE}(1, N T O T-1)$

permitg summation below to go up to NTOT

DO $1000 I=1$, NTOT

IF (COMBSAMPLE $(1, I)$. NE.COMBSAMPLE $(1, I+1)$ ) GOTO 950

IF (NTIED.EQ.O) INITRANR = I

NTIED $=$ NTIED +1

GOTO 1000

950 IF(NTIED.EQ.0) GOTO 980

AVRANK $=($ INITRANK $* 2$. + NTIED $) / 2$.

DO $970 \mathrm{~J}=$ INITRANK, (INITRANK+NTIED)

IF (COMBSAMPLE $(2, J) \cdot$ EQ.1) RANK1 = RANK1 + AVRANK

970 IF (COMBSAMPLE $(2, J) \cdot E Q \cdot 2)$ RANK2 = RANK2 + AVRANK

TIESUM $=$ TIESUM $+(\operatorname{NTIED+1)*(NTIED+1)*(NTIED+1)-NTIED~-~} 1$

NTIED $=0$

GOTO 1000

980 IF (COMBSAMPLE (2,I) .EQ.1) RANR1 = RANK1 + I

IF (COMBSAMPLE $(2, I) \cdot E Q .2)$ RANK2 = RANK2 + I

1000 CONTINUE 
C
C
C
C
C

Check for final unresolved tie group.

IF (NTIED.EQ.O) GOTO 1100

AVRANK $=($ INITRANK $* 2 .+$ NTIED $) / 2$.

DO $990 \mathrm{~J}=$ INITRANK, (INITRANK + NTIED)

IF (COMBSAMPLE $(2, J) \cdot$ EQ.1) RANK1 = RANK1 + AVRANK

990 IF (COMBSAMPLE (2,J).EQ.2) RANK2 = RANK2 + AVRANK

TIESUM $=\operatorname{TIESUM~}+(\operatorname{NTIED+1)} *(\operatorname{NTIED}+1) *(N T I E D+1)-\operatorname{NTIED}-1$

C

C

Calculate Wilcoxon parameters.

C

CONTINUE

LBOUND $=0.5 *$ NSAMPLE $1 *($ NSAMPLE $1+1)$

UBOUND = NSAMPLE1 * NSAMPLE2 + LBOUND

WILMEAN $=0.5$ * NSAMPLE 1 * (NSAMPLE $1+$ NSAMPLE $2+1)$

WILSD $=($ NSAMPLEI * NSAMPLE2) $/(12$. NTOT* $($ NTOT-1) $)$

WILSD $=$ SQRT (WILSD * (NTOT*NTOT*NTOT - NTOT - TIESUM))

PRINT *, 'CALCULATING $z$ '

$\mathbf{Z}=$ (RANK1 - WILMEAN) / WILSD

CHECKSD $=$ SQRT $($ NSAMPLE1*NSAMPLE2* $($ NTOT +1$) / 12$.

C

C

C

C

Print out input data.

FORMAT(' Enter desired output option:')

1200

1210

1220

1230

1240

1250

1260

1270

1280

1300

FORMAT(' (C)onsole, (P)rinter (serial) or type in file name: '\$)

FORMAT (' Wilcoxon test for significant differences'/)

FORMAT(" performed on the following samples:'/)

FORMAT (" Sample1 Sample2 '/)

FORMAT ( 5X, F8.2, 10X, F8.2)

FORMAT ( $5 X, F 8.2)$

FORMAT ( $23 X, F 8.2)$

FORMAT (/" Filenames: "/)

FORMAT(' Sample1 ',A12)

FORMAT(' Sample2 ',A12)

WRITE $(*, 1110)$

WRITE ( *, 1120)

READ (*,' (A)') FNAME3

IF (FNAME 3. EQ. ' C

?) FNAME3 $3=1 \mathrm{CON}$

') FNAME3 $=$ ' CON

') FNAME3 3 = PRN

IF (FNAME 3 . EQ. ' $P$

') FNAME3 = 'PRN

IF (FNAME 3 . EQ. 'p

'

OPEN $(12$, FILE $=$
WRITE $(12,1200)$

WRITE $(12,1210)$

WRITE $(12,1220)$

DO $1300 I=1$, NSAMPLE 1

IF (I.LE.NSAMPLE2) WRITE $(12,1230)$ SAMPLE I (I), SAMPLE2 (I)

IF(I.GT.NSAMPLE2) WRITE $(12,1240)$ SAMPLE1(I)

IF (NSAMPLE2.LE.NSAMPLE1) GOTO 1400

DO $1350 \mathrm{~J}=$ (NSAMPLE $1+1$ ), NSAMPLE2

WRITE $(12,1250)$ SAMPLE2 (J)

1400

IF (IFILE.NE. 'R' . OR . OFILE . EQ. 'C') THEN

WRITE $(12,1260)$

WRITE $(12,1270)$ FNAME1

WRITE $(12,1280)$ FNAME2

ENDIF 


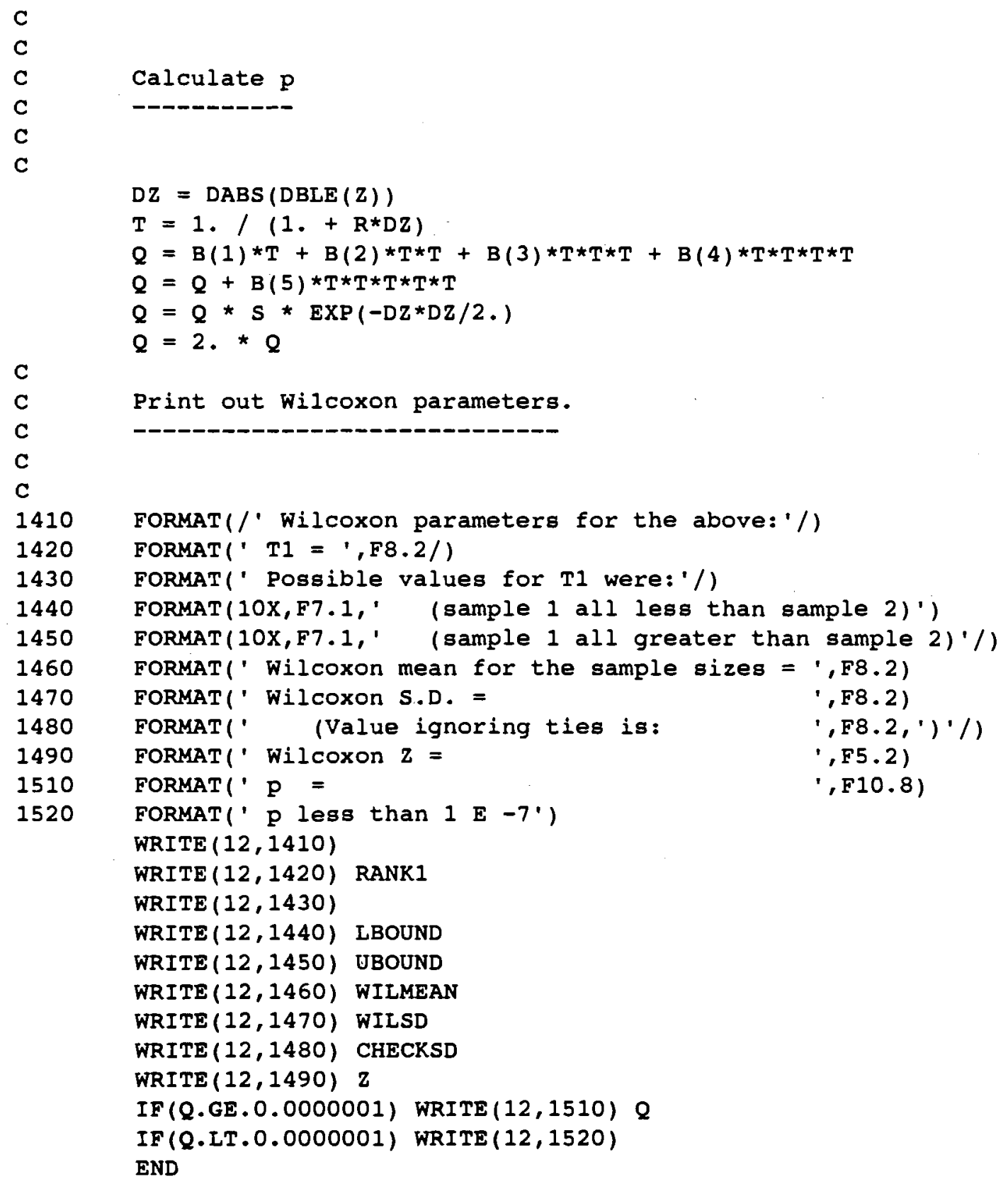




\section{Program CORREL: Nonparametric Correlation \& Linear Regression.}

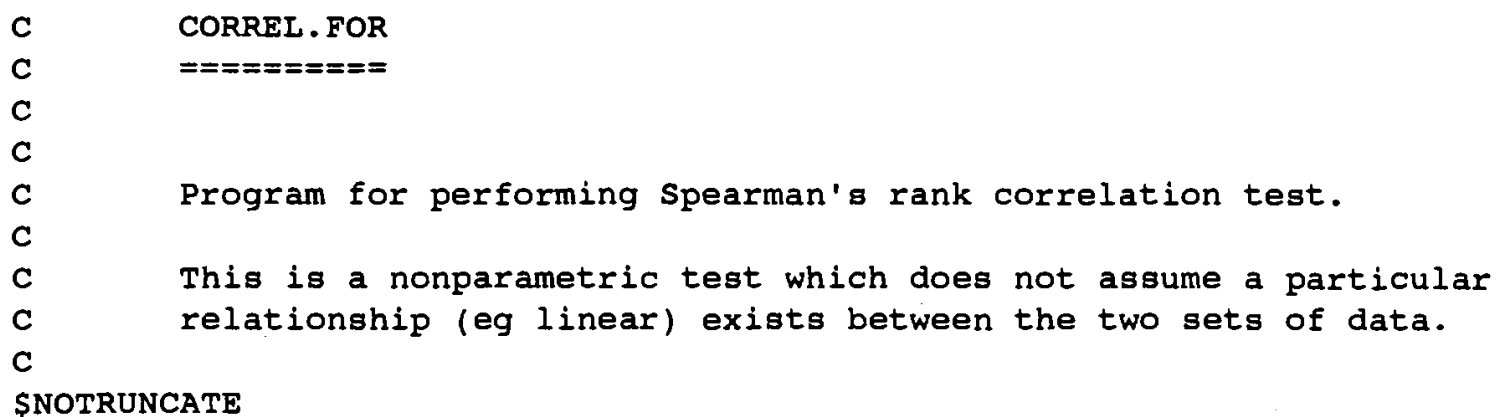

INTEGER IFILE

INTEGER OFILE

CHARACTER*12 FNAME 1

CHARACTER*12 FNAME2

CHARACTER*12 FNAME 3

REAL SAMPLE1 $(2,100)$

REAL SAMPLE2 $(2,100)$

REAL ORDER1 $(2,100)$

REAL ORDER2 $(2,100)$

INTEGER* 4 NTOT

INTEGER FLIPS

REAL TEMP

REAL DIFF

REAL DIFFSUM

REAL RS

REAL Z
: 'D' for input from disk files, : otherwise input is from keyboard.

: 'C' to copy keyboard data entries : to a disk file.

: Name of disk file containing first

: data set.

: Name of disk file containing second : data set.

: Name of disk file for output of results.

: Values of first data set (value, rank).

: Values of second data set (value, rank).

: First data set (value, item number).

: Second data set (value, item number).

: Number of data pairs.

: Control variable for ranking process.

: Records whether exchanges have taken

: place in current pass.

: Temporary store for flips.

: Difference in rankings for each data : point.

: Sum of (rank difference) $* \star 2$.

: spearman rank correlation coefficient.

: Normal variate calculated from Rs

: and NTOT. 
C

C

C

*

*

C

C

C

C

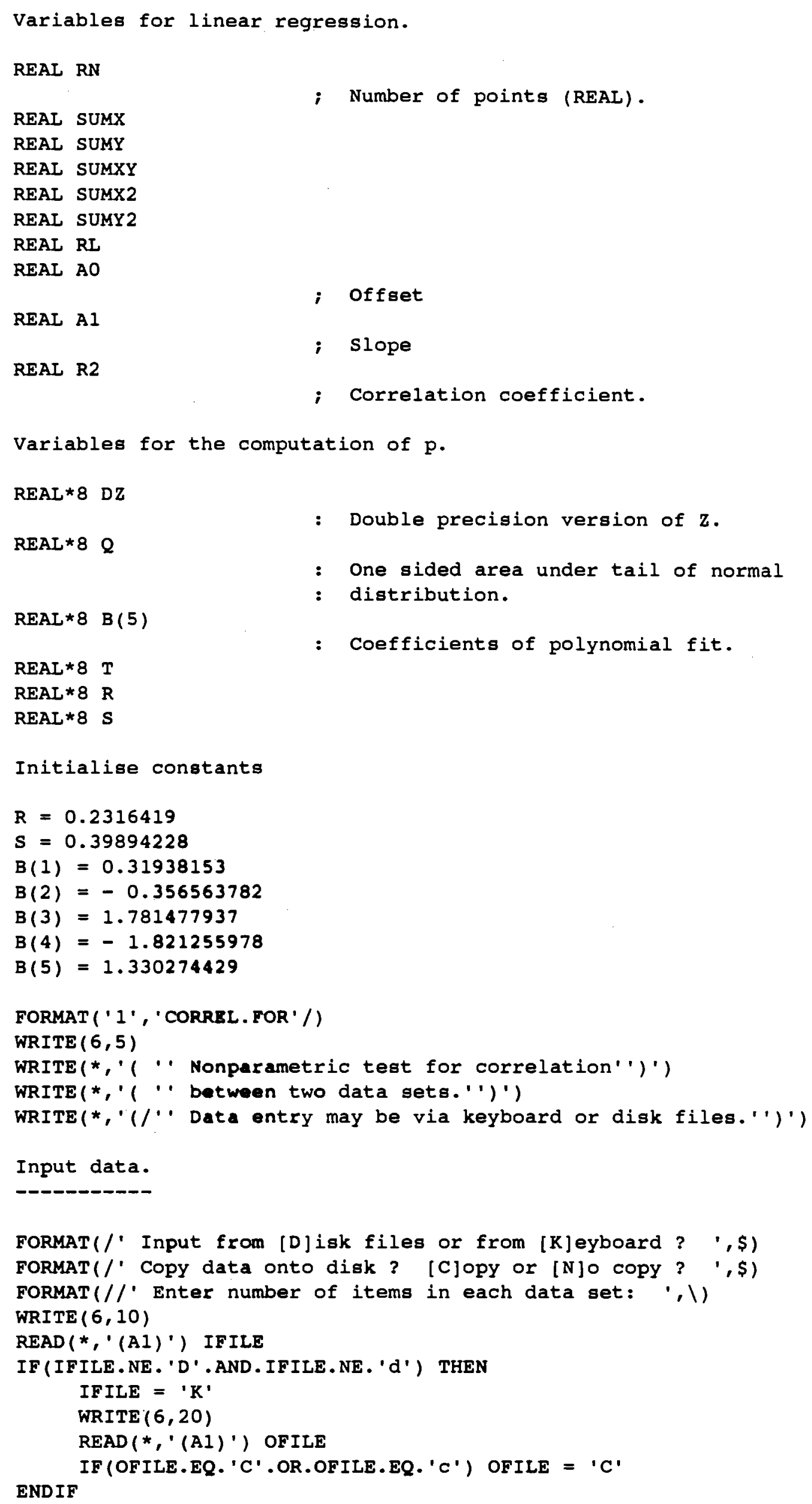




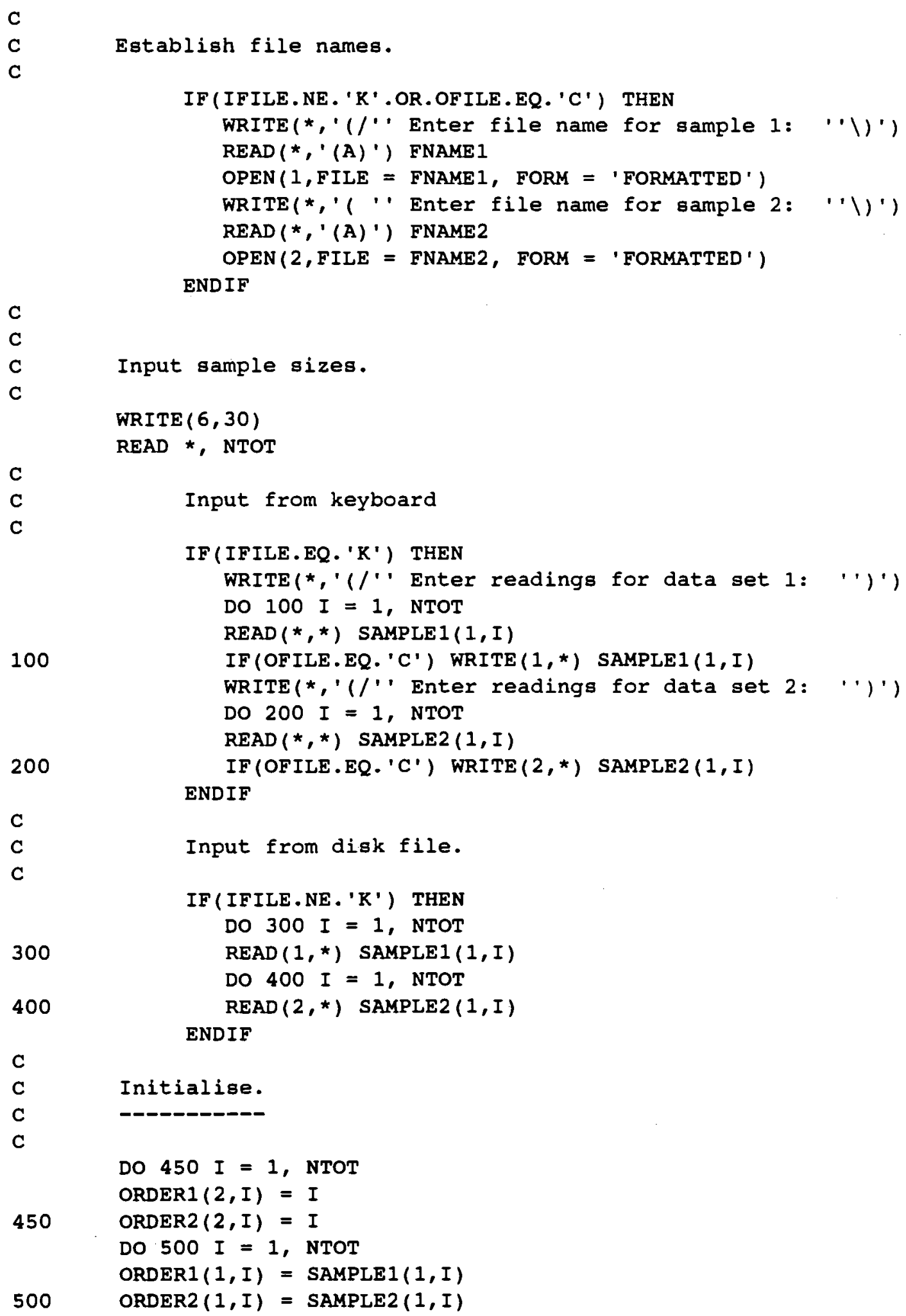




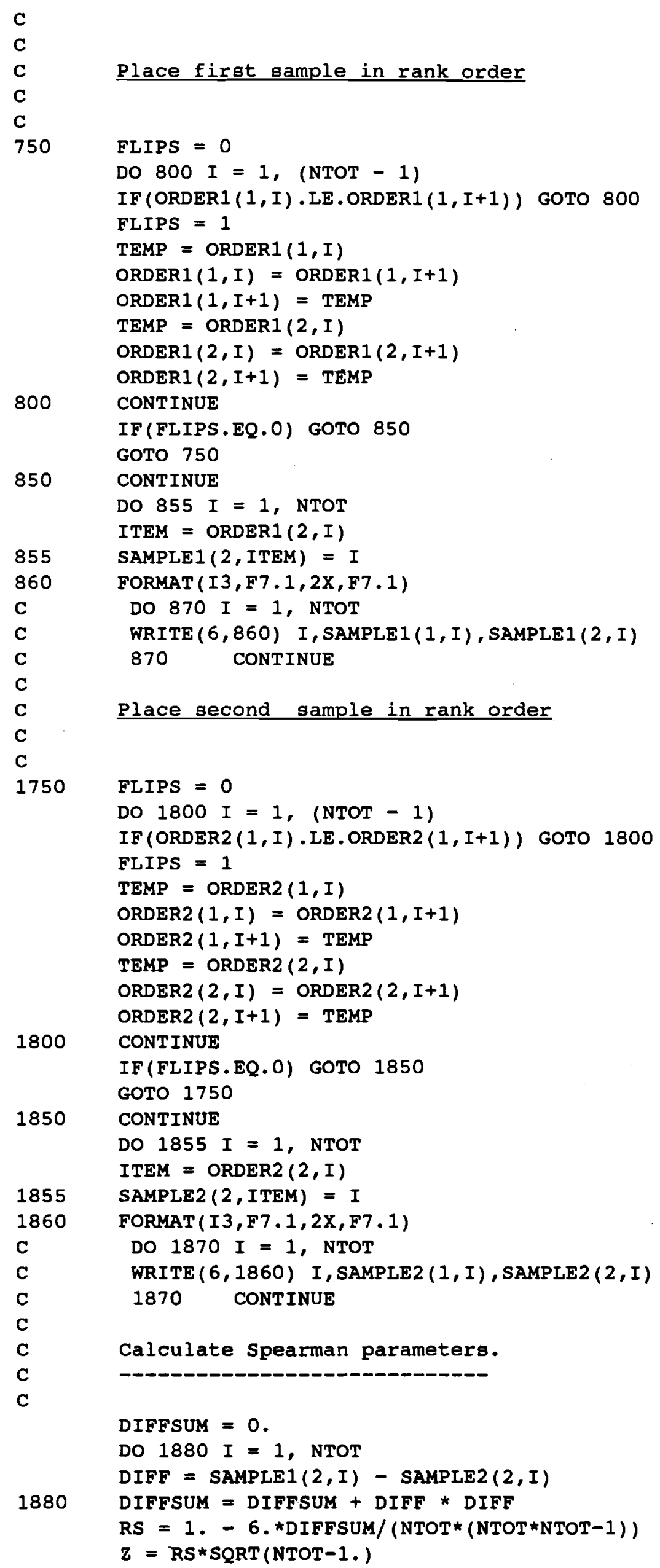

C

Calculate spearman parameters.

C

DIFFSUM $=0$.

DO $1880 I=1$, NTOT

$\operatorname{DIFF}=\operatorname{SAMPLE} 1(2, I)-\operatorname{SAMPLE} 2(2, I)$

1880 DIFFSUM $=$ DIFFSUM + DIFF * DIFF

RS $=1 .-6 . *$ DIFFSUM/(NTOT* $(N T O T * N T O T-1))$

$\mathrm{z}=\mathrm{RS} * \mathrm{SQRT}(\mathrm{NTOT}-1$. 


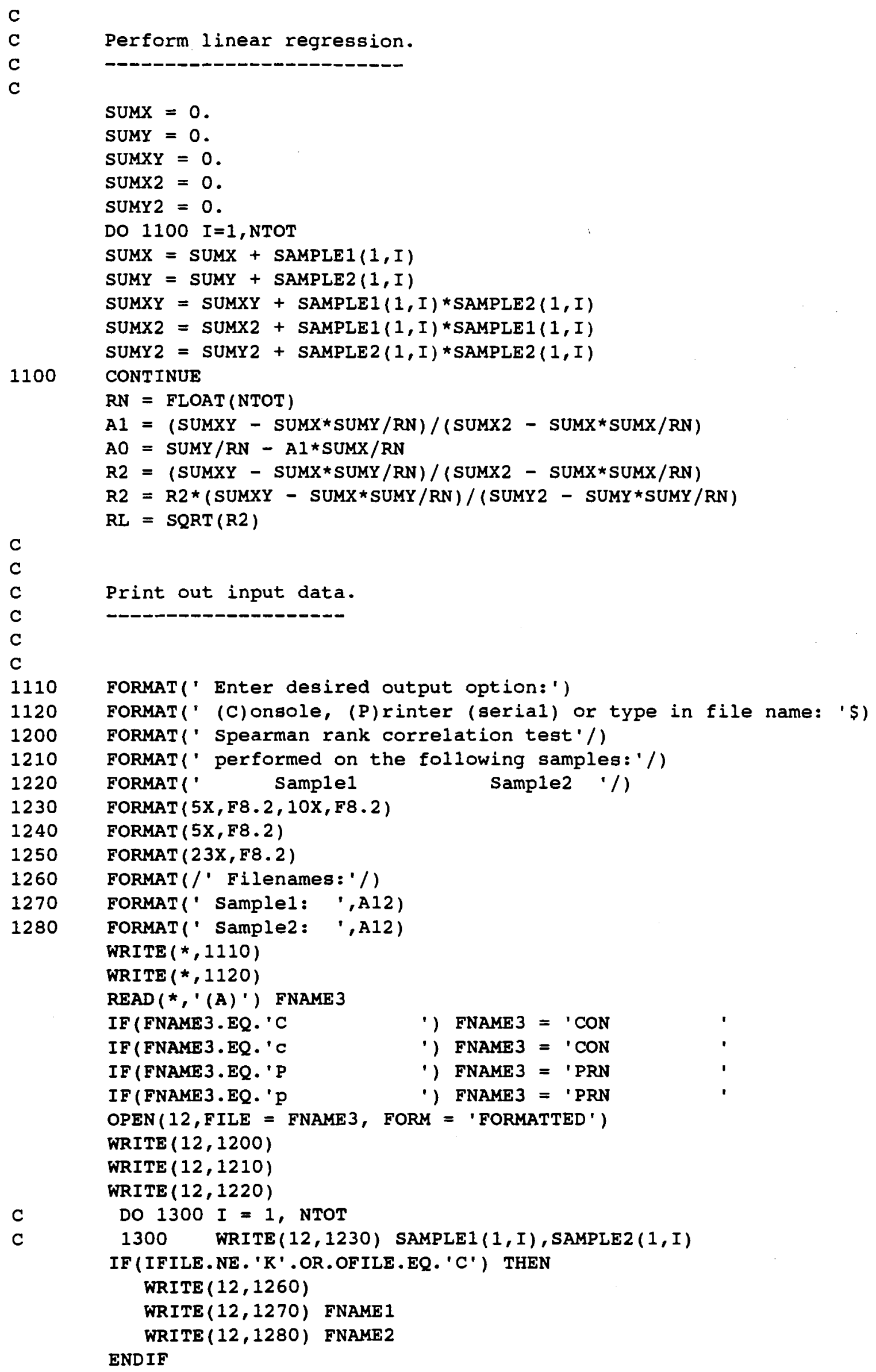




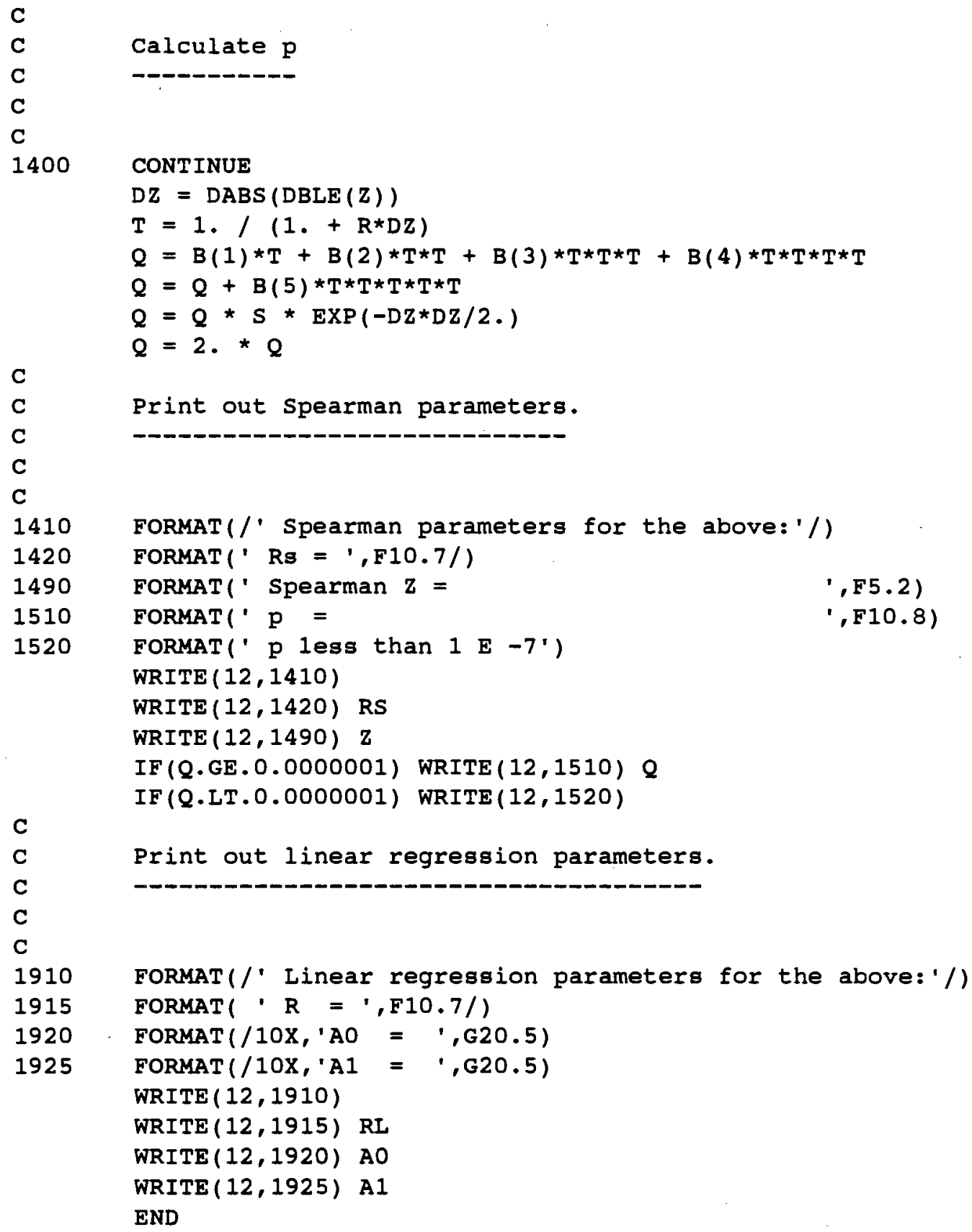




\section{Program MULTREG: Multiple Linear Regression.}

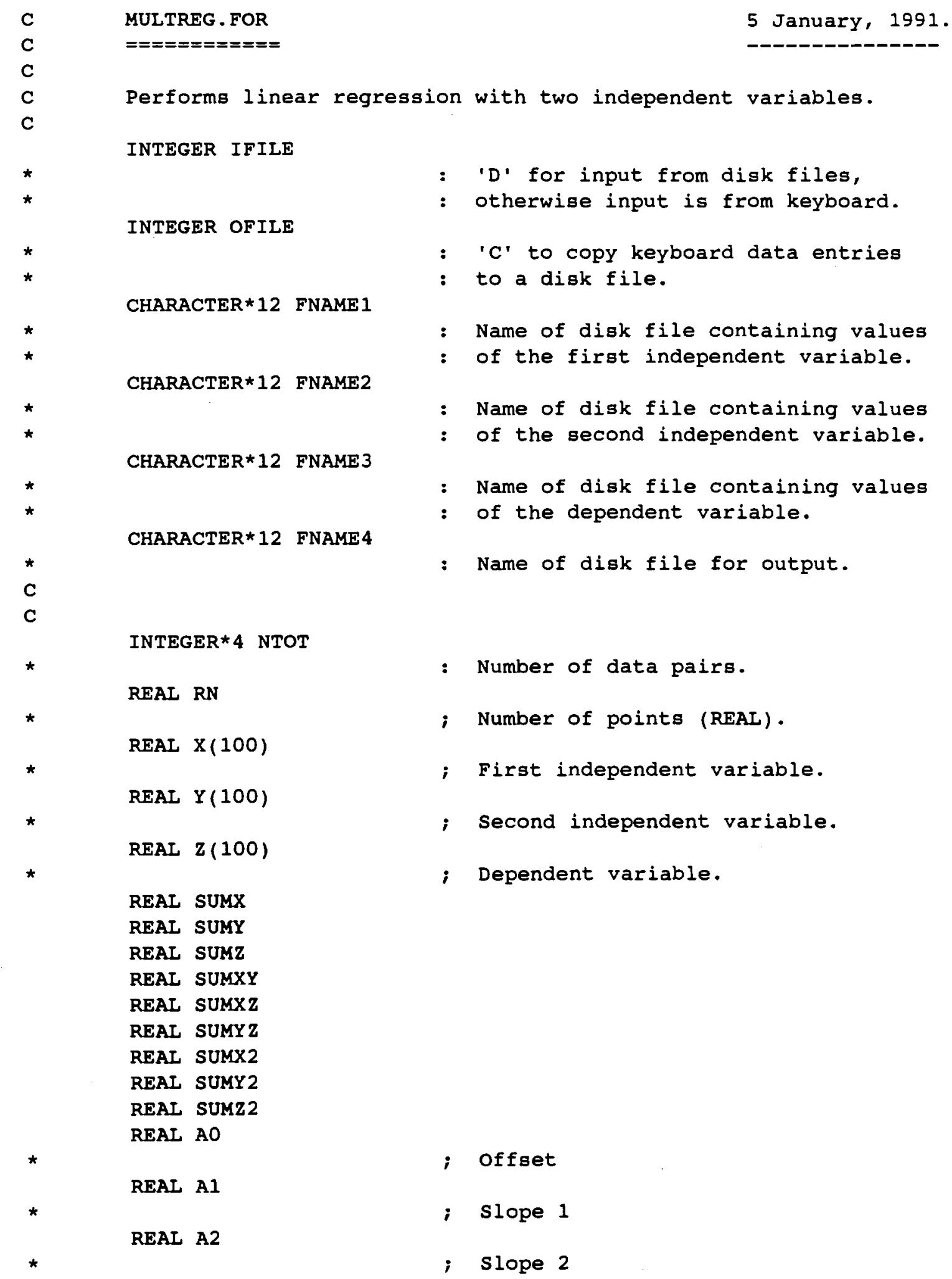


REAL VARX

REAL VARY

REAL VARZ

REAL VARXZ

REAL VARYZ

REAL VARXY

REAL RXZ

REAL RYZ

REAL RXY

REAL RMULT
; Variance of first indept variable.

- Variance of second indept variable.

- Variance of dependent variable.

- Covariance of dependent variable

; \& first indept variable.

; Covariance of dependent variable

; \& first indept variable.

i Covariance of independent variables.

; Correlation coefficient between

- dependent variable \& first

i independent variable.

; Correlation coefficient between dependent variable \& second

independent variable.

; Correlation coefficient between

; independent variables.

; Multiple linear regression coefficient.
C

C

C

Input data.

FORMAT(/' Input from [D]isk files or from [K] eyboard? ', ) FORMAT (/" Copy data onto disk? [C]Opy or [N]o copy ? ', FORMAT(//, Enter number of items in each data set: ', /) WRITE $(6,10)$

READ (*,' (A1)') IFILE

IF (IFILE.NE.'D'.AND. IFILE.NE.'d') THEN

IFILE $=$ ' $\mathrm{K}$ ' WRITE $(6,20)$

Establish file names.

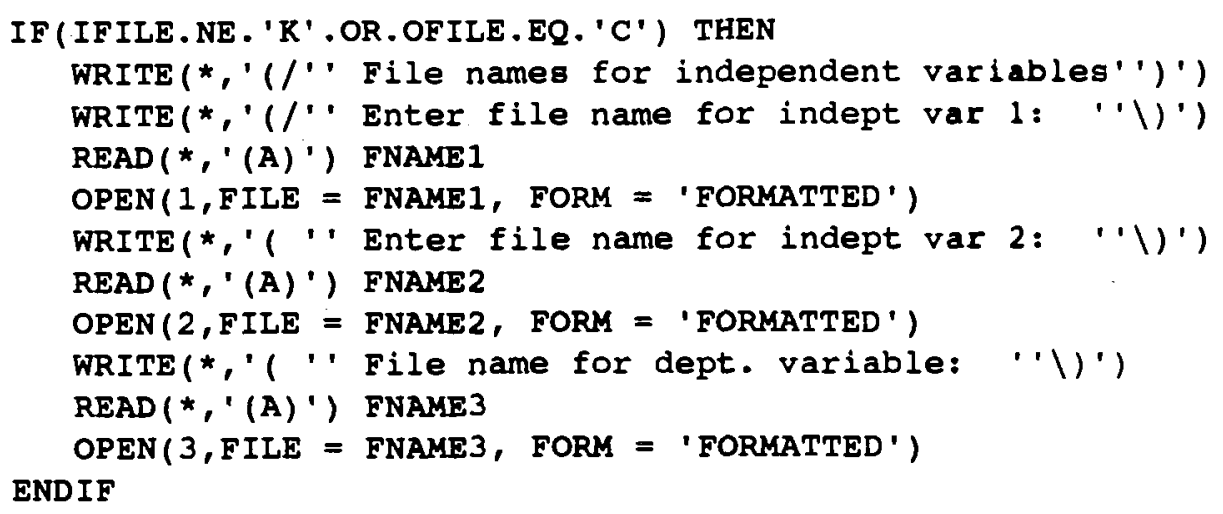




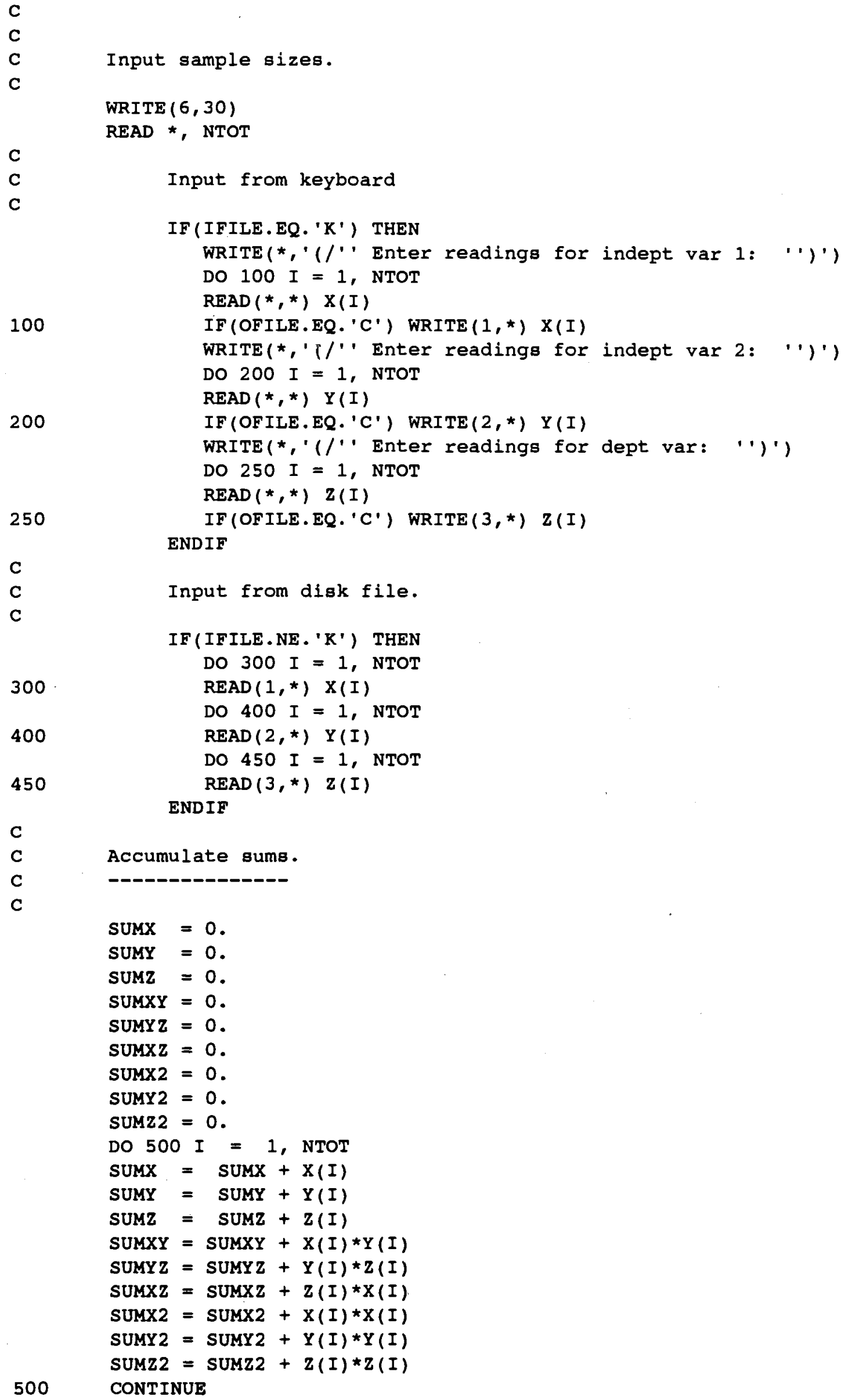


c

C

C

C

C

C

C

C

C

C

C

Compute regression coefficients.

$\mathrm{RN}=$ FLOAT (NTOT)

$A=(R N *$ SUMX $2-$ SUMX $*$ SUMX $) *(R N *$ SUMY Z -SUMY *SUMZ)

$B=(R N * S U M X Y-S U M X * S U M Y) *(R N * S U M X Z-S U M X * S U M Z)$

$A 2=A-B$

$A 2=A 2 /((R N *$ SUMX2-SUMX * SUMX)* $(R N *$ SUMY 2-SUMY *SUMY $)$

$-\quad-(R N * S U M X Y-S U M X * S U M Y) *(R N * S U M X Y-S U M X * S U M Y))$

$A 1=((R N * \operatorname{SUMXZ}-$ SUMX $*$ SUMZ $)-A 2 *(R N * \operatorname{SUMXY}-\mathrm{SUMX} *$ SUMY $))$

/ / $/(R N * \operatorname{SUMX} 2-\operatorname{SUMX} * \operatorname{SUMX})$

$A O=(1 / R N) *(S U M Z-A 2 *$ SUMY $-A 1 *$ SUMX $)$

Compute correlation coefficients.

$\operatorname{VARX}=(\operatorname{SUMX} 2-(\operatorname{SUMX} * \operatorname{SUMX} / \mathrm{RN})) /(\mathrm{RN}-1$.

VARY $=($ SUMY $2-($ SUMY *SUMY $/$ RN $)) /($ RN -1.$)$

VARZ $=($ SUMZ2 $-($ SUMZ $*$ SUMZ $/$ RN $)) /($ RN -1.$)$

VARXZ $=($ SUMXZ $-($ SUMX $*$ SUMZ RN $)) /($ RN -1.$)$

VARYZ $=($ SUMYZ $-($ SUMY*SUMZ /RN $)) /($ RN -1.$)$

VARXY $=($ SUMXY $-($ SUMX*SUMY $/ R N)) /(R N-1$.

$\operatorname{RXZ}=\operatorname{VARXZ} /(\operatorname{SQRT}(\operatorname{VARX}) * \operatorname{SQRT}(\operatorname{VARZ}))$

RYZ $=$ VARYZ / (SQRT (VARY)*SQRT $($ VARZ))

RXY $=$ VARXY / (SQRT (VARX)*SQRT (VARY))

RMULT $=\operatorname{SQRT}(A 1 * S Q R T($ VARX/VARZ) *RXZ + A2*SQRT $($ VARY/VARZ) *RYZ)

Print out input data.

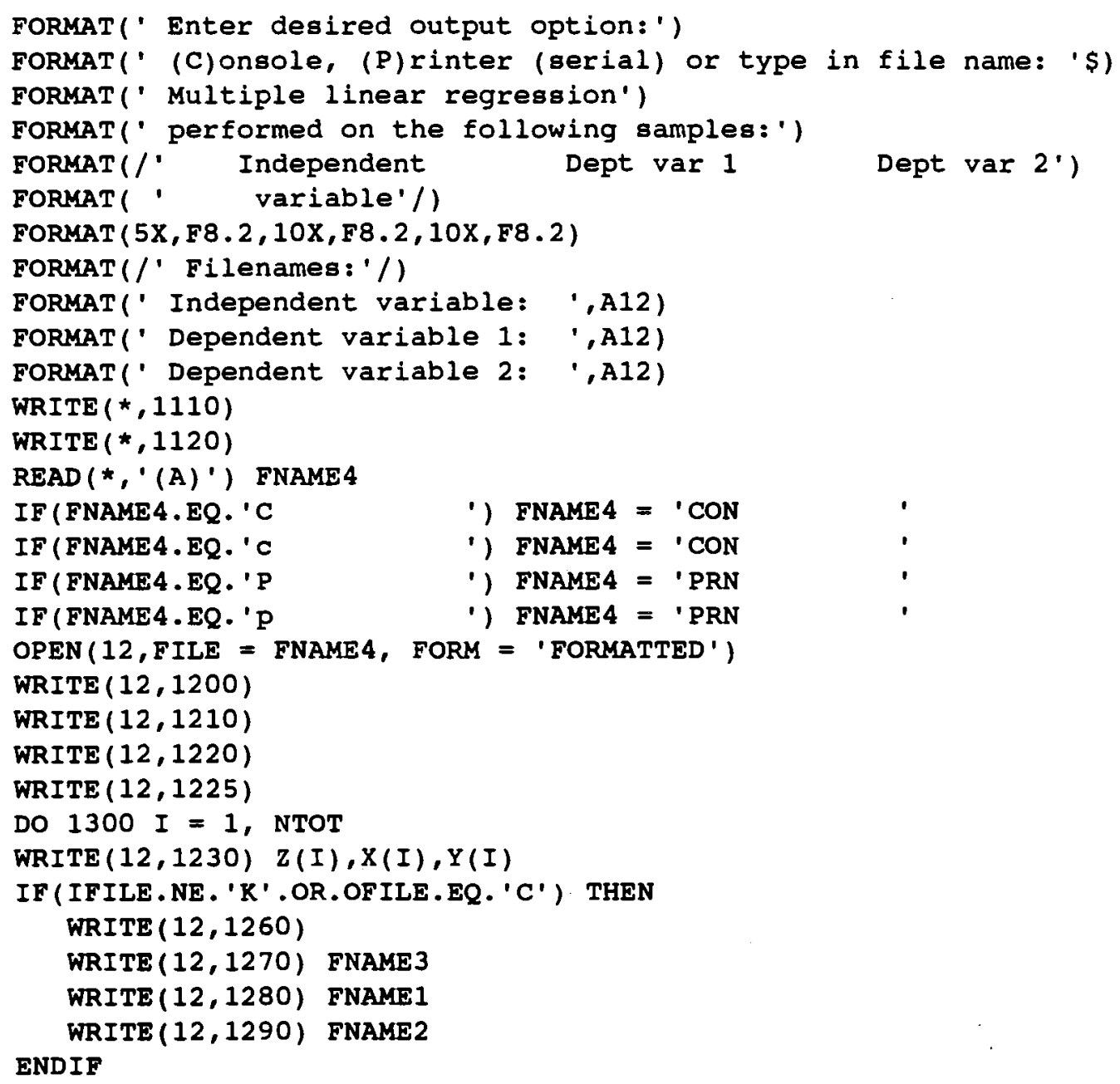




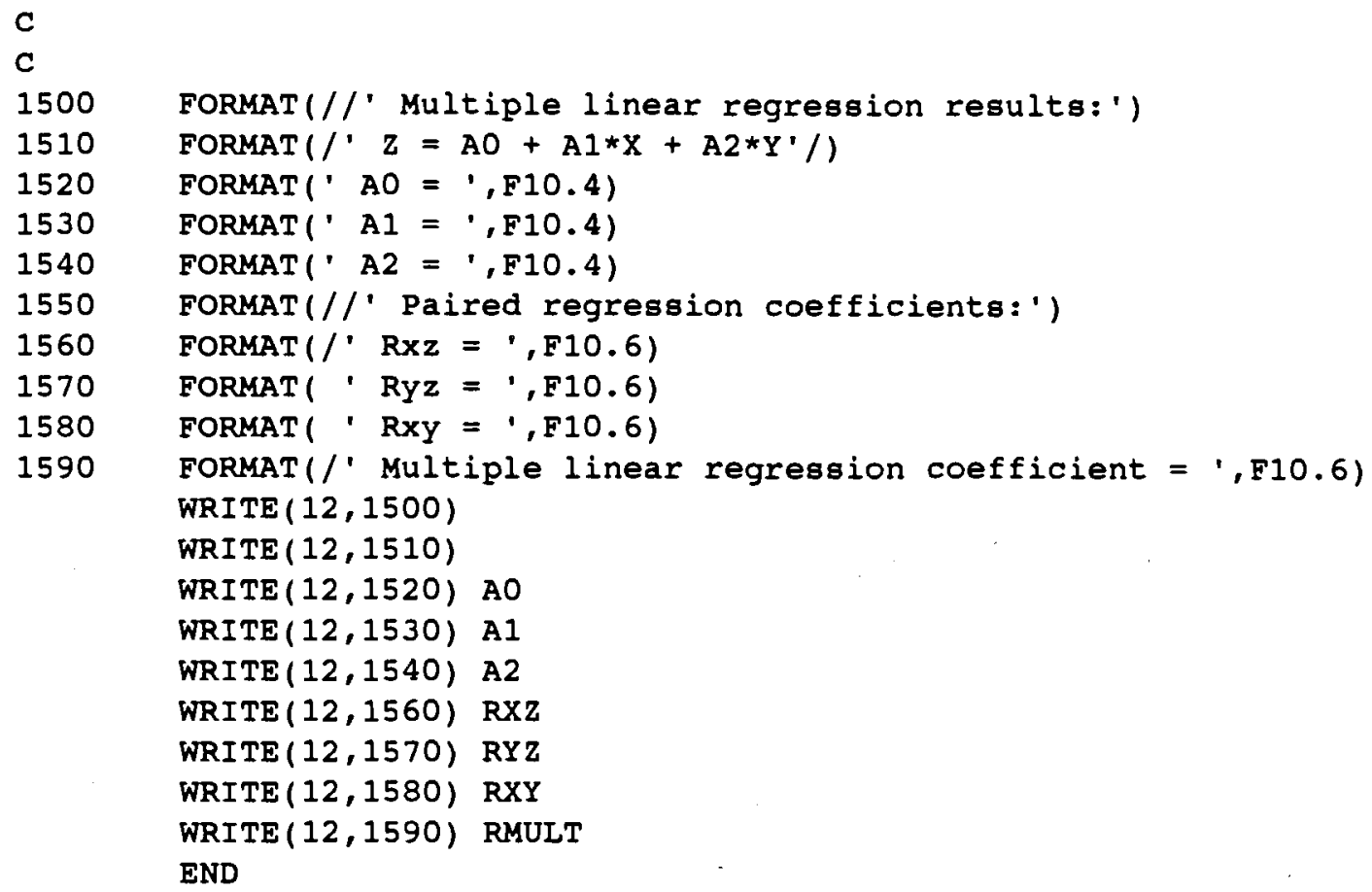




\section{APPENDIX J: Correlations of Bone Measurements with Clinical}

\section{Information.}

Table J.1: Clinical variables.

Table J.2: Statistics for controls.

Table J.3: Statistics for Anorexics.

Table J.4: Clinical Correlations for Controls. page 301

Table J.5: Clinical Correlations for Anorexics. page 302 page 298

page 299

page 300 
Table J.1: Clinical variables.

\begin{tabular}{|c|c|}
\hline $\begin{array}{l}\text { CB8O } \\
\text { CMIN } \\
\text { RHO }\end{array}$ & $\begin{array}{l}\text { Single energy measurement. Average for L2, L3 \& L4. } \\
\text { Dual energy mineral measurement. Average for L2, L3 \& L4. } \\
\text { Dual energy density measurement. Average for L2, L3 \& L4. }\end{array}$ \\
\hline AGE & Age at time of $\mathrm{x}$-ray examination. \\
\hline WGTX & Weight at $\mathrm{X}$-ray $(\mathrm{kg})$. \\
\hline HGTX & Height at $\mathrm{x}$-ray (cms). \\
\hline BMIX & Body Mass Index at X-ray (weight / height2). \\
\hline $\begin{array}{l}\text { PARITY } \\
\text { EHOSTEOP } \\
\text { ERACT }\end{array}$ & $\begin{array}{l}\text { Number of childbirths. } \\
\text { Family history of osteoporosis } 0=\text { no, } 1=\text { yes. } \\
\text { Number of bone fractures. }\end{array}$ \\
\hline $\begin{array}{l}\text { CAMGX } \\
\text { CACATX } \\
\text { CACATPX } \\
\text { CAHIADOL }\end{array}$ & $\begin{array}{l}\text { Daily calcium intake (mgms). } \\
\text { Daily calcium intake. } 0 \text { to } 3 \text { scale. } \\
\text { Time in months at current calcium intake category. } \\
\text { Number of months of dietary calcium above } 900 \mathrm{mg} / \text { day } \\
\text { between the ages of } 10 \& 20 \text {. }\end{array}$ \\
\hline $\begin{array}{l}\text { VITS } \\
\text { DURTOB } \\
\text { CAFFEINE } \\
\text { ALCNO } \\
\text { SALT }\end{array}$ & $\begin{array}{l}\text { Taking vitamin tablets. } 0=\text { no, } 1 \text { = yes. } \\
\text { Total? number of months of cigarette smoking? } \\
\text { Number of cups of coffee per day at x-ray? } \\
\text { Number of alcoholic drinks per day? }\end{array}$ \\
\hline $\begin{array}{l}\text { PILLT } \\
\text { PILLX } \\
\text { PILLPX }\end{array}$ & $\begin{array}{l}\text { Total number of months on estrogen contraceptive pill. } \\
\text { On estrogen pill at } x \text {-ray. } 0=\text { no, } 1=\text { yes. } \\
\text { Number of months on estrogen pill immediately before } x \text {-ray. }\end{array}$ \\
\hline $\begin{array}{l}\text { EXCATX } \\
\text { EXCATPX } \\
\text { EXSEVT }\end{array}$ & $\begin{array}{l}\text { Exercise category at } x \text {-ray. } 0 \text { to } 3 \text {. } \\
\text { Time in months on current exercise category. } \\
\text { Total number of months at exercise category } 2 \text { or more. }\end{array}$ \\
\hline $\begin{array}{l}\text { AGEMENAR } \\
\text { AMENT }\end{array}$ & $\begin{array}{l}\text { Age at which menstruation commenced (menarche). } \\
\text { Total number of months of amenorrhoea since menarche. }\end{array}$ \\
\hline $\begin{array}{l}\text { DURAN } \\
\text { AMENPX } \\
\text { MENSPX } \\
\text { ONSETD } \\
\text { ONSETA } \\
\text { ONSETG }\end{array}$ & $\begin{array}{l}\text { Total number of months of anorexia nervosa. } \\
\text { Number of months of amenorrhoea immediately prior to x-ray. } \\
\text { Number of months of menstruation immediately prior to x-ray. } \\
\text { Age of onset of dieting leading directly to anorexia nervosa. } \\
\text { Age at onset of amenorrhoea caused by anorexia nervoaa. } \\
\text { Years difference between AGEMENAR \& ONSETA. }\end{array}$ \\
\hline $\begin{array}{l}\text { DIARRH } \\
\text { VOMIT } \\
\text { BULPX } \\
\text { ANBULT }\end{array}$ & $\begin{array}{l}\text { Total months of diarrhoea. } \\
\text { Total months of vomiting. } \\
\text { Months of bulimia immediately prior to x-ray. } \\
\text { Total number of months of anorexia or bulimia. }\end{array}$ \\
\hline $\begin{array}{l}\text { BMI 16PX } \\
\text { BMI16T } \\
\text { BMI 18PX } \\
\text { BMI18T }\end{array}$ & $\begin{array}{l}\text { Number of months BMI } \leq 16 \text { prior to } \mathrm{X} \text {-ray. } \\
\text { Total number of months BMI } \leq 16 \text {. } \\
\text { Number of months BMI } \leq 18 \text { prior to } \mathrm{x} \text {-ray. } \\
\text { Total number of months BMI } \leq 18 \text {. }\end{array}$ \\
\hline $\begin{array}{l}\text { CALOWT } \\
\text { HOSPADM } \\
\text { PREDICT }\end{array}$ & $\begin{array}{l}\text { Total number of months for which calcium intake }<700 \mathrm{mg} / \mathrm{day} \\
\text { Number of hospital admissions for anorexia nervosa. } \\
\text { Investigators' prediction of low bone mass ( } 0 \text { to } 2 \text { sale). }\end{array}$ \\
\hline
\end{tabular}


Table J.2: Statistics for Controls.

\begin{tabular}{|c|c|c|c|c|c|c|}
\hline & Mean & S.D. & Skew & Min & Max & Median \\
\hline CB80 & 148.5 & 24.0 & 0.4 & 113.4 & 206.3 & 144.9 \\
\hline CMIN & 196.2 & 28.0 & 1.0 & 151 & 290 & 199.3 \\
\hline RHO & 1.092 & 0.041 & 0.3 & 1.003 & 1.196 & 1.088 \\
\hline AGE & 30.9 & 5.8 & -0.1 & 20 & 40 & 32 \\
\hline WGTX & 63.0 & -10.5 & 0.8 & 47.0 & 90.0 & 61.0 \\
\hline HGTX & 165.5 & 6.9 & 0.6 & 155 & 183 & 165 \\
\hline BMIX & 22.8 & 3.0 & 1.1 & 18.5 & 33.0 & 22.7 \\
\hline PARITY & 1.3 & 1.4 & 0.3 & 0 & 4 & 1 \\
\hline FHOSTEOP & 0.1 & 0.3 & 2.2 & 0 & 1 & $\overline{0}$ \\
\hline FRACT & 0.5 & 1.0 & 1.5 & 0 & 3 & 0 \\
\hline CAMGX & 669.4 & 253.8 & 0.0 & 242 & 1071 & 701 \\
\hline CACATX & 1.5 & 1.1 & 0.0 & 0 & 3 & 2 \\
\hline CAHIADOL & 43.5 & 52.3 & 0.5 & 0 & 120 & 0 \\
\hline VITS & 0.2 & 0.4 & 1.8 & 0 & 1 & 0 \\
\hline DURTOB & 38.1 & 72.2 & 2.0 & 0 & 264 & 0 \\
\hline CAFFEINE & 5.5 & 3.8 & 1.8 & 0 & 20 & 4 \\
\hline ALCNO & 0.4 & 0.6 & 1.2 & 0 & 2 & 0 \\
\hline SALT & 1.8 & 0.9 & 0.4 & 1 & 3 & 2 \\
\hline PILLT & 30.3 & 40.2 & 1.3 & 0 & 132 & 6 \\
\hline PILLX & 0.2 & 0.4 & 1.8 & 0 & 1 & 0 \\
\hline PILLPX & 3.4 & 9.5 & 2.8 & 0 & 36 & 0 \\
\hline EXCATX & 1.1 & 0.7 & 0.6 & 0 & 3 & 1 \\
\hline EXSEVT & 40.6 & 47.3 & 0.7 & 0 & 156 & 12 \\
\hline AGEMENAR & 12.6 & 1.6 & 1.3 & 11 & 18 & 12 \\
\hline AMENT & 1.6 & 5.1 & 3.6 & 0 & 24 & 0 \\
\hline
\end{tabular}


Table J.3: Statistics for Anorexics.

\begin{tabular}{|c|c|c|c|c|c|c|}
\hline & Mean & S.D. & Skew & Min & Max & Median \\
\hline $\begin{array}{l}\text { CB80 } \\
\text { CMIN } \\
\text { RHO }\end{array}$ & $\begin{array}{l}121.4 \\
159.3 \\
1.085\end{array}$ & $\begin{array}{l}25.8 \\
38.1 \\
0.038\end{array}$ & $\begin{array}{r}-0.3 \\
0.0 \\
-0.4\end{array}$ & $\begin{array}{c}53 \\
48 \\
0.95\end{array}$ & $\begin{array}{l}174 \\
246 \\
1.196\end{array}$ & $\begin{array}{l}123 \\
159 \\
1.092\end{array}$ \\
\hline $\begin{array}{l}\text { AGE } \\
\text { WGTX } \\
\text { HGTX } \\
\text { BMIX }\end{array}$ & $\begin{array}{r}27.5 \\
51.7 \\
164.2 \\
19.2\end{array}$ & $\begin{array}{l}5.8 \\
9.1 \\
6.3 \\
3.1\end{array}$ & $\begin{array}{l}0.6 \\
0.3 \\
0.1 \\
0.1\end{array}$ & $\begin{array}{l}20 \\
34.8 \\
149 \\
13.0\end{array}$ & $\begin{array}{l}40 \\
72.5 \\
178 \\
26.7\end{array}$ & $\begin{array}{c}27 \\
51.7 \\
164 \\
19.2\end{array}$ \\
\hline $\begin{array}{l}\text { PARITY } \\
\text { FHOSTEOP } \\
\text { FRACT }\end{array}$ & $\begin{array}{l}0.4 \\
0.1 \\
0.3\end{array}$ & $\begin{array}{l}0.9 \\
0.3 \\
0.7\end{array}$ & $\begin{array}{l}2.3 \\
2.9 \\
2.3\end{array}$ & $\begin{array}{l}0 \\
0 \\
0\end{array}$ & $\begin{array}{l}4 \\
1 \\
3\end{array}$ & $\begin{array}{l}0 \\
0 \\
0\end{array}$ \\
\hline $\begin{array}{l}\text { CAMGX } \\
\text { CACATX } \\
\text { CAHIADOL }\end{array}$ & $\begin{array}{r}640.1 \\
1.3 \\
32.6\end{array}$ & $\begin{array}{r}326.7 \\
1.1 \\
41.9\end{array}$ & $\begin{array}{l}0.5 \\
0.2 \\
0.8\end{array}$ & $\begin{array}{r}63 \\
0 \\
0\end{array}$ & $\begin{array}{r}1600 \\
3 \\
120\end{array}$ & $\begin{array}{r}650 \\
1 \\
0\end{array}$ \\
\hline $\begin{array}{l}\text { VITS } \\
\text { DURTOB } \\
\text { CAFFEINE } \\
\text { ALCNO } \\
\text { SALT }\end{array}$ & $\begin{array}{r}0.4 \\
56.3 \\
4.9 \\
0.4 \\
1.4\end{array}$ & $\begin{array}{r}0.6 \\
72.9 \\
3.3 \\
0.9 \\
0.6\end{array}$ & $\begin{array}{l}1.0 \\
1.3 \\
0.3 \\
2.2 \\
1.4\end{array}$ & $\begin{array}{l}0 \\
0 \\
0 \\
0 \\
1\end{array}$ & $\begin{array}{r}2 \\
300 \\
13 \\
4 \\
3\end{array}$ & $\begin{array}{r}0 \\
24 \\
5 \\
0 \\
1\end{array}$ \\
\hline $\begin{array}{l}\text { PILLT } \\
\text { PILLX } \\
\text { PILLPX }\end{array}$ & $\begin{array}{r}21.0 \\
0.2 \\
7.6\end{array}$ & $\begin{array}{r}32.4 \\
0.4 \\
20.2\end{array}$ & $\begin{array}{l}1.7 \\
1.8 \\
2.7\end{array}$ & $\begin{array}{l}0 \\
0 \\
0\end{array}$ & $\begin{array}{r}132 \\
1 \\
84\end{array}$ & $\begin{array}{l}6 \\
0 \\
0\end{array}$ \\
\hline $\begin{array}{l}\text { EXCATX } \\
\text { EXSEVT }\end{array}$ & $\begin{array}{r}1.4 \\
68.1\end{array}$ & $\begin{array}{r}0.8 \\
64.9\end{array}$ & $\begin{array}{l}0.3 \\
1.1\end{array}$ & $\begin{array}{l}0 \\
0\end{array}$ & $\begin{array}{r}3 \\
240\end{array}$ & $\begin{array}{r}1 \\
48\end{array}$ \\
\hline $\begin{array}{l}\text { AGEMENAR } \\
\text { AMENT }\end{array}$ & $\begin{array}{l}13.3 \\
51.2\end{array}$ & $\begin{array}{r}1.8 \\
52.2\end{array}$ & $\begin{array}{l}1.3 \\
1.5\end{array}$ & $\begin{array}{r}11 \\
0\end{array}$ & $\begin{array}{r}20 \\
228\end{array}$ & $\begin{array}{l}13 \\
36\end{array}$ \\
\hline $\begin{array}{l}\text { DURAN } \\
\text { AMENPX } \\
\text { MENSPX } \\
\text { ONSETG } \\
\text { ONSETA } \\
\text { ONSETD }\end{array}$ & $\begin{array}{r}58.0 \\
22.8 \\
37.5 \\
5.6 \\
18.8 \\
18.3\end{array}$ & $\begin{array}{r}52.5 \\
47.3 \\
54.8 \\
4.5 \\
4.6 \\
4.3\end{array}$ & $\begin{array}{l}1.7 \\
2.8 \\
1.7 \\
1.0 \\
1.0 \\
0.9\end{array}$ & $\begin{array}{r}12 \\
0 \\
0 \\
0 \\
12 \\
12\end{array}$ & $\begin{array}{r}228 \\
228 \\
264 \\
17 \\
31 \\
31\end{array}$ & $\begin{array}{r}36 \\
0 \\
1 \\
5 \\
17 \\
17\end{array}$ \\
\hline $\begin{array}{l}\text { DIARRH } \\
\text { VOMIT } \\
\text { BULPX } \\
\text { ANBULT }\end{array}$ & $\begin{array}{l}25.5 \\
20.1 \\
20.7 \\
78.9\end{array}$ & $\begin{array}{l}49.3 \\
40.8 \\
43.9 \\
59.2\end{array}$ & $\begin{array}{l}2.9 \\
3.1 \\
2.4 \\
1.0\end{array}$ & $\begin{array}{r}0 \\
0 \\
0 \\
12\end{array}$ & $\begin{array}{l}276 \\
228 \\
192 \\
228\end{array}$ & $\begin{array}{r}0 \\
0 \\
0 \\
60\end{array}$ \\
\hline $\begin{array}{l}\text { BMI 16PX } \\
\text { BMI 16T }\end{array}$ & $\begin{array}{r}9.5 \\
26.6\end{array}$ & $\begin{array}{l}27.7 \\
44.4\end{array}$ & $\begin{array}{l}3.3 \\
2.8\end{array}$ & $\begin{array}{l}0 \\
0\end{array}$ & $\begin{array}{l}144 \\
216\end{array}$ & $\begin{array}{l}0 \\
9\end{array}$ \\
\hline $\begin{array}{l}\text { CALOWT } \\
\text { HOSPADM } \\
\text { PREDICT }\end{array}$ & $\begin{array}{r}86.8 \\
1.2 \\
1.0\end{array}$ & $\begin{array}{r}72.3 \\
2.1 \\
0.9\end{array}$ & $\begin{array}{r}0.9 \\
3.4 \\
-\quad 0.1\end{array}$ & $\begin{array}{l}0 \\
0 \\
0\end{array}$ & $\begin{array}{r}312 \\
12 \\
2\end{array}$ & $\begin{array}{r}72 \\
1 \\
1\end{array}$ \\
\hline
\end{tabular}


Table J.4: Clinical Correlations for Controls.

\begin{tabular}{|c|c|c|c|c|c|c|}
\hline \multirow{2}{*}{$\begin{array}{l}\text { Clinical } \\
\text { variable }\end{array}$} & \multicolumn{2}{|c|}{$\mathrm{Cb} 80$} & \multicolumn{2}{|c|}{ Cmin } & \multicolumn{2}{|c|}{ RHO } \\
\hline & $r_{\mathbf{S}}$ & $\mathrm{p}$ & $r_{\mathbf{S}}$ & $\mathrm{p}$ & $r_{s}$ & $\mathrm{p}$ \\
\hline WGTX & 0.06 & 0.76 & -0.11 & 0.53 & 0.21 & 0.25 \\
\hline HGTX & -0.35 & 0.05 & -0.21 & 0.25 & -0.23 & 0.22 \\
\hline BMIX & 0.33 & 0.07 & 0.06 & 0.73 & 0.40 & 0.03 \\
\hline PARITY & -0.12 & 0.53 & 0.03 & 0.89 & -0.11 & 0.56 \\
\hline FHOSTEOP & 0.02 & 0.92 & 0.25 & 0.17 & -0.16 & 0.38 \\
\hline FRACT & -0.31 & 0.09 & 0.00 & 0.99 & -0.37 & 0.04 \\
\hline VITS & -0.11 & 0.56 & 0.04 & 0.81 & -0.14 & 0.44 \\
\hline DURTOB & -0.06 & 0.75 & 0.16 & 0.37 & -0.19 & 0.29 \\
\hline CAFFEINE & -0.03 & 0.87 & -0.20 & 0.26 & 0.19 & 0.30 \\
\hline ALCNO & -0.23 & 0.21 & -0.04 & 0.83 & -0.23 & 0.21 \\
\hline SALT & -0.12 & 0.50 & -0.11 & 0.56 & -0.14 & 0.45 \\
\hline PILLT & -0.04 & 0.83 & -0.04 & 0.84 & 0.05 & 0.79 \\
\hline PILLX & -0.05 & 0.77 & 0.03 & 0.87 & -0.03 & 0.87 \\
\hline PILLPX & -0.06 & 0.75 & 0.01 & 0.96 & -0.02 & 0.91 \\
\hline EXCATX & 0.25 & 0.16 & 0.21 & 0.26 & 0.12 & 0.48 \\
\hline EXSEVT & 0.11 & 0.54 & 0.01 & 0.94 & 0.22 & 0.24 \\
\hline CAMGX & -0.14 & 0.46 & -0.33 & 0.07 & 0.12 & 0.52 \\
\hline CACATX & -0.11 & 0.54 & -0.28 & 0.13 & 0.14 & 0.46 \\
\hline CAHIADOL & -0.13 & 0.48 & -0.08 & 0.68 & -0.03 & 0.89 \\
\hline $\begin{array}{l}\text { AGEMENAR } \\
\text { AMENT }\end{array}$ & $\begin{aligned}- & 0.06 \\
& 0.0\end{aligned}$ & $\begin{array}{l}0.72 \\
1.00\end{array}$ & $\begin{array}{l}0.22 \\
0.35\end{array}$ & $\begin{array}{l}0.24 \\
0.06\end{array}$ & $\begin{array}{l}-0.29 \\
-0.35\end{array}$ & $\begin{array}{l}0.12 \\
0.06\end{array}$ \\
\hline
\end{tabular}


Table J.5: Clinical Correlations for Anorexics.

\begin{tabular}{|c|c|c|c|c|c|c|}
\hline \multirow{2}{*}{$\begin{array}{l}\text { Clinical } \\
\text { variable }\end{array}$} & \multicolumn{2}{|c|}{$\mathrm{Cb} 80$} & \multicolumn{2}{|c|}{ Cmin } & \multicolumn{2}{|c|}{ RHO } \\
\hline & $r_{s}$ & $p$ & $r_{s}$ & $p$ & $r_{s}$ & $p$ \\
\hline $\begin{array}{l}\text { WGTX } \\
\text { HGTX } \\
\text { BMIX }\end{array}$ & $\begin{array}{r}0.29 \\
-\quad 0.12 \\
0.35\end{array}$ & $\begin{array}{l}0.02 \\
0.32 \\
0.004\end{array}$ & $\begin{array}{r}0.02 \\
0.01 \\
-0.02\end{array}$ & $\begin{array}{l}0.90 \\
0.92 \\
0.90\end{array}$ & $\begin{array}{r}0.43 \\
-0.15 \\
0.51\end{array}$ & $\begin{array}{ll}4 & E-4 \\
0.23 \\
2 & E-5\end{array}$ \\
\hline $\begin{array}{l}\text { PARITY } \\
\text { FHOSTEOP } \\
\text { FRACT }\end{array}$ & $\begin{array}{l}0.23 \\
0.10 \\
0.03\end{array}$ & $\begin{array}{l}0.06 \\
0.40 \\
0.79\end{array}$ & $\begin{array}{l}0.23 \\
0.21 \\
0.03\end{array}$ & $\begin{array}{l}0.06 \\
0.08 \\
0.83\end{array}$ & $\begin{array}{l}-0.04 \\
-0.16 \\
-0.08\end{array}$ & $\begin{array}{l}0.72 \\
0.18 \\
0.49\end{array}$ \\
\hline $\begin{array}{l}\text { VITS } \\
\text { DURTOB } \\
\text { CAFFEINE } \\
\text { ALCNO } \\
\text { SALT }\end{array}$ & $\begin{array}{r}0.07 \\
-\quad 0.04 \\
-0.11 \\
0.02 \\
0.19\end{array}$ & $\begin{array}{l}0.55 \\
0.74 \\
0.36 \\
0.90 \\
0.12\end{array}$ & $\begin{array}{r}0.08 \\
-0.01 \\
-0.06 \\
0.07 \\
0.11\end{array}$ & $\begin{array}{l}0.50 \\
0.95 \\
0.60 \\
0.56 \\
0.37\end{array}$ & $\begin{array}{r}-0.07 \\
-0.03 \\
-0.16 \\
0.09 \\
0.04\end{array}$ & $\begin{array}{l}0.57 \\
0.82 \\
0.18 \\
0.53 \\
0.73\end{array}$ \\
\hline $\begin{array}{l}\text { PILLT } \\
\text { PILLX } \\
\text { PILLPX }\end{array}$ & $\begin{array}{r}-0.01 \\
0.14 \\
0.13\end{array}$ & $\begin{array}{l}0.95 \\
0.25 \\
0.27\end{array}$ & $\begin{array}{r}-0.09 \\
0.11 \\
0.10\end{array}$ & $\begin{array}{l}0.46 \\
0.37 \\
0.42\end{array}$ & $\begin{array}{l}0.06 \\
0.01 \\
0.02\end{array}$ & $\begin{array}{l}0.62 \\
0.92 \\
0.90\end{array}$ \\
\hline $\begin{array}{l}\text { EXCATX } \\
\text { EXSEVT }\end{array}$ & $\begin{array}{r}0.08 \\
-\quad 0.15\end{array}$ & $\begin{array}{l}0.51 \\
0.23\end{array}$ & $\begin{array}{r}0.25 \\
-0.06\end{array}$ & $\begin{array}{l}0.04 \\
0.65\end{array}$ & $\begin{array}{l}-0.22 \\
-0.12\end{array}$ & $\begin{array}{l}0.07 \\
0.31\end{array}$ \\
\hline $\begin{array}{l}\text { CAMGX } \\
\text { CACATX } \\
\text { CAHIADOL }\end{array}$ & $\begin{array}{l}0.14 \\
0.17 \\
0.25\end{array}$ & $\begin{array}{l}0.25 \\
0.18 \\
0.04\end{array}$ & $\begin{array}{l}0.03 \\
0.10 \\
0.23\end{array}$ & $\begin{array}{l}0.78 \\
0.42 \\
0.05\end{array}$ & $\begin{array}{r}0.04 \\
-\quad 0.04 \\
0.01\end{array}$ & $\begin{array}{l}0.76 \\
0.72 \\
0.96\end{array}$ \\
\hline $\begin{array}{l}\text { AGEMENAR } \\
\text { AMENT }\end{array}$ & $\begin{array}{l}-0.09 \\
-0.36\end{array}$ & $\begin{array}{l}0.45 \\
0.003\end{array}$ & $\begin{array}{r}0.01 \\
-\quad 0.15\end{array}$ & $\begin{array}{l}0.94 \\
0.23\end{array}$ & $\begin{array}{l}-0.20 \\
-0.36\end{array}$ & $\begin{array}{l}0.10 \\
0.003\end{array}$ \\
\hline $\begin{array}{l}\text { DURAN } \\
\text { AMENPX } \\
\text { MENSPX } \\
\text { ONSETG } \\
\text { ONSETA } \\
\text { ONSETD }\end{array}$ & $\begin{array}{r}-0.45 \\
-0.24 \\
-0.32 \\
-0.05 \\
-0.04 \\
-0.10\end{array}$ & $\begin{array}{l}2 \quad E-4 \\
0.05 \\
0.01 \\
0.67 \\
0.74 \\
0.43\end{array}$ & $\begin{array}{r}-0.13 \\
0.09 \\
0.12 \\
0.09 \\
0.15 \\
0.12\end{array}$ & $\begin{array}{l}0.28 \\
0.46 \\
0.34 \\
0.44 \\
0.22 \\
0.32\end{array}$ & $\begin{array}{l}-0.48 \\
-0.42 \\
-0.26 \\
-0.15 \\
-0.24 \\
-0.29\end{array}$ & $\begin{array}{ll}8 & E-5 \\
5 & E-4 \\
0.04 \\
0.22 \\
0.05 \\
0.02\end{array}$ \\
\hline $\begin{array}{l}\text { DIARRH } \\
\text { VOMIT } \\
\text { BULPX } \\
\text { ANBULT }\end{array}$ & $\begin{array}{r}0.15 \\
0.14 \\
0.12 \\
-\quad 0.40\end{array}$ & $\begin{array}{l}0.22 \\
0.26 \\
0.34 \\
0.001\end{array}$ & $\begin{array}{r}0.07 \\
0.06 \\
-0.03 \\
-0.26\end{array}$ & $\begin{array}{l}0.52 \\
0.62 \\
0.81 \\
0.03\end{array}$ & $\begin{array}{r}0.02 \\
0.09 \\
0.13 \\
-0.25\end{array}$ & $\begin{array}{l}0.87 \\
0.46 \\
0.27 \\
0.04\end{array}$ \\
\hline $\begin{array}{l}\text { BMI 16PX } \\
\text { BMI 16T } \\
\text { BMI 18PX } \\
\text { BMI18T }\end{array}$ & $\begin{array}{l}-0.03 \\
-0.29 \\
-0.17 \\
-0.30\end{array}$ & $\begin{array}{l}0.81 \\
0.02 \\
0.16 \\
0.01\end{array}$ & $\begin{array}{r}0.24 \\
-\quad 0.05 \\
0.11 \\
-0.07\end{array}$ & $\begin{array}{l}0.05 \\
0.68 \\
0.38 \\
0.59\end{array}$ & $\begin{array}{l}-0.32 \\
-0.29 \\
-0.39 \\
-0.31\end{array}$ & $\begin{array}{l}0.01 \\
0.02 \\
0.002 \\
0.01\end{array}$ \\
\hline $\begin{array}{l}\text { CALOWT } \\
\text { HOSPADM } \\
\text { PREDICT }\end{array}$ & $\begin{array}{r}-0.10 \\
-0.07 \\
0.47\end{array}$ & $\begin{array}{l}0.39 \\
0.59 \\
1 \quad E-4\end{array}$ & $\begin{aligned} &- 0.02 \\
& 0.0 \\
& 0.21\end{aligned}$ & $\begin{array}{l}0.85 \\
0.99 \\
0.09\end{array}$ & $\begin{array}{r}-0.11 \\
-0.12 \\
0.39\end{array}$ & $\begin{array}{l}0.36 \\
0.31 \\
0.002\end{array}$ \\
\hline
\end{tabular}




\section{APPENDIX K: Program PIXPLOT.}

Program to plot histograms of pixel values from C.T. images. The program was written in Christchurch by Trevor Knopp [202] as part of a project whose aim was to determine the proportion of fat in live sheep by C.T. scanning. A diagram illustrating the structure of the program is given overleaf. Modifications made for application in Wellington include removal of the section which does the fat assay, modification of routines NPLOT, AXES and ANNOT. A new routine STATS has been added to compute the same parameters as program DIST (see appendix I). 


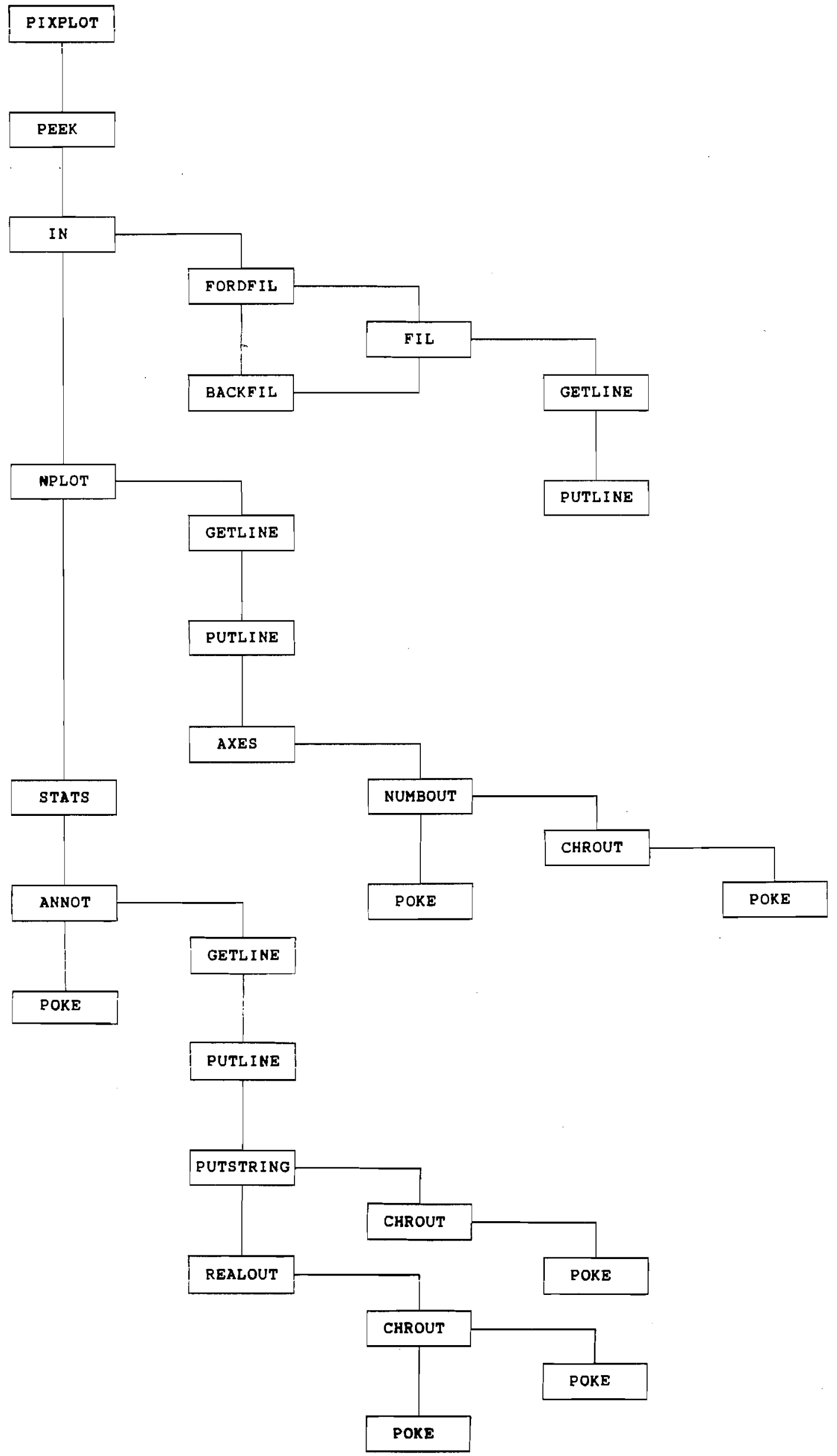




\section{REFERENCES}

1. Ziedses des Plantes, B.G., 1931.

Een bijzondere methode voor het maken van rontgenfoto's van schedel en wervelkolom. N.T.V.G., 75: 5218 .

2. Villafana, T., 1983.

Physics and Instrumentation, chapter 1 in

Cranial Computed Tomography Ed. Lee S.H. \& Rao K.

McGraw-Hill, New York.

3. Curry, T.S., Dowdey, J.E., Murry, R.C., 1984.

Christensen's Introduction to the Physics of Diagnostic Radiology.

Lea \& Febiger, Philadelphia.

4. Radon, J., 1917.

On the Determination of Functions From Their Integrals Along Certain Manifolds.

Ber. Sachs. Akad. Wiss., 69: $262-277$.

5. Bracewell, R.N., 1956.

Strip Integration in Radioastronomy.

Aust. J. Phys., 9: $198-217$.

6. Cormack, A.M., 1963.

Representation of a Function by its Line Integrals, with some Radiological Applications. J. Appl. Phys., 34: 2722.

7. Kuhl, D.E., Edwards, R.Q., 1963.

Image Separation Radioisotope Scanning.

Radiology, 80: 653-661.

8. Hounsfield, G.N., 1973.

Computerized Transverse Axial Scanning (Tomography): Part 1. Description of System.

British Journal of Radiology, 46: 1016 - 1022.

9. Wijdeveld, B., 1979.

Geometrical Enlargement in C.T. Scanning.

Medicamundi, 24: 41 - 44.

10. Marshall, C. (Editor), 1982.

The Physical Basis of Computed Tomography.

Warren H. Green, St. Louis.

11. Ruegsegger, P., Elsasser, U., Anliker, M. et al, 1976.

Quantification of Bone Mineralization Using Computed Tomography.

Radiology, 121: $93-97$.

12. Ter-Pogossian, M.M., 1983.

Physical Principles and Instrumentation, chapter 1 in

Computed Body Tomography, edited by Lee, J.K.T., Sagel, S.S. and Stanley, R.J.

Raven Press, New York.

13. McCullough, E.C., Baker, H.L., Houser, O.W. et al, 1974.

An Evaluation of the Quantitative and Radiation Features of a Scanning X-ray Transverse Axial Tomograph: The E.M.I. Scanner.

Radiology, 111: $709-715$.

14. Davison, M., 1982.

$X$-ray Computed Tomography, chapter 2 in

Scientific Basis of Medical Imaging, edited by P.N.T. Wells.

Churchill Livingstone, Edinburgh.

15. Brooks, R.A., Di Chiro, G., 1976.

Principles of Computer Assisted Tomography (CAT) in Radiographic and Radioisotopic Imaging. Phys. Med. Biol., 21: $689-732$.

16. McCullough, E.C., 1975.

Photon Attenuation in Computed Tomography.

Medical Physics, 2: $307-320$.

17. Joseph, P.M., Spital, R.D., 1978.

A Method for Correcting Bone Induced Artifacts in Computed Tomography Scanners.

J. Comput. Assist. Tomogr., 2: $100-108$.

18. Brooks, R.A., Di Chiro, G., 1976.

Beam Hardening in X-ray Reconstructive Tomography.

Phys. Med. Biol., 21: 390 - 398. 
19. McDavid, W.D., Waggener, R.G., Payne, W.H. et al, 1977.

Correction for Spectral Artefacts in Cross-sectional Reconstruction from X-rays.

Medical Physics, 4: $54-57$.

20. Coleman, A.J., Sinclair, M., 1985.

A Beam-hardening Correction using Dual-Energy Computed Tomography.

Phys. Med. Biol., 30: 1251 - 1256.

21. Stonestrom, J.P., Alvarez, R.E., Macovski, A., 1981.

A Framework for Spectral Artifact Corrections in X-ray C.T.

IEEE Trans. Biomed. Eng., BME-28: 128 - 141.

22. Goitein, M., 1972.

Three Dimensional Density Reconstruction from a Series of Two Dimensional Projections.

Nud. Instrum. Meth., 101: $509-518$.

23. Gilbert, P.F.C., 1972

Iterative Methods for the Three-dimensional Reconstruction of an Object from Projections.

J. Theor. Biol., 36: 105 - 117.

24. Gordon, R., Bender, R., Herman, G.T., 1970.

Algebraic Reconstruction Techniques (ART) for Three dimensional Electron Microscopy and X-ray Photography.

J. Theor. Biol., 29: $471-481$.

25. Kuhl, D.E., Edwards, R.Q., 1968.

Reorganising Data from Transverse Section Scans of the Brain using Digital Processing.

Radiology, 91: 975 - 983 .

26. Macovski, A., 1983.

Medical Imaging Systems.

Prentice-Hall, Englewood Cliffs, New Jersey.

27. Ramachandran, G.N., Lakshminarayanan, A.V., 1971.

Three-dimensional Reconstruction from Radiographs and Electron Micrographs: Applications of Convolutions instead of Fourier Transforms.

Proc. Nat. Acad. Sci. U.S.A., 68: 2236 - 2240.

28. Shepp, L.A., Logan, B.F., 1974.

Reconstruction of Interior Head Tissue from X-ray Transmissions.

I.E.E.E. Trans. Nucl. Sci., 21: $228-236$.

29. Bracewell, R.N., 1965.

The Fourier Transform and its Application.

McGraw-Hill, New York.

30. Mitchell, A.W., Zyskowski, A.L., 1988.

Image Processing Software for the G.E. 8800 Scanner.

Radiology Departmental Report.

31. Leksell, L., Jemberg, B., 1980.

Stereotaxis and Tomography: A Technical Note.

Acta Neurologica, 52: $1-7$.

32. Dettori, P., Colombo, F., Pinna, V. et al., 1982.

C.T. Control of Stereotactic Surgery in the Diencephalon.

Neuroradiology, 23: $91-94$

33. Mitchell, A.W., Martin, G.

Stereotactic Needle Biopsy using Computed Tomography.

Presented to N.Z.I.P. Conference, May 1983.

34. Arimitsu, T., Di Chiro, G., Brooks, R.A. et al, 1977.

White-Gray Matter Differentiation in Computed Tomography.

J. Comput. Assist. Tomogr., 1: 437 - 442.

35. Di Chiro, G., Arimitsu, T., Brooks, R.A. et al, 1979.

Computed Tomography Profiles of Periventricular Hypodensity in Hydrocephalus and

Leukoencephalopathy.

Radiology, 130: 661 - 666.

36. Mitchell, A.W., Zyskowski, A.L., 1985.

Computed Tomography: A Graph Plotting Program.

Radiology Departmental Report.

37. Cardew, A.P., et al., 1980.

Dosimetry of C.T. Scanners.

Australasian Radiology, 24: 182 - 191.

38. Mathews, I.P., Rogers, K., 1984.

Malignancies Induced by Low Dose lonizing Radiation: Consequences for Diagnostic Radiology.

Radiography, 50: $277-280$. 
39. Brooks, R.A., Di Chiro, G., 1976.

Statistical Limitations in X-ray Reconstructive Tomography.

Medical Physics, 3: $237-240$.

40. Southon, F.C., 1981.

C.T. Scanner Comparison.

Medical Physics, 8: $62-75$.

41. Cohen, G., 1979

Contrast-Detail-Dose Analysis of Six Different Computed Tomographic Scanners.

J. Comput. Assist. Tomogr., 3: $197-203$.

42. Goodenough, D.J., Rossman, K., Lusted, L.B., 1974.

Radiographic Applications of Receiver Operating Characteristic Curves.

Radiology, 110: $89-95$.

43. Goodenough, D.J., Rossman, K., Lusted, L.B., 1974.

Factors Affecting the Detectability of a Simulated Radiographic Signal.

Invest. Radiol., 8: 339 - 344.

44. Judy, P.F., 1976.

The Line Spread Function and Modulation Transfer Function of a Computed Tomographic Scanner.

Medical Physics, 3: $233-236$.

45. Wagner, R.F., Brown, D.G., Pastel, M.S., 1979.

Application of Information Theory to the Assessment of Computed Tomography.

Medical Physics, 6: $83-94$.

46. Metz, C.E., Doi, K., 1979.

Transfer Function Analysis of Radiographic Imaging Systems.

Phys. Med. Biol., 24: $1079-1106$.

47. Sorenson, J.A., 1979.

Technique for Evaluating Radiation Beam and Image Slice Parameters of CT Scanners.

Medical Physics, 6(1): $68-69$.

48. Brooks, R.A., Di Chiro, G., 1977.

Slice Geometry in Computer Assisted Tomography.

J Comput Assist Tomogr 1(2): 191 - 199.

49. Hubbell, J.H., 1969.

Photon Cross Sections, Attenuarion Coefficients and Energy Absorption Coefficients from $10 \mathrm{keV}$ to $100 \mathrm{GeV}$.

National Standard Reference Data Series,

N.B.S. - 29, U.S. Government Printing Office.

50. Jackson, D.E., Hawkes, D.J., 1981.

$X$-ray Attenuarion Coefficients of Elements and Mixtures.

Physics Reports, 70, $169-233$

51. Birch, R., Marshall, M., Ardran, G.M., 1979.

Catalogue of Spectral Dara for Diagnostic X-rays.

Hospital Physicists' Aseociation (U.K.).

52. Cho, Z.H., Tsai, C.M., Wilson, G., 1975.

Study of contrast and modularion mechanisms in X-ray/photon transverse axial tomography.

Phys. Med. Biol., 20(6): 879 - 889.

53. Scofield, J.H., 1973.

Theoretical Photionization Crass Sections from 1 to $1500 \mathrm{keV}$.

Lawrence Livermore Laboratory Report, U.C.R.L. 51326.

54. Hubbell, J.H., Veigele, W.J., Briggs, E.A. et al, 1975.

Atomic Form Factors, Incoherent Scattering Functions, and Photon Scattering Cross Sections.

J. Phys. Chem. Ref. Datn, 4: 471-538.

55. Hubbell, J.H., Overbo, I., 1979.

Relativistic Atomic Form Facrors and Photon Coherent Scattering Cross Sections.

J. Phys. Chem. Ref. Data, 8: $69-105$.

56. McMaster, W.H., Kerr del Grande, N. et al, 1969.

Compilation of X-ray Crass Sections.

University of California Radiation Laboratory,

Report UCRL - 50174, Sec. II, Rev. 1.

57. Veigele, W.J., 1973.

Photon Cross Sections from $0.1 \mathrm{keV}$ to $1 \mathrm{MeV}$ for Elements $Z=1$ to $Z=94$.

Atomic Data Tables, 5: $51-111$.

58. Hubbell, J.H., 1982.

Photon Mass Attenuation and Energy-Absorption Coefficients from $1 \mathrm{keV}$ to $20 \mathrm{MeV}$.

Intl. J. Appl. Rad. Isot., 33: 1269 - 1290. 
59. Hubbell, J.H., 1977.

Photon Mass Attenuation and Mass Energy-Absorption Coefficients for $H, C, N, O, A r$, and Seven Mixtures from $0.1 \mathrm{keV}$ to $20 \mathrm{MeV}$.

Radiation Research, 70: 58 - 81

60. Millner, M.R., Payne, W.H., Waggener, R.G. et al, 1978.

Determination of Effective Energies in C.T. Calibration.

Medical Physics, 5: 543 - 545.

61. Nonweiler, T.R.F., 1984.

Computational Mathematics: An Introduction to Numerical Approximation.

Ellis Horwood Ltd.

62. Bevington, P.R., 1969

Data Reduction and Error Analysis for the Physical Sciences.

McGraw-Hill Book Company.

63. White, D.R., Speller, R.D., 1980.

The Measurement of Effective Photon Energy and 'Linearity' in Computerized Tomography.

British Journal of Radiology, 53: 5 - 11 .

64. Weber, J., van den Berge, D.J., 1969

The Effective Atomic Number and the Calculation of the Composition of Phantom Materials. British Journal of Radiology, 42: $378-383$.

65. Henson, P.W., 1983.

Attenuation Coefficient and Atomic Number Calculation Involving Elements Between Hydrogen and

Zinc in the C.T. Scanner Energy Range of 50 to $100 \mathrm{keV}$.

Aust. Phys. Eng. Sci. in Med., 6: $20-25$.

66. Mitchell, A.W., Johnson, P.B., 1989.

Methods for the computation of attenuation coefficient data in the diagnostic

energy range.

presented to the Engineering \& Physical Sciences in Medicine conference,

Hamilton.

67. Eisberg, R.M., 1961

Fundamentals of Modern Physics.

John Wiley \& Sons, Inc.

68. Hawkes, D.J., Jackson, D.F., 1980.

An Accurate Parametrisation of the X-ray Attenuation Coefficient.

Phys. Med. Biol., 25: $1167-1171$.

69. Birch, R., Marshall, M., 1979.

Computation of Bremsstrahlung X-ray Spectra and Comparison with Spectra Measured with a Ge(Li)

Detector.

Phys. Med. Biol., 24: 505 - 517.

70. Le Heron, J.C., 1985.

Measurement of X-ray Spectra Using a High Purity Germanium Detector System.

National Radiation Laboratory Report 1985/4.

71. Archer, B.R., 1982.

A Laplace Transform Pair Model for Spectral Reconstruction.

Med. Phys., 9: $844-847$.

72. Rubio, M., Mainardi, R.T., 1984.

Determination of X-ray Spectra Including Characteristic Line Intensities from Atsenwarion Data.

Phys. Med. Biol., 29: 1371 - 1376.

73. Meredith, W.J., Massey, J.B., 1977.

Fundamental Physics of Radiology.

Bristol: John Wright \& Sons Ltd.

74. Phillips, G.M., Taylor, P.J., 1973.

Theory and Applications of Numerical Analysis.

Academic Press.

75. Green, M., Cosslett, V.E., 1968.

Measurements of $K, L$ and $M$ Shell X-ray Production Efficiencies.

Brit. J. Appl. Phys., 1: 425 - 436.

76. G.E. Technical Support Staff.

Maxiray 100-CT Tube Unit.

Service Manual SM D1180A/B, General Electric, Milwaukee.

77. Hill, D.R. (Editor), 1975.

Principles of Diagnostic X-ray Apparatus.

Philips Technical Library. 
78. Henshaw, E.T. (Editor), 1976.

The Physics of Diagnosis.

Hospital Physicists Association, London.

79. Levi, C., Gray, J.E., McCullough, E.C. et al, 1982.

The Unreliability of C.T. Numbers as Absolute Values.

A.J.R., 139: $443-447$.

80. Zatz, L.M., 1976.

The Effect of the kVp Level on E.M.I. Values.

Radiology, 119: 683 - 688 .

81. Zatz, L.M., Alvarez, R.E., 1977.

An Inaccuracy in Computed Tomography: The Energy Dependence of C.T. Values.

Radiology 124, $91-97$.

82. Lange, S., Grumme, T., Kluge, W., Ringel, K., Meese, W., 1989.

Cerebral and Spinal Computerised Tomography.

Schering AG., Berlin.

83. Reich, N.E., Siedelmann, F.E., Tubbs, R.R. et al, 1976

Determination of Bone Mineral Content Using C.T. Scanning.

Am. J. Roentgenol., 127: 593 - 594.

84. Genant, H.K., Boyd, D., 1977.

Quantitative Bone Mineral Analysis Using Dual Energy Computed Tomography.

Invest. Radiol., 12: 545 - 551 .

85. Rutherford, R.A., Pullan, B.R., Isherwood, I., 1976.

Measurement of Effective Atomic Number and Electron Density Using an E.M.I. Scanner.

Neuroradiology, 11: $15-21$.

86. Rutherford, R.A., Pullan, B.R., Isherwood, I., 1976.

X-ray Energies for Effective Atomic Number Determination.

Neuroradiology, 11: $23-28$.

87. Drost, D.J., Fenster, A., 1980.

Experimental Dual Xenon Detectors for Quantitative C.T. and Spectral Artefact Correction. Med. Phys., 7: $101-107$.

88. Brooks, R.A., Di Chiro, G., 1978.

Split-Detector Computed Tomography: A Preliminary Report.

Radiology, 126: 255 - 257.

89. Ritchings, R.T., Pullan, B.R., 1979.

A Technique for Simultaneous Dual Energy Scanning.

J. Comput. Assist. Tomogr., 3: 842 - 846 .

90. Rutt, B., Fenster, A., 1980.

Split-Filter Computed Tomography: A Simple Technique for Dual Energy Scanning.

J. Comput. Assist. Tomogr., 4: 501 - 509.

91. Kalender, W.A., 1984.

Dual Energy Methods for SOMATOM DR.

Siemens Technical Publication.

92. Hay, G.A., 1982.

Traditional X-ray Imaging, chapter 1 in

Scientific Basis of Medical Imaging

Edited by P.N.T. Wells.

Churchill Livingstone, Edinburgh.

93. Latchaw, R.E., Payne, J.T., Gold, L.H.A., 1978.

Effective Atomic Number and Electron Density as Measured with a Computed Tomography Scanner:

Computation and Correlation with Brain Tumour Histology.

J. Comput. Assist. Tomogr., 2: 199 - 208.

94. Di Chiro, G., Brooks, R.A., Kessler, R.M. et al, 1979.

Tissue Signatures with Dual-Energy Computed Tomography.

Radiology, 131: 521 - 523 .

95. Brooks, R.A., 1977.

A Quantitative Theory of the Hounsfield Unit and its Application to Dual Energy Scanning.

J. Comput. Assist. Tomogr., 1: 487 - 493.

96. Alvarez, R.E., Macovski, A., 1976.

Energy-Selective Reconstructions in X-ray Computerized Tomography.

Phys. Med. Biol., 21: $733-744$.

97. Avrin, D.E., Macovski, A., Zatz, L.M., 1978.

Clinical Application of Compton and Photo-Electric Reconstruction in Computed Tomography.

Invest. Radiol., 13: $217-222$ 
98. Alvarez, R., Seppi, E., 1979.

A Comparison of Noise and Dose in Conventional and Energy Selective Computed Tomography.

I.E.E.E. Trans. Nucl. Sci., 26: 2853 - 2856.

99. Marshall, W.H., Alvarez, R.E., Macovski, A., 1981.

Initial Results with Prereconstruction Dual-Energy Computed Tomography (PREDECT).

Radiology, 140: 421 - 430.

100. Christ, G., 1984.

Exact Treatment of the Dual-Energy Method in C.T. Using Polyenergetic X-ray Spectra.

Phys. Med. Biol., 29: 1501 - 1510.

101. Mitchell, A.W., Harper, G.D.J., 1984.

Dual Energy Computed Tomography: A Progress Report.

Presented to the annual conference of the N.Z. branch of the Australasian College of Physical

Scientists in Medicine.

102. Hawkes, D.J., Jackson, D.F., Parker, R.P., 1986.

Tissue Analysis by Dual-Energy Computed Tomopgraphy.

British J. Radiology, 59: $537-542$.

103. Talbert, A.J., Brooks, R.A., Morgenthaler, D.G., 1980.

Optimum Energies for Dual-Energy Computed Tomography.

Phys. Med. Biol., 25: 261 - 269.

104. Gore, J.C., McCullough, E.C., 1980.

Signal Variance in Computed Tomography: The $\sqrt{ } N$ Syndrome.

Phiys. Med. Biol., 25: $153-155$.

105. Le Heron, J.C., 1982.

Transfer functions and Noise in Diagnostic Radiological Imaging - Basic Principles and

Applications.

N.R.L. Report 1982/1.

106. Coltman, J.W., 1954.

The Specification of Imaging Properties by Response to a Sine Wave Input.

J. Opt. Soc. Am., 44: 468 - 472.

107. Droege, R.T., Morin, R.L., 1982.

A Practical Method to Measure the M.T.F. of C.T. Scanners.

Med. Phys., 9: $758-760$.

108. Poletti, J.L., 1985.

Performance Assessment of C.T. X-ray Scanners.

N.R.L. Report 1985/8.

109. Judy, P.F., Balter, S., Bassano, D. et al, 1977.

Phantoms for Performance Evaluation and Quality Assurance of C.T. Scanners.

A.A.P.M. Report No. 1.

110. Southon, F.C., 1981.

C.T. Scanner Comparison.

Medical Physies, 8: 62 - 75.

111. Delahunt, J.W., 1982.

Disorders of Calcium.

Teaching notes, Department of Medicine,

Wellington Clinical School of Medicine.

112. Avioli, L.V., 1977.

Osteoporosis: Pathogenesis and Therapy, chapter 6 in

Metabolic Bone Disease, edited by Avioli, L.V.

Volume 1, pp $307-369$.

113. Genant, H.K., 1986.

Osteoporosis: Current Assessment, chapter in

Diagnostic Radiology, edited by A.R. Margulis and C.A. Gooding.

C.V. Mosby.

114. Lachman, E., 1955 .

Osteoporosis: The Potentialities and Limitations of its Roentgenologic Diagnosis.

Radiology, 74: 712 - 715 .

115. Riggs, B.L., Wahner, H.W., Dunn, W.L. et al., 1981.

Differential Changes in Bone Mineral Density of the Appendicular and Axial Skeleton with Aging.

J. Clin. Invest., 67: 328 - 335.

116. Dunnill, M.S., Anderson, J.A., Whitehead, R., 1967.

Quantitative Histological Studies on Age Changes in Bone.

J. Path. Bact., 94: $275-291$. 
117. Snyder, W.S., Cook, M.J., Karhausen, L.R. et al. 1974.

Report of the Task Group on Reference Man. (I.C.R.P. Report 23).

Pergamon Press, Oxford.

118. Genant, H.K., Block, J.E., Steiger, P., Glueer, C.C. et al, 1989.

Appropriate use of bone densitometry.

Radiology, 170: $817-822$

119. Andresen, J., Nielsen, H.E., 1986.

Assessment of bone mineral content and bone mass by non-invasive radiologic means.

Acta Radiologica Diagnosis, 27: 609 - 61

120. Cann, C.E., 1988.

Quantitative CT for determination of bone mineral density: a review.

Radiology, 166: $509-522$.

121. Mazess, R.B., Barden, H., Vetter, J., Ettinger, M., 1989.

Advances in noninvasive bone measurement.

Ann. Biomed. Eng., 17(2): 177 - 181.

122. Tothill, P., 1989.

Methods of bone mineral measurement.

Phys. Med. Biol., 34(5): 543 - 572.

123. Sartoris, D.J., Resnick, D., 1990.

Current and innovative methods for noninvasive bone densitometry.

Radiologic Clinics N. Am., 28(2): 257 - 278.

124. Singh, M., Nagrath, A.R., Maini, P.S., 1970.

Changes in Trabecular Pattern of the Upper End of the Femur as an Index of Osteoporosis.

J. Bone Joint Surg., 52: 457 - 467.

125. Singh, M., Riggs, B.L., Beabout, J.W., Jowsey, J., 1972.

Femoral trabecular-pattern index for evaluation of spinal osteoporosis.

Ann. Intern. Med., 77: 63 - 57.

126. Poznanski, A.K., 1974.

The hand in radiologic diagnosis.

W.B. Saunders Company, Philadelphia.

127. Horsman, A., 1976.

Measurements of the Skeleton in vivo, chapter in

Calcium Phosphate and Magnesium Metabolism. Clinical Physiology and Diagnostic Procedures.

Edited by B.E.C. Nordin.

Churchill Livingstone, Edinburgh.

128. Colbert, C., Bachtell, R.S., 1981.

Radiographic absorptiometry (photodensitometry). in

Noninvasive measurements of bone mass and their clinical application. Edited by S.H. Cohn. CRC Press, Boca Raton, Florida.

129. Sorenson, J.A., Cameron, J.R., 1967.

A Reliable in Vivo Measurement of Bone Mineral Content.

J. Bone Joint Surg., 49: 481.

130. Mazess, R.B., Barden, H.S., Ettinger, M., 1988.

Radial and spinal bone mineral density in a patient population.

Arthritis Rheum., 31(7): 891 - 897.

131. McDonald, S.P., Cormack, J., Evill, C.A., Sage, M.R., 1990.

Factors affecting the precision of bone mineral measurements.

Part 1: Review of experimentally derived results obtained from single photon absorptiometry.

Aust. Phys. Eng. Sci. Med., 13(1): $18-24$.

132. Sartoris, D.J., Resnick, D., 1989.

Dual-Energy Radiographic Absorptiometry for Bone Densitometry: Current Status and Perspective.

AJR, 152(2): $241-246$.

133. Greenfield, M.A., Craven, J.D., Shukla, S.S., 1989.

Bone Mineral Measurement Using the Ratio of Coherent to Compton Scattered Photons in the Calcaneus. Chapter 14 in Osteoporosis and Bone Mineral Measurement. Edited by Ring et al. IPSM, York.

134. Beddoe, A.H., Zuidmeer, H., Hill, G.L., 1984.

A Prompt Gamma in vivo Neutron Activation Analysis Facility for Measurement of Total Body

Nitrogen in the Critically Ill.

Phys. Med. Biol. 29(4): 371 - 383. 
135. Ryde, S.J.S., Morgan, W.D., Cobbold, S., Sivyer, A., Dutton, 1989.

Measurement of Total Body Calcium By Prompt Gamma Neutron Activation Analysis: Instrument

Performance and Preliminary Clinical Experience. Chapter 13 in

Osteoporosis \& Bone Mineral Measurement. Edited by Ring et al.

IPSM, York.

136. Evans, W.D., 1989.

Broadband Ultrasonic Attenuation of the Os-calcis. Chapter 16 in

Osteoporosis and Bone Mineral Measurement. edited by E.F.J. Ring et al.

IPSM, York.

137. Ruegsegger, P., Elsasser, U. et al., 1976.

Quantification of Bone Mineralization Using Computed Tomography.

Radiology, 121: 93 - 97.

138. Reich, N.E., Seidelmann, F.E. et al., 1976.

Determination of Bone Mineral Content Using C.T. Scanning.

Am. J. Roentgenol., 127: 593 - 594.

139. Posner, I., Griffiths, H.J., 1977.

Comparison of C.T. Scanning with Photon Absorptiometric Measurement of Bone Mineral Content in the Appendicular Skeleton.

Invest. Radiol., 12: 542 - 544.

140. Genant, H.K., Boyd, D., 1977.

Quantitative Bone Mineral Analysis Using Dual Energy Computed Tomography.

Invest. Radiol., 12: 545 - 551 .

141. Weissberger, M.A., Zamenhof, R.G., Aronow, S., Neer, R.M., 1978.

Computed Tomography Scanning for the Measurement of Bone Mineral in the Human Spine.

J. Comput. Assist. Tomogr., 2: 253 - 262.

142. Genant, H.K., Boyd, D. et al., 1981.

Computed Tomography, chapter in

Non-invasive Measurements of Bone Mass and their Clinical Application, edited by S.H. Cohn.

C.R.C. Press, Boca Raton, Florida.

143. Genant, H.K., Turski, P.A., Moss, A.A., 1983

Advances in C.T. Assessment of Metabolic \& Endocrine Disorders.

Adv. Int. Med., 28: $409-447$.

144. Laval-Jeantet A.M., Cann, C.E., Roger, B. et al., 1984

A Postprocessing Dual Energy Technique for Vertebral C.T. Densitometry.

J. Comput. Assist. Tomogr., 8: 1164 - 1167.

145. Rohloff, R., Hitzler, H., Arndt, W., Frey, K.W., Lissner, J., 1982.

Influence of fat content of bone marrow on bone mineral measurements by $C T$ and photonabsorptiometry in trabecular bones.

J. Comput. Assist. Tomogr., 6: 212 - 213.

146. Vetter, J.R., Permun, W.H., Kalender, W.A., Mazess, R.B., Holden, J.E., 1986

Evaluation of a protorype dual-energy computed tomographic apparatus.

II. Determination of venebral bone mineral content.

Med. Phys., 13(3): $340-343$.

147. Laval-Jeantet, A., Roger, B., Bouysse, S., Bergot, C., Mazess, R.B., 1986.

Influence of vertebral or comens on quantitative C.T. density.

Radiology 159: 463 - 466.

148. Laval-Jeantet, A.M., Roger, B., de Vernejoul, M.C., Laval-Jeantet, M., 1985.

Testing of a dual-energy partprocessing method of QCT densitometry. (abstract)

J. Comput. Assist. Tomos., $9(3): 616-617$.

149. Rosenthal, D.I., Hayee, C.W., Rosen, B., Mayo-Smith, W., Goodsitt, M.M., 1989

Fatty replacement of spinal bone marrow due to radiation:

Demonstration by dual energy quantitative CT and MR imaging.

J. Comput. Assist. Tomogr., 13(3): 463 - 465.

150. Mayo-smith, W., Rosentha, D.I., Goodsitt, M.M., Klibanski, A., 1989.

Intravertebral Fat Measurement with Quantitative CT in Patients with Cushing Disease and Anorexia Nervosa.

Radiology, 170: 835 - 838 .

151. Rosenthal, D.I., Mayo-Smith, W., Goodsitt, M.M., Doppelt, S., Mankin, H.J., 1989. Bone and bone marrow changes in Gaucher disease: evaluation with quantitative CT. Radiology, 170: 143 - 146.

152. Scalzetti, E.M., Bassano, D.A., 1986.

Bone mineral measurement in the human spine using computed tomography.

Invest. Radiol., 21: 858 - 863 . 
153. Burgess, A.E., Colborne, B., Zoffmann, E., 1987

Vertebral trabecular bone: comparison of single and dual-energy CT measurements with chemical analysis.

J. Comput. Assist. Tomogr., 11(3): 505 - 515.

154. Mazess, R.B., 1984.

Errors in Measuring Trabecular Bone by Computed Tomography Due to Marrow and Bone Composition.

Calcif. Tissue Int., 35: $148-152$.

155. Gluer, C., Reiser, U.J., Davis, C.A., Rutt, B.K., Genant, H.K., 1988.

Vertebral mineral determination by quantitative computed tomography (QCT): Accuracy of single and dual energy measurements.

J. Comput. Assist. Tomogr., 12(2): 242 - 258.

156. Goodsitt, M.M., Rosenthal, D.I., Reinus, W.R., Coumas, J., 1987.

Two postprocessing CT techniques for determining the composition of trabecular bone.

Invest. Radiol., 22: 209-215.

157. Kalender, W.A., Perman, W.H., Vetter, J.R., Klotz, E., 1986.

Evaluation of a prototype dual-energy computed tomographic apparatus. I. Phantom studies.

Med. Phys., 13(3): 334 - 339.

158. Rao, G., Yaghmai, I., Wist, A.O., Arora, G., 1987.

Systematic errors in bone-mineral measurements by quantitative computed tomography.

Med. Phys., 14(1): 62 - 69.

159. Nickoloff, E.L., Feldman, F., Atherton, J.V., 1988.

Bone Mineral Assessment: New Dual Energy Approach.

Radiology, 168: 223 - 228.

160. Van Kuijk, C., Grashuis, J.L., Steenbeek, J.C.M., Schutte, H.E., Trouerbach, W.T., 1990. Evaluation of Postprocessing Dual-Energy Methods in Quantitative Computed Tomography. Investigative Radiology, 25(8): 876 - 889.

161. Genant, H.K., Cann, C.E., et al., 1983.

Vertebral Mineral Determination by Quantitative C.T. Clinical Feasibility and Normative Data. J. Comput. Assist. Tomogr., 7: 554.

162. Genant, H.K., Turski, P.A., Moss, A.A., 1983.

Advances in C.T. Assessment of Metabolic and Endocrine Disorders.

Adv. Intern. Med., 28: $409-447$.

163. Genant, H.K., Cann, C.E., Ettinger, B. et al., 1982.

Quantitative Computed Tomography of Vertebral Spongiosa: a Sensitive Method for Detecting

Early Bone Loss After Oophorectomy.

Ann. Intern. Med., 97: 699-705.

164. Firooznia, H., Rafii, M., Golimbu, C., et al., 1986.

Trabecular Mineral Content of the Spine in Women with Hip Fracture: C.T. Measurement.

Radiology, 159: $737-740$.

165. Cann, C.E., 1981.

Low Dose C.T. Scanning for Quantitative Spinal Mineral Analysis.

Radiology, 140: $813-815$.

166. Mahaworasilpa, T., Southon, F.C.G., 1986.

C.T. Vertebral Bone Mineral Measurement - Intrinsic Errors of Single kV.

Aust. Phys. Eng. Sci. Med., 9: 23 - 28.

167. General Electric Technical Support Staff.

CT/T 8800 Service Manual: Chapter four: Theory of Operation.

General Electric.

168. Cann, C.E., Genant, H.K., 1980

Precise Measurement of Vertebral Mineral Content Using Computed Tomography.

J. Comput. Assist. Tomogr., 4: 493 - 500.

169. Odvina, C.V., Wergedal, J.E., Libanati, C.R., Schulz, E.E., Baylink, D.J., 1988.

Relationship Between Trabecular Vertebral Body Density and Fractures:

A Quantitative Definition of Spinal Osteoporosis.

Metabolism, 37(3): $221-228$.

170. McBroom, R.J., Hayes, W.C., Edwards, W.T., Goldberg, R.P., White, A.A., 1985.

Prediction of Vertebral Body Compressive Fracture Using Quantitative Computed Tomography.

J. Bone and Joint Surg., 67A(8): 1206 - 1214.

171. Jones, C.D., Laval-Jeantet, A-M., Laval-Jeantet, M.H., Genant, H.K., 1987.

Importance of Measurement of Spongious Vertebral Bone Mineral Density in the Assessment of

Osteoporosis.

Bone, 8: 201 - 206. 
172. Farrell, T.J., Webber, C.E., 1989.

The error Due to Fat Inhomogeneity in Lumbar Spine Bone Mineral Measurements.

Clin. Phys. Physiolo. Meas., 10(1): 57 - 64.

173. Valkema, R

Single and Dual-Gamma Photon Absorptiometry: Relative Merits. (abstract)

Brit. J. Radiol., 63: $370-372$.

174. Sartoris, D.J., Resnick, D., 1989.

Dual-Energy Radiographic Absorptiometry for Bone Densitometry: Current Status and Perspective. AJR, 152: $241-246$.

175. Sowby, F.D. (editor).

Recommendations of the International Commission on Radiological Protection: Publication 26. Pergamon Press, Oxford.

176. Pye, D.W., Hannan, W.J., Hesp, R., 1990.

Effective Dose Equivalent in Dual X-ray Absorptiometry. (letter)

Brit. J. Radiol., 63: 149.

177. Poletti, J.L., 1985.

Performance Assessment of the Wellington Hospital GE 8800 CT X-ray scanner.

National Radiation Laboratory, Christchurch.

178. Wall, B.F., Harrison, R.M., Spiers, F.W., 1988.

Patient Dosimetry Techniques in Diagnostic'Radiology.

I.P.S.M., York.

179. Kathren, R.L., 1985.

Radiation Protection.

Adam Hilger, Bristol.

180. Battista, J.J., Bronskill, M.J., 1981.

Compton Scatter Imaging of Transverse Sections.

Phys. Med. Biol., 26(1): 81 - 99.

181. McCullough, E.C., Holmes, T.W., 1985.

Acceptance Testing Computerized Radiation Therapy Treatment Planning Systems: Direct Utilization of C.T. Scan Data.

Med. Phys., 12(2): $237-242$.

182. Huizenga, H., Storchi, P.R.M., 1985.

The Use of Computed Tomography Numbers in Dose Calculations for Radiation Therapy.

Acta Radiologica Oncol., 24: 509 - 519.

183. White, D.R., Martin, R.J., Darlison, R., 1977.

Epoxy Resin Based Tissue Substitutes.

Brit. J. Radiol., 50: $814-821$.

184. Genant, H.K., Turski, P.A., Moss, A.A., 1983.

Advances in CT Assessment of Metabolic and Endocrine Disorders.

Adv. Intern. Med., 28: 409 - 447.

185. Genant, H.K., Cann, C.E., Pozzi-Mucelli, R.S., Kantner, A.S., 1983.

Vertebral Mineral Determination by QCT: Clinical Feasibility and Normative Data.

J. Comput. Assist. Tomogr., 7: 554.

186. Firooznia, H., Rafii, M., Golimbu, C., Schwartz, M.S., Ort, P., 1986

Trabecular mineral content of the spine in women with hip fracture: CT measurement.

Radiology, 159: 737 - 740 .

187. Marel, G.M., 1987.

Letter to Assoc Prof A. Hall.

188. Compston, J.E., Evans, W.D., Crawley, E.O., Evans, C., 1988.

Bone mineral content in normal UK subjects.

Brit. J. Radiology, 61: 631 - 636 .

189. Hay, P.J., Hall, A., Delahunt, J., Harper, G., Mitchell, A., Salmond, C., 1989

Investigation of osteopaenia in anorexia nervosa.

Austral. \& N.Z. J. Psych., 23: 261 - 268.

190. Smith, R.R.L., Spivak, J.L., 1985.

Marrow cell necrosis in anorexia nervosa and involuntary starvation.

Brit. J. Haematology, 60: 525 - 530 .

191. Mant, M.J., Faragher, B.S., 1972.

The haematology of anorexia nervosa.

J. Haematology, 23: 737 - 749. 
192. Jones, K.P., Ravnikar, V.A., Tulchinsky, D., Schiff, I., 1985.

Comparison of Bone Density in Amenorrheic Women due to Athletics, Weight Loss and Premature Menopause.

Obstetrics and Gynaecology, 66: 5 - 8.

193. Morgan, H.G., Russell, G.F.M., 1975.

Value of Family Background and Clinical Features as Predictors of Long-Term Outcome in Anorexia Nervosa: Four Year Follow-up Study of 41 Patients. Psychological Medicine, 5: 355 - 371.

194. Treasure, J.L., Russell, G.F., Fogelman, I., Murby, B., 1987.

Reversible bone loss in anorexia nervosa.

Br. Med. J., 295: 474 - 475.

195. Singh, I., 1978.

The architecture of cancellous bone.

J. Anat., 127: 305 - 310.

196. Parfitt, A.M., 1988.

Bone remodeling: Relationship to the amount and structure of bone, and the pathogenesis and prevention of fractures. In:

Osteoporosis: Etiology, Diagnosis and Management. Ed Riggs, B.L.

Raven Press, New York.

197. Parfitt, A.M., Drezner, M.K., Glorieux, F.H., Kanis, J.A., et al, 1987.

Bone histomorphometry: standardization of nomenclature, symbols and units.

J. Bone Min. Res., 2(6): 595 - 610 .

198. Parfitt, A.M., 1984.

The cellular basis of bone remodeling. The quantum concept re-examined in light of recent advances in cell biology of bone.

Calcif. Tissue Int., 36(Suppl): S37 - S45.

199. Golder, F.J., 1990.

A Scanning Electron Microscope and Computed Tomography Study of Sheep Vertebral Trabecular Bone.

B.Phil. thesis, Department of Physiology \& Anatomy, Massey University.

200. Gray, H., 1977.

Anatomy, descriptive and Surgical.

American Edition, Bounty Books,

New York.

201. Ratcliffe, J.F., 1981.

The Arterial Anatomy of the Immature Vertebra.

J. Anat., 133(4): 630 .

202. Knopp, T.C., 1985

Quantitative Analysis of Computed Tomographic Images.

M.Sc. thesis, Department of Physics, University of Otago.

204. Beddoe, A.H., 1978.

A quantitative study of trabecular bone in man, rhesus monkey, beagle and miniature pig.

Calcif. Tiss. Res., 25: $273-281$.

205. Whitehouse, W.J., Dyson, E.D., Jackson, C.K., 1971.

The scanning electron microscope in studies of trabecular bone from a human vertebral body.

J. Anat., 108(3): $481-496$.

206. I.C.R.P.

Protection of the Patient in Diagnostic Radiology. Report 34.

Pergamon Press, Oxford. 